
A.

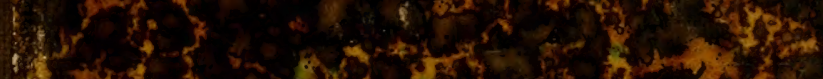
W

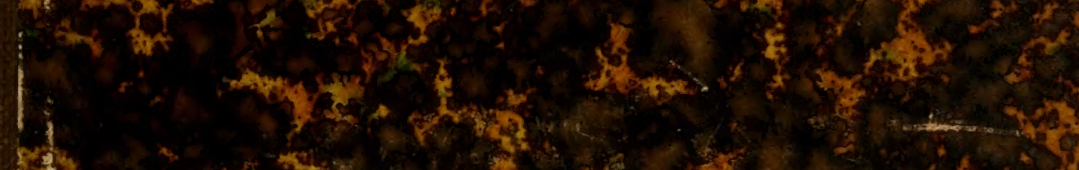

(3)

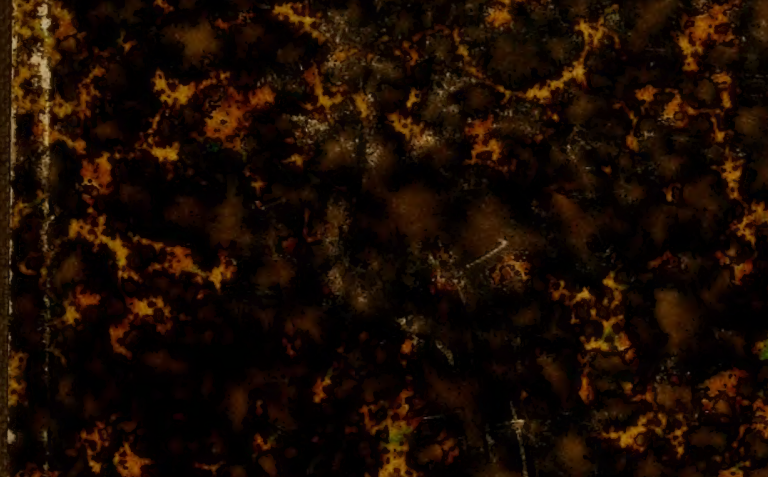
$2+\frac{1}{3}$

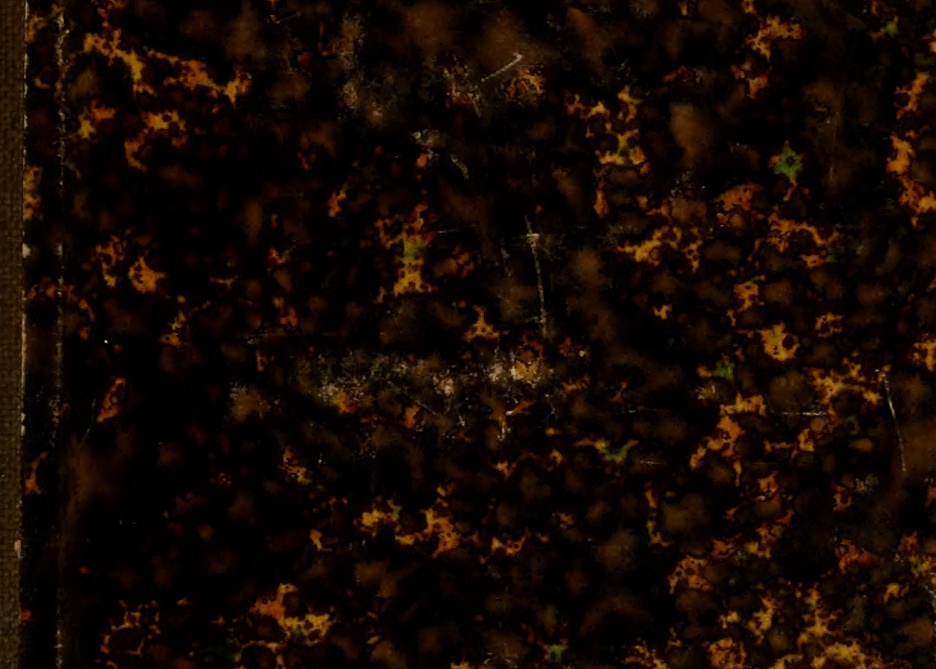

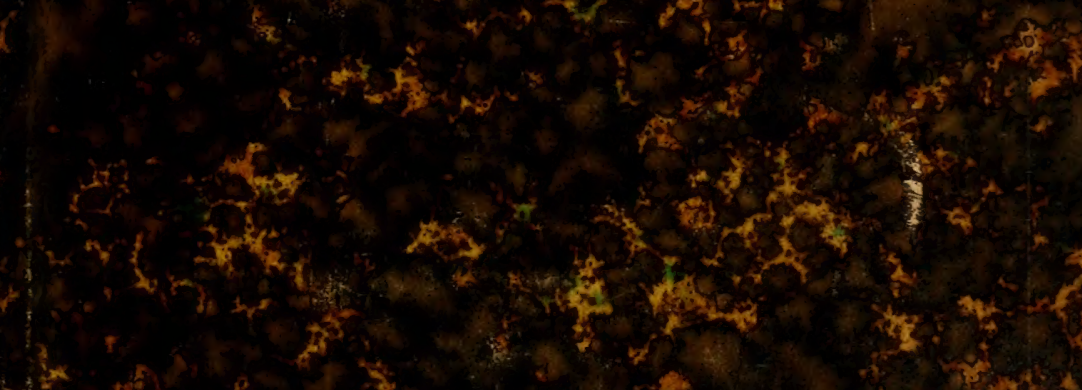

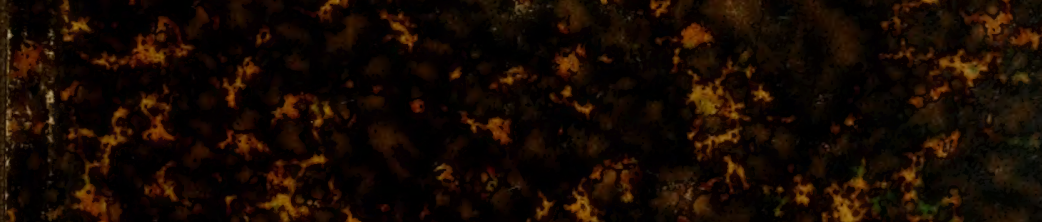
$\frac{1}{3}+x+x^{2}+x^{2}$

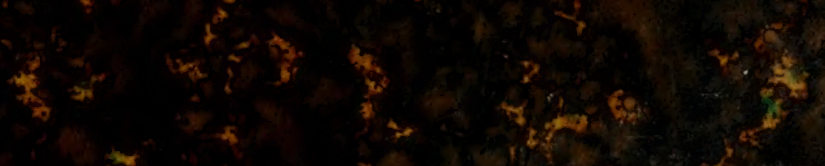

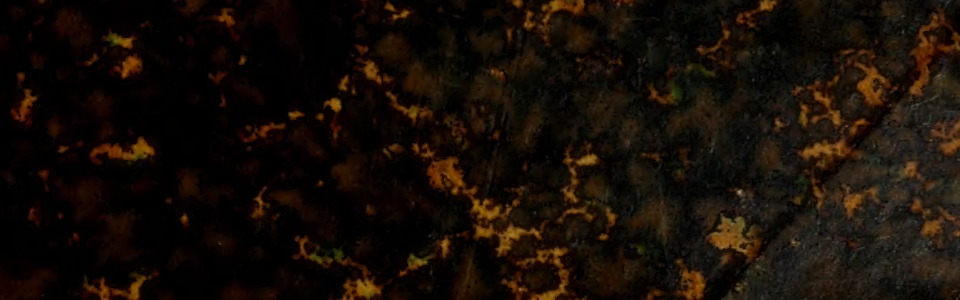

$$
\begin{aligned}
& \text { wh } 2
\end{aligned}
$$




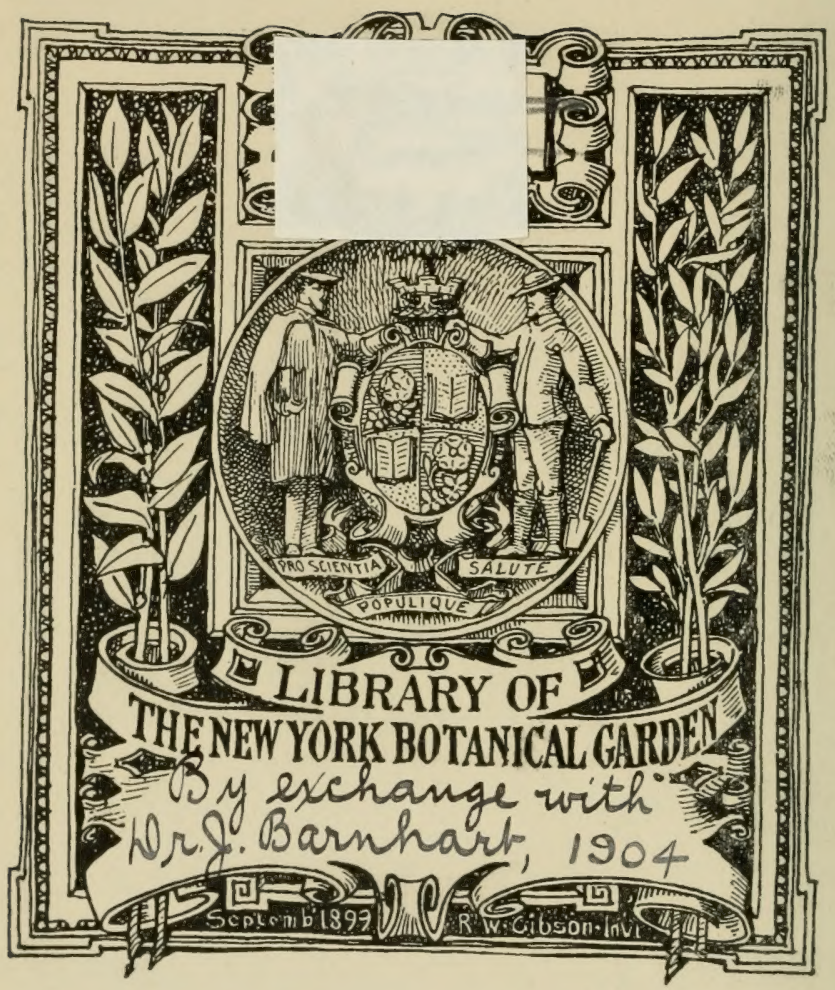






ROYAUME DES PAYS-BAS.

\title{
BULLETIN
}

2

DU

\section{CONGRESS INTERNATIONAL}

\section{DE BOTANIQUE ET D'HORTICULTURE,}

\author{
REUNI A AMSTERDAM
}

les $7,8,10$ et 11 AVRIL 1865.

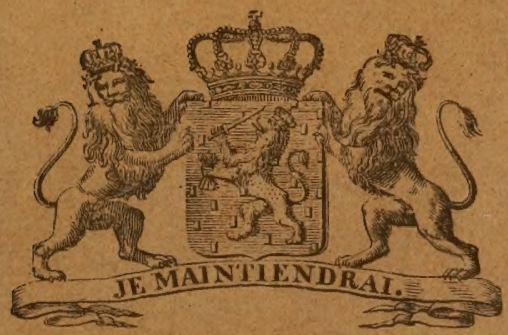

ROTTERDAM

Imprinerie de STEFANUS MOSTERT \& FILS

1866. 

CONGRĖS INTERNATIONAL DE BOTANIQUE ET D'HORTICULTURE. 



\section{BULLETIN}

DU

\section{CONGRES INTERNATIONAL}

\section{IE BOTANIQUE ET D'HORTICULTURE,}

RÉUNI À AMSTERDAM

les 7, 8, 10 et 11 AVRIL 1865,

EN COINCIDENTCE AVEC

L'EXPOSITION UNIVERSELLE D'HORTICULTURE,

ORGANISÉE PAR UNE COMMISSIOI IES DELTGLES DE LA PLUPART DES

SOCLETÉS D'HORTICULTURE ET D'AGRTCULTURE DES PAYS-BAS.

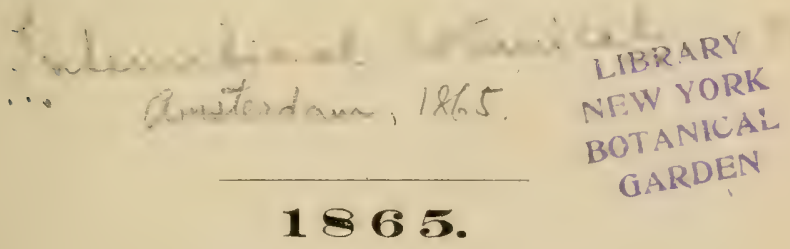

ROTTERDAM

Imprimerie de STEFANUS MOSTERT \& FILS.

1866. 
Les discussions n'ont pas été sténographiées. On at demandé des renseignements anx orateurs, qui presque tous ont cu l'oceasion de corriger eux-mémea les épreuves de leurs mémoires ou de leurs communications. La redaction et l'impression du Bulletin ont été dirigées par M. RavweNhoFr, secrétaire-général du Congrès. 


\section{CONGRES INTERNATIONAL}

\section{DE BOTANIQUE ET' D'HORTIOULIURE,}

convoqué

\section{à AMSTERDAM le 7 du mois d'Arril 1865 .} MN.

\section{Commission organisatrice du Congrès,}

C. A. J. A. OUDEMANS ...... prof. de botanique à l'A thénée Illustre d'An-sterdam, président.

N. W. P. RAUWENHOFF ... Dr. directeur du Jardin Botanique à Rotterdam, secrétaire.

F. A. W. MIQUEL prof. de bot. à l'Université d'Utrecht.

W. F. R. SURINGAR de Leide.

J. C. GROENEWEGEN ...... jardinier en chef du Jardin Bot. à Amsterdam.

J. H. KRELAGE ............. horticulteur it Harlem.

H. WITTE jardinier en chef du Jarlin Bot. à Leide.

Commission directrice du Congrès, nommée par l'assemhlée l.e 7 Avril 1865.

C. A. J. A. OUDEMANS..... prof. 'de botanique à l'Athénée Illastre d'Amsterdam, président.

N. J. ANDERSSON prof. de botanique, délégué du gouv. suède, à Stockholm....

DE CANNAR'T D'HAMALE, sénateur, président de la soc. royale d'horticulture de Malines, à Malines

1R. CASPARY prof. de botanique, directeur du Jardin Bot.

FÉE à Königsbery prof. de botanique au Jardin des Plantes, ì Strasbourg

E. FENZL . prof. de botanique et directeur du Jardin Botanique, à Vienne.

H. R. GÖPPERT prof. de botanique à l'université à Breslau...

K. $\mathrm{KOCH}$ prof. de bot., délégué de la soc. pour l'avancement de l'horticulture en Prusse, à Berlin.

H. LECOCQ prof. d'histoire naturelle, directeur du Jar- 
NiI.

MASTERS

Dr. délégué de la société royale d’horticulture, à South Kensington, Londres

C. F. MEISSNER prof. de botanique, directeur du Jardin Botanique, à Bâle

F. A. W. MIQUEL prof. de botanique à l'université d'Utrecht. .

E. MORREN prof. de botanique à l'université, directeur du Jardin Botanique, à Liège

L. PIRÉ prof. de l'athénée royal, délégué du gouv. Belge, à Birwelles

J. PASSERINI prof. de botanique et directeur du Jardin Botanique, ì Parme

E. REGEL Dr. direet, du Jardin Bot, ì St. Pétersbourg H. J. REICHENBACH fils ... prof. de botanique et directeur du Jardin

Botanique, à Hambourg. .

N. W. P. RAUWENHOFF ... Dr. directeur du Jardin Botanique de Rot. terdan, secrétaire.

W. F. R. SURINGAR ... ..... prof. de botanique à l'université de Leide.

J. C. GROENEWEGEN ...... jardinier en chef du Jardin Bot. d'Amsterdani. J. H. KRELAGE ............. horticulteur à Harlem.

H. WITTE jardinier en chef du Jardin Bot. de Leide.

\section{Section de botanique pure.}

\section{BUREAU:}

FÉE prof. de botanique à Strasbourg, Président.

FENZL

MIQUEL prof. de botanique à Vienne...

MORREN prof. de botanique à Utrecht... $\}$ Vice-Présidents.

OUDEMANS prof. de botanique à Liège.........
prof. de botanique ì Amsterdan. Secrétaires.

Section de botanique appliquée et d'horticulture.

\section{BUREAU:}

K. $\mathrm{KOCH}$.................... prof. de Botanique ì Berlin, Président.

DE CANNART D'HAMAJE, sénateur, à Malines NISSON ..................... directeur du Jardin d'Accli-/

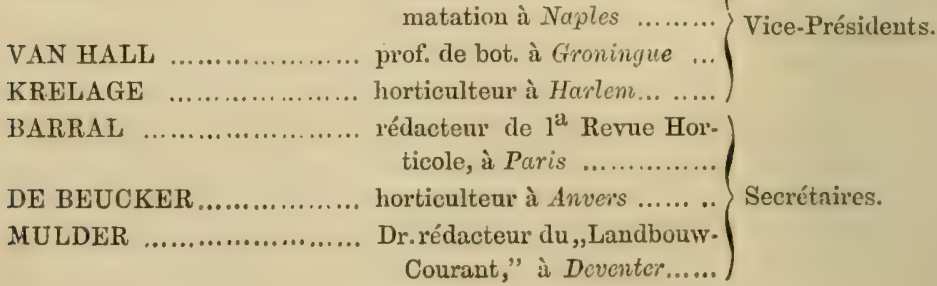




\section{MEMBRES DU CONGRES.}

MII. Pays-Bas.

AMERSFOORDT (Mr. J. 1'.), au Haarlemmermeer.

BACKER (Jhr. Mr. S.), secrétaire-adjoint de la commission dırectrice de l'Expos. Univers. d'Horticulture, à Amsterdam.

BAHLMAN (B.), à Amsterdam.

BACKER née DE WILDT (Madame), à Amsterdium.

BARGE (ROBERT), à Amsterdam.

BARNAART (Jhr. A. E.), à Vogeleusang (près d'Harlem).

BARNAART VAN ZANDVOORT (Jhr. H. G.), à Harlem.

BECKER (C.), ì Amsterdam.

BECKER (Madame J.), ì Amsterdam.

BEELAERTS VAN BLUKLAND (Jhr. Mr. H. A. A.), propriétaire, à Utrecht.

BEEN (MI.), horticulteur, à Rotterdam.

BEMLELEN (J. A. VAN), ì Harlem.

BEYMA (Jhr. O. S.), ì Werdam (Frise).

BLAAS (W.), jardinier de M. A. van Doom, à Koudekerke (Zélande).

BLOKHUIS (W.), ì Noordwyk.

BODDAERT (Jhr. Mr. J. PH.), propriétaire, à Domburg ('Lélande).

BOER (W. C.), arboriculteur, délégué de la Soc. de Pomologie de Boskoop, à Boskoop.

BOII fils ('H. VAN DER), arboriculteur, ì Oudenboseh.

BOM (TH. VAN DER), arboriculteur, ì Oudenbosch.

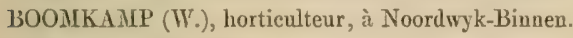

BOS (J. S.), à Amsterdam.

BRANTS (Mr. J. J.), proprićtaire, à Vorden (près de 'Zutphen).

BRAUW (Jhr. Mr. W. M. DE), président de la Société Royal Néerl. pour l'encouragement de l'Hort., président de la comm. dir. de l'Expos. Univ. d'Hort., à la Haye.

BRAUW (Jhr. Mr. W. MAURITZ DE), ì la Haye.

BRAUW (Jhr. E. N. DË), ì la Hayc.

BRINKMAN uće BULTERMAN (Madame $\Lambda$. M.), ì Amsterdim.

BRINK (G. VAN DEN), jardinier en chef du Jardin Botaniquc, à Utrecht. 
Mil.

BRINK (K. R.), horticulteur, ì Leenwarden.

BROOS (H.), ì Harlem.

BR̂UINSMA (J.J.), à Leeuwarden.

BRUNET (L. DE), à Amsterdam.

BRUYNSELLS (J. F.), horticulteur, it I'rinsenlage (Brabant).

BURGERHOUDT (J. J.), propriétaire, ì Utrecht.

BYVOET (M. W.), horticuitcur, ì Overveen (près d'Harlem).

BYVOET ( $\Lambda$. N.), vice-président de la Société „le Bloem van Kenuemerland," ì Overveeu (près d'Harlem).

CANKRIEN (B. E.), propriétaire, ì Rotterdam.

CITTERS (Jhr. Mr. C. VAN), bourgmestre, ì Jeinkenszaud ('Kélande).

COMMELIN Jr. (Mr. J.), notaire, à Amsterdam.

COSTER (Dr. D. J.), ì Amsterdam, Keizersgracht, U, 460.

CRIELLAER'I' (S. B.), propriétaire, ì Rotterdam.

DEDEL (Jur. Mr. C.), à Amsterdam.

DONELA NIEUWENHUIS (F. J.), professeur, à Amsterdam.

DALMAN (RUDOLPHE HENRI), jardinier en chef de Monsieur Mr. J. Liesschert van Vollenhoven, à Berkenrode.

DUYMLER VAN 'TWIST (Mr. A. J.), ì Diepenveen.

DUYMAER VAN 'ITIST (Madame), ì Diepenveen.

EEDEN (F. W. VAN), à Harlem.

EEGIIEN (C. P. VAN), à Amsterdam.

EEGHEN (H. VAN), à Amsterdam.

EGGINK (R.), jardinier de S. A. R. le Prince Frédéric des P'ays-Bas, à la Haye.

EVERWYN (Dr. J.), propriétaire, à Noordwyk-Binnen.

EVERWYN LANGE née RENTE LINSEN (Madame S. A. C.), à Amsterdam.

EVERWYN LANGE (Mr. E. J.), avocat, à Amsterdam.

FABRICIUS (JAC.), agent du chemin de fer Rhénan, à Amsterdam.

FRANCO (F. A.), ì Amsterdam.

FULD (E.), à Amsterdam.

GEUNS (J. VAN), professeur de médecine ì l'Athénée Illustre d'Amsterdam, à Imsterdam.

GEVERS DEYNOOT (Jhr. Mr. D. R.), secrétaire de li Société Hollandaise d'Igriculture;

à Kotterdam.

GLYM (C.), horticulteur, à Utrecht.

GOWS (Mr. C. VAN DER), vice-président de la Société lioyale Néerlandaise pour l'encouragement de l'Horticulture, ì la Haye.

GRAAFF (A. DE), horticulteur ì Lisse (près d'Hariem).

GRANDPRÉ MOLIËE (A. C.), ì Amsterdam.

GROENEWEGEN Jr. (J. C.), horticulteur, à Amsterdam.

GROENEWEGEN (II.), horticulteur, ì Amsterdam.

GROENEWEGEN (J. C.), jardinier en chef du Jardin Botanique, à Amsterdam.

GROENEWEGEN Jr. (J. B.), horticulteur, ì Amsterdam.

GUYE (ANT.), docteur en médecine, à Amsterdam.

GUYE (V. H.), l'un des pasteurs de l'église Wallome, à Amsterdam.

HACKE VAN MYNDEMI (Dr. VAN), ì Amsterdam.

HALL (II. C. VAN), professeur de Botanique à l'Université de Groninguc.

IJEEMSKERK Az. (Mr. J.), cunseiller, ì Amsterdam.

HEYNSIUS (Dr. A.), professeur de médicine à l'Athénice Illnstre, ì Amsterdam. 
MIN.

HOEUFFT TAN VELSEN (Jlır. Mr. II.), vice-président de la comın, dir. de l'Expos,

Univ, d'Hort, ì Amsterdam.

HOOF'MAN Yz. (J.), arboriculteur, président de la Société de Pomologie, à Boskoop.

HOOG (W, ), président de la Sociêté: , Flora ran Noordwijk ," aे Leide.

HUBRECHT (Mr. C. W.), membre des États prov, de la Hollande Mérid., ì Leide.

IIUCHT' (G. L. J. VAN DEIR), propriétaire, 丸̀ Velsen.

JACOMETTI (T. MI. A.), professeur à l'école d'agriculture, à Groningue.

JANSEN (M.), jardinier en chef de Madame la Veuve van der Oudermeulen, 's Graveland. JONCHEERE VAN HARMELEN (A. DE), propriétaire, ì Harmelen (Utrecht).

JONGE VAN ELLENEET' (B. M. DE), étudiant, à Utrecht.

JONGE VAN ELLEMEET (Mr. W. C. M. DE), membre du conseil d'Administration du Palais de l'Industrie, à Oostkapelle (près de Middelbourg).

KALLENBERG VAN DEN BOSCH (R. J. A.), vice-président de la Suciété d'Hort. de l'Arrondissement de Breda, à Teteringen (près de Breda).

KEER Jr. (P.), à Amsterdam.

KENPENAER, Veuve HARINGMAN (E. M. F. DE), à Leide.

KEMPENAER (C. C. A. DE), à Leide.

KEMPER (J. R.), propriétaire, ì Amsterdam.

KEVERBFRG 1)'ALENGOOR (Baron DE), an chattenu d'Aldengoor, près de Roermoud. KISTEMAKER (J. S.), à Amsterdam.

KLUPPEL (J. J.), à Amsterdam.

KNEPPELIIOU't' (J.), à Arnhem.

KNEPPELIIOUT (Madame), à Arnhem.

KNOBELSDORFF VAN DE GELDER (Mr. F. W. A. K. Baron VAN), propriétaire, it Wyhe (Overyssel).

KNUTTEL (S.), à Amsterdam.

KOENEN (Mr. H. J.), à Ansterdam.

KOSTER Azn. (ARIE), arboriculteur, délégućde la Soc. de Pomologie de Buskoop, ì Boskoop..

KRAAYENBhiNK (J. M.), jardinier de S. M. le Roi des Pays-Bas, au château du Loo.

KRELAGE (J. II.), président de la Société générale pour la culture des plantes bulbeuses, premier secrétaire de la comm. dir. de l'Expos. Univ. d'Hort., à Harlem.

KROOK (J. C.), lorticulteur, ì Amsterdam.

KlloOK (J. C.), horticulteur, à Amsterdam.

KRUSEMAN Jr. (H. D.), propriétaire de la maison V. Schertzer et fils, à Harlem.

KRUYFF (II.), horticulteur, ì Sassenhieim.

KRUYFF (L.), fleuriste, à Sassenheim.

KRUYFI' (J. II. G.), fleuriste, à Sassenheim.

KRUYFF (J. J.), fleuriste, ì Sassenheim.

LAAN (W. C. VAN DER), horticulteur, à la Haye.

LAMPSINS VAN DEN VELDEN (Jhr.), à Utreeht.

LANGERHU YZEN L7. (P.), ̀̀ Delft.

LEEUWEN (1). VAN), horticulteur; à Rotterdam.

LENNEP née VAN EEGHEN (Madame VAN), ì Amsterdan.

LENNEY née VAN LENNEI' (Madame A. L. VAN), à Amsterdam.

LENNEP (ARNAUD VAN), à Amsterdam.

LENNEP (MIr. H. S. VAN), trésorier de la comm. dir. de l'Expos. Univ. d'Hort., is Amsterdam.

LEY (B. VAN UER), à Ilallum (Frise). 
MLM.

LOON VAN WINTER (Douairière VAN), à Amsterdam.

LOOPUYT (J.), à Schiedam.

LOOYMLANS (JOSEPH), pépiniériste, à Oudenbosch.

LOOYMANS (JOH.), pépiniériste, à Oudenbosch.

LUDEN Az. (Mr. J.), à Amsterdam.

LUDEWIG (C. A.), à Maestricht.

LUNTEREN (H. VAN), à Utrecht.

LUNTEREN (E. G. VAN), horticulteur, à Utrecht.

LYCKLAMA A NYEHOLT (Jhr. W. H.), à Oldeborn (Frise).

LYCKLAMA A NYEHOLT (Jhr. S.), à Bolsward.

M̈̈̈HL (J. W.), jardinier en chef de M. Willink, aे Amsterdam.

MARITZ VAN CRAYESTEYN (T. W. H.), secrétaire-trésorier et délégué de la Société:

"Dordrechtsche Flora", à Dordrecht.

MARITZ VAN CRAYESTEYN (Mr. J. E. B. L.), président de la Société „Dordrechtsche Flora", à Dordrecht.

MARREE (Dr. L. J. DE), président de la Société d'horticulture de Zúlande, à Miudelbourg. MELVIL (F.), à Amsterdam.

MIENDES (J. A.), à Amsterdam, Keizersgracht, CC, 699.

METELERKAMP (Mr. A. H.), président de la Société d'Agric. et de Bot., à Utrecht.

MEULEN Jr. (JEAN TER), à Amsterdam.

MEULMAN (Js.), membre de la Comm. dir. de l'Expos. Univ. d'Hort., ì Amsterdam.

MEULMLAN (A. W.), à Amsterdam.

MEULMAN ( JAC\&), à Amsterdam.

MIQUEL (F. A. W.), professeur de Botanique à l'Université d'Utrecht.

MONCHY (E. P. DE), à Amsterdam.

MOOY (H. POLMAN), horticulteur, à Harlem.

MOOY (J. POLMAN), horticulteur, à Harlem.

NAAMEN VAN EEMNES (Mr. A. VAN), à Zwolle.

NEUFVILLE (ABRAHAM DE), propriétaire, ì Amsterdam.

OTYTOLANDER (K. J. W.), arboriculteur, délégué de la Société de Pomologie de Bosknop,

à Boskoop.

OTTOLANDER (J. W.), arboriculteur, délégué de la Soc. de Pomologie de Boskoop, ì Boskoopl.

OUDEMANS (Dr. C. A. J. A.), professeur de botanique, directeur du Jardin Botanique,

à Amsterdam.

OUDERMEULEN née DEDEL (Madame la Veure VAN DER), à Amsterdam

OUTEREN (Mr. F. J. VAN), à Amsterdam.

OVEREYNDER (C. G.), arboriculteur, déléguédela Soc. de Pomologie do Boskoop, s̀ Busknop. PAHUD (CH. F.), ancien Ministre des Colonies, ancien Gouverneur-gánéral des Indes

Néerlandaises, membre d'honneur de la Société rovale Néerlandaise pour l'encouragement de l'Horticulture, à la Haye.

PANHorst' (G. P.), à Amsterdam.

PUTMAN CRAMER (Mr. W. J. C.), Notaire, A Zutphen.

POIAK (J. M.), à Amsterdam, Hoogstraat, C, 264.

POPPEL (P. VAN), horticulteur, à Prinsenhage (Brabrint).

PYPER (E. C.), jardinier en chef de Mr. Metelerkamp, ì Brummen.

RAHUSEN (Mr. J. J.), à Utrecht. 
MM.

RAMI (Jhr. H. ML.), membre de la comm. dir. de la Société d'Agric. et de Botanique d'Utrecht, ì Utrecht.

RAUWENHOFF (Dr. N. W. P.) secrétaire de la Société Royale Néerl. pour l'encouragement de l'Hort., directeur du Jardin Botanique, d̀ Rotterdam.

RAVESTEYN (A.), à Amsterdam.

REEPIAKER (A. A.), notaire, trésorier de la Société Royale Néerlandaise pour l'encouragement de l'Horticulture, à Rotterdam.

REINST (Jhr. J. C.), ancien vice-président du Conseil Suprême des Indes Néerlandaises, membre d'honneur de la Soc. Royale Néerl. pour l'encouragement de l'Hort., à la Haye.

RAHDER H\%. (J.), à Amsterdam.

RIVIERE DE VERNINAS (J.G.), directeur de fabrique, à Oudenbosch.

ROCHUSSEN (CH.), artiste-peintre, ì Amsterdam.

RODBARD (J. C.), horticulteur, à Leide.

ROO (G. J. H. DE), meniore du comité et délégué de la Société: "Dordrechtsche Flora", ¿ Dordrecht.

ROYEN (Mr. J. I. M.), propriétaire, à Vreeswyk (Utrecht).

RYNBENDE (S. M. W.), è Amsterdam.

SACHER ( $\mathrm{K}$.), horticulteur, ì Amsterdam.

SANBERG (J.), jardinier au château de Dieren, près d'Arnhem.

SCHIFLER VAN BLEISTIJK (C.), à Wassenaar.

SCINEEVOOGT (C. G. VOORHET,M), trésorier de la Société pour la culture des plantes bulbeuses, ̀̀ Harlem.

SCHOBER (Mr. J. H.), propriétaire, à Utrecht.

SCHIT ARTZENBERG WN HOHENLANDSBERG (G.T.BaronTHOE), ì Beetgum(Frise). SCHIART\%ENBERG FN HOIENLANDSBERG (II. T. H. Baron THOE), à Beetgum (Frise).

SILLEMI (J. G.), à Amsterdam.

SIX (J. P.), à Amsterdam.

STARING (IV. C. A.), référendaire au Ministère de l'intérieur, à la Haye.

STARING (Dr. W. C. H.), membre de la Direction du Palais de l'Industrie, ̀̀ Boekhorst (près de Zutplien).

STIRUM (S. J. Comte DE LIMBUTRG), vice-président de la comm. dir. de l'Exposit. Univ. d'Hort., à Amsterdam

STRAAL Mz. (C. L. VAN DER), à Rotterdam.

SUERMONDT (E.), à Rotterdam.

SURINGAR (Dr. W. F. R.), professeur de Botanique à l'Úniversité de Leide.

STTSEMA (J. D. O. Baron VAN), à Rinsemastate près de Dockum (Frise).

TAACK TRAKRANEN (F. VAN), à Amsterdam.

'TEDING VAN BERKHOUT' (Jhr. Mr. A. V.), à Harlem.

'LEDING VAN BERKHOU' (Jhr. Mr. J. P), 丸̀ Harlem.

TEDING VAN BERKHOUT née VAN HELMONT (Madame), à Harlem.

TIEBOEL SIEGENBEEK (Mr. D.), bourgmestre, à Leide.

'WEENK (H. F.), courtier, à Amsterdam.

TUYLL VAN SEROOSKERKEN (W. R. Baron VAN), à la Haye.

VAILLAN'T (Dr. C. J.), à Schiedam.

VEEN (J. H.), horticulteur, à Harlem.

VEGELIN VAN CLAERBERGEN (Jhr. P. B. J.), à Joure (Frise).

VELSEN (A. J. VAN), horticulteur, à Overveen (près d'Harlem). 
MM.

VERHEY (J.), directeur des travaux publics de la ville d'Amsterdam, ì Amsterdam.

VIRIEU (F. W. DE), directeur des Postes, à Zalt-Bommel (Gueldre).

VISSER (Mr. D.), avocat, à Amsterdam.

VLIET (Mr. W. VAN DER), avocat, ì Amsterdam.

VOLLENHOVEN (Mr. J. MESSCIERT VAN), bourgmestre d'Amsterdam, président de la com. dir. de l'Expos. Univ. d'Hort., à Amsterdam.

VRIESE (F. A.), à Ansterdam.

VROLIK (Dr. A.), ancien Ministre, président de la Société d'exploitation des chemins de fer de l'état, à la Haye.

VRY (Dr. J. E. DE), ̀̀ la Haye.

WALDECK (P. F. L.), à Loosduinen.

WALTER (A. H.), ì Amsterdam.

WATEREN (J. J. VAN DE), jardinier en chef de M. de Jonge van Ellemeet, à Oostkapelle (près de Middelbourg).

WAVEREN (G. VAN), à Hillegom.

WAVEREN Pz。 (J. VAN), à Hillegom.

WAVEREN Lz. (P. H. VAN), fleuriste ì Hillegom (près d'Harlem).

WICKEVOORT CROMMELIX (Mr. A. H. VAN), propriétaire, à Berkenrode (près d'Harlem).

WICKEVOORT CROMMELIN (Mr. J. Y. A.), propriétaire, à Amsterdam.

WILKE (H. J.), horticulteur, à Aruhem.

WHLINK Wz. (J. A.), membre de la comm. dir. de l'Expos. Unir. d'Hort., ̀̀ Amsterdam. WILLINK VAN COLIEN (D.), à Amsterdam.

WIT (C. A. A. DUDOK DE), à Amsterdam.

WITTE (C.), jardinier en chef du Jardin Botanique, à Rotterdam.

WITTE (H.), jardinier en chef du Jardin Botanique ì Leide, secrétaire-adjoint de la comm. dir. de l'Expos. Univ. d'Hort., à Leide.

ZOFS'I' (J. VAN), horticulteur, à Arnhenı.

\section{Hrance.}

ANDRĒ (ED.), jardinier, secrétaire de la Société Impér. et centr. d'Horticulture, 113, rue de la Cour, à Passy-les-Paris.

ANOULH DE SALIES $\left(\mathrm{D}^{2}\right)$, officier en retraite, délégué de la Société Impériale d'Iforticulture pratique du département de Rhône, à Fontannes-les-Lyon.

BALTET (CH.), horticulteur-pépiniériste, à Troyes.

BARILLET-DESCHANPS, jardinier en chef des promenades et des plantations de Paris, 24 enceinte de la Muette, à Passy-les-Paris.

BARRAI (J. A.), rédacteur de la Revue horticole et du Journal d'agriculture pratique, 24, rue Jacob, à Paris.

BAUMANN (A. N.), horticulteur, a Bollwiller (Haut-Khin).

BERGILAN (FERD.), chef des cultures de Monsieur le Baron J. de Rothschild, au Domaine fle Ferrières, à Ferrières.

BER NARDIN (CAMILLE), secrétiare-géníral des Socitíśs d'Hortirulture de ('oulommiers, Melan et Fontainebleau a Brie Comte liobert (Seine et Marne), France.

BOSSIN, grainier pépiniériste, 15, rue de Tivoli, à Paris.

BOUCHARD-HUSARD, premier secrétaire-adjoint de la Société impériale et centrale d'Horticulture de l'aris. 
MIM.

BROGNIART (AD.), membre de l'Institut de France, professeur au Muséun d'Histoire Naturelle, vice-président et délégué de la Société Impériale et centr. d'Ilorticulture, ì Paris.

CrIATIN (A.), professeur de botanique et directeur du jardin botani(pue de l'école supérieure de Pharmacie, délégué de la Soc. Imp. d'Agric. et des Arts, à Paris.

CROUSSE, horticulteur, 1 , rue du champ d'Asile, à Nancy.

DELAIRE, chef du jardin botanique d'Orléans, secrétaire et délégué de la Société d'Horticulture d'Orléans, à Orléans.

DEPOIR (RENÊ), banquier, délégué de la Société d'horticulture de Pontoise.

DESVE, archiviste de Ia Société impériale et centrale d'lorticulture du département de la Seine inférieure, à Rouen.

DURAND, arboriculteur, Bourg-la-Reine, à Paris.

ESTAINTET (Comte D'), à Rouen.

FÉE, professeur de botanique au Jardin des plantes de la faculté des sciences, ì Strasshourg.

FORT (EDOUARD LE), représentant du Journal: „la Patrie", directeur propriétaire du Journal illustré, la Maison de campagne, 8, boulevard des MFartyrs, ì Paris.

GOURIÉ (ALEX.), propriétaire, 22, boulevard poissonière, ì Paris.

GUENOT, Mfd. grainier et horticulteur, quai Napoléon, 35, à Paris.

GUTZBERGER, 91, rue Boithord, à Paris.

GUTZBERGER, (Madame), rue Boithord, à Paris.

HELYE (D.), chef de culture, attaché au Muséun d'histoire naturelle, ì Paris.

HERINCQ (F.), rédacteur en chef de l'horticulteur français, rue Guy de la brosse, 11 , ì Paris.

HOULLET (R.), chef des serres ùu jardin des plantes de Paris (Muséun d'histoire uaturelle), à Paris.

JAMIN, arboriculteur, Bourg-la-Reine, ì Paris.

KETELEER, de la maison Thibaut \& Keteleer, horticulteur, 146, rue de Charonne, à Paris.

LAMBERTYE (Comte LËONCE DE), propriétaire, à Chaltrait (Marne).

LANDRY (JOSEPH), horticulteur, 26, rue de la Croix, à Passy-les-Paris.

LAVRIL (ERN.), propriétaire, rue Hauteville, 23, à Paris.

LE CONTE (CASIMIIR), rue du Rivoli, 238, à Paris.

LECOCQ (HENRI), professeur d'histoire naturelle à la faculté des sciences, correspondant de l'institut, directeur du jardin des plantes, à Clermont-Ferrand.

LEFẼVRE-PONTALIS (Madame), ì Paris.

LEFËVRE-PONTALIS, l'un des rédacteurs du Journal des Débats et de la Revue de Deux Mondes, président et délégué de la Socićté d'A griculture et d'Horticulture de l'arrondissement de Pontoise, rue de Rivoli, 238, à Paris.

LEMOINE (V.), horticulteur, ì Nancy.

LEROY, horticulteur, ì Paris.

LOUESSE, délégué de la Société Impériale et centr. d'horticulture de Paris, ̀̀ la celle de St. Cloud par Bougival, à Bougival.

LUDDEMAN (G.), horticulteur, 22, boulevard des Gobelins, à Paris.

MARTINS (CH.), professeur de botanique et directeur du jardin des plantes, it Montpellier.

MAZEL, propriétaire, délégué de la Sociŕté d’horticulture de Marseille, 74, cours Bonaparte, at Marseille.

MORRIS (GABRIEL), 64, rue Amesat, ì Paris.

MURET' (C.), architecte des jardins, 30, rue de lontoise, à Paris 
MM.

PÉPIN, jardinier en chef au MIuséum d'histoire naturelle, déléguć du Gourernement de France, à Paris.

PINEL (E.), secrétaire de correspondance et délégué de la Société Impériale et centrale d'horticulture du departement de la Seine-Inférieure, à Rouen.

ROSCIAUD, jardinier en chef à Laveng (Seine et Oise), délégué de la Société d'horticult. de l'arrondissement de Pontoise.

ROUGIER CHAUVIËRE, horticulteur, 152, rue de Roquette, à Paris.

SIMON (LEON), horticulteur, pépiniériste, maison Simon Louis frères, à Metz.

THIERRY (G.), conservateur du jardin botanique de la ville de Caen, secrétaire archiviste et délégué de la société centrale d'horticulture de Caen et de Calvados, à Caen.

THOU VENEL (A.), conservateur du jardin de la ville d'Orléans, 92, faubourg Bourgogne, à Orléans.

TISSERAND (EUGENE), chef du département des institutions impériales de l'agriculture, délégué du gouvernement français, à Paris.

TRIANA (J.), botaniste voyageur, rue du Cardinal Lemoine, No. 14, à Paris.

TRUFFAUT fils, horticulteur, rue des Chantiers, No. 40, à Versailles.

VERDIER fils (CHARLES), horticulteur, rue du Marché aux chevaux, No. 32, à Paris.

VERLOT, délégué de la société impériale et centrale d'horticulture de Paris, à Paris.

VILMORIN (IIENRI), de la maison Vilmorin Andrieux \& Co., marchand grainier, quai de la Megisserie, No. 30, ì Paris.

WEISS-SCHIUMBERGER, président de la société d'horticulture de Miülhouse, Mrülhouse (Haut-Rhin).

\section{Belgique.}

AUBAND (CH.), administrateur de la société des conférences horticoles et agricoles, l'un des rédacteurs de l'Indépendance Belge, 7, Montagne du Pare, à Bruxelles.

BAUMANN (J.), horticulteur, président de l'académie royale d'horticulture, nourelle Promenade, No. 5 en 7, Gand.

BAUWENS (L. H.), 19, rue des sables, à Bruxelles.

BEAUCARNE, notaire, vice-président de la société d'horticulture d'Oudenaerde, à Eenaeme.

BECKX (CONSTANT), proprićtaire, délécué de la société rovale d'agric. et d'horticulture de Louvain, ì Louvain.

BEUCKER (J. J. DE), horticulteur, délégué du cercle professoral d'arboriculture de Belgique et du „Antwerpsch Kruytkundig Genootsehap," à Anvers.

BIVORT (ALEXANDRE), secrétaire-redacteur et délégné de la commission royale de Pomologie de Belgique, à Fleurus (Hainaut).

BOMMER (F.), conserrateur des collections des sociétés royales d'horticulture et de botanique de Belgique, à Bruxelles.

BOUCQUEAU, membre du bureau de la félíration des sociétés d'horticulture de Belgique, à Nivelles, près de 13ruxelles.

BRICHY (J.DE), directeur de l'école de l'état, ì Vilrorde.

CANNART DHAMALE (F. DE), sénateur, président de la société royale d'horticulture de Malines et vice-président de la fédération des sociétés d'horticulture de Belgique, à Malines.

CAROLUS (HENRI), négociant, délégué de la société royale d'agric. et d'hortieulture de Louvain, it Louvain.

CATERS (le Baron CONSTANT DF), président et délégué de la société royale d’horticulture et d'agriculture d'Anvers, à Anvers.

DALLTERF (ALEX.), horticulteur, à Gand. 
MM.

DE GRAET BRAEQ, banquier, ì Gand.

DE ZANTIS, rentier, protecteur et délégué de la société horticole de Liège, Faubourg St. Gilles, ì Liège.

DESMOULIN (GASPARD), propriétaire, rue de Niny, 46, à Mons.

DONCKIER, déléguć de la société royale de botanique de Belgique, à Bruxelles.

EECKHAUTE (JACQUES VAN), horticulteur, à Ledeberg-les-Gand.

FUCHS (L.), professeur à l'école de Vilvorde, à Ixelles-les-Bruxelles.

FUNCK, directeur du jardin de la société royale de zoülogie et d'horticulture de Belgiq̨ue, et délégué de la société royale de Flore de Bruxelles, à Bruxelles.

GAILLY, jardinier en chef du jardin botzmique, à Bruxelles.

GASPARD DOZIN, horticulteur, rue du Paradis, à Liège.

GEERT (AUG. VAN), horticulteur, à Gaind.

GEERT (CHARLES VAN), horticulteur pépiniériste, à Anvers.

GROBBEN, jardinier chez le comte Mercy d'Argenteau, délégué de la société horticole de Liège, à Argenteau.

GUILLEAUME, président honoraire de la société royale des conférences horticoles de Liège, Boulevard d'Avray, à liège.

HAESENDONCK (VAN), délégué de la société royale de bot. de Belgique, à Bruxelles.

HECKE DE LEMBECKE (V. VAN DEN), président et délégué de la société d'agriculture et de botanique de Gand et du cercle professoral pour le progrès de l'arboriculture en Belgique, à Gand.

HECKE (D. VAN DEN), rue d'Orléans, 10, à Gand.

HEURCK (HENRT VAN), professeur de botanique, vice-président et délégué du Kruytkundig Genootschap, président de la société phytologique d'Anvers, ì Berchem-Anvers.

IIONSEM-LUNDEN (C. J. VAN), rentier, membre du conseil d'administration de la société d'horticulture d'Anvers, rue Louise, No. 2, à Anvers.

HOUTTE (LOUIS VAN), horticulteur, éditeur de la Flore des Serres et des Jardins, directeur de l'école d'horticulture, ì Gendbrugge-les-Gand.

HOUZEAU DE LEIIALE (L ), professeur au jardin bot. de MIons, à Hyou, près de Mons.

HULLE (H. VAN), jardinier en chef du jardin botanique de l'Unversité de Gand, soussecrétaire et délégué du cercle prof. d'arboric. de Belgique, à Gand.

JANSSENS (E.), trésorier et délégué de la société royale Linnéenne de Bruxelles, rue de T'erre Neuve, 11\%, ì Bruxelles.

KEGELJAN (FERD.), secrétaire de la société royale d'horticulture de Nanur, à Namur. KERCHOVE DE LIMON (CII. DE), représentant, bourgmestre de Gand, président d'houneur de la société royale d'agricole et de botanique de Gand, ì Gand.

KICKZ (J. J.), directeur du jardin botanique de l'université, à Gand.

LEMAIRE (CIIs.), professeur de botanique, rédacteur de l'lllustration Horticole, à Gand

LINDEN (J.), directeur du jardin royal de zoölogie, délégué du gouvernement Belge et de la société 'Toscane d'horticulture à Florence, ì Bruxelles.

LINDEN (II. VAN DER), négociant, trésorier de la société d'horticulture d'Anvers, rue Haute, 43, Anvers.

MARTENS (ED.), professeur de botanique à l'université de Louvain, à Louvain.

MAWET-PORTULA, horticulteur et délégué de la société horticole de Liège, rue Thier de la Fontaine, à Liège.

MEYER (R.), horticulteur, de la maison Stelzner \& Mayer, à Gand (faubourg de Bruxelles).

MORREN (EDOUARD), professeur de botanique à l'université, directeur du jardin botanique, délégué du gouvernement Belge, secrétaire et délésné de la fédération des sociétés d'horticulture de Belgique, à Liège. 
MM.

MUULLER (FELIX), président et délégué de la société royale Linnéenne de Bruxelles, à Bruxelles.

MULLER (T.), conseiller et délégué de la société royale de bot. de Belgique, à Bruxelles.

NECK (C. VAN), administrateur et délégué de la société royale Linnéenne de Bruxelles, Chaussée de Gand, 1, à Bruxelles.

NONDONSAZ, secrétaire et délégué de la société horticole de Liège, rue Basse chevaufosse, 9 , à Liège.

NUYTENS (JEAN), hortıculteur, rue de la Caverne, 43 , à Gand.

OSY (Baron ED.), conseiller provincial, vice-président de la société royale d'horticulture et d'agriculture d'Anvers, ì Anvers.

PIRE (LOUIS), professeur de l'athénée royal, délégué du gouvernement Belge, sécrétaire et délégué de la société royale de botanique de Belgique, rue d'Orléans, 15, à Bruxelles.

PIRLO'T (JULES), propriétaire, administrateur de la société royale d'horticulture de Liège, à Liège.

PYNAERT (ED.), professeur à l'école d'horticulture de l'état ì Gendbrugge, architecte des jardins, 26, quai d'Orton, délégué du cercle prof, d'arboric. de Belgique, à Gand.

RIBAUCOURT (Comte DE), sénateur, vice-président de la société royale de Flore, ancien président de la comm. dir. de l'exposition universelle d'horticulture de bruselles, Avril 1864, à Bruxelles.

RIGOT'TS-VERBERT', professenr de botanique, directeur du jardin botanique, secrétaire de la société royale d'agriculture et d'horticulture, à Anvers.

RODIGAS Père, docteur en médecine, vice-président et délégué dn cercle professoral d'arboriculture de Belgique, ì St. 'I'roud.

RODIGAS (EMI.), professeur ì l'école d'horticulture de l'état ì Gendbrugge-les-Gand, secrétaire-général du cerele professional d'arboriculture de Belgique, à Gendbruggeles-Gand.

ROMBERG, chef de division au département des affaires intérieures de Belgique, délégué du Gouvernement Belge, ì Bruxelles.

ROSSEELS ainé (E.), horticulteur, architecte des jardins, président de section de la société d'agriculture et d'horticulture, ì Louvain.

ROSSEELS (L. J.), géomètre, à Lourain.

ROSSEELS fils (L.), horticulteur, délégué de la société royale d'agric. et d'horticulture de Louvain, ì Louvain.

RY (EMILE DU), propriétaire, à Gaud.

SEVEREYNS $\left(G_{.}\right)$, lithographe de l'acad. royale, 49 , rue de Liedekerke, à St. Josseten-noode-les-Bruxelles.

SIGAUT-CAPOUILLEI', président de la société d'horticulture et de zoölogie au Vauxhall, ì Mous.

SMET (LOUIS DE), horticulteur, it Gendbrugge-les-Gand.

SMITS (CONSTANT), architecte de jardins, it St. Gilles-les-Bruxelles.

SOMERS (L.), à Anvers.

SOMERS (FERD.), négociant, rue aux Laines, 29, à Anvers.

STELZNEIR ( $\Lambda$.), horticulteur, à Gand.

STERCKMANS (CUF.), jardinier en chef du jardin botanique, à Louvain.

THIELENS (A.), botaniste, délégué de la société royale de bot. de Belgique, ì Tirlemont. TILT (LOUIS VAN), propriétaire, délígué de la société royale d'agric. et d'horticulture de Louvain, it Louvain.

VBRSCHAEFELT (AMBROISL), hortieniteur, filiteur de l'Illustration horticole, ì frand. VFRSCHAFHELT (JFAN), horticnlteur, 43, rue de la Caverne, at Gand. 
MII.

WEHGLFOSSE, délégué de la société royale de botanique de Belgique, ì Bruxelles.

WESMAFL (ALFRED), directeur de la société anonyme d'agrément d'horticulture et de zoülogie, ì Mons.

WESTENDORI, vice-président et délégué de la suciété royale de botanique de Belgique, ì Bruxelles.

WIO'T (F.), de lat maisou Jacob Nackoy \& Co., horticulteur, ì Liège.

\section{Suisse.}

MEISSNER (Dr. C. F.), protesseur de botanique, directeur du jardin botanique de l'université de Båle, ì Bâle.

\section{Italie.}

NISSON (MLX.), directeur du jardin d'acelimatation, ì Naples.

PASSERINI (JEAN), professeur de botanique et directeur du jardin botanique, à Parme.

\section{Espagne.}

ROCCA (VICENTA), horticulteur, ì Valencia.

\section{Prusse.}

BENARY (ERNES'1), horticulteur, à Erfurt.

BENDA (CARL), horticulteur, Magazinstrasze, I6́, ì Berlin.

BOESE (E.), jardinier en chef du conseiller L. Reichenlein, 4t, Grabenstrasse, à Berlin, BOUCHE (C.), inspecteur du jarlin hotanique royal, à Berlin.

CASPARY (Dr. ROBERT), directeur du jardin lotanique, professeur de botanique a l'université de Künigsberg, à Königsherg.

COHN (Dr. FERDIN.), professeur de botanique, délégué de la société silésienne d'horti. culture, à Breslau.

ERBSCHLOE-MULLER (CHs.), à Elberfeld.

ESSER ( $\Lambda$.), jardinier en clicf du jardin Schoeller, à Dieren, près de Cologne.

GAERDT, jardinier en chef au jardin Borsic., ì Moabit, près de Berlin.

GOEPPERT (Dr. H. $\boldsymbol{K}_{\text {. }}$ ) professeur de botanique à l'université, délégué de la société silésienne d'horticulture, à Breslau.

HAAGE (J. N.), horticulteur, à Erfurt.

HENEL (ALB.), imprimeur de la cour, à Magdebourg.

HASSKARL (Dr. J. K.), voyageur naturaliste, à Clève.

HEYDER, conseiller secret, ì Berlin.

HILDEBRAND (Dr. '1'), professeur de botanique, ì Bomm.

HOFFMANN (JULIUS), horticulteur, Köpnicherstrasse, No. 131, à Berlin.

JACOBI, général et inspecteur de l'artillerie, ¿̀ Breslau.

JÜHLKE (FERD.), directeur des jardins royaux, président et délégué de la société d'horticulture d'Erfurt, ì Erfurt.

KARSTEN (Dr. HERM.), Constanstrasse, No. 4 , ¿ Berlin.

KNERK, conseiller secret, président de la société pour l'encouringenent de l'horticulture en Prusse, ì Berlin.

KOCH (Dr. KARL), professeur de botaniq̨ue, secrétaire général et délégué cie la société pour l'avancement de l'horticulture eu P'russe, rédacteur du "Wochenschrift," à ßBerlin.

MÜNTER (Dr. S.), professcur de botanique et directeur du jardin butanique de Greifswald, ì Greifswald.

S.ICIS (Dr. JULIUS), professeur de botanique, it $130 m$. 
MM.

SAUER, jardinier en chef du jardin botanique à l'université, ¿̀ Berlin.

SINNING (W.), jardinier en chef du jardin botanique de l'umiversité, à Bonn.

SONNTAG (W.), trésorier de la société pour l'encouragement de l'horticulture, Alexandrizenstrasse, 51 , à Berlin.

STRASBURGER (LEON), étudiant, à Bonn.

WEYHE (J. C.), directeur du jardin botanique royal, Düsseldorf.

\section{Autriche.}

ABEL (LUDWIG), horticulteur, membre de l'administration de la sociétié de l'horticulture de Vienue, Laudstrasse, ì Vienne.

ENTZ (VICTOR), botaniste à Bude (Hongrie).

ENTZ-FERENEZ (Dr. TRATÇOIS), directeur de lia pépinière nationale, ì Bude (Ifongrie). FENZL (Dr. E.), professeur de botanique et directeur du jardin botanique, à Vieune.

HÜGEL (Baron CHARLES DE), envoyé extraordinaire et ministre plénipotentiaire de S. M. Imp. et Royale l'Empereur d'Autriche, à Bruxelles.

LAGLER (EDIOND), jardinier en chef du jardin du Comte François de Thun-IIohenstein, Chateau de Fetschen, près de Bodenbach (Bolı̀me).

WARSCEWICZ (JOSEPH), inspecteur du jardin botanique impérial et royal, ì Cracovie.

\section{Bavière.}

EFFNER, jardinier en chef de la cour, premier secrétaire et délégué de la société d'horticulture de Bavière, à Munich.

- KOLB (MI.), inspecteur du jardin botanique, à Mfunich.

SCHNIZLEIN (Dr.), professeur de botanique à l'université d'Erlangen.

\section{Hanovre.}

GRISEBACH (Dr.), conseiller de la cour, professeur de botanique et directeur de jardin botanique, à Goettingue.

TATTER (W.) jardinier en chef du jardin royal, à Linden, près de Hanovre.

WENDLAND (HERML), inspecteur du jardin royal de Berg Herrenhausen, près Hanovre.

\section{Wurtemberg.}

CALIBBACH (W.), jardinier en chef de la cour, à Stuttgart.

HOCHSTETTER (W.), jardinier en chef du jardin botanique, à 'l'übingen.

NEUBERT (Dr. W.), rédacteur du Deutsches Magazin, à Stuttgart.

\section{Bade.}

MLAYER Jr. (E.), jardinier en chef du Grand-Duc de Bade, à Carlsruhe.

SEUBERT (Dr. M.), conseiller de la cour du Grand-Duc de Bade, professeur de botamique et directeur du jardin Botanique, à Carlsruhe.

\section{Luxembourg.}

SOUPERT, de la maison Soupert \& Nothing, horticulteur, ì Luxembourg.

WILHELM (A.), horticulteur, à Clausen, près de Luxembourg.

\section{Weimar.}

PRINGSHEIMI (Dr. N.), professeur de botanique, rédacteur des ,Jahrbüicher für wissensch. Botanik," à Jena. 
MII.

Saxe.

GEITNER (G.), horticulteur à Planitz, près de 'Zwickau.

WILLKOMM (Dr. MORITZ), professeur de botanique et de zoölogie à l'académie royale forestière et d'agriculture, directeur du jardin botaniqque, délégué du Gouvernement de Saxe, ì Tharand.

\section{Hesse (Griand-Duché).}

BRAUN (J. J.), rentier, membre de l'administration et délégué de la société d’horticulture de Mayence, à Mayence.

HOFFMANN $(\mathbf{H}$.$) , professeur de botanique et directeur du jardin botanique, ì Giessen.$

HUMANN (L.ANT.) président et lélégué de la société d'horticulture de Mayence, ì Najence.

KUNZE (GUSTAV), horticulteur, ì Offenbach a/M.

MARDNER (JOSEPH), de la maison Mardner frères, horticulteur, ì Mrayence.

SCHNITTSPAHN (G.), directeur du jardin du Grand-Due de Hesse, ì Darmstadt.

\section{Hesse (Hlectorat.)}

DEINES (O'TO), horticulteur, à Hanau.

\section{Nassau.}

LADE (ED.) consul-général, à Geisenheim.

THELEMANN, inspecteur des jardins du Due de Nassau, à Biebrich (Rhin).

\section{Sleeswic.}

AHLMANN (Dr. W.), ì Kiel.

\section{Hambourg.}

BOECKMANN (H.), rentier, secrétaire et délégué de la société d'horticulture et de floriculture de Hambourg, Altona et environs, à Hambourg.

BOOTH (LORENZ), liorticulteur-pépiniériste aux pépinières le llottbeck, près de Hambourg.

CRANER (F. B.), jardinier en chef' du jardin de Mad. Jenisch, Flottbecker Park, près de Hambourg.

ERNST $(O . \Lambda$.), de la maison Ernst \& von Spreckelsen, horticulteurs-mds. Grainiers, à Hambourg.

REICHENBACH FILS (II. J.), professeur de botanique et directeur du jardin botanique, à Hambourg.

\section{Francfort s/Main.}

GRUENEBERG FILS (FR.), horticulteur et architecte du jardin, à Francfort s/Main.

\section{Suède et Norvège.}

ANDERSSON (Dr. X. J.), professeur de botanique, membre de l'académie des sciences et d'agriculture Suède, délégué du gouvernement Suède, secrétaire et délégué de la société d'horticulture Suède, à Stockholm.

\section{Danemarc.}

LANGE (JOH.), professeur de botanique, directeur du jardin botmique, ì Copenhaguc. TYGE ROTHE, chef des jardins de l'académie royale d'agriculture et d'horticulture, délégué de la société d'hort. lu Danemare, ì Copenhague. 
MM.

Russie.

NEDZELSKY (A.), docteur en sciences, botaniste de l'université de Moscou (rue du trône, 26, au premier ì Bruxelles), à Moscou.

NORDLiANN (Dr. A.), professeur de botanique et directeur du muséum d'hist. nat. à Helsingförs.

REGEL (Dr. E.), directeur du jardin botanique, vice-présitent đle la société russe d'horticulture, rédacteur du "Garten-Flora”, ì St. Yetersbourg.

TÉPLOUCHOFF (FÉDOR), botaniste et entomologiste, natif du Gouvemement de l'erm, ¿े Tharand en Saxe.

\section{Grande-Bretagne et Irlande.}

BERKELEY (Rev. M. J.), premier délégué de la société royale d’horticulture, ì SouthKensington, Londres.

BULL (WLLLIAM), horticulteur, King's lioad Chelsea, près de Londres, S W.

CU'BUSU (JANES), de la maison Wm. Cutbush \& Son, horticulteurs, Highgate, près de Londres, $N$.

HOGG (Doct. ROBERT), rédacteur du journal of horticulture, ì Londres.

JOHNSON (GEORGE W.), collaborateur du journal of hort., à Londres.

LEE (CHARLES), de la maison John \& Charles Lee, lorticulteurs, Royal Vineyard Nưrsery, Hammersmith, près de Londres.

MASTELS (Dr.), délégué de la société royale d'horticulture, à South Kensington, Londres. MOORE (THOMAS), directeur du jardin botanique de l'hôpital, à Chelsea, près de Londres. PAUL (WILLIAM), horticulteur, Waltham Cross, ì Londres, N.

STANDISII (INO), horticulteur, Royal Nursery, ì Ascot.

VEITCII (JAMES), horticulteur, royal exotic Nursery, King's road, à Chelsea, près de Londres.

WARNER (ROBERT), 8, cressent Cripplegate, à Londres E. C.

WILDING Esq. (THs. F.), 2, Chesterfieldstreet, King's Cross., ì Lond̉res, W. C.

WILLIAMS (B. S.), horticulteur, Paradise \& Victoria Nurseries, Holloway, ì Londres, N. 


\title{
REVUE DES QUESTIONS
}

\author{
sommises
}

\section{AUX DISCUSSIONS DU CONGRÈS.}

\section{QUESTIONS DE BOTANIQUE PURE.}

I. M. Belhomme, directeur du jardin botanique de Metz, regrettant vivement de ne pouvoir assister au Congrès, propose les questions suivantes:

1. Est-il possible de supposer que la présence du Juniperus Sabina, sur lequel vient le Gymnosporangium fuscum, soit la cause de l'apparition de l'Aecidium cancellatum sur les Poiriers?

Dans le cas où le fait aurait été véritablement constaté, y aurait-il métumorphose, comme cela se voit dans certains animaux, du moins comme le supposent quelques botanistes, ou transformation du Gymmosporangium en Aecidium?

2. Peut-on reconnaitre à la forme extérieure des graines de plantes dioïques, du Cammabis par exemple, si lar graine domnera un sexe mâle ou un sexe femelle, comme cela se voit chez certains oiseaux; dans le cas où le fait serait reconnu, quels en seraient les caractères?

"A ce sujet, voici ce qu'une année m'a domné (sauf ¿̀ répéter les expériences pour justifier la question). Dans ime poignée de graines de Cammabis sativa j’ai remarqué deux formes, l'une allongée et elliptique, l'autre presque sphérique; ces dernières sont zébrées et la pellicule est plus pâle. Celles allongées et elliptiques sont maculées de 
points zébrés sur leur surface et sur les deux extrémités aiguës, la pellicule est brune. Les ayant semées, celles elliptiques m'ont donmé des pieds máles, et les sphériques des pieds femelles; sur ces dernières, sur quinze pieds de chanvre, il est apparu un mâle; était-ce une griaine restée dans le sol? Sur les pieds femelles de ce semis on remarque que les graines elliptiques sont en plus grand nombre.

II. M. Ch. Martins, prof. de botanique ì Montpellier, propose an Congrès de mettre ì l'ordre du jour la question suivante:

L'influence de l'échaufiement du sol par les rayons solaires sur la végétation et la distribution géegraphique des végétaux.

III. M. Ch. Lemaire, ¿̀ Gund, rédacteur de l'IlIustration Horticole, annonce un discours contenant:

„Critiques raisonnées et réfutation sur la théorie des boyaux polliniques."

IV. M. Jean Passerini, prot. de botanique ì Parme, se propose de discuter:

1. Sur l'émission de l'acide carbonique par' les racines des plantes et sur lia décompusition du mème acide par les parties vertes.

2. Sur la fonction des stomates.

V. M. le prof. Julius Sachs, de Bonn, traitera le sujet suivant:

Ueber die Wichtigkeit photonetrischer Bestimmungen tür die Physiologie, Geographie und Cultur der PHanzen, und über die Principien der dabei zu befolgenden Methode.

VI. M. le proî. F. Cohn, à Breslau, a indiqué comme questions contentibles ponr la discussion du Congrès:

1. L'influence que la lumière exerce sur les mouvements des plantes.

2. Les métamorphoses que les ravages de certains insectes produisent dans l'ćrolution des cellules des plantes attaquées.

VII. M. Chatin, mof. de botau, à l'École Impériale 
de Pharmacie à Paris, a remis les questions suivantes comme élément de quelques discussions, qu'il ouvrira par un exposé sommaire de ses recherches sur ces sujets:

1. Des caractères anatomiques pour la classification des végétaux.

2. Structure et fonctions du tissu de l'anthère.

3. Du sucre dans les tissus des plantes.

VIII. MT. F. MIuller, président de la Société Royale Linnéenne à Bruxelles, se propose de donner

Lecture d'une notice sur le genre Cuscuta, et il annonce en même temps un travail sur les plantes de plein air sous le climat de la Hollande et de la Belgiqne.

IX. M. le prof. Karl Koch, ì Berlin, fait les propositions suivantes:

1. Jährlich werden grosse Mengen neuer Pflanzen eingeführt und mit beliebigen Namen versehn in den Handel gebracht. Bedient man sich dabei eines schon in der systematischen Botanik gebräuchlichen Namens, um vielleicht damit zu sagen, dass die neu eingeführte Pflanze eine zwar bekannte, aber bisher in den Gärten noch nicht kultivirte interessante Art darstelle, so entsteht nothwendiger Weise eine Confusion, die zu den bedauerlichsten Täuschungen führt. Heut' zu Tage kann selbst der geübteste Botaniler nicht alle Pflanzen mehr kennen und beschränkt sich in seinem Wissen an einige Familien, mit denen er sich bereits eine geraume Zeit beschäftigt hat. Wie wäre es num, wemn die in Amsterdam anwesenden Botaniker sich zu einer Theilung der Arbeit in der Weise vereinigten, dass Jeder bekannt machte, welche Familien er forthin mit besonderer Vorliebe bearbeiten wollte?

2. Es wäre gewiss wïnschenswerth, wenn in Amsterdam Männer der Wissenschaft zusammen'reten wollten, um eine Berichtigung der Namen vorzmehmen und zu gleicher Zeit eine Einigung in der Nomenclatur herzustellen. Es sind dieses z. B. die sogenamnten bammartigen Lilien (Agaveen, Yucceen, Dracteneen), wo die einzelnen 
Arten hinsichtlich der Benennumg sich in der traurigsten Verwirring befinden.

I. II. le prof. Reichenbach Fils, de Hambourg, traitera:

Des Orchidées de la Nouvelle Calédonie.

XI. II. le Dr. J. K. Hasskarl, à Clives, annonce des communications:

„Over eenige nieuwe of min bekende geslachten der Commelynaceae."

XII. M. C. A. J. A. Oudemans, prof. de botanique à l'Athénée Illustre d'Amsterdam, traitera:

1. De I'origine des stomates dans quelques espèces d'Aneimia.

2. De l'origine des spores dans certaines espèces de NIucor.

IIII. M. Fr. Entz, de Bule (Hongrie), se propose d'ouvrir la discussion sur la question suivante:

Quelle est la destination véritable des plantes dans l'économie de la nature?

XIV. II. Herinq, rédacteur en chef de l'Horticulteur français, à Paris, nous propose de porter au programme la question:

La Sève (mouvement et circulation), sur laquelle on ne sait ì peu près rien, et qui némmoins est devenue le pivòt, antour duquel toument toutes les théories de la taille des arbres, etc.

XV. M. Ed. IMorren, prof. à Liège, nous écrit encore qu'il compte faire deux communications:

1. Sur les fleurs doubles, et

2. Sur les fonctions des stomates.

IVI. II. le Dr. Schultz-Schultzenstein, ì Beilin. reugrettint que l'état de sil simnté ne lui permettra pals d'assister au Congrès, nous invite à faire connaître les considérations suivantes:

Durch die nenere Ainchaldüngerthevrie ist die Praxis der Zubereitung des Brdens und der. Comproste für Topf- 
gewächse alterirt worden, indem die Aufimerksamkeit ron den für überflüssig gehaltenen humösen Theilen der Bodenmischnngen abgelenkt worden, und zu viel Hoffinung anf die Ernährung der Pflimzen aus der Luft gesetzt worden ist, während die Praxis immer wieler auf die Bolengüte angewiesen ist. $D_{\text {a }}$ ich mich mit diesem Gegenstande theoretisch und practisch beschättigt habe, so beehre ich mich dem Congress meine Schrift: , über Pflanzenernährung, Bodenbereicherung und Bodenerschöpfung"' mitzntheilen, worin durch nene Versuche die Irrthümer der Lufternährungstheorie gezeigt sind und nachg'ewiesen ist, dass die aus den humösen Bodenbestandtheilen gebildeten organischen Säuren das Hauptnahrungsmaterial der Pflanzen bilden, und dass insbesondere das rom den Blättern ausgehauchte Oxygen seine Quelle nicht in der Kohlensäure der Luft, sondern in den genamnten fixen Borlensäuren hat, indem durch Zusatz solcher Säuren zum Wasser, worin Pflanzenblätter regetiren, die Ausscheidung ron Oxygen mugemein rermehrt wird in dem Masse als die Säuren verschwinden; so dass beispielsweise dreissig Weinblätter, die in reinem Wasser nur einen Kúbikzoll, in mit Weinsiure angesünertem Tisser zehn Kubikzoll Oxygen lieferm. Es scheint hiemach nuzweifelhaft, diss bei der Pflanzenernährung in der Kultur urr insbesondere bei den Bodemmischungen cine grössere Aufmerksankeit auf die humösen Bodenbestandtheile zu richten ist. Meine Versuche haben gezeigt, dass unter den humösen Substanzen, die zur Düngung verwendet werden, gewisse Sorten ron Torf, wie der Torf überhaupt, einen bisher wenig gekamnten Werth haben, und selbst der Heideerde rorzuziehen sind, weil der Torf ansser den humösen Süuren, die sich durch Vermoderung aus ilnm bilden, einen bedentenden Gehalt an Stickstoft zeigt, wesshalb auch das bei der trocknen Distillation des Torf's gewonnene ammoniakhaltige Wasser zur Silmialifabrication verwendet wirl. Gunanere Untersuchungen des Torfs haben mir gezeigt, diss 
der Ursprung des Stickstoffs im Torf nicht bloss von einer Bindung ron Stickstoff aus der Luft, sondern vielmehr von den Leichen kleiner Thiere aus der Klasse der Evertebraten: der Mollusken, Crustaceen, Annulaten, Arachniden, Insecten (besonders Coleopteren, Dipteren, Hemipteren und deren Larven), Polypen, Foraminiferen und Infusorien herrühre, indem sich massenhafte Ueberreste dieser Thiere im Torfe finden, die dem besten thierischen Kunstdünger: an die Seite zu stellen sind. Die Naturdüngung des Torfs möchte sogar für gervisse zarte Pflanzen vorzuziehen sein, weil die humöse Dungmasse darin völlig verrottet ist. In dem Stickstoffgehalt verschiedener Torfsorten ist jedoch eine Verschiedenheit, indem einige Sorten stickstoffïmer, andere stickstoffreicher, jenach dem Gehalt an Thierleichen sind. Die Stickstoffreicheren sind besonders zur Compostbereitung für Landund Topfpflanzen zu verwenden.

XVII. M. le général Jacobi, à Breslau, nous écrit:

Demnächst beabsichtige ich dem Kongress eine ron mir verfaszte Arbeit über die Agaveen rorzulegen und über die systematische Eintheilung dieser Pflanzenfamilie zu sprechen.

En même temps M. ЈасовI nous fait part de son adhésion à la proposition de M. le prof. к. косн, (voir notre Programme, pag. 19, IX, 2), en promettant son secours, quand cette question sera mise' à l'ordre du jour.

XVIII. M. le prof. M. Willkomm, de Tharand, en Saxe, a l'intention de traiter les questions suirantes:

1. Sur la distribution géographique et les formes du Pinus Montana Mill. et de quelques autres pins de l'Europe.

2. Sur le Chêne-liège de l'oceident (Quercus occidentalis $G(a y)$ et sa distribution géographique en Espagne et en Portugal.

3. Welches Verhältniss besteht zwischen der Zahl und Anordnung der Spaltöftinungen und der Ernährung der Pflanze aus der Atmosphäre, und welchen Einftuss 
würde die Ermittelung dieses Verhältnisses auf die Behandlung und Erziehumg bestimmter Culturpflinzen haben?

4. Ueber den schädlichen Einfluss des Hütten- und Steinkohlenrauchs auf die Pflanzen, besonders auf die Laubund Nadelhölzer, und üher dic in dieser Beziehung im botanischen Garten zu Tharand angestellten Versuche.

XIX. M. Meissner, prof. de botanique, à Bûle, annoncera plus tard les communications qu'il pourra présenter au Congrès.

XX. M. D. Clos, prof. à la faculté des sciences, directeur du Jardin Butanique, à Toulouse, regrettant virement que d'impérieuses fonctions le prirent du plaisir de participerau Congrès, nous adresse les communications suivantes:

Il serait bien à désirer notamment, cue l'on pût reconnaître dans les plantes diöques, d'après les caractères extérieurs des graines, celles qui doivent domer naissance à des individus mâles ou ì des pieds femelles, si tant est que ces caractères existent. Les recherches D'A UTENRIETH et DE MaUz derraient être reprises, et M. BELHomme a été heureusement inspiré en portant cette question derant le Congrès.

$\Pi 1$ en est une autre qui touche de très-près à celle-là, et auquel il appartient anssi an Congrès de répondre définitivement; je veux parler de la parthénogénèse, au moins en ce qui concerme le Coelebogyme ilicifolia Sm. Entre M. KARstex qui a trouvé un cinquième des fleurs de cette espèce hermaphrodites, et MM. ALEx. BRAUN et. METTENIUS qui sur plus de 100 fleurs n'ont pu en rencontrer une seule possédant les deux sexes, quelle opinion doit se faire le physiologiste?

J'ai moi-même fait commaitre en 1857 chez plusieurs espèces d'Atriplex l'existence de deux sortes de graines verticales, les unes noires, les antres rouges, toutes renfermant un embryon bien conformé, mais les dernières seules possédant la faculté de grermer (Bullet. de la soc. botanique de France, tom. IV, pag. 441). Quelle est la cause de cette 
différence? les graines noires ne pourraient-elles g'ermer qu'ì une époque plus ou moins éloignée du moment de leur complète formation? C'est ce que j'ignore et ce que peutêtre quelque membre du Congrès sern en état d'élucider.

\section{QUESTIONS DE BOTANIQUE APPLIQUÉE} ET D'HORTICULTURE.

I. M.Ferd.Jühlke, d'Erfurt, a l'intention de parler:

1. Ueber die Ergebnisse der Luft-Drainage, nach HOOIBRINK.

2. Ueber die Naturalisation und Localisation unserer Culturpflanzen.

II. M. J. C. Weyhe, directeur du jardin botanique de Düsseldorf, nous écrit:

" $\mathrm{Si}$ au Congrès international il y a une section pour l'architecture des jardins, ma spécialité, je ne manquerai pas de prendre part aux délibérations."

III. M. Lecocq, de Clermont-Ferrand, prof. d'hist. nat. à la faculté des sciences, corresp. de l'Institut etc., se propose de prendre part à:

La question des fécondations artificielles.

IV. M. Bommer, conservateur des collections de la Soc. Royale d'hortic. et de bot. de Belgique, it Brurelles, prendra part au Congrès en lui communiquant:

Quelques remarques sur la panachure et lit colorition du feuillage au point de vue horticole.

V. M. Chs. Baltet, rédacteur de la Rerne Horticole à Troyes, propose la question suivante:

Y-a-t-il dégénérescence chez les plantes bulbeuses herbacées on ligneuses, les arbres fruitiers on d'ormement?" Duns le cas affirmatif, indiquer les causes de la dégénéres. cence (détérioration) et les moyens de la combattre. 
$\Pi$ se propose de traiter cette question en ce qui concerne les arbres fruitiers et d'ornement.

VI. M. Van Hulle, jardinier en chef du Jardin Botanique de Gand, traitera les questions suivantes:

1. Les systèmes de taille d'arbres fruitiers.

2. La mission des jardins botaniques.

Quant à la première question, M. VAN HULLE tâchera de faire mieux apprécier les tailles raisonnées, si genéralement appliquées en France et adoptées arec tant d'enthousiasme en Belgique, mais qui, à son aris, trourent si peu d'adhérents dans les pays voisins. M. Rodigas père ou M. PYNAERT répondront aux Allemands, M. DE beUCKeR aux Hollandais, et lui-même aux Anglais.

VII. M. Alfred Wesmael, directeur de la Société anonyme d'agriculture, d'horticulture et de zoölogie à Mons, traitera de

Quelques points sur les hybrides des cultures.

VIII. M A. Stelzner, horticulteur it Gand, communiquera au Congrès:

Sesexpériences pratiques surl'hybridation des Fougères.

IX. M. Ed. Pynaert, architecte de jardins, à Gand, à l'intention de parler:

„De la nécessité de recourir à la réproduction par voie de semis pour créer des plantations fruitières rustiques."

X. M. le prof. F. Cohn, de Breslau, se propose de discuter:

Sur la culture des Algues marines.

XI. M. R. Caspary, prof. de botanique à Königstierg, annonce comme sujet sur lequel il propose la discussion:

„Des hybrides obtenus par la greffe, sur l'existence desquelles il a fait des observations décisives et presque inconnues."

XII. M. Chatin, prof. de botanique ì l'école Impériale de pharmacie à Prisis, a l'intention de parler:

1. Du cresson (Nasturtium officinale), sa culture et ses applications. 
2. Du Bromus Schraderi pour la formation des prairies artificielles.

XIII. M. J. M. Kraayenbrink, jardinier au château royal du Loo annonce les questions suivantes:

1. Welke zijn de oorzaken, waardoor de teelt der Oranjeboomen niet meer die goede resultaten oplevert als eertijds; welke middelen kumnen aangewend worden om dit verval te herstellen?

2. Waaraan is het toe te schrijiven, dat de liefhebberij roor Erica's, die bij eene nitgebreide verzameling ons altijd bloeijende toonen, zoo weinig algemeen is en hoe langer hoe meer verflaauwt?

XIV. M. J. Münter, prof. de botanique ì Greifswald, indique la question suivante:

Les malarties des plantes cultives, par exemple les siliques des prumes, la nielle des Graminées (du blé etc.), le nouveau parasite des fenilles acerenses du Pinus Sylrestris L. (Caloma pinitorqum R. Br.), la vraie nature du Sclérotium etc. etc., sur lesquelles il se permettra d'expliquer plusieurs nouvelles études.

XV. M. le Dr. H. Karsten, à Berlin, amonce un discours:

Ueber die Verbreitung und das Clima der Cinchonen, so wie über die daraufzu stützende Culturmethode derselben.

XVI. M. le prof. Karl Koch, à Berlin, nous écrit:

Eine der wichtigsten Fragen ist auf alle Fille die Handhabung der Nomenclatur. Der Gebrauch hat sich schon lange geltend gemacht, dass echte Pflunzen-Arten einen lateinischen oder einen dem Namen eines rerdienten Botanikers oder Pflanzenfreundes entlehnten Namen erhalten, Gartenformen hingegen mit anderen zufälligen Benenmungen ohne lateinische Endungen belegt werden. Ich halte die Sache für ausserordentlich wichtig, dass sie bei einer solchen Gelegenheit als der Amsterdamer Kongress darbietet, zur Sprache lkommt, um definitiv geregelt zu werden. 
XVII. M. F. Rodigas, M. D. prof. à St. Troud, se propose de prendre part aux discussions sur:

La variation de l'espèce.

La panachure.

XVIII. M. le prof. Reichenbach Fils, à Hambourg, nous écrit:

Eine Frage, die wohl zünden würde, wäre die Nomenclaturfrage. Es liesse sich etwa ein allgemeiner Vortrag über die ganze Frage ausarbeiten, vielleicht ein specieller Anhang über die von mir für die Wissenschaft proponirten Aenderungen bei Orchideen.

Et encore:

Eine höchst wichtige Frage wäre Anregung, Unterstützung fähiger Gehülfe der Gärtner:

1. durch Stipendium seitens der Gesellschaft;

2. durch Preise bei Ausstellungen.

XIX. M. Em. Rodigas, prof. de l'Ecole d'Hortic. de l'Etat à Gendlungge-les-Gand, a l'intention de parler:

1. De l'influence de l'humidité sur les végétaux et de la nécessité de régler celle-ci dans les serres.

2. De l'enseignement de l'horticulture.

XX. M. Ed. Morren, prof. à Liège, fera quelques communications concernant:

La coloration des plantes, la panachure, l'acclimatation, l'hybridation.

XXI. M. A. Nedzelsky, Dr. ès sciences, botaniste à l'université de Moscou, nous écrit:

„Je compte présenter un travail sur les progrès de l'arboriculture fruitière et de la pomologie théorique, ainsi que sur l'avantage que pent avoir pour la pomologie et pour la classification des fruits l'étude de la physiologie et de l'anatomie des plantes, en particulier de la famille des Rosacées."

XXIT. M. H. Hoffmann, prof. de botanique à Giessen, propose les questions suivantes:

1. Entstehungsweise der Gartenvarietäten. 
2. Rückschlagen und Permanenz der Racen (Pisum, Oenothera amoena, Brassica, Linnm usitatissimum, Solitnum tuberosum, Triticum vulgare.)

3. Werth der Herbstaussaat im Gegensatze zur Frühlingsaussaat.

4. Woher stammt die cultivirte gelbe-Rübe (Daucus carota)?

5. Woher stammt der Lattich (Lactuca sativa) und seine Formen?

6. Woher stammt der $K$ ohl (Brassica oleracea Napus und Rapa) und dessen Formen?

7. Es ist dringend zu wünschen, dass die Gärtner und Botaniker durch mehrjährige Beobachtungen der Zeit der allgemeinen Blïthe, Blïthentalender für ganz Europa feststellen. Hiedureh wird der relative Character des Sommers einer Gegend ermittelt. Wenige Pflanzen, welche aber durch die ganze Vegetationszeit blühen und überall vorkommen, sind hiezu auszuwählen (1. Aesculus Hippocastanum. 2. Castanea vulgaris. 3. Catalpa syringifolia. 4. Colchicum autumnale. 5. Crocus sativus. 6. Lilium candidum. 7. Persica rulgaris. 8. Prunus avium. 9. Pyrus communis. 10. Pyrus Malus. 11. Ribes grossularia. 12. Sambucus nigra. 13. Syringa vulgaris. 14. Vitis vinifera).

8. Es mögen die Gärtner aus ganz Europa aufgefordert werden, anzugeben, welche empfindlichern Culturpflanzen an ihrem Wohnorte im Freien unbedeckt "iberwintem. Diess ergiebt die sichersten Rühkschlüsse bezug des Winterklima'sderbetreffendenGegenden, und erlaubt wichtige Rückschlüsse bezüglich der Cultur fremder und neuer Pflanzen. Als ein solches Maassstab können dienen: Phönix dactyliferı, Opuntia rulgू., Agare americana, Cupressus sempervirens, Laums nobilis, Cedrus Libani, Viburnum Tinus, Tlex aquifolium, Rhododendrum ponticum.

XXIII. M. Rob. Warner, ì Londies, nous ćcrit: I will propose for discussion a practical question, a 
question on which I desire information, viz: What has been the experience of continental horticulturists as to the nerits and dewerits of donble glaziny plunt and finithouses?

XXíV. H. Ed. André, à Passy-les-Puis, amnonce un mémoire:

Sur les jardins publics et les jardins paysagers.

XXV. J. le Dr. E. Regel, direteur du Jardin Botanique à St. Petersbourg, à l'intention de parler:

1. Sur l'espèce et les variétés des plantes.

2. Ueber die Betulueen, deren Verbreitung, Formenbildung, u. s. w.

XXVI. M. G. Geitner, horticulteur à Planitz, en Saxe, se propose de discuter, s'il y a lieu:

Ueber die Consolidirung des Gärtnerstandes.

XXVII. M. Chs. Erbschloe-Muller, ì Elberfeld, nous adresse:

Quelques communications sur la culture des plantes de serre et sur le chauffige ì eau chande, dont il vante les avantages.

XXVIII. M. T. L. de Puydt, ì Mons, regrettunt vivenent de ne pouvoir assister alu-Congrès, nous promet:

Un petit travail sur une question d'horticulture.

XXIX. M. J. H. Krelage, ler secr. de l'expos. univ. d'hort., it Harlen, se jropuse de discuter, s'il y a lieu:

Sur les avantages l'nne confédération des differentes sociétés d'horticulture d'un méme pays, à l'instar de ce qui se trouve en Belgique.

XIX. M. Bossin, Mul grinirier-pépiniériste à $P a-$ ris, a l'intention de soumettre au Congrès:

Une proposition sur la nécessité d'adapter des adjectifs latins aux variétés fixes des plantes potagères.

XXXI. M. M. Kolb, jardinier en chef du Jardin Bot. i Munich, se propose de faire quelques communications: Sur le système de vigne de Hoorbrink.

XXXI. M. Ch. Auband, de Bincelles, compte traiter la question: 
Des jardins botaniques, leur but, leur peu d'utilité jusqu'à ce jour; les avantages nombreux qui résulteraient pour li botanique d'un meilleur ordre de choses à leux '́gard.

Ensuite, si le temps le permet, il donneri quelques extraits d'un ouvrage en voie de préparation:

Sur les fécondations artificielles.

XXXIII. M. C. Glym, horticulteur à Utrecht, indiquera plus tard les questions qu'il désirerait voir discutées par le Congrès.

XXXIV. M. J. H. Schober, propriétaire à Utrecht, promet également des communications ultérieures.

XXXV. M. T. d'Anouilh de Salies, à Fontainessur-Saône, désire proposer au Congrès les questions suivantes:

1. Des moyens d'arriver avec certitude, dans un délai le plus restreint possible, à la déstruction complète du puceron lanigìre, ce fléan des pommiers dans beauconp de contrées.

2. Des avantages que peut offrir dans bien des cas le greffage en fente, au coin du feu ou sur les genoux.

3. Des moyens de tirer partie, au point de vue de la production, des arbres fiuitiers en général, et notamment de ceux à pépins, arbres plus ou moins âg'és, arrivés à l'infertilité, même à une sorte de décrépitude, par l'effet de mauvais traitements ou par défaut de soins.

4. De l'hibillage des racines dans la transplantation des arbres en général, et notaminent daiıs ceux qui sont adultes et à feuilles caduques.

XXXVI. M. le prof. Reichenbach, de Hambourg, propose la question suivante:

Ob die netren Etiketten ron Porcellan und Schiefer irgend wo und unter welchen climatischen Verhältnissen sich besser bewähren als die alten von verderblichem Eichenholz oder dito Blech und dito Eichenholz? 


\section{P R O J E T}

DE

\section{REGLEMENT DU CONGRES,}

(adopté par les deux Sections, le 7 Avril 1865.)

Article 1.

Le Congrès s'occupe de questions de botanique et d'horticulture.

ART. 2.

Le Congrès se divisera en deux sections, l'une pour la botanique, l'autre pour l'horticulture.

ART. 3.

La première réunion générale aura lieu sous la présidence d'un des membres de la commission-directrice de l'exposition. Dans cette assemblée les membres déclarent dans quelle section ils désirent être inserits. Il est permis aux membres d'me section de prendre part nux clélibérations de l'autre.

\section{ARt. 4 .}

Le bureau de chaque section, composé d'um président, d'un vice-président, de deux ou trois secrétaires et de quelques membres, est nommé par l'assemblée.

Art. 5.

Le choix rles sujets à discuter dans les assemblées dépend de la libre décision de chaque section.

ArT. 6.

Les membres, qui ont l'intention d'ouvrir les délibérations sur une question, sont priés de bien rouloir en informer le président de la section avant l'ouv erture de la sćance. 
ART. 7.

Le bureau décide sur l'ordre dans lequel les différentes questions annoncées seront traitées.

ART. 8.

Les orateurs ne peuvent parler sur une question que durant 15 minutes, à moins que l'assemblée n'en décide autrement. La parole ne pourra etre accordée à la méme personne plus de deux fois sur le même sujet.

ART. 9.

Les secrétaires (auxquels pourront étre ajoutés des bureaulistes de la part de la commission-directrice) se chargent de la rédaction des délibérations et descommunications.

Ils remettent an secrétaire-général le compte-rendu de leur section aussitôt que possible, au plus tard deux mois après la clôture du Congrès.

ART. 10.

La commission-directrice publie en limgue française un compte-rendu du Congrès. Un exemplaire en sera offert à chacun des membres.

\section{ARt. 11.}

Les membres, qui désirent que leur's communications soient insérées in extenso dans le compte-rendu dn Congrès, sont priés d'en déposer la notice sur le bureau de la section, vin bien de la faire parrenir an secrétaire au plus tard un mois après la clôture du Congrès. 


\section{CONGRES INTERNATIONAL}

\section{DE BOTANIQUE ET D'HORTICULTURE,}

CONVIÉ

à AMSTERDAM le 7, 8, 10 et 11 Avril 1865.

Première réunion générale. - Séanue d'ouverture.

Le 7 Avril 1865, à trois hemres de relevée, environ trois cents membres du Congrès se trourent réunis dans la Salle aux tableaux du Palais d'industrie à Amsterdam. La commission organisatrice occupe le bureau.

M. C. A. J. A. Oudemans, président, prononce le discours suivant:

\section{,Messieurs!}

Avant d'entamer les questions de hant intérêt qui nous annènent en ce liei, souffiez que je vous exprime ma vive satisfaction de vous voir assemblés en cette enceinte, où, prenant la science pour guide de ilos explorations, nous allons tâcher d'étendre sun domine par nos entretiens. Non que je me dissimule toute l'importance de la tâche que je me suis imposée, en prenant l'initiative lì où tant d'autres parmi vous, forts de leurs talents éminents, de leur renom et de leur éloquence, auraient pu réclamer à plus juste titre cet honneur; mais, membre de la direction de cette exposition universelle, je n'ai pas osé me soustraire à un devoir, que la dignité dont je suis revêtu en cette ville, semblait me prescrire d'elle-même. Ne croyez cependant pas, messieurs, 
que je ne considère pas comme une faveur insigne d'avoir été élu pour vous adresser le premier la parole dans le cercle plus restreint de nos travaux. Au contraire, je me félicite et je le regarde comme le plus grand honneur auquel puisse aspirer un homme de science. Mais les circonstances qui ont contribué à me le faire obtenir me justifieront, j'espère, à vus yeux, si je viens faire un appel à votre indulgence pour les quelques paroles que je vais vous adresser.

Le plan d'organisation d'une exposition internationale d'horticulture, projeté, mûri et exécuté pour la première fois en Belgique, avait déjà rencontré les sympathies de toutes les nations et disposé plusieurs d'entre elles à l'imiter, comme cela se fait à présent chez nous; mais ce qui en rehausse l'éclat et en étend démesurémerit la tendance, c'est l'idée de lier à cette exposition un Congrès scientifique, idée spécialement patromée par ceux qui avaient assisté au dernier Congrès belge; idée, digne de la nation qui montre par tous ses efforts, qu'elle attache un haut prix aux progrès des sciences et qu'elle ne recule devant aucun sacrifice pour en accélérer la marche.

Nous anssi, nous autres Néerlandais, avons applaudi aux considérations qui ont réglé la tenue du Congrès belge, et nous n'avons pas cru pouvoir mieux lui témoigner nos sympathies que de l'imiter et de joindre, comme à Bruxelles, la partie scientifique à la partie pratique, la botanique à l'horticulture.

Entrer dans l'exposé des raisons, qui justifient cette alliance, serait oisenx devant vous, qui, par votre seule présence dins cette enceinte, domnez la preuve lir plus convaincante que vous êtes pénétrés de ces motifs et que vous les approuvez.

A Dieu ne plaise que j'abuse de votre patience par cette énamération; j'aime bien plutôt constater que les résultats obtenus par les Congrès botaniques en général et spécialement par celui de Bruxelles, non seule- 
ment n'ont pas trompé l'attente qu'on en avait conçue, wais l'ont réalisée en tous points, si non surpassée.

Permettez moi, Messieurs, en citant Bruxelles, d'y ajouter une observation.

De même que chez nos voisins méridionaux, on a désigné aussi chez nous la capitale duroyaumecommel'endroit où deviait se tenir la seconde solemnité consacrée à Flore et à Pomune, et qui, comme nous l'espérons, ne le cèdera en rien à lir première; elle aussi est destincée à voir déposer aux pieds de ces denx divinités tout ce que l'Europe possède de plus rare et de plus intéressant dans ses jardins; à voir l'élite de ceux qui ont acquis quelque gloire dans le domaine de la botanique et de l'horticulture, appelée à scruter avec l'veil de la science les trésors du monde végétal apportés de près et de loin; où tous ceux qui s'intéressent à l'horticulture seraient invités à être témoins des résultats surprenants que la sagacité, l'industrie, la constance des efforts ont su amener dans cette partie de la science.

Mais pourquoi avoir choisi la capitale du royaume? Scrait-ce à cause de son Palais d'Industrie qui, possédant des salles plus spacieuses, plus élégantes que d'autres villes, dispose par là des moyens plus efficaces pour y tenir dignement des expositions internationales? Serait-ce à cause des étublissements importints, des trésors et édifices artistiques, rémis ici en bien plus grand nombre qu'en ancume autre ville du royaume, et qui offiriraient un attrait de plus à la curiosité des visiteurs étrangers? Ou bien parce que sà position géographique pouvait faire espérex un accès plus commode aux produits qu'on nous amènerait du dehors? On ne peut nier, Messieurs, que ces trois considérations ont été d'uin grand poids dans nos délibérations pour nous faire choisir Amsterdam comme le point central de nos conférences, mais on ne peut oublier que ce qui contribua en grande mesure à déterminer notre choix, fut le droit historique de la capitale de la Néerlande à l'homneur do vous recevoir dans ses murs, à celui de voir dresser sous 
ses auspices l'autel où nous sommes tous conviés à porter nos offiandes.

Car, remarquez le bien, Messieurs, s'il est recomnu qu'une des périodes de la renaissance de la belle science que nous cultivons - et elle en a connu beaucoup - date de l'époque, où l'on entreprit des voyages pour aller demander aux régrions lointaines, aux climats inhospitaliers et incivilisés, le tribut de leur monde végétal, où l'on inventa des moyens pour donner à ces produits du sol exutique me seconde patrie artificielle, Annsterdam, parmi toutes les villes de l'Europe, et spécialement parmi celles de la Néerlande, peut sous ce rapport poiter la tête haute et réclamer à juste titre l'honneur d'y avoir le plus efficacement coopéré.

Il ne faut pas s'en étomner; car cette renaissance coïncide avee l'époque, où Amsterdam occupait déjà une place éminente parmi les premières villes commerciales du monde, et où ses flottes marchandes et guerrières, couvrant toutes les mers, faisaient l'envie des autres nations, qui, bien qu'en possession d'un territoire infiniment plus étendu, durent le céder en puissance et en nombre de voiles à la petite république.

Je le répète: il ne faut pas s'en étomer; mais constatons aussi à la gloire éternelle de la capitale, qu'en amassant ses trésors, elle n'a jamais perdu de vue l'intérêt de la science et qu'on pouvait bien appliquer à elle:

"Nulla etenim vel bellica vel oneraria navis ex portu educebatur, quin illius praefectis mandaretur atque praeciperetur, ut, quocunque appellerent, semina legenda, radices effodiendas, surculos depangendos, stirpes vasis imponendas curarent, eaque ommia incorrupta in hame civitatem deferrent, quae sine morầ pro soli coelique nativi naturà colebantur tam diligenter, ut etiam hîc florerent et fructus ferrent."

Ce que fit Amsterdam dans ces temps reculés et ce qu'elle ponmait faire encore plus tard comme reine des 
villes commerçantes, était alors tout-ì-fait impossible aux autres villes de notre royaume, et quoique nous ne puissions faire un reproche à ces dernières, de ne pas s'être montrées aussi actives là où il s'agissait de coopérer ì la découverte de nouvelles formes végétales, il ressort de ce que je viens de dire, que la capitale de la Néerlande peut depuis plus d'un siècle et demi prétendre à juste titre aux hommages de tous ceux qui s'intéressent à la botanique.

Le lieu, où, depuis l'année 1682, les plantes nouvellement importées sont déposées et soignées, est ce même jardin botanique qui orne encore aujourd'hui notre capitale; établissement institué et entretenu non aux frais de l'état, mais à ceux de la ville elle-même, preuve de plus de l'esprit d'indépendance qui a toujours présidé dans Amsterdam, lorsqu'il s'agissait de la propagation de notre belle science. L'on pourra se faire une idée de la richesse de cette collection en plantes exotiques dans les temps écoulés et par le célèbre recueil des commelin, dont les dessins originaux se trouvent à la bibliothèque de la ville, et par les paroles de BREYNIUs qui, ayant visité en 1692 notre Hortus Botanicus, s'exprima ainsi:

" Se per humc hortum spatiantem, exquisitissimas plantas summa cum admiratione et jucunditate conspexisse, atque, utrum in Indiam an vero in Americam esset delatum plane nescire."

Cependant, Messieurs, notre science aurait peu profité, si l'on s'était borné à recueillir et cultiver seulement ces plantes exotiques. Pour rendre le travail rraiment fructueux, il fallait commencer par coordonner et décrire les collections, ouvrir des cours publies, pratigner des échanges, et comment ces pensées auraient-elles jamais pu être réalisées, si l'on n'attachait un homme de la science au jardin botanique? Conraincu de l'urgence et de la laute portée de cette mesure, la régence de la ville ne recula devant aucun sacrifice; elle vota les fonds nécessaires 
pour une chaire de botanique; et grâce à cet excellent esprit, à ce vrai libéralisme, nous pouvons nous glorifier d'avoir vu des hommes tels que JEAN et GASPARD COMMELIN, BLASIUS, RUYSCH, HOTTON, les deUX BURMANN, BONDT, VROLIK, DE VRIESE et MIQUEL, vouer ici leurs labeurs à la propagation de notre science.

Si je ne craignais d'outre-passer le temps dont je puis disposer, je m'étendrais encore sur tont ce qu'Amsterdam a fait depuis un siècle et au dehor's, en faveur de l'agriculture et de l'horticulture; mais je me bornerai seulement it constater que, parmi toutes les villes de notre pays, Amsterdam a vu naître la première société d'agriculture; que jadis on ne trouvait nulle part des villas et des campagnes en aussi grand nombre et aussi somptueuses, et qui, reflets de la grande prospérité de la ville, créées et entretenues par les plus riches commerçants, exerçaient une influence marquée sur le développement de l'horticulture dans la Néerlande; que de nombreux ouvrages fort coûteux, sur la botanique, qu'on ne pouvait publier ailleurs, ont ru le jour dans cette ville; qu'Amsterdam enfin a dans les derniers jours, au moyen d'un subside généreux, puissamment concouru au succès de notre entreprise.

Certes, Messieurs, on ne peut nier qu'Amsterdam a perdu beancoup de son ancienne splendeur, que son commerce n'a pas su se maintenir à sa hauteur primitive et que l'envie de s'occuper de choses auxquelles on n'était pas directement appelé, ne se soit affaibli arec la marche rétrograde du commerce; que la Société d'agricultuxe, quelqu'indépendante que fût sa position, fut dissoute et ne forme maintenant qu'une branche d'une société générale, dont le cercle d'opérations embrasse tout le pays; que les amélinrations dans les moyens de transport et la construction des voies ferrées, en facilitant et encourageant les voyages, entraina la décadence des maisons de plaisance. Mais tout ceci ne nous autorise nullement à oublier les services rendus. Au contraire, la recommaissance exige qu'aujourd'hui encore 
nous rendions hommage à Amsterdam de ce qu'elle a fait daus les siècles écoulés pour la prospérité de la botanique et pour la réalisation de ses belles théories; comme en des temps, déjà fort loin de nous, elle a maintenu sous ce rapport l'honneur national.

Ainsi, nous voilà réunis, Messieurs, non seulement dans la capitale de la Néerlande, mais aussi dans un lieu, qui, plus que tout autre endroit de ce pays, a toujours pris l'initiative là où la botanique et l'horticulture réclamaient du secours. Arec une vive satisfaction, je puis constater que la direction de l'exposition internationale a aussi fait valoir ce motif, quand elle vous convoqua dans cette ville pour nous aider dans l'exécution de nos vastes desseins. Nous souhaitons ardemment que ce choix obtienne aussi votre sanction, et que vous puissiez retourner dans vos foyers arec un agréable souvenir de la capitale de ce pays de liberté, en emportant la conviction que vous laissez ici des cœurs reconnaissants, qui sauront toujours se rappeler que vous avez bien voulu, pour les rendre plus fiuctueux, vous associer à leurs efforts.

Et maintenant, Messieurs, à l'œuvre! Les questions soumises par un grand nombre d'entre vous à notre examen, et les communications promises sur plusieurs sujets intéressants nous prescrivent le devoir d'utiliser nos moments. Eclairez nous du flambeau de vos connaissances. Communiquez vous réciproquement les riches trésors de ros expériences. Servez l'un à l'autre de guides et de soutiens dans le sentier à parcourir, et puisse du sein de nos études mutuelles croître la palme, dont la science aime à se tresser sa plus belle couronne!" (Applaudissements.)

M. le Président annonce à l'assemblée que la commission organisatrice du congrès, jugeant sa tâche remplie, s'est décidée à se retirer. Elle prie les membres de bien rouloir désigner une autre commission, ì laquelle sera confié le soin de diriger les conférences scientifiques. 
M. d'Anouilh de Salies (France) fait la proposition suivànte:

\section{Messieurs!}

„Frappé des magnificences si habilement groupées dans ce palais splendide et si elégamment déroulées sous nos yeux, nos facultés intellectuelles sont vivement excitées. Les sensations, les sentiments se succèdent avec la plus grande rapidité, et sont, jusques à un certain point, un obstacle à la formation complète de nos idées. Nous ne formulerons donc pas aussi bien que nous le désirerions, ce que nous voulons exprimer comme développement de notre proposition. Mais un sentiment de bien général nous réunit ici de toutes les parties de l'Europe dans un but commun: le développement de l'horticulture. C'est là, Messieur's, de la véritable confraternité, qui peut, à juste titre, être appelée pacifique. Osant espérer que ce noble sentiment domnera accès à votre indulgence envers nous, nous rentrons immédiatement dans le vif de la question, sans plus de commentaires.

„Notre proposition est celle-ci: De la nécessité de composer le burean de membres, pris dans la commission organisatrice du Congrès, ou tout au moins parmi les membres du Congrès appartenant au royaume des PaysBas, à l'exclusion des autres états. Cependant des conseillers de ces demiers états pourront être adjoints au bureau, mais sans voix délibérative."

Cette motion étant complexe, divisons-là en deux parties pour en faciliter la discussion:

1. Prendre le bureau uniquement parmi les Hollandais.

2. Adjoindre au burean des conseillers non-délibérants. Examinons la première partie: Bureau exclusivement Néerlandais.

La grande habileté, la science incontestable qui a présidé à cette magnifique exposition universelle, les soins inimaginables, la bienveillante hospitalité, dont ont été l'objet les hommes et les choses, nous sont un sûr garant que nous ne pouvons remettre le soin de recueillir, de 
co-ordonner nos délibérations, ¿̀ des mains plus dignes à tous égards que celles des ordonnateurs de cette fête d'horticulture sans pareille au monde.

D'ailleurs après la clôture du Congrès, un grand trarail restera à faire: rédigger, co-ordonner, imprimer. Dans ce but, Messieurs, les membres du bureau auront fréquemment ì se réunir. Et comment se rétmiraient-ils, s'ils étaient éparpillés dans toutes les parties de l'Europe? Vous voyez déjà, Messieurs, l'impossibilité de cette réunion. Mais objecterait-on pent-être: La correspondance n'est elle pas là? La correspondance? Mais c'est le tiraillement, la lenteur, quelquefois l'avortement, au lieu de l'enfantement. Done, nous devons prier MM. de la commission organisatrice d'ajouter encore ì notre reconnaissance déjà acquise, à celle de leur patrie, en acceptant la difficile tâche de composer seuls le bureau définitif du Congrès. J'arrive maintenant ì la seconde partie de ma proposition: Conseillers adjoints.

Il pourra advenir que le bureau ait accidentellement ì s'éclairer sur des points, sur des objets particuliers i telle ou telle nation. Aussi croyons-nous qu'il serait utile de nommer des conseillers parmi les étrangers au royaume Néerlandais. Ces conseillers, ainsi que je l'ai dit plus haut, n'auraient que roix consultative. Après la clôture du Congrès, si le bureau avait à les consulter, ils devraient répondre dans un bref délai que rous préciseriez. Faute par eux de le faire de suite, le bureau passerait outre.

Les auteurs des questions proposées au Congrès pourraient aussi être consultés, mais seulement sur les sujets relatifs à leurs propositions, et ils auraient dans ce cas, à répondre dans un bref delai.

Tel est, Messieurs, le court exposé que nous tenions ì sommettre ì ros appréciations dès l'ourerture de nos délibérations. Je considère celles-ci comme le germe d'un avenir fécond en résultats des plus utiles. La ville d'Amsterdain a ouvert là un champ immense, qui sera cultivé 
par l'univers horticole, et dans lequel, la Providence aidant, d'immenses moissons seront récoltées."

M. T. Cannart d'Hamale, (président du Congrès intern. de botanique et d'hort. de 1864 à Bruxelles) propose le maintien du bureau actuel. Cette commission, qui a arrangé tous les préparatifs, sera le mieux à même de régler les travaux.

Les acclamations unanimes constatent l'adhésion générale à cette proposition.

M. le président. „Je crois agir dans le sens de tous les membres de notre commission, en rous remerciant de votre bienveillance, que nous apprécions hautement. Cédant ì votre désir, nous nous déclarons prêts à remplir la tâche que volontiers nous aurions vu confiée à une commission nouvelle. Permettez-nous seulement de compter sur votre concours qui nous sera indispensable.

En premier lieu, Messieurs! je vous prie de bien vouloir procéder à l'élection des vice-présidents qui devront compléter le bureau, à moins que, pour gagner $d u$ temps, vous nous autorisiez à rous présenter une liste de candidats."

Cette dernière proposition est adoptée par acclamation.

M. le président. ,Eh bien, Messieurs! conformément à ce vote de confiance, nous arons l'honneur de vous nommer: MM. ANDERSSON, CASPARY, FÉE, FENZL, GÖPPERT, KOCH, LecocQ, jasters, Meissner, Miquel, piré, passerini, REGEL, REICHENBACH fils. (Applandissements prolongés).

Je prie ces Messieurs de bien vouloir prendre place à côté de nous au bureau."

Les acclamations sanctionnent le choix du bureau.

M. Barral (de Paris) propose que MM. cannart D'hamale et ed. morren, le président et le secrétaire du Congrès de Bruxelles, soient également élus vice-présidents et qu'ils premnent place au burean, afin de relier ce Congrès-ci à celui de l'amnée passée. (Appiaudissements). 
En conséquence M. le Président prie MM. CANNART D'HAMALE et MORREN de bien vouloir accepter la distinction, qui leur est offerte et qu'ils méritent à plusieurs titres.

M. Piré (de Bruxelles) demande la parole et prononce le discours flamand, qui suit:

\section{Weledelgeleerde Heeren!}

„,Het zij eenen der gelastigden der Belgische regeering vergund, bij de opening dezer geleerde zittingen, zijnen Noordschen broederen eenen hartelijken groet toe te sturen.

Ofschoon wij ten onzent de Fransche taal gedurig beoefenen, zoo wil ik nogtans eenige woorden uiten in uwe sprak, die tevens de spriak der meerderheid in België is.

Wanneer wij den voet op dezen alouden en alvermaarden grond der wetenschap plaatsten, ging ons het hart open bij de herimnering van al wat Holland voor tuinbour en kruidkunde gedaan heeft. Holland heeft in Europa lust en smaak rerspreid tot het kweken van rreemde gewassen. Holland heeft de eerste plantentuinen gesticht, en, in den beginne der $17^{\text {de }}$ eeuw, werd de Leidensche tuin de stapelhof aller gewassen des aardbols. Zoo heeft Leiden stellig het meest bijgedragen tot de ontwikkeling van land- en tuinbouw, niet alleen door de menigruldigheid zijner gewrassen, maar ook door de geleerden welke daar hun onderwijs gaven.

Laat mij hier tusschenlasschen, dat onze Dodonafus en cuusius aan die vermaarde school leeraarden en dat onze LOBEL als arts aan den persoon des Zwijgers gehecht was.

Inderdaad biedt ons dat groote tijdvak eene heerlijke reeks ran beroemde kruidkundigen, van geleerde tuinbouwers. Ilk noem u enkel BonTIUS, PAEW, vorstius, RHEEDE, RUMPHIUS, COMMELYN, PATL HERMANN, WACHENDORF, BURMAN, MUNTING, de beide RUGEN, BRUGMANS, VAN RoYen en den Europeeschen BoErHave, den beschermer van 
LINNAEUS, den grootsten aller natumrkundigen. Holland bezit den roem, dezen vorst der wetenschap gevormd te hebben; hier werd Linnaeus onthaald, hier vond hij de middelen zich te verheffen. In 1735 ontving hij hier den doctorgraad in de medicijnen. Boerhave die zijn genie erkende, beval hem aan CLIFFORD, en deze benoemde hem tot bestuurder van zijnen plantentuin te Hartecamp. Hier bevond zich uinnaeus in eenen der schoonste hoven Europa's, rijke herbaria en eene uitgebreide boekerij te zijner beschikking hebbende, en kon zich uitsluitelijk met gewasstudiën onledig houden. Ook verschenen zijne eerste werken in Holland, zijn tweede vaderland.

Eenen boERHave tot zijnen zoon gewonnen, eenen uINNAEUs groot gebracht te hebben, zijn die twee titels niet voldoende om een klein land tot den hoogsten adelstand in de wetenschap te verheffen?

Ware ik niet te midden eens volks dat zijne geschiedenis kent, dan zou ik u spreken van Nederlands vermaarde tuinen in Amsterdam, Utrecht, Franeker, Harderwijk, maar een eenvoudig verslag over die gewasverzamelingen en over de mannen die er de natuurwetenschap onderwezen, vereischte een gansch boekdeel.

Omtrent 1752 wilde Keizer Frans I in Duitschland den lust opwekken tot plantenkumde en hofbouw, en riep uit Holland den beroemden JAçUin met twee rerdienstelijke bloemisten, ADRTAAN STECKHOVEN ran Leiden en den Delftenaar van DER schot. Zoo groot was toenmaals Hollands faam op het gebied van Flora.

Sedert dien verliepeene eeuw en Holland heeft niets van zijnen ouden roen verloren; deze werd gestaafd door eene reeks van kruidkundigen, die verdiende faam erlangden en heden nog een sieraad onzer schoone rereeniging zijn.

En thans, nadien wij Amsterdams prachtige tentoonstelling in haar geheel en in hare bijzonderheden bewonderd hebben, thans moeten wij bekennen dat Holland ook op het praktisch gebied zijnen luister bewarde. 
Dit bewijst ook de schitterende vergadering, die uit zoo vele gewesten Europa's hier samenstroomde; dit bewijst de bescherming warmede Humne Majesteiten de Koning en de Koningin der Nederlanden en hum edele zoon, de Prins van Oranje, de matschappijen van kruidkunde vereeren, hier wat de wetenschap nog haar vaderland erkent, walarin wij zoo als vroeger de groote LrNNaEus, zoo gulhartig onthaald worden.

In tegenwoordigheid van zoo vele tolken der wetenschap, kan ik met fierheid zeggen, dat er eene innige verwantschap bestaat tusschen Noord-en Zuid-Nederland; hebben wij vorig jaar in onze hoofdstad de schatten van Gent, Antwerpen en Brussel tentoongesteld, wij vinden heden hier den bloei van Amsterdam, Haarlem, Leiden en vele andere steden. Die verwantschap bepaalt zich niet bij onze gelijke liefde roor dezelfde wetenschap; kunst en wetenschap met haar bekoorlijk schoon, vereenigen en verbinden ook de harten harer vereerderen met eenen heerlijken bloemenband.

Hopen wij dat deze bloemige broederband door den hemel en door de vorsten beider landen beschermd en bij onze toekomende vergadering nog vaster toegehaald worde." (Applaudissements.)

Au nom de la commission M. le Président remercie M. PIRÉ de ses bienveillantes paroles et le prie d'assurer ses compatriotes de l'estime et des bons sentiments des Hollandais.

M. le Président invite les membres à se diviser en deux sections, l'une pour la botanique pure l'autre pour l'horticulture, et à se rendre aux salles, qui leur sont réservées au premier.

Chaque section est priée de procéder d'abord à l'élection de son bureau, afin de pouvoir dès demain ouvrir les déliberations sur les questions posées.

La séance est levée à 4 heures. 


\section{SECTION DE BOTANIQUE PURE.}

SÊANCE du 7 Avril 1865, à 4 heụres de relevée.

La séance-est ouverte par M. le prof. Suringar de Leide, nembre de la commission organisatrice du congrès. Après l'échange de quelques observations de la part de Messieurs Chatin, Féz, Mrquel, Momiren ete. la section nomme:

Président:

Vice-Présidents:

Secrétaires:
M. FEE,

MM. FENZL et MIQUEL,

MM. MORREN et OUDEMANS.

La section adopte le projet de règlement qui lui est soumis par la commission orģanisatrice. Elle règle l'ordre de ses séances.

M. Morren fait hommage au Congrès de quelques opuscules de sa main, savoir:

Détermination du nombre des stomates chez quelques végétaux indigènes ou cultivés en Belgique.

Chorise du Gloxinia speciosa.

Hérédité de la panachure.

Remacue Fosch, su vie et ses oeuvres.

Charles Morren.

L'acclimatation des plantes.

La lumière et la végétation.

Bulletin du Congrès international d'horticulture à Bruxelles, les 24, 25 et 26 Avril 1864.

Bulletin de la fédération des sociétés d'horticulture de Belgique, Gand, 1864.

La séance est levée et l'on ạjourne la réunion an lendemain à 9 heures du matin. 


\section{SECTION DE BOTANIQUE PURE.}

SÉANCE du 8 Avril 1865, à 9 heures du matin.

LE T3UREAU SE COMPOSE DE:

MII. FEE, Président,

FENZL et MIQUEL, Vice-Présidents, MORREN et OUDEMANS, Secrétaires.

M. le Président ouvre la séance.

Le procès-verbal de la séance précédente, lu par M. Morren secrétaire, est approuvé.

M. Louis Bouchard (de Puris), firit hommage au Congrès d'un opuscule, intitulé: Habitations à l'usage des cultivateurs, Paris 1863,8 .

Avant d'aborder les questions ammoncées, M. le Président propose de donner la priorité aux questions présentées par des membres présents et d'observer ensuite l'ordre chronologique.

L'assemblée ratifie cette proposition.

M. Lecoq (de Clemont-Feriand), deminde de ne pas suivre à la lettre l'article du règlement en vertu duquel chaque orateur ne peut avoir la parole que pendant un quart d'heure.

I. M. le Président met à l'ordre du jour la question proposée par M. BelHonne (No. 1 du Programme):

Est-il possible de supposer que la présence du Juniperus Sabina, sur lequel vient́ le Gymnosporangium fuscum, soit la cause del'apparition del'Aecidium cancellatum sur les poiriers?

M. Lecoq, ayant obtenu la parole, fitit connaître sur cette question les observations suivantes:

„Il y a déjà longtemps que l’on a attribué la présence de ce cryptogame sur les fenilles du poirier au voisinage du Juniperus Sabinu. Tous les faits que j'ai été à même d'ob- 
server tendent à confirmer' cette opinion. II n'est pas probable toutefois que l'apparition de l'Aecidium tienne à la présence du Juniperus mème, mais à celle d'un champignon parasite, le Gymnosporanginm fuscum qui vit sur le trone et sur les branches de la sabine et qui s'y montre après les pluies sous la forme de papilles gélatineuses et tremblantes.

Ayant eu occasion d'entrer dans un vaste jardin rempli de poirier's et les royant attaqués par l'Aecidium, j’ai pu trouver directement le pied de sabine en me dirigeant vers les arbres qui étuient le plus chargés de ce champignon.

Limoculation tentée sur les feuilles de poirier par le Gymnosporangium adulte réussit toujours.

Dans une autre circonstance, j'ai trouvé le Gymnosporangium sur le genévrier commun et tout ì côté j’ai rencontré des pieds d'aubépine, (Cratcogus oxyacantha) garnis d'Aecidium laceratum de sowERBr, et je ne serais pas étonné que d'autres f́ccilium de la même section, c'està-dire du Ceratites de LiNk, ne puissent aussi provenir de la transformation du Gymnosporanyium sur des sujets divers. Ce qui tend a me le faire supposer, e'est que l'Aecidium Berberidis peut aussi occasionner sur le blé une transformation nouvelle et se métamorphoser en Uiedo Rubigo ou en Uredo linearis.

J'ai cru devoir sigmaler ces faits pour appeler de nouveau l'attention des naturalistes sur ces singulières nétamorphoses, qui ne sont pas sans rapports avec celles que subissent les vers intestinaux et qui certainement peuvent aussi servir à l'étude de la transformation des espèces selon les milieux dans lesquels elles vivent."

M. Hoffmann (de Giessen) fait observer qu'à moins d'expériences ultérieures on ne pent rejeter l'opinion de M. вецномие, qu'il y ait cohérence entre le Rostelia cancellata et le Gymnosporangium Juniperi. De même les relations, qu'on soupçonnait depuis longtemps entre l'Uredo 
Rubigo ver'a et l'Aecidium Berberidis sont entièrement confirmées par les expériences de M. DE BARY. Pour de plus amples détails l'oriateur renvoie à ses notes mycolugiques, qui se trouvent dans la Botan. Zeitung de vox sонL et SCHLECHTENDAL. (1865 p. 100 et 122).

M. Fée (de Strastourg) croit qu'un mème germe d'organisme peut domner lieu ì des formes différentes suivant le milieu sur lequel il se développe.

II. Sur la $2^{\text {de }}$ question de M. BeLHonne:

Peut-on reconnaître à la forme extérieure des graines de plantes diö̈ques, du Cannabis par exemple, si la graine donnera un sexe mâle ou un sexe femelle comme cela se voit chez certains oisean; dans le cas on le fait serait reconnu, quels en seraient les caractères?

M. Karsten (de Berlin) miésente les observations suivantes:

„,Diese interessante Mittheilung des Herm вецномим, dass das Geschlecht der entwickelten Hanfipflanze schon an dem im Samen eingeschlossenen Keim vorbereitet sei, erregte in mir den Wunsch, die von Herru вецномие zur Begründung dieser Ansicht angestellten Versuche zu wiederholen.

Aber schon bei dem Begim dieses Vornehmens stoutite dasselbe, denn es war mir nicht möglich die von Herrn веLнопив angegebenen Merkzeichen der weiblichen und männlichen Cannabisfrüchte zu erkennen. (Zum Beweise legt Herr karsten der Sitzung einige Hanfsamen vor).

Dennoch bin ich im Stande, zur Beantwortung der Frage, ob das Geschlecht der Pflanzen schon im Samenzustande zu erkennen sei, etwas beizutragen, da ich diesem, für die Physiologie so wichtigen Ġeyenstande seit lïngerer Zeit meine Aufinerksamkeit zuwendete, und aus diesem Grunde auch germ bereit gewesen wäre, die von Herm веLномме vorgeschlagenen und angestellten Versuche zu wiederholen. 
Vielleicht ist es von der Varietät von Cannabis abhängig, ob ihre Früchte schon dieverschiedenen von BELномм erkannten männlichen wnd weiblichen Formen erkennen lassen oder nicht, und ich werde in der Untersuchung derselben fortfahren.

Wem eine solche geschlechtliche Verschiedenheit in den Cannabisfruichten, wie sie Herr BeLHonne angiebt, wirklich stattfindet, was ich für sehr wahrscheinlich halte, so ist ohne Zweifel durch die mehr oder minder kräftige Ernährung des sich entwickelnden Embryo die Anlage dazu vorbereitet, und man wird nicht allein, wie das bei den Vogeleiern auch statthaben soll, an der längeren oder kuglichern Form der Frïchte die männlichen und weiblichen Samen unterscheiden, sondern auch sicherer durch den mehr oder minder kräftig entwickelten Embryo im Samen, und durch die etwa vorhandenen mehr oder minder grossen Mengen von Eiweiss darin.

Und da die Emährung des sich entwickelnden Samens von der Stellung der Frucht an der Pflanzenachse abhängt, so werden, je nachdem die Entwicklung und Entfaltung der Blumen begrenzt oder umbegrenzt (der Blüthenstand eine cyma oder eine umbella ete. ist,) die endständigen oder die untersten seitenständigen Früchte die bestgenährten, kräftigsten und somit, wie ich sogleich begründen werde, weiblichen Keime hervorbringen.

Es sind also schon die Blüthen- und Fruchtstände in dieser Hinsicht zu berücksichtigen, sowie auch andrerseits die Geschlechtsverhältnisse der Blume augenscheinlich schon von der Ernährung während ihrer Entwicklung abhängen, wie ich dies schon in der Limnaea 1856 , pag. 259 und 260 auseinandergesetzt habe, und ich dort, wie folgt, mich ausspreche:

In der Natur der polyganen Palmenblumen liegt es jedoch, dass hierauf nicht zu viel Gewicht gelegt werden darf, da die klimatischen und Bodenverhältnisse einen grossen Einfluss darauf äussern, ob sich mehr männliche oder 
weibliche oder Zwitterblumen bilden, das Eine oder das Andere der beiden Geschlechtsorgane fehlschlägt. Die in Sümpfe: orler in feuchten Wäldern wachsenden Palmen haben a is Regel Zwitterblumen, und werden polygamisch durch Mangel an Zufluss des einen oder des andern Nilurungstoffes, der Kohlensäure durch die Wurzeln oder des Stickstoffes (in Form ammoniakalischer Verbindungen) durch die Blätter, je nachdem die Luft oder der Boden grösserer Trockenheit ausgesetzt ist. Die an trocknen Abhängen oder in wasserarmen Ebenen wachsenden Gattungen sind regelmässig' (nicht gesetzmässig) getrennten Geschlechtes und zwar männliche und weibliche Blumen in getrennten Aehren. Gegen das Ende der Regenzeit und im Anfunge der trocknen Jahreszeit reifen die kohlenstuffreichen, ölhältigen Früchte, und fast nur männliche Blumen entfalten und entwickeln sich, während gegen das Ende der trocknen Jahreszeit und im Anfange der allmählig eintretenden Regenzeit, wo nur dürftige atmosphärische Niederschläge die Blätter, kaum aber den Boden nässen, vorherrschend die Anlage weiblicher Organe begründet wird.

Seitdem ich jene Beobachtungen in dem Tropenklima abschloss, habe ich fortgefahren, mit Hülfe des Herm Reinecke im hiesigen DeckeR'schen Garten das Verhältniss der Samen der Palmenfrüchte zu den daraus entkeimenden männlichen oder weiblichen Pflanzen weiter zu verfolgen, und es hat sich ergeben, dass die weiblichen Pflanzen aus den besternährten, mit vielem Eiweiss versehenen grossen Samen entstehen, während die in dem kleineren, nur wenig nährendes Albumen enthaltenden Samen eingeschlossenen Keime zu männlichen Pflanzen werden.

Auch den jüngst gekeimten Pflänzchen sieht man es schon an, ob sie männlichen oder weiblichen Geschlechtes sind, indem die den eiweissreichen Samen entkeimten jungen Palmen viel dickere Stamm- und Blattstielbasen haben, als die schmächtiger bleibenden männlichen Pflan- 
zen, welche sich aus den an Eiweiss ärmeren Samen entwickelten.

Auch die Grösse des in dem Sameneiweiss enthaltenen Keimlings variirt schon etwas in der Art, ditss die kleineren Samen anch einen kleineren Keim als die grösseren Samen enthalten. Der Unterschied in der Menge des Eiweisses beider Samen ist jedoch viel beteutender als der in der Grösse des Keimes."

M. Miquel (d'Utrecht) demande à M. Karsten, s“il al recueilli lui-même les semences présentées.

M. Karsten répond, qu'il vient de les acheter ce' matin-même à Amsterdam.

M. Morren (de Liége) fait remirquer que, s'il fiat en croire Kлгqнт, les mêmes graines cle plantes diöques, telles que les Concombres et les Potirons produisent plus ou moins de pieds mâles suivant que la chaleur agit plus ou moins sur elles. Il rappelle, comme pouvant éclairer le débat, la théorie sur la production des sexes récemment produite par M. le professeur Thury, de Genève, d'après laquelle le sexe chez les animaux serait déterminé par l'état plus ou moins avancé de développement dans lequel se trouve l'oeuf, au moment où il reçoit l'action fécondante du spermatozo:ide.

M. Lecoq croit avoir vu, que dans le Bombyx les petits veufs domnent des individus mâles, les grands des femelles. Les femelles sont aussi plus considérables.

M. Chatin (de Paris) fait observer que l'opinion de M. Karsten est opposée à celle de M. Belhomie. Selon lui, la même différence se remarque chez les vers-ì-soie, dont les cocuns mâles sont petits et étranglés, les cocons femelles grands et ovales.

M. Fée croit que les lois, qui dominent dans le règne amimal, ne sont pas sous tous les rapports comparaloles à celles du règne végrétal. 
III. La 3e question du Programme est mise à l'ordre du jour.

M. Lemaire (de Gand), qui avait posé la question, la développe en quelques mots, et lépose la note suirante, intitulée:

Critiques raisonnées et réfutations de la théorie des boyaux polliniques.

\section{Messieurs!}

„,Venir en présence de tant d'illustres représentants de la science non seulement critiquer, réfuter même une opinion si répandue parmi les botanistes, mais non généralement adoptée, souvent même contestée, est de ma part quelque peu téméraire; mais l'amour de la science et de la vérité m'oblige à vous soumettre mes objections contre la Théorie des boyaux polliniques: question grave, immense, digne, Messieurs, de toute votre attention, de rotre plus sévère examen.

Je vous prierai tout d'abord, MM., de faire pour un instant abstraction de toute idée préconçue pour ou contre cette théorie, et de m'accorder cette sage indulgence, qu'ont les rrais savants pour leurs adversaires, s'ils sont dans l'erreur, ou, s'il y a lieu, cette désirable approbation, avec laquelle ils accueillent des opinions rationnelles, des faits incontestables.

Il me faudrait, MM. écrire un bien gros volume, vous présenter d'excellentes figures, des dissections bien délicates, pour traiter convenablement la question, que je soulève pour en soumettre la solution à vos lumières; et cependant ne pouvant abuser de vos précieux instants, force me sera de n'y consacrer que quelques mots, et c'est par lì surtout que je dois réclamer votre indulgence, puisqu'il m'est interdit de développer in extenso un aussi vaste sujet.

Des auteurs modernes d'un mérite incontestable, abandonnant l'ancien système qui faisait simplement gonfler et crever le pollen à la surface du stigmate sur 
laquelle coulait le fluide fécondateur, se sont assurés, disent-ils, à l'aide du microscope et de délicates dissections que chaque granule pollinique, quelles que fussent sa forme et sa vestiture, est composé de denx membranes, dont l'interne, au moment du contact avec les papilles stigmatiques, crève l'externe, et s'allonge en un boyau qui pénètre dans les méats intercellulaires entre celles-ci, pour. aller de là rejoindre le canal stylique; que ces tubes, disent la plupart d'entre eux, descendent soit par le canal, soit latéralement à lui et en dehors à travers le tissu cellulaire jusque dans l'ovaire, pour s'aboucher chacun avec l'orifice (micropyle) d'un ovule, afin d'y déposer le fluide générateur, par l'infinence duquel se développera l'embryon ou foetus de l'être futur. Voilà en peu de mots la théorie moderne de la fécondation végétale: théorie résultant d'expériences et adoptée par le plus grand nombre des botanistes, souvent sans vérification personnelle.

C'est cette végétation des boyaux polliniques (on a même avancé qu'ils se ramifiaient!), e'est cet allongement miraculeux qui s'étend jusqu'aux ovaires, dont je viens contester l'existence, à laquelle ma raison se refuse de croire, et voici les objections qu'elle me suggère:

L'œuf végétal, comme l'œuf animal, comme l'œuf humain, adhère au placentaire par un funicule dont il se détache après la fécondation. Eh bien! dans ces trois catégories le mode d'imprégnation est le même. Natura non facit saltus, a dit le maître, et jamais peut-être, selon moi, cet axiome si vrai n'a été mieux appliqué qu'en cette occasion. Le fluide fécondant végétal, comme la liqueur séminale des animaux détermine l'imprégnation, absolument comme cela a lieu chez les êtres animés de tous les ordres. Ceci est incontestable et incontesté jusqu'ici dans la science, qui toutefois ignore encore par quel moyen l'embryon ou fœetus reçoit la vie.

Nous avons rapporté comment le fluide fécondant, ce sperme végétal — permettez-moi cetteexpression — par- 
venait jusqu'aux ovules. Cette végétation, cet alongement du boyau pollinique, je les nie très positivement. Les fleurs, vous le savez tous, MM., n'ont en général qu'une durée fort éphémère. Beaucoup même vivent à peine quelques heures. Et c'est dans un aussi court laps de temps, que l'on veut que les boyaux polliniques acquièrent une sorte de végétation assez puissante pour la faire parvenir jusqu'aux ovules! Mais vraiment, une telle végétation, instantanée pour ainsi dire, serait par trop merveilleuse. Voyez les fleurs des Datura, des Loranthus, des Nicotiana ete., d'une foule de Cinchonées, dont les styles atteignent dix, vingt et même trente centimètres de long'ueur, et se fanent en même temps que les fleurs; celles des dernières surtout durent à peine un jour. J'en ai devant les yeux divers spécimens (Posoqueria de la Guiane), dont le tube et le style dépassent trente centimètres de longueur. Or, on sait que chez ces plantes les fleurs durent ì peine deux ou trois heures le matin; et l'on veut que, pendant ces deux ou trois heures, les boyaux polliniques puissent végéter assez vigoureusement pour gagner les ovules? Nous ne saurions le croire.

Comment un granule pollinique, dont le diamètre n'est guère que le $1 / 10$, le $1 / 20$ d'un millimètre, pourrait-il multiplier longitudinalement ce diamètre des centaines, des milliers de fois, pour parvenir en deux heures aux ovules? Dira-t-on que cet alongement procède encore après la fécondation de la fleur? Mais cette opinion n'est pas soutenable. Chez la fleur qui se fane, tous les organes sexuels se fanent en même temps; l'ovaire seul subsiste et se développe.

On a dit (car l'objection qui précède, les auteurs que je combats ont dû se la faire), que les dits boyaux polliniques, en cas de styles trop allongés, se rompaient plus ou moins près de leur' point d'émission, s'alongeaient, descendaient quand même, en puisant dans les méats intercellulaires et dans le canal du style les sues et la force nécessaires pour 
aller aboutir aux ovules! Mais tout d'abord le premier cas est contre nature. En vertu de quelle force de régétation ces boyaux rompus, inertes dès lors, opéreraient-ils leur long voyage? Par la loi de la pesanteur? Non, sans doute. Par une force d'impulsion? Pas davantage, le fait même de la prétendue rupture leur enlevant toute puissance de progression.

On a dit, qu'un seul granule pollinique peut émettre plusieurs boyaux, jouant le même rôle qu'un seul! Que la rupture du granule ait lieu par plusieurs déchirures, cela est possible; mais l'alongement de chacum d'eux n'est pas plus naturel que celui d'un seul.

On a dit, que chaque ovule était fécondé par un boyau pollinique particulier. N'est-ce pas là quelque chose de tout à fait impossible? Voyez, par exemple, dans les genres Digitalis, Papaver, Nicotiana, ete., dans la plupart des Carctées, dont les ovaires contiennent quelques centaines, je dirai presque des milliers d'ovules, il y aurait done des centaines, des milliers de boyaux polliniques, chacun d'eux allant féconder chaque ovule! Et tous ces boyaux seraient contenus dans le canal de cette grêle colonnette, qu'on appelle le style, qui sourent, nous l'avons dit, atteint à peine le diamètre d'un fil à coudre? Prétendra-t-on aussi expliquer ainsi la fécondation chez les Orchidées, dont les ovaires contiennent des milliers d'orules microscopiques, par autant de boyaux polliniques? Elles, dont le pollen est une masse compacte et céreuse?

Enfin, pour terminer l'exposition de la théorie moderne de la fécondation chez les végétaux, on a dit, que chaque prolongement des boyaux, étant abouché avec le micropyle ovulaire, y pénétrait, s'y rompait, et ... devenait l'embryon!

Telles sont, MM., très sommairement rapportées, les idées généralement admises au sujet de la théorie, que je conteste parce que ma raison se refuse à l'admettre, à laquelle je ne puis ajouter foi, tant à mes yeux l'admirable harmonie de la nature, dont elle contrarie les lois, est une 
et indivisible. J'ai assisté à des expériences microscopiques faites avec le plus grand soin par d'habiles micrographes, bien connus par leurs travaux en ce genre. Eh bien! les uns admettaient, les autres rejetaient la régétation des granules, et aucun n'était d'accord avec les autres pour affirmer l'alongement jusqu'aux ovules; moins encore croyaient-ils à la rupture des granules et surtout à leur pénétration dans le sac ovulaire. Quelques-uns les aperceraient faufilés entre les papilles et les comparaient à des épingles enfoncés dans une pelote.

Ce que plusieurs de ces observateurs (1) ont vu, ce que j'ai vu (et non cru voir), ce sont les granules lancés par une sorte d'explosion, au moment propice, sur le stigmate, s'y gonflunt, s'y rompant et éjuculant arec une certaine force le liquide spermatique, qui pénétrait alors, en rertu de l'impulsion à lui imprimé, et entre les papilles du style, où il se perdait, et dans le canal stylique dilaté, d'où nécessairement il descendait, toujour's en vertu de l'impulsion reçne, jusque dans l'ovaire. Nécessairement en pénétrant entre les méats intercellulaires, il se modelait sur la forme tubulaire des vilisseatux (de même à l'intérieur du style), dans lesquels le firisait aisément distinguer son coloris plus intense que celui des parois cellulaires. Jamais, jamais nous n'avons pu le suivre loin dans celles-ci, mais seulement à son entrée dans l'ovaire, où il baignait, imprignait les orules, ainsi que cela se passe chez tous les anmanx. Nous comparerions volontiers ce mode de fécondation ì celui des Poissons et des Batraciens. De plus, les plus délicates dissections nous ont montré, non des boyaux, mais le fluide, visqueux de sa nature, coagulé lui-même en petits cylindres plus ou moins allongés. Ceux-ci, projetés sur um charbon ardent, ont émis aussitôt l'odeur spermatis humani d'une manière indubitable.

(1) Nous nous sommes fait, dans cet article, une règle de ne citer aucun nom propre. 
Nous avons conclu de ce qui précède, plusieurs de ces observateurs et moi, que l'alongement des boyanx polliniques n'était qu'une simple aberration d'optique, causée, comme je vais l'expliquer, par le contenant et le contenu.

J'ai également remarqué, mais très rarement, les solutions de continuité, signalées par quelques auteurs dans les boyaux polliniques. Je les ai attribuées à une cause analogue à celle qui, par exemple, distance dans un tube de thermomètre les parties de mercure ou d'esprit de vin, telle que celle qui résulte d'un choe subit. Mais je ne puis admettre, ainsi que je l'ai dit, que ces portions de boyaux puissent royager jusqu'aux ovules; encore une fois, en vertu de quelle force?

Mais maintenant, à quoi attribuer l'erreur physiologique que je signale, dont rous admettrez l'existence, ou que vous rejetterez avee moi, MM., selon vos cruyances spéeiales? Trop heureux, si, au moins dans ce dernier cas, j’ai pu par les raisons alléguées faire naitre des doutes dans votre esprit sur le mode de fécondation chez les régétaux, préconisé de nos jours; si, dans l'un et l'autre cas, j'ai pu appeler l'attention des savants sur cette question et provoquer de nourelles expériences contradictoires et définitives. Or, cette cause d'aberration physiologique est, selon moi, tout simplement une illusion d'optique.

Il n'est pas un de vous, MM., qui ne connaisse plus ou moins le maniement du microscope; il n'en est pas un qui puisse nier les illusions d'optique, auxquelles donne lieu cet instrument, surtout en cas de forts grossissements, quelle que soit d'ailleurs l'habilité de l'opérateur. Ces illusions résultent nécessairement de la transparence extrême des tissus cellulaires, de leur irisation inéritable, le tout se confondant avec le fluide des granules polliniques plus ou moins engagé dans les canaux et présentant les mêmes phénomènes. De là tous les faits signalés ci-dessus, sur lesquels j’appelle, MM., rotre plus sérieuse attention et de nouvelles et très rigoureuses expériences. 
Contraint par le temps et l'espace, je conclus, Messieurs, chers et honorés confrères, en vous soumettant ces critiques; réclamant, comme je l'ai dit, votre sévère examen et en même temps votre indulgence si je me trompe, votre approbation dans le cas contraire."

MM. Hoffmann, Miquel et autres déclarent, que ce que l'orateur propose, a été fait depuis longtemps.

Du reste ce mémoire, dans lequel on combat une théorie basée sur des faits incontestables, n'a soulevé aucune discussion, en raison des points nombreux sur lesquels elle eût porté.

IV. M. le Président propose de passer au No. 7 du Programme, vu que MM. Passerini, Sachs et Cohn sont absents. En conséquence la parole est accordée à

M. Chatin, qui donne un exposé sommaire de ses recherches sur la structure et les fonctions du tissu de l'anthère. (1)

M. Reichenbach (de Hambourg) demande à M. CHAтIN, s'il a fait des observations sur les gemres des Ericacées d'affinité un peu donteuse, comme les Monotropées et les Pyrolacées.

M. Chatin répond, qu'il n'a pas manqué de comprendre les Monotropées dans ses recherches, et qu'elles lui ont rlémontré, que les Monotropées comme les Ericacées, etc., manquent de cellules fibreuses.

Sur quoi M. Reichenbach fait remarquer, que ces observitions ajoutent me nouvelle preuve à celles, sur lesquelles on se fondait pour rapprocher les Monotropées des Ericacées.

(1) Nous regrettons vivement, que MI. Ciraris n'ait pu jusqu'd̀ présent nous faire parvenir le résumé de son intéressant discours. Dans le cas où il viendrait encore à temps pour être mis sous presse, nous le publierons sous forme de supplénent.

RED. 
V. M. le Président accorde la parole à M. PASserini de Parme, qui s'est proposé de discuter:

Sur l'émission de l'Acide Carbonique par les racines des plantes et sur la décomposition du même acide par les parties vertes.

M. Passerini s'exprime ainsi:

On connaît déjà les expériences au moyen desquelles M. Lifbic et d'autres, tant en Italie qu'ailleurs, démontrèrent que les plantes émettent de l'acide carbonique de leurs racines.

Après avoir vérifié les expériences par rapport à cette émission de l'acide carbonique, mon collègue M. le prof. GIORGInI et moi, nous avons répété les mêmes observations sur la Lemna minor qui, étant une plante flottante, excluait tout doute, les racines n'ayant pu ĉtre blessées, comme il arrive assez facilement aux plantes qu'on a enlevées du sol.

Or, ayant mis à flot une couche de Lemna sur la teinture de tournesol, celle-ci rougissait d'autant plus vite et plus fortement qu'on exposait darantage l'appareil aux rayons solaires; mais elle reprenait bientôt sa couleur azurée par l'action de la simple ébullition.

M. le prof. Bertint de Sienne (1) oppose à cette expérience, que la couleur rouge de la teinture était due à l'ozone, émis par les feuilles de Lemna, après l'action de lia lumière solaire; mais il est érident, que dans ce cas là l'ebullition ne serait pas en état de rendre à la teinture la couleur blene, ce qui nous fait croire que la conleur rouge est dûe à l'acide carbonique, émané des racines de Lemna et de la surface inférieure de ses feuilles.

Ensuite nous arons plongé dans la teinture de tournesol l'extrémité inférieure de jeunes branches, détachées de plusieurs plantes lignenses, et nous arons obtenu le rouge tant dans l'obscurité qu'à la lumière directe du soleil,

(1) V. Atti della Societi italiana di Scienze naturali, Vol. V, Milano 1863. 
toutes les fois que ces branches avaient des feuilles. Des branches analogues, mais sans feuilles, laissaient toujours la teinture dans son état naturel.

C'est de là que nous avons déduit:

1. Que l'acide carbonique descend de la partie supérieure de la plante à la partie inférieure.

‥ Que les feuilles, ainsi que les autres parties vertes, penvent dommer de l'acide carbonique, même sous l'action de la lumière solaire.

3. Que, supposé mème que, sous l'action de la lumière du soleil, une partie de l'acide carbonique introduit dans les feuilles ne soit pas decomposée, la théorie sur la force décomposante des feuilles est toutefois sensiblement ébranlée.

M. Hoffmann (de Giessen) rappelle les nombreuses expériences de M. Krop, publiées dans les journaux, dits: „Chemisches Centralbiatt," et , die landwirthschaftlichen Versuchsstationen." (Voir de même les „Annalen der Chemie u. Pharmacie," Tom. CXXIX, pag. 287, Mars 1864). A ces recherches se rattachent les expériences de M. Nobbe (Landw. Versuchsstat. 1864, I, pag. 61) et celles de M. Sachs sur la corrosion du marbre par les secrétions des racines. (Bot. Zeit. 1860, pag. 117).

M. Chatin fait observer que, vu que les résultats énoncés sont en opposition avec les idées généralement reçues, il faudrait en donner des preuves plus conchuantes. Il pourrait rester quelques doutes sur la nature de l'acide, par l'action duquel la teinture de tournesol rougissait, parce que l'ozone produit le meme effet sur cette teinture. C'est pourquoi M. Chatun recommande de faire l'essai avec l'eau de baryte, qui pourrait mettre en toute évidence la nature de l'acide en question.

M. Lecoq croit que les racines peurent dégager de l'acide carbonique ou au moins fommir du carbone au sol, mais que la source de l'acide carbonique se trouve dans l'atmosphère. La luzerne en est la preure. Malgré ses profondes racines, cette plante vit aux dépens de l'atmos- 
phère. Ce qui le prouve, c'est qu'un champ qui, pendant dix ans, a donné chaque année 4 coupes de luzerne, en tout 40 coupes, se trouve encore amélioré. Il fuut done, que la plante ait puisé łans l'air de quoi fommir les 40 coupes enlevées et de plus l'amélioration du champ.

M. Chatin ne conteste pas, que les ricines puissent exhaler de l'acide carbonique, mais il ne samait voir là qu'un phénomène secontaire. Pour lui, de même que les feuilles, et en général les parties rertes des plantes, ont pour fonction essentielle de décomposer le gaz carbonique (pendant le jour), et pour fonction secondaire d'en produire ou d'en exhaler (durant la muit), ainsi les racines absorbent en forte proportion, avec l'eau du sol, le gaz carbonique que M. Boussingault a prouvé y exister, tandis qu'elles ne rejettent qu'une proportion relativement minime de ce gaz. M. Chatrin se plaît d'ailleurs à féliciter M. PAsserini de la délicatesse de ses observations.

M. Passerini déclare n'avoir voulu prouver qu'une chose, l'exhalation de l'acide carbonique par les racines des plantes.

VI. MM. Cohn, Muluer et KocH étant absents, II. le Président met ì l'ordre du jour la $10^{\mathrm{e}}$ question du Programme, amoncée par M. le Prof. Reichenbach FILS de Hambourg.

M. Reichenbach s'étuit proposé de traiter des Orchidées de la Nonvelle Calédonie, mais, faute de temps, il cutretient la section de la différence entre Selenipedium caudatum et Uropedium Lindenii.

„Ce fut en 1846 que M. Lindery avertit les botanistes de l'existence d'une Orchidée tris étrange, découverte par M. Linden sur les bords du lac de Maracaybo à me hautenr de 8500 ', sur le sol de petites forêts qui se trourent dans les Savannes, de l'Uropedium Lindenii. Il la décrit en ces termes: ,Omnia Cypripedii, serl labellum planmm et petalia longissime caudata, anthera sterilis trilobo-hastata." 
La plante ayant été lécoltée par M. Funck, arriva vivante dans l'établissement de M. Linden, d'où un pied passa chez M. Pescatore à la Celle, près St. Cloud. Elle y fiemit en 1850, cultivée par Mi. Luddeman. Heureusement une des fleurs fut mise à la disposition de M. Brongniart, qui en révéla les caractères étranges dans unn ménoire admirable, publié dans les Annales des sciences naturelles. Il se servit de cet exemplaire pour"en déduire le diagramme normal des Orchidées, que R. Brown avait déjà indiqué mais sans connaître les nouvelles bases, que M. Brongniart a su mettre si bien à profit.

M. Broneniart trouva l'Uropedium différant par son labelle, à peine distinct des deux autres pétales; par la présence de trois étamines fertiles, presqu'entièrement libres et distinctes, appartenant au rang intérieur et opposées aux pétales, et d'une stérile opposée au sépale médian; par son style ì peu près libre dès sa base, terminé par un stigmate bilobé; par son ovaire triloculaire. M. Brongniart saisit de même heureusement l'occasion pour fair sentir, que l'Uiopedium avait an Cypripedium des aftinités analogues à celles des Apostasiées aux Neottiées. Enfin, M. Broneniart avait fini par se demander, si l'Uropedium forme un véritable type, oi s'il n'est qu'un monstre du Cypripedium caudatum. Loin de se décider pour la dernière opinion, comme on l'a souvent assuré, l'illustre savant paraissait hésiter à abaisser à l'état de monstre cette superbe plante. Ce qui, dit-il, le rend peu probable ̀̀ mes yeux, c'est que l'Uropedium, dont M. Linden a mis en vente plusieurs pieds, ne formait pas un individu mique, comme cela a lieu ordinairement pour le cas de monstruosité; c'est en outre, que la transformation du périanthe et du système staminal n'aurait probablement pas déterminé les différences, que j’ai signalées dans la structure de l'ovaire. Quoiqu'il en puisse être: soit que l'Uropedium représente une forme constante et bien définie de la famille des Orchidées, soit qu'il ne constitue qu'une 
modification accidentelle et monstrueuse d'un C!ypripedium, son organisation n'en est pas moins propre ì jeter beaucoup de jour sur la symmétrie florale de cette famille remarquable."

(L'orateur montre à l'assemblée une fleur desséchée de ce même pied, dont une fleur avait suffi à M. Brongniart pour écrire le mémoire dont il a été question. Il fait voir aussi des échantillons spontanés, recueillis par MM. Funck, Schlim et W Agener, dont un en boutons lui donne l'occasion de rappeler à l'assemblée la vitesse, avec laquelle les sépales et le labelle s'alongent. Puis il montre des capsules bien mûries et des graines, recueillies dans la Nouvelle Grenade, ainsi que le dessin d'une monstruosité de l'Uropedium ayant trois lobes stigmatiques très-bienséparés).

„, J'ai eu le plaisir de recomnaître, ajoute-t-il, que tous les Cypripedium de l'Amérique tropicale (excepté le seul C. Irapaeanum Lex.) ont un ovaire tout aussi bien triloculaire que l'Uropedium, ce qui m'a foumi le caractère du genre Selenipedium, dans lequel j'ai fait rentrer grand nombre d'espèces." L'orateur montre divers échantillons tant spontanés que cultivés de Selenipeclium caudatum (Cypripedium caudatum Linäl.), et continue:

„, Je n'ai jamais pensé, que l'Uropedium fut un état monstrueux du Selenipedium. L'Uropedium a été ramassé par centaines de spécimens, mais jamais on n'a rencontré dans les mêmes lieux un seul Selenipedium. Jamais ni Warszewicz, ni LobB, ni Bridges n'ont rencontré un Uropedium entre les S. caudatum. Or, l'Uropedium se trouve toujours par terre, somvent entre les Gleichenia, tandis que le Selenipedium caudatum se bouve sur des chênes. Enfin cette dernière espèce (tant qu'on réunit les Warszewicaii et caudatum) n'a été rencontrée qu'au Pérou et en Costa Rica, jamais en Nourelle Grenade. Les deux espèces portent toutes les deux des graines mûres.

Mais l'été passé un fait nouvean et étonnant vint se révéler. Je fus averti que, dans les riches serres 
de M. Schiller à Neumühlen sur l'Elbe, au delà d'Altona, un épi monstre d'un vieux pied de Selenipedium caudatum montrait deux boutons très différents, dont l'un avait la taille de ceux de l'Uropedium. L'épi fut visité de jour en jour, mais lorsqu'il se fut développé, le 17 Juin, sa fleur anormale se trouva ne pas être celle d'un Uropedium. Le labelle en effet avait pris peu à peu quelque chose de sépalique, car il était laminiforme et non sacciforme; il était ondulé et retourné plusieurs fois, portant à sa pointe un petit endurcissement, comme il s'en trouve à l'orifice du labelle sacciforme. Si cette fleur avait montré, comme l'Uropedium, une anthère devant le labelle, elle aurait prouvé l'identité des deux types et j'en aurais conclu, que le développement de cette anthère avait été la suite de la transformation du labelle en sépale (1). Mais cette fleur ayant, malgré la nature pétalique du labelle, montré un gynostème de Selenipedium, elle fouxnit un argument de plus, pour regarder les deux genres comme différents. (La dite inflorescence est montrée par M. Reichenbach fils séchée et dessinée).

VII. M. Caspary (de Königsberg) obtient la parole, pour traiter la question qu'il avait annoncée, (Voir le programme, section d'horticulture No. 11):

„Sur les hybrides obtenus par la greffe."

L'orateur s'exprime en allemand en ces termes:

,Ich erlaube mir die Aufmerksamkeit dieserVersammlung auf eine sehr dunkle Erscheinung des Pflanzenlebens

(1) Il se trouve souvent, que le développement d'organes dormants est combiné arec la réduction d'organes d'un autre verticille. Ainsi la plupart des Orchidées ì deux labelles possédent un sépale à l'endroit, où devrait être le labelle propre. Ce sépale, c'est le lahelle primitif réduit. Les deux labelles ne sont autre chose, que les deux anthères ordinairement réduites à des staminodes ou non développées, mais réduites en ce cas à l'état inférieur de labelles. Ici l'organe dormant, l'anthère impaire ne s'était pas développée, comme cela a lieu ordinairement dans l'Uropedium. Quelquefois, mais très rarement, trois labelles se développent; alors le labelle normal n'est pas réduit. 
hinzulenken, die so beschaffen ist, dass die Gegenstände durch deren Beobachtung Licht über sie verbreitet werden könnte, häufiger dem praktischen Gärtner, der mit Veredelung von Bäumen und Stränchern im Grossen sich beschäftig.t, als dem Botaniker vorkommen dürften. Es ist diess die Fähigkeit der Pflanzen, duss durch Pfropjen ähnliche, wenn auch nicht gunz ebenso sich verhaltencle Mischlinge von zwei Arten oder Spielarten gebildet werden können, wie durch geschlechtliche Befinchtung. Der Gegenstand ist bisher leider so beschaffen, dass sich über ihn gar nicht experimentiren lässt, sondern dass darüber Beobachtungen nur dann gemacht werden können, wemn er uns gelegentlich aufstösst. Liesse er sich auf's Experiment bringen, so würde ich mich für verpflichtet halten ron ihm noch nicht zu reden, sondern erst völlige Klarheit und Gewissheit über die Gesetze, denen er miterliegt, durch Versuche zu erlangen. So jedoch bin ich genüthigt von ihm fast mehr als einer Hypothese, die ich aus subjectiver Ueberzeugung hege, als einer für Alle beweisbaren Thatsache zu sprechen, schene mich aber nicht diess zu thun, weil die Sache an sich sehr wichtig und es daher sehr wünschenswerth ist, dass sie durch vervielfältigte Beobachtungen entweder constatirt oder widerlegt werde.

Wem im Allgemeinen der Einfluss des Phroptreises auf die Unterlage und der Unterlage auf das Pfropfreis ein dumkles Capitel ist - es hat Carl Friedrich yox Gärtner in seinem ausgezeichneten Werk über Bastarderzengung (Stuttgart 18.19, Seite 606) die hierher gehörigen Erscheinungen möglichst vollständig zusammengestellt — so ist das Extrem dieses Einflusses: die gänzliche Hischung der Unterlage mit dem Pfropfreise so äusserst selten bemerkt worden, dass die Gesetze, welche diese Erscheinung zeigt, nur erst in einigen Zügen sich andeuten lassen.

Sichere Fälle solch hïchst auffallender Mischung scheinen bei den Aurantiaccen, Leguminosen (zwischen Cytisus Laburnum L. und mupureus L.) und Rosaceen 
(Gattung Rosa) gefunden zu sein. Ich selbst habe nach mehrjähriger Bekanntschaft mit der Sache erst im Sommer 1864 dadurch die Ueberzengung von dem Vorkommen dieser äusserst merkwürdigen Erscheinung erlangt, dass ich einen Fall der Art zwischen einer weissen Moosrose und einer gärtnerisch werthlosen, nicht moosigen rothen Centifolie beobachten konnte.

Was die Aurantiaceen anbetrifft, so ist der Erste, der über einen dieser Familie angehörigen Pfropfinischling ausführliche und für die Zeit genaue Beobachtungen giebt, der Professor der Pharmacognosie an der Universität zu Pisa, Pretro Nati, im Jahr 1674. In diesem Jahr veröffentlichte er ein jetzt aüsserst seltenes Buch zu Florenz unter dem Titel: „,De malo limonia citrata-aurantia, Florentiae vulgo la Bizzarria." Das Buch ist so selten, dass Pritzel (Thesaurus, 207) nur in der Bibliothek von Jussiev ein Exemplar sah, welches nach Jussrev's Tode von der Königl. Bibliothek zu Berlin angekauft wurde, wo ich es benutzte. Natr beschreibt in diesem Werk einen Orangenbaum, der etwa un das Jahr 1640 in dem Garten Torve degl' Agli, der edlen Familie der Paxcraticr gehörig, bei Florenz, entstanden war, welcher sowohl im Laube als in den Früchten olme Ordnung hie und da eine dreifache Form zeigte:

1. Die bittere Pomeranze, Citrus Bigaradia Risso. Die Pflanze nennt Nati Aurantia, die Frucht Aurantium.

2. Die Cedrate, Citrus medica Risso. Die Pflanze wird von NaTr Limonia citrete, die Frucht Limon citratus genannt.

3. Eine Mischung oder Mengung beider in allen möglichen Verhältnissen, als Pflanze Limonia citrataaurantia Nati, als Frucht Limon citratus-aurantium Nati. Die Frucht beschreibt Nati so, dass man sieht, dass zwei Fonnen dabei vorkommen, nämlich, dass es erstens Früchte giebt, die nach der Weise der Frïchte geschlechtlich gebildeter Bastarde die Natur beider Arten so innigst vereinigt zeigen, dass in keinem ihrer Theile eine Art rein 
da ist, sonder'n überall imnigstes Gemisch beider, und dass zweitens der Baum Natr's Früchte brachte, die ein fast mechanisches Gemenge beider Arten, einige Karpelle ganz von der Beschaffenheit der Citrus Bigaraclia, und andere ganz von der Beschaffenheit der Citrus medica Risso in derselben Frucht hatten. Die Misch- und Mengfrüchte bringen keine keimfähigen Samen. Die Bizzamia kann nur durch Pfropfen erhalten werden.

Was die Entstehung dieser wunderbaren Pflanze anbetrifft, so hat der Gärtner, welcher sie zuerst beobachtete, nach Nati angegeben, , dass er durch kein Aufpfropfen anderer Arten, durch kein Kunststück der Aussaat, durch keine geschickt angebrachte Krenzung, sonderm als ganz freiwilliges Naturerzeugniss jenen Baum erhalten habe" (p. 17). NATi jedoch erklärt sich dafür, dass der Baum entstanden sei durch Pfropfung von der Cedrate (Citrus medica Risso) auf die bittere Pomeranze (Citrus Bigaradia Risso), wodurch eine Mischung der Natur beider in einem Spross entstanden sei, der entweder an der Stelle, wo ein gut angewachsenes Pfropfreis der Cedrate auf dem bittern Pomeranzenstock aufsass und sich die Säfte beider begegneten, aufgewachsen sei, oder, welcher da zum Vorschein gekommen wäre, wo ein aufgepfropftes Auge, das unterste von 2 oder 3, nicht getrieben, sondern eingezogen hätte, olme jedoch gestorben zu sein. NATI weist zwei Ansichten über die Entstehung der Bizarria, ohne weitere Gründe anzugeben, ab, die später noch öfters wiederholt sind. Nach der ersten seien zwei Samen, einer von der Cedrate, der andere von der bittern Pomeranze, zugleich gesät und die Stämmchen der Keimlinge eng mit einander nach Weise der Pfropfung verbunden (, tenerrimis caudicibus arcte insitionis lege colligentis"); nach der zweiten seien zwei Aeste beider Arten von Orangen der Länge nach zerschnitten und so auf einander veredelt. Ueber die Entstehung der wunderbaren Pflanze konnte also historisch Gewisses nicht gegeben werden. 
Die Kunde ron der in Mittel-Italien zuerst bekannt gerrordenen Bizarria zieht sich bis in die neueste Zeit him. Tanara, Volkammer, die philosophical Transactions, vax Steerbeck, Zucagni, Gallesio, Poiteau und Turpin, Michel, Risso, Calvel und Andere erwähnen ihrer und bilden sie in mannichfachen Formen ab. Aber in den letzten 30 Jahren scheint sie mehr und mehr unbekannt geworden zu sein und genau, unserem heutigen botanischen Bedürfniss entsprechend, ist sie nie beschrieben. Ich habe bisher keine Bizarria zu Gesicht bekommen, obgleich ich in Deutschland, Frankreich, England rielfach nach ihr fragte.

Durch einige Engländer ist 1841 die sehr dunkle Kunde ron einer anders zusammengesetzten Bizarria zu uns gelangt, die im Orient aufgefunden wurde und jedenfalls verschiedenen Ursprungs ron der mittel-italienischen ist, die den englischen Berichterstattern unbekannt blieb. Lindley giebt (Gard. chron. 1841, 36.5, 379, 471, 613, rrgl. auch Gard. chron. 1854, 583; 1855, 627. LINDLEY Theory and practice of horticulture, 2 edit. 1855, 357) Nachricht ron einer Bizarria, die Sт. John in Alexandrien in dem Garten eines gewissen Boghos Bey fand, bei der in einer Frucht drei verschiedene Arten sein sollten, nämlich die sanve Citrone (,Lemon" engl., Citrus Limonum Risso), die süsse Apfelsine (,, sweet Orange" engl., Citrus aurantium Risso) und die Cedrate ("Citron" engl., Citrus medica Risso) und G. C. Renodard berichtet (Gard. chron. 1841, 396), dass er schon 1812 bei Smyrna aus dem Dorf Hajilar eine Frucht gesehen habe, die nur 2 Arten in sich vereinigte, die saure Citrone (,Lemon" engl.) und die süsse Apfelsine (,sweet Orange" engl.). Renouard ist der Ansicht, dass möglicher Weise die bei Alexandrien ron $\mathrm{ST}$. Јонм beobachtete dreiartige Frucht ron Smyrna aus dorthin gelangt und identisch mit der von ihm daselbst untersuchten, nach seiner Beschreibung jedoch bloss zweiartigen Frucht sei. Ueber die Entstehung der Alexandrinischen Bizarria 
wird gläubig die Sage von ST. JoHn nacherzählt, dass man 3 Samen nehme von Citrone, Apfelsine und Cedrate, einen von zwei Seiten beschneide, die beiden andern bloss von einer Seite, den ersten in die Mitte lege, die beiden andern seitlich an den ersten, alle 3 mit dünnem Grase zusammenbinde und in die Erde senke. Diess Verfahren dreiartige Orangen zu bilden sollte Jussuf Bey in Smyrna gelernt haben, wo es seit unvordenklicher Zeit geübt werde.

Ich habe die Ueberzengung, dass die Angabe ST. Joнn's, dass 3 Arten von Citrus in der Bizarria von Alexandrien enthalten seien, umrichtig ist, sondern dass sie bloss aus 2 Arten besteht: der Cedrate und süssen Apfelsine. Was Sт. John und ihm folgend Linduey für eine dritte Art, die saure Citrone, erklären, ist nach meinen Dafürhalten diess nicht, sondern nach Analogie klarerer Pfropfmischlinge nur eine Mischform von Cedrate und süsser Apfelsine.

Weit bekannter als die Bizarria und uns Allen zugänglich ist der Pfropfbastard zweier Papilionaceen aus der Gattung Cytisus, der Cytisus Adami Poiteau (nicht Poiret), welcher durch Pfropfen ron Cytisus purpureus auf Cytisus Laburnum entstanden ist. Die erste Nachricht von Cytisus Adami gab Prévost der Sohn, Handelsgärtner in Rouen 1830 (Annal. soc. d'hortic. de Paris VII, 1830, 93). Da die Pflanze sehr bekannt ist, scheint es überflüssig, sie genauer zu beschreiben. Sie steht durch ihr glattes, mässig tief-grünes Blatt, welches grösser als das von Cytisus purpureus, aber kleiner als das von Cytisus Laburnum ist, und durch die lange, lockere, hängende, schmutzig karmoisinroth-gelbliche Blüthentraube so in der Mitte zwischen Cytisus purpureus und Laburnum, dass man einen geschlechtlich erzeugten Bastard beider vor sich zu haben glaubt. Aber die Angabe des Handelsgärtners JeAn Lours AdAr, zu Vitry bei Paris, über die Entstehung der merkwürdigen Pflanze -- denn in 
seiner Gärtnerei hat sie sich gebildet - lautet ganz anders. Wir verdanken die Mittheilung darüber Porteau (Annal. soc. d'hort. de Paris 1. c.), der auch den Namen: Cytisus Adami gab. Adasr's Bericht lautet: ,1825 habe ich eine gewisse Zahl von Cytisus purpureus auf ebensoviel Stämme von Cytisus Laburnum als Unterlage mit dem Schilde (en écusson) gepfropft; eins der Schilde ruhte ein Jahr lang, wie das oft genug geschieht und das Auge vermehrte sich während dieser Zeit sehr, wie das ebenfalls oft genug vorkommt. Im folgenden Jahre trieben alle Augen von diesem Schilde und ich bemerkte unter den Aesten, die sich daraus entwickelten, einen, der sich von den andern durch seine stärkere Entwicklung, durch senkrechte Richtung und durch viel grössere Blätter unterschied, die denen des Cytisus Laburnum ziemlich ähnlich waren. Ich habe dann diesen Zweig verpfropft und vermehrt, indem ich hoffte, dass er eine interessante Spielart liefern würde; indem ich die Bäume aber immer sofort nach der Vermehrung durchs Pfropfreis verkaufte, habe ich niemals die Blüthe gesehen." Der Bericht Adan's über die Entstehung des Cytisus Adami enthielt so höchst Abweichendes über das sonstige allgemein bekannte Verhalten ron Pfropfreisern, durch welche gerade am Sichersten Spielarten fortgepflanzt und erhalten werden, dass Ads neinen Glauben fand. Selbst Portend, der den Bericht Adarr's bekannt machte, hielt ihn nicht für richtig' und erklärt Cytisus Adami für ein zufälliges Erzeugniss (,,un développement accidentel") des Cytisus Laburnum, gerade wie die Bizarria ein solches bei den Orangen sei und das, was den Cytisus Adami so höchst merkwürdig machte, wäre ohne Zweifel bald vergessen worden, wenn die Pflanze nicht durch neue ausserordentliche Erscheinumgen die Aufmerksamkeit sehr zahlreicher Beobachter auf sich gezog'en hätte. Cytisus Adami zeigte nämlich überall, wo er angepflanzt war und gedieh, nach einigen Jahren Zweige auf sich, die theils nach Laub, Blüthen und Früch- 
ten reinen Cytisus Laburnum, theils reinen Cytisus purpureus darstellten. Diese unerhörte Erscheinung wurde bemerkt und mehr oder weniger umständlich beschrieben von Rivers, Loudon, Hénon, Seringe, Herbert, Dillwyn, Schnittspahn, Noack, Kirschleger, Reisseck, A. Bradn, Steetz, Lindley, le Jolis, Sinning, Treviranus, Kurr, mir und Anderen. Da die Angabe Adan's über die Entstehung der Pflanze von den meisten Beobachtern nicht geglaubt wurde, wurden andere Hypothesen über ihren Ursprung aufgestellt. Viele hielten die Pflanze für einen auf geschlechtlichem Wege entstandenen Bastard. Andere hatten andere Erklärungen, auf die ich, der Kürze der Zeit weg'en, nicht näher eingehen kann. Ich hatte Gelegenheit früher die Pflanze genauer in der Rheinprovinz an verschiedenen Orten $\mathrm{zu}$ untersuchen, habe sie auch jetzt 5 Jahre unter dem ungünstigen Klima Königsberg's, obgleich hier ohne die Zweige des Cytisus purpureus und Laburnum auf ihr zu erzielen, gezogen. Gleich der Bizarria bietet Cytisus Adami eine vollständige Verschmelzung, eine mechanische Mengung und eine völlige Trennung beider in ihm vereinigter Arten. Cytisus Adami zeigt nach Laub und Blüthe folgende verschiedene Zweige: 1. solche, in denen die Natur des Cytisus Laburmum und purpureus, wie bei geschlechtlich gebildeten Bastarden innigst verschmolzen ist, so dass die Eigenschaften keiner Art rein hervortreten; solche Zweige und Blüthen stellen den reinen Cytisus Adami Poiteau dar. Die Blüthen sind an allen Orten unfruchtbar gewesen; sie verhalten sich also wie die der Mehrzahl eigentlicher, geschlechtlich erzeugter Bastarde. Ich erwartete daher, dass der Pollen bei ihmen nach Art der geschlechtlich gebildeten Bastarde mangelhaft entwickelt sein würde. Wie erstaunte ich aber, als ich die Kömer nicht bloss reichlich an Zahl, sondern alle einzelnen sehr g'ut nach Form und Inhalt ausgebildet fand. Sie trieben, sowohl auf der Narbe des Pistills, als auf Zuckerwasser gestreut, sehr leicht Schläuche. 
Der Pollen verhielt sich mithin ganz abweichend von dem der geschlechtlich gebildeten Bastarde. Eben diess war der Fall mit der grössern Zahl der Samenknospen. Bei geschlechtlich gebildeten Bastarden ist der weibliche Theil: das Pistill, namentlich die Samenknospen, meist gut entwickelt, bei Cytisus Adami nicht; die meisten Samenknospen sind ganz monströs, nur wenige zeigen normale Form; ich werde darüber ausfülırlicher anderwegen sprechen. 2. Es giebt zahlreiche Blüthen, die aus Cytisus Laburnum und Cytisus purpureus fast mechanisch gemengt erscheinen. A. Braun (Verjüngung 1851, S. 340) hat sie ausführlich beschrieben. Auch sie bringen keine Frucht. 3. Zweige und Blüthen, die vollkommen entwickelter Cytisus Laburnum und 4. solche, die rollkommener Cytisus purpureus sind; beide tragen Frucht, deren Samen keimen. Ich zog: aus solchen, auf Cytisus Adami gewachsenen Samen von Cytisus Laburnum, junge Pflanzen (von Cytisus Laburnum), die leider bei Königsberg's rauhem Klima nur wenige Jahre alt wurden und im harten Winter von 1863/1864 erlagen. Hofgärtner Sснмпт (Verhandlungen des Gartenbauvereins zu Dessau 1859 und 1860, S. 46) zog aus Samen der Blüthen von Cytisus purpureus, die auf Cytisus Adami erschienen waren, Cytisus purpureus.

Es ist einleuchtend, dass es ron hoher Wichtigkeit wäre, geschlechtlich erzeugte Bastarde zwischen Cytisus Laburnum und purpureus zur Vergleichung mit Cytisus Adami zu gewinnen (1). Es ist diess eine Aufgabe, die

(1) AUg, NAP. BaUManN, Handelsgärtner in Bollwiller, (Haut-Rhin), zeigt in seinem Catalog von 1847 Cytisus purpurco-elongatus als ,une nouvelle hybride superbe" an. Auf eine Anfrage von ALEX. BraUx in Betreff der Entstehung und Beständigkeit dieser Pflanze gab Herr Bavmann unter dem 24 Mai 1850 folgende Antwort: „Mein Cytisus purpureoelongatus ist von meinen eignen Aussaaten, allein nicht durch künstliche Befruchtung. Die MIutter dieser Pflanze ist Cytisus clongatus, von welcher ich den Samen sammelte; neben dieser stand ein Cytisus purpureus und wahrscheinlich ist der Pollen durch Insekten von einer Pflanze zur andern übertragen worden und so wurde dieser Bastard erzeugt. Veränderung der Form dieser Pflanze habe ich noch niemals bemerkt und ich glaube bestimmt sagen zu können, dass dieses nicht so, wie bei Cytisus Adami, stattfindet." Die letztere Angabe Baumanv's spricht nicht dafür, dass Cytisus Adani ein geschlechtlich erzeugter Bastard sei. Ich hatte nie Gelegenheit, Cytisus purpureo-elongatus zu sehen. 
ich den Anwesenden, deren Heimath sich eines milderen Klima's als Königsberg erfreut, besonders empfehle. Es scheint schwierig zu sein den geschlechtlichen Bastard zwischen beiden Pflanzen zu erzeugen. 12 Blüthen von Cytisus Laburnum, die ich mit dem Pollen von Cytisus purpureus im botanischen Garten zu Königsberg bestäubte, setzten keine Frucht an. Dr. ReIsseck in Wien hat auch 2 Jahre hintereinander vergeblich Cytisus Labumum mit Cytisus purpureus und umgekehrt zu befruchten versucht, wie er mir brieflich mittheilte.

Ich lhabe Beobachtungen an einer Rose, deren Geschichte sich klar ermitteln liess, gemacht, die der Art sind, dass sie mich bestimmen der Ansicht Natr's über die Entstehung der Bizarria und dem Bericht Adan's über die des Cytisus Adami Glauben zu schenken und die mir die Frage zu entscheiden scheinen: giebt es Pfropfimischlinge oder nicht?

Am 31 Juli 1864 brachte mir Herr Kaufmann A. E. Loreк, Haus- und Gartenbesitzer in Königsberg, Alte Gasse No. 23, einen abgeschnittenen Ast einer weissen Moosrose, der zwei Zweige besass; einer derselben trug eine weisse Moosrose (wahrscheinlich Quatre saisons) und der andere zwei gemeine, gärtnerisch werthlose, nicht moosige rothe Centifolien. An Aufgepfropftsein war nicht zu denken. Sofort fiel mir die Analogie mit der Bizarria und Cytisus Adami auf. Herr Loren hatte den Stock, von dem jener Ast abgeschnitten war, vor 6 Jahren vom Handelsgärtner C. L. Kasenir in Königsberg als weisse Moosrose gekauft und in seinen Garten ins Freie gepflanzt. Der Stock hatte bis dahin weisse Moosrosen getragen, hatte durch Frost viel gelitten und seine Triebe waren dann bis auf den Boden abgeschitten worden, aber er hatte stets von unter der Erde her neue Triebe gemacht. Erst 1864 bemerkte Herr LoREK, dass auch rothe nicht moosige Centifolien auf den Zweigen, die weisse Moosrosen trugen, erschienen und glücklicher Weise war ihm diess so auffal- 
lend, dass er mir einen solchen Zweig zustellte. Als ich den Stock selbst am folgenden Tage in Herrn Lorek's Garten untersuchte, fand ich 6 aufrechte Triebe von 3 bis $3 \frac{1}{2}$ Fuss Höhe aus der Erde kommend vor. Einer derselben war ganz und gar eine nicht bemooste rothe Centifolie mit Stacheln ron 5 bis $5 \frac{1}{2} / / /$ Länge; die andern 5 , bemooste weisse Rosen. Der Trieb, welcher neben den weissen Moosrosen auch die rothen nicht moosigen Centifolien trug, von dem Herт Loneк die Spitze für mich abgeschnitten hatte, kam aus einem horizontalen in der Erde halb verborgenem Stammtheil herror, der in $\frac{1}{2}$ Zoll Entfernung den Trieb, der durchweg nicht moosige rothe Centifolien brachte, erzeugt hatte. Der die zweierlei Rosen führende Trieb schien nur ziveijährig zu sein, war unten $6^{\prime \prime /}$ dick und besass auf dem alten Holze keine Stacheln, sondern bloss auf dem diessjährigen. Bei näherer Untersuchung der weissen Moosrosen der übrigen 4 Triebe des Stocks fand ich, dass 2 dieser Triebe wiederum eine neue Erscheinung zeigten, nämlich weisse Moosrosen, in denen eimzelne Petala mechanische Mengung von Roth und Weiss zeigten. Entweder war die Hälfte eines Blumenblatts roth, die andere weiss, oder der rothe Theil war grösser als der weisse, oder der weisse grösser als der rothe und in den letzteren Fällen bildete oft der verschieden gefärbte Theil einen Keil zwischen gleichartig gefärbten Seitenstücken. Der unterirdische Theil des Stocks gestattete weiter keine Untersuchung. Es war sehr günstig dass die Geschichte des Stocks, seine Art der Veredelung, sich völlig ermitteln liess. Herr Kasenir theilte mir mit, dass der an Herrn LoReK verkaufte weisse Moosrosenstock, wie alle anderen, die er in jener Zeit veredelt habe, auf die ,Wurzel" von nichtmoosiger rother Centifolie von ihm veredelt sei. Er habe zu dem Zweck, aus Augen oder Stecklingen, sich Stöcke ron nicht moosigen rothen Centifolien angezogen und wenn diese gut und kräftig bewurzelt waren, dicht über der Wurzel unter der Erde den Haupttrieb und die unterirdi- 
schen Nebentriebe abgeschnitten und dann das Edelreis der weissen Moosrose auf die Stelle des ausgeschnittenen Haupttriebes gepfropft. Er ziehe diess Verfahren der Veredelung auf die Wurzel der Veredelung auf den Stamm vor, denn bei letzterer seien ihm viele, bei ersterer kein Auge fehlgeschlagen. Die weisse von ihm zur Veredelung angewandte Moosrose habe die Eigenthünlichkeit gehabt, dass sie stets in der Knospe auf der Spitze blassroth gewesen sei, und erst nach dem Aufbrechen völlig weiss geworden.

Da es häufig rorkommt, dass auf demselben Stock Blüthen ron verschiedener Färbung oder verschiedener Grösse und Färbung auftreten, z. B. bei Viola tricolor, Viola lutea Sm. (V. calaminaria Lej.), wie ich diess bei Moresnet bei Aachen beobachtete, bei Georginen, Phlox, Rosa Eglanteria, Mirabilis Jalapa u. s. W., musste natürlich neben der Vermuthung, dass der beschriebene Rosenstock ein Pfropfblendling sei, auch die andere entstehen, dass vielleicht nur ein jenen Fällen analoges Variiren auf demselben Stock vorliege. Dass Letzteres stattfinde, ist höchst unwahrscheinlich, ja erschien mir bei näherer Betrachtung als unmöglich, da nicht bloss die Farbe der Rosen verändert war, sondern ihre Bedeckung im höchsten Grade, demn die eine hatte jene rieldrüsigen dicht stehenden langen Anhänge, die Moos genannt werden und nichts ron Stacheln, die andere hatte kein Moos, aber weitläufig stehende lange Stacheln. Da mir kein Beispiel eines so gewaltigen Umfings des Variirens der morphologischen Erscheinung auf demselben Stock bei einer andern Pflanze bekannt ist, schien mir dieser Fall auch hier nicht eingetreten zu sein, und es blieb dann nichts übrig, als dass hier einer der seltenen Fälle rorliege, in welchem durch Pfropfen eine Mischumg der Natur zweier Pflanzen, diessmal zweier Spielarten, nicht Arten, wie bei der Bizarria und dem Cytisus Adami, erzeugt sei.

An dem beschriebenen Pfropfblendling ron Rosa centifolia L. fl. rosen und Rosa centifolia $\beta$. muscosa Lindl. 
flore albo bemerkte ich übrigens nicht die wirkliche, imnige Mischung beider Varietäten, sondern entweder die schon vollendete Scheidung beider oder die anfangende Scheidung in den fast mechanisch aus beiden gemengten Blïthen. Eine Mischung nach Art geschlechtlich gebildeter Blendlinge würde hier vorhanden gewesen sein, wenn der Stock des Herr'm LoREx, ausserdem noch nicht-moosige weisse Rosen und moosige rothe getragen hätte. Beides sah ich an ihm nicht. Jedoch kanm meine Beobachtung auch durchaus nicht als vollständige gelten. Aber es berichtet mir ein zuverlässiger Gartenliebhaber, Herr Commerziemrath Burdach, dass er in seinem Garten, Weidendamm No. 3, vor etwa 15 Jahren auf eine rothe, nichtmoosige Province-Rose hochstämmig 3 Augen einer weissen Moosrose aufpfropfen liess, die gut trieben und mehrere Jahre weisse Moosrosen brachten. Nach einigen Jahren hatte eines der 3 Edelreiser jedoch weisse Moosrosen, die auch rothe Blumenblätter besassen, so dass einige der weissen Moosrosen bis zur Hälfte ganz roth waren, und im folgenden Jahre kamen auf diesem selben Triebe völlig rothe Moosrosen zum Vorschein. Auch das Laub dieser rothen Moosrosen-Zweige wurde dunkler grün, das der weissen Moosrosen war und blieb heller. Diese Beobachtung der innigen Mischung beider Rosenspielarten ergänzt die meinige.

In Gardn. Chron. (1860, p. 672) wird ein durchaus hierher gehöriger Fall berichtet, dass im Garten eines Herrn Robert H. Pornter in Taunton auf einem Stock von der weissen Rosa Banksiae ein Auge von ,Rosa Devoniensis," die mir nicht einmal dem Namen nach bekannt ist, gepfropft sei. Von der Veredelungsstelle sind dann neben reiner Rosa Devoniensis einige Zweige aufgesprosst, welche die Natur beider Pflanzen vereinigen. „Die Blüthen” (des Mischlings) , sind gleich, jedoch besser als ,Lamarque" und haben etwas vom Geruch der Theerose, während die Art des Wachsthums sehr ähnlich dem von Rosa Bank- 
siae ist. Die kleinen Sprosse und die langen starken, nicht blühenden Zweige sind ganz die ihrigen, jedoch (nur mit Ausnahme der kleinen Zweige) mit Stacheln besetzt." In diesem Pfropfinischling der Rosa Banksine und Devoniensis, ist es, so weit der Bericht vorliegt, zwar zur Mischung, aber noch nicht zur Trennung beider Faktoren gekommen.

Wäre Jemand geneigt diese von Rosen berichteten Fälle, welche verschiedene Formen von Blüthen auf demselben Stamm zeigten, als blosses Variiren aufzufassen, so steht dem ein Bedenken entgegen, welches diese Auffassunga als unstatthaft erscheinen lässt, dass nämlich dann der Umfang des Variirens ganz und gar innerhalb der Eigenschaften der Unterlage und des Pfropfreises fällt. Wie kommt es denn, dass die Variation nicht neue Merkmale zeigt, die beide nicht besitzen? Es ist geradezu ein Wunder in allen diesen Fällen, dass die Variation bloss innerhalb der Eigenschaften der Unterlage und des Pfropfreises geblieben ist und, meine ich, es kann gar keine andere Erklärung für ein so auffallendes Faktum gegeben werden, als dass Unterlage und Edelreis sich gemischt haten.

Die Pfropfmischlinge, die unalog den geschlechtlich gebildeten Mischlingen, welche ich als Bastarde beztichne, wenn sie ans Arten, als Blendlinge, wenn sie aus Spielarten entstanden sind, in Pfropfbastarde und Pfropflblendlinge sich theilen, haben nach dem Wenigen, was mir als zuverlüssig beobachtet erscheint, mit den geschlechtlich gebildeten Mischlingen das Gemeinsame, dass sie eine Form darstellen, die eine völlige Verschmelzung der Natur beider Pflanzen, aus denen sie entstanden, zeigt. Diese Form ist, wie meist bei den geschlechtlich gebildeten Bastarden, bei den Pfropfbastarden auch unfruchtbar (Bizarria Natr's, Cytisus Adami), jedoch ist wenigstens bei Cytisus Adami die von der Weise der eigentlichen Bastarde sehr abweichende Eigenschaft da, dass der Pollen vollkommen gebildet, dagegen die Samenknospen unvollkommen sind 
und um ihretwillen die Unfruchtbarkeit einzutreten scheint. Dann zeichnen sich aber die Pfropfmischlinge sehr vor den geschlechtlich entstandenen Mischlingen durch eine Eigenschaft aus, die bei letzteren bisher nie beobachtet ist, dass auf demselben Stamm der Mischform zugleich eine Trennung in ihre Aeltern eintritt und zwar in verschiedenen Graden; einige Zweige bilden Laub, Blüthen und theilweise Früchte, welche die Natur beider Aeltern mechanisch nebeneinander gestellt zeigen; ich nenne diese unvollendete Trennungserscheinung: Mengform; und neben diesen Mengformen zeigen andere Zweige dis Extreme der Trennung, nämlich die vollkommene Entwicklung der beiden Aeltern in Laub, Blüthen und Früchten, die sogar keimfähige Samen enthalten.

Ob einige Beobachtungen von zweierlei Früchten auf demselben Stamm bei kultivirten Pflanzen anderer Arten als den von mir aufgezählten, z. B. von Nectarinen und

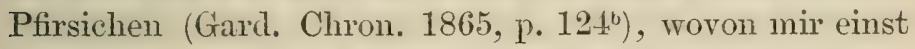
Decaisne brieflich Mittheilung machte, von rothen und gelben Stachelbeeren (Gard. Chron. 1855, p. 596 und 612), von verschiedenen Birnen (Dureau de la Malle, in Compt. Rend. LI, 18ǎs̆, p. 805), Aepfeln (Gaudichaud, in Compt. Rend. XXXIV, 1852, p. 746., Treviranus Sitzung'sberichte der Niederrhein. Gesellschaft in Bomn 1858, p. CVII) und von Andern, auch durch Mischung in Folge von Veredelung zu erklären sind, lässt sich nicht sicher sagen, đa die Geschichte jener Stämme d. h. was bei ihnen Pfropfreis, was Unterlage war, nicht mitgetheilt ist. Jedoch ist es mir sehr wahrscheinlich, dass wenigstens der von GAudicHaud erwähnte Apfelbaum, der zweierlei Fruchtformen und eine dritte, aus beiden gemischte trug, hierher gehöre. Möge in Zukunft genauere Beobachtung ähnlicher Fälle stattfinden. Es wird damn auch vielleicht dahin kommen, dass die bis jetzt verborgenen Bedingungen erkannt werden, unter welchen sich, wemn eine Pfropfung vollzogen ist, ein Pfropfnischling bildet und diejenigen, unter welchen das 
Pfropfreis seine Natur ohne Mischung mit der Unterlage beibehält."

(Pour illustrer les faits divers, cités dans son discours, l'orateur fait circuler plusieurs planches et dessins.)

M. Morren ne saurait se rallier à la théorie, qui vient d'être produite. Le fait du Cytisus Adami lui paraît fort simple et il ne comprend pas, comment cette plante ait pu occuper si longtemps les Congrès de Bruxelles et d'Amsterdam. On ne peut mettre en doute son origine hybride, dérivée des Cytisus purpureus et Laburnum. Son histoire est bien connue et a été souvent publiée. S'il fallait une explication de son hétéromorphie, on pourrait supposer qu'il doit son origine ì la soudure de deux tubes polliniques, l'un de Cytisus purpureus, l'autre de Cytisus Laburnum, qui seraient venus féconder conjointement le même ovule, en dommant lieu ainsi à un embryon dans lequel l'impulsion de deux pères se serait réunie. Mais cette hypothèse n'est pas nécessaire. Le Cytisus Adami présente simplement un bel exemple de la loi générale, aujourd'hui bien établie, de la disjonction des types originaux chez les hybrides; que cette disjonction se manifeste par les graines dans la progéniture, ou par les bourgeons dans les rameaux, il importe peu, le principe est le même. Les cultivateurs savent que, pour multiplier le Cytisus Adami, il faut greffer l'un ou l'autre de ses rameaux qui montre les caractères intermédiaires de l'hybride. Au bout d'une année ou plus, la disjonction se manifeste dans les ramifications de la nouvelle plante ainsi obtenue. M. Morren cite les résultats des expériences de M. NAUDin sur la disjonction des hybrides de Primula, Nicotiana, etc., et montre que la loi est la même dans le Cytisus Adami. Il relate un second fait analogue, manifesté par le Syringa Rothomagensis ou $S$. sinensis, en d'autres termes par le Lilas Varin (1) et

(1) Voir la Belgique horlicole 1863, pag. 108. 
dit, qu'on l'observe encore assez communément dans le genre Datura. C'est aussi par la disjonction qu'il explique les jeux et les bizarreries, qui se montrent dans beaucoup de variétés horticoles, telles que les Rosiers, les Azalea etc., sur lesquels on voit des fleurs de couleur et, même, de forme toutes différentes. Après avoir rappelé encore le Renanthera Lowii et d'autres Orchidées, M. Morren dit en terminant, que, dans son opinion, les hybrides sont comme le résultat d'un mélange physique et non comme le produit d'une combinaison chimique entre les deux ascendants. Il veut exprimer par cette comparaison, que, suivant lui, le sang paternel et le sang maternel sont toujours plus ou moins distincts dans l'hybride et susceptibles de se séparer.

M. Meissner (de Bâle) dit que, pour parvenir à des conclusions sûres dans cette question, il lui semble nécessaire de faire en premier lieu des expériences nombreuses et variées sur différentes espèces de plantes, en greffant une espèce sur une autre du même genre (genus), et qu'il faut choisir des arbustes ou des arbres de culture facile, des espèces à la fois très-voisines les unes des autres (proxime affines) +et pourtant bien tranchées et faciles à distinguer soit par la couleur des fleurs, soit par d'autres caractères, comme p. e. les Ribes sanguineum et floridum ou flavum, les Aesculus blancs, rouges et jaunes, les Robinia Pseudacacia, glutinosa et hispida, les Spiraea salicifolia, tomentosa, Douglasii, etc., les Lonicera tatarica, sinensis, xylosteum. Il finit en conseillant de faire les mêmes expériences sur quelques uns de nos arbres fruitiers, p. e. sur les cerisiers à fruits aigres (les merisiers) ou doux (rouges et noirs), sur les pommiers, ete."

M. Regel (de St. Petersbourg), ayant obtenu la parole, fait en allemand les observations suivantes:

„Die Beobachtungen des Herrn Prof. Caspary haben einen hohen Werth für die Wissenschaft; ich kann aber die auf diese Beobachtungen gegründeten Schlüsse nicht 
theilen; dass der Wildling einen Einfluss anf die eingepfropften Edelreiser ausüben könne. Dass hieraus sogar eine andere Form des aufgesetzten Edelreises hervorgrehen könne, das ist eine Amnahme, die durch Tansende von guten Beobachtungen widerlegt wird. Eins der auffallendsten Beispiele, dass der Wildling keinerlei Einfluss auf das Edelreis ausübt, greben die sogenamnten Sortenbäume, oder diejenigen grossen Exemplare unserer Obstbämme, wo auf ein einziges Exemplar eine grössere Zahl verschiedener. Sorten aufgesetzt worden, um solche zu erproben. Auf dem gleichen Wildstanme erlangen alle die verschiedenen aufgesetzten Sorten ihre besondern Eigenthümlichkeiten, in Bezug auf Form und Farbe der Frucht, in Berug auf Reifezeit, in Bezug auf Geschmack; mo so kamn man beispielsweise rom gleichen Baume frühe und späte, grüne und rothe, süsse und saure, grosse und kleine Aepfel erndten, je nach den Sorten welche aufgesetzt wurden. Wenn num aber der Wildstanm nicht einmal einen Einfluss anf solche Eormen der Art besitzt, die, genau genommen, nicht eimmal Formen genannt werden können, sondern, richtiger ausgedrückt, als auf ungeschlechtlichem Wege fortgepflanzte Individuen bezeichnet werden müssen, damn kann noch viel weniger von einem Einfluss desselben auf Unbildung eines viel grössere Verschiedenheiten zeigenden Edelreises, der in der Rückbildung des Bastardes zи einer der elterlichen Arten bestehen soll, die Rede sein.

Wo Tausende von richtigen Beobachtungen ein Gesetz festgestellt haben, kann ein vereinzelter Fall dieses Gesetz nicht umstossen, und es muss für solche einzelnen Fälle eine andere Erklärung geben, durch welche anerkannte Gesetze nicht umgestossen werden."

M. Lecoq (de Clermont-Ferrand) signale un fait remarquable, qui se rattache aux observations de M. CAsPARY. Un Lilas ou Syringa chinensis, qu'il a acheté, lui domne tous les ans une branche de Lilas de Perse, entièrement conforme à cette dernière espèce et se continnant 
très bien avee la branche du Lilas de Chine, dont elle s'échappe.

M. Regel fait encore les observations qui suivent:

„Erlauben Sie mir noch einige Worte, um mein zuvor abgegebenes Votum zu ergänzen, und zugleich eine Erklärung für die vom Herrn Prof. CAspary angeführten Fälle zu greben. Ein sehr bekanntes Gesetz ist der sogenannte Dimorphismus, den Darwin in nenester Zeit bei einer grossen Menge von Pflanzenarten nachgewiesen hat. Als Dimorphismus bezeichne ich überhaupt die dimorphe Ausbildung bestimmer Organe an gleichen Individuen, oder auch an verschiedenen Individuen der gleichen

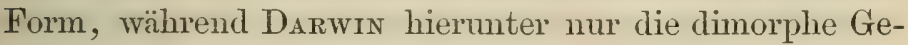
stalt der Geschlechtsorgane versteht. Der Dimorphismus tritt in sehr verschiedener Richtung auf. So sind die buntblättrigen Abarten unserer Kulturpflanzen grösstentheils in der Weise entstanden, dass einzelne Aeste der Zweige sich anormal mit bunten Blättern ausbildeten. Diese wurden ungeschlechtlich fortgepflanzt, und so eine durch Dimorphismus entstandene Form festgehalten. Dass die buntblättrigen Abarten in ähnlicher Weise zur grünblättrigen Stammform zurücklielıren können, ist eben so bekannt. Nicht bloss an den Blattorganen, sondern noch häufiger an den Blüthenorganen zeigt sich der Dimorphismus der Pflanzenart. Den Gärtnern bekamnt ist zum Beispiel bei den Aurikeln die Form, mit aus der Blumenröhre hervorragendem und in der Blumenröhre eingeschlossenem Griffel.

Die Limneischen Classen Monoecia, Dioecia und Polygamia sind nur auf den Dimorphismus der Pflanzenart gegründet. Die umregelmässigen, zur regelmässigen Blüthenform zurückkehrenden Blumen (Pelorien der Calceolarien) sind bekannt genug. Die so verschiedene Form der sterilen oder eingeschlechtlichen Blumen in dem Umkreis der Blüthenstände der Compositen, der Hydrangeaceen, etc., ist eine so bekannte Erscheinung, dass solche nicht auf- 
fällt, während die in die gleiche Categorie gehörende, aber seltener auftretende, auffallend verschiedene Form der sterilen und fruchtbaren Blumen mancher Orchideen (Cycnoches Egertonianum, etc.) Vielen jetzt noch als ein unbegreifliches Faktum erscheint.

So wie num aber die Pflanzenart dimorphe Gestaltungen ihrer einzelnen Organe zeigen kann, - so giebt es andererseits manche Formen, oder besser gesagt Individuen der Pflanzenart, bei denen verschiedene Formen des gleichen Gebildes fast regelmässig neben einander auftreten. Ich will hier nur an die sogenannten nicht beständigen, panachirten Blumen erinnern, wo auf dem gleichen Individuum, z. B., roth und weiss panachirte Blumen, neben einfarbig roth oder einfarbig weiss gefarbten auftreten können, so dass solche also eine trimorphe Gestaltung, nämlich die der beiden Grundtöne und die der gemischten Form zeigen.

Der Bastard hält bekanntlich in der Ausbildung seiner Organe gemeiniglich die Mitte zwischen den beiden elterlichen Pflanzen, und auch den auf ungeschlechtlichemWege fortgepflanzten Individuen bleibt diese Eigenthümlichkeit. Wenn aber von den Pflanzen mit panachirten Blättern einzelne Aeste zur Stammform zurückkehren können, so scheint es auch Bastarde zu geben, deren auf ungeschlechtlichem Wege erhaltene Nachkommen zu einer der Stammarten zurückkehren kömnen, indem sie am gleichen Individuum einzelne Aeste bilden kömnen, die nach einer der beiden elterlichen Pflanzen zurückschlagen. Das Beispiel eines solchen Trimorphismus, wo am gleichen Individum, neben der normalen Form des Bastardes, auch zufällig noch die beiden Stammformen auftreten, würde nun Cytisus Adami, der Bastard zwischen Cytisus Laburnum und Cytisus purpwreus, sein, an dem diese Erscheinung schon vielfich beobachtet und beschrieben worden ist. Ein Einfluss des Wildlings kamn hier aber ebensowenig wie in andern Fillen nachgewiesen werden, indem im letzteren 
Falle, nicht bloss einzelne Aeste des aufgesetzten Edelreises, sondern das ganze Edelreis sich zurückbilden müsste, da ja der Wildling auf alle Theile desselben einen jedenfalls gleichartigen Einfluss ausübt. Wer die dimorphen Blumen einer Orchidee beobachtet hat, wird nicht mehr über die dimorphe oder selbst trimorphe Gestalt (ähnlich den panachirten Blumen) eines Bastardes erstaunen, sondern solche in ein und dieselbe Reihe von Erscheinungen setzen, ohne desshalb an den Einfluss des Wildlings zu glauben. Nur in einer einzigen Richtung existirt ein gewisser Einfluss des Wildlings auf das Edelreis, nämlich in Bezug auf kräftigeres oder schwächlicheres Wachsthum, je nach dem Verhältniss des Wildlings in dieser Beziehung."

VIII. La 12e question du Programme étant mise à l'ordre du jour, M. Oudemans lit la notice suivante:

Sur l'origine des Stomates de quelques espèces d'Aneimia.

„Quand on examine l'épiderme des feuilles de certaines espèces d'Aneimia, p. e. de l'Aneimia fraxinifolia, on est frappé de deux particularités, dont cependant l'une me paraît beaucoup plus intéressante que l'autre. La dernière, moins étrange, consiste en ce que les cellules épidermiques contiennent des grains de chlorophylle; mais puisque ce phénomène a été rencontré aussi dans d'autres fougères et qu'il doit être rangé parmi les choses connues, il me semble superflu de m'y arrêter.

L'autre particularité, qui n'a pas encore été décrite que je sache, et qui ne s'est jamais présentée à moi, lorsque je me suis occupé de l'examen de l'épiderme d'autres plantes, consiste en ceci. Les stomates ne sont pas répandus parmi les cellules épidermiques, de manière que celles-ci les entourent de tous côtés, mais ils occupent le centre de ces cellules, de sorte que chaque cellule épidermique forme un anneau fermé de toutes parts, 
au milieu duquel le stomate se laisse isoler au moyen de la solution de Schultz.

Non content d'avoir observé ce fait tout-à-fait singulier, je me suis demandé quels moyens la nature pourrait avoir employés pour assurer aux stomates de notre Aneimia cette place toute particulière entièrement en désaccord avec celle que ces organes occupent d'ordinaire.

Quatre solutions se sont présentées à mon esprit. On peut les formuler en ces termes:

1. Les stomates sont originaires d'un nucléus, qui après avoir occupé le centre des cellules épidermiques annuliformes, s'est transformé d'abord en cellule-mère, puis en deux cellules-filles, séparées par une fente.

2. Les stomates sont originaires d'une cellule parenchymateuse sous-épidermique, qui, pénétrant par le milieu de certaines cellules épidermiques, les a transformées en cellules annuliformes et s'est divisée en deux autres semilunaires.

3. Les stomates doivent être considérés comme provenant d'un poil, composé primitivement de deux cellules, l'une basilaire et l'autre apicale, et dont la dernière, tout en grandissant dans sa moitié inférieure, aura fini par détruire la cellule basilaire, en perforant la cellule épidermique du centre de laquelle le poil aurait pris naissance.

4. Les stomates ne sont que des cellules épidermiques, divisées comme de coutume en deux moitiés semilunaires par une cloison longitudinale. Leur position extraordinaire au centre de cellules épidermiques doit être expliquée par l'absorption des parois latérales qui, primitivenent, divisaient les cellules annuliformes en plusieurs portions et maintenaient le rapport entre la circonférence externe et interne de l'anneau.

Il va sans dire que le principal moyen, par lequel je pouvais être mis ì même de décider, laquelle de ces solutions présentait le plus de probabilité, consistait dans l'étude microscopique des différentes périodes d'évolutions, que les 
cellules épidermiques de notre Aneimia parcomrent avant d'atteindre leur forme ultérieure et invariable. Cette étude cependant présente les plus grandes difficultés, à cause de l'extrême délicatesse des différentes parties, dont les feuilles se composent. Aussi suis-je seulement parvenu à établir que les cellules, qui plus tard formeront les stomates, font saillie en dehors longtemps déjà avant leur séparation en deux moitiés semilunaires et qu'elles se présentent sous forme de ballons reposant sur l'épiderme lui-mềme. Cette observation toutefois nous force à recommaitre tout de suite, que les deux premières suppositions, énoncées plus haut, doivent être abandomées; de sorte qu'il ne nous reste qu'ì discuter les deux autres.

Quant à la 3e supposition, qui admet la probabilité que les stomates de notre Aneimia seraient les restes de poils, je dois avouer que je ne me souviens d'aucun fait, qui plaide en sa faveur; tandis que pour la $4_{e}$, fondée sur l'absorption des parois cellulaires, on pourrait alléguer beaucoup d'observations analogues. Ajoutons, que nous arons eu la bonne fortune d'en constater presque la justesse, en observant un stomate, occupant le milien d'une cellule, laquelle, au lieu d'être continue et parfaitement annuliforme, laissait roir une de ses cloisons primitives, vraisemblablement échappée ì la déliquescence, par laquelle il est juste de dire que les autres ont été atteintes.

Ce qui donne encore plus de vraisemblance à notre dernière explication, c'est que dans d'uutres espèces d'Aneimia et dans d'autres fougères en général, ces cloisons persistent toutes, de manière que les stomates, dans ces cas, sont entourés de toute une rangée de cellules épidermiques inaltérées.

J'aime à croire, Messieurs, que les considérations que j'ai eu l'honneur de soumettre ì rotre attention, rous auront domné, comme à moi-mème, la conriction que la $4^{\text {me }}$ supposition est la seule, qui puisse donner une explicationsuffisantedel'étrangephénomèneque jeviens de décrire." 
IX. M. le Président accorde le parole à M. PASSERINI de Parme, qui, conformément à l'annonce du Programme (Q. IV, 2), se propose de discuter:

Sur la fonction des Stomates.

M. Passerini fait connaître une observation fort intéressante, qui démontre que l'exhalaison des gaz a lieu directement par l'intermédiaire des stomates.

,Même après les travaux très importants de M. MoHL, dit-il, de M. Ed. Morren et d'autres auteurs sur les stomates, on ne peut admettre que leur vraie fonction dans l'économie végétale soit bien connue.

N'ignorant pas que certaines plantes arrosées avec une solution de sulfite de soude exhalent bientôt de leurs feuilles de l'acide sulfhydrique, qui noircit le papier imbibé d'acétate de plomb, j'ai étudié le moyen de découvrir par où s'exhalait cet acide.

Après avoir arrosé un jeune individu de Pereskia Bleo avec une solution de sulfite de soude (100 parties d'eau, 5 de sel) j'ai appliqué sur les deux faces des feuilles de minces bandelettes de papier très delié et transparent, imbibé d'acétate de plomb et conservées toujours humides.

Ayant vérifié la tache noire des bandelettes quelques heures après, je les ai soumises à l'examen microscopique; et j'ai constaté que cette tache n'était ni continue ni uniforme comme on aurait pu le croire à l'oeil nu; mais elle était formée, au contraire, de points circonscrits et disséminés, de façon à simuler à peu près une réproduction de la disposition et de la grandeur des stomates. Les interstices blanes du papier entre ces points n'étaient pas altérés le moins du monde.

J'aroue que je n'ai pas eu le temps de répéter cette observation, qui suffirait pour nous prouver que l'acide sulfhydrique est sorti directement par les stomates.

Une telle recherche, qui du reste me parait neuve, pourrait bien nous conduire, selon moi, à d'importantes découvertes sur un point encore bien obscur de la physio- 
logie végétale, et c'est précisément pour cette raison que j'en ai fait part à cette docte assemblée."

M. Morren a écouté la communication de M. PASSERINI avec le plus vif intérêt et il admire l'expérimentation si ingénieuse, qui a permis à ce savant de déterminer d'une manière précise et directe que l'exhalaison des gaz s'opère par les stomates. M. Morren s'est spécialement occupé de cet organe. Il en a déterminé le nombre dans un certain nombre de plantes (1) et il a communiqué à l'académie royale de Belgique les résultats, auxquels il estarrivé. Il a conclu d'expériences qu'il a poursuivies pendant plusieurs amnées, relativement à l'influence de divers gaz sur la végétation, que l'absorption de ces gaz, spécialement ceux des acides carbonique et sulfureux s'opére par les stomates. L'acide sulfurenx étant fort nuisible à la végétation, même en proportion minime, il a pu s'assurer que la sensibilité des plantes, variable suivant les espèces, est directement proportionnelle au nombre des stomates. En d'autres termes, que, toutes choses égales d'ailleurs, une plante absorbe d'autant plus d'acide sulfureux qu'elle présente plus de stomates. Lorsqu'il bouchait ces organes en enduisant avec de la cire le tout ou une partie des feuilles, les parties ainsi protégées n'absorbaient plus ni l'acide sulfureux, ni l'acide carbonique.

M. Wesmael confirme les conclusions de M. Morren. Il s'exprime en ces termes:

\section{Messieurs!}

,Les observations de notre savant confrère, M. ED. Morren, sur le nombre des stomates des feuilles de Poirier et de Prunier, viennent rendre compte de l'observation d'un phénomène, dont j'ai été témoin an mois de Juillet de l'année dernière. Dans un verger, voisin d'une exploitation charbonnière, je remarquai que la presque totalité

(1) Détermination du nombre des stomates. Bull. de l'Acad. roy. des Sc. de Belgique 2e série, tome LVI, No. 12. 
des arbres fruitiers était inalade; les feuilles étaient jaunes et certaines d'entre elles présentaient des taches, comme si un acide les avait touchées. Renseignements pris, on me dit qu'un tas de schiste houiller, très riche en pyrite (sulfure de fer) brûlait depuis plusieurs mois dans les environs. Ce seul renseignement me suftit pour me rendre compte du singulier phénomène que j'avais observé sur les feuilles. En effet, par suite de la combustion des pyrites, une notable quantité de gaz sulfureux se répandait dans l'atmosphère et était directement absorbé par les feuilles ou indirectement par l'eau, donts'emparaient ces mêmesorganes. J'avais constaté, que les pruniers étaient beaucoup plus malades que les poiriers, et jusqu'à ce jour je ne me rendais pas bien compte de cette différence. Les observations de M. Morren sur le nombre des stomates viennent me donner une solution du problème. Si le nombre de ces petits organes est beaucoup plus considérable chez le prunier que chez le poirier, il doit en résulter, que le premier de ces arbres doit absorber une plus grande quantité de rapeurs sulfureuses.

X. M. Hasskarl (de Clèves), présente les communications suivantes sur les Commelinacées. (Voir le Programme, quest. XI, p. 20).

„,Die Familie der Commelinaceae, - meine Herren über welche ich Thnen heute einige Mittheilungen zu machen gedenke, ist eine von denen, welche noch am meisten der monographischen Bearbeitung bedarf; der Grund ihrer Vernachlässigumg ist wohl hauptsächlich darin zu suchen, dass die Blüthen der Commelinaceen meistentheils ungemein vergänglich sind, so dass man sie in den Herbarien nur selten in gehörig entwickeltem Zustande vorfindet. Hierzu kommt noch, dass die Mitglieder dieser Familie rorzugsweise Kinder der Tropenländer und nur in rerhältnissmässig geringer Zahl in unsern Gärten vorhanden sind, wodurch es denen, welche sich 
mit deren Untersuchung befassen wollen, nur in wenig Fällen gelingt, zu diesem Behufe frische Blüthen- oder Frucht-Exemplare zu erlangen. Letztere, die FruchtExemplare, finden sich auch nur sparsam in den Herbarien, obgleich nicht so sparsam, als es auf den ersten Anblick den Anschein hat, da die Früchte meist nur bei sorgsamer Untersuchung der Blüthenstände gefunden werden und sich dem nur flüchtigen Blicke des oberflächlichen Beschauers meist entziehen. Olme Beachtung der Früchte aber ist eine gute und scharfe Characterisirung der Gattungen kaum zu ermöglichen.

Wenden wir vorerst einen kurzen Ueberblick auf die Geschichte dieser Familie, die als solche allerdings erst längere Zeit nach dem Bekanntwerden der ersten Repräsentantin derselben in Eriropa zur Geltung und Anerkennung gekommen ist. Es war im Jahre 1622, als der Baseler Arzt Georg Spörlin eine Pflanze aus dem berühmten Garten von Јон. Roвin in Paris an den Schweizer Botaniker Caspar Baumin sandte, und zwar unter dem Namen Phalanginm virginianum; C. BadHIN war grade mit der Herausgabe seines $\Pi$ Iiv $\boldsymbol{\xi}$ beschäftigt und beschrieb diese ihm zugesandte Planze im Anhange zu diesem Werke,(p. 520) unter dem Namen Allium seu Moly virginianum; - es ist dies die jetzige Tradescantia virginica $\mathbf{L}$. Zwar hatte Hernandez in seinem grossen Werke über die Pflanzen Mexico's schon 1615 die Commelina tuberosa L. unter dem Namen Matlalytztic Tetzcoana s. Triorchis mexicana abgebildet und nach seiner Weise beschrieben; wie bekannt, blieb dieses Werk aber im Escurial verborgen, wo es gegen Ende des siebenzelnnten Jahrhunderts vom Feuer rerzelnt wurde; nur ein von Recchi in Rom veranstalteter Auszug aus diesem Werke gab auf Tab. 253 eine Darstellung der erwähnten Pflanze; da dieser Auszug aber erst 1651 erschien, so war es die virginische Pflanze, die der wissenschaftlichen Welt in Europa zuerst einen Repräsentanten der Commelinaceen vorführte. 
Vergeblich sucht man in dem späteren (1650) grossen Werke JоH. BAбHrs's, Historia plantarum, nach einer auf diese Familie bezüglichen Spur, während doch Bontrus (1642) schon einige Jahre früher, in seiner Historia naturalis (p. 154), unter dem Namen Gentianella indica die Abbildung einer Pflanze lieferte, die zwar, was Deutlichkeit betrifft, vieles zu wünschen übrig lässt, in welcher ich aber demnoch eine Commelina zu erkennen glaube. Erst ein halbes Jahrhundert später (1705) erscheinen unter dem Namen Ephemerum mehre Repräsentanten dieser Familie im dem Paradisus batavus (p. 140 sqq.) von Hermann.

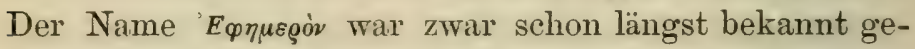
wesen; die Alten hatten darunter alle Gifte verstanden, welche - einmal ins Innere des menschlichen Körpers gelangt - innerhalb eines Tages tödtliche Wirkungen hervorriefen. Später hatte man diesen Namen jedoch auch unschädlichen Pflanzen gegeben, so dass bald Pflanzen der verschiedensten Verwandtschaften ron den verschiedenen Pflanzenkennern unter diesem Namen aufgeführt wurden. Hermann num beschränkte diese Zahl auf einen engeren Kreis und beschrieb unter Ephemerum eine Zahl (17) von Pflanzen unserer Familie so deutlich und klar, dass die Commelinaceen darin nicht zu verkennen sind; dass er den erwähnten Namen dafür wählte, hat wohl in der grossen Flüchtigkeit der Blüthen der damals bekannten Arten seinen Grund. Thm folgte (1719) Tounnefort, welcher in seinen Institutiones rei herbariae (p. 365) den Namen Ephemerum für diese Pflanzen beibehielt, die Gattung näher characterisirte und 10 Arten davon aufzählte, von denen jedoch die 8 ersten nur Varietäten der obenerwähnten virginischen Pflanze waren, wie dem auch Herma w unter seinen Arten ebenfalls 5 Varietäten der genamnten Art aufzählte.

Inzwischen kam die Zeit des grossen Lrnvé; am Schlusse (1771) seiner botanischen Laufbahn kannte er bereits 19 Arten; der Name Ephemenum aber, welcher gewissermassen die ganze Familie bezeichnete, findet 
sich nicht mehr; Linné hatte dieselbe in 3 Gattungen vertheilt, von denen eine, Commelina, schon (1703) von Puumier (Nov, plant. gener. 48) den keiden Brüdern ConMELxN zu Ehren diesen Namen erhalten hatte, und ron welchem sich bei Dillenius (Herb. Elthamense p. 91) schon (1732) drei Arten abgebildet fanden. Linné hatte diese Gattung adoptirt und 11 Arten davon aufgezählt; Callisia trat mit einer Art und Tradescantia mit 7 Arten auf; er zählte sie zu seinen Ensatis. Auch BernH. Jussiev brachte die Pflanzen dieser Familie (1759) mit vielen andern zu seinen Juncis und erst Batsch (1802) stellte dieselben in seinen Tabul. affinit. (p. 125) zuerst als eigene Familie unter dem Namen Ephemereae auf, nach der von Möмcн (1784) noch festgehaltenen Gattung Ephemerum, worunter dieser die Tradescantia verstand, wie demn auch LAмarck in seiner Encyclopaedie (II, 370) diese Gattung mit dem französischen Namen: Ephemerine belegte; zu dieser Familie rechnete MöNcH aber auch noch einige jetzt nicht mehr dazu gehörigen Pflanzen.

Mit raschen Schritten vergrösserte sich bald die Anzahl der hiezu gerechneten Pflanzen; WILLDENow (1797) erwähnte in seinen Species plantarum bereits 31 Arten, noch in die đrei Linné'schen Gattungen vertheilt, aber nur wenige Jahre später (1805) zählte Persoon in seiner Synopsis plantanum schon 46 Arten in 5 bis 6 Gattungen, wobei zu den bereits genannten Gattungen noch Pollia Thnb. und Flos-scopa Lour. kamen, während er die PLUxren'sche Gattung Zanonia zwar aufstellte, sie aber von Commelina noch nicht zu trennen wagte. 20 Jahre später kannte C. Sprenger (1825) in seinem Systema vegetabilium schon 109 Arten in 8 Gattungen, und in dem, theils einige Jahre früher, theils nur einige Jahre später erschienenen gleichnamigen Werke von Roener und Schoutes $(1817$ und 1827) war die Zahl der Arten bereits anf 164, die der Gattungen auf 11 gestiegen, indem zu den früher genannten moch Aclisia E. Mey., Cyanotis Don., Campelia L. C. Rich., 
Dichorisandra Milnn., Cartonema Lndl. und Lamprocarpus Bl. hinzugetreten waren, von denen die letztgenannte sich später freilich als mit Pollia Thnb. zusammenfallend erwies. S. Kuntr endlich lieferte (18t3) in seiner Enumeratio plantarum eine monographische Zusammenstellung dieser Familie, die einzige bis auf den heutigen Tag, welche daher gegenwärtig noch bei Bestimmung der' Pflanzen der Commelinaceen als Richtschnur dienen muss, unerachtet seitdem schon fist ein Viertel Jahrhundert darüber hingegangen ist, und in dieser Zeit sich die Zahl der bekannt gewordenen Pflanzen dieser Familie in Vergleich mit der früher bekamnt gewesenen zu einer ungemein grossen angesammelt hat. Schon Kunтн hatte in kürzerer Zeit über humlert Arten mehr gekamnt (269), die er in 17 Gattungen vertheilte, von denen einige abermals mit bereits bestehenden, jedoch nicht genügend bekannt gewesenen, zusammenfallen. Vergeblich sucht man aber in dieser Monographie nach sorgfältiger Verarbeitung des vorhandenen Materials; Kunтн begnügte sich damit, dies Material nur zusanmenzutragen, und wemn er auch selbstständige Untersnchungen vornahm, so wurden dieselben doch nicht inaassgrebend für die Aufstellung neuer Gattungen, die er wohl ahnend hier und da andentete, aber doch den bekamnten feststeheuden Gattungen unterordnete und sie höchstens als Unterabtheilungen hervortreten liess. Ebenso benutzte Kunтr auch die von verschiedenen Autoren oft nach sehr verschiedenen Gesichtspunkten aufgestellten Diagnosen der Arten, so dass die verschiedenen oft zu einer Art zusammengestellten Pflanzen gar nicht zu den gegebenen Diagnosen passen. Allerdings ist nicht zu rerkemen, dass manche Arten dieser Fanilie, welche R. Brows (1810), in seinem Prodromus, zuerst genaner characterisirte mul Commelinucute nannte, je nach der Verschiedenheit des Stancortes mol äusserer Verhältnisse einem grossen Formenreichthum zeigen, der sich schwer in die fiüher beliebten kurzen 
Diagnosen zusammendrängen liess; wie aber kann eine Diagnose, die of nur nach einer dieser Formen und häutig genug noch dazu in sehr unrollikommenem Zustande angefertigt ist, - wie kann eine solche Diagnose dazu dienen, die formenreiche Art in ihrer Mamnichfiltigkeit zu erkemen? Es müssen daher neue Diagnosen, den ganzen Formenkreis der Arten umfassend, gebildet werden, zugleich auch unter Berücksichtigung der vielen neuen inzwischen bekannt gewordenen oder neu anfzustellenden Arten. Wie sehr aber der Reichthum der Gattungen an Arten zugenommen hat, möge aus einigen Beispiclen entnommen werden: $C y$ anotis Don, zählte bei Kuntr 11 Arten, bei meinen Untersuchungen bin ich bereits auf ungefähr 50 Arten gekommen; Campelia, welehe früher zum Theil mit Forrestia verwechselt wurde, von welcher nur eine Art bekannt war, zählte bei Kunтн 6 Arten, während mir jetzt davon 8 Arten bekannt sind und Forrestia 6 Arten erlangt hat. In nachstehender Tabelle habe ich die Zniname an Arten bei den einzelnen Gattmingen nach den verschiedenen Zeitepochen zusammengestellt:

\begin{tabular}{|c|c|c|c|c|c|c|c|c|c|c|c|c|c|c|c|c|c|c|}
\hline ANNo. & AUTOR. & OPUS. & 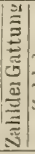 & 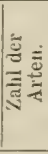 & 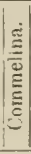 & . & 赵 & 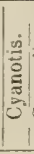 & 这. & 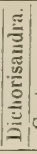 & 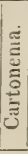 & 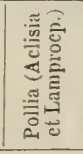 & 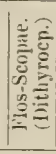 & 施 & 吾 & 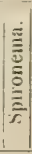 & 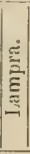 & 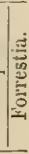 \\
\hline $\begin{array}{c}1771 \\
1797 / 99 \\
1805 \\
1825 \\
3817 / 27 \\
1813\end{array}$ & $\mid \begin{array}{l}\text { LINNE ........... } \\
\text { WILLDENOW ... } \\
\text { PERSOON ......... } \\
\text { SPRENGEL ...... } \\
\text { RöM, et SCHIULT } \\
\text { KUNTH............ }\end{array}$ & $\begin{array}{l}\text { (Spec.plant.\&Mant.II) } \\
\text { Species plantarum ... } \\
\text { Synopsis plantarum... } \\
\text { Systema vegetabilium } \\
\text { Eouneratio plautar... }\end{array}$ & \begin{tabular}{|r|} 
\\
3 \\
3 \\
5 \\
8 \\
11 \\
17
\end{tabular} & $\begin{array}{l}19 \\
31 \\
46 \\
109 \\
164 \\
269\end{array}$ & $\left\{\begin{array}{l}11 \\
13 \\
22 \\
69 \\
80 \\
81\end{array}\right.$ & $\left\{\begin{array}{l}1 \\
1 \\
3 \\
1 \\
3 \\
3\end{array}\right.$ & $\begin{array}{l}17 \\
17 \\
19 \\
31 \\
12 \\
62\end{array}$ & $\begin{array}{l}- \\
- \\
- \\
- \\
9 \\
11\end{array}$ & $\begin{array}{l}- \\
- \\
- \\
1 \\
4 \\
6\end{array}$ & $\mid \begin{array}{c}- \\
- \\
4 \\
4 \\
21 \\
29\end{array}$ & $\begin{array}{l}- \\
- \\
1 \\
1 \\
1\end{array}$ & $\begin{array}{c}\frac{-}{1} \\
1 \\
1+1+1 \\
1+1+1\end{array}$ & $\begin{array}{c}- \\
\frac{1}{1} \\
1 \\
1 \\
1+8\end{array}$ & $\begin{array}{l}- \\
- \\
- \\
- \\
- \\
60\end{array}$ & $\begin{array}{l}- \\
- \\
- \\
- \\
\overline{1}\end{array}$ & $\left\{\begin{array}{l}- \\
- \\
- \\
- \\
1\end{array}\right.$ & $\begin{array}{l}- \\
- \\
- \\
\overline{1}\end{array}$ & $\begin{array}{l}- \\
\overline{-} \\
\overline{1}\end{array}$ \\
\hline
\end{tabular}

In gleichem Maassstabe, wie sich hier die Zumahme der Arten herausstellt und solche bei Cyanotis, Campelia und Forrestia oben nachgewiesen wurde, in gleichem Maassstabe haben auch die anderen Gattungen an Artenreichthum zugenommen, wälnend andrerseits auch die Zahl der schon bis jetzt bekannten Gattungen nicht mubetriichtlich gewachsen ist und bei sorgfältiger Untersuchung sich immer mehr vergrössert, so dass wohl mit einiger Sicherheit 
deren Zahl auf 30 bis 40 angegeben werden darf. Von verschiedenen .Seiten sind schon tüchtige Vorarbeiten zur näheren Erkemntniss der Commelinaceen-Gattungen geliefert worden; ich brauche nur an die Namen: Wigнt, Edgeworth, Scheidweiler, Schnitzlein, v. Schlechtendal, Bentham, Lindley, C. Косн zu erinnern. Wiederholt wurde durch von Schlechtendal in seiner Linnaea einerseits darauf hingewiesen, dass die Unterabtheilungen der Gattung Tradescantia bei Kunтн wohl selbstständige Gattungen bilden, andrerseits dass die Untersuchung auch der Früchte wesentliche Unterscheidungskennzeichen zur Bildung neuer Gattungen zu liefern geeignet sein dürfte. Auch der unter uns anwesende Professor C. KocH hat im 2ten Jahrgange seiner Wochenschrift wichtige Andeutungen in dieser Richtung gegeben. Meine noch erst schwachen Anfänge der Untersuchung haben mir bei dieser Familie schon reiche Ausbente geliefert und noch mehr in Aussicht gestellt.

Ehe ich aber zur Untersuchung selbst überging, habe ich eine Sichtung des vorhandenen Materials vorgenommen, wie solches in den verschiedenen Werken zerstreut vorkommt. In der hierbei folgenden Uebersicht habe ich die Resultate dieser Nachlese zugleich mit den bis dahin gemachten eigenen Untersuchungen in möglichst lklarer Weise zusammengestellt; es war mir dabei besonders wichtig, alle angegebenen Unterschiede, die sich zu Gattungscharacteren verwerthen liessen, hervorzuheben, um bei der Untersuchung der betreffenden Pflanzen darauf hingewiesen zu werden, diese Kennzeichen genauer zu würdigen. Manche werden vielleicht als nicht stichhaltig erkannt werden; anch ein solch' negatives Resultat hat seinen Werth. Ich habe diese Uebersicht in Duplo angefertigt, ohne dass beide vollkonmen übereinstimmen; denn während die eine dieser Uebersichten die Commelinaceen in zwei grosse Gruppen theilt, je nachdem alle Staubgefässe fruchtbar, oder theilweise unfruchtbar und 
umgebildet sind, enthält die andere cine neue Theilung der mit nur 3 fruchtbaren Staubgefässen versehenen Gruppe, nach dem Vorgange von Wigнт. Es können nämlich dieser Ansicht zufolge entweder die Staubgefässe der einen Seite fehlschlagen, wie bei den Aneilemateen, oder die alternirenden Staubgefässe sind es, die unfruchtbar werden, wie bei den Commelineen; während die Gruppe der Tradescantieen, bei welchen alle Staubgefässe fruchtbar sind, hierbei unberührt bleibt. Bis dahin haben meine Untersuchungen vorzüglich letztgenannte Gruppe umfasst und fehlt mir über die Beständigkeit des WIgнт'schen Eintheilungsprincips die Erfahrung; sollte es aber, wie sehr wahrscheinlich ist, von durchgreifender Bedeutung sein, so würde dadurch die Uebersichtlichkeit der Gattungen dieser Familie nur gewinnen können.

Gestatten Sie mir, an einem Beispiele nachzuweisen, wie sehr selbstständige Untersuchung bei dieser Familie Noth thut, und wie wenig man sich mit blosser Zusammenstellung des vorhandenen Materials begnügen kann. Die Gattung Forrestia wurde vor etwa 25 Jahren von A. RICHARD nach einer Pflanze von Neu-Guinea aufgestellt, seitdem aber von Niemandem wieder erkannt, und doch hatte man in den Tropenländern der östlichen Hemisphäre verschiedene zu dieser Gattung gehörige Pflanzen gefunden; man brachte diese zu der Gattung Campelia, die freilich im unfruchtbaren Zustande ihrer Arten eine habituelle Uebereinstimmung mit denen der Forrestia zeigten, die aber im blühenden oder fruchttragenden Zustande damit gar nicht verwechselt werden können, auch wenn man absieht von den feineren Unterschieden, welche die Gattungscharaktere beider Gattungen darbieten. Das einzige, welches beide gemein haben, ist, dass die Blüthenstände bei ihrer Entwicklung von dem Blatt- oder Scheidewinkel aus die Blattscheide selbst durchbrechen; während bei Campelia die Blüthenstiele aber lang sind und an der Spitze zwei Blätter tragen, welche die dolden- 
kopffömig gredrängten Blïthen unterstiitzen, bilden dieselben bei Forrestiu einen Knäuel, der bei genanerer Untersuchung meist eine sehr verkürzte Trugdolde oder Rispe ist, die sich ohne Blüthenstielverlängerung ummittelbar auf der Blattscheide selbst ausbreitet und dioselbe bedeckt, "supra vaginam exserta (inflorescentia)" wie A. RICHARD sich ausdrückt.

Bei Erwähnung der Gattung Campelia, tritt eine andere Frage in den Vordergrund. Schon 1703 hatte Plumier (Nov. plant. amer. gener., 38) eine Pflanze dieser Gattung als Zanonic yiaminea perfoliata aufgestellt. Als nun später Linné dieselbe in seinem Systeme einreihen wollte, fund er deren Unterschied von der ebenfalls PLUnIER'schen Gattung Commelina, die er adoptirte, nicht so bedeutend, dass er sie glaubte bestehen lassen zu können; er ordnete sie letztgenamnter Gattung unter und nannte sie Commelina Zanonia. Um aber den Gattungsnamen Zanonia nicht untergehen zil lassen, gab er denselben einer dieser Familie gar nicht verwandten Pflanze (Cucurbitacee). Als man später aber die Plumer'sche Zanonia näher kenmen lernte, fand man, dass sie doch nicht mit Commelina vereinigt bleiben könne und Cramer brachte (1803) die alte Gattung grade hundert Jahre nach ihrer Aufstellung wieder zu Ehren. PErsoon führte den Gattungscharakter unter Zanonia wohl auf, konnte sich aber nicht entschliessen, sie ganz von Commelinc zu trennen (Synops. I, 347, 19), so dass sich also bei ihm zwei Zanonia's vorfinden (Syn. II, 618). Wenige Jahre später (1808) stellte L. C. Richard (Analys. d. fruct. 46) diese Gattung auch als selbstständige wieder auf und gab ihr den Namen Campelia. Wollte man bloss nach dem Rechte der Priorität urtheilen, dann müsste allerding's die Plunier'sche Zanonia bestehen bleiben; wir müssten damn aber der Linsé'schen Zanonia einen neuen Namen geben, was doch wohl manche Unzuträglichkeiten mit sich bringen dürfte. 
Ganz anders scheint es sich mit dem Gattungsnamen Cyanotis zu verhalten. R. Brown hatte nämlich diese Gattung in seinem Prodromus (I, 269, ed. Nees, 125) sehr deutlich charakterisirt, aber nicht benannt; diess that num D. Don (1825) in seinem Prodr. Fl. nepal., 45; allein schon jo Jahre früher hatte Salisbury (Horticult. Transact. I) eine andere Art dieser Gattung unter dem Namen Zyyomenes axilluris aufgeführt. Mit einem Scheine von Recht bemerkte daher schon vor mehren Jahren Prof. Косн (Wochenschr. II, 3336), dass dieser letztere Name Zygomenes aus Prioritäts-Riicksichten dem Don'schen Cyanotis vorzuziehen sei; allein SALIsBury hat nur einen Namen und keine Beschreibung dieser Gattung gegeben, weshalb wohl der Don'sche Gattungname beizubehalten sein dürfte.

Eine sorgfältige Untersuchung dieser Gattung lehrt, dass die beiden Hauptgruppen, in welche Kunтн (Enum. IV, 102 u. 105) sie theilt, nicht so scharf die Arten von einander scheiden, dass man sie in besondere Gattungen trennen dürfte; Uebergänge sind gar nicht selten; dagegen giebt die Fruchtbildung wohl Anleitung zu weiteren Unterabtheilungen, wie Sie diess aus der folgenden Uebersicht erkennen werden:

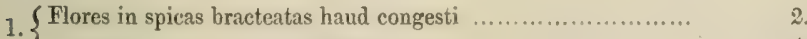

(" " " sane $"$ ".....................

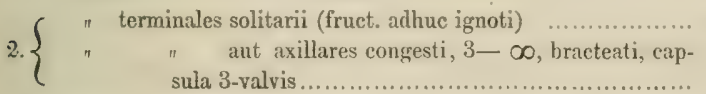

I. Monanthae. sula 3 -valvis .....................................

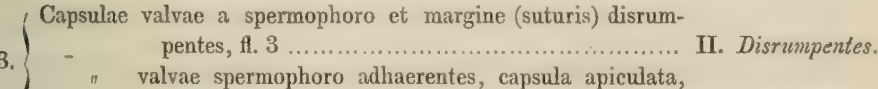
flores $\infty$........................................ III. Glomeratae.

4. $\left\{\begin{array}{cccc}\| & \text { valvae a spermophoro haud sejunctae ................. } \\ " \text { " } & \text { sejunctae } & \text { centrali columnaeformi } 3 \text {-fido }\end{array}\right.$

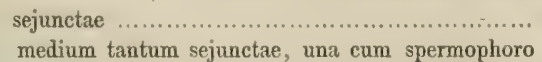

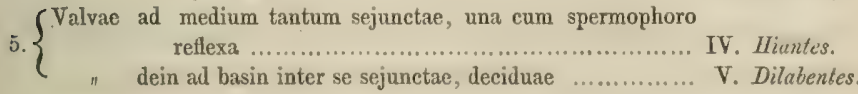

Je nachdem die Blüthen nämlich in einer mehr oder weniger langen, gestielten, zweizeilig mit Deckblättern 
versehenen und von einem Blatte unterstützten Aehre stehen oder nicht, zerfallen die Arten in zwei Gruppen, von denen die letztere wieder nach der Fruchtbildung in zwei Abtheilungen getheilt werden unuss, je nachdem sich die Fruchtklappen vom Samenträger und ihren Nähten trennen oder damit verbunden bleiben, wonach die Abtheilungen Dismumpentes und Glomeratue genamnt wurden, während die einzige Art mit einzelner endständigen Blüthe, deren Früchte bis dahin unbekannt geblieben sind, zur Abtheilung Monanthae gerechnet wurde. Die zweite grosse Gruppe zeigt Früchte, die nur bis zur Mitte sich theilen und deren Scheidewände ebenfalls nur bis zur Mitte getrennt sind, übrigens aber der Mitte der Fruchtklappen aufsitzen: Hiantes; bei denjenigen Arten, deren Früchte sich bis anf den Grund in 3 Klappen theilen, unterscheiden sich einige dadurch, dass die darauf eingefügten Scheidewïnde sich von den zu einer oben dreitheiligen Sänle verwachsenen Samenhaltern tremen, so dass diese Achse der Frucht stehen bleibt: Columnifercae; oder dass diese Scheidewände auf ihrem inmeren Rande von dem Samenträger eingefasst sind, so dass die Frucht hernach in 3 gleiche Theile zerfällt: Dilabentes. Ich konnte, besonders mit Rücksicht auf die Uebereinstiumung der dazu gehörigen Arten im Habitus, diese Verschiedenheiten nicht als so wesentliche anerkennen, um darauf Gattungscharaktere zu begründen. Ganz anders dagegen steht es mit der Cyanotis vivipara Dalz., welche sowohl durch Fruchtbildung als durch ihren Habitus als etwas so Eigenthümliches erscheint, dass ich selbige als neue Gattung, die ich Dalzellia namnte, glaubte betrachten zu müssen. Achselständige Blüthenstiele entwickeln sich hier mitunter zu kleinen blättertrag'enden wenigblüthigen Träubchen, mitunter tragen sie nur eine einzelne Blüthe; die Staubgefässe sind bartlos; die Kapsel theilt sich bis auf den Grund in 3 Klappen, die auf der Mittellinie die mit dem Samenträger verbundenen Scheidewände tragen; 
die beiden Hälften der Klappen legen sich zurück in eine Fläche mit der Scheidewand und drehen sich sodann widderhornähnlich zusammen, wobei sie zwischen den äusseren stehenbleibenden Blüthenhüllblättchen am Grunde in eigenthümlicher Gestaltumg selbst stehen bleiben. Ich nannte diese Pflanze Dalzellia vivipara.

Indem ich hiermit meine Mittheilungen schliesse, hoffe ich über die oben atusgesprochenen Zweifel von Thnen eine Lösung erwarten zu dürfen. 


\section{TABULA SYNOPTICA COMMELINACEARUM.}

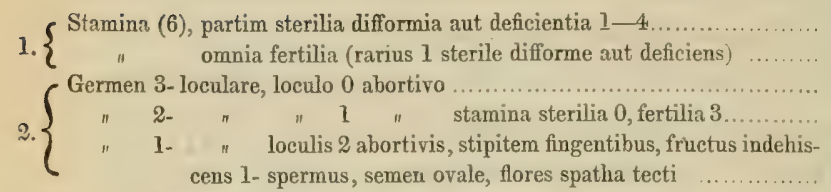

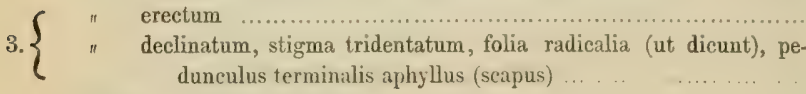

4. $\left\{\begin{array}{c}\text { Gemmulae in loculis solitariae } \\ \| \text { ". " }\end{array}\right.$

Inflorescentia paniculata, perigonium marcescens, filamenta imberbia

5. $\begin{cases}\text { spatha turbinata tecta, perigonium caducum, pedicelli soli- } \\ \text { " }\end{cases}$ tarii (Commel, polyspatha Wght. Ic. 2066.).

6. $\left\{\begin{array}{r}\text { Stamina sterilia 3, fructus stipitatus tomentosus, bracteae cuculliformes } \\ \text { persistentes (Aneilema protensum WII. \& A. riticnse Secm.) ... 1. Piletocarpus Hsskl. } \\ \text { sterilia 2, fructus sessilis laevis nitidus, bracteae apertae deciduae 2. Dictyospermum Wg }\end{array}\right.$

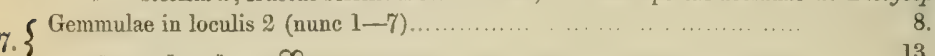

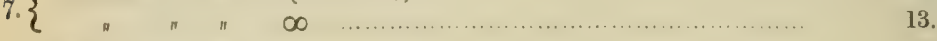

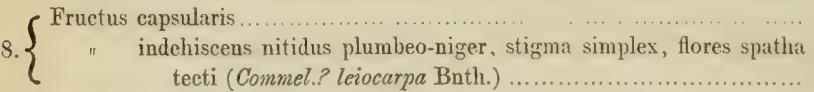

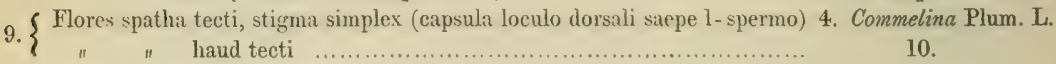

$\{$ Stigma simplex (P), gemmulae et semina in loculis plerumque 2 (nunc $l-7$ )

Stigmata 3 penicillata (Callisia Schlchtd.).......

11. Flores in racemis secundis, rhachis serraeformi-exsculpta ...........5. Prionostachys Hsskl.

11" " " " hand dispositi, rhachis haud serraeformi-exsculpta 6. Aneilema RBr.

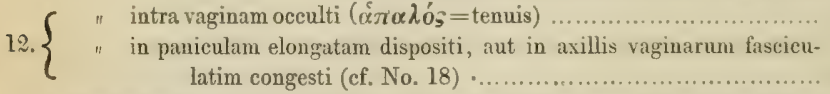

13. $\{$ Filamenta stricta, perigonii foliola interna integra, genmulae orthotropae

13. $\{$ spiraliter torta, " " fimbriata, ". anatropae... 14. Cochliostemma Lenoir.

14. $\{$ Gemmulac in loculis 1- seriatae, fructus indehisceus, dein coeruleo-piumbeus

Stigma capitatum, flores paniculati, perigonii foliola interna coerulea, folia parva ..................................................... folia lata congesta.

7. Hapalanthus Ieq.

8. Callisia Loeffl.

14.

15.

16.

12. Dichoesperma Wght.

13. Palisota Rehb.

16. Flores solitarii aut bini ad apicem pedunculi, spatha cordata tecti. (Commel. pallida Wld.)... .............................................

10. Athyrocarpus Schlchtd.

11. Pollia Thnb.

17. $\left\{\begin{array}{c}\text { Gemmulae in loculıs solitariae } \ldots \ldots \ldots \ldots \ldots \ldots \ldots \ldots \ldots \ldots \ldots \ldots \ldots \ldots \ldots \ldots \ldots \ldots \ldots \ldots \ldots \ldots \ldots \ldots \ldots \ldots \ldots \ldots \ldots \ldots \ldots \ldots \ldots \ldots \ldots \ldots \ldots \ldots \ldots \ldots \ldots \ldots \ldots \ldots \ldots \ldots \\ " \text { " geminae } \ldots \ldots \ldots \ldots \ldots \ldots \ldots\end{array}\right.$

18.

19. 
$\{$ Perigonii foliola interna longissime unguiculata.....

16. Polyspatha Buth.

18. $\{$ " " breviter " capsula stipitata obovato-

19. $\{$ Stamina 3-1 fertilia, sterilia 0 , antherarum loculi ad marginem con-

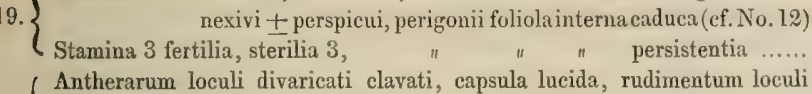

19b. $\left\{\begin{array}{r}\text { Antherilis } 0 \text {, spatha nulla ....................................... } \\ \text { "loculi basi divergentes, capsula opaca, rudimentum loculi }\end{array}\right.$ sterilis umbiliforme, spatha complicata ...................... 18b. Omphalotheca Hsskl.

20. $\{$ Stamina diversifornia aut eorum antherae tantum .......................

$\left\{\begin{array}{cc}\| & \text { conformia } \ldots \ldots \ldots \ldots \ldots \ldots \ldots \ldots \ldots \ldots \ldots \ldots \ldots \ldots \ldots \ldots \ldots \ldots \ldots \ldots \ldots \ldots \ldots \ldots \ldots \ldots \ldots \ldots \ldots\end{array}\right.$

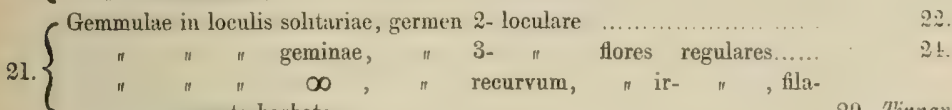
" " $\infty$, " recurvum, " ir- ", fila-

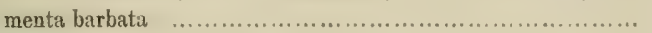

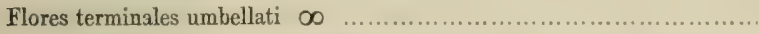

29. Tinnantia Schdw.

23.

22. " axillares in spicam umbellatam congesti (cf. No. 28) (Tradescantia

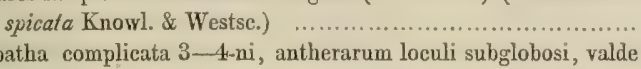
disjuncti, connectivo filiformiarcuato(Tradesc.commelinoid.RS.) 24 $23 .\left\{\begin{array}{c}\text { " spatha haud tecti .................................................. 21. Descantaria } \\ \text { " duplici " fructus plerumque bilocularis, stylus filiformis, } \\ \text { stigma obtusum, herbae acaules (ut dicunt) .................. 22. Rhoco Hanc. }\end{array}\right.$

24. $\left\{\begin{array}{c}\text { Perigonii foliola interna linearia, herbae volubiles ......................... 25. Streptoli } \\ \| " \text { latiora obtusa, herbae haud volubiles ........... }\end{array}\right.$

23. Knowlesia Hsskl.

1. Descontaria Schlehtd.

Stylus longus, stigma minutum, fructus perigonii foliolis externis inclusus,

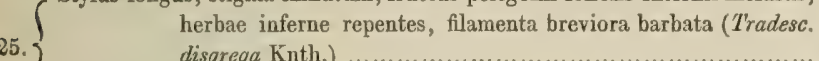

" brevis .....................................................................

26. Disgrega Hssk].

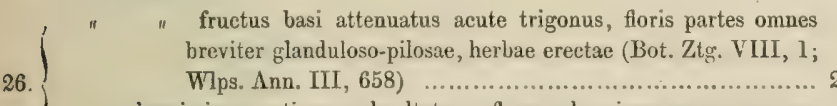

brevissimus, stigmi subpeltatum, flores ad apicem ramorum umbellati, herbae repentes......................................

$\left\{\begin{array}{c}\text { Gemmulae in loculis solitariae } \ldots \ldots \ldots \ldots \ldots \ldots \ldots \ldots \ldots \ldots \ldots \ldots \ldots \ldots \ldots \ldots \ldots \ldots \ldots \ldots \ldots \ldots \ldots \\ \text { " " }\end{array}\right.$

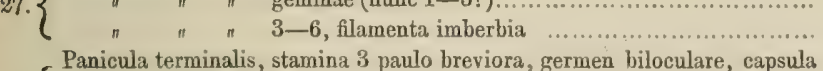

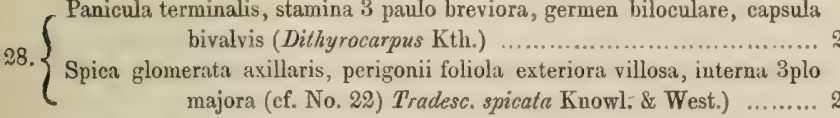

29. $\{$ Perigonium profunde 6 - partitum ....................................... 30.

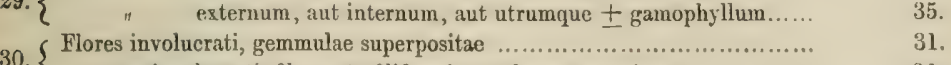

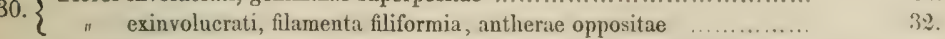

Perigonii foliola interna unguiculata persistentia, connectivum latum ..... 29. Tradescantia I.

32. $\left\{\begin{array}{l}\text { Flores umbellati } \\ n \text { spicato-umbellati, s. spicati, s. condensato-cymosi, aut pauiculati, }\end{array}\right.$ sum, curvatum) vix connata, eoque potius suffulta (ef. No. 36) 30. Campelia L. C. Rich.

eapituliformem inflorescentiam sistentes

34. 
Umbella sessilis, herbae erectae aut procumbentes, folia lanceolata aut

33. $\begin{aligned} \text { linearia } \\ \text { Umbella pedunculata, herbae repentes, folia cordata, flamenta imberbia }\end{aligned}$

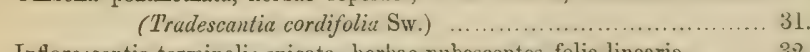

29. Tradescantia I. 1. -

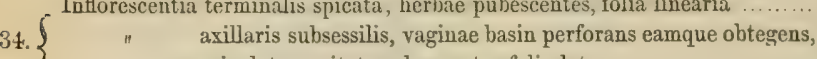
paniculato-capitata, glomerata, folia lata

33. Forrestia Less et A. Rich.

Perigonii foliola externa, basi tantum paulo aut vix connata. 35. $\left\{\begin{array}{l}\text { " " } " \text { in tubulum } 3 \text {-dentatum, interna in tubum } 1 \frac{1}{2}-2 \text { plo } \\ \text { longiorem, apice 3fidum connata, capsula inversa, perigonium }\end{array}\right.$ lateraliter perforans, stamina fauci perigonii interni inserta ... foliola exterua basi nunquam in pedicellum connata, interna ca
duca, in tubum 3-lobum connata, stamina hypogyna ...........

36. Zebrina Schnizl.

36.

foliola externa, basi sane (aut vix cf. No. 31) in pedicellum brevem, crassum, curvatum, inflexum connata, internum 3-partitum, inflorescentiate pedunculi vag. perforans apice 2- phyll, caps. inversa, fol. lata.

30. Campelia L. C. Rich.

Flores congesti sessiles aut subsessiles raro solitarii, filamenta plerumque

37. $\left\{\begin{array}{r}\text { barbata, capsulae valvae haud revolutae (Zygomenes } \text { Slsb.) ... } \\ \text { " solitarii axillares, pedicellati aut subracemosi, filamenta imberbia, }\end{array}\right.$ capsulae valvate circinatim revolutae (Cyanot. vicipara Dalz)... 35. Dalzellia Hsskl.

35. Perigonium haud tubulosum, inflorescentia terminalis

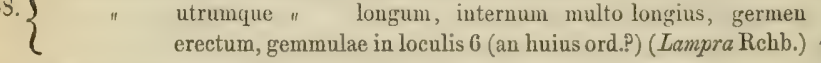

39. $\left\{\begin{array}{c}\text { Filamenta longa, spiraliter torta, antherae connectivo foliaceo............... } \\ \pi \text { stricta, imberbia }\end{array}\right.$

40. Weldenia Schlt. fil. 37. Spironema Lindl. stricta, imberbia " " angusto, loculi contigui 40.

(" natae, loculi lunulati, perig. fol. int. obovat. undul.-lobu-

40. lata, herb. ferrug.-hirsutae (Trad. fuscata Kth.) breviuscula, in phalanges 2 disposita (? Kch.), antherae elongatae, apice poro hiantes, herbae nunc scandentes. 


\section{TABULA SYNOPTICA COMIELINACEARUM}

\section{ALTERA.}

1. $\left\{\begin{array}{cc}\text { Stamina (6), partim sterilia, dilformia aut deficientia } 1-4 \\ \text { omnia fertilia (rarius deficiens ant difforne }\end{array}\right.$

, $\{$ " 3 anteriora fertilia et 3 posteriora sterilia (nune 0), stamen anterius perigonii foliolo oppositum, fertile.

3 alterna fertilia et 3 alterua sterilia (nune 0 )

Germen 3- loculare, loculo 0 abortivo.

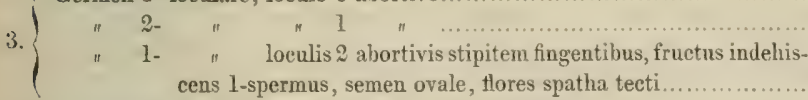

4. $\{$ Gemmulae in loculis solitarine, fructus capsularis

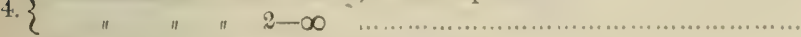

$5 .\left\{\begin{array}{c}\text { Perigonium marcescens, flamenta imberbia ............................... } \\ " \text { internum caducum, flores spatha turbinata tecti, pedicelli }\end{array}\right.$ solitarii (Commelina polyspatha Wght.)

$\int$ Stamina sterilia 3, fructus stipitatus tomentosus, bracteae cuculliformes

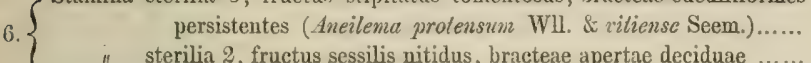

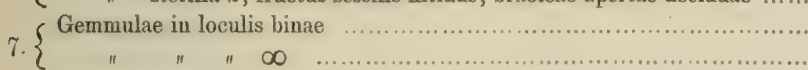

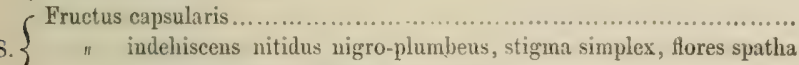

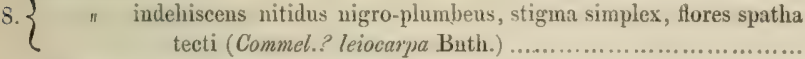

Stigma simplex obtusum, flores spatha tecti .............................

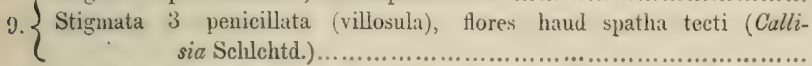

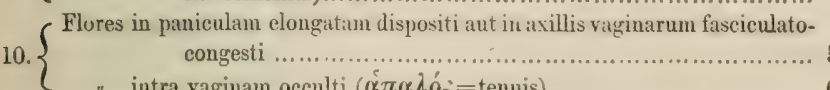

" intra vagiuan occulti ( $\alpha \dot{c} \pi \alpha^{\prime} \lambda_{o_{3}}=$ tenuis)

$\{$ Filamenta stricta, perigonii foliola interiora integra, gemmulae orthotropae

11. $\{$ " spiraliter contorta, perigomi foliola interiora fimbriata, gem-

(Gemmulae in loculis 1-seriatae, fructus indehiscens, dein coeruleo-plumbeus nulae anatropae...........................................

12. $\{$ " " $"$ " " , Hores subirregulares, perigouii foliola interna paulo majora.

S Flores ad apicem pedumculi solitarii aut bini, spatha cordato-ovata tecti 13. $\left\{\begin{array}{r}\text { (Commelina pallida Wlld.) ................................. 8. Alhyrocarpus Schlchtd. } \\ \text { " paniculati aut thy rsoidei (Aclisin C. A. Meyer, Lampocarpus BI.) 9. Pollia Thnb. }\end{array}\right.$

14. $\{$ Gemmulae in loculis solitariae .................................... 15.

15. $\{$ Perigonii foliola interiora sessilia, minura, semina radiato-rugosa ........ $16 \mathrm{~b}$.

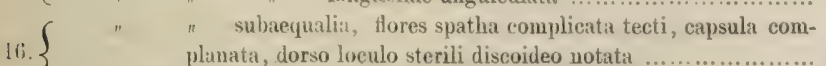
foliolit interiora sessilia minora (ef. No. 10)
2.

II. Tradescattieae 22.

I. Commelineaz 3.

II. AYEILEMATEAE, 17 . 4.

14.

14. Heterocarpus Wght.

7.

6.

1. Piletocarpus Hsskl.

2. Dictyospermum Wght. 8.

11.

9.

7. Phaeosphaerion Hsskl.

4. Commelina Plum, L.

10.

. Callisia Loeff.

6. Hapalanthus Ieq.

12.

11. Cochliostemma Lenoir. 13.

13. Polyspathe Bnth.

13b. Omphalotheca IIsskl. 5. Callisia Loeftl. 
C Capsula sessilis 2-3-locularis, flores sparsi, nunc fasciculati, pedicelli nunc gemini, herbae repentes debiles..........................

5. Callisia Loefft. obovata, flores paniculato-corymbosi (Flor. 1864,, 58 )

12. Ropalophora Hsskl.

17. $\{$ Gemmulae et semina 1- seriata in loculis.

18.

18. $\{$ Germen et capsula 3- (nune 1- (?)) locularis, semina in loculis plerum.

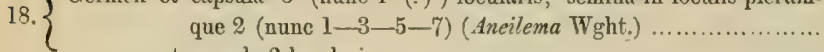
20. Dichoesperma Wght.

19.

21.

20 . (ut dicunt) radicalia, pedunculus terminalis aphyllus (scapus). 17. MIurdania Royle.

$19 .\left\{\begin{array}{l}\text { " } \\ \text { " dectinatum (Rxb. ? Wght.), stigma minute 3dentatum, folia }\end{array}\right.$

$20\left\{\begin{array}{c}\text { Flores in racemis secundis, rhachis serraeformi-exsculpta .................... } \\ \text { " " " haud secundis dispositi, rhachis haud serraeformi- }\end{array}\right.$

exsculpta ................................................. 16. Aneilema $\mathrm{R} . \mathrm{Br}$.

21. $\{$ Fructus loculi 3 -spermi, capsula 4-cuspidata (Aneil. aequinoctiale W11.).. 18.

19. Lamprodithyros Hsskl.

22. $\{$ Stamina diversiformia aut eorum antherae tantum 23.

Gemmulae in loculis solitariae, germen 2- loculare $\ldots . . . . . \ldots \ldots \ldots \ldots \ldots . . . . . .$.

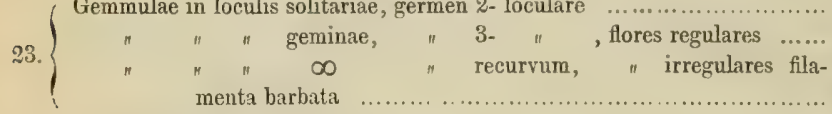

Flores terminales umbellati $\infty$

\section{Tinnantia Schdw.}

25.

\section{Knowlesia Hsskl.}

24.

21. Descantaria Schlchtd.

22. Rhoeo Hanc.

25. Streptolirion Edgew. 27.

26. $\{$ Perigonii foliola interna linearia, herbae volubiles

(Stylus longus, stigina minutum, fructus perigonii foliolis externis inclusus,

27. herbae inferue repentes, filamenta breviora barbata (Tradesc.

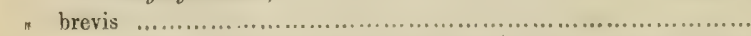

\section{Disgrega Hsskl.

\section{Heterachtia Knze.}

21. Descantaria Schlchtd.

31.

40.

\section{Flos-scopae Lour.}

23. Knowlesia Hsskl. 


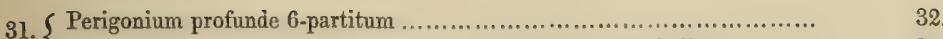

$\{$ "externum, aut internum, aut utrumque gamophyllum .........

32. $\{$ Flores involucrati, gemmulae superpositae ............................ 33 .

$\{$ " exinvolucrati, filamenta filiformia, antherae oppositae ............ 34 .

( Perigonii foliola interiora unguiculata persistentia, connectivum latum... 29. Tradescantia L.

33. " " sessilia, exteriora basi vix in pedicellum (brevem, crassum, curvatum, inflexum) connata, eoque potius suffulta
(ef. No. 38) $\ldots \ldots \ldots \ldots \ldots \ldots \ldots \ldots \ldots \ldots \ldots \ldots \ldots \ldots \ldots \ldots \ldots \ldots \ldots \ldots \ldots \ldots \ldots \ldots \ldots \ldots \ldots \ldots \ldots \ldots \ldots \ldots \ldots$

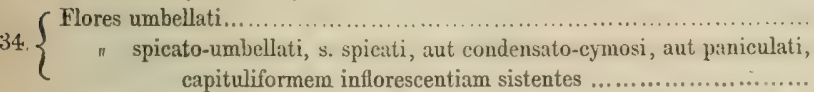

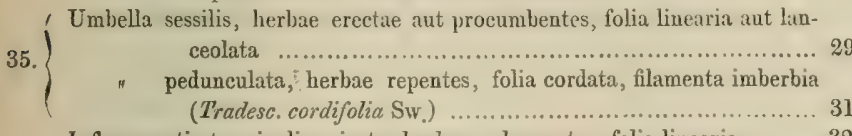

36. $\left\{\begin{array}{c}\text { Inflorescentia terminalis spicata, herbae pubescentes, folia linearia ......... } \\ \text { nxillaris subsessilis, vaginae basin perforans eamque obte- }\end{array}\right.$ gens, paniculato-capitata, glomerata, folia lata..............

( Perigonii foliola externa basi tantum paulo aut vix conuata ................. 30. Campelia L. C. Rich. 35.

36.

29. Tradescantia L.

31.

32. Cartonema R. Br.

33. Forrestia A. Rich.

38. longiorem, apice 3fidum counata, capsula inversa, perigonium lateraliter perforans, stamina fauci perigonii interni inserta ... 36. Zebrina Schnizl.

38. $\left\{\begin{array}{r}\text { foliola externa basi nunquam in pedicellum conuata, interna ca- } \\ \text { duca, in tubum 3-lobum connata, stamina hypogyna ............ } \\ \text { foliola externa basi sane (aut vix ef. No. 33) in pedicellum con- }\end{array}\right.$

38. $\left\{\begin{array}{r}\text { foliola externa basi nunquam in pedicellum conuata, interna ca- } \\ \text { duca, in tubum } 3 \text {-lobum connata, stamina hypogyna ............ } \\ \text { foliola externa basi sane (aut vix ef. No. 33) in pedicellum con- }\end{array}\right.$ nata brevem, crassum, curvatum, inflexum, internum 3-partitum, pedunculi vag. perforant. apice 2-phyll., caps. inversa, folia lata......

30. Campelia L. C. Rich.

(Flores congesti sessiles aut subsessiles raro solitarii, filamenta plerumque barbata, capsulae valvae haud revolutae (Zygomenes Slsb.)..... 34. Cyanotis D. Don.

39. "solitarii axillares, pedicellati aut subracemosi, filamenta imberbia, capsulae valvae circinnation revolutae persistentes (Cyanot. vivipara Dalz.)

35. Dalzellia Hsskl.

41.

40. $\left\{\begin{array}{c}\text { Perigonium haud tubulosum, } \\ " \text { inflorescentia terminalis ...................... }\end{array}\right.$ erectum, gemmulae in loculis 6 (an huius ord.?) (Lampra Rchb.) 40. Weldenia Sehlt. fil. 41. $\left\{\begin{array}{cl}\text { Filamenta longa spiraliter torta, antherae connectivo foliaceo............... 37. Spironema Lindl. } \\ " \text { stricta imberbia }\end{array}\right.$ alterna paulo longiora, " suborbiculares utrinque emarginatae, loculi lunulati, perig. int. fol. obovata undul.-lobulata, herb. ferrug.-hirsutae (Trad. fuscata Kth.) ............ 38. breviuscula, in phalanges 2 disposita (? Kch.), antherae elongatae, apice poro hiantes, herbae nunc scandentes.

\section{Dichorisandra Mchx.}


XI. Passant à la $17^{\mathrm{me}}$ question du Programme (Voir pag. 22) M. le Président accorde la parole à M. le général $J_{\triangle \mathrm{ACOBr}}$.

M. le général Jacobi (de Breslau) fait connaitre le principe de sa classification des Agavées, en faisant hommage de son ouvrage sur ce sujet, dont la première partie est imprimée, l'autre manuscrite. L'orateur dit qu'on a souvent fait l'essai d'une classification des Agavées. Il se borne à indiquer celle du prince Salm-Reifferscheidt-Drck, ainsi que la monographie publiée par le prof. K. Kocr de Berlin. En appréciant d'ailleurs pleinement le mérite de leurs travaux, il n'a pu souserire aux principes qui les ont guidés. Aussi la théorie qui leur a servi de base, fait elle défut dans l'application, de sorte que leurs systèmes manquent de conséquence.

Il a done tâché de trouver quelque chose de plus satisfaisant, et se basant sur des observations et des études souvent réitérées et bien longtemps poursuivies, il a établi les caractères distinctif's de ces espèces sur la forme des épines et sur l'aspect général de la plante. Par conséquent son système est celui du prince SALII, complété par celui du professeur Косн.

D'après le dit principe il y a quatre groupes principaux, savoir:

\section{Agavae keratacanthae.}

Ce groupe contient toutes les Agavées, dont l'épine se termine en pointe cornée et piquante.

II. Agavae chondracanthae.

Les feuilles de cette classe se terminent en une pointe molle.

III. Agavae inermes.

Sans épines ou, s’il y en a, elles sont à-peu-près imperceptibles.

IV. Agavae herbaceae.

Les feuilles des plantes, appartenant à ce groupe, tombent chaque anmée pour se former de nouveau. 
Toute la famille des Agavées se divise done en quatre classes; les subdivisions se font d'après l'aspect général et d'après la forme des feuilles.

Les quelques espèces de Fourcroya ne different qu'en ce que les unes ne portent pas d'épines, tandis que les autres sont épineuses; les Beschorneria sont si peu nombreux qu'il n'y aucun avantage à les séparer.

Voici comment M. JАсові a tâché de rapporter la plupart des espèces connues aux quatre classes susdites:

AGAVE开 Salisb. Endl.

Agaviformes Herb. Am. 57. 69. 126.

Agavae Lin. Vent. Herb. Endl.

Perianthium superum, basi tubulosum, sexpartitum. Stamina tubo corollæ affixa, æque ac stylus filiformia, ante anthesin inflexa (exserta). Zucc.

\section{Keratacanthae.}

Folia crassa vel subcoriacea, margine repando excavata aut recta, dentato aut filifero; dentibus corneis vel interdum spinescentibus valicis aut minutis; spina terminali scepe validissima omnino comea aut spinescente, semper dura pungensque.

\section{§ 1. MARGINAT尼 (I.)}

Folia angustata, ensiformia aut lanceolata, rigidissima plerumque crassa, margine plus mimusve distincte solubili cincta fibroso liguoso ant corneo; dentibus rigidis spinaque terminali valida, canaliculata.

$$
\text { * Margine Frumere. }
$$

Agave filifera $S l m$. (1.)

" filamentosa Slm. (2.)

" Schidigera Lem. (3.)

$$
\text { * * Margine Dentate. }
$$

Agave Funkii C. Koch. (4.)

" cœrulescens Slm. (5:)

" lophantha Schiede. (6.)

" Poselgerii Slm. (7.)

" univittata Haw. (8.) 


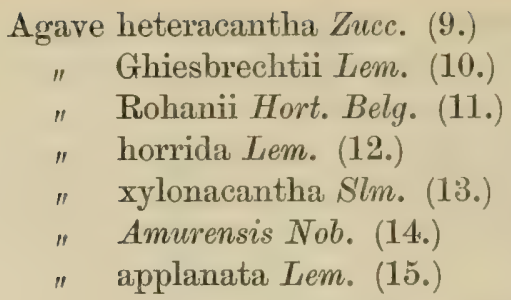

\section{§ 2. CARNOS无. (II.)}

Folia basi semper percrassa carnosa, margine dentata, dentibus validis vel minutis, spina terminali omnino cornea, valida.

\section{* Latifoliz.}

Folia crassa plerumque rigida sublanceolata, basin versus sæpe attenuata, superne plus minusve longe acuminaia, dentibus marginalibus validis aut minutis, spina terminali canaliculata.

\section{† Semimarginate.}

Folia plerumque longissime acuminata, in suprema parte margine integro discolore subcorneo sed non solubili. Dentes marginales validi basi perlati ibique interdum confluentes, vel sæpe minores in interstitiis profunde excavatis.

Agave latissima $N o b .-S y n$. A. atrovirens $C . K o c h(16$.

" cinerascens Nob. (17.)

" inæquidens $C$. Koch. (18.)

" Schlechtendalii Nob. (19.)

" atrovirens Karw. (20.)

" Jacobiana Slm. - an Syn. A. Montezumæ et A. Fernand Cortez Hort. Belg. (21.)

" Salmiana Otto. (22.)

" $\quad$ " $\quad$. recurvata Nob. (23.)

" tehuacanensis Karw. (24.)

" asperrima Nob. (25.)

$\dagger+$ Substantia totius Marginis folis mqualis.

* Folitis lanceolatis.

Spinæ marginales minutiores ac spina terminalis brevior sed crassior.

Agave Americana Lin. (26.)

\begin{tabular}{|c|c|c|}
\hline & " & $\beta$. intermedia $C \cdot$ Koch. (27.) \\
\hline & " & $\gamma$. foliis luteo-marginatis. \\
\hline & " & $\delta$. foliis luteo-striatis. \\
\hline & " & $\varepsilon_{0}$ foliis luteis viride-marginatis. \\
\hline
\end{tabular}


Agave Milleri Haw. (28.)

" picta Slm. (29.)

" latevirens marginata Hort. Belg. (30.)

" Theometel Roem. (31.)

" Xalapensis Roezl. (32.)

" polyacantha C. Koch. (33.)

\section{* * Foliss spathulatis (V.)}

Folia crassa rigidissima spathulata, plus minusve brevia apiculata, dentibus marginalibus æque ac spina terminali flexuosa plerumque validissimis.

Agave ferox $C$. Koch. (34.)

" coccinea Roezl. (35.)

" potatorum Zucc. (36.)

" Scolymus Karw. (37.)

" Schnittspahnii Nob. (38.)

" amœna Lem. (39.)

" Verschaffeltii Lem. (40.)

" seabra Slm. (41.)

" cucullata Lem. (42.)

* * * Foliis angustatis apiculatis (VI.)

Folia plus minusve crassa recta, rigida angustato-apiculata, interdum cylindraceo- aut semitereti-compressa; inter dentes marginales pane recta, spina terminali abreviata pervalida tereti-conica.

Agave flavescens Hort. Monac. (43.)

" " $\quad$. macracantha Karw. (44.)

" Besseriana Hort. Belg. (45.)

" Karwinskii Zuce. (46.)

" angustifolia Haw. - Syn. A. rigida Mill. (47.)

"Ixtly Harw. (48.)

\section{§ 3. SUBCORLACE正. (VII.)}

Folia subcoriacea subrigida, margine recta rel minime sinuata plus minusve corneo aut subspinescente dentata, dentibus multo minutioribus plerumque triangularibus, spina terminali conoidea recta, valida aut tenui, omnino corneo aut spinescente. 
* Folits angustatis elongatis sibflaccidis mox

A MEDIO DEPENDENTIBUS.

Agave Fourcroydes Nob. - Syn. A. Ixtly C. Koch. (52.)

" elongata Nob. (53.)

" lurida Ait. (54.)

" Veræ Crucis Mill. (55.)

" pallida Nob. (56.)

* Folits latioribus erectis.

Agave Jacquiniana Gawl. (49.)

"Mexicana Lamk.-Syn.A.polyphyllaC.Koch.(50.)

" uncinata Nob. - Syn. A. multiflora Hort. Kew. et A. polyacantha Hort. (51.)

\section{§ 4. SUBCARINAT瓜. (VIII.)}

Folia plus minusve molliora carnosa crassa, plerumque pluricarinata, sæpe nitida, margine minus aut minime dentata, dentibus corneis vel cartilagineis apice solum corueis, spina terminali spinescente subcanaliculata aut tereti-conica.

\section{* Dentibus corneis.}

Agave Martiana C. Koch. (57.)

" Offoyana Hort. Belg. (58.)

" melanacantha Lem. (59.)

"Rudis Lem. - Syn. A. Malinezii C. Koch. (60.) Wochenschr. 1862, p. 198.

" Bouchéi Nob. Hort. Berol. (61.)

$$
\text { * * Dentibus spinescentibus. }
$$

Agave sobolifera Herm. (62.)

" densiflora Hook. . (63.)

" Noackii? Syn. A. aloina C. Koch.? (64.)

" Keratto Mill. (65.)

" lucida Nob. - Syn. A. viridis Hort. Belg. (66.)

" pendula Schnittspahn. (67.)

" vivipara $\operatorname{Lin}$. (68.)

" rupicola Regl. (69.) 
Agave mitis Hort. Monac. (70.)

" micracantha Slm. - Syn. A. glaucescens Hort. Berol. A. concinna Hort. Belg. an Lem.? (71.)

" Ehrenbergii Nob. (72.)

" albicans Nob. (73.)

Syn. A. micracantha $\beta$. albidior Slm.

\section{$\S 5$. MARGINE INTEGERRIM屟.}

Folia molliora carnosa lato-lanceolata in spinam corneam subvalidam excurrentia, margine pergameneo integerrimo omnino inermi; scapo simplici, spica crassa densiflora demum copiose vivipara.

Agave glaucescens Hook. (74.)

\section{§ 6. CANALICULAT压. (X.)}

Folia angusta recurvata, interdum laxa, pergameneo-coriacea, canaliculata, margine spinescente dentata vel serrulata, spina terminali tenui nonnunquam spinescenti.

Agave pugioniformis Zucc. (75.)

" serrulata $S l m$. (76.)

" rubescens Slm. - Syn. A. punctata Slm.

" Rumphii Hassk. (78.)

" laxa Karw. (79.)

"Yuccæfolia Redout. (80.)

\section{§ 7. LORIFORMES. (XI.)}

Folia numerosissima lori- aut ensiformia, interdum apicem versus tenua applanata et attenuata, magine serrata, in spinam tenuem longissime acuminata.

Agave Dasylirioides Nob. et Bouché. Hort. Berol. (81.) " dealbata Lem. (82.)

\section{§ 8. JUNCINE无. (XII.)}

Folia lineari-lanceolata, subcylindraceo-, subrhombeo- aut semitereti-compressia, ancipitia, longitudinaliter asperule striata, ad margines integras plus minusve serrata, seniora interdum filifera.

Agave stricta Slm. (83.)

" striata Zucc. (84.)

" recurva Zucc. (85.)

" geminiflora Brande. - Syn. Bonapartea juncea Wild. (86.) 


\section{Chondracanthae. (XIII.)}

\$. Folia plus minusve lato lanceolata aut angustata, interdum canaliculata et maculis discoloribus instructa, margine spinescente aut membranaceo minute vel minutissime dentata aut serrulata, absque spina terminati.

* Folia subcoriacea loriformia aut lineari lanceolata margine spinescente dentata.

Agave bulbifera Slm. (87.)

" bromeliæfolia Slm. (88.)

* * Folia crassa carnosa lanceolata, subspinescente aut membranaceo minutissime dentata vel serrulata, in mucronem mox marcescentem desinentia.

Agave Celsiana Hook. (89.)

" chloracantha $\mathrm{Sml}$. (90.)

" Sartorii C. Koch. (91.)

" pruinosa Lem. (92.)

* * Folia mollia canaliculata maculata, margine subinermia.

Agave maculosa Hook. (93.)

" maculata Regl. (94.)

" virginica Lin. (95.)

\section{Inermes. (XIV.)}

$\S 10$. Folia molliora fragiliaque, lato-lanceolata aut ensiformicanaliculati in mucronem mollem excurrentia, margine integerrimo omnino inermi.

Agave attenuata Hort. Berol. (96.)

" $\quad$ " $\quad$. compacta Hort. Belg. (97.)

" Ellemetiana Hort. Paris. (98.)

" Saponaria Hook. (99.)

" variegataNob. - an Syn.A. saponariaDietr.(100.)

IV. Herbaceae. (XV.)

Folia herbacea marcescentia e rhizomate perenni, ensiformi-canaliculata in mucronem mollem excurrentia; perangusto albo- vel rubescenti-marginata, margine minutissime serrata, asperula aut lævia.

Agave brachystachis Cav. - Syn. A. pulyanthoides

Hort. nec Schlecht. (101.)

" spicata Cav. (102.) 
Agave undulata Klotzsch. (103.)

" revoluta Klotasch. (104.)

" guttata Nob. et Bouché. Hort. Berol. (105.)

Fourcroya. Vent. Zuce. (XVI.)

Perianthium superum hexapetalum, tubo nullo. Stamina e fundo floris, a petalis libera, basi aeque ac stylus valde incrassata, ante anthesin erecta (inclusa) Zuce.

Fourcroya longæva Zucc. (106.)

" gigantea Vent. (107.)

" atroviridis Nob. et Goeppert. (108.)

" tuberosa Ait. (109.)

" cubensis Haw. (110.)

". Commelyni Kunth. - Syn. A. Commelyni Slm. (111.)

" Selloa C. Koch. (112.)

" Bedinghausii C. Koch. (113.)

Beschorneria. Kunth. (XVII.)

Perianthium sexfidum tubulosum. Stamina perigyna ante anthesin erecta semper inclusa, filamenta basi ventricosa apicem versus attenuata linearia. Stylus basi incrassatus.

Beschorneria tubiflora Kunth. (114.)

Yuccoides Schlecht. (115.)

Tonelii Nob. (116.)

Parmentieri Nob. - Syn Yucca Parmentieri Roezl. (117.)

M. Entz-Ferenez (de Bude) demande si le nom de Chondracanthae est bien choisi, puisque ce même nom sert déjà à indiquer certains animaux.

M. Jacobi ne croit pas nécessaire de se tenir à la règle de ne jamais adopter dans le règne végétal une dénomination qui existe déjà dans le règne animal. 
XII. M. Cohn (de Breslan), ayant obtenu la parole, présente les observations suivantes: (1)

"Sur la rulture des Algues marines."

(question amnoncée par le Programme p. 25, No 10).

W:ihrend durch die glïckliche Vereinigun der theoretischen und practischen Botaniker die Cultur der Phanerogamen in neuster Zeit solche Fortschritte gemacht hat, dass kamm eine Pflanzenfamilie in unseren Gärten der Reprïsentanten völlig entbehrt, so sind die Kryptog"amen in dieser Beziehung bis jetzt minder begünstigt gewesen. Nur von den Farnen und den Lycopodiaceen beherbergen unsere Gärten eine Anzahl der schönsten Arten; auffallend dagegen ist die Vernachlässigung der Equiseten, obwohl manche Speries, wie Ennisetum Telmateja und silvaticum, in hohem Grade decorativ sein würden. Die Rhizocarpeen eignen sich ausgezeichnet zur Ausschmückung von Süsswasser-Aquarien. Ich selbst cultivire in einem gewölnnlichen Cylinderglase mit höchstens 10 Kubikzoll Wasser, dessen Boden mit einer Sandschicht belegt ist, Pilularia pilulifera, Isoetes lacustris und Salvinia natans seit länger als einem Jahre, wobei das Wasser durch Auflegen eines Glasdeckels vor dem Verdunsten geschützt, aber nie gewechselt wird; nichtsdestoweniger bleibt dasselbe, je länger je mehr, krystallklar. Salvinia ging im Winter ein, erzeugte sich aber im Frühling von neuem durch Keimung seiner auf der Oberfläche schwimmenden Macrosporen, doch blieben die Exemplare der zweiten Generation zwergartig und fructificirten nicht wieder; Isoetes, der im selben Glase wächst, keimte ebenfalls reichlich und erneuerte seine Blätter in Frühling, doch blieben anch diese kleiner als das Jahr vorher. Offenbar reichen die in einer so kleined Wassermenge enthaltenen Nahrungsstoffe (hauptsächlics

(1) L’auteur a complété ces observations pur le rísultat de ses expériences prolongées pendant l'été et l'automne de cette ammée.

REv. 
wohl Salze) nicht aus, um die darin vegetirenden Pflanzen auf unbestimmte Zeit in normaler Weise zu ermähren. Dagegen haben sich in denselben Glase die prächtig. spangrünen Bälle der Tolypothrix coactilis bedeutend vermehrt. Zeitweise Emeuerung des Wassers würde wahrscheinlich der Erschöpfung der darin gelösten Nahrungsstoffe vorbengen.

Von den übrigen kryptoyamischen Familien sind die Laub- und Lebermoose wohl nur zum Zweck wissenschaftlicher Studien, von den Pilzen nur der essbare Champignon der Cultur unterworfen worden; die Cultur der Flechten scheint bisher, trotz mehrfacher Versuche (vergleiche Goeppent im Jahresbericht der Schlesischen Gesellschaft, 1859), missglückt. Die Süsswasseralgen lassen sich zwar vielleicht mit Ausnahme von Lemania und Batrachospermum - leicht in kleinen Gläsern in allen ihren Entwicklungszuständen erhalten; doch bieten dieselben bei ihren geringen, meist mikroskopischen Dimensionen nur ein wissenschaftliches Interesse. Anders ist es mit den Algen des Meeres, deren eigenthümliche oft majestätische, noch läufiger aber überaus zierliche Formen anch dem Laien das lebendigste Interesse abgewinnen, ganz abgesehen von der Bedentung, welche das Studium derselben dem eigentlichen Forscher gewährt. Dennoch ist meines Wissens noch in keinem öffentlichen Institut der Versuch gemacht worien, durch Cultur einer gewissen Zahl ron Meeresalgen ein Bild der marinen Vegetation in ähnlicher Weise zu gewähren, wie dies in unseren Gärten für die Flora fast aller Länder der Erde in mehr oder minder vollständiger Weise möglich ist. Die Ursache dieser anffallenden Thatsache liegt offenbar in der Voranssetzung, dass die Algen des Meeres im Binnenlande sich gar nicht oder doch nicht ohne grosse Umstände lebend erhalten lassen. Diese Voraussetzung ist jeduch grossentheils michtig.

Die grossen See-aquarien in London, Paris und insbesondere das in Hamburg haben gezeigt, mit welchem 
Erfolge und in welcher Reichhaltigkeit die Fauna des Meeres in grossen Glasgefässen cultivirt und in ihrer Lebensweise und Entwicklung beobachtet werden kann. In allen diesen Aquarien kommen zwar Meeresalgen vor, aber sie finden sich darin nur zufällig, und werden meist als ein Uebelstand betrachtet, da in der That ihre Vermehrung der Thierwelt der Aquarien in gewissem Grade hinderlich ist. Gerade dieser Umstand regte mich an, die Cultur der Meeresalgen zur Hauptsache zu machen. Ich setzte mich zu diesem Zwecke mit dem Custos des Aquarium in Hamburg, Mr. W. Alford Lloxd in Verbindung, einem Manne, der nicht nur die ausgebreitetste Erfahrung in diesem Gebiete besitzt, sondern der auch über die Theorie und die Principien gründlich nachgedacht hat. Durch Herrn Luoyd erhielt ich am 1 November 1864 etwa einen halben Centner Nordseewasser von der Insel Helgoland, so wie später eine Anzahl Steine, die mit verschiedenen Algen bewachsen waren, und von den Südküsten Englands (Dorsetshire) stammten. Ausserdem verdanke ich Herrn LLord mehrere ausfülırliche Briefe, in denen er seine Ansichten und Erfahrungen über die Bedingungen dieser Cultur auf das freundlichste mittheilt. Aus diesen Briefen glaube ich einige Stellen von allgemeinerem Interesse hier in der Uebersetzung wieder greben zu dürfen, deren Reichthum an Beobachtungen für sich selbst spricht, wenn auch nicht ausser Acht gelassen werden darf, dass Herr Licoyd nicht Botaniker vom Fach ist:

,Eine systematische Cultur der Seealgen, so dass jegliche Art mit Sicherheit sich erhalten liesse, ist gegenwärtig noch nicht möglich. Ich arbeite in diesem Theile meiner Aufgabe noch ganz im Dunkel. Manchmal gedeihen einige Arten vortrefflich; aber oft sind es gerade Species, die ich nicht wünsche, und diese erscheinen im Ueberfluss, während die verlangten Arten nicht fortkommen; im Winter sind die Felsen meines Aquarium fast leer 
ron Algenwuchs. Diese Beobachtungen gelten namentlich von den grünen Chlorospermeae und den rothen Florideae. Niemals kann ich mit Sicherheit auf die Entwicklung einer so nützlichen Pflanze, wie Ulva latissima oder Enteromorpha intestinalis rechnen; obwohl ich oft zufällig bei beiden gutes Gedeihen antraf, so wusste ich doch nie, warum sie gerade damals fortkamen, noch konnte ich eine Wiederholung des Versuchs mit Absicht bewirken. Auch Zostera marina lässt sich nicht eultiviren; sie stirbt sofort ab und verdirbt das Wasser."

, Zu verschiedenen Zeiten erhielt ich nachstehende Algen: Rytiphlaea, Laurencia, Chylocladia, Corallina, Jania, Delesseria, Hyphea, Gelidium, Chondrus, Phyllophora Gymnogongrus, Polyides, Furcellaria und Iridaea. Bryopsis plumosa wächst sonderbarer Weise besser in künstlichem als in natürlichem Seewasser. Vaucheria (Derbesia) marina entwickelt sich reichlich an Madreporen (Balanophyllia), wenn sie dem Licht im Aquarium ausgesetzt sind; wenn die Exemplare frisch aus der See kamen, habe ich diese Alge nie bemerkt. In Hamburg wuchs Ulva latissima üppig, jedoch in einer Zwergform, ebenso Laminaria saccharina während des letzten Sommers (1864) auf den Röhren von Sabella Ventilabrum. In demselben Gefäss erhielt ich eine grosse Masse ron Delesseria sanguinea in gutem Zustande, wenn ich sie in ein ganz finstres Loch im Felsen setzte; im Lichte bedeckt sich die Pflanze mit orangerothen Flecken und stirbt in 1-2 Stunden. Vor einigen Jahren hatte ich ein Glasgefäss, in welchem durch Aussetzen ans Licht die Sporen einiger grümen Algen sich in solcher Menge entwickelten, dass das Wasser ganz und gar röthlichbraun wurde, fast wie Kaffee, so dass man durch eine zolldicke Schicht nicht hindurch sehen konnte. Ich überliess das Ganze bis zum Herbste sich selbst; da aber das Wasser nicht klar geworden war, so zog ich es $a b$ und fand nun zu meiner grossen Ueberraschung und Freude, dass viele Arten schöner Florilleen, von der Dun- 
kelheit des trüben Wassers begünstigt, sich ïppig entwickelt hatten, mit ihrer klaren rothen Farbe und dem metallischen Schimmer, den man in der See selbst findet (1). Um die Algen besser zu studiren, füllte ich das Gefäss wieder mit reinem Seewasser; aber in wenig Tagen machte das vermehrte Licht sie wieder rerschwinden; sie wurden schnell überwuchert und entstellt durch Massen von Conferven und Oscillarien."

,ROBERT WARRINGTON in London experimentirte und schrieb über das Wachsthum der Florideen in Aquarium; er benutzte farbiges Glas von purpurbläulicher Farbe, frei von Roth, um das richtige Licht zu erhalten. Auch P. H. Grosse hat sich mit der Cultur der Seealgen beschäftigt. Auf Muscheln und Felsstïcken mit Serpula finden sich die besten Algen; andere Arten leben auf Schnecken und wandern mit ihnen im Wasser umher. Rothe Algen rverden im Licht stets von grünen Parasiten überzogen."

„Das bisherige, nur temporäre Erscheinen so vieler Algen ist für mich höchst unbefriedigend. Ich wünsche Thiere wie Pflanzen durch längere Zeit im Aquarium zu erhalten, und wenn sie leben und sterben, will ich wissen, warum. Aber bei den Algen ist noch alles rom Zufall abhängig."

Ich komme nun zur Beschreibung meiner eigenen Erfahrungen. Das mir von Helgoland zugesendete Seewasser goss ich in ein rundes Glasgefäss (grosse Goldfischglocke) von 1 Fuss Höhe, 12 Zoll Oeffnung und 20 Zoll grösstem Durchmesser, das auf einem 14 Zoll hohen hölzernen Fusse (2) in meinem nach Westen gelegenen Arbeitszimmer etwa 2 Fuss von einem Fenster aufgestellt wurde. Das Seewasser roch beim Eingiessen stark nach Schwefelwasserstoff, doch verlor sich dieser Geruch in kurzer

(1) Diese Bemerkung gilt nur von einigen Arten: Chondrus crispus, Cystosira abrotanifolia, ete.

(2) Vergleiche die speciellere Abbildung und Beschreibung in meinem Aufsatz über eeaquarien in Trewend's Volkskalender für 1866. 
Zeit; es bildete in der Glasglocke, deren Boden etwa 1 Zoll hoch mit grobem reingewaschenem Kies und einigen Tuffstücken belegt ward, eine Schicht von ca. 7" Höhe, war ganz krysstallklar, jedoch in dickeren Schichten von dentlich topasgelber Firbe. Um die Verdunstung zu verringern, wurde die Oeffinung der Glasglocke mit einer Glasplatte lose zugedeckt, so dass nur sehr selten zum Ersatz des Verlustes das Nachgiessen von etwas destillirtem Wasser erforderlich ward. Vier Wochen sich selbst überlassen, vermehrten sich unsichtbare Keime im Seewasser dergestalt, dass die dem Fenster zugekehrte Glasfläche wie alle zum Lichte gewendeten Steine sich mit dickerem goldbraunem Ulebrizug färbten und in Sonnenlicht reichlich Gasblasen entwickelten. Die Ursache waren zahllose Diatomeen und zwar vorzugsweise Amphiprora alata, die sich ins Unendliche, sowohl durch Theilung als auch durch sehr kleine, aber der Mutterzelle gleich gestaltete Keime vermehrte. An einzelnen Punkten bildete sich ein schwarzgrüner Anflug von Phormidium Julianum var. marinum; auch kleine Entomostraceen hatten sich massenhaft entwickelt. Ansserdem lebten und vermehrten sich durch Embryonen einige grössere Actinia Mesembryanthemum, die ich selbst am 12. September an den Klippen von Helgoland gesammelt und in einer Glasflasche, aber ohne Wasser, glücklich nach Haus gebracht hatte.

Am 18. December erhielt ich von Herrn Ltoxn eine grosse Anzahl (ca. 100) Seethiere, welche meist aus England stammten, und theils frei, theils auf Steinen festsitzend und in gröbere Seealgen (namentlich Fucus canaliculatus, Cladophora mpestris und Enteromorpha) eingepackt, ganz ohne Wasser, in einer Blechkiste versandt waren, auch fast sämmtlich (nur mit Ausnahme einiger Fische und Krebse) unversehrt hier ankamen. Es waren grösstentheils Actinien in zahlreichen Arten, aber auch Madreporen (Balanophyllia regia), Sabellen, Serpula, Balanus, Mytilus, mehrere Schnecken (insbesonlere Nassa retienlatu. Purpura 
Lapillus, Litorina litorea) so wie eine Unzahl kleinerer, zum Theil mikroskopischer Infusorien, Polythalamien, Schwämme, Würmer, Antho- und Bryozoen, und Entomostraceen. Eine speciellere Darstellung der Geschichte dieser Thiere gehört nicht hierher, und ich erwähne nur, dass durch die Verwesung eines abgestorbenen Mytilus und Seeschwamms mehre Tage nach dem Einsetzen der Thiere das ganze Wasser in Fäulniss gerieth, durch Infusorienentwicklung sich trübte und einen widerlichen Geruch entwickelte, in Folge dessen auch ein grosser Theil der übrigen Thiere abstarb; dass jedoch nach Entfernung der todten Körper der Fäulnissprocess bald zum Stillstand kam, das Wasser wieder ganz klar und geruchlos wurde, und die zahllreichen Thiere, welche jener Katastrophe glücklich entgangen waren, sich durch mehrere Monate in normalem Zustande lebend erhielten.

Die Schnecken und Muscheln wurden eine nach der andern durch Purpura Lapillus getödtet, welche ihre Colleginnen anbohrt und aufzehrt, worauf die Purpuren selbst aus Mangel an Nahrung zu Grunde gehen mussten; ein Theil der kleineren. Thiere (namentlich Balanus und Serpula) wurden allmählich durch das Ueberwuchern der Algen erstickt; besonders verderblich wirkte die grosse Hitze dieses Sommers, indem das täglich durch mehrere Stunden der directen Somne exponirte Aquarium sich bis zu 22 und mehr Graden erhitzte, wobei namentlich dienordischen Arten bald abstarben, vielleicht ebensosehr wegen der allzuhohen Temperatur, als weil das warme Wasser zu wenig Sauerstoff aufgelöst enthält. Gleichwohl sind noch gegenwärtig, nach länger als einem Jahre, mehrere Actinien, Balanophyllion, Austern, Nassa lebendig, und insbesondere ist das Wasser, welches rollständig klar und rein geblieben ist, obwohl es nie ernevert, filtrirt, gelüftet oder gereinigt wurde, noch jetzt eine unerschöpfliche Fundgrube mikroskopischer Seethiere, namentlich Würmer und Infusorien. 
Die in das Aquarium eingelegten Steine waren mit verschiedenen Secalgen bewachsen, welche ebenfalls fast sämmtlich lebensfähig hier ankamen. Folgende Arten habe ich längere oder kürzere Zeit zum grössten Theil durch ein ganzes Jahr lebendig erhalten: (1)

A. Von Phycochromeae.

1. Beggiatoa mirabilis n. s. 4. Phormidium Julianum,

2. Beggiatoa pellucida n.s. var, marinum.

3. Beggiatoa alba var. marina.

\section{B. Von Diatomeae.}

6. Amphiprora alata.

10. Grammotophora marina.

7. Amphora salina:

8. Pinnularia peregrina.

11. Rhabdonema arcuatum.

9. Navicula pygmaea.

12. Ceratoneis Arcus,

so wie mehrere nicht bestimmte Arten von Schizonema und andern Gattungen.

\section{Von Chlorosporeae.}

13. Confervae spec. tenuis.

19. Enteromorpha spec.

14. Chaetomorpha Linum.

15. Cladophora rupestris.

16. Derbesia marina.

17. Bryopsis furcellata.

18. Valonia pusilla.

20. Ulva latissima.

21. Chlamydomonas marina n. $\mathrm{s}$.

22. Protococcus crepidinum Thur.

D. Von Melanosporeae.

*23. Ectocarpi spec. plures. $\left.\right|^{* 26 . ~ L a m i n a r i a ~ s a c c h a r i n a . ~}$

24. Sphacelaria cirrhosa.

27. Aglaozonia repens.

25. Cladostephus spongiosus. 28. Fucus vesiculosus.

E. Von Florideue.

29. Hildenbrandtia rubra.

35. Corallina officinalis.

30. Spermothamnion'Turneri

31. Bornetia secundiflora.

*32. Ptilota plumosa.

*33. Ceramium rubrum.

*34. Melobesia pustulata.

36. Polyides rotundus.

37. Phyllotylus membranifolius.

*38. Delesseria alata.

*39. Delesseria sanguinea.

(1) Nur die mit einem * versehenen $\Lambda$ rten sind jetzt (December 1865) eingegangen 
Die hier gegebene Liste beweist:

1. Dass es möglich ist, Repräsentanten aus allen Abtheilungen der Algen ohne besondere Vorbereitungen mehrere hundert Meilen von ihrer. Heimath in lebensfähigem Zustande zu versenden, und

2. Dieselben ohne alle Schwierigkeiten mit einem geringen Seswasservorruth, der nie erneuert oder gereinigt zu werden braucht, eine für wissenschaftliche Untersuchungen wie für Demonstrationen durchaus ausreichende Zeit hindurch, ja vielleicht Jahre lang, mitten im Binnenlande lebendig zu erhalten.

Die Ursache dieser fïr Viele gewiss überraschenden Thatsache liegt offenbar darin, dass die Algen in Aquarium im Allgemeinen ein dem marinen analoges Klima geniessen. Dem da eine grössere Wassermenge sich nur langsam erwärmt und abkühlt, so ist das im Aquarium eingeschlossene Seewasser den Schwankungen der Lufttemperatur um so weniger ausgesetzt, als selbst die durch den Glasdeckel abgeschlossene Luft im Innern der Glasglocke nur langsam den thermischen Bewegungen der Zimmerluft folgt. Es besitzt daher das Wasser im Aquarium eine ziemlich constante Temperatur, welche der mittleren Temperatur jedes Tages ungefähr entspricht, und dureh drei Viertel des Jahres, nämlich im Herbst, Winter und Frühling, von der des Meeres auch nicht sehr wesentlich abweichen mag. Ich habe durch längere Zeit die Temperatur des Aquarium mit der des Zimmers verglichen und lasse ein Paar Auszüge folgen:

(Zt. Zimmer Temperatur. At. Aquarium Temperatur, in Reaumur'schen Graden):

4. Dec. 1864. 8 h. a. m. Zt. $8^{\circ}$ At. $7^{\circ}, 5$.

3 h. p. m. " 13 " 9,5 .

5 " $" 8$ h.. a. $11 . " 10 " 7$.

11 h. a. m. " 16 " 8.

1 h. p. m. " 17 " 9 .

2 h. p. m. " 15,5 " 10.

5 h. p. m. " 14 " 10,5

8 h.p.m." 12 " 10.

6 " " 8 h. a. m. " 15 " 8,5 . u. s. f. 
Während in sehr kalten Nächten die Zimmer-Temperatur bis auf - $3^{\circ}$ sank, und beim Heizen bis zn $20^{\circ}$ stieg, schwankte die Temperatur des Aquarium den ganzen Winter durch nur zwischen $6^{\circ}$ und $12^{\circ}$. Sie war des Nachts wärmer, des Tages kälter als die der Luft. Daher war am Vormittag das Aquarium an seiner Aussenseite mit Wasserdïnsten beschlagen, die gegen Mittag verschwanden, während gegen Abend der Glasdeckel und die Innenseite des Gefässes mit Wassertropfen sich bedeckten, die in kalten Nächten in einem förmlichen Regen wieder in den Seewasserspiegel zurückflossen, - im Kleinen ein Abbild der über dem grossen Ocean stattfindenden meteorologischen Processe. Im Frühling und Herbst, wo anch die Luftemperatur ziemlich constant ist, war dies im Aquarium noch weit mehr der Fall; doch stieg natürlich mit den wärmeren Tagen alhmählich auch die letztere; Mitte Mai betrug sie $15^{\circ}$. Erst als in den heissen Tagen des Juni und Juli die Wassertemperatur über $20^{\circ}$ sich erhob, geriethen die Algen in ein Klima, für das sie offenbar nicht organisirt waren, und viele der höheren Formen, namentlich Ptilota, Laminaria, Delsseria, ete. gingen zu Grunde, auch Bornetic litt etwas; nur die Ectocarpen und Sphacelarien, die Oscillarien und Derbesien accommodirten sich dieser Temperatur und vermehrten sich um so reichlicher. Durch Placiren des Aquarium in einen temperirten Ort, etwa in einen Keller, wie dies Herr A. MEY ER in Hamburg mit den seinigen gethan, oder durch regelmässiges Einführen von Eis würde sich vielleicht einer allzuhohen Erwärmung im Sommer vorbeugen lassen.

Der verderbliche Einfluss des Lichtes auf die Seealgen ist offenbar überschätzt worden, da die Algen, namentlich die der Tiefe, sich allerding's mit geringerer Lichtintensität begnügen, aber dasselbe sicher nicht ganz entbehren; zahlreiche Arten kommen ja auch nahe der Oberfläche vor, wo sie dem intensivsten Lichte ausgesetzt sind. Immerhin mag Anwendung von Blendungen oder 
rothem Glase in manchen Fällen nützlich sein. Der Hauptübelstand des ungebrochenen Lichtes besteht jedoch nicht darin, lass es gewisse Arten zerstört, sondern dass es die allzu üppige Entwicklung einzelner Species auf Kosten der übrigen übermässig begünstigt. Dies gilt namentlich von den als Chlainydomonus marina bezeichneten Schwärmzellen, die sich in Mai so mermesslich vermehrten, dass das ganze Wasser dadurch trübgrün wurde, und sich nach dem Zurruhekommen der Zellen mit einem grünen Häutchen bedeckte, welches die Durchsichtigkeit des Wassers wesentlich beeinträchtigte (vergleiche meinen Aufsatz über Chlamydomonas marina, Hedwigia 1865, pag. 9 c. ic). Erst im October dieses Jahres sind die letzten Spuren dieser Volvocinee wieder verschwunden. Vielleicht noch lästiger ist die, von mir als Spirulina versicolor bezeichnete, höchst zierliche Oscillarie (siehe RABEnHorst Flora Algarum europaea II, p. 292), welche alle Gegenstände, die Felsstücke wie die Glaswände, die grösseren Algen und selbst Thiere mit dicken schwarzgrünen oder purpurrothen Häuten überspinnt, die im Lichte massenhafte Gasblasen entwickeln, auch wohl von diesen emporgehoben, als schwarze Gallertklumpen an die Oberfläche kommen, oder aufdemWasser in dünnerer Schicht schwimmend, eine Art Wasserblüthe bilden. Nur wo ihre Vermehrung, wie im grossen Hamburger Aquarium, sorgfältig in Schranken gehalten wird, dient sie mit ihren prachtvollen Farben demselben zur Zierde. In der Gesellschaft der Spirulina gedeiht nur die Derbesia, welche ebenfalls gern andere Algen mit lichtgrünem Buschwerk überzieht und verunstaltet.

Verderblicher noch als die Spirulina sind die Beggiatoen, welche ich mur in den Wintermonaten als kreideweisse Schleimmassen über todte Thiere und andere Algen sich hinwegspinnen sah, die dimn im Sommer verschwanden und in Herbst sich aufs neue einfanden. Sie überwuchern nicht bloss die feineren Formen, sondern da sie 
reichlich Schwefelwasserstoff' aushauchen, tödten sie auch alles Organische in ihrer Ungebung (vergleiche meinen Aufsatz: Zwei neue Beggiatoen in Hedwigia 1865, p. 81, c. tab.)

Nachtheilig ist dem Gedeihen der Algen auch die gleichzeitige Cultur von Seethieren; denn, abgesehen davon dass sich im diesem Falle die Sorgfalt der Pflege zu sehr zersplittert, so schaden die Thiere direct, durch ihre Ausscheidungen (Schleimsecrete der Actinien, Excremente) wie durch ilure Bewegungen; viele Thieren nähren sich auch von den Algen, namentlich weidet die überaus gefrässige Litorina die grösseren Arten ab und zerstört sie völlig. Ein Paar Litorinen hatten in wenig Stunden die innere Glaswand, die von Diatomeen ganz undurchsichtig braun geworden war, rein abgeputzt.

Viele Algen sind einjähırig und gehen im Winter ein, oder verschwinden umgekehrt in Sommer; es darf uns daher nicht verwundern, wem sie auch im Aquarium nicht perennirend werden; dies gilt unter andern von den Delesserien, die sich nur kurze Zeit erhielten. Andere Arten sind so zart, dass sie nur schwer ohne Verletzung einen weiteren Transport aushalten; natürlich können solche, schon in Fäuhniss begriffene, Exemplare auch in Aquarien nicht weiter wachsen. Manche Alge stirbt ab, weil ihre Basis von einem Schwamm oder einer Bryozoe überzogen ist, welche faulend auch die Pflanze ansteckt. Obwohl im Allgemeinen die Algen nur mit ihrer natürlichen Befestigung an Steinen ein weiteres Fortkommen verheissen, so fand ich doch, dass losgerissene Bornetia und Ptilota noch Monate lang' sich lebend erhielten; selbst schwimmende Bruchstïcke von Polyides sprossten fort.

Einige Arten dagegen erhielten sich Monate hindureh ganz unverändert, ohne zu verderben, aber auch olne weiter zu wachsen. Dies beobachtete ich namentlich an einer kleinen Laminaria saccharina, und an keimendem Fucus vesiculosus. Bei den meisten Arten scheint zwar 
die vegetative Zellbildung im Aquarium fortzugehen, aber die neuen Theile bleiben kleiner als normal, und sie fructificiren nicht. Ersteres kmme ich insbesondere bei Bornetia wahrnehmen, wo die jungen Gliecterzellen weit kürzer und anders gefurint waren als die äteren, vom Ocean kommenden Aeste. Die Verkümmermg im Aquarium kann um so weniger Wunder nehmen, da ja selbst die Algen der Ostsee viel kleiner sind, als die gleichen Arten in der Nordsee. Die Ursache liegt wahrscheinlich in gewissen anorganischen Nalnungsstofien, die nur in geringen Proportionen in Seewasser enthalten sind, und deren Vorrath sich dinher in begrenztem Ramme des Aquarium leicht erschöpft. Ist lies der Grund, so hönnte nur häufiger Wechsel des Seowassers abhelten. An den in meinem Aquarium lebenden Austern hibe icl: beobachtet, dass sie zwar neue Anwachsstreifen an ihren Schalen absondern, aber diese nicht mit Kalk incrustiren kümnen, von dem wahrscheinlich nicht genügenie Quantitäten sich in Lösung befinden.

Wirkliche normale Fortphthnzung habe ich direct nur bei den Derbesien und Ectorurpen (durch Zousporen), den Sphacelarien (durch Bruthnospen), den Ptiloten (durch Tetrasporen) beubachtet; alle andem Arten fand ich nur steril. Die Sporen von Ptilota, Ceramium. Syermothamion, Fucus und Laninaria keimten zwar, entwickelten sich aber nicht zu vollkommenen Pflanzen. Vielleicht würde sich ein besserer Erfolg erzielen lassen, wem nicht, wie es bei mir der Fall war, zahlreiche Arten in einer grossen Glasglocke, sondern jede Species isolirt in einem kleineren Gefässe, aber mit hinreichendem Scetwasser versehen, cultivirt würde. Oftenbar würlen wir anch in unseren butanischen Gärten nicht so gute Resultate anfzuweisen haben, wenn wir die feineren Arten, statt jede Pflanze in einem besonderen Topf, alle durch einander in einem Beete aufzögen.

Obige Bemerkungen werken genügen, un die Bedeu- 
tung darzulegen, welche den Seewasseraquarien unter den botanischen Unterrichtsmitteln meiner Ansicht nach zukommt. Nur wenige Institute werden ohne Zweifel im Stande sein, so vollkommene und grossartige Einrichtungen zu treffen, wie sie z. B. in Paris und Hamburg für die Fauna des Meeres bestehen, und wie sie bei entsprechender Einrichtung sicher auch im Stande wären, ein getreues Bild der Meeresvegetation darzubieten. Aber keine öffentliche Sammlung wird sich vor der Anschaffung eines oder einiger flachen Schieferkasten zu scheuen haben, die oben offen und an der Vorderseite mit einer Glaswand versehen, in einer nur wenige Zoll tiefen Seewasserschicht zahlreichen Meeresalgen ein zuträgliches und dauerhaftes Gedeihen sichern. Für solche flache Kasten können die complicirten Apparate für Circulation des Wassers und Eintreiben von atmosphärischer Luft vollständig entbehrt werden, da die grosse Oberfläche des Wassers die erforderlichen Gase (Sauerstoff, resp. Kohlensäure) durch Diffusion aus der Atmosphäre aufnimmt.

Der einzelne Gelehrte wird sich mit einem einfachen Zimmeraquarium begnügen, wie ich selbst es in diesem Aufsatz beschrieben habe. Selbst wemn es nicht gelingen sollte, zahlreiche Arten durch mehrere Jahre im Leben zu erhalten, so wird doch die Erneuerung des Algenvorraths, da sie höchstens jährlich zu geschehen hat, nirgends grosse Schwierigkeiten darbieten. Und wenn wirklich selbst die grösseren Algen dem Phykologen vom Fach die normale Entwicklung ihrer Früchte hartnäckig verweigern sollten, so wird derselbe dafür durch die Beobachtung zahlreicher mikroskopischer Formen entschädigt werden, die auch im beschränkten Raume sich vollständig zu entfalten vermögen, und die zum grossen Theil noch ganz umbekannt sind, da ihre Beobachtung am Meere selbst oft an unüberwindlichen Schwierigkeiten leidet.

M. Reichenbach (de Hambourg) craint que la lumière ne soit nuisible à la culture des Algues marines. 
M. Cohn ne partage pas cette crainte.

M. Münter demande si l'eau ne se putrifie pas bientôt.

M. Cohn le rassure à cet égard. Avec quelques précautions, on peut conserver l'eau assez longtemps fraîche.

M. Regel demande quelle est la température de l'eau la plus favorable aux aquaires.

M. Cohn répond qu'un hectolitre d'eau est mainteriu dans la salle à une température moyenne de 8 à $12^{\circ}$

M. Reichenbach fait remarquer que M. Lloyd, le conservateur du bel aquarium de Hambourg qu'on dit le plus beau du monde après la mer, n'avait réussi jusqu'à présent qu'à faire éclore le Delesseria sanguinea, les Rhodospores lui ayant opposé des obstacles insurmontables.

XII. M. Fée prend la parole et entretient la section sur les sécrétions des Fougères. L'orateur dépose un mémoire sur ce sujet, intitulé:

Quelques particularités ielatives à la famille des Fougères.

Les études microscopiques et les belles découvertes qui en sont résultées, ont singulièrement élevé en dignité les êtres des deux règnes que l'on qualifiait si mal à propos d'inférieurs. On les croyait doués d'une organisation simple et elle s'est trouvée compliquée, on ve leur accordait qu'une importance médiocre et elle est devenue immense, on les dédaignait et ils se sont rendus redoutables. Arjourd'hui, ce ne sont plus seulement les naturalistes qui les suivent dans leur évolution, les médecins, les horticulteurs, les industriels veulent les connaître, afin d'en combattre les effets pernicieux. Ils s'attaquent à l'homme, aux animaux domestiques, aux plantes économiques et alimentaires. Ce sont des ennemis, et il a fallu chercher à les vaincre. Sous un autre rapport cette étude a soulevé de graves et importantes questions, par exemple celle de l'hétérogénie et cette autre non 
moins grave qui concerne les règnes, dont les limites semblent de plus en phs vagues et indécises. Je n'ai point l'intention, dans ce petit écrit, d'aborder les sujets aussi élevés qui demandent de longs développements, sans qu'il soit toujours possible de conchure. Ma tâche sera plus modeste et plus en rapport avec ce qu'il m'est permis de faire; aussi sera-ce dans le règne végétal que je puiserai les matériaux de cette note et les Fougères, si fécondes en particularités curieuses, vont me les fournir.

Depuis un quart de siècle environ, ces plantes ont été l'objet de nombreux travaux; elles se sont multipliées dans les jardins et les herbiers se sont enrichis d'espèces nombreuses, la plupart aujourd'hui décrites et figurées. Le fait le plus considérable de leur histoire est sans contredit la découverte de MM. NAEgeli et Suminski, qui ont constaté l'existence de deux appareils regardés comme sexuels, tantôt réunis sur une même expansion et tantôt séparés, circonstance qui fait reconnaître des espèces monoïques et diöques. La spore, telle qu'elle se comporte pendant la germination, ne serait qu'un organe préparatoire, seulement destiné à produire une sorte d'ovule, sans qu'elle-même en remplisse le rôle, étant destinée à périr. C'est un développement en deux actes, une sorte de végétation alternante. Dans les Phanérogames, la graine se complète; dans les Fougères, la spore ne serait qu'un simple agent de fécondation qui doit produire des anthérozoïdes et des archégones; là se borne sa puissance qui ne saurait aller au-delà, e'est un organe de transmission, comme le boyau pollinique ou l'agent, quel qu'il soit, qui opère sur l'ovule. Plusieurs botanistes pensent que la présence des anthérozoïdes, constatée dans certaines Algues, dans les Mousses et les Équisétacées, se généralisera, de manière à se manifester aussi bien dans les Phanérogames que dans les Cryptogames; les spermatozoïdes existent dans la liqueur séminale de tous les animaux sexués, pourquoi n'existerait-il pas des anthérozoïdes qui 
sont leurs analogues, dans turtes les plantes? Mais où les trouverait-on? La végétation qui n'est pour les plantes embryonnées que le développement de parties déjà existantes, sauf la fleur, ne peut produire de spermatozoïdes qui n'auraient aucun rôle à remplir; il faudrait les chercher dans le pollen, et, si ces corps y existaient, on les y aurait depuis longtemps trouvés; les granules sont peut-ètre leurs analogues.

Une particularité tr'ès curieuse de l'histoire des Foug'ères et d'une explication difficile est celle qui nous montre sur une fronde fructifère les sporothèces et les sporanges dans un même état de développement; cette évolution ne suit aucune des lois observées dans les phanérogames ou même dans les Equisétacées dont l'inflorescence est celle de l'épi. Les sporothèces du sommet, ceux du centre, ceux de la base, semblent résulter d'une fleuraison simultanée, et il s'en suit d'assez grandes difficultés dans la diagnose des espèces représentées dans les herbiers par un spécimen unique. On voudrait voir les sporothèces ì tous les degrés de maturité, et on ne le peut pas. Si les indusiums sont caduques, tous se détachent en même temps, et c'est à peine si l'on en trouve quelques-uns pour en déterminer la forme et en constater la présence.

Dans les Lycopodiacées, les capsules sont attachées sur le tissu vasculaire; il n'en est pas toujours ainsi pour les Fougères. Les sporothèces sont très souvent cuticulaires, ex. les Acrostichées, parmi lesquelles il en est dont les deux lames sont fructifères. Ce sont donc des organes épidermoïdes, comme les poils, les glandes et les écailles; c'est là ce qui explique comment il arrive si souvent que dans les sporothèces les mieux circonscrits, les spores sont mêlées avec des poils, ordinairement intestiniformes, étranglés d'espace en espace. Les poils pareils à ceux des phanérogames n'existent guère sur les frondes des Fougères, et ils tirent presque toujours leur origine des écailles.

De toutes les plantes connues, ce sont celles qui se 
trouvent le plus fréquemment à l'état vivipare ou radicant. Certains groupes, et dans ceux-ci certains genres en fournissent des exemples remarquables et nombreux. Ce développement anormal donne naissance à de petites plantes complètes, pourvues d'un rhizome; elles ne s'accroissent pas au point de fructifier. Un spécimen de Polypodium plantagineum (Drynaria plantaginea) est doublement prolifère; la plantule étant à son tour prolifère, il y a trois générations: père, fils et petit-fils. Nous ne possédons en Europe aucune Fougère qui soit prolifère ou radicante; il en est de même pour les Fougères en arbre des tropiques.

Les frondes des Fougères, dès la première période de leur développement, sont, comme on sait, roulées en crosse; dans les grandes espèces multipinnatifides toutes les parties étant roulées, l'ensemble présente l'apparence d'une élégante rosace. On pourrait sous ce rapport les diviser en Fougères à évolution indéfinie et en Fougères à évolution définie; dans les premières, la crosse ne se déroule jamais complètement, ainsi qu'on peut le voir dans les Jamesonia et dans une espèce de Polypodium que nous avons décrite sous le nom de $P$. jamesonoides; dans les autres, le contraire arrive. Il va sans dire qu'il en serait de cette classification comme de celle qui voulait partager les métaux, suivant qu'ils domnent ou qu'ils ne donnent pas, en brûlant, l'odeur alliacée: l'arsenic était d'un côté, et tout le reste de l'autre.

Plusieurs Fougères ont reçu spécifiquement les noms de glandulifères et de visqueuses; mais la plupart ne fournissent pas de sécrétions. En général, elles sont trèsrares et de nature peu variée, visqueuse, résineuse et calcaire. La nature de la sécrétion qui a valu à un Acrostichum, à deux Cheilanthes et à un Aspidium les noms spécifiques de viscosum et de viscidulum, n'est pas visqueuse, mais résineuse. Le système adénique dans l'Hypolepis helenensis (FÉE Gen. Filic. p. 147) est représenté par des glandes courtes, arrondies, succinoïdes, portées sur un pédicelle très- 
large, articulé; le produit sécrété est insoluble dans l'eau. Dans les Cheilanthes viscosa et glandulosa, les glandes sont pédicellées et visibles à l'oeil nu, la sécrétion est de nature résineuse. Nous croyons donc que ces Fougères ne justifient pas le nom spécifique qu'elles portent.

Le genre Ceropteris de LrNк a reçu ce nom dans la persuasion où se trouvait cet auteur, que la sécrétion jaune, dorée ou blanche, qui recouvre si abondamment les lames inférieures de ces plantes curieuses, était analogue à la cire. Un assez grand nombre de Fougères en présentent une semblable, tantôt d'une manière générale pour les genres Ceropteris, Trismeria, Cincinalis, Aleuritopteris, tantôt exceptionnellement: Adiantum sulfureum, Drynaria glanca, Pteris argyraea, craesus et Anogramma rosea. Dans toutes ces plantes, les glandes sont en massue, trèscourtes et très-faiblement adhérentes à l'épiderme. La matière sécrétée présente sous le microscope l'aspect de petits filaments d'une extrême ténuité(Ceropteris). L'alcool et l'éther la dissolvent avec la plus grande facilité, et se colorent légèrement en jaune. Cette dissolution, qui se trouble quand on l'étend d'eau, reprend sa limpidité par l'action des alcalis, ce qui a lieu pour les résines et non pour les cires.

Gaudichaud, dont le nom et le souvenir sont chers aux amis de la botanique, est le fondateur d'un genre Adenophorus, ainsi qualifié parce qu'il est chargé de glandes. Si ce sont en effet des glandes, du moins est-il certain qu'elles ne produisent aucune sécrétion, du reste elles sont fort belles, de couleur dorée, pyriformes, tantôt sessiles, tantôt pédicellées.

Mais de toutes les sécrétions connues la plus curieuse est, sans contredit, celle qui dépose du carbonate de chaux sur l'épiderme, fait unique dans le règne végétal, tel du moins qu'il se présente dans les Fougères, où il est localisé, et de forme déterminée.

Willdenow a décrit (Filic. p. 242), d'après Bory, un 
Aspidium albo-punctatum, ainsi nommé parce qu'il est. marqué de points blanes, sur la nature desquels il ne s'explique pas. Cette même plante, ou peut-être une de ses formes, a été présentée comme nouvelle par Kunze (Linn. XXIII, p. 301) sous le nom de leucostichon; cet auteur constate qu'elle est, in apice venarum albo-squamosa," ce qui veut dire qu'il regarde ces petits points comme des écailles. M. HоокеR (Spec. filic. IV, p. 85) n'en dit pas d'avantage.

Ayant soumis au microscope cette production épidermoïde, qui se détache avec une très-grande facilité, il ne nous a pas été difficile de voir qu'elle était absolument inorganique; nous avons pu constater qu'elle faisait effervescence avec les acides, et nous l'avons reconnue comme étant un earbonate calcaire.

Ces sortes de concrétions, exceptionnelles parmi les Aspidium, existent dans presque toutes les espèces des genres Chrysopteris, Nephrolepis et Campyloneuron. On les trouve sur les lames supérieures, au sommet des nervilles prolifères. Ce sommet s'épaissit, devient elliptique et translucide. La présence de cette excrétion coïncide, mais non toujours, avec l'époque de la formation des sporothèces et leur situation est pareille, l'une en dessus, les autres en dessous. Ainsi bordée de ces points d'un blanc de neige, la plante prend un aspect tout-à-fait curieux.

Rien n'est plus ordinaire que de trouver la chaux dans les plantes, toutes en contiemnent, mais la voir telle qu'elle se montre dans les Fougères est un fait vraiment singulier.

L'apparition de ces points calcaires, coincidant, ainsi que nous l'avons dit, avec le développement des sporanges, dont ils ne sont séparés que par l'épaisseur de la fronde, permet de conclure que le sommet de la nerville renflée fait office de glande, et qu'il est donc de la propriété singulière de séparer du liquide nourricier le carbonate de chaux qui s'y trouve à l'état de dissolution; le liquide salin traverse l'épiderme de la lame supérieure, dépose, après 
son évaporation, une couche mince de calcaire, limitée dans son étendue aux dimensions de la nerville elle-même, organe de sécrétion. L'absence du calcaire sur la lame inférieure fournit un exemple de plus, qui permet de constater l'indépendance physiologique des deux lames.

La nature glanduleuse du sommet des nervilles est justifiée, non seulement par la fonction, mais aussi par l'organisation anatomique, ainsi que l'a démontré la diagnose microscopique.

L'histoire des Fougères présente encore d'autres singularités; nous ne ferons que les indiquer.

Rien n'est plus rare que la production de tubercules; il n'en existe à notre connaissance qu'un seul exemple, fourni par le Nephrolepis tuberosa; nous en avons donné ailleurs l'analyse.

Parmi les frondes il en est de pendantes, qui ont des frondules organisées dans le sens de leur suspension, et qui seraient inverses, si elles étaient dressées. Les frondes dissimilaires ne sont pas rares; on connaît dans les Polybotrya des frondes stériles pinnées-pinnatifides, tandis que les fertiles sont surdécomposées. Assez fréquemment dans les genres Lomaria, Olfersia, Chrysodium, Neurocallis, Heteroneuron les frondes deviennent mixtes, stériles par le bas et fertiles dans le haut, quoiqu'il soit dans leur nature d'être complètement indépendantes. Le genre Photinopteris, qui présente à la base des pétioles des espèces d'écussons semblables à des stipules, a sur une même fronde des frondules stériles et ovales, fort grandes, et, sans aucune transition, des frondules linéaires fertiles. Certaines espèces du gemre Lomariopsis ont des frondes hétérophylles à un degré que n'atteint aucune phanérogame; les $L$. ludens, variabilis, spinescens, sorbifolia se modifient si profondément, que si l'on royait séparément leurs frondes, on les décrirait comme espèces distinctes. Les frondes de premier développement des Neuroplatyceros (Acrostichum alcicome L.) sont persis- 
tantes et deviennent scarieuses; le même fuit se reproduit dans le Drynaria quercifolia.

La situation endophylle des sporanges dans quelques espèces de Vittaria est digne d'être remarquée, ainsi que les arrêts de déreloppement des sporanges ou capsules, tantôt réduites à l'anneau, tantôt même au pédicelle, circonstance qui acquiert parfois l'importance d'un caractère générique.

Quoique nous n'ayons pas encore épuisé notre sujet, nous n'irons pas plus loin; l'intéret qui s'attache à ces plantes est assez grand pour qu'il ne soit pas nécessaire de parler en leur faveur. Soit qu'on les étudie, soit qu'on se contente d'admirer leur's formes, elles plaisent également, et les botanistes aussi bien que les horticulteurs sont d'accord pour les placer au premier rang des familles les plus intéressantes du règne végétal.

La seconde séance est fixée à $3 \frac{1}{4}$ heures de l'aprèsmidi. La séance est levée à 1 heure. 


\section{SECTION DE BOTANIQUE PURE.}

SEANCE du 8 Avril 1865, à $3 \frac{1}{4}$ heures de relevée.

LE BUREAU SE COMPOSE DE:

MM. FÉE, Président.

FENZL et MIQUEL, Vice-Présidents.

MORREN et OUDEMANS, Secrétaires.

Le Séance est ouverte à $3 \frac{1}{4}$ heures de l'après-midi.

Le procès-verbal de la séance du matin, lu par M. Morren, est adopté.

M. Wesmael (de Mons) fait hommage au Congrès de quelques-uns de ses opuscules, savoir:

Catalogue raisonné des Arbres forestiers et d'ornement de pleine terre en Belgique.

Monographie des Saules de la Flore Belge et des espèces les plus répandues dans les cultures.

Annotations à la Flore de la partie septentrionale du Brabant, par A. Thielens et A. Wesmael.

Synanthie chez le Symphytum officinale.

Observations sur la nature de la cavité ovarienne chez les trifolium.

Notes sur quelques Plantes rares, nouvelles ou critiques de la Flore de Belgique.

Notice sur la nature de l'utricule des Carex.

Observations sur la culture de plantes hybrides.

Transformation des étanines en carpelles chez le salix caprea.

De la Fécondation au point de vue des croisements et des hybridations en horticulture.

Les Ormes forestier's et d'ornement, leur histoire et leur culture.

Monographie des Groseilliers et de leurs variétés propres aux jardins fruitiers et d'agrément. 


\section{MM. Herincq, Schultz-Schultzenstein et} Clos étant absents, et M. Meissner renonçant à la parole,

M. Oudemans traite la seconde question annoncée par lui (voir le Programme p. 20, No. 12, 2) en donnant lecture d'une notice: Sur l'origine des spores dans certaines espèces de Mucor.

M. Oddemans n'ayant pu apercevoir dans plusieurs espèces de Mucor la formation d'une cloison entre le pédicelle et la vésicule sporifère se croit autorisé à émettre l'hypothèse, qu'il y aurait dans les Mucors une formation de spores entre la membrane primitive d'une cellule, qui formerait la paroi de la vésicule et une production ultérieure, qui jouerait le rôle de membrane secondaire. Il convient que cette hypothèse est quelque peu téméraire tant parce que de cette manière ni la membrane primitive, ni la membrane secondaire ne forment une vésicule fermée de toutes parts, que parce qu'il n'a rencontré nulle part un fait analogue à celui qu'il vient de décrire. Aussi l'orateur ne veut-il pas trop insister sur son explication; mais il exprime l'espoir qu'un temps viendra où l'on découvrira une cloison entre le pédicelle et la vésicule sporifère, ce qui jettera une grande lumière sur plusieurs points encore obscurs de l'histoire des Mucorinées.

M. Schnizlein (d'Erlangen) demande la parole et dit: Voyant que mon collègue M. Hoffrann de Giessen, qui est un maître en mycologie, n'est point présent, je crois cependant devoir dire quelques mots sur le sujet intéressant que M. Oddemans nous a comminiqué. Si j’ai bien compris, cette exposition contiendrait un fait inoui jusqu'ici et contre toute analogie en anatomie végétale, qu'une membrane cellulaire pourrait se fendre et former entre ses lames quelque chose de nouveau. Je dois exprimer mes doutes au sujet de cette explication, mon silence pourrait faire croire que je voudrais l'accepter.

M. Suringar (de Leide) demande de même, si le fuit 
signalé ne pourrait pas s'expliquer d'une manière plus plausible. Selon l'explication de M. Oddemans le contenu liquide dans l'intérieur de la cellule aurait été résorbé, et entre les couches secondaires de la membrane un autre liquide se serait formé. Cette supposition est tellement contraire à tout ce qu'on sait des fonctions physiologiques de la cellule végétale, que l'orateur désire des preuves plus concluantes pour oser l'admettre. Selon lui, le fait s'explique beaucoup mieux, en supposant la formation d'un dissépiment qui se forme en résicule, comme la partie supérieure de la cellule primaire.

II. M. ie Président accorde la parole à M. Suringar, de Leide.

M. Suringar présente le résumé suivant d'un travail qu'il vient de publier sur la Sarcina ventriculi.

\section{Messieurs!}

„J'ai demandé la parole pour quelques moments, afin de présenter à votre savante assemblée un ouvrage publié récemment sur la Sarcine de l'estomac (1). II contient des recherches sur la nature végétale, la structure anatomique et les lois qui président au développement de cet organisme. Je tâcherai d'indiquer en peu de mots le but que je m'étais proposé dans ce travail et quelques-uns desrésultats obtenus.

On sait que la sarcine a été découverte en 1842 par M. Goodsir à Edimbourg. En examinant au microscope les matières rejetées par un malade souffrant de romissements périodiques, il y trouva des corpuscules d'une nature particulière, qu’il a décrits comme constituant une nouvelle espèce parmi les plantes inférieures. Les corpuscules, à faces rectangulaires, et divisées très-régulièrement en com-

(1) DE SARCINE (Sarcina Tentriculi GooDsin). Onderzoek naar de plantaardige natuur, den ligchaamsbouw en de ontwikkelingstretten ran dit organisme, door Dr. W. F. R. Suringar, Hoogleeraar te Leiden. Leeuwarden, G. T. N. Suringar, 1865. 
partiments plus petits, mais de même forme, ressemblaient assez bien à des ballots, serrés par des cordes dans deux directions perpendiculaires entre elles. De là le nom de Sarcina ventriculli (ballot de l'estomac) qu'il assigna à ce parasite microscopique. Quand cette décourerte fut connue sur le continent de l'Europe, on douta d'abord de la nature végétale de cette production. Quoiqu'on soit bien d'accord aujourdhui sur ce point, en raison de sa ressemblance intime avec quelques Algues d'un ordre inférieur, notamment avec les espèces du genre Merisnopoedia, une cause de doute, soulevée par le célèbre VIRchow et déduite de l'absence de la réaction de l'iode sur la cellulose, avait persisté.

En traitant la sarcine avec l'iode et l'acide sulfurique, on n'avait obtenu qu'une coloration jaunâtre, et non pas cette coloration bleue, propre à la cellulose. En répétant cette expérience, j'ai trouvé en effet que la sarcine, traitée avec ces mêmes réactifs sans préparation antérieure, ne présente qu'une très-faible coloration bleue au contour interne de la paroi des cellules. Il parut nécessaire, comme en beaucoup d'autres cas où la réaction de la cellulose est empêchée par la présence de matières étrangères, de traiter d'arance le tissu avec l'acide nitrique ou la potasse caustique. Un traitement avec cette dernière, poursuivi pendant quelques heures, suffit pour rendre la sarcine accessible à la réaction sur la cellulose, et les figures 26-35 de la planche II en rendent témoignage.

Les auteurs, dont on trouve les mémoires au nombre de 50 environ cités à la fin de l'ouvrage, ne sont pas du tout d'accord entre eux sur la structure anatomique de la sarcine, sur la nature des parties élémentaires du tissu, sur l'absence, la précence ou le nombre des cytoblastes etc. Leurs opinions sont discutées plus spécialement de la page 110 à la page 117 de ce mémoire. Cette discussion était nécessaire; des parties très-différentes étant souvent désignées par le même nom chez les différents auteurs, il 
en était résulté une confusion de languge, impossible à dissiper sans se rendre compte de la terminologie de chacun d'eux en particulier. En expliquant mes propres vues, et afin d'éviter tout équivoque, j'ai pris pour point de départ la description de la sarcine donnée dans l'ouvrage classique de M. Roвin sur les parasites qui croissent sur le corps de l'homme et des animaua vivants, description accompagnée de figures, dont quelques unes ont été reproduites (Plınche II), pour servir de moyen de comparaison. A l'aide d'un objectif très-fort et très-clair (le $\frac{1}{8}$ inch de Sнiтн \& BEск) et avec le secours de la réaction chimique sur la cellulose, il me fut démontré, que les parties, décrites généralement comme cellules simples, étaient des groupes composés de 4 (dans l'espace 8 ) cellules. On avait assigné à ces cellules une forme très-particulière, leur attribuant des faces quadrilobées, chacune d'elles étant parcourue par deux sillons perpendiculaires entre eux et sortant des sinus entre les lobes de cette même face, tandis qu'au centre ils se rencontraient dans une petite cavité. Généralement on considérait ces sillons comme signe d'une division à venir, d'une multiplication des cellules, que la plupart des auteurs se représentaient comme ayant lieu au moyen des cytoblastes, par formation libre et simultanée de 4 (8) cellules-filles dans la cellule-mère.

Or, les quatre lobes de la cellule ainsi construite correspondant à quatre cellules dejà toutes formées et séparées l'une de l'autre par de vraies cloisons, il parut nécessaire de chercher dès l'origine la multiplication des cellules dans chacun de ces compartiments. C'est là en effet que peut s'observer la multiplication des cellules, ayant lieu de la manière la plus simple et la plus générale, c. à. d. par division et au moyen d'une cloison nouvelle au milieu de chaque cellule. Cette division s'opère alternativement dans les trois directions de l'espace et par là il en résulte la forme cubique ou plutôt prismatique qui est propre aux individus de la sarcine. 
Quand ces corps ont atteint un certain degré de composition et, relativement à cette composition, ume certaine grandeur, ils se divisent en deux moitiés qui par cela même deviennent deux corps distincts. Cette segmentation, qui donne lieu à une multiplication par progression géométrique, se fait constanment selon le plan correspondant à la cloison cellulaire la plus ancienne du corps. J'en trouve la cause dans une dissolution successive de la paroi des cellules-mères vieillies, qui, emboitant les générations de cellules qui leur succèdent, sont forcées par là de s'étendre sur une surface toujours plus grande. L'influence de cette destruction des membranes-mères au fur et à mesure qu'elles vieillissent se retrouve de même en ce qui concerne la grandeur relative des méats intercellulaires, dont l'histoire détaillée est donnée aussi complètement que la petitesse des objets l'a permis, de la page 89 à la p. 110 .

Quant aux cytoblastes, il était évident, qu'il fallait les chercher à l'intérieur des parties, appelées noyaux dans la description de M. RoBIn et qui ne sont autre chose que le contenu entier de chacune des cellules constituant le groupe regardé geénéralement comme cellule simple. J'ai trouvé dans chacune de ces cellules un cytoblaste central, entouré à chaque face par quatre petits points opaques, dont la nature ne pouvait être déterminée avec certitude, vu leur extrème petitesse. En général il fant un éclairage très-favorable pour voir bien distinctement ces parties. On les distingue plus nettement après le traitement de la sarcine par une solution de carmine daws de la potasse diluée. Lors de la division des cellules, ces cytoblastes s'allongent d'abord, pour se diviser ensuite en formant, de cette manière, un nouveau cytoblaste pour chacune des cellules nouvelles. (Voir pour les cytoblastes les fig. $34-45$ de la planche II).

La forme et le développement si réguliers, pour ainsi dire mathématiques, de la sarcine mont engragé à faire l'épreuve d'une détermination exacte des lois-mêmes de ce 
développement. Une série de mesures (pag. 26) accompagnée des observations nécessaires sur le degré de composition, sur l'état des cellules quant à la division, sur la direction de celle-ci etc., sert de base à cette recherche, dont la voie a été retracée pas à pas dans le mémoire, afin de permettre de porter un jugement complet sur la méthode suivie et sur les résultats obtenus. Il n'est guère possible de rendre compte de cette partie du travail sans le reproduire textuellement. Il faut se contenter de relever un seul point. L'accroissement du corps de la sarcine a lieu simultanément dans les trois directions perpendiculaires entre elles, et suit, dans chacune de ces trois directions, la même loi. Malgré cela les dimensions dans cés trois directions ne sont jamais égales entre elles. La forme cubique, qui peut être considérée comme le type, n'est jamais atteinte en réalité. Voici quelle en est la cause. $n$ existe entre le développement qui s'opère dans ces trois directions une différence non de loi, mais de phase, correspondant à la durée d'une période de division des cellules. Ces lois, et leur identité pour les trois directions, étaient déduites de la considération d'une seule surface des corps de la sarcine, c. à. d. de la plus grande, sur laquelle ces corps viennent reposer naturellement, lorsque le liquide qui les contient, d'abord agité, rentre lentement à l'état de repos. Après avoir déduit l'histoire des autres surfaces de l'histoire de cette première, un certain nombre de sarcines fut une à une renversée sous le microscope, au moyen d'une legère pression sur le couvre-objet. De cette manière une des surfaces qui se trouvaient d'abord perpendiculaires au champ de vue, vint se présenter à l'observateur. Une série de mesures prises sur ces surfaces (pag. 44) fut trouvée parfaitement conforme à celle qui avait été déduite antérieurement du calcul.

Les cellules, tout comme le corps entier de la sarcine, présentent dans les trois directions des dimensions inégales, inégalité qui s'explique absolument par la même 
cause. Mais pour les cellules il y a un autre gemre d'inégalités qu'on trouve en considérant de plus près la forme spéciale de ces parties elémentaires. En général, les six faces qu'on peut listinguer à chaque cellule, se trouvent ètre toutes différentes l'une de l'autre. Les dunze côtes, les huit angles s'arrondissent ì divers degrés, ce que la grandeur différente des méats intercellulaires, traversant le tissu dans les trois directions, rend évident. Encore doit-on dire que ces parties présentent une forme qui varie selon la place que les cellules occupent, an coin, ou au bord d'un groupe de cellules plus ou moins composé. Ces différences sont discutées de la page 99 à la page 110. Qu'il sutfise ici de dire, qu'il a été possible de réduire toute cette diversité de phénomènes à quatre phénomènes fondamentaux:

1. à une division des cellules qui se succède alternativement dans trois directions perpendiculaires entre elles.

2. à un accroissement continu et simultané dans ces trois directions, selon la loi exprimée pour l'une d'elles par la formule

$$
\mathrm{A}_{t}=2^{\frac{1}{3} \cdot \frac{\mathrm{t}}{\mathrm{T}}} \cdot \mathrm{A}_{\circ} \text {, dans laquelle } \mathrm{A}_{0}=4.25 \mathrm{mmm} .
$$

( $T$ étant l'espace de temps entre deux divisions successives des cellules dams le corps de la sarcine, done la durée moyenne d'une génération de cellules) et identique pour les deux autres directions, hors une différence de phase correspondant à ce même espace de temps $T$; ainsi:

$$
\begin{aligned}
& \mathrm{A}_{t}=2^{\frac{1}{3} \frac{\mathrm{t}}{T}} \cdot 4.25 \text { mmm. } \\
& \mathrm{B}_{\mathrm{t}}=2^{\frac{1}{3}\left(\frac{\mathrm{t}}{T}-1\right)} \cdot 4.25 \quad " \\
& \mathrm{C}_{\mathrm{t}}=2^{\frac{1}{3}\left(\frac{\mathrm{t}}{T}-2\right)} \cdot 4.25 \quad "
\end{aligned}
$$


3. à un défaut d'accroissement à l'équateur de la cellule-mère pendant la période qui suit sa division.

4. à une dissolution successive des membranes-mères avec leur âge.

Partout identité de loi, et différence seule de phase rendant compte de la diversité des phénomènes. Sans doute on trouverait des résultats semblables pour d'autres tissus végétaux, si, par une méthode analogue, on réussissait à déterminer d'une manière rigoureuse les lois qui président à leur développement et si l'on pouvait réduire ainsi les phénomènes immédiatement observés aux phénomènes fondamentaux qui leur servent de base. Sous ce rapport le mémoire présente un essai, fait sur un tissu très-simple et très-régulier, mais par lequel il me semble qu'il fuudra toujours commencer, pour passer ensuite à ceux qui paraissent plus compliqués."

En texminant M. Suringar signale deux cas de monstruosités, observés par lui, l'un sur la Digitalis purpurea, l'autre sur la Matricaria Chamomilla.

M. Miquel fait observer que la monstruosité de la Digitale est héréditaire.

III. M. Van Hulle (de Gand), ayant obtenu la parole, fait hommage de quelques-uns de ses opuscules, savoir:

1. Dinifkweek onder glas voor Jan en Alleman, Gent 1863.

2. De Moeshovenierderij, 2 ${ }^{\circ} d r u k, 229 \mathrm{bl}$. met platen, Gent 1863.

3. De Boomteelt, 3e vitgauf, 200 bl. 88 fig., Gent 1864.

L'orateur développe la question amnoncée par lui (voir Progr. pag. 25, No. 6, 2.) dans la note suivante, qu'il dépose sur le bureau:

La mission des jardins botaniques.

,Tout homme professant $m$ état, tout fonctiomaire public surtout, a non seulement le droit, mais le devoir de rechercher constamment tout ce qui peut tommer à 
l'avantage des affaires qui lui sont confiées. C'est pourquoi j'ai cru devoir profiter de l'oceasion que m'offire ce congrès, pour appeler votre attention sur la mission des jardins botaniques. Cette question me semble intéressante à plus d'un titre. Aussi l'avais-je signalée depuis longtemps à la commission directrice, dans l'espoir que l'un ou l'antre directeur-professeur de botanique, plus compétent que moi, jardinier en chef, se serait fait inscrire pour la traiter. Puisqu'il n'en a point été ainsi, ces Messieurs préférant peut-être répondre à mon discours, qu'il me soit permis de vous dire quelles sont mes vues à ce sujet.

L'histoire horticole nous laisse deviner combien l'origine de l'horticulture a dû être modeste, son application restreinte et son développement peu rapide. L'horticulture primitive était culinaire et officinale, et s'adaptait aux besoins peu considérables de l'époque. Elle n'a progressé un peu, n'est devenue florale et ormementale que plus tard, quand la civilisation a fait naitre le luxe et a rendu les échanges possibles. C'étaient d'abord les princes et les rois, les grands seigneur's et les riches commerçants seuls, qui recueillaient, le plus souvent au prix d'énormes sacrifices, tout ce que leurs priviléges ou leurs relations leur permettaient de se procurer de plantes. Ils en agissaient ainsi par pure fantaisie, si non par ambition.

Les gouvernements envisagèrent la question suus un tout autre point de vue et comprirent aussitôt l'immense utilité de l'étude et de la connaissance intime des plantes. Ils fondèrent auprès de leurs établissements d'instruction publique - guidés en cela par les hommes de science qui y furent attachés, - ce qu'on nommait dès lors et qu'on nomme encore de nos jours les jardins botaniques. Ce n'est que depuis que l'horticulture a commencé à se développer rapidement, à s'enrichir de nombreuses introductions nouvelles et à entrer dans la véritable voie du progrès.

En effet ces jardins, qui ne venaient que de naître, 
jouissaient alors de toute la sollicitude des gouvernements; ceux-ci, ayant pour ainsi dire seuls les moyens de faire venir des pays lointains des plantes et des graines inconnues jusqu'alors, pour être essayées et cultivées dans les jardins botaniques, avaient non seulement le monopole des introductions, mais les moyens de posséder des collections plus riches que les particulier's. Ensuite, ayant pour directeurs des hommes distingués qui classaient les plantes d'après leur's caractères, leurs propriétés et leurs vertus, et des jardiniers-chefs non moins habiles, qui pouvaient d'autant mieux rechercher la vraie culture d'me plante, que le nombre de celles-ci était encore relativement restreint, les jardins botaniques jouissaient à juste titre d'une grande réputation à cette époque. Aussi amateurs et jardiniers venaient s'y approvisionner et s'y instruire, jusqu'à ce que, excités par les résultats obtenus, et profitant des voies de communication rendues de plus en plus faciles, ils purent faire explorer à leur tour les pays luintains. A partir de ce moment, les gouvernements diminuant leur action dans la même mesure, comme s'il leur suffisait d'avoir donné l'élan — sont devenus tributuires de ceux dont ils ont été si longtemps les fournisseurs.

D'après cet exposé on serait porté à dire que les jardins botaniques ont fait leur temps; et en effet bien souvent leur mission se borne à conserver les vieilles reliques végétales dans des collections méthodiquement classées! Cependant que de services pouraient-ils rendre encore!

D'abord ils doivent posséder et conserver à l'état vivant, non pas toujours ce qui est vieux, mais aussi ce que les récentes introductions offrent d'intéressant ou de curieux, et toujoux's de façon à avoir des représentants du plus grand nombre possible de familles végétales; celles-ci doivent se suivre d'après leurs affinités naturelles. Tous les directeurs de jardins botaniques tâchent d'atteindre ce but, qui est bien plus important qu'on ne le croit géné- 
ralement; car, n'est-ce pas par l'étude des affinités que l'on apprend à trouver le nom d'une plante, sa culture, le choix du sujet pour la greffe, les parents pour les hybridations, etc.? Si les jardins botaniques ne remplissaient pas plus ou moins cette utile mission, quel est le particulier qui pourrait ou voudrait le faire?

Une autre mission des jardins botaniques est de cultiver séparément et par séries, les plantes qui sont spécialement économiques, médicinales, industrielles, maraîchères, fruitières, ornementales, vénéneuses et nuisibles même. Cette culture aurait pour objet de faire connaître ces plantes et de rendre les comparaisons et le choix plus faciles quand il s'agit d'en faire. Quel est le particulier qui consacrera son jardin à ce but, et ne sont-ce pas encore une fois les jardins botaniques qui doivent avoir cette mission?

Pour ce qui concerne l'arrangement des plantes d'ornement, une partie d'agrément d'une bonne étendue esi indispensable aux jardins botaniques, afin de pouvoir y planter les arbres et les arbrisseaux les plus variés possibles, à une distance qui leur permette de prendre quelque développement. Le plan en devrait être conçu d'après les règles de l'art, et la plantation faite, si non géographiquement, du moins harmonieusement et ne jurant pas avec la nature. Les corbeilles fleuries, sans abonder, devraient cependant avoir aussi leur place et être composées de façon à pouvoir servir de modèle aux particuliers, qui fréquenteraient alors ces jardins avec plaisir. Les pares privés seuls peuvent offrir tous ces avantages; mais cormme ils sont fermés pour le public, ne sont-ce pas les jardins botaniques qui doivent remplir cette mission?

Il va sans dire, que les plantes doivent-être non seulement bien étiquetées, mais surtout exactement dénommées. Il va encore de soi, que dans les villes universitaires les jardins botaniques doirent servir principalement et arant tout à l'instruction des étudiants; mais cela n'empê- 
cherait nullement qu'ils fussent en même temps des champs d'expérience, d'exercice et de démonstrations ì une toute autre catégorie d'élèves. Et en effet, quel est aujourd'hui l'homme qui ne s'intéresse pas à l'horticulture et ne veut pas s'y instruire! Où donc un cours public de taille d'arbres p. e., ou tout autre cours cultural, qui tous exercent une si heureuse influence, pourraient-ils être mieux organisés? Où donc la rusticité d'ume plante, les résultats d'un essai horticole quelconque, pourraient-ils être mieux constatés, mieux suivis par chacun, que dans les jardins botaniques? N'est ce pas à eux encore, qu'appartiennent spécialement ces différentes missions?

Nous renons de toucher à l'enseignement horticole. Puisqu'il ne suffit plus d'être routinier, de n'être capable qu'ì un âge avancé, lorsqu'après bien des échecs on ne sait encore que ce que l'expérience nous a appris à nos dépens, il faut aujourd'hui faire marcher la pratique avec la théorie, pour être de son temps et arriver plus vite à un résultat; puisque, en un mot, l'utilité des écoles d'horticulture est reconnue, où les établirait-on plus convenablement que dans les jardins botaniques, puisqu'on ne trouve pas, d'ordinaire, des horticulteurs assez capables, assez riches et surtout assez dévoués pour prendre cette charge?

Je ne finirais pas si je voulais examiner tout ce dont les jardins botaniques sont susceptibles de faire; je dois cependant émettre encore un vou, c'est de voir annexer aux jardins des espèces de musées, où les produits bruts et fabriqués de toutes les plantes utiles seraient constamment visibles pour le public. Une telle institution existe déjà au jardin de KEw et dans quelques autres, et personne n'en contestera l'utilité. Eh bien, ici encore, qui pourrait mieux imiter cet heureux exemple, que les jardins botaniques?

Il suit de ce qui précède que les jardins botaniques sont encore loin d'avoir payé tout leur tribut. Il est vrai que les temps ont bien changé depuis un siècle; 
les jardins qui jadis prochaient d'exemple, sont anjonrd'hui dépassés par des particuliers, parce que ces derniers, atin de pouvoir soutenir la concurrence, ont du élargir le cercle de leurs opérations, modifier et perfectionner leurs systèmes; plus libres dans leur allure, ils se sont maintenus à la hauteur du progrès, tandis que les jardins botaniques, dont les ressources sont restées les mêmes ou sont diminuées, n'ont pu suirre cette marche progressire et sont par conséquent restés stationnaires ou ont même rétrogradé.

C'est d'une part, parce que je suis si intimement convaincu de cette vérité, et d'autre part en considérant les missions élevées dont les jardins botaniques sont susceptibles, que j'ai cru devoir remuer cette question.

Je n'examinerai pas jusqu'à quel point les jardins botaniques sont à même de progresser d'après ces bases, mais je dirai seulement, que celui de l'université de Gand, qui n'est certes pas un des moins bien partagés, est cependant loin de pouvoir répondre aux exigences que j’ai ćnumérées tout à l'heure. Quelle doit être alors la position de tant d'autres jardins, qui n'ont ni locaux convenables pour loger leurs plantes, ni assez de combustibles pour chauffer leurs serres, ni les moyens de prendre les ouvriers nécessaires etc.?

La question que je viens de traiter sommairement, est donc des plus importantes, et, pour finir, je demande à tous les hommes éminents qui s'intéressent à l'horticulture, aux directeurs-professeurs de botanique surtont, si le temps n'est pas venu d'appeler la sérieuse attention des gouvernements - dont la plupart des jardins botaniques dépendent - sur des établissements qui pourraient devenir si éminemment utiles. Si à leur égard on prenait d'autres mesures, si, à l'aide d'encouragements et des ressources nécessaires, on les mettait à même de progresser, leur développement ne se ferait pas attendre.

En effet, les gourernements encouragent bien les ar- 
tistes et dépensent de très-grosses sommes pour les fêtes et les réjouissances publiques, pourquoi ne montrent-ils pas plus de sollicitude pour les jardins botaniques? L'on ne peut admettre qu'ils le font par indifférence, mais c'est uniquement, faute d'être suffisamment et constamment renseignés.

A rous donc, directeurs-professeurs de botanique, à vous mettre à l'œurre, à nous, jartiniers-en-chef, de vous seconder de toutes nos forces."

M. Fée applaudit aux bonnes intentions du préopinant. Il ajoute que les directeurs des jardins botaniques partagent les mêmes sentiments et tâchent autant que possible d'améliorer les jardins, afin de les faire marcher d'un pas égal avec les progrès du siècle. Les gouvernements ne manquent pas de documents qui les éclairent, car les directeurs cherchent partout à démontrer la nécessité d'augmenter les subsides, mais les gourernements ont beaucoup à faire et les budjets n'y peuvent toujours suffire.

M. Morren attire l'attention sur la transformation des jardins des simples en jardins horticoles, et sur la nécessité de s'appliquer de préférence à quelques spécialités.

M. Lecoq appuie cette dernière observation, et cite comme exemple les Cycadées du jardin botanique d'Amsterdam, cultivées là depuis longtemps et célèbres à juste titre.

M. Miquel fait remarquer que ces Cycadées ne sont pas des reliques, mais pour la plupart des introductions nouvelles, faites à grands frais.

M. Regel fait les observations suivantes:

,Ich hatte nicht im Sinne, mich an der Discussion über den vom Herrn van Hulue angeregten Punkt zu betheiligen. Da die Discussion aber so lange schon angedauert hat, so erlaube ich auch mir noch einige Worte in dieser Beziehung. Herr van Hulle verlangt ron botanischen Gärten Cultur der in früherer Zeit, wie der in neuerer Zeit eingeführten Pflanzen, ferner aller Nutzpflanzen, Obstkulturen und alle damit in Verbindung 
stehenden Manipulationen etc., kurz ein botanischer Garten sollte nicht bloss ein Herbarium vivum, nein, auch ein Versuchsgarten und eine pomologische Anstalt sein.

Ich will nun durchaus nicht läugnen, dass es gut wäre, wenn botanische Gürten nach allen Richtungen in wissenschaftlicher, wie praktischer Beziehung, Musteranstalten sein könnten, die gleichmässig für wissenschaftliche, wie praktische Zwecke Belehrung bieten würden. Dazu würde aber Geld und immer wieder Geld gyehören, das den botanischen Gärten meist sehr kärglich zugemessen ist. Da es mir nun aber selnr mwahrscheinlich ist, dass die verschiedenen Gouvernements in Folge unserer heutigen Discussion die Mittel dieser Institute in einer so bedeutenden Weise vermehren sollten, so wird nichts anderes übrig bleiben, als dass jeder botanische Garten seine Leistungen den vorhandenen Mitteln anzupassen sucht.

Die erste und wichtigste Aurgabe eines botanischen Gartens ist nach meiner Ansicht die wissenschaftliche, die praktische kann dam erst berücksichtigt werden, wenn jener genügt ist. Denn für die Wissenschaft nur allein sind die botanischen Gïrten gegrindet, und es ist eigentlich kein gutes Zeichen unserer Zeit, dass man so oft bemüht ist, einem rein wissenschaftlichen Institute durch untergeschobene praktische Zwecke anf die Beine zu helfen. Wie im praktischen Gebiet durch immer weiter gehende Theilung der Arbeit so Grosses bewirkt worden ist, so kann auch der kleinste botanische Garten in seiner Weise für die Wissenschaft arbeiten. Man strebe nicht darnach, grössere und reichere Sammlungen zu erwerben, als nach den gegebenen Mitteln auch gut mnterhalten werden kïnnen, und betrete im Uebrigen den schon rom Herm Professor Morren bezeichneten Weg, zu dessen Durchführung die Mittel eines jeden Institutes noch ausreichen werden. Durch derartiges vorzugsweises Sammeln einzelner Familien oder Pflanzengruppen und deren wissenschaftliche Bearbeitung, oder durch Unterstützung 
anatomischer und physiologischer Arbeiten etc., hat so mancher kleinere botanische Garten sich bleibende Verdienste un die Wissenschaft erworben und wird auch in der Zukunft sich solche noch reichlich erwerben können! Den wenigen bevorzugten, gut dotirten, derartigen Central-Instituten grösserer Reiche überlasse man gerne die dornenvolle A ufgabe, möglichst vollständige Sammlungen in allen Richtungen anzubahnen, alle Bestrebungen für wissenschaftliche Zwecke dureh die vorhandenen vollständigen Sammlungen kräftigst zu unterstützen, und endlich auch für die Praxis wichtige Versuche zu machen, soweit letztere auch gut controlirt und durchgeführt werden können, damit auch wirklich ein Nutzen aus solchen Versuchen entstehe. Aber auch der grösste und am. reichsten dotirte botanische Garten wird in vollkommener Weise nicht allen den von Herru vax Hulue gestellten Anforderungen nachkommen können. Besser einzelne Specialitäten musterhaft, ferner lieber weniger zahlreiche Sammlungen lebender Pflanzen, aber was rorhanden unter richtiger Benennung, als vieles mangelhaft. Ich schliesse mich daher dem von Herm Professor Morrex gegebenen Votum mit voller Ueberzeugung an."

M. Hoffmann fait observer que le jardin botanique doit être aussi un Institut physiologique et qu'il doit posséder les moyens pour servir à l'étude du professeur. I se félicite de ce que le jardin botanique, auquel il est attaché, lui fournit ces moyens.

IV. M. le Président accorde la parole à M. Bommer (de Bruxelles), qui fait les remarques suivantes sur $L a c o$ loration des plantes, qu'il accompagne d'expériences pour montrer diverses réactions chimiques.

„La plupart d'entre rous, Messieurs, commaissent les recherches de M. Freny sur la matière colorante verte des feuilles. Selon ce sarant, cette matière colorante aurait 
pour éléments constitutifs deux principes colorants: l'un bleu, phyllocyanine, l'autre jaune, phylloxanthine. Ayant répété consciencieusement les expériences de M. FrEMr, je suis convaincu que la substance verte des feuilles renferme parfois d'antres matières colorantes que celles qui viennent d'être citées.

Malgré la minutie que j’ai mise dans mes opérations, pour obtenir des résultats parfaitement iđlentiques à ceux de M. Fremy, jamais il ne m'a été possible d'obtenir ce qu'il annonce comme étant immanquable. Comme M. Fremy n’a pas indiqué l'espèce végétale sur laquelle il a opéré, j'ai été amené à conclure que le choix n'est pas indifférent pour réussir de prime abord.

L'Ilea: aquifolium a été en grande partie le sujet de mes expériences parce que son feuillage, d'un vert foncé, semblait devoir contenir une assez forte proportion de phyllocyanine. Beaucoup de végétaux contiennent, outre la matière verte, une matière colorante brune soluble dans l'eau. Lorsque l'on a fait une dissolution alcoolique de chlorophylle, du houx (Ilex aquifolium) par exemple, elle offre: 1. une forte fluorescence d'une couleur rouge sang; 2. une teinte brunâtre à la lumière solaire transmise; 3. une teinte rouge vive à la lumière artificielle. L'intensité de cette teinte est en proportion avec la matière colorante brune mêlée à la chlorophylle. Je désignerai cette matière colorante sons le nom de matieve extractive, parce que c'est à sa présence que l'on doit attribuer la coloration de la plupart des extraits que l'on emploie en pharmacie. La matière extractive peut être facilement séparée de la matière colorante verte par une addition d'ammoniaque, qui immédiatement s'empare de la première et isole la seconde.

Voilà le procédé qui m’a toujours bien réussi pour la chlorophylle provenant du houx (Ilex aquifolium).

Après avoir neutralisé l'ammoniaque contenu dans la solution verte, on la soumet seule au réactif indiqué par M. Fremy, c'est-ì-dire, ì l'action de 1 partie d'acide 
chlorhydrique étendue d'une petite quantité d'eau et de 2 parties d'éther sulfurique, qu'on agite fortement dans un flacon de manière à saturer l'acide chlorhydrique d'éther. Dès que l'on a ajouté la substance verte, et après avoir agité de nouveau le liquide pendant quelques instants, il se produit une réaction qui donne lieu à deux colorations bien distinctes. La première, d'un beau jaune (phylloxanthine) dissous par l'éther; la seconde, d'un vert bleuâtre pâle, qui, lorsqu'on laisse reposer le liquide, produit à sa partie supérieure une zône d'un bleu brillant (phyllocyanine). La matière extractive traitée par l'acide chlorhydrique ne donne lieu à aucune réaction.

De toutes mes expériences, c'est celle que je viens de citer qui a le plus de rapport avec les faits énoncés par le savant chimiste français. Mais il en est une autre, dont le résultat, sans le qualifier de négatif, est cependant tout à fait contraire à la règle établie par M. Frenr. Il a été produit par une solution éthéro-alcoolique de feuilles du Coleus Verschaffeltii, traitée par le procédé de M. Freny pour dédoubler la chlorophylle. Dans ce cas, l'acide chlorhydrique a isolé une matière colorante d'un rouge vif au lieu de la matière colorante bleue qu'indique M. Fremr.

- Pour ne laisser aucun doute à cet égard, je reproduirai devant rous, Messieurs, cette expérience qui me semble de nature à vous intéresser. (Démonstration).

Avant de terminer, je me permettrai, Messieurs, de signaler encore à votre attention le sulfate de zine, comme réactif qui m'a fort bien réussi pour la chlorophlylle, lorsqu'elle contenait une matière colorante rouge qui dérivait de la série cyanique. Pour obtenir ce résultat, il suffit de faire une addition d'éther sulfurique à une solution de chlorophylle, et d'y ajouter ensuite une solution concentrée de sulfate de zinc pur. Si la matière colorinte verte ne contient ancune matière colorante ronge, la solution de sulfate de zinc reste incolore, et dans le cas contraire, elle prend une teinte bleuâtre plus ou moins foncée, selon 
la quantité de matière colorante rouge contenue dans la chlorophylle, qui alor's se masse en me couche très-dense à la partie supérieure du liquide.

Voilà en résumé, Messieur's, les faits que je tenais à vous communiquer et auxquels je donnerai de plus amples développements dans un travail destiné aux Bulletins du Congrès (1)."

M. Chatin fait ì ce sujet les remarques suivantes:

„La matière, d'abord incolore, et qui, en s'altérant chimiquement et se colorant, donne la teinte brune (les teintes jaune et rouge sont d'origine différente) aux feuilles d'automme, a été nommée provisoirement matière $\mathbf{A}$ dans mon mémoire présenté à l'Académie des sciences (2). Depuis, je lui ai donné le nom de protextrine qui rappelle à la fois ses rapports avec l'extractif qui en dérive et sa transformation rapide, quand il n'est plus protégé par la vie des organes.

Relativement ì la matière verte des feuilles, ma remarque n'a d'autre objet que d'amener l'énoncé des recherches qui me sont communes arec mon ami le professeur Finhor, de Toulouse, sur la matière colorante des feuilles (3), et les recherches encore inédites de FinHor seul, recherches qui l'ont amené ì établir, que la chlorophylle (dissolution alcoolique) se dédouble soit par les acides végétaux, soit par une trace d'acide minéral, en une matière noiruttre, azotée et insoluble (qu'on sépare par la filtration) et une matiere jaune non azotée, qui n'est autre que la xantline. Le dédoublement de la chlorophylle s'opère fort bien aussi par la simple agitation de la solution alcoolique avec le charbou animal, lequel retient la matière noire.

La xanthine des feuilles, comme celle des fleurs,

(1) Voir ce mémoire, publić sous forme de supplément après le compte rendu des séances de la section de botanique pure.

(2) Comptes rendus de l'Académic des Sciences, Tome 51, pag. 810, année 1860.

(3) Comptes rendus de l'Acadénie des Sciences, 'Tome 57, pag. 39, année 1863. 
donne par l'acide chorhydrique du vert, qui agité avec l'éther se dédouble en jaune (solution éthérée) et en bleu (solution acide sousjacente).",

M. Rauwenhoff fait quelques observations au sujet des expériences de M. Frenr, qui, en traitant par un mélange d'acide chlorhydrique et d'éther le résidu de la solution alcoolique ou éthérique, a séparé la chlorophylle en deux matières, l'une bleue, l'autre jamne. L'orateur a répété ces expériences et a trouvé qu'elles exig’ent un temps assez long avant d'obtenir la séparation des deux parties et encore la partie jaune a-t-elle été souvent légèment brunâtre. Il a soumis ensuite les deux solutions à l'analyse spectrale (qui domne dans la solution de la chlorophylle ces remarquables raies d'absorption que chacun comnait), et il a vu dans la solution blene de phyllocyanine à peu près les mêmes raies que dans la chlorophylle, mais dans la solution jaune de phylloxanthine il a remarqué plusieurs autres raies, qui mnanquent dans la chlorophylle. Quoique ces phénomènes demandent encore des études ultérieures, M. RAUWENHofF se croit autorisé à déduire de ses expériences que la phylloxanthine n'est pas identique à la matière jaune qui se forme dans les parties étiolées, comme le veut M. SAchs, car la solution de cette dernière ne présente aucune raie dans l'analyse spectrale.

V. M. le Président ammonce que M. NARDY ainé à Lyon a présenté in mémoire intitulé: La lune et le règne végétal (1).

La séance est levée et remise à lundi 10 Avril, à 10 heures du matin.

(1) Voir le supplément anx comptes rendus des séances de la section. 


\section{SECTION DE BOTANIQUE PURE.}

SEANCE du 10 Avril 1865, à 10 hemres du matin.

LE BUREAU SE COMPOSE DE:

MM. FÉE, Président.

FENZL et MIQUEL, Vice-Présidents.

MORREN et OUDEMANS, Secrétaires.

La Séance est ouverte à 10 heures.

Le procès-verbal de la seconde séance de Samedi est lu et adopté.

Des dames honorent la séance de leur présence.

I. M. le Président accorde la parole à M. Regel (de St. Pétersbourg), qui prononce en ces termes un discours allemand:

Sur la valeur de l'espèce. (Ueber die Idee der Art).

\section{Einleitung.}

„Der Begriff der Art im Pflanzenreiche hat im Laufe der Zeit mancherlei Deutumg erhalten, indem die einen die Gränzen der Pflanzenart weiter, die andern dieselben immer enger und enger steckten. Eine Folge dieser verschiedenen Begränzung der Art ist die Masse der verschiedenen Namen, welche eine und dieselbe zu Variation geneigte Art im Laufe der Zeit erhalten hat.

Ferner ist in der nenesten Zeit, seitdem Darwin sein berühmtes Werk ,Die Entstehung der Arten im Thier-und Pfanzenreiche" veröffentlicht hat, die Frage: was ist Art? und wie ist solche entstanden? gleichsam in eine neue Phase getreten.

Bei der Behandlung dieses Themas von hohem Interesse für die Wissenschaft wird der Referent nur die 
Pflanzenwelt berïcksichtigen, mu so mehr als in der Pflanzenwelt Verhältnisse vorhanden sird z. B. die ungeschlechtliche Fortptianzung), die in der. Thierwelt nicht in analoger Weise vorkommen.

Um ferner den Standpunkt zu bezeichnen, von dem aus der Referent sich seine Ansicht über die PHanzenart gebildet hat, erlaubt sich derselbe anzudenten, dass der Artbegriff ein um so engerer und begränzterer sein wird, in je engeren Gränzen die Plimzenart studirt wurde; dass solcher aber um so mehr sich exweitern wird, je reicher das Material, das zur Beobachtung vorlag, und in je weiterer Verbreitung die Pflanenart beobachtet werden konnte.

Der Referent geht ferner von der Ansicht aus, dass bei der Feststeliung derartiger Begriffe, sei es nun der Begriff der Pfhanzenart in Allgemeinen oder im Speciellen, nur direkte Beobachtumgen entscheiden können, dass ferner dieser Begriff für den speziellen Fall ein um so richtigrerer sein wirl, auf je zalhlreichere Beobachtungen solcher basirt ist, und dass endlich der allgemeine Begriff für die Pflanzenart nur als Resultat aus vielen gut beobachteten speziellen Fällen gezogen werden kimn.

Wenn wir nun mit DaRwry einen noch allgemeineren Standpunkt ammehmen wollen, indem wir uns nicht damit begnügen, nur den Begriff der Art festzustellen, sondern aus der Gesammtheit aller bekannten Fälle auch noch auf die Entstehung der Art zurückschliessen wollen, so kann ein solcher Schluss nur da mit Sicherheit gemacht werden, wo er sich auf keine einzelnen Bruchstücke, sondern auf ganze Reihen gut beobachteter und im genauen Zusammenhange stehender Thatsachen in der Lebensgeschichte vieler Arten basirt. Mit andern Worten: der Referent kam nur denjenigen Sclılüssen oder Theorien in dieser Beziehung sich anschliessen, die sich immer und immer wieder auf zahlreiche Beobachtungen innerhalb des Begriffes der speziellen Art basiren, muss 
aber allen denen seine Anerkemnung versagen, die, wenn sie sich auch auf Thatsachen stützen, doch diese gerade in einer Art und Weise benutzen, welche die auf solche gestützten Schlïsse nichts weniger als rechtfertigen. Dass dieses für alle die Folgerungen des Darwrs und dessen Vertreter, zum Beweise ihrer Ansicht, ,dass einerseits die vollkommensten Organismen unserer Jetzhvelt, im Laufe der Zeit, sich allmühlig aus den einfachsten Organismen hervorgebildet hïtes, und andierseits cuch unsere jetat lebenden Pfanzen in einem bestündigen, wenn gleich sehr langsamen Fortbildungs- oder Umbildungsprozess zu andern Typen begriffen seien," wirklich der Fall ist, das sei der Zweck meines heutigen Vortrags.

Darwin und dessen Vertreter entnahmen die Beweise für ihre Ansicht theils der Geschichte der Pflanzenwelt in der Vorwelt, theils der Formbildung der Art in der Jetztwelt, wesshalb wir in beide Vorgänge zumächst eintreten müssen.

Die Entwickhungs-Geschichte der Pflanzen der Vorvelt.

In den Zeiten, als sich die Anfänge des Pflanzengeschlechtes auf unserer Erde ansiedelten, in der Silurischen, Devonischen und Ueberyungsperiode, da entwickelten sich nur blüthelose PHanzen, die theils im Wasser lebten und zur Familie der jetzt noch existirenden Tange gehörten, theils als Kräuter und Bämme das Land bedeckten. Von den letztern ist nur die Familie der Farne auf unsere Zeiten ïbergegangen, während von allen andern selbst die Familien (Calamites, Clatoxylon, Sigillaria) ganz uncergegangen sind.

Auf die ältesten Zeiten folgt jene lange Periode eines überaus üppigen Pflanzenwuchses, die wir als Steinkohlenperiode bezeichnen, weil wir die Pflanzenreste jener Zeit noch jetzt als Steinkohlen aus dem Schoosse der Erde hervorholen. Noch finden sich einzelne Pflanzenformen der frühern Perioden, neben ilmen treten aber als die Baumriesen der Waldungen, die Lepidodendien, gabelig. 
verästelte Bäume mit einem Schopf langer linearer Blätter auf den Spitzen der Zweig'e, die gleichsam als Bindeglied zwischen den Selaginellen und Cycadeen stehen; damn gegen das Ende der Periode, auch die noch in unserer Jetztwelt vertretene Familie der Cycadeen, auf.

Jahrtausende und Jahrtausende sind wieder vergangen, die Pflanzen der Kohlenperiode sind grossentheils in den Schooss der Erde eingebettet, und in den folgenden Perioden des Bunten Sandsteins und IInschelkalkes treten, neben einzelnen aus den frïheren Perioden übergetretenen Typen (Calamiten, Fantu, Cycadcen) die Coniferen als Herrn der Waldungen auf', während an die Monocotyledonen erimmernde schilfartige Pflanzen die Oberfläche des Bodens decken. Erst in den folgenden Perioden des Keupersandsteins, der Oolith- und Wealden-Periode treten, gemischt mit den Bäumen der vergangenen Perioden, wirkliche Monocotyledonen und zwar in Formen, die unsern Pandanu-Arten verwandt sind (Podocarpa), auf.

Es folgt die Kreideperiode, und mit ihr verschwinden die letzten der untergegangenen Baumformen der frühern Perioden, die Calamiten. Farne und Cycadeen herrschen nicht mehr. Eine Fächerpalme vom Aussehen unserer Chamaerops tritt auf und auch schon einige Laubbäume aus der Gruppe der Apetalen (Salicites).

Bis jetzt, und auch noch zu Anfing der folgenden, unserer Jetztwelt voransgẻhenden Periode der Tertiärzeit, herrschte auf der ganzen Erde ein tropisches Klima.

Ein reicher Pfanzenwuchs, dessen Reste als Bram- kohlen genugsam beliamnt, deciste in der Tertiärzeit wiederum die Erde. Die Vegretation ist aber schon der unserer Jetztwelt sehr ähnlich, indem unter den Pflanzenformen jener Zeit fast alle die Familien der Jetatwelt vertreten sind. Das am Anfang tropische Klima ward in unsern Breiten gregen Ende der Tertiärzeit zu einem subtropischen.

Nun erheben sich die Kallialgen, weite Gebiete sinken 
zurück ins Meer, mächtige Bimnenseen bilden sich, und so beginnt die in insere Jetztwelt reichende Diluvialzeit.

Diluvialperiode.

Die Diluvialzeit ist zum Verständniss der Verbreitumg. der PHanzen in der Jetztwelt von hoher Wichtigkeit. Wir müssen uns desshalb etwas einlässlicher sowohl die Diluvialzeit, sowie die Verbreitung der Pflanzen von der Diluvialzeit auf unsere Zeit betrachten.

Wasser hatte während der Diluvialzeit wieder einen grossen Theil unserer Erde gedeckt. Damit trat in unsern Breiten ein noch kälteres Klimat als das unserer Jetztzeit ein, unter dessen Einfluss in Europa die subtropischen Pflanzen der Tertiärzeit grossentheils ansstarben, mnd in unsern Breiten uu die kleine Zahl solcher erhalten blieb, die auch jetzt noch im warmen, wie in kältern Klimaten heimisch sind. Als ein fiappantes Beispiel der Art möge unser Adlerfarm (Pteris Aquilina) genannt werden.

Dagegen wurden in den nildern Klimaten Japans, ferner der südlichen Stuaten Nordamerika's und selbst Neuholland's viele zur Tertiärzeit läufigen Bäume auch der Jetztwelt erhalten. Nur ein auffallendes Beispiel der Art, das Taxodium distichum, wollen wir anführen. Dasselbe war bis zu Ende der Tertiärzeit einer der gemeinsten Waldbäume Europa's, starb aber in der Diluvialzeit in Europa ganz aus, während es in den südlichen Staaten Nordamerika's erhalten blieb. Dass aber in unsern jetzigen klimatischen Verhältnissen dieser schon im mittleren Europa wieder existiren kamm, zeigen die mächtigen Exemplaré dieser Pflanze in den Gärten des westlichen Deutschlands, ja selbst noch in Berlin. Nur dureh bedeutende Wärmeabnahme lässt sich daher das Aussterben dieses Baumes selbst für Südeuropa erklären.

Die tropischen Pflanzen waren in Europa schon zu Ende der Tertiärzeit ansgestorben, wesshalb die grosse Mehrzahl der der Jetztwelt erhaltenen Pflanzenformen ans den der Tertiärzeit vorausgehenden Epochen jetzt auf 
die tropische und subtropische Zone beschränkt ist. So die Baumfarne, die Cycadeen, die Araucarien, die Palmen etc. Jetatwelt.

Es beginnt die Jetztzeit; die Wasser der mächtigen Binnenseen brechen ihre Dämme und thürmen mächtige Schuttwälle auf. Die Sahara steigt aus dem Meeresboden auf, und so bilden sich die Zonengürtel Europa's aus, unter' deren Einfluss nach der Diluvialzeit die über einen grossen Theil Europa's verbreiteten Gletscher zurückweichen.

Im östlichen Asien scheinen diese Vorgänge andrer Art gewesen zu sein, als in Europa. Wohl versank dort zur Zeit der Diluvialperiode ein Continent, der Japan und die Ostküste Asiens nördlich von Japan mit Nordamerika verband (1), das Klima blieb aber noch verhältnissmässig mild, wie dies das noch sehr späte Vorkommen des Mammuth in Sibirien beweist. Erst mit der wohl in unsere Jetztwelt fallenden Durchbrechung der Landverbindung zwischen dem nördlichsten Asien und Amerika (Behringstrasse) mögen die Meeresströmungen aus dem Becken des Eismeeres, die längs Ostasien herabziehen, das Klima jener Gegenden so plötzlich verändert haben, dass hierdurch der merkwürdige Fall des Autfindens der in Eis eingebetteten noch frischen Körper des Mammuth in Sibirien sich erklären mag.

Verbreitung der Pflanzen in der Jetztwelt.

Mit Berücksichtigung dessen was wir über die Pflanzenwelt früherer Epochen, sowie über die frühere Gestaltung der Erdoberfläche wissen, hätten wir uns die Verbreitung der Pflanzen in der Jetztwelt ungefähr in der folgenden Weise vorzustellen.

Zu Anfang unserer Jetztwelt hatten sich die Reste der Pflanzenwelt der Tertiärzeit nur auf den über die

(1) In meiner Flora des Ussurigebiets habe ich gezeigt, dass die Verbreitung der Pflanzen von Ostasien nach Nordamerika eine solche Verbindung voraussetzen lïsst. Seitdem hat Schмmp an der Ostküste $\Lambda$ siens die tertiären Schichten mit Pflanzen- und Thierabdrïcken auf dem Boden des Mecres von der Kï̈ste aus übergehen sehen. 
weite Wasserfläche emporgehobenen Parthien des Festlandes erhalten können. Zn diesen hier angesiedelten Typen früherer Epochen scheinen sich aber auch noch eine grosse Anzahl anderer Pflanzenarten gesellt zu haben, die in der Tertiärzeit noch nicht vorhanden waren, und deren Entstehung daher in den Anfang unserer Jetztwelt gesetzt werden müsste.

Wir erinnern daran, dass die in frühern Perioden auf dem ganzen Erdball ziemlich gleichmässige Flora, schon zu Ende der Tertiärzeit, in unsern Breitengraden den Charakter der' warmen gemässigten Zone erhalten hatte, und bei dem Uebergang zur Diluvialzeit in unsern Breitengraden, mit der steigenden Erkaltung des Klimas, auch noch die grosse Melirzahl der Tertiärpflanzen ausstarb und theils durch neu auftretende Formen ersetzt ward.

Von den über die Wasserflächen empor gehobenen Parthien traten nun, während und nach der Diluvialzeit, die Pflanzen ihre Wanderung über den Erdball an, soweit klimatische Verhältnisse und Weltmeere dieser Wanderung keine unübersteiglichen Gränzen entgegenstellten.

Es ist dies nicht bloss eine Annahme, sondern es wird das noch jetzt beim Studium grösserer Florengebiete tausendfach bewiesen. Noch jetzt bilden die höher gehobenen Parthien gleichsam die Centren der Flora; noch jetzt sind solche am reichsten an eigenthümlichen und mannigfachen Pflanzenarten, und darum das Eldorado für -den Botaniker, denn sie haben so manche Pflanzenart in ihrem Bereich gebannt erhalten, die in dem Gebiete der angränzenden Ebenen nicht die Bedingungen für ihr Gedeihen finden.

Die Art wie die scheinbar auf ihre beschränkte Wolnnstätte angerviesenen Pflanzen wandern, ist schon oft und hinlänglich klar besprochen worden. Als Leiter der Verbreitung sind Luft, Wasser, Thiere und Menschen zu nennen. 
Die Luftströmungen tragen die leichten Samen der Pflanzen oft auf weite Strecken fort. Wer lätte nicht schon die mit Haarkronen versehenen Samen unserer Hieracien, des Löwenzalıns und anderer Compositen einem Ballon gleich durch die Luft schweben selien; Sturmwinde tragen aber auch andere leichte Samen auf weite Strecken fort. Ausschliesslich auf Verbreitung mittelst der Luft sind die Sporen vieler kleiner Schmarotzerpilze angewiesen, und es ist eine bekannte Thatsache, dass die die Kartoffel- und Weinlirankheit erzeugenden Pilze binnen wenigen Jahren ihre Wanderung durch ganz Europa gemacht haben.

Die Strömungen des Wassers sind ein anderes Mittel zur Verbreitung der Pflanzen. Die Flora bestimmter Flussgebiete ist daher meist auch eine ziemlich gleichförmige, so weit limatische Verhältnisse nicht Unterschiede bedingen. Wer aus der Ebene längs eines Gebirgsbaches in das Gebirge aufsteigt, findet an den Ufern desselben schon lange vorher einzelne Bergpflanzen, bevor deren eigentliche Zone beginnt, dem die Strömungen des Wassers bringen Samen und auch ausgespülte lebende Exemplare mit, die sich dann tiefer unten längs der Ufer festsetzen.

Thiere, und namentlich die gefiederten Bewohner der Luft, sind Verbreiter, indem theils mit Haaren, Borsten oder Wiederhaken versehene Früchte im Fell und den Federn derselben häng'en bleiben und verschleppt werden, oder indem solche die Früchte fressen, und die Samen mit den Excrementen der Thiere verstrent werden.

Wie schnell und leicht derartige Verbreitung statt findet, dazu wollen wir nur ein Beispiel geben, das uns einer unserer Freunde aus dem Innern Russlands, Herr Jung, kïrzlich mittheilte. Auf einem grossen Gute des Fürsten Bäriatinsкy, zu Ivanowskoe, im Gouvernement Kursk, ward ein grosser See gegraben; dieser hatte ursprünglich ganz klares reines Wasser. Jetzt aber ist derselbe theils ganz ron üppigen Wasserpflanzen aller 
Art dicht erfüllt, die nur von Wasserrögehn aus entfernten Sümpfen dorthin rerschleppt worden sein kömmen.

Der Mensch verändert nicht nur durch seine Kulturen den Karakter der Flora ganzer Gegenden, sondern es folgen ihm auch gleichsam, olne sein Zuthun, eine Masse von Pflanzen, unter welchen ror allen die längs der Wege und Schutthaufen wachsenden Unkräuter zu nennen sind. Ueberall wohin die Kultur gedrungen, sind diese Unkräuter mitgewandert, indem sie ganz zufällig eingeschleppt wurden, und stellenweis die einheimischen Pflanzen verdrängten. So ist es z. B. von Neuseeland bekannt, dass manche der aus Europa wegreschleppten Pflanzen sich dort so eingebürgert haben, dass sie dominiren und die einheimischen Pflanzen verdrängen.

Lassen wir aber den Menschen ganz bei Seite, so ist doch durch Luft, Wasser und Thiere der scheinbar auf ihre Lokalität gebannten Pflunzenart hinlänglich die Gelegenheit geboten, im Laufe der Zeit von einzelnen Lokalitäten ausgehend, soweit zu wandern, als dies die Statur der Pflanzenart oder Terrainrerhältnisse erlauben.

Diesem Wanderungsgesetz widerspricht scheinbar eine Thatsache, die wir schon erwähnten, nämlich die auffallende Verschiedenheit der Flora der Gebirge und der Hügel ron der der angränzenden Ebenen. Hierzu kommt noch die andere Thatsache, dass so manche Pflanze der Gebirge Mitteleuropa's in den Ebenen des höhern Nordens wieder vorkommt, deren klimatische Verhältnisse ung'efähr ilirem Standorte im Gebirge entsprechen, während auf den weiten zwischenliegenden Gebieten die gleiche Pflanzenart keine Verbreitung besitzt.

Es sind das fü Europa so bekamnte Thatsachen, dass wir dafür keine Beispiele anzufüluren brauchen. In Asien wiederholt sich dasselbe, ja es sind selbst in den höhern Gebirgen des südlichen Japans noch manche Bewohner des entfernten Sibiriens heimisch. 
Dieser scheinbare Wiederspruch wird aber wieder durch die folgenden Betrachtungen gehoben:

Die Pflanzenarten waren in ihrer grossen Mehrzahl, zu Anfang unserer Jetzwelt, sehr wahrscheinlich nicht bloss auf einzelne Lokalitäten beschränkt, sondern hatten sich auch schon in jener Zeit auf rerschiedenen Punkten der über das Wasser emporragenden Parthien angesiedelt. Thre Verbreitung in die Ebenen fand daher von den zunächst gelegenen Gebirgen aus Statt. Als aber immer mehr Land über die Oberfläche des Meeres allmählig herrortrat, als die grossen, das mittlere Europa deckenden Eismassen unterm Einfluss höherer Wärmegrade zu weichen begannen, ward das Klima der angränzenden Ebenen milder als das der Gebirge. Die Folge davon musste die sein, dass in den Ebenen alle für solche nicht geeigneten Pflanzenarten bis zu den Breitegraden ausstarben, wo das Klima ihnen wieder die Bedingungen zum normalen Wachsthum gestattete.

Ferner nahmen hohe Waldungen und üppige Prairien. Besitz von den Ebenen und erstickten so manchen hier angesiedelten Bewohner freier Lokalitäten der Gebirge, und nur in den weiten Moorbrüchen, wo eine ärmliche Vegetation herrschte, da erhielt sich noch bis auf unsere Zeiten, in derartigen Lokalitäten der Ėbenen, mancher ursprüngliche Bewohner höherer Gebirge.

Endlich nahm auch der Mensch mit seinen Kulturen so ausschliesslichen Besitz von den fruchtburen Niederungen, dass er den ursprünglichen Charakter der Flora gänzlich veränderte, so dass wir, zum Beispiel, das ursprüngliche Heimathland vieler unserer wichtigsten Kulturpflanzen gar nicht mehr mit Sicherheit nachzuweisen vermögen.

In unserm übervölkerten Europa bewahrten daher nur der Kultur unzugängliche Steppen, Moräste und Gebirge noch einigermassen den ursprünglichen Charakter der Flora, während in andern Erdtheilen noch weite 
Gebiete derartigen Forschungen ein günstigeres Feld darbieten.

Verbreitung ein und derselben Pflanzenart von verschiedenen Centren, Erwärmung des Klimas der Ebenen nach dem Weichen der Gletschermassen, Erstickung der Pflanzen des Hochgebirges in den dichten Waldungen der Niederungen, und Einfluss des Menschen waren mithin Ursachen jener bedentenden Lücken in Verbreitungsgebiet vieler Pflanzenarten.

Gränzen der Verbreitung der Pflanzenart.

Der Wanderung der Pflanzenart in unserer Jetztwelt waren aber in zwei Richtungen mübersteigliche Gränzen gesetzt. Es sind dies einestheils weite Weltmeere, und anderntheils unsere Zonengürtel.

Wo weite Weltmeere jetzt Continente tremnen, da zeigt denn anch die Flora, ungeachtet ganz ähnlicher Temperaturverhältnisse, in den gleichen Breitengraden meistentheils einen ganz andern Charakter. Wo aber dies nicht der Fall ist, wie z. B. in Bezug auf das östliche Gebiet Mittel- und Nordasiens einerseits, und des nordwestlichen Amerika's andrerseits, da können wir mit ziemlicher Sicherheit auf das Versinken ron Landverbindungen erst zu Anfang unserer Jetztwelt zurückschliessen.

So viel uns fermer die Erfahrung geleht hat, giebt es unter den zahlreichen Planzenarten nur wenige, die zugleich in warmen wie in kialten Klimaten gedeihen. Die unendlich grosse Mehrzahl derselben ist gegentheils so organisirt, dass eine bestimmte Pfanzenart, sich selbst überlassen, nur in bestimmten Zonengürteln leben und sich sellständig fortpflanzen kamn. So verbreitete sich denn anclı die Pflanzenart von bestimmten Vegetationscentren nur soweit nach Süd und Nord, als dies je nach der speziellen Natur der Pflanzenart möglich war.

Was unsere von. Akklimatisation träumenden Sanguiniker in wenigen Jahren und wenigen Generationen umzuändern hoffen, indem sie eine Pflanze wärmeres 
Klimate allmählig an ein bedeutend kälteres anzugewöhnen hoffen, das hat Mutter Natur schon seit Jahrtausenden in gleicher Weise gethan, indem solche ganz allmählig Schritt für Schritt die Pflanzenart bis an ihre äussersten Gränzen nach Süd und Nord, und in zahllosen neuen Generationen, rorschob. So hat dem die Pflanzenart, schon lange ehe der Mensch mit solcher manipulirte, ihre natürlichen Verbreitungsgränzen' in Bezug auf klimatische Verhältnisse erhalten, und der Mensch, mit aller seiner Kunst, wird in dieser Beziehung nie mehr erreichen können, als dass er der Pflanzenart über den natürlichen Verbreitungsbezirk hinaus nur noch einen schwachen weiter ausgreifenden Verbreitungsbezirk, den kïnstlichen Terbreitungsbezirk, anweisen kamn, wo die Pflanzenart sich selbst überlassen wieder nussterben würde, aber künstlich vermehrt und gepflegt, doch noch gedeihen kann.

Für die Pflanzen der gemässigten und der kalten Zone der Jetztwelt ist der Aequator eine unübersteigliche Schranke der natürlichen Verbreitung gewesen, und in Folge dessein der grelle Unterschied der gleichen Zonengürtel jenseit und diesseit der Wendekreise.

Wo aber dennoch einige Pflanzenarten der gemässigten und kalten Zone in unserer Jetztwelt auf der südlichen und nördlichen Halbkugel gleichzeitig rorkommen, da können wir mit Sicherheit den Schluss machen, dass solche entweder aus frühern Epochen, wo noch eine gleichmässige Temperatur die Wanderung der Pflanzenart über den ganzen Erdball und in alle Breitegrade desselben ermöglichte, auf unsere Jetztwelt herabgekommen, oder dass solche erst in den letzten Jahrtausenden durch den Menschen auf beide Halblingeln rerpflanzt wurden.

Pflanzenarten von beschionliter und weiter Verbreitung.

Ueberblicken wir num noch eimmal alles das, was über die Art der Pflanzenwanderung, seit dem Beginn der Jetztwelt, gestützt auf Erscheinumgen und Thatsachen, die wir jetzt noch beobachten, gesagt wurde, so liegt es 
auf der Hand, dass Pflanzen, die Früchte oder Samen besitzen, welche von der Luft leicht fortgetragen werden können, oder solche, deren Samen und Früchte von den Thieren vorzugsweise anfgesucht werden, anch viel leichter befähigt waren kleincre Hindernisse, als die Wasserscheiden der Flussgebiete, ferner Wasserflächen von nicht zu grosser Ausdehnung etc. zu überspringen, welche für andere Arten, die mehr nur auf zufällige Verbreitung durch Wasserströmungen, oder vielleicht gar nur auf ihr eigenthümliches langsames Fortschreiten mittelst der Sprossbildung angewiesen waren, schon eine unübersteigliche Gränze bildeten.

Dies bedingt es, dass wir Pflanzenarten ron sehr beschränkter Verbreitung besitzen, und wieder andere, die allmählig in ilırem Zonengebiet alle Hindernisse bewältigend, ihre Wanderung durch Europa, Asien und Nordamerika schon beendet hatten, bevor noch der Mensch durch seine Eingriffe berlentende Ungestaltungen in den natürlichen Florengebieten bedingte.

Formbildung der Pfanzenart in Folge der Wanderung.

Es liegt nun anf der Hand, dass die Pflanzenart, je weiter sie ron ihren ursprünglichen beschränkten Standorten vordrang, auch unter um so verschiedenern äussern Einflüssen, welche durch Licht, Lage, Boden und Feuchtigkeit bedingt werden, zu leben gezwungen war.

Die mannigfach verschiedene Combination dieser Verhältnisse wird für die spezielle Pflanzenart noch um so verschiedenartiger, je mehr solche, je nach ihrer Organisation, anch unter verschiedenen klimatischen Einflüssen oder, mit andern Worten, in einem mehr oder weniger breiten Breitegürtel, gedeihen kann.

Die Pflanzenart von weiterm Verbreitungsbezirk erlitt, in Folge dessen, unter den verschiedenartigen äussern Einflüssen in ihrer äussern Gestaltung mancherlei Abänderungen.

Derartige durch Klima, Standort und Boden bedingte 
Abänderungen können oft sehr bedeutend sein, indem z. B. der hohe Baum zum Zwerge, der Stranch zur kriechenden Pflanze, die kahle Pflanze zur mehr oder weniger stark behaarten Pflanze werden kann, und indem anch noch andere untergeordnete Differenzen in Bezug auf Grösse und Farbe der Blumen, Theilung der Blätter u. s. w., sich ausbilden können.

Diese Unterschiede zwischen den extremen Formen der Art bedingen zuweilen so auffallende Differenzen, dass dem Monographen einer Gattung von weitem Verbreitungsbezirk es häufig leichter wird, die extremen Formen der Art durch scharfe Diagnosen auseinander zu halten, als andrerseits scharfe Charaktere zur Unterscheidung der eimzelnen Arten und zwar mit Inbegriff aller ihrer Formen aufzustellen.

Hieraus sind zweierlei Uebelstände für die Wissenschaft entstanden. Erstens sind fast alle extremen Formen der Art ursprünglich als eigene Arten aufgestellt worden, und erst nachdem die Flora eines Landes genaner bekannt ward, und damit anch die Uebergänge ron einer Form zur andern zur Beobachtung vorlagen, komnten derartige sehr natürliche Irrthümer aufgedeckt werden. Sehr natürliche Irrthüner nenne ich aber mur dann das Anfstellen solcher Endformen als Arten, wemn dem betreffenden Botaniker bei seinen Untersuchungen die Uebergänge noch nicht vorlagen.

Zu geisseln ist dagegren das so mmütze, den Begriff der Art so gänzlich verwirrende Aufstellen neuer Arten, nach Formen, deren Uebergänge so leicht gefunden werden konnten oder sogar absichtlich bei Seite geschoben wurden. Ist es doch noch nicht lange her, dass manche Systematiker in die Herbarien gar keine im Garten gewachsene Pflanzen aufnehmen wollten, weil diese nicht genugsam characteristisch seien, d. l. mit andern Worten, nicht die speziellen Charaktere einer Lokalität zeigten. 
Verhalten der Formen der Pfanzenart in Kultur.

Die obige Ansicht über die in den Kulturzustand übergegangenen Pflanzen lhat sich jetzt glücklicher Weise geändert, ja von jedem nach Wahrheit forschenden Systematiker muss sogar fortgesetzte Kultur so mancher zweifelhaften Arten einen der wichtigsten Prüfsteine für ihr Verhalten als Form oder Art abgeben.

Die verschiedenen Formen der Art müssen nämlich in Anbetracht ihrer Entstehung, schon vom theoretischen Standpunkte aus betrachtet, in Folge fortgesetzter Kultur unter gleichartigen Verhältnissen und mehreren auf einander folgenden Generationen, allmählig wieder zu einem einzigen Typus übergehen.

Wie sich in Wirklichkeit die Formen der Art bei der' Kultur in dieser Beziehung verhalten, will ich, auf 30jährige Erfahrung gestützt, mit kurzen Worten zu schildern versuchen.

Bei solchen Kulturversuchen werden leichtere, lediglich dureh den Standort veranlasste Formen, meist schon als Individuum, immer aber in der folgenden Generation zur normalen Form zurïckichren. So z. B. kümmerliche Formen von Sand und sterilem Boden, Formen des schattigen Standortes etc.

Viel fester mit ihren besondern Eigenthümlichkeiten verbunden sind schon die Formen, die durch lange Angewöhnung an ein besonderes Klima entstanden sind. Diese behalten gemeiniglich als Individuen ihre besonderen Eigenthümlichkeiten oder Angewöhmungen, und werden exst durch die folgenden, auf geschlechtlichem Wege erhaltenen Generationen zu dem Normaltypus zurïckgeführt.

Nehmen wir hier, um ganz verstanden zu werden, ein Beispiel von Formen des wärmeren Klimas und ein anderes von Formen des kältern Klimas.

Der Apfelbaum (Pyrus Malus) gehört noch im Petersburger Klima zu den ohne jede Deckung im freien Lande ausdanernden Pflanzenarten. 
Verpflanzen wir aber, z. B., eine der in südlichern Breitegraden entstandenen Formen desselben, wie z. B. ein Exemplar einer edlen Reinette aus dem wärmern Klima nach Petersburg, so wird dieses schon im ersten Winter oder doch in einigen Jahren gänzlich erfirieren. Dies ist nicht bloss der Fall, wenn eine edle Reinette als Pflanze aus einem mildern Klima bezogen wird, sondern auch dann noch, wenn wir nur die Pfropfreiser beziehen und solche auf einen hiesigen Apfelbamm aufsetzen.

Säen wir dagegen die Samen einer Reinette in Petersburg aus, so erhalten wir daraus junge Pflanzen, die unser Klima ertragen, freilich aber anch bei eintretender Fruchtbarkeit verschiedene Früchte liefern.

In diesem Faile ist also die Angewölnunng eines Individuums von einer Form des wärmern Klimas an das letztere so gross, dass es, ins kältere Klima verpflanzt, als Individuum nicht fortbestehen kann; oder mit andern Worten, es kann dasselbe nur unter Einwirkung eines längern, wärmern Sommers seine Vegetation so weit beenden (reifes $\mathrm{Holz}$ bilden), um cler Winterkälte Widerstand leisten zu können.

Der Referent wählt dieses Beispiel, weil ihm gerade in dieser Beziehung hunderte von übereinstimmenden Erfahrungen, die er in den letzten Jahren zu machen Gelegenheit hatte, vorliegen. Was die Veränderungen betrifft, die eine Reinette als Individum, sofern es wirklich gelingen sollte solche in einem kältern Klima anzugewöhnen, in diesem erleiden dürfte, darüber liegen uns keine direkten Beobachtungen vor. Schliessen wir aber aus analogen Fällen von andern Aepfeln zurück, so würden die Früchte wohl ihre Form behalten, ohne jedoch die ihnen eigene Güte zu bekommen.

Als Beispiele von Formen, die, ans kälterm Klima in ein wärmeres übergeführt, ihne Eigenschaften erst in den folgenden Generationen verändern, dient ein grosser Theil der Pflanzen unserer Gebirge. 
Greifen wir ans diesem als einzehnes Beispiel den Alpenmohn (Pupuver ulpimu L.) heraus. In den höhern Alpen Europa's kommt derselbe als kleine Pflanze, mit kleinen 1-2fach tiederschnittigen Blättern mit schmalen Lappen min mit haum spamnenhohen, weisse oder hellgelbe Blumen tragenden Blüthenschäften, vor. In Sibirien wächst die gleiche PHanzenurt, die 1-2fach fiederschnittigen Blätter sind aber grösser, die Lappen derselben breiter, die Schäfte bis $1 \frac{1}{2}$ Fuss hoch, und die Blumen gelb oder weiss. Die erstere Form ward von Linné als $P$. alpinum, die andere ebenfills von Linsé als $P$. mudicaule beschrieben. Die zithlreichen Uebergänge zwischen den beiden Formen stellte der Referent auf pag. 130-133 seiner Flora Ostsibiriens zusammen.

Nehmen wir nun ein Individuum der Alpenform Europa's von der Höhe des Gebirges und verpflanzen solches in den Garten, so behält dias Exemplar seine Charaktere, die Pflanze bleibt aber kümmerlich, und zeigt kein rechtes Gedeihen. So wenigstens verhielten sich Exemplare, die wir früher in den Alpun der Schweiz sammelten. Säet man aber Samen, welche von Exemplaren der Alpen der Schweiz entnommen sind, in Garten aus, so erhält man Pflanzen, die im ersten und zweiten Jahre in Blatt und Blüthe noch der Stammfurm ziemlich ähnlich sind; aber schon in den folgenden Jahren werden die gleichen Pflanzen allmälig üppiger und üppiger, die Blätter bekommen fast die Form und Grüsse der Form Sibiriens und die Blüthenschäfte werden über einen Fuss lang.

So wenigstens verhielten sich Exemplare, die im hiesigen Garten aus von den Alpein der Schweiz stammenden Exemplaren erzogen wurden und num in der, der Kultur der Alpenpflanzen gewidmeten Parthie unseres Gartens üppig vegretiren. Damit will dei Referent keineswegs die Behauptung anfistellen, duss nicht auch das Individuum derartiger Formen, in Folge lany fortgesetzter aufmerksamer Kultur und bei allmühligem Vorrïcken nach külterm oder 
wärmerm Klima zu; Form eines andern Klimas übergehen könnte.

Im Allgemeinen nur wird das schon desshalb selten der Fall sein können, weil es Erfahrungssarche ist, dass aus wärmern Klimaten in kältere versetzte Individuen dem Erfrieren so ausgresetzt sind, dass sie, selbst unter güustigen Unständen, doch schon in den ersten Jahren durch den Frost geschädigt werden.

In Folge dessen werden sie von Jahr zu Jahr schwächlicher wachsen und so wird die Hotfuung auf normalen, dem Klima angemessenen Wuchs immer geringer. Dass aber in Wahrlieit solche Formen des Klimas, bei ganz allmähligem Vorrücken nach anderm Klima, auch ihre Angewöhnungen und die aus kimatischen Verhältnissen entspringenden Eigenschaften, in den folgenden durch ungeschlechtliche Vermehrung erzielten Generationen, verlieren können, das wird durch eine Masse von Beispielen belegt, wie z. B. durch die vom Süden bis zum $61^{\circ} \mathrm{N}$. Breite verbreiteten Aepfel Russlands (Belui Nalio, Aport, Borowinka,etc.) so wie ferner durch unsere nordischen Klaräpfel, die in mildern Klimaten niemals die Eigenschaft des Glasigwerdens behalten, etc. Andrerseits zeigen ans dem Hochgebirge in die Ebene versetzte Individuen selten eine längere Dauer, sonder'n siechen meistens bald dihin, wenn nicht durch Entziehung von Nahrung gleichsam die zu lang andauernde Sornmerwärme paralisirt wird.

Es liegen mir aber einzelne Erfahrungen ror, dass selbst sehr auffallend verschiedene Alpenformen schon als Individuen zur Form der tiefern Regionen sich umbilden kömmen. So sah ich vor num 15 Jahren die aus den Alpen in den botanischen Garten zu Zürich rerpflanzte Möhringia polygonoides allmählig zur M. muscosa werden.

Einzelformen und deren Verhalten in Kultur.

Wie die Formen, die durch anderes Klima enzeugt sind, so gehen auch die Formen mit anders gefärbten Blumen und Blätter'n, mit ander's gestalteten Blätter'n, 
mit gefüllten Blumen etc., selten als Individumu zurück zur Stammart. Beruhen doch auf dieser Eigenschaft der Beharrlichkeit des Individuums die so verschiedenartigen in den Gärten ausgeführten Arten der ungeschlechtlichen Vermehrung, um solche Formen unverändert zu exhalten.

Erinnern wir in dieser Bezielnung als Beispiel nur an eine leichte Form eines unserer Waldbäume, an die Blutbuche. Hunderttausende derselben sind, auf ungeschlechtlichem Wege vermehrt, in die Gärten des mittlern Europa's übergegangen, und alle haben, so viel mir bekannt, ihre Eigenthümlichkeit, nämlich die tief rothen Blätter behalten. Mache man aber den Versuch, säe man die von einer Blutbuche stammenden Samen aus, und unter Hunderten von jungen Samenpflanzen sah der Referent, wenigstens bei einem derartigen Versuche, alle vollkommen zur Stammform zurückgehen. Freilich haben wir es hier in der Mehrzahl der Fälle nicht mit Formen zu thun, wie solche in Folge der Wanderung der Pflanzenart entstehen, sondern vielmehr nur mit auf geschlechtlichem Wege erzeugten einzelnen Individuen der Art, welche, ohne von dem Arttypus abzuweichen, in einzelnen Richtungen eine anormale Ausbildung zeigen, wesshalb wir derartige Formen als Einzelformen bezeichnen. Wo derartige Formen nicht durch Kultur erzeugt sind, da rommen sie in der freien Natur als einzelnes anormales Individuum zwischen Tausenden von normal gebildeten Individuen vor. Der Mensch nur hat solchen in seinen Kulturen eine grössere Bedeutung gegeben, indem er gerade die anormale Form durch ungeschlechtliche Vermehrung fortpflanzte. Derartigen Ursprungs ist z. B. anch die als Beispiel gebrauchte Blutbuche, von der ein einzelnes Exemplar vom Forstmeister Winter im Thüringer Walde aufgefunden und, durch Veredlung vermehrt, der Kultur übergeben wurde. Schlitzblättrige Erlen, Birken, Alorne und andere unserer beliebtesten Laubbäume haben eine ähnliche Entstehung und lassen sich, wenn man deren 
Geschichte verfolgt, auf ein einzelnes Individumm zurückführen, das zwischen den andern normalen, sei es in der freien Natur, sei es in unsern Kulturen, zufällig entstanden ist, dessen Eigenthümlichkeit uns aber nur durch Anwendung der ungeschlechtlichen Vermehrung erhalten wurde. Aehnlich verhalten sich Formen mit gefüllten und verschiedenartig gefärbten Blumen, mit grössern Blumen und Früchten, mit monströs gebildeten Stengeln, Blättern und Blumen.

Wir müssen hier noch eimmal des Beispiels der Aepfel gedenken. Wir sprachen oben von den Formen derselben, die solche unter'm Einfluss des wärmern und kältern Klimas bilden und zeigten, wie der Geschmack der Frucht und die Eigenschaft in Bezug auf Bedarf an Wärme eine Eigenschaft del Form sei. Wir haben es aber bei den Aepfeln unserer Gärten nicht bloss mit den Eigenschaften der Form, sondern zugleich auch mit den Eigenschatten der Einzelform zu thun. Dem während Geschmack der Frucht und Angewöhnung ans Klima Eigenschaften der Form sind, so sind all die andern zahlreichen Unterschiede, die unsere Apfelsorten unter einander zeigen, Eigenschaften der Einzelform. Während nun die Eigenschaften der Form, schon bei den folgenden aus ungeschlechtlicher Vermehrung gewonnenen Generationen, bei ganz allmähliger Ueberführung in ein anderes Klima, zurückschlagen können, bleiben dagegen alle die Charaktere der Einzelform, auch bei allen folgenden auf ungeschlechtlichem Wege erzielten Generationen, unverändert, so die vom Bau der Frucht, vom Wuchs des Baumes, von Färbung, Behaarung ete., genommenen Kennzeichen.

Während nun also bei der Fortpflanzung auf ungeschlechtlichem Wege die Einzelform viel constanter als die Form sich verhält, ist ungekehrt bei geschlechtlicher Fortpflanzung durch Samen die Einzelform, in Bezug auf die solcher zufillenden Charaktere, stets unbeständig, während die Forn unter gleichbleibenden äussern Verhält- 
nissen auch bei geschlechtlicher Fortpflanzung eine gewisse Beständigkeit zeigt. Hieraus resultirt, dass in der freien Natur, sich selbst überlassen, nur die Form eine Bedeutung erhält, während die Einzelform, bei ihrem sporadischen vereinzelten Vorkommen, fast ganz verschwindet.

In Kultur dagegen spielt auch die Einzelform, durch ihre Eigenschaft bei ungeschlechtlicher Fortpflanzung ihre speziellen Charaktere zu behalten, eine wichtige Rolle.

Racebildung durch Einfluss der Kultur.

In der freien Natur bildete sich die Form unter Einfluss bestimmter äusserer Verhältnisse und zeigte, auch bei der geschlechtlichen Fortpflanzung und gleichmässiger Andaner gleichartiger Verhältnisse, eine gewisse Beständigkeit. In der Kultur dagegen entsteht, unter Andauer gleichartiger Verhältnisse und Auswahl, die Race aus der Einzelform.

Nehmen wir als Beispiel die Reihe der Formen mit gefüllten Blumen, mit monströsen Blättern oder Stengeln, mit verschiedenartig gefärbten Blumen. Alle diese sind ursprünglich nur Einzelformen. Es werden daher in der Kultur bei melnjährigen Pflanzen derartige Formen auch nur auf ungeschlechtlichem Wege fortgepflanzt und erhalten.

Dagegen besitzen wir eine grosse Zahl einjähriger Kulturpflanzen, wo derartige Eigenschaften auch auf die geschlechtlich erzeugten folgenden Generationen vererbt werden können. Unsere einjährigen Florblumen, Gemüse, Getreide sind da genugsam bekannte Belege. Hier haben wir es aber schon nicht mehr mit der Pflanzenart in ihrem natürlichen Zustande, sondern mit der Pflanzenart in ihrem cultivirten Zustande zu thum, wo der Mensch, durch den Einfluss seiner Kulturen und sorgfälige Auswahl des zur Aussaat bestimmten Samens, inehr oder weniger konstante Formen gebildet hat, die am besten durch Racen bezeichnet werden. 
Sie verhalten sich aber in einer Beziehung ganz ähnlich, wie die bei der Wanderung der Pflanzenart entstandenen Formen der Art, indem auch sie nur unter den gleichen Kulturbedingungen, unter denen sie entstanden, und unter fortdauernder Auswahl der zum Tragen des Samens bestimmten Individuen, ihre speziellen Eigenschaften, wegen deren man sie schätzt, behalten, während sie, unter veränderten Kulturbedingungen oder sich selbst überlassen, schon in wenigen Generationen wieder ausarten, d. h. nach dem Normaltypus allmählig zurückfallen. Sprossformen.

Schliesslich haben wir noch der Formen mit bunten Blättern zu gedenken. Wir haben schon an andern Orten darauf hingewiesen, dass diese nur zufällige Entstehung haben, ja dass sie sogar am einzelnen Individuum in Folge einer dimorphen Ausbildung einzelner Zweige oder Sprossen entstehen, und nur durch ungeschlechtliche Vermehrung festgehalten werden. Wie sie sich zufällig an einzelnen Individuen ausbildet, ebenso kann auch ein buntblättriges Individuum häufig, in einzelnen Aesten oder auch als ganze Pflanze, wieder zum normalen Typus zurückkehren.

Aus Samen pflanzen derartige buntblättrige Formen in der Regel sich nicht fort. Aber hier giebt es einzelne auffallende Ausnahmen von dieser Regel, wie z. B. die buntblättrigen Abarten mancher Farne (von Pteris cretica und quadriaurita), und ferner die buntblättrige Abart der Barbarea vulgaris, die sich ziemlich konstant aus Samen fortpflanzen, was man also als Racenbildung von buntblättrigen Pflanzen zu bezeichnen hätte.

Basturde im Pflanzenreiche, deren Eigenschaften und Einfluss auf die Art.

Ausser der Variation, die die Pflanzenart in Folge der äussern Einflüsse und in Folge anderer mehr zufälliger Umstände erleidet, giebt es aber noch ein anderes Moment, durch welches manche Pflanzenarten, und zwar vorzüglich 
solche, die mit einander in vielen ihrer Charaktere übereinstimmen, abgeändert oder scheinbar in einander übergeführt werden. Es ist das die Bastardbildung. Auch in Bezug auf Bastardbildung liegen dem Referenten ganze Reihen eigner Versuche und Beobachtungen vor.

Er erinnert daran, dass er der erste war, der der Annahme, Aegylops ovata sei die Mutterpflanze unseres Weizens, entgegen trat, und die Ueberführung des Aegylops ovata in den Weizen durch Bastardbildung nicht nur erklärte, sondern auch zum Beleg später den Bastard erzog.

Ebenso war er es, der es mit Beispielen belegte, dass es auch durchaus fruchtbare Bastarde gebe und die Resultate seiner Versuche in zahlreichen Artikeln mittheilte (1). Der Referent kann sich um so mehr jetzt auf diese frühern Versuche berufen, als auch spätere genaue Beobachter alle von ihm gefundenen Resultate in dieser Beziehung bestätigt haben.

Für die Diskussion der wichtigen Frage, die wir heute besprochen, resultirt aus diesen Beobachtungen das folgende:

a. Es giebt zwei unter einander verwandte Arten der gleichen Gattung, oder auch unter einander noch verwandter Gattungen, die durch gegenseitige Befruchtung einen Bastard erzeugen können.

b. Dieser Bastard hält in seinen Charakteren die Mitte zwischen den beiden elterlichen Arten.

c. Der Bastard besitzt meistentheils vollkommen ausgebildete und befruchtung'sfähige weibliche Geschlechtsorgane. Der Pollen der Bastarde ist aber in der Mehrzahl der Fälle verkümmert und zur Befruchtung unfähig, in

(1) Vergleiche Gartenflora, Jahrg. 1853, pag. 269-273, 280; Gartenflora, Jahrg. 1854, pag. 61, 116-126, 240, 247-250, 303; Bonplandia, Jahrg. 1854, pag. 286-293; Gartenflora, Jahrg. 1855, pag. 278-281; Bonplandia, Jahrg. 1855, pag. 53, 163-171; Botanische Zeitung, Jahrg. 1855, pag. 269; Gartenflora, Jahrg. 1856, pag 153, 191-192; Bonplandia, Jahrg. 1856, pag. 298, 243; Gartenflora, Jahrg. 1857, pag. 163-168, tab. 97; Gartenflora, Jahrg. 1860, pag. 60-62. 
der Minderheit der Fälle aber theilweis oder selten durchgängig vollkommen und befruchtungsfähig.

d. Der von einer der elterlichen Pflanzen befruchtete Bastard kehrt schon gänzlich oder gleich einer leichten Form in der folgenden Generation zu dieser zurück.

e. Der ron andern (nicht den elterlichen Arten) befruchtete Bastard bildet in der folgenden Generation eine Form mit jenen, die jedoch nicht in der Mitte stehet, sondern mehr zu den eigentlichen Arten als zum Bastard hinneigt.

f. Die folgenden Generationen des im Pollen und den weiblichen Organen fruchtbaren Bastardes kehren in der Mehrheit der Individuen zu einer der elterlichen Arten zurück. Einzelne der Nachkommen des fruchtbaren Bastardes zeigen dagegen in unwesentlichen Charakteren zuweilen Variationen, die von keiner der elterlichen Arten hergeleitet werden kömmen. So ist in der Kultur der fruchtbare Bastard der Erzeuger einer grossen Mamnigfaltigkeit von Einzelformen zwischen je zwei Arten geworden, so in Bezug auf Färbung und Form der Blumen (Gesneriaceen, Rosen, Verbenen, Geranien, Petunien, etc.), Grösse der Frucht (Erdbeeren), ete. Diese Mannigfaltigkeit ist durch fernere Befruchtung der erzielten Formen mit andern Arten oder Bastarden noch rermehrt worden; immer sind, es aber nur Formen geblieben, die sich zwischen bestimmten Arten bewegen, die niemals eine Fortbildung zu einem. höhern Typus gezeigt haben, und, sich selbst überlassen, bald wieder aussterben oder zu einer der elterlichen Arten zurückkehren würden.

q. In der freien Natur spielt der Bastard nur eine sehr untergeordnete Rolle, indem er hier überhaupt nur vereinzelt und verhältnissmässig nur in wenigen Gattungen rorkommt, und indem er ferner selten Samen bildet, und, wo dies geschieht, meist nur durch Befruchtung mit einer der elterlichen Arten, zu der er damn in den folgenden Generationen zuriickkehrt. So ist der Bastard in der 
Natur gleichsam nur eine vereinzelte und zwar vorübergehende Erscheinung, durch den nur in wenigen Gattungen dem treuen Beobachter die Erkennung der Arten, so wie der Bastardformen, erschwert wird. Einen scheinbar wichtigern Platz nimmt der Bastard schon in dem Herbarium ein, da namentlich von den rereinzelten Exemplaren von Bastarden unserer Holzpflanzen mancher Florengebiete jührlich Massen von Exemplaren gesammelt und oft mehr als die gemeine Stammart verbreitet werden.

Wo endlich der Bastard, im Gebiete mancher Flora, auch noch mit andern als den elterlichen Arten sich mischt und Formen bildet, wie z. B. bei den Weiden (Salix), ist dieses doch nur eine Folge der Einmischung des Menschen, durch künstliche massenhafte ungeschlechtliche Fortpflanzung mancher Arten, wodurch häufig das eine Geschlecht dieser zweihäusigen Pflanzen eine vorwaltende unnatürliche Verbreitung erhält.

h. Der Bastard verhält sich, ung'eschlechtlich fortgepflanzt, ungefähr so wie die Einzelform, d. h. er behält alle seine Eigenthümlichkeiten, ohne eine allmählige Umbildung nach einer der elterlichen Arten einzugehen. Es giebt aber Fälle, wo der Bastard neben seiner normalen Form einzelne Zwreige bildet, die vollständig der einen oder andern der elterlichen Pflanzen gleichen.

Das frappante Beispiel eines solchen Dimorphismus, bietet Cytisus Adami, der Bastard zwischen Cytisus Laburnum und purpureus.

$i$. Die vom Bastarde gefallenen Einzelformen verhalten sich bei ungeschlechtlicher Vermehrung ganz wie die Einzelform der Form.

Nachdem wir im Obigen in der Bastardbildung einen andern Vorgang kemnen gelemt, durch welchen einzelne Arten scheimbar zu andern übergeführt und zu fernerer Formbildung veranlasst werden, wiederholen wir jedoch, 
dass der Bastard in der freien Natur durch sein sporadisches Auftreten und schnelles Zurückgehen zu den Stammeltern nichts weniger als eine wichtige Rolle bei der Formbildung der Art spielt. Wo dagegen durch die Eingriffe des Menschen die natürlichen Verhältnisse verändert worden sind, d. h. die männlichen und weiblichen Exemplare dioiker Pflanzen ganz umnatülich gemengt worden sind, da kommt wohl auch scheinbar in der freien Natur Bastardirung in mehreren Generationen nach einander vor, und erzengt ein buntes Gemisch von Einzelformen, die die Gränzen zwischen manchen Arten gänzlich verwischen.

Der Bastard und dessen Nachkommen im Kulturzustande.

Wichtig ist die Rolle, welche der Bastard in unsern Kulturen spielt. Wie durch Vermischung und fortgesetzte Bastardbildung in mehreren auf einander folgenden Gliedern eine Masse von Formen entstehen, die, bald wieder mittelst ungeschlechtlicher Vermehrung fortgepflanzt, eine sehr wichtige Rolle in unsern Blumen- und Gemüsegärten spielen, das zeigen die erst in neuerer Zeit vorgenommenen Experimente in den Gattungen Verbena, Fuchsia ete. einerseits und der Erdbeeren anderseits. Dem Referenten ist es aber sehr wahrscheinlich, dass auch bei vielen derjenigen unserer Kulturpflanzen, wo wegen der Länge der Zeit sich dies nicht mehr mit Sicherheit nachweisen lässt, denmoch die Masse der Formen derselben durch geschlechtliche Vermischung zweier oder selbst mehrerer, ursprünglich gut geschiedener Arten entstanden ist, so, z. B., bei den Weinreben, den Pflaumen, den Bohnen, ete.

Dass aber auch bei in den Geschlechtsorganen vollkommen ansgebildeten Bastarden, durch fortgesetzte Kultur und Auswahl, eine Racebildung ermöglicht werden liamn, das zeigen z. B. die Herbst-Levkoien, die Pensées, und vielleicht haben auch die Fornen vieler unserer Getreide, ferner die der Bohnen, eine ähnliche Entstehung. 
DARwin's Theorie über Entstehung der Arten und deren

\section{Beleuchtung.}

Nachdem wir num im Vorhergehenden zuerst die allmälige Entstehung und Vervollkommnung der Pflanzenwelt auf unserm Erdball, dann Formbildung der Art in Folge der Wanderung und zufälliger $(d . h$. bis jetzt nicht erklärter) Einwirkungen, und endlich in Folge von Kultur und Bastardbildung, kennen gelernt haben, wollen wir es nun versuchen, DARw In's Ansichten auf die im Vorhergehenden geschilderten Thatsachen zu prüfen, um dann schliesslich unsere Ansicht über die Pflanzenart und deren Formen zu geben.

DARWIN stelt in seinem berühmten Werke, ,Die Entstehung der Arten," keine netre Idee auf, sondern er ist nur der geistreiche Vertreter einer schon von LAmaRCK und Andern ausgesprochenen Ansicht, dass die Arten im Thierreich und Pflanzenreich nicht als geschaffene Typen zu betrachten seien, sondern dass sie durch allmählige Veränderung eines oder einiger Urtypen entstanden seien. DARwIN stützt sich dabei auf allgemeine Gründe, d. h. aus Zusammenstellung aller bekannten Erscheinungen und Thatsachen gewonnene Schlüsse, und auf spezielle Gründe.

Als allgemeine Gründe für die Annahme, dass die Arten im Thier- und Pflanzenreiche sich, von der niedrigsten Stufe anfangend, durch divergirende und allmählig erblich werdende Fortentwicklung zu anderm Typus, zu höherer Form, im Laufe der Zeit aus einem oder wenigen sehr einfachen Urtypen gebildet haben, werden von DARwin und dessen Vertsetern die folgenden angeführt:

1. Die Thatsache, dass in den frühesten Perioden der Schöpfung nur niedrige Pflanzen- und Thierformen auftraten, und dass die Pflanzen- und Thierwelt im Laufe der Jahrhunderttausende, die seit den ersten Anfängen pflanzlichen und thierischen Lebens bis zur Jetztzeit über unsern Erdball dahingezogen, immer vollkommnere und vollkommnere Organismen aufzuweisen hat. 
2. Die Neigung der Arten beider Reiche zur Varietätenbildung und die hieraus resultirende Schwierigkeit der Unterscheidung der Arten, Gattungen, Familien u. s. f.

Als spezielle Berreise führt $D_{\text {ARwin ganz besonders }}$ seine Beobachtungen über die in Kulturzustand übergegangenen Pflanzen und Thiere, so wie über Bildung von erblichen Racen durch den Einfluss der Kultur auf.

Ferner bespricht er die Bastardbildung und die in Folge derselben eintretende Formbildung und kommt dabei zu dem Schluss dass ein Theil der massenhaften Formen mancher in Kulturzustande befindlichen Arten von Pflanzen und Thieren durch die Vermischung mehrerer ursprünglich rerschiedener Typen entsprungen sein dürften, während andere nur durch die Formbildung der Art zu erklären seien.

Diese theils unter unsern Augen in der Kultur sich ausbildenden neuen Formen, durch Tausende von Generationen hindurch potenzirt gedacht, liefern nach DARwis's Ansicht den Bereis für die allmählige Ausbildung ganz neuer Arten oder Typen.

Die Sprünge oder das Fehlen der Mittelformen erklärt DARwin durch das, was er als Kampf um das Dasein bezeichnet. Stets nämlich werden viel mehr Embryonen gebildet, als zur Entwicklung kommen können. DARWIN nimmt num an, dass die für gewisse Verhältnisse geeignetsten Organismen bei diesem Kampf um die Entwicklung stets obsiegen mussten, und damit gingen die Mittelformen verloren, und nur die für die speziellen Verhältnisse am zweckmässigsten gebildeten, einen neuen Typus darstellenden, Organismen erhielten sich.

Es würde zu weit führen, noch näher auf DARwin's geistreiche Entwicklungen einzugehen; nur das sei noch erwähnt, dass el selbst sich der Schwächen seiner Darstellung sehr wohl bewusst ist, und wiederholt zugiebt, dass aus den ron ihm selbst aufgeführten Thatsachen oft auch gerade der entgegenstehende Schluss gezogen werden kann. 
Der Referent hat den Standpunkt, den er bei der Beurtheilung derartiger Fragen schon immer eingenommen hat, schon Eingangs bezeichnet, d. h. für ihn gilt nur das als Beweis, wo Thatsachen eben nur einen einzigen Schluss, wie zweimal zwei ist vier, zulassen.

Wie und auf welche Weise die Pflanzen in der Vorwelt entstanden sind, dazu werden wir in unserer Jetztwelt nie und nimmermehr derartige Beweise finden können. Wir können für die Vorwelt nur als Thatsachen constatiren, dass einmal eine Urerzeugung, also ein Schafjen, wenn auch nur der einfachsten Organismen stattgefunden haben muss, während in unserer Jetztwelt die Urerzeugung auch für die eimfachsten Organismen erIoschen ist. Wir haben ferner die allmählige Weiterentwicklung der Pflanzenwelt von den ältesten Zeiten bis auf unsere Jetztwelt constatirt.

Daraus aber Schlüsse auf die allmälige Umbildung und Weiterentwicklung der einfachsten Organismen zu den vollkommensten der Jetztwelt zu ziehen, dazu liøfern uns am allerwenigsten die Bruchstücke, die wir von den Organismen der Vorwelt besitzen, das Material.

Constatirt ist es, dass die grosse Mehrzahl gerade der Pflanzen, die ror und während der Kohlenperiode unsere Erde berölkerten, jetzt ganz ausgestorben ist. Gerade die ausgestorbenen Pflanzentypen jener Zeit (Calamiten, Lepidodendren) stehen in der Schöpfungsgeschichte der Pflanzenwelt so vereinzelt und barock da, dass noch keinerlei Uebergänge nach vollkommern Pflanzen weder unter den jetzt lebenden, noch unter denen früherer Perioden bekannt sind.

Ferner ist es eine Thatsache, dass unserer Jetztwelt gerade die einfachsten Pflanzenarten neben den vollkommensten erhalten blieben. Müssten sich nicht, wenn DARwin's Theorie die richtige wäre, von den einfachsten Stufen ausgehend, auch all die unserer Jetztwelt verlorenen Mittelstufen wieder ron Nenem nachgebildet haben? 
DARwrN giebt selbst wiederholt $z u$, dass einmal untergegangene Pflanzenformen sich niemals von Neuem erzeugen können. Wenn wir daher in Bezug auf untergegangene Pflanzenarten einen Schluss machen wollen, sind wir nicht viel eher zu dem berechtigt, dass mit einer untergegangenen Art auch zugleich ein Princip verloren ging, welches in das erste Glied der Kette, das zur ganzen Bewegung den Anstoss gab, gelegt war, nämlich die Idee der Art verloren ging? Werfen wir nun aber gar von der Pflanzenart, wie uns solche die Beobachtung in der Jetztwelt kennen lehrte, einen Blick auf die Bruchstücke die wir von den Pflanzenarten der Vorwelt kennen. Aus der Beobachtung unserer noch lebenden Pflanzen wissen wir, dass wir uns den Begriff der Art nicht nach einzelnen undeutlichen Blumen und Früchten oder vereinzelten Zweigfragmenten etc. bilden können, sondern dass dazu die Kenntniss der ganzen Entwicklungsgeschichte, ihre Formbildung in früherer Jugend und späterm Alter, ihre Formbildung je nach Boden und Lokalität und Klima gehört.

Wie oft und häufig ist die gleiche Pflanzenart der Jetztwelt, nach Exemplaren vom gleichen Individuum in verschiedenen Entwicklungsperioden, so wie bei unvollkommenen Pflanzen, unter ganz verschiedenen Benennungen beschrieben worden, und wie noch viel häufiger giebt die Formbildung der Art zu ähnlichem Versehen Anlass. Wie und mit welchem Recht könmen wir also auf die uns gebliebenen Bruchstücke von Pflanzenresten der Vorwelt Theorien für die viel schwierigere, und nach meiner Ansicht überhaupt nicht zu lösende, Frage über Entstehung der Pflanzenart aufstellen?

Auch wir sind durchaus nicht der Ansicht, wenn wir die Schöpfungsgeschichte der Pflanzenwelt ron ihrem ersten Entstehen an überblicken, dass unsere vollkommnere Arten plötzlich als fertige Organismen in die Welt gesetzt worden seien. Die Erzengung derselben hat sich viel mehr 
sehr wahrscheinlich an andere vorhandene Organismen angelehnt.

Auf welche Weise das aber geschehen, dafür giebt uns weder das, was wir ron der Vorwelt, noch das was wir von der Jetztwelt wissen, einen Aufschluss. Uns muss es genügen zu wissen, dass es Zeiten gegeben haben muss, wo wenigstens die einfachsten Organismen durch Urerzeugung auf unserer Erde geschaffen wurden.

$\mathrm{Ob}$ nun aber die vollkommneren Organismen aus diesen hervorgingen, indem solche sich in frühern Perioden (in der Jetztwelt ist das sicher nicht der Fall), wie DARwIN annimmt, allmählig höher und höher entwickelten, oder ob es Zeiten gab, wo das in der Jetztwelt herrschende Gesetz der Erhaltung der Art nicht gültig' war, sondern die vorhandenen Organismen Embryonen ausstreuten, aus denen ganz differente Pflanzenarten hervorgingen, oder ob durch Dimorphismus aus einzelnen Knospen vorhandener Organismen neue Arten gebildet wurden, oder ob endlich vielleicht noch ein ganz anderes Verhältniss die Bildung oder Erschaffung neuer Pflanzen in der Vorwelt und zu Anfang der Jetztwelt bedingte: das wissen wir nicht, und werden es auch sinwerlich mit allem unserm Scharfsinn unzweifelhaft sicher ergrïnden können.

Gehen wir zur Jetztwelt über, so sind die Beweise, welche Darwin für seine Ansicht aus dieser nimmt, nur für den verführerisch, der nicht selbst derartige Beobachtungen, in der freien Natur, wie bei unsern Kulturpflanzen, zu machen Gelegenheit hatte.

Wir haben oben ebenfalls die Racebildung der Pflanzenart durch den Einfluss der Auswahl und Kultur besprochen. Wir haben oben gesehen, dass die Racebildung nicht die Folge der Formbildung der Pflanze, im gewöhmlichen Sinne, jst, sondern dass nachweislich einzelne Individuen, welche an irgend einem ihrer Organe eine abweichende Bildung zeigen, den ersten Anstoss zu solcher geben. Von solch einem Individuum wird nun der Same 
ausgesäet. Finden sich in der folgenden Generation einzelne Pflanzen, die die gleiche Eigenthümlichkeit zeigen, so werden diese isolirt gestellt, von ihnen wieder. Samen gesammelt, und von den hieraus hervorgehenden Pflanzen wieder die Exemplare gewählt, welche jene Eigenthümlicbkeit am stärksten ausgebildet besitzen.

In dieser Weise fortgefahren, gelingt es, gewisse Eigenthümlichkeiten so festzuhalten, dass sich solche auch bei den meisten Individuen der folgenden Generation zeigen. Durch sorgfältige Auswahl wird diese Eigenthümlichkeit nun immer fester und fester gelegt, ungefähr ähnlich wie in manchen Familien Krankheiten erblich werden. Auf diese Weise, entweder in Folge der Neigung der Pflanzenart zur Formbildung, oder durch fortgesetzte Vermischung zweier oder selbst mehrerer typischer Arten, sind alle unsere Racen von Kulturpflanzen entstanden, und werden deren auch für die Zukunft in Folge einsichtiger Kultur immer mehr und mehr entstehen.

DARWIN denkt sich nun durch natürliche Züchtung einen derartigen Racenprozess durch Tausende ron Generationen hierdurch fortgesetzt, denkt sich durch den Kampf ums Dasein die Rolle des Menschen in Bezug auf Auswahl ersetzt, und erhält auf diese Weise, in irgend einer Eigenthümlichkeit, von Generation zu Generation mehr divergirende neue Generationen, bis zuletzt eine ganz neue Art daraus hervorgeht.

Dieses Bild, das Darwin, gestützt auf die Racebildung, über Entstehung der Arten giebt, ist recht geschickt gewählt, aber der Natur nicht entnommen, sondern nur der Kultur entlehnt und daher nach unserer Ansicht um so weniger von Beweiskraft, als Darwın's ganzer Beweis nur auf einer Annahme basirt, dass nämlich die Varietätenbildung sich auch in der freien Natur, von Generation zu Generation, in Folge natürlicher Züchtung in gevissen Richtungen mehr potenziren müsse, während doch gegentheils in der freien Natur dieser Neigung zur Form- 
bildung schon eben so bald der Grenzpfahl gesetzt ist, sobald sich die Pflanzenart an veränderte äussere Einflüsse, denen sie ausgesetzt ward, vollständig angeschlossen hat.

Die äussern Eiuflüsse sind aber das einzige Moment, welches wir zur Herstellung der natürlichen Züchtung', wie sich Darwin ausdrückt, an die Stelle des Menschen setzen können.

Alle zufälligen Verschiedenheiten, welche bei der Pflanzenart vom Menschen zur Festhaltung von Formen, mittelst ungeschlechtlicher Vermehrung, oder mittelst Bildung von Racen, in der oben besprochenen Weise benutzt werden, kömmen dagegen in der freien Natur niemals festgehalten werden, sondern treten da nur sporadisch gleich dem Bastard auf, um später wieder in den Arttypus zurückzufallen.

Dass dies so ist, das zeigt gerade die von Darwin als Beweis für seine Ansicht benutrte künstliche Züchtung von Racen. Allerdings erhalten viele der künstlich gebildeten Racen auch in den folgenden Generationen ihre Charaktere, so lange die zu ihrer Hervorbildumg nothwendigen Verhältnisse der Kulturbedingungen und Auswahl andauern.

Schon wenn noch Auswahl durch den Menschen stattfindet, aber die Kulturbedingungen durch Wechsel der Lokalitäten etc. geändert wurden, arten viele unserer künstlichen Racen von Kulturpflanzen in den folgenden Generationen wieder aus, d. h. sie fallen wieder mehr nach dem Arttypus zurück.

Ueberlassen wir solche aber der freien Natur, so werden sie, als künstliche Produlite der Kultur, gar keine Lebensfähigkeit zeigen und bald ganz aussterben oder zur Stammart zurückfallen, oder wie DARwin sagen würde, beim Kampf um's Dasein untergehen.

Es haben mithin für die Natur im Grossen die zufällig gebildeten Variationen an einzelnen Individuen, die wir künstlich zur Racebildung benutzen, gar keinen Werth 
und keine Dauer, sondern alles was hier durch natürliche Züchtung, ohne Zuthun des Menschen, in Bezug auf Formbildung von der Pflanzenart erlangt werden kann, das sind jene Formen, die wir als Formen des wärmern oder kältern Klima's, als Formen des Gebirges und der Ebene, des Sumpfs, des Sands, des Schattens etc. bezeichnen, welche bei der Verbreitung der Pflanzenart über den Erdboden entstanden sind.

Wir zeigten oben, wie auch diese Formen in den folgenden Generationen, oder auch schon als Individuen unter gleichartigem Einfluss, in einen Normaltypus zurückgeführt werden können. Ebenso wenig kann, wie wir oben zeigten, in der freien Natur, durch den Einfluss der Bastardbefruchtung von der Bildung neuer Arten die Rede sein.

Angenommen endlich, es könnten in der freien Natur durch lange Angewöhnung aus der Pflanzenart eimige Formen hervorgehen, die sich auch bei unsern Experimenten in den folgenden Generationen als vollständig konstant erweisen würden, was, fragen wir, wäre damit für die Erklärung der Entstehung der Arten gewonnen? Nichts, gar nichts; denn solche Formen bewegen sich noch innerhalb eines sehr engen Kreises, und wemn sie auch in einzelnen Beziehungen eine veränderte Gestaltung der Organe angenommen, so sind damit doch solche in keiner Beziehung einer höhern Entwicklung entg'eg'en gegangen, sondern in dieser Beziehung vollständig geblieben was sie waren.

In Tausenden von Generationen werden in schon geschichtlicher Zeit unsere Getreide kultivirt. Wohl haben solche in dieser Zeit manche ziemlich konstante Racen gebildet, aber sie haben sich doch in dieser Zeit nicht weiter von ihrem Artbegriff entfernt, als dass es vielleicht nur bei einzelnen Racen zweifelhaft bleibt, ob solche von nur einer Art, oder durch zwei mit einander vermischte Arten entstanden sind. Sie weisen also trotz 
der Kultur in Tausenden von Generationen keine Umbildung, sondern sogar umgekehrt ein Verharren der Art im bestimmten Formenkreis nach.

Die Pflanzenart soll aber, nach den auf die Vorgänge der Vorwelt aufgebauten Schlüssen, von den einfachsten Formen durch allmählige Weiterbildung zu den höchst entwickelten Formen sich umgebildet haben. Wohl rechnen wir di nicht mehr nach Tausenden von Generationen, sondern mach Millionen von Generationen. Wenn ich aber mit o potenzire, so erhalte ich auch in Milliarden von Generationen kein Resultat, denn unsere Jetatwelt, unsere bis auf sechs Jahrtausende hinaufreichende Kulturgeschichte einzelner Pflanzenarten, und alles, was ferner vorurtheilsfreie Beobachtung an den Pfanzen der Jetztwelt zusammenstellen kann, enthült nur Belege für die Formbildung der Pflanzenart, aber keinen einzigen Beleg dafür, dass irgend eine Pflanzenart, auch mu in einem kleinen Bruchtheil, einer vollkommeren Stufe der Ausbildung entgegengeführt worden sei, ja wir vermögen es uns nicht einmal zu denken, wie es möglich sei, dass die blïthenlosen Pflunzen dwich eine Uebergangsstufe zu den Phanerogamen übergeführt worden seien.

So in der vollen nur auf Thatsachen ruhenden Ueberzeugung, dass weder die Vorwelt noch die Jetztwelt uns sichere Anhaltspunkte giebt, wie die Pflanzenart entstanden ist, begnügen wix uns damit, schliesslich für dieselbe und deren Formen die folgenden Begriffsbestimmungen zu geben.

Die Pflanzenart und die aus solcher hervorgehenden Formen.

Als PFLANzenaRT (species) unserer Jetztwelt bezeichnen wir einen Gesammtbegriff von Individuen, die unter allen Verhältnissen auch in allen folgenden Generationen von diesem Gesammtbegriff nicht abweichen und nur innerhalb desselben variiren können.

Die Pflanzenart, in dieser Weise definirt, stammt von einem oder auch vielen mit diesem Artbegriff ansgerüsteten Individuen, die auf unsere Jetztwelt herübergekommen 
sind. Dieser Artbegriff umfasst nicht nur den ganzen Entwicklungsgang, den jedes einzelne Individuum vom ausgestreuten Keim bis zu seinem Absterben zu durchlaufen hat, sondern anch seinen imern und äussem Bau, ferner seine Formbildung unter veränderten äussern Einflüssen, und dieser Artbegriff wirkt auch in gleicher stetiger Kraft von der ersten Mutterpflanze, als dem ersten Glied der Kette, bis in die entferntesten Generationen hin. Wenn alle Individuen der Pflanzenart aussterben sollten, ohne neue Generationen zu hinterlassen, so muss mit ihnen auch der Artbegriff einer bestimmten Pflanzenart verloren gehen. Weder die Wanderung der Pflanzenart über einen Theil des Erdballs und die mit solcher veränderten äussern Einflüsse, noch die Kultur der Menschen vermag diesen Artbegriff abzuänderm, denn die Formbildung der Pfinzenart immerhalb bestimmter Gränzen gehört ebenso wesentlich zum Begriff der Art, wie andrerseits auch deren Abhängigkeit von klimatischen Verhältnissen.

Auch in letzterer Beziehung ist vom Artbegriff der Pflanze eine so feste Gränze gezogen, dass während der Erkaltung Europa's zur Diluvialzeit die Pflanzenarten der gemässigt warmen Zonengürtel grossentheils ausstarben, und auch der Mensch mit aller seiner Kunst und allem seinem Scharfsimn diese natürlichen Gränzen für die Verbreitung der Pfianzenart nur um ein weniges durch liünstliche Nachhülfe hinausrücken kann.

Die Pflanzenart, in diesem Simne aufgefisst, ist der Inbegriff aller jener Formen, die solche bei ihrer Wanderung über einen Theil des Erdballs bildete. Dass solche Formen meist als besondere Arten in unsern botanischen Werken beschrieben worden sind, ward schon erwähnt.

Die beschreibende Botanik hat daher, für die grösste Zahl der Fälle, erst noch in der Zukunft die Aufgabe zu lösen, die Pflanzenart aus diesem Gesichtspunkt zu studiren, und alles Zusammengehörige, olme Furcht vor 
Autoritäten, zusammenzufassen. Den für die Wissenschaft gegründeten Gärten wird in zweifelhaften Fällen die ehrenvolle Aufgabe zufillen, zweifelhafte Arten durch Generationen hindurch zu prüfen.

Unterarten (subspecies) werden am geeignetsten jene Endformen einer sehr vielgestaltigen Pflanzenart genannt, die sehr wahrseheinlich ursprünglich verschiedene typische Arten bildeten, jetzt aber in Folge der fortgesetzten Bastardbildung durch zahlllose Mittelformen so vollständig zu einander übergeführt sind, dass solche nur eine vielgestaltige Art zn bilden scheinen. Solche Unterarten sind z. B. von unserer Betula alba, die B. alba typica, B. glutinosa (pubescens), B. papyracea und B. populifolia.

Als Varietäten (varietas) würden alle jene Formen innerhalb des Begriffs einer Pflanzenart gelten, welche bei der Wanderung derselben über einen Theil des Erdballs in Folge veränderter äusserer Einflüsse entstanden sind.

Wir haben oben zu solchen das Beispiel von Paprever alpinum aufgeführt, und gezeigt, dass solche Varietäten unter veränderten Einflüssen, oft schon als Individuum, immer aber in den folgenden Generationen, zu einer bekannten Normalform zurückgehen.

Einzelform (lusus) remen wir alle jene zufällig, ohne nachweisbare besondere Einwirkungen, aus Samen fallenden Einzelpflanzen, die in irgend einer unwesentlichen Richtung, aber doch auffallend von den gewöhnlichen Formen abweichen. Wir namnten oben als Beispiel die Blutbuche; hierher gehören aber auch die Formen mit hängenden Zweigen, nit geschlitzten Blättern, gefüllten und anders gefärbten Blumen, rerschiedengestaltigen Früchten und Knollen etc. Die so entstehende Einzelform besitzt die Eigenschaft als Individum und ebenso in allen durch ungeschlechtliche Fortpflanzung gewonnenen folgenden Generationen, ihren abweichenden Charakter beizubehalten in den aus Samen erzogenen Nachliommen, 
solchen aber bei der grössten Masse zu verlieren. Die Einzelform tritt daher in der freien Natur nur sporadisch auf, und bekommt erst in der Kultur, wie bei unsern Obstsorten, Kartoffeliı ete, eine grössere Bedeutung. durch zahlreiche Nachkommen.

.Racen (stirpes) nemmen wir mit DaRwin alle jene Einzelformen, die durch fortgesetzte Kultur unter unveränderten Verhältnissen, mit der sorgfältigen Auswahl verbuulen, zu einer begränzten Erblichkeit gebracht wurden, d. h. einer Erblichkeit auf die folgenden aus Samen erzeugten Generationen, in der grossen Mehrzahl der Individuen, so lange ähnliche Verhältnisse andanern; aber Zurïckgehen der besondern Charaktere der Race, sobald veränderte Verhältnisse eintreten. Die Race ist also mit anderen Worten die künstliche Varietät der Kultur. Unsere Gemüse, Getreide, viele unserer Sommerblumen des Gartens gaben Beispiele für solche. Die Charaktere der Race sind ähnlich erblich, wie zuweilen Krankheiten in Familien.

Sprossformen (cymatoplasmu). Als sulche bezeichnen wir die Reile jener Formen, die, wie die Mehrzahl der Formen mit bunten Blättern, durch dimorphe Ausbildung eines einzelnen Astes oder Sprosses, an eimem sonst normalen Individuum entstanden sind. Sie spielen nur in der Kultur eine Rolle, wo sie durch ungeschlechtliche Vermehrung und Ausbildung festgehalten werden, aber auch in ähnlicher. Weise, wie sie entstanden, in einzelnen Aesten und Sprossen zurïckschlagen lï̈nnen. Formen mit bunten Blättern, die sich durch Samen fortptimzen (Farne, Barbarea) gehören zu der Racenbildung.

Formen der Entwicklung (varietates regetationis). Als solche wäre die Reihe jener Formen zn bezeichnen, die durch den rerschiedenartigen Gestaltungsprozess der gleichen Pflumenart in den verschiedenen Stadien ihrer Entwicklung entstehen.

Bei den niedrigen Plinzentimilien liommt dieses Ver- 
hältniss häufig vor und ist drort zum Theil mit dem Namen ,Generationswechsel " belegt worden, eine Bezeichnung. die zu vielfachen Missverständnissen geführt hat, und auch von den Anhängern DARwis's als einer der Beweise für DARwin's Exklärung der Entstehung der Pflanzenart gebraucht worden. Darwiv selbst ist aber viel zu umsichtig, um die Entwicklungsformen der Pflanzenart mit unter den Beweisen für seine Ansicht aufzuführen; denn der sogenannte Generationswechsel der niedern Pflanzenarten gehört eben so sehr zu dem Artbegriff, wie die verschiedenen Entwicklungsperioden des monocotyledonen oder dikotyledonen Baumes, oder wie Larve, Puppe und das rollkommene Insekt nur Entwicklungsstadien der gleichen Art bezeichnen.

Nicht bloss bei den niedern Pflanzen, sondern selbst auch bei den höher entwickelten Pflanzenarten sind die Entwicklungsstadien der Art zuweilen als besondere Arten beschrieben worden. So z. B. sind Betula pubescens und glandulosa, im Sinne der meisten Autoren, nur die Entwicklungsformen von $B$, alba glutinosa und $B$. alba typica. In einzelnen wenigen Fällen spielen die Entwicklungsformen auch in unsern Gärten eine Rolle. So z. B. sind die Formen unseres Epheus mit nicht gelappten Blättern, zum Theil wenigstens, aus der ungeschlechtlichen Fortpflanzung der obersten fruchtbaren Aeste aller zur Fruchtbarkeit gelangten Epheupflanzen entstanden.

Bastard (planta hybrida) ist das Mischlingsprodukt zweier Arten. Wir haben uns oben über das Wesen des Bastardes und seine Eigenschaften schon genügend ausgesprochen.

Mischlinge (miscellus) sind die aus Vermischung des fruchtbaren Bastards mit den elterlichen oder andern Arten, oder endlich aus Selbstbefruchtung des fruchtbaren Bastards entstehenden fernern Formen.

Als Mischlingsracen (stirpes miscellaneae) wären endlich alle jene Mischformen za bezeichnen, die durch Aus- 
wahl und fortgesetzte Kultur die bedingte Erblichkeit der Racen erhalten haben."

M. Fée, président, dit qu'il lui semble que l'espèce oscille, que les formes de l'espèce se modifient, mais, sans que les formes essentielles varient, jamais une espèce n'envahit une autre. L'espèce elle-même est immuable, mais il existe des modifications qui ont été décrites comme espèces; de là les différentes opinions sur la valeur de l'espèce.

II. M. de Nordmann (de Helsingförs en Finlande) ayant obtenu la parole, discute en allemand sur les mérites $d u$ professeur Christian Steven, le Nestor des botanistes. Il dépose le livre qu'il a écrit sur ce sujet.

III. M. Coster (d'Amsterdam) adresse à la section la communication suivante:

Sur la métamorphose des plantes.

\section{Messieurs!}

Nous respectons tous les idées d'un Linné, d'un Goethe, d'un de Candolle, etc. sur la métamorphose. Cependant il peut sembler utile d'examiner, si de nos jours, tant de scrutateurs ayant étudié le développement des organes, il y a encore lieu d'admettre cette théorie. D'après les résultats de la science moderne, il y a lieu de diviser les organes de presque toutes les plantes vasculaires en axiles et en foliacés. Nous savons tous pourquoi: le mode de développement indique la nature axile ou foliacée d'un organe.

Pour nous borner aux organes foliacés (les mêmes observations s'appliquent aux organes axiles), la théorie veut par exemple que les bractées, les périanthes, les étamines, les feuilles carpellaires soient, dans l'ordre où je viens de les nommer, des organes supérieurs les uns aux autres 
et des transformations de feuilles caulinaires. Or, je le demande MM., quel est donc le type de la feuille caulinaire? On pourrait me répondre: chaque espèce de plante a le sien propre. Mais seraient-ce done les feuilles cotylédonaires, radicales ou caulinaires stricto sensu, qu'on choisira pour type? On pourrait s'entendre là-dessus en se figurant un type moyen de ces trois diversités. Soit! mais j'ai deux objections à faire:

D'abord beaucoup de plantes dont les feuilles caulinaires ont des dimensions très étendues, produisent des fleurs dont le périgone ou le périanthe, ou plutôt chaque feuille de ce verticille, est relativement très-petit, très simplement organisé, si on le compare aux vastes feuilles caulinaires. Cependant la théorie y voit un organe foliacé d'un ordre supérieur à celui de la feuille caulinaire, dont il serait une transformation. Combien d'exemples ne pourrais-je pas vous citer de calices à peine visibles chez des plantes, dont les feuilles caulinaires ont d'énormes dimensions ou une structure beancoup plus compliquée. Néanmoins on continue à décrire les calices comme des verticilles de feuilles caulinaires transformées et d'un rang beaucoup supérieur au rang de celles-ci. Mais, MM. y a-t-il des raisons suffisantes pour adhérer encore à cette théorie? Pour cela, il nous faudrait un critérium afin de distinguer les ordres; - mais, où le trouver? Quant à moi, e'est en vain que je le cherche, soit dans la diversité des formes, soit dans la structure, soit même dans les fonctions physiologiques. Le seul fait que je vois, c'est que, par exemple, les calices, comme les autres parties de la fleur, s'épanouissent ordinairement plus tard que les feuilles caulinaires. Mais est-ce-lì une raison pour y voir des organes d'un ordre supérieur? Je n'hésite nullement à répondre négativement.

Laissons de côté la question, si nous avons le droit ou non d'adopter une métamorphose progressive, quand nous avons affaire à des parties florales; posons tout simplement, 
que les parties de la fleur sont des feuilles caulinaires transformées, soit même en ordre rétrograde. Eh bien, MM., et voici ma seconde objection, qui de vous a observé et pu constater la métamorphose, c'est-à-dire le changement d'une forme en une autre chez les organes d'une plante? Très souvent, dans les premières périodes du développement des organes, leur forme se prononce clairement et reste la même, sauf l'accroissement des dimensions à un âge plus avancé des mêmes organes. Mais, me direz vous, où sont done les monstruosités, les fleurs doubles ou pleines, les corolles caliciées, les étamines entièrement ou partiellement transformées en pétales, les carpelles foliacés, etc.? Mais ces mêmes étamines avaient-elles donc, à leur origine, une autre forme que celle qu'elles ont eu plus tard? Ces carpelles ne montraient-ils pas de bonne heure ce qu'ils deviendraient, ou plutôt ce qu'ils seraient un jour? Je sens que l'admission d'une métamorphose rétrograde est alors un moyen très simple pour expliquer ces monstruosités. Mais j'ose avancer que, tant que l'on n'aura pas observé la transformation même, on ne sera pas autorisé à l'admettre.

Regardons donc les faits en face. De temps à autre (et chẹ quelques plantes même très-fréquemment) nous voyons à l'endroit où l'on aurait attendu, selon la règle commune, un organe quelconque, se montrer un organe sous une forme, ressemblant absolument ou en partie à celle d'un autre organe. Et voilà tout! Vous comprenez bien qu'il y a là une différence très-marquée: un organe, dont la forme est semblable à celle d'un autre, est autre chose qu'un organe transformé, e'est-à-dire, qui aurait parcouru (selon la théorie de la métamorphose) les formes de divers organes.

N'allez pas croire cependant, que je n'admets la transformation sous aucun rapport. Il y a des cas où le fait est prouvé par l'observation. Comparez, par exemple, entre elles les feuilles caulinaires, qui finiront par être 
des feuilles pinnées ou palmées. Dans la première période de leur existence, le bourgeon étant très jeune, elles se ressemblent tout-à-fait. La forme pinnée ou palmée est done une vraie métamorphose de la forme primitive du même organe. Souvent les très-jeunes pétales et les étamines du même âge se ressemblent; la forme que ces parties auront plus tard, est une métamorphose de cette forme primitive dans l'alabastre. Mais dans nos fleurs doubles ou pleines, où l'on trouve des pétales à l'endroit ordinairement réservé aux étamines, il n'y a jamais eu d'étamine; dès le commencement il s'y développait des pétales et rien que des pétales. Pourquoi done les nommer étamines pétalisées? Ce sont (qu'on me permette cette expression) des pétales pétalisées dans le verticille, où l'on aurait attendu des étamines. Je veux même passer sous silence les fleurs, pourvues d'un nombre multiple de pétales, quoique le nombre d'étamines ne soit pas diminué. Les adhérents à la théorie de la métamorphose sauront trouver, même dans ces cas, des argumens spécieux à l'appui de leur opinion; quant à moi, je n'aime pas à avoir recours, pour expliquer les phénomènes de la vie végétale, à une théorie facile, il est vrai, mais non constatée par l'observation. Résumons, MM.!

1. On n'est pas fondé à donner aux verticilles floraux le nom de feuilles caulinaires transformées.

2. L'apparition ultérieure d'un organe ne justifie pas le tître d'organe supérieur.

3. Un organe, se présentant sous une forme inattendue, n'est pas pour cela transformé.

4. Pour admettre une métamorphose, il faut l'avoir vu se développer successivement."

Après la lecture de cette note, l'orateur rappelle la théorie analogue, qui prétend que beaucoup des organes des animaux supérieurs seraient des vertébres métamorphosés. Le bon sens a déjà jugé, ou plutôt condamné cette théorie." 
M. Fée ne trouve pas sérieuses les objections présentées par M. Coster contre la théorie de la métamorphose des plantes de Goethe. Entrevue par Wolff de Saint Pétersbourg, trouvée simultanément à Genève et à Gotha, elle s'est fortifiée par les travaux d'Aubert-DU perit Thouars, de Gaudichaud et de Turpin; il semble qu'elle est dans ses principales bâses tout-à-fait inébranlable. M. Coster cherche le point de départ ; il est dans l'embryon et les feuilles cotylédommaires. Tout commence par la feuille et tout y revient. La même force d'érolution qui, dans des circonstances domnées, produit une feuille, peut dans d'autres circonstances produire un sépale, un pétale, une étamine, un carpelle et enfin une graine. En suivant de près toutes les phases de développement de la plante, il devient facile de se convaincre que tous les organes axiles dérivent les uns des autres. M. FÉE, en terminant, exprime le regret de roir juger en si peu de mots et d'une manière si absolue les travaux de Geofrror St. Hruarre sur l'identité des parties osseuses du squelette des vertébrés, aperçus ingénieux qui ont valu à l'illustre auteur une célébrité si bien justifiée.

M. Morren dit que e'était tout naturellement au vénérable et savant président de l'assemblée qu'il appartenait de dégager le génie de Goztre des langes dont on semblait vouloir l'entourer. M. Martins a publié, en France, une belle édition des oeuvres scientifiques du poète de Weimar, dont il a ainsi popularisé les grandes conceptions. Cependant M. Morren ne peut s'empecher de joindre sa faible voix à l'autorité de M. FÉE, pour faire remarquer que la théorie de Goethe peut être mal comprise, mais ne saurait être ébranlée. Il ne s'agit pas de saroir si les organes floraux ont été feuilles dans leur enfance, mais bien de ramener tous les organes des végétaux phanérogames ì deux types: l'axe et le phylle $\left(\boldsymbol{\varphi}\left(\lambda_{0} \boldsymbol{v}\right)\right.$, et de reconnaitre l'existence de deux types seulement, la tige et la feuille, dans cette prodigieuse di- 
versité apparente des organes végétaux; de proclamer ce principe, que la forme et même la fonction ont peu ou point d'importance morphologique, que l'insertion détermine seule la nature d'm organe. Les organes axiles présentent un ensemble de caractères, tels que leur situation terminale ou axile, leur formation au moyen de faisceaux fibro-vasculaires indépendants, leur développement centrifuge, leur accroissement et leur durée, leur faculté de domner naissance aux organes appendiculaires, etc. Les organes appendiculaires, au contraire, sont infraaxillaires, se forment par de simples embranchements des faisceaux préexistants, se déreloppent en geénéral suivant un mode centripète, ont un accroissement défini et une durée limitée, n'émettent jamais d'autres organes etc. Mais suivant leur insertion et leurs rapports ces organes sont nommés cotylédons, feuilles, bractées, sépales, pétales, étamines ou carpelles. La métamorphose est dans la pensée, elle n'est pas dans la matière.

M. Masters (de Londres) fait observer qu'en Angleterre on se sert généralement du mot substitution au lieu de métamorphose. On admet que l'organe théorique, le type n'existe pas.

IV. M. Willkomm (de Tharand) traite la question annoncée par lui (voir Progr. pag. 22, No. 18, 2), en prononçant le discours suivant:

Sur le Chêne-liége de l'occident (Quercus occidentalis Gay) et sa distribution géographique en Espagne.

„C'était en 1857 que feu. M. Gax attira le premier les regards des botanistes français sur un fait bien remarquable, en découvrant qu'on avait confondu jusqu'alors sous le nom du Quercus Suber deux espèces différentes du genre Quercus. Dans l'une de ces deux espèces la maturation des glands est ammelle, dans l'autre au contraire 
bisannuelle. La première espèce est le vrai Quercus Suber L., elle est répandue sur une grande partie du bassin de la Méditerranée; la seconde reçut de M. Gar le nom de Quercus occidentalis, pour la raison qu'elle n'avait été observée que dans l'occident de l'Europe, saroir dans les landes de Bayonne et près de Cintra en Portugal. Dans la notice, que M. GAr domne sur cette curieuse espèce dans le bulletin de la Société botanique de France (IV, 1857, p. 449), i] dit qu'on ]a retronverait probablement sur toute la côte intermédiaire de l'Espagne et du Portugal. Cette supposition de feu M. GAr a été confirmée d'une manière positive par un naturaliste allemand, qui m'a communiqué des rameaux en fleurs de cet arbre, provenant de la province de Santander. C'est. M. Schaufuss de Dresde qui, en 1860 , fit un royage au nord de l'Espagne, pour r collecter des insectes et autres animaux. Il existe dans les montagnes de la province de Santander un canton, appelé la Liébana. Sur les pentes inférieures des hautes montagnes, dites Picos de Europa, est située la vallée de Potes, chef-lieu du canton, où M. Schaufuss observa des forêts entières d'un chêne-liége, qu'exploitait à cette époque un Français établi ì Potes. Celui-ci avait assuré à M. Schaufuss que le chêne de Potes était identique avec celvi des Landes. En effet les rameaux fleuris, que j'ai reçus de M. Schaufuss, diffèrent notablement par la forme des feuilles, par la disposition des châtons et surtout par la structure des fleurs mâles du chêne-liége du midi et sont parfaitement conformes à la description du Quercus occidentalis, que M. Mathieu donne dans sa Flore forestière de la France. Je regrette virement de n'avoir pas reçu des rameaux fructifères, mais déjà les feuilles et surtout les fleurs mâles offrent assez de différences, comparées avec celles du Quercus Suber. M. GAY n'ayant pu étudier les châtons mâles, il me paraît assez important de domner ici une description exacte de ces organes. 
Quercus Suber L.

Folioles du périgone ordinairement 5 , orbiculaires, très concaves, presque cuculliformes, tomenteuses sur le dos et sur les bords.

Etamines ordinairement 5 , à filets à peine une fois plus longs que le périgone, à anthères globuleuses, en coeur à la base, émarginées au sommet.
Quercus occidentalis GAY.

Folioles du périgone ordimairement 7 , de la même forme, mais entièrement glabres.

Etamines ordinairement 7 , à filets $1 \frac{1}{2}-2$ fois plus longs que le périgone, à anthères ellipsoïdes, en coeur ou bipartites à la base, apiculées au sommet.

A ces notions sur les fleurs du Quercus occidentalis, je crois devoir joindre l'indicution des principaux caractères qui distinguent cette espèce du Quercus Suber. Les plus remarquables sont $1^{\circ}$. la disposition des fruits mûrs, et 20. la structure de la cupule. Dans le Quercus Suber les glands se trouvent ì l'extrémité des rameaux feuillés, dans le Quercus occidentalis on les trouve sur les rameaux défeuillés de l'année précédente, surmontés par les pousses feuillées, provenunt des bourgeons de ces mêmes rameaux qui portent les fiuits. Le Quereus Suber a la cupule allongée, conique inférieurement, à écailles toutes appliquées; dans le Quercus occidentalis la cupule est hémisphérique, à écailles supérieures dressées et appliquées, tandis que les écailles infériemres sont coniques et réfléchies.

De plus la durée des feuilles du Quercus occidentalis est à peine anmuelle, tandis que celles du Quercus Suber persistent 2-3 ans. Quint is la forme des feuilles, elles varient autant dans le Quercus occidentalis, que dans le vrai Chêne-liégre. On en rencontre sur les deux espèces à bords entiers et dentés, inermes et épineux. Je ne sais pas, s'il y a des différences quant à la croissince et à la qualité du liégre. Senlement je puis constater, que les conches du liégre ne sont jamais aussi épaisses dans le Quercus occidentulis, que sur des vieux trones du Quercus 
Suber. Enfin le Quercus occidentalis, au moins l'arbre des Landes, a la cime pyramidale-conique; c'est un arbre de taille moyenne, tandis que le Quercus Suber est un arbre souvent majestueux, au moins en Espagne, à cime trèsample et très-irrégulière.

Revenons maintenant à la distribution géographique du.Quercus occidentulis en Espagne. Déjà en 1850, au mois de mai, j’ai vu moi-même, en allant en diligence de St. Sebastien à Bilbao, dans le voisinage de la ville de Durango, des pieds d'un chêne-liégge entremêlés aux chênes (Quercus pubescens W.) qui forment les forêts de cette contrée. Ces arbres avaient tout-ì-fait le port des chênesliége que j’avais observés quinze jours auparavant dans les Landes. Je ne doute done pas que l'arbre de Durangone ne soit identique avec celui de la Liébana. M. PAstor, ingénieur de forêts espagnol, indique aussi le chêneliége dans un mémoire qu’il a publié sur les Asturies, et M. Planeluas, auteur d'une Flore de la Galicie (Ensayo de une Flora fanerogama gallega) dit, que l'alcornoque, c'est le chêne-liége, est très-fréquent dans les bois de cette partie de l'Espagne. Le Quercus occidentalis croissant encore près de Cintra en Portugal, je suis bien persuadé que le chêne-liége de la Galicie et des Asturies appartient aussi à l'espèce décrite par M. GAY. Par conséquent cette curieuse espèce occupe un assez grand espace, car elle s'étend depuis les landes situées entre la Gironde et l'Adour, le long du Golfe de Gascogne et de la côte septentrionale de l'Espagne jusqu'à l'embonchure du Tage sur la côte occidentale de la péninsule ibérique, c'est-à-dire depuis le $44^{\circ}$ jusqu'au de là du $39^{\circ} \mathrm{L}$. N., formant dans la dịection du NE. au SO. une zône arquée de plus de $9^{\circ}$ de longitude.

Contemplons encore le climat des contrées, où croit le - Quercus occidentalis. Suivant M. PAstor la température moyenne de l'aunée est 1\%。C., dans la pleine d'Oviédo, c'est-à-dire dans la zône centrale des Asturies à environ 100 mètres d'élévation au-dessus du niveau de l'Océan At- 
lantique. La température moyeme de l'hiver est de 7 à 8 , celle du printemps 12 ì $13^{\circ}$, celle de l'été 18 à $20^{\circ}$, celle de l'automne 11 à $144^{\circ}$. En hiver le mercure descend quelquefois jusqu'à $4^{n}$ an dessous de zéro; en été il s'élève rarement jusqu'à 31․ En 1859 la température moyenne de l'amée était $12^{\circ}, 7$, la plus haute $32 \circ, 9$, la plus basse $5^{\circ}$, 2. Dans la même année, il y avait à Bilbao une température moyenne de $18,9,9$, un maximum de $35^{\circ}, 5$, , un minimum de $3 \circ, 9$; à Santiago de Compostella une température moyeme de $13^{\circ}, 1$, un maximum de $39^{\circ}, 0$, un minimum de $2^{\circ}, 0$. La température moyenne de l'hiver était à Bilbao $7^{\circ}, 7$, à Santiago $7^{\circ}, 9$, celle du printemps à Bilbao $13^{\circ}, 7$, à Santiago $11^{\circ}, 7$, celle de l'été à Bilbao 210,8, à Santiago $18^{\circ}, 6$, celle de l'antomne ì Bilbao $15^{\circ}, 8$, à Santiago $14^{\circ}, 3$. Dans la méme année la quantité totale de la pluie importait à Oviédo 2614 millim., à Bilbao 1195,7 millim., à Santiago 1453,7 millim. Dans la capitale des Asturies il y avait 151 jours pluvieux, dans celle de la Biscaye 134, dans celle de la Galicie 175. Ces domnées méteorologiques du Nord de l'Espagne sont bien d'accord avec celles du climat de Dax en France (températ. moyenne de l'année $13,66^{\circ} \mathrm{C}$., de l'hiver 6,70 , du printemps 13,58 , de l'été 20,34, de l'automne 13,89; seulement la quantité de l'eau annuelle est bien inférieure, 625 millim.).

Mais le Quercus occidentalis prospère encore dans un climat beancoup moins doux. Suivant M. GAY cet arbre fleurit et fructifie à Trianon, où l'on en voit en pleine terre un pied agé qui mesure 14,5 mèt. de hanteur, sur 1,45 de circonférence du tronc à la base. Le chêne-liége du midi ne peut pas végéter en pleine terre sous le climat de Versailles; le Quercus occidentulis est done benucoup plus rustique que le Quercus Suber. Or, je crois que dans le canton de la Liébana, sur les pentes des Pics de l'Europe couromnés de neige presque toute l'année, à une hauteur. d'environ 700 mèt., le climat doit ĉtre moins doux qu'au Trianon. Par conséquent il me parait assez probable qu'il 
soit possible d'acclimater cet arbre utile dans le nord de la France, en Belgique et peut-être aussi dans le midi des Pays-Bas. Il serait du plus haut intérêt pour la botanique comme pour le monde forestier que messieurs les directeurs des jardins botaniques belges et néerlandais fissent des semis et des plantations du Quercus occidentalis. Mais je crois qu'il serait plus sûr encore de faire venir les glands du canton de la Liébana; car assurément l'arbre des montagnes de cette contrée est plus rustique que celui des landes de Bayonne."

V. M. Münter (de Greifswald) ayant obtenu la parole, communique à la section des observations sur les maladies des plantes (1).

Cette communication, appuyée de faits propres à l'éclairer, intéresse vivement la réunion. Elle est suivie de développements donnés par MM. Münter, Fée, Mrquel, Morren, ete.

VI. M. Morren (de Liège) communique ses remarques sur la Panachure et la Duplicature, question annoncée par lui (voir Progr. pag. 27, No. 20).

M. Morren rappelle que dams une note qu'il a récemment communiquée à l'académie royale des sciences de Belgique (2), sur l'Hérédité de la panachure, il a fait connaître que dans son opinion la panachure du feuillage (variegatio) et la duplicature des fleurs constituaient deux phénomènes respectivement exclusifs l'un de l'autre. En d'autres termes, qu'il n'existe pas de plantes à feuilles

(1) Nous regrettons vivement, que M. Mü̈TER n'ait pu jusqu'à présent nous faire parvenir le résumé de son intéressant discours. Dans le cas où il viendrait encore à temps pour être mis sous presse, nous le publierons sous forme de supplément.

RED.

(2) Voyez Bullet. de l'Acad. roy. des sciences de Belg., 34e année, 2c série, tome 19 (1862) p. 224. 
panachées portant des fleur's duubles. Dans cette note il a cité les quelques exceptions qu'il croyait exister à cette loi de physiologie végétale. La plus importante était celle du Kerria Japonica qui, dans nos jardins, donne toujours des fleurs doubles, et dont M. von Siebold a récemment importé une variété à feuilles panachées (var. foliis argenteis variegatis). MM. Lemaire et Амв. Verschaffelt en décrivant et en figurant cette nouvelle race dans l'Illustration Horticole (1) lui attribuent des fleurs doubles. Mais M. Morren montre à la section, d'après des spécimens vivants et flemis qui viemment de lui être transmis avec la phis grande obligeance par M. Krelage d'Harlem, que les redacteurs de l'Illustration Horticole ont été induit en erreur et qu'en realité le Kerria Japonica à feuilles panachées a des fleurs simples. Une antre variété caulibus aureo-striatis, dont les tiges seules sont striées de panachure, est dans le même cas. Cette plante, loin d'être une exception au principe avancé par M. Morren, en devient donc une des plus remarquables confirmations.

Il ajoute que l'examen judicieux des autres exceptions qu'il avait cru devoir consigner dans sa première notice, conduit à un même résultat. Ainsi l'Amaryllis Kwanso à fleurs ordinairement doubles ne montre qu'une panachure fort instable et rentre souvent, comme disent les jardiniers. Quant aux Camellias et aux tulipes doubles, c'est plutôt une chlorose accidentelle qui peut atteindre leur feuillage qu'une véritable panachure. On pourrait encore lui opyoser l'Aesculus Hippocastamun, dont on dit qu'il existeunevariété à fleurs pleines et à feuillage panaché, mais M. Morren ne croit pas que cette panachure soit fixée. En terminant il dit qu'il ne croit pas devoir, devant un anditoire aussi compétent, s'arrêter à la distinction fondamentale qui existe entre les feuillages panachés et les feuillages colorés (folia variegata et folia colorata).

(1) Voir l'Illustration IIorticole, 1862, tome 1X, tab. 336. 
Il croit à peine nécessaire de faire voir que le principe qu'il vient d'établir d'après les faits, est en tous points conforme aux lois générales de la physiologie végétale, d'après lesquelles la panachure doit être considérée comme une affection pathologique du système de nutrition, tandis que la duplicature des fleurs est un phénomène de pléthore, et qu'ainsi il est tout naturel que ces deux phénomènes soient exclusifs l'un de l'autre.

Enfin il attire l'attention sur l'utilité qu'il y aurait à dresser le catalogue des fleur's doubles connues en horticulture. M. le Dr. Seeman a pris l'initiative en ce travail dans son Joumal of Botany, et lui-même s'y est associé dans sa Belgique horticole.

VII. M. le Président dome lecture d'une lettre de M. de Cannart d'Hanale, Vice-président de la $2^{\mathrm{e}}$ section, transmettant le voeu, exprimé par M. le Prof. Косн en ces mots:

„In der Gartenbau-Section ist der Beschluss gefasst worden, die Herren Botaniker zu bitten, sich zu erklären, welche Familien sie besonders bearbeiten und desshalb in diesen am meisten vertraut sind, damit Gärtner, welche nene Pflanzen eingefüht haben, sich mit ihnen in Verbindung setzen können, um dieVerzeichnisserichtiger zu haben.

Mim. Fenzl, Miquel et autres font remarquer que les persomes qui s'occupent spécialement de certaines familles, sont bien connues.

VIII. M. Fée, président, remereie les membres de la section des témoignages de contiance qu'il a reçus d'eux, ainsi que de la facilité avec laquelle ils Jui ont permis de remplir sa tâche, ag'réable et nullement difficile. Il se félicite d'avoir présidé des séances toujours bien remplies, aussi remarquables par l'importance des communications 
qui ont été faites que par la manière cordiale dont elles ont été accueillies et discutées.

M. Miquel adresse au nom des membres de la section des remerciments à M. Le Président, qui a si bien réglé les discussions et dirigé les débats.

Le procès-verbal, lu séance tenante par M. Morren, est adopté.

La séance est levée à midi. 


\section{RESUME}

\section{DU COMPTE-RENDU DES SÉANCES DE LA SECTION DE BOIANIQUE PURE.}

SEANCE du 7 Avril 1865, à 4 heures de relevée.

La séance est ouverte par M. le professeur Surivgar (de Leide)

T'ag.

Constitutiou du Burean.

46.

46.

La Section adopte le projet de règlement qui lui est soumis par la conlmission organisatrice et règle l'ordre de ses séances

M. Morken (de Liége) fait hommage au Congrès de quelques opuscules de sa composition

La séance est levée et remise au lendemain à 9 heures du matin......

46.

SÉANCE du 8 Avril 1865, à 9 heures du matin.

\section{Présidence de M. FÉE.}

Ouverture de la séance

Le procès-verbal de la séance précedente, lu par M. MoRren secrétaire, est approuvé.

II. L. Bovcrianv (de Paris) fait lommage au Congrès d'unde ses opuscules.

MI. le Président fait une proposition relative à l'ordre des questions ì traiter. L'assemblée la ratifie.

M. Lecoq (de Clermont-Ferrand) désire que l'article du règlement, n'accordant cu'un quart d'heure à chaque orateur, ne soit pas suivi à la lettre.

La question, proposée par M Benioma (No. I du Programme), est mise à l'ordre du jour 
M. LECOQ fait connaître ses idées sur ce sujet

M. Hofemain (de Giessen) et M. TÉE (de Strasbourg) y ajoutent quelques observations

M. Karsten (de Berlin) présente des observations sur la 2de question de M. BЕLHотме

49.

mis. Miquel, Morrex, Lecoq, Chatin et F'ée discutent sur l'opinion émise par l'orateur.

La 3e question du Programme est mise à l'ordre: MI. Lemaire (de Gand) qui l'a annoncée, la développe en déposant une notice, intitulée: „Critiques raisonnées et réfutations de la théorie des boyaux polliniques." .........................

MM. Hoffmava, Miqued, FéE et autres déclarent que ce que l'orateur propose, ne leur parait pas aumissible

M. Cirativ (de Paris) donue un exposé de ses recherches sur „la structure et les fonctions du tissu de l'anthère." (Voir Supplément)

M. Reichenbacu (de IIambourg) adresse une question à ce sujet ì M. Cilatin

M. Passerini (de Parme) discute ,sur l'émission de l'acide carbonique par les racines des plantes et sur la décomposition du même acide par les parties vertes."

MM. Hoffmann, Ciratin et Lecoq y ąoutent quelques observations... 61 et62.

M. Reichenbacir, entretient la section sur la différence entre , ,le Selenipedium caudatum et l'Uropedium Lindenii." . ...............................

II. Caspary (de Königsberg) traite la question qu'il avait posée, "sur les hybrides obtenus par la greffe."

M. Morren ne saurait se rallier à la théorie, développée par l'orateur. M. Meissner (de Bále) voudrait des expériences plus nombreuses et plus variées. M. REGEL fait plusieurs observations se rattachant au discours de M. CASPARY; M. LECOQ signale un fait concernant la question

M. OUdemans lit sa notice, , sur l'origine des stomates dans quelques espèces d'Aneimia."...

Liz parole est accord́́e à M. PAsserini qui diseute "sur la fonction des stomates."

MI. Morriex communique ses expériences sur ce sujet. M. Wesmael (de Mons) confirme les conclusions de M. Moniex

M. II Asskarl (de Clèves) présente ses observations "sur les Commelinicées."

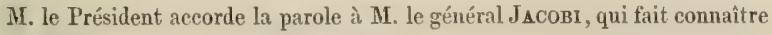
le principe de sa , classification des Agavées."

108.

M. Entz-Ferenez (de Bude) fait une observation sur le uom de , chondracanthae," appliqué à des plantes ......

M. Corn (de Breslau) présente ses observations sur "la culture des Álgues marines."

MiM. Reichenbach, Münter et REgEI font quelques réflexions, auxquelles l'orateur répond 
II. FÉE entretient la section de faits relatifs aux secrétions des Fougères et dépose un mémoire sur ce sujet, intitulé: „Quelques particularités relatives à la famille des Fougères."

130.

La séance est levée à 1 heure. La seconde séance est fixée à $3 \frac{1}{4}$ heures de l'après-midi

\section{SEANCE du 8 Avril 1865, à $3 \frac{1}{4}$ heures de relevée.}

\section{Présidence de M. FÉE.}

Ouverture de la séance 138.

Le procès-verbal de la séance du matin, lu par M. MorREN, est adopté.

M. Wesmael (de Mons) fait hommage au Congrès de quelques-uns de ses opuscules

M. Oudemans traite la seconde question annoncée par lui, et donne lecture d'une notice: „Sur l'origine des spores dans certaines espèces de Mucor.”

MM. ScirvizLein et Surivgar combattent l'opinion émise par l'orateur. 139 et 140.

La parole est accordée à M. SURrvgar, qui présente le resumé d'un travail sur ,la Sarcina ventriculi."

M. Miqueu fait une observation sur la monstruosité de la digitale ......

M. VAN Hulle (de Gand) fait hommage de quelques-uns de ses opuscules et développe la seconde question annoncée par lui; il dépose sur le bureau sa notice sur ce sujet, intitulée: „La mission des jardins botaniques." "...........

MiM. Fée, Morren, Lecoq, Miquel, Regel et Hoffmann discutent sur les idées émises par M. Var Hulde ..................................... des plantes."

MM. Chatin et RAUWENHoff y ajoutent quelques observations.

M. le Président annonce que M. NARDY ainé, à Lyon, a présenté un mémoire intitulé: „la lune et le règne végétal.” (Voir Supplément)

\section{SEANCE du 10 Avril 1865, à 10 heures du matin.}

\section{Présidence de M. FÉE.}

Ouverture de la séance 
M. REGEL prononce un discours allemand sur „la valeur de l'espèce."...

MI. FÉE émet son opinion à l'égard de l'espèce

159.

M. DE NoRdravy (de Helsingförs) discute sur les mérites du professeur Cirr. Steven. Il dépose le livre qu'il a écrit sur ce sujet

198.

198.

M. Coster (d'Amsterdam) adresse ì la section une communication sur „la métamorphose des plantes."

198.

MM. FÉE et Morren combattent les idées produites par l'orateur. M. Masters (de Londres) fait une observation sur ce sujet

M. Wilckomm (de Tharand) lit sa notice sur „le Chêne-liége de l'Occident et sa distribution géographique en Espagne.".

203.

M. Müvter (de Greifswald) fait des observations sur „les maladies des plantes.".

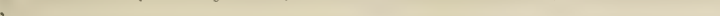

Une conversation s'engage à ce sujet entre MM. Müvter, Fée, Miquel, Morrex, ete .

208.

208.

M. Morrex communique ses remarques sur ,la panachure et la duplicature."

208.

M. le Président donne lecture d'une proposition de la part de M. le professeur Kocir concernant la classification de plantes nourelles

M. FÉE, président, remercie l'assemblée de la confauce dont elle l'a honorée

M. Mrquel adresse des remarcîments à M. le Président au nom des membres de la section

Le procès-verbal, lu par M. Monren, est adopté ..................... 


\section{SECTION DE BOTANIQUE}

\section{APPLIQUEE ET D'HORTICULTURE.}

SÉANCE du 7 Avril 1865, à 4 heures de relevée.

La séance est ouverte par M. le docteur RAUWEnhoff, Secrétaire général de la commission directrice.

Après l'échange de quelques observations de la part de MM. de Cannart d'Hamale, Tisserand, van Hulle, RAUWenhofF et autres, sont nommés par acclamation:

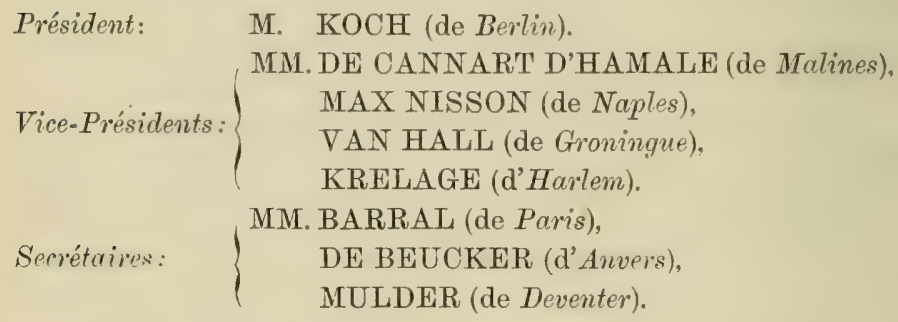

Après quelques objections de la part de MM. vas HaLL et DE BEUcker, ces messieurs acceptent la nomination.

M. Косн étant absent, M. de Cannart d'Hamale, 1 er Vice-président, occupe le fauteuil de la Présidence.

M. de Beucker fait observer qu’il serait désirabie que le procès-verbal fût rédigé en hollandais et en français (1). Il est résolu que M. G. Francken, d'Amsterdam, fera partie du bureau en qualité de secrétaire-adjoint.

M. de Cannart d'Hamale prend la parole pour remereier l'assemblée du vote qu'elle a émis en sa faveur.

(1) Nous n'avons pu, ì notre regret, nous conformer au voeu exprimé par M. DE BEUCKER, le congrès ayant résolu que la rédaction du procis-verbal se ferait en français. (Voir l'art. 10 du règlement, adopté par les deux sections.) 
Il espère que M. Косн acceptera la présidence. M. Косн a rendu les plus grands services ì la botanique appliquée et à l'horticulture. (Applaudissements).

La section règle l'ordre de ses séances. Après quelques discussions auxquelles prennent part, outre M. le Président, MM. van Hall, van Hulle, Overeynder, Tisserand, de Beucher, Krelage ete, elle artête que la question annoncée par M. VAN HuLte, ,sur la taille des arbres firuitiers," sera abordée la première, afin que le sujet puisse se truiter ì fond, et que la théorie soit suivie immédiatement de la démonstration pratique. A cet effet on aura soin de faire apporter à la section quelques exemplaires d'arbres fruitiers.

M. Nedzelsky désire que la question, qu'il a posée sur les progrès de l'arboriculture fruitière et de la pomologie théorique, soit traitée après celle qui a été présentée par M. van Hulle.

M. le Président fait observer que l'ordre du jour pourra être arrêté demain.

La séance est close à 5 heures pour être reprise le lendemain à 9 heures du matin. 


\section{SECTION DE BOTANIQUE}

\section{APPLIQUEE ET D'HORTICULTURE.}

SÉANCE du 8 Avril, à 9 heures du matin.

LE BUREAU SE COMPOSE DE:

MM. KOCH, Président.

$\left.\begin{array}{l}\text { DE CANNART D'HAMALE, } \\ \text { VAN HALL et KRELAGE, }\end{array}\right\}$ Vice-Présidents.

BARRAL, DE BEUCKER et MULDER, Secrétairts.

FRANCKEN, Secrétaire-adjoint.

M. de Cannart d'Hamale ourre la séance.

Le procès-verbal de la séance précédente, lu par M. Francken, secrétaire-adjoint, est approuvé.

M. De Cannart D'Hamale cède le fauteuil à M. Koch qui déclare accepter la Présidence.

Il est donné lecture d'une lettre concernant la nomination des Vice-présidents du Congrès.

M. de Cannart d'Hamale, Vice-président, donne lecture d'mue lettre de M. C. BrIDes, propriétaire à Gaimont (Genève), écrite dans le but de provoquer une discussion sur la question suivante:

L'Igname de la Chine (Dioscorea Batatas) est-elle une plante dont la culture doive être recommandée?

I. D'après l'ordre du jour la première question à traiter est celle posée par M. van Hulle (de Gand) concernant les systèmes de taille d'arbres fruitiers.

L'orateur s'exprime de la manière suivante:

Messieurs,

Vous connaissez la question sur laquelle j'ai demandé à appeler votre attention. Elle est relative à l'arboriculture fruitière et notamment à la taille. 
Si ce ne sont pas les Français qui ont inventé le système de la taille raisonńe, ce sont eux du moins qui ont porté le plus loin ses perfectionnements; c'est à eux que les Belges ont pris l'exemple qu'ils mettent en pratique depuis quelques années. Ils le font avec tant de succès que celui qui vous parle et qui, après feu M. DE Bavay fils, a été le premier chargé d'enseigner publiquement, en Belgique, les meilleurs systèmes de taille, a cru de son devoir de saisir l'occasion que lui offre ce Congrès, pour exposer aux nations ici représentées, ce qu'on entend par taille raisonnée. Je ne la prétends pas exclusivement supérieure aux autres, mais je me demande pourquoi ce qui est bon en France et en Belgique, ne vaudrait rien en Angleterre, en Allemagne et chez les Hollandais, nos roisins immédiats. Dans ces pays, en effet, la taille raisonnée n'est pas en vigneur. Elle n'y paraît être même que très peu connue.

Comme je ne désire pas parler maintenant pour les Français ni pour les Belges qui comprennent la langue française - auxquels du reste je n'ai rien à apprendre mais principalement pour les autres étrangers ici présents, je ferai un effort pour m'entretenir avee quelques-uns d'entre eux dans leur langue maternelle. De cette façon je serai mieux compris de cenx que la question intéresse spécialement.

S'il n'y a pas d'inconvénient à agoir ainsi, je m'adresse d'abord à Messieurs les Anglais.

\section{On pruning fruit-trees.}

\section{Gentlemen,}

You most likely know already that, convinced as I am of the general superiority of what we call the rational system of pruning fruit-trees, my intention is to explain in a few words what is meant by it, and further to submit the question to your consideration, if it could not be as successfully practised in your own country, as in ours. 
In England you have generally small, though much fruit; you obtain it however often more by accident than by art. If the soil is stony, as in most places, permeable or made so by draining, you get, if not perfectly formed trees, at least beautiful ones and loaded with fruit. But if the soil is damp, rich and consequently the growth rigorous, scarcely any fine fruit can be got, if trees are not treated according" to their requirements.

To be enabled to do this, it is necessary in every case to consider: Firstly, the form to be given to the tree; secondly, the different parts of which the tree is composed; and thirdly, the functions which each of these parts have to fulfil.

It is by means of the leading-or parent-branches, that the form of the tree is giren. These forms may rary more or less, but should nevertheless not be too much complicated, but as simple as possible. The leading-lyranches ought to be very straight, and instead of spreading all out firm the same point on the mother-stem, or nearly so, a sufficient space should be left between each of them, and so on successively, each space, being greater or smaller, according to the nature of the tree. In all cases, the spaces between each leading-branch should be as equal as possible. If, in order to fill up the space, some of them are required to ramify or be forled, it is with the lowest leading-hranches that the bifureation should be formed, and these must also be the strongest. Symmetry ought to exist everywhere.

Now, as I have observed, the leading-branches give the form of the tree; but they bear another kind of wood, namely the bearing-branches, which gire the fruittwigs, and often also the reserve fruittwigs for the following year; so that in every tree, to be pruned, only three or four sorts of branches exist. This being admitted, the task of the pruner is much simplified. Indeed, knowing that each of these four parts has its own functions to perform, and may not, but by exception, be charged with that of another part, it will be much easier 
to operate than if no proper distinction at all were made, as in most old systems of fruit-tree pruning.

It now remains to be seen what the function of each of the above mentioned branches is, and how the desired result can be obtainerl: that is the essential point which the pruner ought to know and to wbserve. Firstly, the leading-branches must not give any fruit, but alter having been inly established by former short prunings, they must becume longer every year (more or less so, according to the vigour of growth); they must biturcate where necessiry, as has already been explained, and give at the same time, on their last year's woul, the begimning of the bearingbranches of the following years, which, in order to be of good shape, should rather be too weak than too strong. If then the leading-hranches are left too long, the lowest eyes do not break open, but remain dormant, and the following year leave empty places. If, on the contrary, they are eut too short, the beariny-branches shout out so vigourously, that even by pinching them in summer and by leaving the leading shoot (the leader) free, as is generally done, the growth cannot be stopped.

Such are the most important considerations with respect to the leating-lranches. Along. these, the bearingbranches ought to be placed at regular distances, and be as short as possible, in order to prevent confusion and to admit the free action of the sum and air, not to require too much space, and also to produce and bear the firuit better. All that remains to be done, in order to have them so, is to cut them the first year down to the length of two or three eyes only, whether they have buds or not. This capital point is too often neglected, and empty spaces are sooner or later the consequences.

Now, on the bearing-branches the fruittwigs exist single and isolated, and list several years, as in almost all kemelfruit-trees. They should also be kept (by pinching, breaking off', etc.) as short as possible. In all cases, these 
operations ought to be done according to the strength of the tree. But if the fruit-twigs fructify only one year, as in almost all stonefruit-trees, and if, of course, it is necessary to renovate them every year, care should be taken that the fruiting or fruitgiving twig itself, or still better another (a reserve finittwig) left on the same bearing branch, be destined (by short pruning) to replace the former fruiting twig, which is ent off.

Such are a few interesting points of the rational system of fruit-tree pruning with us in Belgium. For as far as I have seen, as well from your authors as in some of your gardens, this system is not much in practice in England, except in the horticultural gardens at Chiswick etc., where, on iny last visit, Mr. Thonpson began to form some trees, after the so-called new models. I should be most happy, Gentlemen, to have, on this occasion, your appreciation on the subject. In the mean time, permit me to say also a few words to our German friends.

\section{Baumschnitt.}

In keinem Lande vielleicht ist die Banmzucht auf dem Felde so ausgebreitet, so allgemein, wie bei Thnen. Die Spatiorbäume findet man aber weniger, und obschon Sie auf beiden vieles Obst haben, wenn die Gegend günstig ist, möchten Sie wohl besseres haben, wenn Sie den Schnitt anders verständen. Denn ich kann nicht begreifen warum das, was ihre Nachbarn, die Belgier, gut finden, bei Ihmen schlecht sein sollte.

Da es möglich sein könnte, dass Sie den sogenannten raisonnirten Baumschnitt nicht annehmen, weil Sie ihn nicht kennen oder für zu complicirt halten, wie es bei uns früher auch der Fall war, so erlauben Sie mir zu rersuchen, Ihnen in wenigen Worten zu sagen, wie einfach und rationnell er dagegren ist. Iclı werde auf Kron-Obstbäume weniger zurücksehen.

Wenn ein gewöhnlicher Baum nur eine schöne Krone 
hat, so ist es genug. Man bekïmmert sich wenig, woher sie kommt und wie sie zusammengesetzt ist. Ein Obstbaum aber muss bestehen aus ganz genan bestimmten Theilen; es giebt nämlich $1^{\circ}$ die HaUpтzveige, welche die Baumform ausmachen, gleich kräftiç sein müssen und nie Früchte geben dürfe $i$; ${ }^{\circ}$ die rRAGzveige, die regelmässig. und nicht zu nah auf den ersteren stehen und kurz sein müssen, selten Früchte, sondern meistens nux einen, zwei oder höchstens drei lileine Zweige tragen sollen, welche $3^{0}$ eigentuiche Fruchtzweige, oft zum Theil nur to zUnünFтIGE Fruchtzweige sind. Im Ganzen also vier Sorten Holz.

So verstehen Sie es wahrscheinlich auch; aber was Sie nicht scheinen zu beobachten, ist, dass jeder Theil eine gewisse Bestimmung hat und diese immer behalten muss, sodass in keinem Falle die Hauptzweige für Frïchte benutzt werden dürfen. Ebenfalls müssen die Fruchtzweige in demselben Zustand bleiben und nie (oder sehr selten) in Hauptzweige verändert werden.

Die Hauptaweige und ihre Verlängerung sind daher immer von weitem zu unterscheiden vom Fruchtholz, und viel stärker als dieses, das liurz, mager und mehr oder weniger knorrig ist. Es ist weiter nöthig, dass die Zweige weit genug von einander stehen, damit Luft, Wind und Sonne zwischendurch können.

Der Zweigen-Unterschied, den wir beobachten, ist das besondere Keunzeichen unserer Bäume, und es erleichtert ungläublich den Schnitt, weil jeder Zweig immer dasselbe zu thun hat. Die Hauptsache ist demnach den Unterschied fest $\mathrm{zu}$ halten; damit dies gęliuge, lässt man während des Sommers die Verlïngenung und öfters Vergabehung der Hauptziveige fiei wachsen, solange sie gleich kräftig bleiben, sonst müssen die stärksten ein wenig gebogen werden. Alle die anderen Zweige aber, die Fruchtholz werden müssen, sollen früher oder später ein oder mehrere Male eingekneift werden. 
Bei dem Winterschnitt jedoch muss man zusehen: einerseits, dass das Fruchtholz sich fortwährend erneuere, und andrerseits, dass zwischen den verschiedenen Hauptzweigen das Gleichgewicht bestehen bleibe. Beim ersten Punkt ist zu beobachten, dass mittlerweil diese Zweige Frïchte geben, andere, in ihrer Nähe, nene Zweige fur das nächste Jahr bilden, was nur durch ihren kurzen Schnitt zu erreichen ist. Wem jetzt das Gleichgewicht zwischen den verschiedenen Hauptzweigen nicht vollkommen besteht, muss man die schwächeren lünger schmeiden, die stïrkeren viel kiurzer. Sie thun gerade das Gegentheil. Hier noch einmal ist ein Unterschied zu machen: wirklich zu schwache Fruchtzweige sollen besser kurz als lang geschnitten werden; aber wem es Hauptzweige gilt, welche immer Holzzweige sind, je länger man sie schneidet (wenn sie zwischen stürkeren stehen, die man hurz schneidet) je mehr Triebe sie geben, und natürlich um so mehr Blätter. Und sind es nicht diese, welche den Saft nach sich ziehen und ihre allgemeine Mutter, auf welcher sie stehen, kräftig machen?

Die Zeit fehlt mir, jetzt mehr über Bammschnitt zu sagen. Es ist aber schon viel, wemn man weiss: $1^{\circ}$. wie ein Obstbaum gebildet sein soll; $2^{\circ}$. wie einfach und unveränderlich seine Theile sind; $3^{\circ}$. wie man sie im Sommer kneift und im Winter schmeidet. Natürlich ist es mir umöglich hier das in einigen Minuten zu sagen, wozu ich mehrere Wochen brauche um meine Gehülfen zu belehren. Ich hotfe wenigstens die Aufmerksamkeit der Liebhaber angeregt zu haben und bin bereit Thnen, wenn Sie es rerlangen, weitere Erklärungen zu geben, hier oder bei den Bäumen, welche wir auf der Ausstellung antreften.

\section{Over het snoeijen der Boomen.}

En nu, Mijne Heeren Nederlanders, vergunt mij dubbel verschooming dat ik mij het laatst tot $u$ wend (hetgeen ik overigens doe, om dat ik u minder dan 
anderen als vreemdelingen aanzie) en omdat ik mij veroorloof ook eenige aanmerkingen over uwe wijze van boomsnoei te maken. Ik haast mij te rerklarern dat ik uve handelwijze niet wil bedillen, maar gii zult mij toch wel toelaten van ze niet goed te keuren, zoclang ik van hare voortreffelijkheid niet overtnigd ben en u tevens te vragen, of ons boomsnoeistelsel, het zoo gennend beredeneerd stelsel, niet met voordeel bij u eene algemeene toepassing zoude kunnen vinden.

Drie punten, welke voolnamelijk den beredeneerden boomsnoei kenschetsen, worden bij $n$ anders verstatu dan bij ons; namelijk $1^{\circ}$ de zamenstelling uwer boomen; 20 hume winter-snoeijing; $3^{\circ}$ hunne zomer-snoeijing. Voor $\mathrm{u}$ is een fruitboom goed, als hij maar cen regelnatige kruin of waaijer heeft en verder gezond en vruchtdragend is, terwijl gij u weinig bekommert op welke twijgen of takken de vruchten komen. Voor u bestaat de wintersnoei in het om zoo te zeggen willekeurig uitdunnen en afkorten van alle takken en twijgen, zoodat zelden drie hoveniers denzelfden boom op dezelfde wijze zonden snoeijen. Voor $u$ is de zomer-snoeijing niet ander's dan, hetzij wegsnijden wat men niet inbinden kan, hetzij cenvoudig alle krachtige scheuten innijpen.

Voor ons is dit geheel anders: Voor ons zijn de boomen zamengesteld $1^{\circ}$ uit BOEDER- of (iESTELtatitien; $2^{\circ}$ hierop de DraAgtakiten of hum beginsel; $3^{\circ}$ op deze, hetzij enkel vruchthout, of $4^{\circ}$ vervangings-twijgen. De eerste, de gesteltakken, nemen wij niet weg, waar zij zich zoo mar vertoonen, maar op bestemde en, ingevolge den vorm, onveranderlijke plaatsen, op geregelde en regelnatige afstanden. De tweede, de draugtakken, stam un op hume geschikte plaats, niet te digt bij elkander, zij zijn micldenkrachtig en voortdurend kort. De derde, de rmehtuwijgen, worden behouden zoo lang zij liort genoco blijien; zoo niet, dan worden zij, des noods iecier jaar, rerrangen do nieuwe, die men in hume onmiddellijke nabijheid det 
ontstaan. De vierde eindelijk, de vervangingstwijgen, zijn in sommige boomen van het grootste belang; men benuttigt ze niet, naarmate men ze vindt, maar men doet ze daar ontstaan, waar men het heyeert, dat is te zegrgen aan den voet van den te lang geworden vruchttwijg.

Mogelijk zult gij mij opmerken, mijne heeren, dat al die deelen in uwe boomen ook bestaan. Dit kan eenigermate waar zijn, maar terwijl gij meermalen uwe gesteltakken voor vinchthout neemt, of ze deels voor vervangingshout gebruikt, of ook van uwe vruchttwijgen yestelhout maakt, heeft bij ons ieder deel zijne afzonderlijke en voortdurend dezelfde rol te vervullen, zoodat zelden of nooit het eene deel van den boom met de verrigtingen van het andere belast wordt. Ziedaar een gewigtig verschil.

Het spreekt nu van zelf, dat, daar gij zoo weinig acht geeft op het onderscheid dat wij in onze verschillende takken en twijgen maken, uwe manier van snoeijen eveneens moet verschillen, niet alleen van den eenen boom tot den anderen, maar op denzelfilen boom, van den eenen boomsnoeijer tot den anderen, zoo als ik straks zeide. Dit komt omdat de een voor vuchthout zal nemen, hetgeen de ander voor gestelhout zou verkiezen.

Maar al hadt gij ook te doen met zoo gezegde nieuwmodische boomen, dan nog zoudt gij watarschijnlijk anders dan wij te werk gaan, omdat gij over het kort en lang snoeijen anders schijnt te denken dan wij. Gij snijdt uwe flaauwe twijgen, onverschillig welke, kort, uwe sterkere lang; wij doen het tegenovergestelde, en wij heweren gelijk te hebben, wammeer het flatuwe en sterke houttwijgen geldt, die op denzelfden boom staan; maar wamneer het vruchthout of ander kreupelhout is, dan doen wij als gij, en snijden al wat flaauw is het kortst, en wat sterker is het langst. Verder snijden wij sterkgroeijende boomen langer in al humne deelen, ofschoon wij nogtans het ook voor zeker houden dat kortgesnoeide boomen sterker doorschieten dan lang'gesnoeide. Dit in het geheugen hou- 
dende, snijden wij onveranderlijk deze twijgen altijd lang, gene altijd kort; wij weten waarom wij zoo moeten en niet anders mogen te werk gaan.

De zomersnoeijing eindelijk houdt gij voor eene afloende, volledige bewerking; wij integendeel houden ze voor eene voorbereidende, noodige en zoo zeer gewichtige behandeling, dat zij den wintersnoei des noods kan onnoodig maken, of ten minste dien uitermate vereenvoudigen. Gij behoudt uwe boomen zoo wel als wij in evenwicht, maar doet het meest bij inknijping; wij, die onze boomen verdeelen in moeder- of gesteltakken en in klein hout, laten de eerste, de gesteltakken, sterk doorschieten zonder knijpen (dit zou valsche schenten doen ontstaan), en wanneer de eene veel sterker dan de andere dreigt te worden, nemen wij liever onze toevlucht tot de nederbuiging. Het tweede, het klein hout, integendeel wordt, voor zooveel het niet te zwak is, achtereenvolgens een of verscheidene malen geknepen (rrezenlijk snoeijen doet men 's zomers niet), ten einde het zoo niet mager en tenger, dan toch merkelijk dunner dan de moedertak-verlengsels te hebben, om het alzoo beter tot draaghout geschikt te maken.

Wat onze wijze van boomsnoei nog kenmerkt, is datwij van onze jonge boomen niet alleen geene vruchten vragen, maar er geene van willen. Eerst vormen wij onze boomen, om later meer en duuramer opbrengst te bekomen, want wanneer boomen zoo ras dragen, is dit een slecht voorteeken; zij leven gemeenlijk niet lang.

Ziedaar, mijne heeren, waarin, zoo ik mij niet bedrieg, onze ziens- en handelwijze voornamelijk van de uwe verschillen. Ik zou geluklkig zijn hierover uwe gedachte te mogen vernemen.

Het voorgaande had ik reeds opgesteld, toen mij het werk over boomsnoei in handen kwam, vervaardigd door den heer baron van Hoëvelu Nyenuuis van Wijhezigt, bij Deventer, die het bekende geschrift van den heer 
HARDx heeft overgezet. Ziedaar alzoo een uwer landgenooten die den beredeneerden boomsnoei roor aanbevelenswaardig moet houden in Nederland. $\Pi k$ moet dit zoo veel te meer gelooven, daar die heer er eigen aanteekeningen, Nederland betreffende, heeft bijgevoegd welke nicts weerleggen noch afkeuren. Il weet echter niet, in hoeverre de heer van Hoëvel Nyenhuis beoefenaar is, of een man van praktijk heeft geraadpleegd, en daarom vraag ik thans ook uve denkwijze. Ik ook, mijne heeren, heb een werk over beredeneerden boomsnoei nitgegeven, 't welli reeds zijne $3^{\text {de }}$ uitgaaf' beleeft en hier ter uwer inzage ligt. Daar ik het in de Nederduitsche taal geschreven had, heb ik het indertijd aan verscheidenen uwer gezonden. Tot heden heb ik nog van geene tegenspraak gehoord, doch ook nog weinig van toepassing der snoeistelsels (de beredeneerde) daarin ontwikkeld. Wat mag toch de reden daarvan zijn? dat mogt ik gaarne rreten."

M. d'Anouilh de Salies se déclare cuntre la taille des arbres en éventail et démontre que les branches horizontales dépérissent beancoup plus tôt que les branches verticales. Les premières font plus de bois et de feuilles au détriment du fruit; il recommande la forme, dite palmette. La forme pyranidale telle qu'elle est représentée par un sujet qui se trouve dans la salle de la section, n'est pas selon lui une pyramide, mais ce que l'on nomme communément une quenouille. Il en démontre les défauts: l'arbre est privé d'air, domne peu de fruits et cépérit. Il expose brièrement quel est le système français et en constate les avantages.

M. Pynaert (de Gand) prétend que la forme de l'arbre dépend nécessairement du sujet sur lequel il est greffé.

M. d'Anouilh de Salies réplique.

M. de Beucker (d'Aners) demande alors la parole et dit: 
Mijne Heeren!

,Wij hebben over de verbeterde behandeling en vorming der vruchtboomen te spreken. Dit is eene stof van het hoogste belang, doch eer ik het woord daarover voer, moet ik aan mijne vrienden en beroepsgenooten van Noord-Nederland verklaren, dat ik niet gekomen ben om tot hen uit de hoogte te spreken; ik wil mij in hun midden begeven, en onder den kunstbroederlijken handdruk wil ik met hen over die nuttige wetenschap handelen. Laat mij toe aan mij zelven te vragen: waarom moet de vruchtboom gesnoeid worden? En hierop moet ik antwoorden: om de natuur te helpen en door de tusschenkomst van ons verstand de sappen en groeilirachten zoo te leiden, dat op alle plaatsen eene zekere maat van hoeveelheid ten nutte verbruikt worde, om den boom zoo spoedig mogelijk groot en vruchtbaar te maken en zijne vruchtbaarheid en levenskrachten zoo lang mogelijk te behouden.

De tuinier is in dien zin de bestierder van de sappen en levens-verrigtingen, zoodanig dat de stam van den boom den bestierder moet gehoorzamen en deze de levenssappen aan alle zijne takken regelmatig en zuinig zal verdeelen. De stam moet dus niets doen dan onder toezigt van den bestierder de sappen vervoeren en verdeelen (de stam moet geene rruchten dragen); de takken moeten in de rigting van hun oorspronkelijk vertrekpunt regt uitgestrekt blijven loopen en over geheel hunne lengte moeten zij van afstand tot afstand regelmatig met vruchthout bekleed zijn: dit is alles wat men bij het snoeijen der vruchtboomen in acht moet nemen. Mar, zal men zeggen, dit wordt overal gedaan; neen, mijne heeren, het spijt mij dat ik het zeggen moet, maar ik bid $u$ uit liefde voor de wetenschap, laat mij toe de warheid uit te spreken. De wetenschappelijke waarheid zal u misschien wel wat hard vallen, gij zult ze echter welligt aannemen en er roordeel voor u uit trekken. Ziet hier de zaak: 
Bij de behandeling van vruchtboomen moet men de natuurlijke ontwikkeling, den natuurlijken groei in het oog houden; nooit mag een tuinier de natuur door zijne handelwijze verhinderen of belemmeren, hij moet door zijne verstandige tusschenkomst den boom op den weg van de natuur trachten te helpen; de boom, die greene willekeurige beweging of gevoel bezit, moet door den bewerker beschermd en ondersteund worden. Niemand mag den boom snoeijen zonder de reden te weten waarom men snoeit; alvorens men snoeit, moet men weten wat er van het af te snijden deel zou geworden zijn, indien het op den boom bleef, en in de tweede plaats moet men kunnen zeggen, welke verbetering men door die snede heeft voortgebragt, en welke gevolgen daarvan men in al de deelen te wachten heeft. In al die opzigten is de boom, welken wij hier voor ons hebben, en welke in Nederland, zelfs te Boskoop, behandeld is, toch zeer gebrekkig bewerkt. Daarover moet gij, mijne heeren, $\mathrm{u}$ niet verwonderen.

Het is in Nederland niet alleen dat men de vruchtboomen slecht snoeit; neen, in België, Frankrijk, Engeland, Duitschland en elders snoeit men op vele plaatsen, in vele tuinen de boomen zoo slecht en onkundig, als hier. Er is hier geene kwestie van Fransch of Belgisch stelsel tegenover dat van Nederland, neen, wij kunnen maar ééne zaak bepleiten, en dat is, wat men het beste, het verkieslijkste vindt. Den boom te snoeijen met reden van alle oorzaken en gevolgen, of wel werktuiglijk zonder gedachte of reden? Ik meen dat alles, wat de mensch te behandelen en te bewerken heeft, met oordeel en verstand moet geschieden; en nogtans moet men bekennen dat deze pyramide-peereboom tot op zijn zevenjarigen leeftijd nog geen enkele maal goed gesnoeid is geweest; al de takken zijn van hun oorspronkelijken weg afgeleid, en door eene onkundige hand tot eene andere rigting gedwongen.

Daar vind ik een tak, welke door vergaffelingen ver- 
scheidene andere takken dragen moet, terwijl hij niets dan vuchthout zou moeten voortbrengen; hier heeft een tak gepoogd zich te ontwikkelen, en door de verkeerde leiding der toppen is hij verzwakt en verdroogd; men ziet, elke tak is grebrekkig, elke snede is slecht gedaan, en zelfs zoo dat de gemaakte wonden niet genezen kumnen. Dat is een ongelukkige handelwijze! Doch ik moet het bekennen, ook ik heb de boomen zoo gesneden, ook ik heb die fruitopbrengende voortbrengselen van den milddadigen Schepper in mijne jeugd helpen verminken en vermoorden. Het kan dan geen verwijt voor $\mathbf{u}$ zijn, geachte Nederlanders, als ik zeg, dat het eene slechte behandeling is, gelijk ik ze zelf vroeger uitoefende.

Ik weet het, gij wordt voor den meerderen arbeid tot nu toe door de koopers niet beter betaald; gij werkt om den boom zoo spoedig mogelijk groot te hebben, zonder na te denken of de boom in later leven het niet moet bezuren; gij maakt voor den verkoop een zwak gestel, dat groot en krachtig schijnt te zijn; gij denkt voor eenen goeden boom niet genoeg betaald te worden, en daarom maakt gij hem slecht. Dat mag zoo niet blijven voortduren; ik spreek hier niet tot mijn voordeel, ik ben gekomen in de hoop om nuttig aan het nijvere en werkzame Nederland te wezen, en ik verklaar aan de boomkweekers van Boskoop en andere plaatsen, dat het tijd wordt, om op den waren weg van wetenschap te komen. Nederland heeft vele boomkweekerijen, zij bezitten goede gronden: is het dan niet te betreuren, dat men met die groote opoffering van arbeid, met dat voorregt van een goeden grond te bezitten, nog zulke verminkte natuurtelgen vormt?

Op de eene plaats kan er geen lucht of licht tusschen de takken komen, om de rruchten smakelijk en aangenaam te maken, en op de andere plaats is eene groote en nuakte ruimte. Dat de Hollanders de handen ineenslaan en met eene welgemeende wilskracht zeggen: wij 
zullen ons beroep reredelen, wij zullen van al ons werk, van al ons doen, ons rekenschap trachten te geven, waarom wij zoo en niet anders handelen. Van dat oogenblik, mijne heeren, zijt gij geene eenvoudige tuiniers meer, gij wordt tuinbouwkundigen, en, zijt er zeker van, geheel de matschappij van de geringe tot de hoogste standen zal u dankbaar zijn, en uw eigen belang zal er bij wimnen. Gij zult boomen vormen, die gij, in plaats dat gij er één gulden voor bekomt, verkoopen zult aan ware liefhebbers roor 3, 4, 5, ja zelfs voor 20 gulden en nog meer; ik kan u verzekeren dat ik verscheiden boomen van 30, 40 en 50 francs te Antwerpen verkocht heb; want nuarmate de liefhebberij in de wetenschap aangroeit, wordt ook uwe zaak bevorderd. Ilk moet hier eindigen ondat ik van uw geduld misbruik zou maken, maar ik wensch Maandag nog eenige oogenblikken over dit belangrijk onderwerp te mogen spreken."

M. Hooftman (de Boskoop) remercie l'orateur. Il est persuadé que les arboriculteurs néerlandais profiteront des observations, que M. DE Bevcker leur a communiquées. Il prétend turtefois que la taille hollandaise des pếchers et des abricotiers est bonne. Il ajoute encore ce qui suit:

,Het snoeijen en vormen der pyramiden, ofschoon ook bij de meesten onzer reeds aanvankelijk naar de betere methode in beoefening gebragt, is helaas nog niet van zulk een gevolg als wenschelijk en noodig is, daar dit werk bijna uitsluitend door onze arbeiders geschiedt en daar het verkrijgen van geregeld onderwijs, zoo wel voor de meeste patronen als voor hume onderhoorigen, een zaak is die dringend roorziening behoeft. Wij hopen dat ook bij ons te lande de hooge Regering de nuttigheid diarvan zal erkemnen, zoodat door haar doeltreffende scholen in het leven zullen worden geroepen."

M. Van Hulle ne doute nullement que la discussion n'ait déjì porté des fruits. Il s'adresse ensuite spécialement aux pépiniéristes de Boskoop et dit en hollandais: 
,Gij schijnt niet te kunnen aannemen dat het met uwe perzikboomen ook zoo gelegen is als met de andere vruchtboomen. Stat mij toe u zullss te bewijzen, niet door ijdele woorden, waaraan gij overigens weinig waarde hecht, maar door de bewerking uit te voeren op dezen perzikboom, dien gij hier zekerlijk hebt doen ambrengen als type van volkomenheid. Wat wij in uwe boomen meest bestrijden, is niet alleen het uitgang'spunt en de rigting uwer roorname takken, uw gebrek van onderscheid tusschen gestel- en vruchthout, maar voornamelijk moeten wijafkeuren, en in uwe perzikboomen vooral, de groote hoeveelheid hout die gij hum laat. Indien gij zeidet zulks te doen, om den boom een fraaijer aanzien te geven en dien aan de onkundige koopers beter te doen bevallen, dat zou waarheid wezen, maar gij houdt integendeel staande dat de perzikboom in Nederland zoo digt van hout zijn moet, ondat hij bij u te lande zooveel aan houtverdorring onderhevig is. Maar hebt gij wel eens nagedacht, dat zijn hout verdort, juist omdat het in Nederland nog minder dan in België volledig kan rijp worden; dat het nietirijp kan worden, juist omdat de invloed onzer zomerlucht en warmte zoo flaauw is, en dat gij dien weldoenden invloed dan nog meer vermindert, juist door het te digt laten bestaan uwer rerschillende twijgjes, wanr noch wind, noch lucht, noch zon tusschen door kan? Zulke twijgen, gelijk overigens alle ander hout, dat bij gebrek aan warmte en lucht róór den winter niet behoorlijk heeft kunnen rijp worden, moet spoedig bevriezen of verdorren. Wij hebben daarvan een bewijs gehad na den winter vam 1861. De schade, welke hij in de tuinen heeft aangerigt, is niet alleen toe te schrijren gerveest aan zijne eigenlijke gestrengheid, maar veeleer daaraan, dat hij volgde op een kouden regenachtigen en aan zommeschijn karigen zomer, die de scheuten belet had volkonen te sluiten, volkomen rijp te worden. Nu, mijne heeren, zelfs nog veronderstellende dat al het hout van dezen perzikhoom zich eenigzins heeft kumıen sluiten, 
dan nog zou het, noch om zijne rruchten noch om andere redenen, behoorlijk kumnen behouden worden; niet omdat er op den mur plaatsgebrek is, maar omdat er plaats dient behouden te worden, om de scheuten in te binden, die welhaast roor het rolgende jaar gaan uitschieten. Om die redenen voornamelijk en om andere, te lang om alle te rermelden, geloof ik te mogen zeggen dat in dezen perzikboom een derde zoo niet de helft te veel hout is, 't welk ik, indien gij mij zulks veroorlooft, zal uitsnijden. $(\mathrm{Op}$ toestemmend teeken gaat de heer var Hulut tot de daad over).

Dans le cours de la discussion, l'orateur soumet à l'assemblée la proposition suivante, sur laquelle on n'a cependant pris aucune décision:

„,Daar de fruitkundige matschappij ran Boskoop den beredeneerden boomsnoei voor Nederland ontoepasselijk, zoo niet onmogelijk houdt (zonder haar te verdenken van in hare proesen met rooringenomenheid te zijn te werk gegaan), ware het niet wenschelijk dat, hetzij de Boskoopers, hetzij een bijzonder liefhebber in Nederland, een door ons roor te stellen gediplomeerd Belgisch boomsnoeijer in dienst namen, aan wien zij een hoekje gronds en eenige boomen zouden afstaan, welke hij volkomen meester zoude zijn, buiten zijn diensttijd te rerzorgen en te leiden volgens het zoogenaamd nieuwe stelsel (1)?"

M. Hooftman réplique encore de la manière suivante:

,Ik neem de vrijheid te doen opmerken dat de groote prachtige perzik-leiboom, die zich buiten het tentoonstellingsgebouw bevindt en schier door allen bewonderd wordt, onze rertegenwoordiger is ran het idee bij de behandeling der leiboomen, en dat zull een boom aan de vereischten voldoet, die wịj meenen dat men bij de vor-

(1) MMI. vax Hulle et DE Beucker avaient en outre proposé de donner une leçon pratique de taille; cette leçon, fixée à lundi 10 Avril, avant l'ouverture de la séance, a eu lieu plus tard. 
ming in het oog houden moet, namelijk bij fraaijen vorm roldoende ruchtbaarheid. Dat dit nu zoo is, hiertoe kan ik mij beroepen op bewijzen, die voldingend onzen rorm tegenorer den Franschen en Belgischen verdedig'en, en wel voornamelijk dáár waar men een koud en dus minder roordeelig klimat roor steenrruchten heeft dan in België of Frankrijk, en waar dus rooral op dien rorm het meest behoort gelet te worden, die bewijzen levert ran goede of roldoende vruchtbaarheid, bij geschiktheid van vorm. Пk beroep mij dan op tweeёrlei vormen: die à la Montreuil vond ik voor een paar jaren op het Rosenborg-Slot te Kopenhagen, waarvan men eenige groote willige 10 à 12 jarige exemplaren slechts matig met rruchten bezet had staan. Op een andere plaats en wel bij den Heer Handelsgärtner Hansex, in den zoogenaamden Schloss-Garten bij Kopenhagen, werd ik getroffen door eenige uitmuntend groote en schoone willige 10-12jarige exemplaren met heerlijke en rele ruchten als overladen. Deze boomen naar de zuiver Hollandsche minier geleid, zoo als wij die thans ook nog op vele onzer buitens vinden, waren bij regelmatigen rorm goed met fijn en vruchtbaarhout, zoowel van onder als overal elders belegd. Op mijne vraag, hoe het mogelijk was hier zulke schoone naar Hollandsch model gevormde boomen te vinden, daar men algemeen geen begrip schijnt te hebben om leiboomen, en rooral die der steenvruchten, naar het oorspronkelijke te bewaren en roort te bewerken, werd mij door den Heer Hasser gezegd, dat hij in zijn jeugd rijf jaren in Haarlem bij een bloemist was werkzaam geweest, waar hij de snoeijing en vorming der Hollandsche leiboomen had geleerd en ze thans nog wel meende in praktijk te hebben gehouden; hij liet mij hierover oordeelen en ik moest bekennen dat de vorm niets te wenschen orerliet, en de vruchtbaarheid niet alleen, maar ook de vruchten zelre zeer schoon waren en beide tegenorer die, welke ik bij den Heer Petersen op het 
Rosenborg-Slot had gezien (die naar de Fransche methode waren gekweekt), zeer gunstig afstaken (1)."

M. d'Anouilh de Salies présente encore quelques observations contre le système hollandais, lequel a le grand inconvénient pour les arbres fruitiers de trop les dégarnir en bas.

M. de Cannart d'Hamale fait observer que M. de Beucker a parlé dans le même sens, mais qu'au lieu de donner ì son système le nom de système français, il le nomme système ratiomnel. Ensuite il présente encore quelques observations critiques sur le système de taille adopté en Hollande.

M. Overeynder (de Boskoop) prend la défense du système de taille hollandais pour les abricotiers et les pêchers, surtout en rue du climat. La forme en éventail est selon lui la meilleure; la forme, dite palmette, est moins favorable.

M. Ch. Baltet (de Troyes) fait observer qu'il faut prendre en considération la diversité du climat et les bons terrains de la. France, qui ont beancoup favorisé l'arboriculture. Jadis en France on avait aussi adopté la forme en érentail, mais on l'a quittée plus tard, parce qu'on a reconnu qu'une autre forme était plus avantageuse. Il fait brièvement l'historique des changements opérés dans la taille en France. Pour la Hollande M. Baltet approuve les formes aplaties on érasées des aruoriculteurs néerlandais, lesquelles favorisent l'influence de l'air et de la lumière; il admet aussi la forme en éventail, parce

(l) Ik kan hier nog bijoegen, schrijit de heer HoofTux, dat, toen ik ran Amsterdam huiswatsts ben gegaan, mij de eer te beurt viel, bij nijn aankomst aldaar cenige Dnitsche heeren en ook den heer 'TYGe Rotrie, chef van den akademischen tuin te Kopenhagen, te ontmoeten. Sprekende over het verhaudelde omtrent het snoeijen en vormen der leiboonen, inzonderheid over hetgeen ik ter bevestiging van het blijvende en onveranderlijk goede, dat onzen leivorm boven dien van Montreuil kemmerkt, had gezegd, vernam ik ran den heer Rotnis, dat het hem leed deed deze zitting niet te hebben bijgewoond, daar ZijinEd. de waarheid ran mijn beweren, omtrent hetgeen ik te Ko penhagen had oygemerkt, ten volle had kunnen en willen berestigen. 
qu'me branche mourante peut être ficilement remplacée. Mais le traitement de la branche à fruit pourrait être assimilé aux méthodes française et belg’e.

M. van Hall (de Groningue) remercie les orateur's précédents qui ont exposé les systèmes de taille en usigge en France et en Belqique. Cette démonstration a confirmé en pratique ce que les livres nous ont appris en théorie. Il espère que les Hollandais prendront en considération la question, qui vient d'être développée et qu'ils consulteront surtout la nature.

Après quelques paroles échangées entre MM. va.N Hulle et Overeynder la discussion sur cette question est close pour être continuée lundi.

M. de Zantis (de Liège) fait observer que l'on a négligé de traiter la question principale. Il demande, si le pêcher sur lequel les démonstrations ont eu lieu, est greffé et sur quel sujet, ou s'il est gagné de noyau.

II. de Cannart d'Hamale fait observer au préopinant que la discussion sur cette question est close.

II. MI. de Cannart d'Hamale commmique aux membres de la section, que M. N. J. Andersson, de Stockholm, met à la disposition des membres qui les désirent des greffes de bommes sortes d'arbres fruitiers de Suède. Plusieurs membres s'inscrivent.

III. M. Triana (le Puris) truite des Mélastomacíes et dépose son travail, qui est renvoyé à la première section (1).

(1) Nous supposons que ce travail n'est pas parvenu ì la première section; le procìs-verbal des sénnces n’en fait point mention. 
IV. M. Koch (de Berlin) développe la question qu'il a posée (No. XVI du Programme) concernant la nomenclature des plantes. L'orateur s'exprime de la sorte:

, Ich erlaube mir einen Gegenstand zur Sprache zu bringen, der für Gürtnerei nicht weniger als für die Botanik von der grössten Wichtigkeit ist; ich meine die Synonymie der Pflanzen. Es werden alljährlich eine Menge Pflanzen aus fremden Erdtheilen eingeführt, es werden aber auch in vielen Gärtnereien Ab- und Spielarten neu gezüchtet und ebenfalls in den Handel gebracht. Der eine Liebhaber und Gartenbesitzer ist aber mehr für reine Arten, wie sie fremde Länder uns bringen, eingenommen, während der andere vorherrschend den Blumen seine Aufmerksamkeit zuwendet. Beide haben also gleich Anfangs verschiedene Interessen und sehen sich deshalb oft beim Ankaufe neuer Pflanzen getäuscht. Sollte es hier nicht möglich sein, in den Verzeichnissen der Pflanzen durch den Namen selbst schon darauf hinzuweisen, ob er eine neue Art, oder nur eine Form bedeute?

Im Allgemeinen findet man schon den Gebrauch dass, wie Linné es angegeben, reine Arten einen Geschlechtsnamen besitzen, dem ein lateinisches Adjectivum beigegeben wird, welches die bestimmte Art anzeigt, während die Gartenform durch irgend einen Beisatz nicht in lateinischer Sprache ausgedentet wird. Dieser Gebrauch ist ganz geeignet, wenn el: allgemein durchgeführt wïrde, jede Täuschung zu ersparen; ich erlaube mir deshalb die Herren Gärtner ganz speciell darauf aufmerksam zu machen und sie zu ersuchen, ihn bei Ausgabe ihrer Verzeichnisse festzuhalten. Fuchsia globosa, coccinea bedeuten demnach Arten, Fuchsia Mad. Comelissen, Crinoline u. s. w. hingegen Gartenformen. Namen von Männern, die sich um die Wissenschaft oder Gïrtnerei verdient gemacht haben, oder denen man gärtnerischer Seits eine Ehre anthun will, werden heut' zu Tage viel angewendet, nicht allein um Arten, anch um nur. Formen anzuzeigen; 
in diesem Falle müsste man dem Namen aber eine lateinische Endung geben, wenn er eine Art anzeigen soll, hingegen müsste er mverändert bleiben, sobald er zur Benennung einer Gartenform gebraucht würde. Agave Verschaffeltii zeigte demnach eime Art an und Camellia $A m b r$.Verschaffelt wäre die Benennung für eine Gartenform."

M. Nedzelsky (de Moscou) obtient la parole et s'exprime en ces termes: „Ce que $\mathrm{M}$. le professeur $\mathrm{KocH} \mathrm{a}$ dit concernant la nomenclature des fleurs, doit également avoir lieu pour la pomologie. Il faut cependant qu'ici la. délimitation soit plus prononcée encore, et c'est à quoi on a fait trop peu d'attention jusqu'à présent. Il faut adopter certaines règles pour les dénominations des espèces des fruits; il est nécessaire d'étudier à cet effet l'histoire des espèces nouvelles et de celles qui sont depuis longtemps dans le commerce. Ces descriptions historiques, qui existent déjà dans plusieurs ouviages pomologiques, nous font connaître les véritables noms des espèces et peuvent nous servir à rectifier les noms falsitiés par la transcription. Il n'est pas toujours facile d'obtenir l'une ou l'autre forme sous son vrai nom, c'est pourquoi on ne peut accepter l'une ou l'autre dénomination comme la vraie.

Ces falsifications de noms ne sont pas senlement la conséquence de la culture de quelques sortes dans les pays étrang'ers, mais elles ont lieu dans les pays mêmes d'où l'espèce est originaire. Ce changement dans la nomenclature est souvent la conséquence d'une orthographe fautive, ainsi au lieu de Grafeinstein, quelques-uns écrivent Gravenstein; l'histoire seule de l'espèce nous apprend quel est le nom véritable. Les noms des espèces que nous voyons exposées ici, nous prouvent comment on falsifie ces noms. Cette manière d'agir est cause qu'il est très-difficile de trouver le vrai nom de certaines espèces. Il y a beaucoup d'espèces qui ont plusieurs noms; il y en a qui ont même jusqu'à 15 synonymes. En premier lieu l'influence du climat joue un rôle important; ensuite c'est l'intérêt com- 
mercial qui est en jeu. Tout ceci est cependant trèsincommode pour parvenir à faire un certain choix dans les véritables dénominations.

M. van Hall demande si l'intention de M. Косн est, que l'on doive donner une dénomination latine aux espèces, et non aux variétés.

M. Koch répond affirmativement et ajonte encore ce qui suit:

Ein grosser Uebelstand wird ferner dadurch herrorgerufen, dass Gärtner Pflanzen, welche sie aus fremden Ländern erhalten haben und in den Handel bringen wollen, willkürlich neue Namen geben. Eine benennte Pfanze verkäuft sich bekanntlich besser als eine ohne Namen. In diesem Falle wird gewöhnlich ein Name irgend eines bereits bestehenden Geschlechts benützt und ihm ein beliebiges Adjectirum in lateinischer Sprache zugefügt. Die Frage, ob es wirliklich eine nene Pflanze ist oder nicht, wird gar nicht weiter erörtert. Noch schlimmer ist es, wenn zufällig der lateinische Artname schon vorhanden ist und der Käufer num glaubt, eine bestimmte Pflanze zu erhalten, sich also bald getüuscht fühlt. So entstehen sugenammte Garten-Namen, die, wemn schliesslich die Pflanze erkamnt wird und ihren richtigen Namen erhält, man nur mit Mühe wiederum der Vergessenheit übergeben kann. Die Gewöhnung ist manchmal so gross, dass es schlechterding's grar nicht möglich ist, den richtig'en Namen einzuführen.

Leider wird aber oft eine in den Handel gebrachte Pflanze gar nicht aus fremden Ländern eingeführt. Man sieht irgendwo eine längst bekannte Art, die sich zufällig in Habitus etwas rerschieden zeigt, oder wirklich auch eine mehr abweichende Form darstellt, sucht die zu acqueriren und bringt sie, um die Verkïufer anzulocken, als eine aus fremden Lündern eingeführte Pflanze in den Handel. Ich bin fer'n daron, durch diese absichtliche T:̈uschung dem ganzen Gärtnerstande einen Vorwurf 
zu machen; dergleichen Unredlichkeiten kommen, weun auch in anderer Gestalt, in allen Geschäften mehr oder minder vor; es sind stets nur einzelne Personen, für die unmöglich ein ehrenwerther Stand verantwortlich sein kann. In dem Handel befinden sich mehre Formen der Juniperus virginiana, welche zufällig entstanden, von denen aber der betreffende Gärtuer behauptete, dass er sie direct aus dem Vaterlande erst bezogen habe. Es kann aber auch zufällig geschehen, dass in dem einem Lande eine Pflanze schon längst kultivirt wird, während man sie in einem andern erst zu verbreiten anfängt. In der Ansstellung des Industrie-Gebäudes habe ich auf diese Weise mehre Pflanzen gefunden, die mit neuen Namen versehen auch für neu ausgegeben wurden, in Dentschland aber schon längst bekannt sind.

An diesem Uebelstande der falschen Benennungen ist aber der Gärtner nur zum Theil Schuld; diese trifft auch den Botaniker, der leider sich gerade dem Stande, von dem aus ihm für seine Wissenschaft sehr viel geboten wird, gegenüber abschliesst. Würden Botaniker und Gärtner mehr Hand in Hand gehen, und sich gegenseitig unterstützen, so könnten beide Theile nur um grösseren Nutzen davon haben. Allerdings ist die Zahl der Botaniker welche Lust dazu haben, jetzt, wo der grösste Theil sich mehr der anatomischen und physiologischen Seite zugewendet hat, sehr gering, aber doch gibt es deren immer noch, um gemeinschaftlich zu jener wichtigen Arbeit sich zu vereinigen.

Das Material ist in den letzten Tagen so ungemein herangewachsen, dass selbst der befähigste und kemntnissreichste Botaniker nicht alle Pflanzen kemen kann. Man hat sich von Seiten der Botaniker schon längst daran gewöhnt, sich bei der Bearbeitung zu theilen und mit Vorliebe mit der einen oder anderen Familie sich zu beschäftigen. Das Schwierige ist, dass die eingeführten Pflanzen anfangs auch nicht gleich in einem Zustande 
sind, wo man sie leicht erkennen kamn; es fehlen die Blüthen und Früchte, welche schliesslich doch am sichersten leiten. Hat man sich aber mit bestimmten Pflanzenfamilien längere Zeit beschäftigt, dann wird es schliesslich oft doch möglich, wenigstens annähernd der neu eingeführten Pflanze in einer Familie einen Platz anzuweisen.

Es ist deshalb vor Allem wünschenswerth, dass sich die Gärtner, was ich nicht genug hervorheben kann, mit Botanikern in Verbindung setzen. Dazu ist es nothwendig, dass die Gärtner aber wissen, mit welchen Familien sich der eine oder andere Botaniker beschäftigt, um gerade bei diesem die gewünschte Auskunft über eine Palme, Orchidee, Melastomacee, Pomacee, u. s. w. zu erhalten. Ich mache deshalb folgenden Vorschlag:

,Que la seconde section adresse à la première cette question, que MM. les botanistes déclarent la famille ou les familles qu'ils veulent traiter, qu'ils fassent connaître ensuite la nomenclature qu'ils auront arrêtée aux horticulteurs qui pourront les consulter en cas de doute, afin d'arriver ainsi à une bonne nomenclature et prévenir les fraudes et les mauvaises pratiques."

La proposition est adoptée et la question renvoyée à la première section.

M. de Beucker applaudit ì cette proposition. Il y a beaucoup de charlatans parmi les horticulteurs; ils viennent surtout de Grenoble. Ils font voyager des personnes qui oftrient en vente des plantes d'une seule espèce, sous différents noms. Cette manière d'agir est cause que les horticulteurs hounêtes perdent la confiance du public. Ces derniers jour's encore il y avait à Anvers un de ces voyageurs, qui se disait représentant de la maison Giradd. M. DE Beucker a écrit à cette honorable maison et la réponse qu'il reçut, contirma ses soupçons: ce marchand qui avait déballé à Anvers, n'appartenait pas à la maison Greaud. M. de Beucker a fait publier par les journaux la lettre de M. Giraud, afin d'avertir le publie. 
M. Nedzelsky fait remarquer en réponse à M. KocH que les talsifications sont plus nombreuses encure pour les arbres fruitiers, surtout pour les pommiers et les poiriers.

M. Baltet fait observer qu'il y a aussi des horticulteurs belges qui se rendent coupables des mêmes fraudes en France.

M. Overeynder remercie MM. КосH et DE BEucken d'avoir traité cette question, qui sera de grande utilité pour le public Hollandais. Il fait observer que la Société de Boskoop pour l'amélioration des arbres fiuitiers a déjà tâché d'obrenir à cet inconvénient par l'édition d'un ouvrage avec planches. Il ajoute que les notices de M. Nedzelskr, insérées dans le Trochenschrift de M. Косн, ont été l'objet de l'attention de la Société, et qu'elle en a tiré grand profit. Il invite M. Nedzelsky à venir voir les vergers de Boskoop et l'institution pomologique de la Société.

M. Nedzelsky fait remarquer que la pomologie a fait de grands progrès dans les derniers temps, mais que l'on a besoin d'autre chose encore que d'ouvrages avec des planches; il faut qu'un système soit adopté pour ces ouvrages. En Allemagne on a de bons ouvrages, mais les planches ne sont pas coloriées. La Société de Boskoop y a apporté une excellente amélioration en donnant des planches coloriées. II désirerait que l'on donnât aussi des planches représentant les fruits coupés transversalement.

L'orateur adresse ensuite aux délégués de la Société de Boskoop les paroles suivantes:

Je vous remercie infiniment, MM., de l'attention que vous avez daigné prêter à mes notices pomologiques insérées dans le Wochenschrift. Cet onvrage n'est que le premier résultat de mes études de la pomologie. Ce travail m'a déjà conduit à de nouvelles déeouvertes, et m'a appris comment on peut par exemple distinguer les espèces des variétés en coupant les fruits transversalement. Votre attention, qui proure que mes études sur la pomologie 
n'ont pas été infructuenses, m'encourage à persévérer dans l'étude de cette branche de l'horticulture fruitière, à laquelle je me suis voué avec zèle. Je vous remercie également de l'aimable invitation que vous me faites de visiter vos vergers et votre institution. J'espère pouvoir y répondre et je ne donte nullement que je n'y trouve bien des choses intéressantes et instructives, dont je pourrai profiter pour la pomologie et l'horticulture fruitière. Je me flatte que plus tard je povrrai obtenir de votre part des espèces fruitières qui pourront être ntilisées dans mon pays.

Après quelques paroles de M. D'Anouilh de Salies sur cette question, la séance est close pour être reprise à 2 heures de relevée. 


\section{SECTION DE BOTANIQUE}

\section{APPLIQUÉE ET D'HORTICULTURE.}

SEAANCE du 8 Avril 1865, à 2 heures de relevée.

LE BUREAU SE COMPOSE DE:

MM. KOCH, Président.

DE CANNART D'HAMALE,
VAN HALL et KRELAGE, Vice-Présidents.

BARRAL, DE BEUCKER et MULDER, Secrétaires.

FRANCKEN, Secrétaire-adjoint.

M. le Président ouvre la séance et propose de renvoyer la lecture du compte-rendu de la séance précédente à celle de Lundi. Cette proposition est acceptée par acclamation.

I. La première question à l'ordre du jour est la cinquième du programme; elle est posée en ces termes par M. Сн. Вацtet (de Troyes):

„, Y a-t-il dégénérescence chez les plantes bulbeuses, herbacées ou ligneuses, les arbres fruitiers ou d'ornement? Dans le cas affirmatif, indiquer les causes de la dégénérescence (détérioration) et les moyens de la combattre."

M. Baltet obtient la parole pour développer sa thèse. Il s'exprime en ces termes:

„Nous entendons souvent dire: Les végétaux dégénèrent; moi, je ne pense pas que la dégénérescence existe.

On confond la dégénérescence générale avec une sorte de décadence individuelle, provoquée par l'absence des milieux qui faverisent la vie et la reproduction de la plante dans des conditions normales.

D'abord l'esprèce dégénère-t-elle? Non, car s'il en était 
ainsi, combien déjà auraient disparu du globe! Si des essais de naturalisation ont été infructueux, c'est que l'homme n'a pas su domner aus sujets expérimentés le sol, le climat, etc. propres à leur existence.

Maintenant est-ce la variété qui dégénère? Du premier abord, il semblerait qu'elle y est plutôt disposée; mais en y réfléchissant bien, on reconnaît encore que par le fait d'une culture défectueuse, d'une multiplication mal raisonnée, il peut en résulter une sorte de détérioration apparente, chronique même, mais non générique, nous voulons dire inconnue chez les sujets de la même variété, qui ne sont pas nés de l'individu altéré.

Prenons pour exemple les panachures, les maculatures, les écorces dorées, les feuillages pourprés, etc., ce sont des écarts de végétation que nous attribuons an hasard. L'horticulteur les propage par voie de multiplication artificielle, marcottage, bouturage, greffige, et recherche alors les variations plus ou moins accentuées, afin de reproduire une nouveauté plus ou moins distincte du type. Les collections se trouvent ainsi augmentées d'une sousvariété qui vivra aussi longtemps que les multiplicateurs sauront reproduire l'anomalie.

Mais cette déviation dans l'organisme naturel de la plante est visible; on la juge, on la constate facilement. Maintenant supposons qu'au lieu d'être pour ainsi dire externe, elle soit interne, ou pour être plus exact, supposons que ceite déviation ne soit pas risi引le, qu'arriverat-il? Si vous prenez comme élément de multiplication un fragment de végétal vicié de cette sorte, vous reproduisez la détérioration sans vous en aperceroir. En conséquence, votre arbre sera susceptible de donner une végétation rabougrie, une floraison irrégulière, une fructification défectueuse, suivant la nature du vice inhérent au fragment multiplié.

Par le fait d'une multiplication considérable, comme celit se passe dans les grands établissements, un pareil 
régétal infirme, sans que l'on s'en doute, deviendra à son tour le point de départ d'une série de plants avariés, parce que, je le répète, la défection est invisible et ses effets n'ont point encore paru. C'est alor's qu'à la suite de plusieurs geénérations, où l'écart s'est transmis de mal en pis, on s'écrie: La plante a dégénéré.

On ne saurait invoquer ici la régénération par le semis, puisqu'il est recommu que non seulement les panachures ne se perpétuent pas au moyen de la graine, mais la majorité des variétés et sous-variétés jardinières exigent la multiplication artificielle pour être bien fixées. Le malaise n'est donc ni héréditaire par le semis, ni contagieux par la greffe.

Le mot dégénérescence est surtout prononcé en ce qui concerne les arbres fruitiers; déjà parce que l'on consulte les moindres nuances dans la robusticité du sujet, ou dans la saveur du fruit, et que l'on ne tient pas assez compte des modifications entrainées par la nature du terrain et l'état de température sur les arbres de la même sorte. D'ailleurs, les fruits ne varient-ils pas dans la même récolte, ou d'une année à l'autre, sans que pour cela il y ait une dégradation radicale?

D'après certains auteurs, les anciennes sortes de poires ne seraient plus ce qu'elles étaient jadis. Sur quels principes, sur quels faits base-t-on une pareille erreur? Le poirier n'est-il pas un arbre de notre région? Il ne saurait donc y dépérir. Est-on bien sûr par exemple que le Beurré gris, le Doyenné, la Crasanne ne soient plus ce qu'ils étaient à une époque antérieure? Je soutiens qu'ils sont les mêmes.

En l'absence de toute confrontation, figurez-vous qu'un auteur décrive la Royale d'hiver ì Marseille, le Bon-Chrétien à Auch, le Saint-Germain à Beaune, il ne manquera pas de leur décerner un brevet de fiuit de première qualité en plein vent. Cultivez-les ailleurs, avec des conditions moins favorables, vous serez entrainé à découvrir une dégénérescence. 
Autrefois, les rapports entre les producteurs manquaient; le monde horticole ne jouissait ni de sociétés, ni d'expositions, ni de publications périodiques, et encore moins de congrès; les observateurs ne se communiquaient guère le résultat de leurs recherches, de sorte que, les auteurs écrivant sous leur inspiration personnelle, la postérité sur plus d'un point doit infailliblement se trouver trompée.

Ajoutons encore: 10. les carpographes ont souvent le tort de poser leur description au point de vue le plus avantageux; 20 chez nos ancêtres, la majeure partie des arbres fruitiers étaient plantés en espalier, dans les couvents et manoirs; ils produisaient davantage et des fruits plus beaux; 3o. en recourant au type de la variété, toutes les fois qu'il n'est pas décrépit, on lui reconnait les mêmes qualités viriles de son origine, témoin le dessin des poires Bézy de Chaumontel, produites par l'arbre-mère à Luzarches et figurées par Dumanel en 1765, cent ans après la naissance de l'égrin.

En examinant le genre Rosier, on reconnaitrait également que les praticiens minutieux savent choisir leurs greffons et boutures sur les parties vigoureuses des sortes délicates, et au sommet des branches remontantes chez les variétés peu florifères à l'arrière-saison, ete. Done s'il y a dépravation, l'homme doit s'en prendre à lui seulement.

Je suis heureux de voir ici de célèbres fleuristes affirmer d'un signe de tête mon opinion sur cette question importante; et je suis certain que les fameux cultivateurs néerlandais nous diront que le climat et surtout le sol des Pays-Bas, aidés de bons soins de culture, sont les causes de la réputation universelle des oignons et bulbes à fleur de la Hollande qui, dit-on, ne s'y dénaturent jamais."

M. de Cannart d'Hamale justifie ce qu'a avancé M. BALTE'T concernant les poires, surtout la Beurrée royale. 
Il en a vu de très belles chez M. le Baron de Stennhault; il en a envoyé des greffons à M. Dequesne, à Paris, qui les a multipliées, mais elles ont dégénéré. L’arbre-mère avait été trouvé près d'un vieux château et il n'avait pas dégénéré. M. de Cannart D'Hamale croit que la dégénérescence n'est pas fortuite et que la faute en doit être le plus souvent imputée aux arboriculteurs.

M. van Hall (de Groningue) mentionne quelques cas de dégénérescence; il cite entr'autres des feuilles d'if, sur un arbre qui avait été imprégné de la préparation ferrugineuse du Dr. Boucherie, et qui étaient panachées. Selon l'orateur la panachure est un état maladif des plantes. Quant aux arbres fruitiers, il y en a plusieurs qui ne se soutiennent pas regulièrement par le semis; il vaut par conséquent mieux les multiplier par le greffage, la marcotte ou le bouturage. Il est vrai cependant que l'on a vu de bonnes sortes, obtenues de cette manière, donner un produit inférieur; il croit néanmoins devoir en attribuer la cause à ce que l'on avait pris les greffons sur de vieux sujets.

II. M. le Vice-président de Cannart d'Hamale donne lecture d'une lettre, transmise par le Président de la première section de la part de MM. HARDY frères, qui proposent la question suivante:

Si la fécondation artificielle joue souvent un rôle inportant dans la création des races et des variétés, le rôle le plus important n'est-il pas celui, demandé à la sélection des portegraines, ̀̀ leur culture, au traitement auquel on les soumet?

III. M. Pynaert (de Gand) ayant posé la neuvième question du programme, ainsi conçue: De la nécessité de recourir à la reproduction par voie de semis, pour créer des plantations rustiques, demande la parole afin de traiter cette question si intimement liée à celle posée par M. BaLtet. 
La section ne voyant aucun inconvénient à ce que lès deux questions soient traitées simultanément, M. le Président domne la parole à M. Prnafrt, qui s'exprime ainsi:

\section{Messieurs!}

„,J'ai demandé maintenant la parole pour développer ma thèse, parce que celle-ci se lie intimement à l'importante question que vient de soulever mon honorable ami, M. Charles Baltet, à savoir ,s’il y a dégénérescence chez les plantes bulbeuses herbacées ou ligneuses, les arbres fruitiers ou d'ornement." Pour ce qui concerne spécialement les plantes bulbeuses, je pense qu'on désigne sous le nom de dégénérescence un phénomène très naturel et qui n'a aucun rapport arec la dégénérescence proprement dite. Les oignons de Jacinthes, provenant d'Harlem, cultivés en France et dans d'autres pays, n'y conservent pas longtemps, nous dit-on, les éminentes qualités qui les font rechercher. A la deuxième année les fleurs se montrent déjà plus petites, et le plus souvent l'oignon se divise en domnant naissance à plusieurs hampes maigres et rabougries. Ce sont là des faits très-naturels et très-faciles à expliquer, même en admettant que les Jacinthes soient partout cultirées avec les soins, avec la perfection qu'on y apporte aux environs d'Harlem, ce qui n'est que rarement le cas. Il n'existe en Belgique qu'un seul établissement où cette culture soit pratiquée avec succès, e'est l'établissement de M. L. van Hout'te à Grendbrugge-lez-Gand. Il y a à peine dix ans que la spécialité de ce qu'on appelle articles d'Harlem y a été introduite; elle y réussit aujourd'hui tellement bien que chaque année on y consacre une plus grancie étendue de terrain. L'étude, que nous y arons faite du mode de croissance des Jacinthes, me permet aujourd'hui d'expliquer la prétendue dégénérescence de ceslantes et de celles qui se cultivent et se propagent d'une façon analogue.

Comme chacun sait, les Jacinthes sont reproduites 
par semis et par cayeux; dans le premier cas, on a en vue la création de variétés nouvelles, dans le second il s'agit tout bonnement de multiplier une variété connue. Dans l'un et dans l'autre cas, la jeme génération doit traverser une assez longue période avant d'avoir formé des oignons adultes. Cette période est de 3 ou 4 années au moins pour les cayeux et de cinq ou six pour les semis. Elle n'est pourtant pas invariable, on le conçoit, elle varie selon les variétés et surtunt selon la richesse du sol, le mode de culture, etc. Ce n'est que lorsque les bulbes sont arrivés à leur complet développement qu'on les livre au commerce. Ils fournissent alors les plus belles fleurs. On ne doit pas croire que les oignons continuent à se perfectionner et à croître avec l'âge; une fois qu'ils ont dépassé le terme de leur accroissement, ils arrivent tôt ou tard à la décadence. La plante, obéissant ì une loi de la nature, cherche à se multiplier; elle n'a pas pu former de graines, chaque année les fleurs ayant été enlevées après la floraison, elı bien, l'oignon se divise en donnant naissance à plusieurs bulbes. On peut séparer ceux-ci, mais ils ne produisent jamais, quoi que l'on fasse, des fleurs aussi parfaites que les oignons provenant de cayeux.

Une autre cause qui tend à faire décliner encore plus rapidement que la nature la floraison des Jacinthes, réside dans une culture inintelligente. On se figure généralement que le repos de ces plantes commence dès que les fleurs se passent; c'est justement le contraire. Les arrosements sont alors le plus nécessaires; c'est après la floraison que la Jacinthe a besoin de la plus grande somme de nourriture, car dès cette époque elle forme le rudiment, le germe de ses fleurs qui ne viendront au jour que l'année suivante. Si l'humidité manque ì cette période, et e'est le cas ordinaire, le germe est mal nourri, l'oignon lui-même perd de sa grosseur et il n'y a rien d'étommant à ce que la floraison s'éloigne de plus en plus de la perfection qu'elle avait montrée la première année. 
J'arrive maintenant aux arbres fruitiers. Dans le développement de ma proposition je vais nécessairement aborder plusieurs des faits qui viemment de vous être signalés par l'habile pépiniériste de Troyes; je tâcherai d'en expliquer les causes et de vous montrer par quels moyens on peut combattre, au moins en partie, les inconvénients qui en résultent. De cette démonstration découlera, MM., la nécessité de recourir au semis pour établir des plantations fruitières rustiques.

A ne considérer que superficiellement le nombre considérable de variétés fruitières, répandnes dans les jardins et dans les vergers (1), on peut se demander s'il y a utilité réelle à créer encore des variétés nouvelles; on peut se demander surtout s'il ne serait pas beaucoup plus simple de rechercher, parmi ces nombreuses variétés, celles qui conviemnent le plus particulièrement au terrain, au climat, ì la localité où l'on veut établir une plantation. Et dans le fait cette manière de procéder paraît d'autant plus raisommable que, à de très rares exceptions près, les moyens généralement suivis jusqu'à ce jour pour obtenir de bonnes variétés par voie du semis n'ont fourni de résultats satisfaisants que par l'effet du hasard, et sans que l'on puisse les regarder comme le produit d'une combinaison ou d'une méthode bien déterminée.

Je suis loin de contester la possibilité de créer aujourd'hui, par un choix judicieux des variétés, un jardin

(1) Dans un onvrage publié, il y a peu d'annćes par un pomologue allemaud très connul, M. Docmxar, (Der sichere Führer in der Obstlunde) on trouve la description de:

1263 variétés de pommes.

1053 " "poires.

258 " " cerises

343 " " prunes.

45 "d'abricots.

137 . de pêches et brugnons.

32 "d'amandes.
108 variítés de noisettes.

\begin{tabular}{|rcl}
40 & " - & " châtaignes. \\
42 & " & "figues. \\
65 & " & "framboises. \\
540 & " & "groseilles ì maquereau. \\
71 & " & " \\
311 & " " & " raisius.
\end{tabular}

Il y en a certainement, dans le nombre, quelques-unes qui font double emploi, mais d'un autre côté beaucoup de variétés locales ne sont pas mentionuées et ne sont pas cependant dépourvues de mérite. 
fruitier, un verger dans n'importe quelles circonstances (en ne sortant pas toutefois de nos régions tempérées); j'admettrai même, ̀̀ la rigueur, que le domaine pounologique est assez riche, qu'il y a suffisamment de bons fruits de toutes sortes aux différentes saisons où il est possible de les obtenir en état de maturité parfaite, et que nous avons seulement le tort de ne pas les connaître, ou d'ignorer les conditions dans lesquelles leur réussite offre les meilleures chances. Eh bien, malgré cela, je crois à l'impérieuse nécessité de provoquer par la voie du semis la formation de variétés nouvelles, destinées à remplacer les anciemnes, attendu que celles-ci, renouvelées exclusivement par reproduction artificielle, c'est-à-dire pargreffes, parboutures, ou par marcottes, et surtont en raison même du mode de culture de plus en plus artificiel anquel nous les soumettons, subissent, au bout d'une période plus ou moins longue, une certaine altération dans quelques-uns ou dans l'ensemble de leurs caractères, altération que la plupart des écrivains, qui se sont occupés de cette question, ont désigné sous le nom de dégénérescence ou de dégénération. De l'emploi de ces expressions, qui ne sont pas tout-ì-fait exactes, est provenu, sans aucun doute, la, divergence d'opinion entre quelques savants et les pomologues arboriculteurs; les premiers niant la possibilité de la dégénérescence des arbres fruitiers, les seconds constatant par une foule de faits le dépérissement progressif, l'état de langueur, de souffrance que manifestent les meilleures variétés anciennes. Dans une discussion de ce genre, il convient avant tout de s'entendre sur des mots. C'est ce que M. de Bouttevilue, président de la Société d'horticulture de la Seine Inférieure, à Rouen, expose clairement dans un mémoire très-remarquable, inséré dans le Bulletin de cette Société (1). ,La dégénération, dit-il, doit s'entendre

(1) De l'existence limitée et l'extinction des régétaux propagés par division. Ce travail témoigne d'une grande érudition et d'une comnaissance approfondie des nombreux écrits qui traitent de cette partie si difficile de la science pomologique. 
spécialement d’une détérioration du procinit d’une génération comparé anx parents. L'étymologie répugne à admettre une dégénération sans génération.

Dans le cas où une semblable détérioration, conséquence d'uu acte générateur, provient d'un croisement vicieux, il y a abâtardissement.

$\mathrm{Si}$ le changement de bien en mal, de mal en pis arrive dans le cours de l'existence d'un être vivant par' l'effet de circonstances éventuelles, il y a détérioration, dégradation, dépravation ete.

Mais si ce changement arrive par le seul effet du progrès des ans, c'est de la vieillesse, de la caducité, c'est, si l'on veut, de l'afficiblissement, du dépérissement sénil, rien autre chose."

Pour nous en tenir à la lettre, ne disons donc plus que les variétés sont sujettes i la dégénérescence; elles se détériorent, se dégradent, s'affaiblissent, suivant les circonstances, car les causes qui déterminent leur décadence, sont très variées. Et c'est parce que ces causes sont si variées que les effets produits le sont aussi.

Cette doctrine est contraire à celle de van Mons, qui attribue exclusirement à l'âge les changements observés chez les variétés soumises à une longue culture.

Voici comment il s'exprime à cet égard dans son Traité des arbres fruitiers (1).

„On se plaint généralement et avec raison de la décadence des espèces fruitières anciennes; tout le monde remarque les vices des anciens fruits, tout le monde les avoue, s'en plaint et s'en désole, je ne commais rien d'aussi déplorable, en fait de culture, que d'élever un arbre dans l'espoir de jouir de son fruit et de le voir tomber en ruine au moment ù cet espoir devrait se réaliser. Est-il rien de plus décourageant que de perdre ses peines, dauns l'édu-

(1) Arbres fruitiers. Lew culture on Belgique et leur propagation par la graine ou Pomonomie belge, e.rpérimentale et raisonnée, 2 vol, 1835-36, Louvain, Dusart et VAN DEN BROECK. 
cation des arbres greffés, sans espoir d'être récompensé de ses soins par Jeur beauté future? Ils sont assaillis dès leur berceau par toutes les souffrances dé la vieillesse; on accuse le sol, on en veut au temps, le sujet reşoit sit part des reproches; enfin, ce qui est senl répréhensible, l’âge le la variété reste à l'abri du soupçon.

Quand la cause provient de l'âge, aucun remède ne peut le guérir; le mal est persistant.

Quand des causes étrangères, et qu'il dépend de nous d'éviter, rendent le mal passager, l'effet cesse dès que la cause discontinue d'agir, car l'art peut faire disparaitre ce qu'il a contribué à faire naître. Une forme vicieuse qu'on s'obstine à faire prendre et garder à l'arbre; une taille intempestive, trop longue ou trop courte; un sol infécond ou trop humide; une exposition où le jour et l'air ne peuvent circuler librement, sont antant de causes de la souffrance artificielle que l'arbre et son fruit peuvent éprouver.

L'âge d'une variété date du moment où elle a pris naissance de graine; son premier pas vers la vieillesse ou la décadence date de l'époque où elle rapporte son premier fruit; la qualité bonne ou manvaise de ce fruit, la fertilité plus on moins grande de l'arbre qui le porte, font seules une différence dans la longueur de la vie d'un arbre fruitier. Celui qui produit abondanment et chaque année des fruits excellents, s'épuise bien plus tôt que celui qui ne produirait que de mauvais fruits et en petite quantité."

MM. Royer et Bivort partagent entièrement l'opinion de van Mons. M. DE Boutteville aussi considère comme une loi de la natmre la fin des variétés propagées par greffes, boutures on marcottes. „Tous les individus, dit-il, issus du pied-mère, souche primitive de la variété, et reproduits par séparation, constituent une collection d'êtres également éloignés de l'acte générateur qui a présidé à la naissance du tronc commun, dont ils ont été séparés. Il se troure, il est vrai, parmi les êtres multi- 
pliés de la sorte des petits et des grands, mais non des jeunes et des vieux, puisque l'acte de naissance de tous porte la même date ou mieux est unique; M. DE BoutreVILLE en conclut que tous s'avanceront à-peu-près parallèlement ver's la vieillesse, bien que des circonstances diverses en hâtent les approches pour les uns et l'éloignent pour les autres. Pour cette variété il arrirera une époque fatale, inévitable où l'ensemble des êtres qui la constituent ressentira les effets de l'affaiblissement sénil et mourra tout entier de décrépitude."

Il n'entre pas dans mon intention de discuter à fond au point de vue physiologique, ni au point de vue philosophique, la manière de voir des savants pomologues que jai cités plus haut. La question ne me semble pas encore assez avancée pour que l'on puisse émettre sur ce point autre chose que des hypothèses. Quant à moi, les nombreuses observations que j'ai recueillies, tendent ì nie faire admettre en principe que la détérioration des variétés trouve son origine dans les procédés artificiels de culture, lesquels éloignent de jour en jour davantage les arbres fruitiers de leur's conditions naturelles de prospérité.

Cette opinion concorde avec celle de de Candolle sur l'immuabilité de la variété. Selon l'illustre botaniste, les variétés sont permanenteswet ont une durée illimitée aussi longtemps que l'homme en prend soin d'une manière convenable.

Avant de poursuivre ma démonstration, il convient de spécifier les caractères de la détérioration des variétés fruitières. Ceux-ci se montrent tantôt sur les fruits seuls, tantôt sur le bois seul, tantôt enfin sur l'ensemble de la végétation. Certains fruits ne mûrissent plus que difficilement; ils se gercent, crevassent et deviennent pierreux, tandis que le bois paraît encore sain et vigoureux. A mesure qu'ils avancent en âge, ils exigent plus impérieusement l'abri de l'espalier pour acquérir leurs qualités. Dans un climat plus chaud, ils semblent se retremper. 
Ainsi certaines variétés anciennes, qui ne sont plus d'aueun rapport en Belgique quand elles ne sont pas cultivées en espalier, sont vigoureuses et productives en haut vent et en pyrumide dans certaines parties de la France (Brvort, Annales de Pomologie).

D'antres fois la décadence se manifeste par une diminution de vigueur et une prédisposition à contracter des maladies. Dans cet état, l'arbre peut encore produire quelquefois de bons fruits. Il n'en est plus ainsi, lorsquela dégradation devient générale et se fait sentir dans l'ensemble du sujet; alors le bois devient chancreux, il est attaqué de gangrène sénile, les branches se dessèchent par leurs extrémités, les fleurs elles-mêmes, s'il s'en montre encore, deviennent stériles on ne donnent que quelgues rares fruits sans aucune valeur.

On voit évidemment que la décadence des variétés fruitières peut affecter des caractères tre̊ss variés, et cela me confirme encore dans l'opinion qu'il faut l'attribuer, en général, à des causes purement artificielles et variées. Examinons rapidement les plus importantes d'entre elles, ainsi que les moyens à mettre en œuvre pour en arrêter les funestes effets.

Tout le monde connait l'infiuence que le climat exerce non seulement sur la saveur des fruits, mais aussi sur la croissance des arbres, sur leur santé et leur fertilité. Personne n'ignore, par exemple, que la Poire Figue d'Alençon, fine et savoureuse en France, peut n'être que fade et insipide dans certaines parties de la Belgique, où cependant beaucoup d'autres sortes développent parfaitement leurs excellentes qualités. Malgré l'étendue de la région tempérée propre à la culture du poirier, il s'en faut de beaucoup que ses nombreuses variétés puissent vivre dans les mêmes circonstances. Je dirai plus, dans une seule variété il y a des individus qui contractent par habitude ou par suite de l'éducation reçue, certains besoins, certaines prédilections, on pourrait presque dire des caprices, 
qui se perpétuent par la voie de la greffe et constituent ainsi de véritables sous-vuriétés. Placez-les ensuite dans des conditions qui ne leur conviemment plus, vous n'en obtiendrez que des résultats négatifs's ou peu satisfaisants.

Il n'est pas de jardinier qui s'avisera de planter un Doyenné d'hiver contre un mur exposé au nord. Il est de règle aujourd'hui que les bomes poires d'hiver ne peuvent réussir contre un mur privé de soleil, et que l'exposition du nord convient tout au plus à quelques variétés hâtives ou de demi-saison. Pourtant, je comnais un vieux Bré Diel dans ce cas; il s'y montre d'une rare fertilité et les fruits ne laissent rien à clésirer. On m'a parlé d'un Glou-morceau ou Bré d'Hardempont offrunt le mème exemple, mais je ne l'ai pas vu. Je ne reux pas conclure de ces faits isolés que l'on puisse toujours cultiver ces variétés dans des conditions analogues, mais je veux seulement arriver à ne pas faire considérer omme une faute d'impression le conseil d'un auteur qui recommandait, tout an commencement de ce siècle, de planter au nord le Passe Colmar et le $B^{r e ́} d^{\prime} H a r-$ dempont. L'auteur que nous citons, est un de ceux dont on ne récusera jas la cumpétence, c'est van Mons lui-mème qui parlait ainsi dins une note adressée au redacteur du Fruitkundig Woordenboek. An reste la plupart des rariétés qui réclament le plus impérieusement aujourd'hui l'abri protecteur d'un mur favorisé du soleil, réussissaient fort bien, du temps de uA Quintinie, sous la forme en buisson, forme. qui tenait lien, ì cette époque, de celles en quenouille, en fuseau et en pyramide, lesquelles n'ont été inventées que plus tard.

Les partisans de la cégénérescence, telle que l'entendait van Mons, ont déduit de cette observation un argument en faveur de leur théorie. Je n'y vois, pour ma part, autre chose qu'm affaiblissement, me sorte d'énervement. Ainsi, presque tontes nos variétés anciennes de fruits se sont tellement affaiblies par ne longue culture en espalier, elles ży sunt tellement habituées au surcroit 
de chaleur, produit par la réverbération des rayons solaires contre la muraille, qu'elles ne peuvent plus s'en passer. Dès qu'on les prive de celle-ci, elles ne résistent que difficilement aux intempéries, leurs fleurs ne nouent plus, et leurs fruits, si exceptionnellement elles en portent, n’atteignent qu'une maturité imparfaite. On peut avec raison les comparer, avec certain auteur français, à ces chevaux anglais pur sang, qui, à force d'habiter l'écurie depuis un grand nombre de générations, ne peuvent supporter le plein air. Tel est du moins l'effet que la longue culture en espalier a produit sur nos meilleures variétés de fruits à pépins. Chez les espèces à noyau, l'effet apparent n'est peut-être pas toujours le même, mais il n'y a pas moins afficiblissement réel. Un seul exemple suffira pour le démontrer. Nous possédons en Belgique quelques variétés de pêches dont la culture en plein vent est possible, - bien entendu dans une situation privilégiée; - mais pour cela il faut que l'arbre soit franc de pied, e'est-à-dire issu de noyau, ou bien qu'il ait été obtenu de greffe prise sur un pied cultivé lui-même en plein vent. Si la greffe provenait d'un individu soumis depuis quelque temps à l'espalier, l'arbre qui en résulterait, ne conviendrait pas à la culture en plein vent, quoique appartenant à une variété propre à ce genre de culture. Voilà évidemment une preuve concluante d'affaiblissement causé par la culture en espalier.

Cet énervement graduel progressif de nos meilleures variétés (j’insiste là-dessus parce que ce sont précisément celles-là qui sont le plus communément soumises ì une culture raffinée, à une haute culture, comme disent les Anglais) explique facilement leur extrême sensibilité aux intempéries de l'air, sans devoir recourir à un abaissement supposé de la température du globe, comme le font quelques auteurs. Cette détérioration est déplorable, mais il serait difficile de s'y soustraire complètement. C'est ici le lieu de dire qu'il n'y a pas de médaille sans revers; l'énervement de la race ou de la variété (ou plutôt des individlis 
soumis à une culture intensive), voilà le revers de la médaille en fait d'arboriculture fruitière perfectionnée.

Je viens d'employer à dessein l'expression de culture intensive; j'entends par là un ensemble de procédés ayant pour but d'augmenter au maximum la dimension et la qualité des fruits, la précocité et la fertilité des arbres, tels qu'une exposition chande et abritée, un terrain fécond, une forme naine, contenue. Croit-on que toutes ces circonstances réunies n'influent pas sur un individu, et que cette influence continuée pendant plusieurs générations successives de sujets propagés par la greffe ne doive pas finir par s'inoculer pour ainsi dire à leur existence? Voilà pourquoi les variétés anciennement les plus estimées comme fruits à couteau, le $B^{r e ́}$ gris, le Saint-Germain, le Doyenné, la Virgouleuse, la Bergamotte crassane, le Pépin d'or, lir Reinette grise, le Calville blanc, etc., sont celles qui paraissent aujourdhui les plus sensibles aux diverses causes d'affaiblissement.

On m'objectera peut-être ici que ce n'est pas seulement aux arbres à fruits soumis à la culture intensive que l'on peu reprocher la détérioration, qui finira inévitablement par les faire exclure de nos jardins. Dans les vergers, dans les champs les variétés à fruits à cidre comme celles à fiuits de table, cultivées depuis longtemps, dépérissent ou deviennent improductives, et les jeunes pieds qu'on greffe pour les remplacer, vieillissent avant d'avoir atteint le quart des dimensions on de l'âge de leurs devanciers (1).

Je répondıai à cela d'abord, que si l'on doit admettre que les arbres dans les vergers et dans les champs ne sont pas exempts de l'affaiblissement qui s'observe chez les arbres de nos jardins, du moins l'effet se produit chez eux d'une manière bien plus lente. Je connais dans plusieurs localités en Belgique des Bézy de Chaumontel en plein vent ayant acquis un très-fort développement et produisant régulièrement une abondante récolte de beaux et de bons

(1) De Bourteville, mémoire cité. 
fuits. Je puis citer des $B^{r e ́}$ d'Hardempont dans les mêmes conditions, ainsi qu'un $B^{\text {re }}$ rance qui existe a Etichore au haut d'une colline dans la propriété de M. Thienpont, qui, chaque année, semblent protester par leur produit considérable contre cette aveugle habitude de couvrir les murs de poiriers, alors que cette place serait souvent occupée plus utilement par d'autres espèces qui ne peuvent s'en passer. M. Royer lui-même, un des défenseurs de la théorie van Mons, dit qu'il possède dans son jardin un poirier de Virgouleuse en haut vent, énorme, séculaire, se chargeant régulièrement de magnifiques récoltes. Je cite sa phrase: ,ses fruits sont exempts de gerçures, parfaitement sains, lisses et sans taches, tandis que nos poiriers de Virgouleuse en espalier, au midi ou au levant, nous donnent si rarement un fruit remarquable, que nous sommes obligés de le réformer." Tous ces faits ne corroborent-ils pas mon appréciation, quant à l'action affaiblissante d'une longue culture en espalier? Il est peu probable que d'ici à un demi-siècle on trouve encore, comme aujourd'hui, en Belgique des Bergamotte Esperen et des Fortunée d'Enghien en haut vent. On dira alors, comme on le dit actuellement de tant d'autres variétés, qu'elles ne réussissent qu'en espalier et que leur culture en plein vent est impossible. On peut me demander pourquoi. Parce qu'à l'espalier le fruit devient plus gros, plus beau, plus fondant parfois et que naturellement tout le monde finira par consacrer à ces variétés les murs les mieux exposés. C'est en peu de mots l'histoire de la plupart de nos bons fruits anciens.

Je n'opposerai pas aux faits cités plus haut par M. DE Boutteville, concernant le dépérissement des fruits de verger et à cidre, l'exemple de quelques variétés locales, cultivées de temps immémorial, et dont la vigueur, la fertilité et la rusticité ne laissent rien à désirer jusqu'à ce jour. Je le répète, il y a plus d'une cause qui puisse déterminer la décadence, et de ce qu'une plante a long- 
temps prospéré dans une localité, dans un terrain donné, il ne s'ensuit pas qu'elle y doive prospérer toujours.

Je ne trouve rien d'étonnant à ce que dans ces vergers, auxquels fait allusion cet auteur, les jeunes pieds, destinés à remplacer les vieux, acquièrent à peine le quart des dimensions et de l'âge de ces derniers. Par cela seul que les anciens sont morts de vétusté, il paraît probable qu'ils avaient dépouillé le sol de la plus grande partie des principes nutritifs, qui leur convenaient, et cela suffit pour expliquer la langueur des jeunes. Il est incontestable qu'en général on n'apprécie pas assez, pour les plantes ligneuses, qui occupent parfois le terrain une longue suite d'années, l'importance des assolements, si bien comprise cependant dans la culture des plantes annuelles.

Lorsqu'un jardinier soigneux doit remplacer, dans un jardin, une pyramide ou un espalier qui a atteint un certain âge, il prendra, dans le milieu des carrés, de la terre neuve, pour renouveler totalement celle qui avait été occupée par les racines de l'arbre à remplacer. Des engrais, même en quantité, mais sans renouvellement de la terre, ne suffiraient point et ne fourniraient qu'une végétation chétive. Je comprends parfaitement que dans un verger, où les racines de chaque arbre s'étendent dans un espace de 20 à 30 mètres cubes et même davantage, il est impossible de procéder comme dans un jardin; aussi lorsqu'un verger devient trop vieux et doit être renouvelé la seule chose à faire c'est de le rétablir dans un sol vierge, qui n'a pas encore porté d'arbres.

Voilà déjà une première preuve de l'influence du terrain sur la prétendue dégénérescence des arbres fruitiers. En ce qui concerne la composition chimique du sol, cette influence est plus considérable qu'on n'est généralement porté ì l'admettre. L'auteur d'un excellent livre sur Les fruits et les arbres fruitiers de l'Amérique, M. Downing (1),

(1) 'The fruits and fruit-trees of America, by A. J. Downing, revised by Crns. Downing, New Fork, 1862. 
attribue la non-réussite de certaines variétés à l'absence ou à la présence en trop faible quantité, dans le sol, des éléments inorganiques, tels que la chaux, la potasse et les phosphates, éléments absolument indispensables, dit-il, à la formation des poires fines. C'est ainsi que partout sur le littoral, où le sol est léger et où ces éléments ont été épuisés par une culture antérieure, beaucoup de variétés, qui avaient prospéré dans le principe, sont arrivées promptement à un dépérissement prématuré. Et il ajoute: ,le complet rétablissement d'arbres malades (dégénérés) dans des sols légers et appaurris, à la suite d'un emploi abondant de cendres de bois, d’os pulvérisés, de chaux ainsi que d'engrais ordinaires, proure incontestablement, que ce n'est pas l'âge de ces variétés qui est cause de leur décadence apparente, mais tout simplement l'absence de diverses substances indispensables à la production du bon fruit."

L'observation suivante du même écrivain fortifie encore cette opinion, que je partage entièrement. „, Des vergers entiers de Doyenné donnent actuellement les produits les plus beanx et les plus abondants, dans l'intérieur de l'état de New-York et dans quelques états de l'Ouest, et chacun peut voir, en Septembre, près de la ville de Hudson, sur le North-River, et cela dans un sol en apparence froid et argileux, des spécimens de ce fruit pesant trois quarts de livre, d'un blond doré, d'une beauté d'aspect et d'une suavité d'arôme dignes du jardin des Hespérides et qui, nous en sommes certain, n'ont jamais été surpassés en France, à l'épouque de la plus luxuriante jeunesse de cette variété."

Je le demande maintenant, est-il déraisonnable, d'après cet exemple, de supposer que, dans certains cas, la détérioration des fruits soit purement accidentelle et qu'ils puissent se restaurer en fournissant au sol, sous la forme d'amendements, les principes qui y font défaut?

C'est dans les sols légers, on peut s'en convaincre aisément, que les effets de la décadence se font sentir le 
plus rapidement, sans doute parce que les éléments solubles y sont plus vite assimilés par les végétaux.

Ils se produisent, au contraire, d'une manière beaucoup plus lente dans les terres un peu argileuses, soit que d'ordinaire ces éléments s'y trouvent naturellement en plus grande abondance, soit que la ténacité du sol les retienne plus facilement. A Gand, où le terrain est sablonneux, rien n'est plus rare que de rencontrer un Doyenné d'hiver dommant de beaux fruits; presque toujours ils sont crevassés, restent petits et se remplissent de concrétions pierreuses. Mais dans les sols argileux, notamment dans le Hainaut, où il est très répandu, le Doyenné d'hiver est encore dans tous les jardins le roi des poiriers.

Ce qu'il y a de plus remarquable dans tout ceci, c'est de voir à Gand, à côté de ces Doyenné qui ne donnent que du désappointement, de superbes espaliers et pyramides de Bré. Clairgeau, de Louise bonne d'Avranches, de Poire Durondeau et de beaucoup d'autres se charger, tous les ans, de nombreux fruits que ne le cèdent en rien, sous le rapport de la beauté et de la qualité, aux meilleures sortes connues. Il y a aussi certaines variétés qui dans nos terrains légers viennent mieux que dans les terres plus fortes. Cela ne prouve-t-il pas l'utilité qu'il y aurait à étudier plus sérieusement qu'on ne l'a fait jusqu'ici les aptitudes et les goûts particuliers à chaque sorte, afin de pouvoir donner, du moins à celles qui se distinguent ainsi, le terrain, la situation et l'exposition qui leur conviennent spécialement?

L'effet produit dans un terrain humide sur la végétation des pommiers est connu de tous les arboriculteurs et l'on s'accorde pour lui attribuer, en grande partie, les chancres et la carie qui attaquent prématurément ces arbres. Les arboriculteurs reconnaissent, disent-ils, deux espèces de chancres. Le premier est accidentel, qu'il provienne d'une lésion ou d'un terrain défavorable, et on peut le guérir d'abord en supprimant les causes qui l'ont déter- 
miné, puis à l'aide de quelques soins et d'un peu d'onguent de St. Fiacre. Quant au second, le chancre originel, il est héréditaire et on prétend qu'on ne peut pas le guérir. Il y a du vrai dans cette distinction, qui est pourtant plus apparente que réelle. Les maladies, chez les végétaux, peuvent devenir chroniques tout aussi bien que chez les animaux; il suffit pour cela de maintenir assez longtemps ces végétaux sous l'influence des agents qui ont provoqué les maladies. Une fois que celles-ci sont devenues chroniques, elles sont naturellement plus difficiles à combattre que peu de temps après leur apparition, et bien souvent elles deviennent incurables. D'autre part, les maladies peuvent se transmettre aux descendants par la greffe et se perpétuer ainsi tout aussi facilement que les anomalies et les panachures qui du reste, aux yeux de beaucoup de savants, ne sont elles-mêmes que des maladies.

Cette transmission de certaines altérations maladives par la voie de la greffe ne justifie que trop les grandes précautions dont il faut s'entourer dans le choix des scions à greffer. Et pourtant beaucoup de jardiniers se montrent encore à cet égard d'une négligence impardonnable.

C'est un point de la plus haute importance pour l'avenir d'une plantation. Ne sait-on pas que le jeune arbre issu d'une greffe pent hériter, jusqu'à un certain degré, des moindres particularités individuelles du pied-mère. Je l'ai déjà démontré en citant l'exemple de pêchers réussissant en plein vent en Belgique et dont la culture sous cette forme n'est possible que pour autant que les arbres soient issus de noyaux on de greffes prises elles-mêmes sur des pieds cultivés en plein vent. Qui ne connaît ces notables différences entre individus de la même variété, ce qui fait dire à quelques jardiniers qu'il y a plusieurs sortes de Beurré rance, de $B^{r e ́}$ Diel, de $B^{r e ́} g r i s$ etc., différences qui sont dues uniquement au traitement cultural subi par ces arbres, et qui se fixent plus ou moins à la longue et deviennent presque héréditaires? „Il y a," dit un praticien 
bien accrédité, M. NoIserte, ,des Reinette franche moins grosses, plus allongées, dénuées de côtes, plus ou moins marquées de taches rousses; d'autres sont aplaties, jaunâtres, tirant sur le gris, piquetées de brun, marquées de taches rousses; enfin cette Reinette a autant de rariétés que la Reine Claude, et il faut choisir avec autant de soin l'arbre sur lequel on prend des greffes pour ne pas s'exposer à greffer une qualité inférieure." Il est bien possible que quelques-unes de ces sous-variétés soient issues de semis, mais il n'en est pas moins vrai que beaucoup d'entr'elles sont dues à l'influence de la culture.

Voilà pourquoi il n'est pas indifférent de prendre pour porte-greffe un jeune ou un vieux pied; les rameaux de celui-ci ne fourniront pas d'arbres aussi vigoureux et d'une durée aussi longue que ceux provenant d'un jeune arbre. En revanche, ces derniers ne se mettront pas aussi vite à fruit qui ceux issus de greffes, prises sur des arbres ayant atteint l'âģe de puberté et déjà entrés dans la période de production régulière. Même d'un seul portegreffe on peut obtenir des résultats tout différents, selon que les greffes sont détachées d'une branche à direction droite et verticale ou de l'extrémité horizontale des ramifications épuisées par de grandes récoltes, comme cela se rencontre fréquemment sur les arbres des vergers.

Dans le premier cas, on obtiendra des arbres rigoureux et sains; dans le second, les jeunes sujets hériteront de la faiblesse végétative de la branche sur laquelle les greffons ont été cueillis. Il y a certainement des exceptions à cette règle, mais elles ne la détruisent pas.

Le palissage contre un mur bien exposé étant une cause d'énervement, il en résulte clairement aussi la nécessité de ne jamais prendre de greffes sur des arbres élevés dans ces conditions, encore qu'ils se présentent avec tous les caractères désirables de santé et de vigueur et que les jeunes pieds qui en proviendront, soient destinés eux-mêmes à la culture en espalier. 
$\nabla_{\text {AN }}$ Mons n'admet pas que la détérioration des arbres fruitiers puisse être attribuée à la multiplication répétée par la greffe. Je suis également d'avis que l'opération de la greffe elle-même n'a pas cette conséquence; mais on ne peut raisonnablement nier qu'un choix inintelligent des scions à greffer ne doive contribuer à cette détérioration dans un grand nombre de cas. Je dirai plus. Pour moi l'influence réciproque du sujet et de la greffe ne fait pas de doute, en ce sens que la détérioration sera d'autant plus rapide que la différence entre le sujet et la greffe sera plus grande sous le rapport de la végétation, comme sous celui de l'analogie botanique. Je m'explique. En règle générale, les variétés de poires cultivées sans interruption sur sujet de coignassier, lequel appartient à une espèce différente du poirier, arrivent beaucoup plus vite à la décadence que celles qui ne se multiplient que sur franc ou sur sauvageon.

Si l'on trouve aujourd'hui une variété du siècle dernier, ou du moins un individu de cette variété n'offrant pas les caractères de la décadence, on peut être certain que c'est un arbre sur franc ou sur sanvageon. On peut observer, dans nos jardins, un grand nombre de variétés de création relativement récente, parmi les gains de vaN Mons entre autres, portant tous les signes de dépérissement. Etudiez les faits, remontez à leur origine, vous verrez presque chaque fois qu'il s'agit de variétés greffées trop exclusivement sur coignassier.

D'un autre côté, l'emploi continuel de sujets de constitution plus faible que la greffe, doit finir également par exercer une action affaiblissante sur la constitution des individus. Il n'y a rien d'étonnant à ce qu'une variété de pommier cultivée sur paradis pendant une série de générations ne fournisse plus dans la suite qu'une croissance langoureuse, rachitique, si on la porte sur haute tige de pied franc. Il est vrai qu'il y a ici une double cause d'affaiblissement; car les arbres nains, élevés dans nos jardins 
sur paradis, doucin et coignassier, se trouvent évidemment plus favorisés par rapport ì l'action des agents météoriques que les arbres de haut vent et l'on a vu que les végétaux s'habituent à ces conditions privilégiées.

Le fait qui précède a été également recomnu par van Mons; mais Poiteau l'explique autrement dans sa Notice historique sur la théorie du pomologue belge. Voici comment il s'exprime: „Une greffe prise sur un pommier greffé sur paradis, sur un poirier greffé sur coignassier, réussit mal placée sur un arbre franc. L'examen démontre en effet que si le paradis et le coignassier rendent les greffes plus précoces, et donnent souvent un plus gros volume au fruit, ils altèrent la vigueur de l'arbre et abrégent sa vie en ne lui fournissant pas assez de nourriture, et il est aisé d'en conclure qu'une greffe prise sur un tel arbre est déjà altérée."

Quelle que soit d'ailleurs l'explication que l'on admette, la conséquence pratique à tirer de l'observation précédente est qu'il faut, autant que possible, prendre des greffes sur des arbres greffés sur francs ou sur sauvageons, de préférence à ceux greffés sur sujet différent.

Les faits d'altération que je viens d'énumérer ne sont pas exclusifs aux variétés qui sont propagées par la voie de la greffe; ils se font également sentir chez celles qui se reproduisent habituellement par les autres procédés de multiplication artificielle. Je n'étonnerai personne en disant que, parmi les sortes de vignes de table les plus répandues, on peut aussi bien que chez certaines poires distinguer des sous-variétés, ou si on l'aime mieux, des formes à fruits plus ou moins gros, plus ou moins savoureux et d'une fertilité plus ou moins grande.

Il est vrai que ce sont bien là des particularités individuelles produites sous l'influence de la culture, mais il n'en est pas moins certain qu'elles se sont pour ainsi dire fixées à l'aide du bouturage et par un traitement favorable longtemps continué et analogue à celui qui a développé 
ces particularités. L'impression qu'un mode de culture peut exercer sur la constitution des végétaux est très-profonde. Oserait-on mettre en doute, par exemple, qu'une vigne de Frankenthal, obtemue de bouture d'un pied cultivé pendant un grand nombre d'années dans une serre à châssis fixes, mûrira plus difficilement ses fruits, si on la plante à l'air libre, même à bonne exposition, qu'un autre individu de la même variété provenant de bouture d'un arbre n'ayant jamais joui de l'abri artificiel d'un vitrage?

Tous les auteurs qui ont traité de la culture des arbres fruitiers déconseillent l'emploi, comme sujets, des drageons qui poussent au pied des pruniers, parce qu'ils présentent toujours eux-mêmes dans la suite l'inconvénient de drageonner constamment. Ne serait-ce pas encore un effet de l'habitude? Voyez à la souche des anciennes variétés de framboisiers, les nombreux rejets qui affament chaque année le sol et les branches à fruits. Chez les variétés nouvelles ce drageonnement est beaucoup moins considérable, ce qui met souvent un obstacle à leur multiplication rapide; mais précisément le mode adopté pour celle-ci tend à développer de plus en plus l'inconvénient qui vient d'être signalé.

Il résulte donc de là que le choix des pieds-mères pour les arbres propagés par bouturage et par marcottage, n'est pas moins important que celui des porte-greffes pour ceux que l'on multiplie par greffage.

De l'ensemble des faits que je viens de passer rapidement en revue, on ne peut, il est vrai, tirer la conclusion que toutes les variétés sont inévitablement destinées à s'éteindre un jour et à disparaître de nos jardins, accablées sous le poids des infirmités qu'elles auront contractées à la longue dans nos cultures artificielles; car une variété comprend d'ordinaire un certain nombre d'individus, et il faudrait que tous ces individus, tous indistinctement, fussent soumis à la fois aux causes qui peuvent 
provoquer leur décadence. Or, c’est précisément parceque ces causes agissent, suivant les circonstances et le mode de culture, d'une manière plus intime et plus rapide sur les uns que sur les autres, que nous voyons dans la même variété, ainsi que cela a été constaté, des individus ressentir tous les effets d'un décrépitude avancée, tandis que d'autres, plus rapprochés des véritables conditions naturelles, semblent être soustraits aux mêmes effets. Voilà pourquoi il est de toute impossiblité, quelle que soit d'ailleurs l'opinion de van Mons, de déterminer la durée d'une variété, fût-ce même l'une manière approximative. Mais d'autre part, comme les procédés horticoles tendent de plus en plus à nous éloigner de la nature et augmentent ainsi dans la même proportion les causes d'affaiblissement auxquelles les arbres fruitiers sont sujets, je suis arrivé à cette conviction qu'il est de toute nécessité de renouveler continuellement les variétés au moyen du semis, qui est la seule voie de reproduction naturelle. Les procédés artificiels de propagation produisent les. végétaux avec leurs qualités, mais aussi avec tous leurs défauts; le semis seul possède la faculté de donner des sujets de nouvelle formation, exempts de ces infirmités prématurées, causées par une culture intensive et par cela même affaiblissante.

On doit encore considérer la question sous un autre point de vue. S'il y a des variétés qui se comportent très bien dans des conditions tout opposées, il y en a beaucoup plus qui se localisent, e'est-à-dire, qui ne s'adaptent qu'à certaines localités et qui ne réussissent qu'incomplètement dans des contrées voisines, quoique placées dans des conditions non moins favorables. L'excellente pormme de court-pendu, si renommée en Belgique, n'est pour ainsi dire pas connue en France, où d'ailleurs on l'apprécie bien moins que chez nous; la Ribston Pippin, la pomme de prédilection des Anglais n'a pas non plus en France les hautes qualités qui la distinguent de l'autre côté de la Manche. Et pour citer un dernier exemple dans le même grenre de 
fruits, nous sommes loin de nous enticher comme les Allenunds de leur pomme de Borsclort, dont ils font tant de cas.

Eh bien, c'est parce que le déplacement, on pourrait presque dire l'acclimatation d'une excellente variété, d'un climat, d'une localité, d'un terrain, dans un autre climat, dans une antre localité, daus un autre terrain offre tant de mécomptes, qu'il y aurait, suivant nous, tont an moins utilité à créer des variétés plus en rapport avec les conditions de chaque climat, le chaque localité, de chaque terrain, en d'autres termes, des variétés essentiellement rustiques, puisque ce seraient des variétés locales. Il n'y a qu'un seul moyen d'arviver à ce résultat, c'est toujours le semis. Je pose en fait qu'un arbre issu de pépin, élevé en verger ou en plein champ et nun dans une situation privilégiée, et qui réunirait par supposition toutes les qualités du Passe Colmar serait infiniment préférable à un individu quelconque de cette variété, obtenu de greffe. Cenx qui admettent à quelque titre que ce soit la décadence des variétés fruitières, doivent évidemment admettre ce principe. Mais on dira: tout ceci est de la théorie, comment appliquer ce principe en pratique? C'est ce qui me reste à démontrer.

Et d'abord, ne doit-on pas convenir qu'il est vraiment fâcheux de voir recourir si rarement au semis pour propager les pêchers, les abricotiers, les prumiers et les cerisiers, dont plusieurs variétés se reproduisent plus ou moins identiquement de noyau. Dans certaines parties cies EtatsUnis de l'Amérique, où le mode de propagation par semis est pour ainsi dire suivi exclusivement, la culture du pêcher a pris une extension considérable et certains cultivateurs y possèdent des vergers plantés de 10,000 à 20,000 pieds.

Il est vrai que la plantation n'exige pas de grands frais. On se contente tout bonnement de confier au sol des noyaux de sortes commues, cultivées elles-mêmes en francs de pied. On a obtenu ainsi des races de pêchers 
d'une vigueur et d'une fertilité extraordinaires, parmi lesquels on voit fréquemment fleurir et fructifier de jeunes sujets de semis âgés de deux ans à peine.

Pour ce qui concerve les arbres à fruits à pépins, ne pourrait-on obtenir de même, à l'aide de certains procédés, des variétés se reproduisant exactement de graines, ou à peu près? Je pense que cela n'est nullement impossible. Cette manière de voir est cependant loin d'être partagée par les arboriculteurs et par les pomologues; ils sont au contraire pour la plupart du même avis que certain professeur dont parle M. DE LiRon D'Airolles dans un des volumes de ses Notices pomologiques, lequel étant consulté par un amateur sur la question de savoir s'il n'était par possible que deux ou plusieurs arbres fruitiers venus de semis, - des poiriers ou des pornmiers en particulier, pussent donner identiquement le même fruit que l'arbre mère des pépins semés, ou de tel autre fruit dont le pollen étranger aurait fécondé le fruit-mère, avait répondu: „Non, cela ne se trouve pas."

Et pourquoi, je le demande, la reproduction identique serait-elle impossible chez les fruits à pépins, tandis qu'elle serait possible chez les fiuits à noyau? Peut-on supposer que la nature se mette ainsi en contradiction avec ellemême? Cela n'est pas admissible. Si donc il y a contradiction apparente, elle doit avoir évidemment sa source, ou bien dans une observation inexacte des faits, ou bien dans une fausse appréciation des faits observés.

Pour mieux faire comprendre ce point, il est nécessaire de rechercher quelles peuvent être, chez certaines espèces de plantes cultivées, les causes de la dissemblance que l'on observe entre les produits d'un semis et la plantemère, et même entre tous les individus provenant de ce semis.

La reproduction identique constitue la règle chez les êtres à l'état de nature. Végétaux et animaux sont dans le même cas. En règle générale tous les individus issus 
du semis d'une plante sauvage sont semblables entre eux. Il en est de même chez les animaux que l'homme n'a pas soumis à la domestication. Ici également tous les individus de la mème espèce et du même âge se ressemblent au point de ne pouvoir être distingués les uns des antres. Voyez les renards, les lièvres, les perdreaux, les cailles ete. Tous ont le même pelage, le même plumage. Les exceptions sont excessivement rares. Dans l'état domestique, c'est tout le contraire qui se présente. Chez le lapin, le chien, le chat tous les petits de la même portée sont dissemblables, quoique provenant des mêmes parents et il en est ainsi chez tous nos animaux domestiques. Si chez certains individus quelque caractère, quelque forme ou couleur se montre avec constance, c'est que les deux parents possédaient aussi ce même caractère. Au surplus, c'est l'hérédité qui constitue les races chez les animaux, mais pour la maintenir il faut l'intervention de l'homme, ou bien il faut que les races soient isolées daus une région de façon à rendre le croisement impossible.

Les causes, qui font varier les animaux domestiques, peuvent être assimilées à celles qui établissent la variation chez les végétaux. Ceux-ci toutefois sont beancoup plus faciles à soumettre à la culture, j'allais dire à la domestication, ce qui est en définitive une seule et même chose. Quand on commence à cultiver une plante nouvelle, on n'observe que peu on pas de changements dans les premiers semis, mais dès que la culture s'élargit, dès que la plante vient à être placée dans des sols de nature différente et sous des climats différents, on verra se produire peu à peu des variations, par suite de la tendance du végétal à se mettre en harmonie avec le milieu nouveau où il se trouve transporté. Les variations, très légères d'abord, s'accentueront de jour en jour davantage, à mesure que les conditions culturales s'éloignent des conditions naturelles, qui provoquent la végétation spontanée. On voit que la variation est un premier résultat de l'intervention 
humaine. Si plus tard plnsieurs de ces rariétés sont réunies dans un même lien, ou se trourent rapprochées de telle manière qu'ì l'éporpe de la florajson les abeilles, les insectes, puissent opérer entre elles toutes sortes de croisements, en transportant d'une fleur à l'autre le pollen qui s'attache à leurs corps, la tendance de la nouvelle génération à produire des formes variées sera considérablement augmentée. De plus cette tenrlance, cette variabilité - c'est ainsi qu'on la désigne - ne fera que s'accroitre, au point de continuer à se produire même après que la fécondation croisée amra été interrompue. C'est ce qui a dû se passer et se passe encore chez nos arbres fruitiers. Ici malheureusement, comme chez tuntes les plantes ligneuses où les semis mettent un grand nombre d'années à fructifier, les observations de cette nature sont extrêmement difficiles à suivre; mais les résultats obtenus avec les plantes ne peuvent laisser aucun doute à cet égard.

J'insiste surtout sur ce point, que la viariabilité excitée à un certain degré par plusieurs croisements consécutifs peut continuer ses effets, alor's même que les individus sư lesquels on opère ont été isolés, rappelant ainsi l'étrange fécondité des femelles de pucerons, qui peuvent donner naissance à dix générations successives par une seule fécondation. Qui ne comnait les Calcéolaires herbacées mises en vogue par l'établissement van Houtte depuis une vingtaine d'années? En semant des graines de la collection vas Houtre, on obtient dans le même semis toutes les panachures de couleur, tous les tons possibles du jaune le plus vif au ronge le plus sombre. On trouverait difficilement deux plantes identiques. Cette immense diversité est la conséquence de la fécondation croisée, opérée avec soin chaque ammée sur des centaines de porte-graines choisis parmi les types les plus opposés. Eh bien, si l'on isole un individu quelconque d'un semis pareil, atin de le soustraire au croisement par un pollen voisin, et qu'on en sème les graines, on obtirndra encore l'amnée suivante une 
générution très-variée. Ce qui prouve incontestablement que l'influence de la fécondation croisée ne se manifeste pas uniquement sur une seule grénération. On conçoit néanmoins que plus on s'en eloigne, plus cette influence va s'affaiblissant, et voilà pourquoi les plus belles collections de fleurs, propagées par graines, dégénèrent lorsque les porte-graines ne sunt pas suumis à une sélection judicieuse et fécondés les uns par les autres.

Par ce qui précède on peut s'expliquer l'extrême variabilité de nos arbres fruitiers, dont on réunit habituellement plusieurs variétés dans le même enclus. Il n'est pas difficile non plus de compremire comment les graines d'un arbre isolé peuvent foumir.par le semis toute une génération de sujets aussi différents d'aspect entr'eux que $\mathrm{du}$ pied-mère, en admettant mime que ses fleurs n'aient pu recevoir le contact d'un pollen étranger apporté par le vent ou par les insectes.

Les effets de la variabilité sont réellement surprenants, et van Mons a pu croire que la descendance d'une variété produite de graine ne ressemble jamais à la mère; seulement il a eu tort d'ériger cette opinion en principe.

Il est vrai que dars le semis de pépins d'une poire d'hiver on rencontre des individus arrivant à maturité en été et en automne aussi bien qu'en hiver. M. le comte Odar' (1) eite le fait d'un plant issu par semis d'un cepage à grains nuir's, qui a donné des raisins blancs. La variabilité a produit des métamorphoses plus curieuses encore. Ainsi des observations positives faites en Angleterre et aux Etats-Unis ont fourni la preuve que toutes les races de pêches sont de simples variétés d'une même espèce; les brugnons et les violettes ou pêches lisses ne sont done nullement des hybrides comme certains auteurs l'ont supposé. La pêche lisse de Boston, la plus grosse et la plus belle des pêches lisses, provient d'un noyau de

(1) Ampélographie universelle, Introduction, pag. 25. 
pêche ordinaire. La contre-épreuve a été faite. MI. Rrvens, l'habile pépiniériste anglais, qui se livre à des semis nombreux dans les serres-vergers, a pu constater que des noyaux du Brugnon blanc et de la Pavie de Pomponne ont domné naissance de part et l'antre à des pêches proprement dites (1).

Je pense qu'il existe pour nos arbres fruitiers encore une autre cause de variabilité dont la tendance serait de les éloigner de l'améliorntion, et cette cause n'est autre que le greffage r'épété sur sujetśs de nature différente. Voici une observation qui me le fait supposer. Nous possédons en Belgique quelgues variétés de pêches qui se reproduisent de noyau, entre autres la pêche d'Oignies et le Brugnon de Féligny. Toutefois la reproduction ne serait identique que lorsque les noyaux proviemnent d'arbres francs de pied et l'on observerait an contraire une certaine variation chez les individus issus de noyaux récoltés sur pieds greffés. Bien plus, cette variation aurait le plus souvent pour effet d'éloigner le fruit de la voie de perfectionnement. On sait qu'en Belgique les pêchers sont généralement greffés sur prunier. Ce serait donc à l'influence de celui-ci qu'il faudrait attribuer l'espèce de déggénérescence qui s’imprime au fruit, ou plutôt ì la graine. Si le fait que je viens de relater est positif et, en tous cas, il mérite d'être confirmé par de nouvelles expériences, il nous domnerait le mot de ces assertions si contradictoires de la part de nos praticiens les plus consciencieux, dunt l'mu assure qu'mne telle viriété se reproduit exactement de graines ou de noyaux, tandis qu'un autre prétend tout le contraire.

Quoiqu'il en soit des causes de la variabilité, il en résulte toujours que les conlitions ordinaires de la culture ont nécessairement pour conséquence d'exciter sans cesse

(1) Pendant que je corrige les épreuves le ce traviil, les fiits que je viens de citer reçoivent une confirmation toute nouvelle de la part de M. CARrière, l'habile chef des pépinières du Museum de Paris, dans un article publié dans le No. du 10 Février du Journal de la ferme et des maisons de campanne. 
cette variabilité et que, si l'on tient à ce que les plantes d'un semis conserrent quelque chose des qualités du piedmère, il faut avant tout empêcher celui-ci d'être fécondé par le pollen d'autres variétés; en second lieu, qu'il faut récolter la graine sur un arbre franc de pied ou bien greffé sur une essence de même nature, ainsi poirier sur poirier, abricotier sur abricotier, etc. Quant à l'influence de eroisements antérieurs, il n'y a naturellement rien à y faire, mais cette influence diminue à chaque génération, et sur certains individus il se peut même qu'elle soit à peu près nulle. Il faut qu'il en soit ainsi, par exemple, chez les rariétés d'arbres à fruits à noyau qui se reproduisent identiquement par semis. Chez les arbres à fruits à pépins cette influence ne reste pas non plus toujours prépondérante. Ainsi certaines variétés de poires montrent de la tendance à conserver dans leur génération les qualités qui les caractérisent. Des semis de l'Orpheline d'Enghien, opérés dans l'établissement de M. Rrvers cité plus haut, ont donné des sujets différant du pied-mère à peine par l'époque de la maturité des fiuits. Une remarque analogue a été faite pour le Passe-Colmar, dont les descendants ont présenté pareillement les excellents caractères. Je sais très bien que l'on révoquera en doute la possibilité des faits avancés par M. Rivers. La production de fruits identiques sur arbres de semis différents, chez les arbres à fruits à pépins, est contestée encore aujourd'hui par la majorité des pomiculteurs. Mais cela ne signifie rien, on a longtemps nié le mouvement de la terre. On finira aussi par s'incliner devant les preuves, et celles-ci ne manquent pas.

L'identité remarquable (1) entre la Médaille d'or et le Frédéric de Wurtemberg ne prouve-t-elle pas, ainsi que le

(1) Je dois faire olsserver ici qu'en parlant d'identité je n'entends nullement une identité absolue, rigoureuse, mais simplement une ressemblance dans l'ensemble des caractères. On sait parfaitement que l'identité complète est impossible; on ne trouverait pas sur le même arbre deux feuilles identiques dans le sens absolu du mot. 
constate M. Royer, que la nature se répète quelquefois malgré ses ressources infinies pour varier ses productions. M. De Lrron d'Airolues dit qu'un de ses amis de la Vendée a obtenu un Bézy Quessoy d'hiver identique d'un semis de pépins de cette poire. Comment d'ailleurs expliquer l'analogie frappante du Beurré comice de Toulon, obtenu de semis par M. Flory de Nantes, avec la Poire de Curé, et celle de la poire $I d a M$ Muller, gagnée en Allemagne, avec notre Fondante des Bois trouvée sauvage aux environs de Grammont? Celle-ci avait déjà une doublure dans le gain que van Mons dédia au savant chimiste anglais Davy. Elle vient d'en trouver une autre dans une nouveauté, récemment mise dans le commerce par un horticulteur de Stuttgart sous le nom de Neue Stuttgarter Butterbirne et qui ne diffère de notre Fondante des Bois que par une maturité plus précoce de quelques jours. Contestera-t-on à van Mons le gain de la Calebasse Bosc, parce qu'il se trouve qu'elle reproduit entièrement le $B^{\text {rt }}$ d'Apremont, dont l'arbre-mère plus que séculaire existe encore à ce qu'on dit dans la localité de ce nom. Il me semble que si l'on doit admettre que le même fruit puisse provenir du semis de variétés différentes, ainsi qu'il résulte des expériences du savant professeur du muséum de Paris, M. Decaisne, relatées dans son beau mémoire, sur la Variabilité du Poirier," à plus forte raison les graines de la même variété pourront-elles donner naissance à des individus -offrant plusieurs caractères analogues. On n'a même nullement besoin de supposer l'intervention du pollen d'une variété d'hiver pour expliquer l'analogie de la Louise bonne de printemps de M. Borsbunes avec la Louise bonne d'Avranches, dont elle a exactement les feuilles, la couleur du bois, la vigueur, la fertilité et le fruit (à part quelques légères différences).

Ces cas de reproduction identique ou à-peu-près identique chez les poiriers sont peut-être beaucoup moins rares qu'on ne pense, mais par malheur, jusqu'à présent, on n'en 
a pas tenu note avec assez de soin. C'est ainsi que la poire Président Payen, nouvellement gagnée en France, a pour type le $B^{\text {re }}$ Capiaumont, qui jouit depuis si longtemps d'un grande estime dans notre province du Hainaut et que la Gloire de Binche n'est pas autre chose qu'un B' r' $^{\prime}$ 'Har. denpont renouvelé, mais beancoup moins délicat que ce dernier.

On doit le dire, il est regrettable que les observations à ce sujet n'aient pas toujours été consignées. Souvent même lorsqu'un semis reproduit une variété déjà connue, on le supprime pour éviter le double emploi. M. BorsBUNEL, qui est un des plus heureux semeurs français, a obtenu dans ses semis une poire qui présente les plus grands rapports avec le Bézy d'été ou Milan de la Beuvière. Il n'a pas voulu la nommer, dit-il, parce qu'il est persuadé que mise en culture on la confondrait facilement avec l'ancienne variété. A ce point de vue, il a parfaitement raison; c'est embrouiller la nomenclature, qui est déjà un vrai labyrinthe, que de donner un nom nouveau à une variété offrant une analogie complète avec un fruit déjà connu, mais, d'un autre coté, il est à remarquer que si la décadence des anciennes variétés est un fait dont il faille tenir compte, il y aurait évidemment avantage à conserver ces nouveaux gains, sauf à les désigner sous une dénomination rappelant leur parenté, par exemple Bézy d'été de Boisbunel ou bien Bézy d'été nouveau.

Je trouve dans les Notices pomologiques de M. DE LIRoN D'Arrolles un autre fait de reproduction identique, que je considère comme un argument de grande valeur. ,Un jardinier de Nantes a obtenu, dit cet auteur, du même semis de pépins variés de poires deux sujets qui ont donné identiquement le même produit, à deux années de distance; l'un a commencé à fructifier en 1859, l'autre en 1861. Les fruits de ces deux arbres ont été tellement semblables qu'on a décidé qu'un des sujets serait sacrifié et que celui qui resterait, conserverait la dénomination de 
Poire Chaigneau qui avait été donnée au moment du premier rapport en 1859, par la Société d'horticulture de Nantes (1)."

Je le répète, il est plus que probable que de pareils exemples de reproduction identique se sont présentés à différentes reprises, mais que la remarque en aura été négligée. Il serait beaucoup plus difficile encore de trouver quelques faits semblables chez le pommier, espèce fruitière dont on s'est moins occupé que du poirier au point de vue scientifique. Faut-il en tirer la conséquence que dans cette espèce la reproduction identique est impossible et que l'observation relative à la Pomme Reinette de Cuzy, se reproduisant par le semis avec toutes ses précieuses qualités sur le domaine des Chapuis (commune de Cuzy dans le département de la Nièvre), ne peut être que le résultat d'une erreur? Pour moi les circonstances culturales expliqueraient au contraire parfaitement la particularité remarquable qui distingue les pommiers de cette localité.

Le domaine des Chapuis est situé dans la commune de Cuzy sur un petit plateau rocailleux, au fond d'une vallée étroite, fermée de trois côtés par des montagnes et ouverte seulement au sud. Selon la tradition la Reinette de Cuzy y aurait pris naissance. C'est une pomme de haute qualité, paraît-il, pouvant se conserver d'une année à l'autre; sa réputation s'étend au loin. Aux Chapuis elle trouve les conditions qui lui conviennent le mieux, ce qui se comprend en quelque sorte vu qu'elle y est née. A Cuzy même on reconnaît que les meilleures pommes viennent $\mathrm{du}$ domaine des Chapuis. De tout temps il y eut là beaucoup de pommiers et selon toute probabilité tous de la même variété.

(1) C'est là, à mon avis, une précaution pour le moins superflue; car si les deux arbres se ressemblaient tellement dans toutes leurs parties, pouvait-on craindre que les produits de leur multiplication eussent différé? Et pourquoi supprimer un des deux arbres? A la rigueur on pouvait se contenter de ne prendre des greffes que sur l'un on sur l'autre. 
Les fruits ne se vendant pas, firute de débouchés, on en faisait autrefois du cidre, et le marc mélangé au fumier et répandu sur les terres a donné naissance à une quantité de jeunes sujets, dont la plupart étaient détruits par la culture, mais dont quelques-uns ménagés par la charrue, préservés par une roche ou défendus par une haie, existent encore. Un examen minutieux a montré en effet que ces arbres n'offrent aucune trace de greffe, tandis que ceux du reste de la commune de Cuzy sont tous greffés. Eh bien! voilà une situation pour ainsi dire isolée, où n'existe qu'une variété unique, où par conséquent la floraison se passe à l'abri de toute influence étrangère, circonstance excessivement rare dans nos jardins et dans nos vergers. Qu'y aurait-il d'étommant à ce que les semis aient pu y montrer une forte tendance à perpétuer les caractères du pied-mère et même à les perfectionner? Toutefois ce serait une erreur de s'imaginer, comme le fait M. Dolivat, l'auteur de la notice sur la Reinette de Cuzy, dont je viens d'emprunter les détails qui précèdent, qu'on obtiendrait partout le même résultat. Il me semble que la reproduction identique offrira beaucoup plus de chances avec les pépins provenant du domaine des Chapuis qu'avec ceux de toute autre origine.

On comprend quelle serait l'importance qu'offrirait pour la culture la fixation des variétés, ou du moins de certaines d'entr'elles, chez les arbres fruitiers. La reproduction par semis, le seul procédé vraiment naturel, car tous les autres sont artificiels, deviendrait générale et les meilleures sortes pourraient être cultivées dans les localités les plus opposées. La création de fruits nouveaux ne serait plus pour ainsi dire abandonnée au hasard; on pourrait déterminer d'avance la forme, la saveur, l'époque de la maturité des produits auxquels tendent les recherches des semeurs. Alors seulement la fécondation croisée aurait un résultat certain, tandis qu'aujourd'hui elle n'oftire pas plus de chances furorables 
que le simple semis, du moins chez les fruits à pépins. Comment admettre en effet que le produit d'un croisement puisse avoir de l'analogie avec ses parents, aloi's que le produit direct d'un semis présente les caractères de la plus grande variation. КNıGHт, qui a préconisé le premier le croisement pour l'amélioration des variétés fruitières, a mis en principe que ce procédé avait en général pour conséquence de mieux fixer, dans la génération issue de ce croisement, les caractères des deux parents, en constatant néanmoins qu'elle uffre toujours plus de ressemblance avec l'un des ascendants qu'arec l'autre. La manière de voir de KNıGHT s'explique en ee sens qu'il a pratiqué ses expériences en grande partie sur les diverses espèces de fruits à noyau, lesquelles présentent fréquemment, comme nous l'avons dit, une certaine tendance à la reproduction identique. Cela expliquerait aussi pourquoi, ainsi que cela paraît avoir été constaté aux Etats-Unis, les produits d'un croisement ont plus d'analogie avec le parent dont les caractères sont les plus permanents.

Je le répète, la fécondation croisée ne peut être appelée à jouer un rôle sérieux pour l'amélioration des variétés fruitières, que pour autant que l'on travaille sur des variétés dont les caractères montrent une certaine fixité dans le semis. Alors, mais alor's seulement on peut espérer un résultat certain, qui ne soit pas l'œurre du hasard. Ainsi je suppose un fruit à saveur particulièrement agréable, mais de dimension trop petite; si nous désirons retrouver cette saveur dans un fruit plus gros, il fuudra féconder les fleurs de cette variété avec le pollen d'une autre à fruits plus gros et vice versa. De même, si l'on désire obtenir un fruit tardif ayant quelque chose des qualités d'un excellent fruit d'été, on pourra croiser ce dernier, avec quelque chance de sucè̀s, au moyen d'un fiuit à maturité tardive. Et comme les semences, qui résultent d'un croisement, ne domnent pas précisément des arbres identiques entre eux, quoique participant des caractères de l'un 
et de l'autre des parents, il s'ensuit que ce procédé offre encore une assez large marge à lia variété des produits.

Il me reste à dire deux mots de la manière d'élever les sujets de semis, parce que le grenre d'éducation adopté par quelques semeurs peut aroir une action défarorable sur la constitution des variétés qu'ils obtiennent.

Van Mons, se basant sur sa propre expérience, a prétendu qu'une greffe ne fleurit pas plus tôt que le jeune pied-mère sur lequel on l'a prise. La plupart des semeurs belges, qui ont continué l'œuvre du célèbre pomologue, partagent la même opinion. Les arboriculteurs et les pépiniéristes sont au contraire presque tous d'avis que la greffe avance de plusieur's années la fructification des arbres. Je crois avoir trouvé l'explication de ce désaccord. Un arbre de semis, un poirier par exemple, abandonné à lui-même, atteindra l'âge de 15 à 20 ans avant de fructifier, plus qu'il n'en faut pour lasser la patience du plus enthousiaste semeur. On emploie lonc toutes sortes de moyens pour hâter la mise à fruit, et on y arrive le plus ordinairement en soumettant le jeune semis à une déplantation fréquente au moins tous les deux ans, en raceourcissant ses grosses racines, en supprimant même toutes celles qui montrent de la tendance à pivoter. Les semis ainsi traités peuvent fructifier au bout de 6 ì 8 ans. Eh bien, ce traitement exerce sur les jeunes arbres à peu près le même effet que la greffe d'un rameau sur un vieux pied; car si, au lieu de leur faire subir une déplantation fréquente, on les laissait tranquillement en place, nul doute que tout le monde ne fut d'accord sur l'influence exercée par la greffe sur la fructification.

Cette mutilation du système radical des pieds-mères de semis devrait être déconseillée. Pratiquée sur plusieurs générations successives, elle doit avoir évidemment pour conséquence d'affaiblir de plus en plus la taille et la constitution des individus. J'y rois la seule et unique cause d'un fait déjà remarqué par vas Mons que ses, ,nourelles variétés 
de poiriers ne possèdent pas la rusticité, la longévité des anciennes variétés.” Il dit aussi: „Je remarque que les plus jeunes variétés, les plus fines surtout, résistent moins aux ravages de la vieillesse, sont plus tôt vieilles que les variétés dont la naissance a précédé la leur; elles ne peuvent atteindre au-delì d'un demi siècle, sans que des symptômes de décrépitude se manifestent." En effet telle doit être la conséquence inévitable du mode d'éducation essentiellement affaiblissant adopté par lui et par ses imitateurs. Ces caractères de décadence prématurée ne s'observent pas chez les variétés qui ont été trouvées à l'état spontané dans une haie ou au coin d'un bois. Il ne faut donc pas ériger en système la déplantation, la mutilation des racines. Si l'on vent avancer la première fructification des arbres de semis, on en portera des greffes sur un arbre adulte qui est entré dans la voie de la production régulière.

Je me résume:

1. Les variétés fruitières ne conservent pas indéfiniment les qualités qu'elles possédaient dans le jeune âge. A mesure qu'elles vieillissent, elles s'énervent, se détériorent, s'affaiblissent, se présentent enfin avec tous les caractères d'une véritable décadence.

$2^{\circ}$. La décadence est d'autant plus rapide que les procédés de culture sont plus artificiels, et comme ils le sont tous plus ou moins et d'autant plus que la culture est plus perfectionnée et plus productive, il est de toute impossibilité d'assigner des limites à la durée des variétés.

$3^{\circ}$. La restauration des anciennes variétés ne paraît pas impossible en certains cas; mais en général il est plus simple et plus facile de créer par le semis des variétés de qualité au moins égale.

$4^{\circ}$ Le semis offre aussi le seul et unique moyen d'obtenir des variétés rustiques, d'un tempérament en harmonie complète avec les conditions spéciales de chaque localité.

5. Pour rendre praticable la création de plantations 
rustiques, il fandrait commencer par fixer la variation chez les fruits à pépins, comme cela existe déjà chez plusieurs variétés de fruits à noyan, c'est-à-dire, créer des races se reproduisant par la graine, identiquement ou à peu près.

6. Les procédés généralement usités pour hâter la mise à fruit des arbres de semis unt aussi pour résultat de hâter la décrépitude des variétés qui en proviemnent.

M. d'Anouilh de Salies fait observer que les arbres fruitiers, transplantés des autres parties de la France dans les environs de Paris, dégénèrent. Selon lui le meilleur moyen de prévenir la dégénérescence est le greffage. Il fait remarquer à M. van HaLl qu'il a eu de bons greffons pris de vieux sujets. Chaque partie d'un végétal a une vie qui lui est propre, et par conséquent on peut avoir de bons greffons pris de vieux sujets, fussent-ils même dans un certain état de décrépitude.

M. Koch (de Berlin) fait de même quelques remarques. L'orateux s'exprime en allemand à peu près en ces termes:

,Ich glaube, dass vor Allem der Begriff des Degenerirens festzustellen sei; nach dem, was ich annehme, ist man über die Deutung keinesweg's einig und verwechselt ganz verschiedene Zustände. Herr Pynaert hat zwar gelegentlich eine Definition gegeben, schliesslich sich aber doch nicht daran gehalten: die Etymologie kann hier keine Richtschnur geben, da mit der Zeit bekanntlich die Worte im Munde des Volkes ihre Bedeutung ändern. Es wäre demmach die Frage, was versteht man jetzt unter Degeneration? und was hat vor Allem der Fragesteller darunter verstanden? Nach meiner Meinung' ist unter Degeneration ein allnähliges Abweichen in der Form, verbunden mit einer Abnahme der regelrechten Funktionen, welche schliesslich zum Untergange führen, zu verstehen. Im Verlauf cler Debatte hat man aber auch die Fille bei unseren Obstbämnen als Degenerationen betrachtet, wenn ein Zurükgehen auf die ursprüngliche Form, wo diese dem wilden Zustaude der Pflanze 
sich nähert, vorhamden ist. Im naturhistorischen Simne findet hier aber gerade das Gegentheil statt, die Pflanze kehrt aus jhrem abnormen Kulturzustande zu ihrer natürlichen Beschatfienheit zurück. Anders wird freilich der Obstzüchter denken, dem gerade diese mehr oder weniger abweichende Form seiner Kulturpflanze Norm ist. In so fern kam er freilich auch das Zurückgehen in die Normal-Forn in seinem Simne eine Degeneration nennen.

Eine Degeneration der wahren Species, wobei diese schliesslich zu Grunde geht, giht es nicht, ist wenigstens noch nirgends nachgewiesen; dagegen können vermeintliche Species, also Abarten mit wesentlichen und hartnäckig anhaltenden Abweichungen, mit dem Augenblicke wo ihnen die Berlingungen zu der Abweichung fehlen, aufhören zu sein und sich verlieren. Alle unsere Kulturpflanzen haben ihren Normaltypus mehr oder weniger verändert, können demnach auch im Simne des Cultivateurs degeneriren, d. h. diesem sich nähern, resp. zu ihmm zurückkehren, sobald die Beding'ungen ilnrer Existenz aufhören. Die Species bewegen sich mit ilmen einzelnen Exemplaren in einem bestimmten Formenkreise, der durch die Kultur selbst noch grösser werden kann.

Die specielle Frage, ob unsere Obstbäume, in so fern sie bestimmte Sorten darstellen, degeneriren, resp. aussterben können, beantworte ich ebenfalls mit nein, in so fern man ihnen nur die Bedingungen, unter denen sie als solche existiren, gibt; ich stimme in dieser Hinsicht vollständig mitHerm Batter ä̉erein. Wenn ich nicht ire, hat KNrGHT zuerst die Behauptung aufgestellt, dass eine Obstsorte nicht länger danere, als der ursprüngliche Stamm, von dem die ersten Pfropfreiser genommen sind, existire, und nicht durch Altersschwäche \%u Grunde gehe. Wenn demnach der ursprüngliche Stamm einer Sorte eine Existenz von vielleicht 150 Jahren habe, so sei damit auch nach dieser Zeit die Sorte zu Grunde gegangen. Es 
würden, un ein Beispiel zu geben, alle Giavensteiner Apfelbäume nach 150 Jahren mit einem Male absterben, weil anzunehmen ist, dass der erste Baum, von dem die Gravensteiner stammen, in Folge von Altersschwäche nach dieser Zeit nicht mehr zu existiren vermag.

Die Erfahrung hat gelehrt, dass dieses ein ganz unrichtiger Grundsitz ist. Obwohl der Boi'sdorfer Apfel bereits mehre Jahrhunderte existirt, vegetiren unsere diese tragenden Bäume noch auf gleich kräftige Weise und werden sicherlich nach melneren hunder.t Jahren noch existiren, wenn man sie nur auf gleiche Weise pflegt. Dabei läugne ich nicht, dass gewiss mehre unserer älteren Obstsorten verloren gegangen sind, aber nicht aus Altersschwäche, sondern weil man sich, wo die Neuzeit bessere Sorten hervorgebracht hatte, nicht mehr die Mühe gab wie früher, und sie ihrem Schicksale überliess. Man sieht allerdings auch oft, dass Sorten nicht mehr gedeihen wollen. Untersucht man aber die Grïnde, so finden sich allerhand Ursachen, nur die nicht, dass sie sich überlebt haben. Klima und Boden haben mächtigen Einfluss auf die Entwickelung unserer sämmtlichen Kultuxpflanzen. Noch auffalliger ist dieses beim Gemüse, wo gerrisse Sorten sogar an bestimmte Lokalitäten grebunden sind. Die Teltower Rüber erhalten nur in der Ungegend ron Berlin ihre vorzüglichen Eigenschaften und degeneriren, (d. h. weichen von der Norm ab) in Paris und London, wo man sich grosse Mühe gegeben hat, un sie zu kultiviren. Wir wissen ebenfalls, dass einige Obstsorten in einer Gegend vorzügliche Früchte liefern, wälnend man in einer anderen behauptet, sie seien degenerirt. Anch dieses Lukalisiren, worüber ich mich schon früher an anderer Stelle ausgesprochen habe, ist ausserordentlich wichtig und rerdient unsere ganze Aufmerksamkeit. Hat man doch behauptet, dass auch unsere Kirtoffel degenerirt sei und almählig ganz und gar zu Grunde gehe. Unses'e Sorten hätten sich überlebt und man müsste sich neue aus Samen der wilden Pflanze 
erziehen. Die Erfahrung hat aber gelehrt, dass die auf diese Weise erhaltenen Sorten eben so der Krankheit unterliegen, als die anderen, während man neuerding's in Schottland gerade aus solchen sich selbst überlebten Sorten so vorzügliche neue erzogen hat, wie es aus SamenKartoffeln aus den ursprünglichen Vaterlande der Fall sein würde.

M. Nedzelsky (de Moscou) a la parole. Il fait observer que depuis longtemps il est admis dans la science que l'on peut multiplier les espèces pas le semis et les variétés par le greffige, la marcotte, le bouturage, etc. Il faut cependant se demander encore ce qui, parmi les Pomacées et les Anygdalinées, doit être considéré comme espèce, comme sorte, comme variété. Il n'est pas encore certain que les espèces, considérés jusqu'à présent comme telles, ne soient pas dégénérées, et que nos variétés aient été depuis leur origine toujours des variétés, ce qui semble impossible d'après les lois de la reproduction. D'après des indices qu'il a découverts dans la structure intérieure des fruits, l'orateur est parvenu à distinguer les espèces des Pomacées des variétés. D'après ces indices, certaines sortes doivent être considérées comme des variétés: ainsi la pomme d'étoile (Sternapfel) est une sorte, (et nous n'avons presque pas d'antres sortes qui aient quelque affinité avec elle) mais lorsqu'on en obtient de beaux et gros fruits, on les considère comme variétés. Par contre plusieurs espèces ne sont que des sortes, par exemple le Pyrus astracanica, dont on a beancoup de variétés. M. Косн vient de dire qu'il a vu en Pologne des fruits de Pyrus monifolia, qui étaient aussi gros que ceux de la Calville, mais cette comparaisun n'est que superficielle, apparente; elle est fondée sur la grosseur extérieure, mais non d'après la véritable distinction des caractères des espèces et des variétés. L'orateur lni-même a vu en Allemagne beaucoup de gros fruits, qu'on lui a présentés sous le nom d'espèces, mais qui n'étaient que 
des sauvageons, produits de pépins de variétés. Il est connu que, parmi les espèces, il $y$ en a dont sont issus le plus grand nombre de variétés, par exemple: Pyrus Malus tomentosa Косн, Pyrus Malus glabra; d'autres espèces comme Pyrus cerasifera, Pyrus prunitolia, Pyrus praecox ne donnent qu'un petit nombre de variétés, et Pyrus coronaria n'en donne aucune.

M. Battet a dit que, pour conserver de bonnes nouvelles espèces par le semis, on doit prendre des pépins de fruits bien formés de bonnes espèces. Il est cependant très-difficile de dire quel fruit oil doit considérer comme normalement formé, puisque presque tous les caractères, qui peuvent nous guider poụ distinguer les espèces l'une de l'autre, diffèrent beaucoup d'après l'influence du climat, du terrain etc. Le meilleur indice est le nombre normal des pépins dans chaque loge du finit. Ce nombre est de deux. Cet indice est considéré comme le meilleur par tous les pomolog'res qui s'attachent à obtenir de nouvelles variétés.

Revenant à la question qui est à l'ordre: la dégénérescence des arbres fruitiers, l'orateur se permet de faire observer qu’il a trouvé dans le discours du préopinant deux principes contradictoires. Le premier que, par une longue continuité de leur multiplication, surtout par le greffage sur des sauvageons qui n'ont pas grande affinité avec eux, les arbres fruitier's perdent leurs qualités caractéristiques pour les sortes, et en continuant encore cette multiplication, ces sortes nous donnent des variétés, qui sont fort souvent de beancoup plus mauvaises que le pied-mère dont on a pris les greffons. Le second, quand il dit que l'état maladif de l'arbre-mère se propage par le greffage.

Nous voyons done d'abord, que le sujet sur lequel on a greffé, a une grande influence sur la dégénérescence des sortes, mais que cette dégénérescence ne serait possible que si la sève du sujet pouvait transmettre ses propriétés 
aux fruits des surtes greffées. Ceci n'a pas lieu dans la nature.

Il est notoire jusqu'à présent que les poires greffées sur Pyrus Cydonia (le coignassier), et les pommes sur Pyrus pracox, nous domnent des arbres rabougris, parce que les sujets sur lesquels ils sont greffés, ne peuvent d'après leur nature former assez de racines pour tirer de la terre les sucs nécessaires à la nourriture des plantes. Il est reconmu que, quand on veut améliorer une espèce à bois noir ou brunâtre sur sauvageon ou sur bois blanc, la greffe reste toujours la limite entre les deux espèces de bois; mais si nous voulons améliorer des sortes qui ont été cultivées depuis Jongtemps comme arbres nains sur Cydonia on douçain, par greffage sur poiriel sauvageon, nous revenons pour certaines sortes à l'état naturel.

On a dit que l'état maladif des arbres fruitiers se propage par le greffage; ceci n'a lieu que lorsqu'on cultive les plantes dans des conditions qui ne conviennent pas à leur nature. Les plantes panachées peuvent sous ce rapport nous servir d'exemple. Si la maladie pouvait toujours se propager, si nous ne connaissions atucun moyen pour arrêter cette propagation, si elle était inhérente à lia nature des plantes, nous aurions vu disparaître déjà bien des sortes. Et cependant nous avons encore en culture les mêmes espèces qui le sont déjà depuis bien des siècles; nous avons encore des sortes qui sont toujours maladives par nature et très sensibles à l'action d'un climat qui ne lem convient pas. Nous voyons done que la propagation de la maladie a ses limites, à savoir l'amélioration des conditions sous lesquelles la plinte subit telle on telle maladic. Ces conditions sont le climat, le terrain, le sujet sur lequel on greffe etc.

M. de Beucker dit que la dégénérescence est souvent la conséquence du transplantement des plantes dans un autre terrain, quoique dans leur propre pays. Par le semis 
on obtient des variétés, car la vie du végétal est affaiblie non pas extérieurement, mais intérieurement.

M. Pynaert répond brièvement aux observations présentées par M. Nedzelskr sur l'influence du sujet sur le greffon et du greffon sur le sujet. Il soutient que le semis est le meilleur moyen de conserver les espèces intactes. Après une courte réplique de MI. Nedzelsix , M. Baltet dit que la liscussion, qui vient l'avoir lieu, cunfirme son opinion. Il présente encore quelques observations pour réfuter les orateurs précédents. Il clit entre autres que c'est une faute de greffer pêcher sur mirobolant; abricutier sur prunier réussit très bien. Le pêcher de Syrie ne se soutient pas lorsqu'il est produit de noyau. Il réfute brièvement M. Pynaert. Il admet que l'on peut obtenir de bonnes reproductions de pruniers par le semis, mais il croit que la reproduction par le greffage vaut mieux.

M. d'Anouilh de Salies fait observer qu'il est rare que les arbres reproduits par le semis domnent des fruits identiques.

M. Pynaert répond que la règle générale est que senlement les espèces se reproduisent exactement par des moyens artificiels.

M. Baltet mentionne un fait bizarre; il a vu un pêcher dont les fruits étaient demi prune demi pêche.

M. Koch certifie le cas; il a vu également des arbres dont les fruits étaient demi prune demi pèche.

M. Kolb rappelle que M. Косн, au commencement de la discussion, a parlé de la maladie des pommes de terre. Il mentionne que, dans les années citées par le préopinant, les plantes nutritives en Suisse étaient inaladives, tandis que les autres étaient saines. Il en conclut que la maladie ne doit être imputée qu'au terrain.

M. Nedzelsky fait encore observer que ce ne sunt pas seulement les fruits à pépins, mais aussi les fruits à noyaux qui varient. 
IV. MI. Stelzner (de Gand), dépose un travail sur „l'Hybridation des fougères" (1).

V. M. Karsten (de Berlin) domne un aperçu de ses travaux sur "la méthode de culture des Cinchona" et dépose le mémoire suivant:

Die Cinchonen, diese Gruppe reich und schön blühender Pflanzen, welche sich durch ihren ausgebreiteten Blïthenstand, so wie durch Frucht und Saamenbau characterisiren, haben schon seit längerer Zeit die Aufmerksamkeit der Blumenfreunde und Pflanzencultivatoren auf sich gezogen und sind schon mit Erfolg aus ihrem Vaterlande, sowohl wegen ihrer duftenden Blumen in unsere Pflanzenhäuser, als anch wegen ihrer heilkräftigen Rinden selbst, im Grossen unter entsprechenden climatischen Verhältnissen nach Asien übergesiedelt und angebaut worden.

Meistens bewohnen die Cinchonen die tropischen Gegenden und zwar vorzugsweise diejenigen Südamerika's, so anch ganz besonders die wichtigste von allen, die artenreiche Gattung Cinchona, welche, wie ich dies schon an anderen Orten nachwies (2), und wie dies auch Martius bestätigte (3), in mehrere nahe verwandte, von älteren Systematikern als Gattungen betrachtete Untergattungen gruppirt werden kann.

Diese, durch die Aehnlichkeit ihrer morphologisehen Charactere in die eine grosse Gattung. Cinchona vereinigten, Untergattmigen sind von einander dureh ihre physiologischen Charactere und dureh ihre Lebensbedingungen in so weit verschieden, dass einer jeden dieser Untergattungen ein bestimmter, wemn auch nicht scharf abge-

(1) Voir le Supplément.

(2) Kooı's Wochenschrift, 1859.

(3) Buchner's Repertorium, Band XII. 
grenzter, durch Wärme und Fenchtigkeit bedingter Verbreitungsbezirk angewiesen ist.

Die interessanteste, schon von Linné benannte und als Gattung bezeichnete Untergattung Cinchona (Quinaquina ENDL.) sucht die Nähe der beeisten Gebirgsgipfel, von deren äussersten, unter dem Aequator etwa 10,500 Fuss hoch belegenen Baumyrenze sie bis 6000 Fuss (unter dem $8^{\circ} \mathrm{N}$. Br. und $14^{\circ} \mathrm{S} . \mathrm{Br}$.) herabsteigt, in einer etwa $12^{\circ} \mathrm{C}$. betragenden mittleren Temperatur.

Die wärmste Gegend nehmen von den Pflanzen dieser Gattung die von de Candolle Remijia genannten Arten ein, welche, indem sie die heissesten und trockensten Gegenden verschmähen, in einer von etwa 600 bis zu 3000 Fuss Höhe über dem Meeresspiegel belegenen Zone vorkommen, die die Nebelregion liebende Cinchonen in einem Abstande von etwa 3000 Fuss umgürtet. Die mittlere Temperatur dieses breiten Gürtels, in welchem warme Regengüsse und heisser Sonnenschein in längeren Perioden wechseln, dürfte durch $18^{\circ} \mathrm{C}$. bezeichnet werden.

Zwischen diesen beiden verschwisterten CinchonenTribus wohnen die mit grossen, prächtigen, duftenden, meist weissen Blumen geschmückten Arten von Cascarilla Endl., Buena Poнl und Muzonia Wedd., welche die benachbarten Grenzen beider, je nach den Localitäten, mehr oder minder überschreitend einen etwa 2500-6500 Fuss hoch über dem Wasserspiegel belegenen Gürtel von 4000 Fuss Breite zwischen beiden eimnehmen. Die mittlere Temperatur dieser Zone beträgt gegen $15^{\circ} \mathrm{C}$.

Eben so wenig wie die Angabe der mittlern Temperatur dieser ausgुedehnten, vielen verschiedenen Arten angehörenden Zoneu ein auch nur einigermassen anschauliches Bild von dem eigentlichen Clima desselben giebt, eben so wenig kann sie dem Cultivateur als brauchbares Maass für die Behandlung seiner Pfleglinge dienen; denn nicht das mittlere Maass der Wärme, sondern die Art der Vertheilung derselben, bei direct oder zerstreut wir- 
kendem Lichte, und die gleichzeitig herrschende Feuchtigkeit der Luft und des Bodens sind die Factoren, welche in der lebensthätigen Pflanze das wechsehreiche Spiel der chemischen Combinationen ihrer sie zusammensetzenden Grundstoffe leiten und regeln.

Die für die menschliche Gesellschaft interessantesten der genannten Cinchonen-Tribus sind die Limneischen Cinchonen, welche sich durch die specifische Wirksamkeit ihrer Rindenbestandtheile geg'en alle intermittirenden Nervenleiden unseres Organismus als die grössten Wohlthäter des von Krankheiten heimgesuchten Menschengeschlechtes erwiesen und bewährt haben, und die sich wegen eben dieser unschätzbaren Eigenschaften des besonderen Schutzes der hohen Regierung erfieuen, deren Sinn für Kunst und Wissenschaft uns hier rereinigt.

Die letzt bezeichnetén Pflanzenarten, welche von Reisenden und Botanikern in neuerer Zeit mit besonderer Vorliebe beobachtet und studirt wurden, lassen sich in zwei, durch morphologische und physiologische Charaktere kenntliche Gruppen sondern, deren natürliche Standorte ich schon früher einmal zu schildern unternahm.

Die an organischen Basen reicheren Cinchonen, welche meist schon an ihren harten, mit Grübchen in den Aderachseln versehenen Blättern kemntlich sind, als deren Typus die C. Calisaya und C. lancifolia betrachtet werden kann, bewohnen die eigentliche Nebelregion der Andenkette, unter den bezeichneten geographischen Breiten, wo während der neun Monate anhaltenden Regenzeit beständiger Regen mit mehr oder minder dichtem Nebel wechselt, der nur bei Tage durch vorübereilende Sommenblicke unterbrochen wird; während in der dem Winter entsprechenden Jahreszeit kalte sternklare Nächte, in denen die Lufttemperatur unter den Gefrierpunkt herabsinkt, mit Tagen wechseln, in denen die bis auf $25^{\circ}$ die Atmosphäre erwärmenden Sonnenstrahlen dichte Nebel hin und wieder durchdringen, welche fist 
beständig auf dem mit Than benetrten Laube des Waldes lagern.

Untersuchen wir mun, welche Mittel uns zu Gebote stehen, ciesen Wechsel feuchter Niederschläge und erwärmenden Lichtes bei einer Lufttemperatur, die sich zwischen $0-20^{\circ}$ bewegt, welche Extreme aber nur von kurzer Dauer sind, hervorzubringen, so mag es erlaubt sein, zuerst die für die gesammte menschliche Gesellschaft so wichtige Cultur im Grossen, dann die von Kunst und Wissenschaft geforderte in den PHanzenhäusern zu betrachten.

Hinsichts der ersten Aufgabe, welche den Zweck hat, die kostbaren, in ihrem Vaterlande der Verwüstung preisgegebenen, medicinisch-wirksimen Cinchonen zu erhalten und zu vervielfaltigen, ist es, wie ich früher gezeigt habe, für die Erzielung einer an organischen Basen reichen Rinde von der grössten Wiclitigkeit, den Pflanzen den bezeichneten Wechsel ron Nebel und Sommenschein bei einer möglichst kühlen Lufttemperatur zu geben. Es wird dies am einfachsten erreicht, wemn die Pflanzungen am Abhange eines hohen, wo möglich auf dem Gipfel beeisten, von constanten, heftigen Luftströmungen möglichst freien Berges, oder einer Gebirgskette angelegt werden. Hier tritt der oben bezeichnete fortwährende Wechsel aufsteigender warmer, feuchter und herabfallender kalter Luft ein, durch deren Mischung die Nebel erzeugt werden, welche die ganze Pflanze allseitig benetzen, um bald darauf wieder, sobald die eine oder die andere der Luftströmungen die Oberhand gewann, von neuem durch die Sonnenstrahlen wieder erwärmt und getrocknet $\mathrm{zu}$ werden.

Je näher der beeisten Gebirgskuppe, desto häufiger wird der bezeichnete Wechsel eintreten, und desto grösser wird die Differenz der Erwärmung durch die Somnenstrahlen und der darauf folgenden Abkühlung durch Nebel und Reif. Unter diesen Bedingungen werden den Pflanzen mit den Thautropfen die luftförmigen Nahrungsmittel 
aus der Atmosphäre stets von neuem hinzugefülnt, und wird alsbald durch Luft und Wärme der Somne die Assimilation derselben ermöglicht und beschleunigt; dies sind die günstigsten Bedingungen, unter denen die Pflanze aus dem durch die warmen Luftströmungen zugeführten Gasen (Ammoniak und Kohlensäure) die stickstoffreichen, organisehen, krystallisirbaren Materien zu bilden vermag, welche sie für die Zeiten des Mangels aufspeichert, um dann dieselben für die Vermehrung ilırer organisirten Theile zu verwenden.

Wollte man die Pflanzungen nicht am Abhange, sondern auf dem Gipfel eines die Schneegrenze nicht erreichenden Berges anlegen, so würde hier gleichfalls ein Wechsel von Nebel, Wolken und Somnenlicht die Pflanze treffen. Aber in der Regel würde dieser Wechsel nur ein einmaliger während des Tages sein; denn die nach dem Erwärmen der Gebirgskuppe mit dem aufsteigenden Luftstrome aufwärts geführte Feuchtigkeit wird bald auf dem Gipfel selbst erst verdichtet und als Wolke so lange auf oder über demselben lagern, bis je nach der Höhe des Berges, unter verschiedener geographischer Breite, entweder die Kraft des aufsteigenden Luftstromes sich verringert und die Sonnenstrahlen, welche die Wolke treffen, diese auflösen und zerstreuen, oder bis dieselbe mit sinkender Somne als Regen herabfällt, um noch am Abend oder erst am folgenden Morgen den bildsam thätigen Sonnenstrahlen Zutritt zu den hungernden Blättern der Cinchona zu gewähren.

Die Cultur der Cinchonen in den Häusern, welche nicht in erster Linie das Aufspeichern von Nahrungsstoffen in der Pflanze bezweckt, sondern nur schöngeformte Blätter und Blumen zu erzielen beabsichtigt, verlangt wegen des lange verschleierten Himmels unseres nordischen Winters, gleichfills das Erstere nicht ganz unberücksichtigt zu lassen. 
Es möchte scheinen, dass diese Cultur für Blumen und Blätter nicht so energisch den oben beschriebenen Wechsel der atmosphärischen Verhältnisse erfordere, und allerdings entwickeln sich Cinchonen auch ohne die vollständige Erfüllung der von der Natur gelösten Aufgabe zur Blüthe; dennoch muss der Cultivatemr die natürlichen Lebensbedingungen, so viel es die Kunst vermag, seiner Pflanze zuführen, wrenn er eine vollkommene und vollständige Entwickelung derselben erzielen will.

Die eine der Bedingungen, die Wärme in der bezeichneten Menge zuzuführen, ist bei der jetzigen Einrichtung unserer Häuser olne Schwierigkeit zu erfüllen; anders ist es mit dem Sonnenlichte, welches unsere Cinchonen mit ihren Genossen, während des langen schaurigen Winters, in der Reg'el nur zu linge entbehren müssen. Spendet aber die Somne ihre wirksamen Strahlen auch unsern Pflanzen von neuem, wie vermitteln wir dann denselben die übrigen natürlichen Bedürfnisse?

Vielleicht erinnert sich bei dieser Frage mancher meiner Herren Zuhörer seiner vortrefflichen Patentspritze, mittelst welcher ex seine Pfanzen gleichfalls mit den zartesten Thautröpfchen zu bedecken vermag, und glaubt auf diese Weise mit Pröвus Hülfe der Pflanzennatur Genüge leisten zu können.

Dass aber dieser Glaube im Irrthume rubt, zeigt uns die Analyse des natürlichen Vorganges im Vergleiche mit der künstlichen Operation. Denn sind wohl die aufgespritzten Wassertropfen so völlig mit der Kohlensäure und deren Ammoniakverbindung gesättigt, wie die durch natürliche Abkühlung in der Luft verdichteten Nebeltröpfchen? Dies ist nicht zu rermuthen, vielmehr das Gegentheil vormuszusetzen, auch wenn die Luft der Pflanzenhäuser durch den stickstoffreichen Dünger ihrer Treibbeete mit jenen gasfömigen Nahrungsstoffen des Pflanzenreiches geschwängert wäre, und zweitens, was mehr noch in Betracht zu ziehen, wirken die während des Son- 
nenscheines auf die Pflanzenblätter gespritzten Wassertropfen, auch abgesehen ron ihrer chemischen Constitution, physikalisch ebenso wohlthätig wie jene nach und nach im Schatten der Nebelwolken auf die erkaltenden Blätter sich senkenden Thautropfen, die ebensowenig plötzlich von den durch die Nebelmassen wieder hindurchdringenden und sie endlich wieder zerstrenenden Sonnenstrahlen getroffen und verflüchtigt werden?

Beides, ganz besonders aber Letzteres, ist sicher nicht der Fall!

Das auf die Pflanzen gespritzte Wasser kamn auch, wenn es in noch so kleinen Tröpfchen durch die Brause gespritzt wird, und besonders wemn es, wie es doch für die Pflanzen wünschenswerth ist, annähernd dieselbe Wärme hat wie die ron der Some beschienenen Blätter, nicht die Quantitüt gasförmiger Stoffe, während sie die Luft durcheilen, aufnehmen, wie die sich langsam aus der Luft, in der sie gasförmig verbreitet waren, condensirenden Nebeltröpfchen.

Die Wassertröpfchen ferner, welche auf die von der Somme beschienenen Blätter gelangen, üben auf dieselben, wie dies durch die Praxis längst erkannt wurde, statt eines wohlthätigen, nur einen nachtheiligen Einfluss; sie führen sehr häufig die sehr treifend als, ,Verbrennen” bezeichnete Erscheinung herbei.

Wir sehen uns demmach genöthigt, Mittel aufzusuchen, durch die wir die im Freien stattfindende Nebelbildung nachahmen können; und in der That ist dies ohne grosse Schwierigkeit zu erreichen, da, wie bekannt, die Aufnahmefahigkeit der Luft für Wassergas bei zunehmender Erwärmung derselben zunimmt, und daher ein Theil des gelösten Wassergases ausgeschieden wird, wemn zwei mit demselben gesättigte Luftströme von verschiedener Temperatur mit einander sich mengen.

Je grösser die Temperaturdifferenz der beiden mit Wassergas gesättigten Luftströmungen ist, desto grösser 
wird die Quantität des sich tropfenförmig verdichtenden Wassers sein.

Durch das Oeffinen der Thüre und Fenster in den Pflanzenhäusern und Hineintretenlassen der äussern Luft wird im Winter daher sehr wenig, im Sommer noch weniger von der bezweckten Wirkung erreicht werden.

Schattendecken, angefertigt aus breiten, in bestimmten Abständen von einander entfernten Leisten, - welche wo möglich zu zweien, durch einen geringen Zwischenraum getrennt, übereinander liegen —, sind von grossem Nutzen um die anhaltende ïberreizende Wirkung unserer Sommersonne zu mässigen; sie werden jedoch nicht hinreichen, Nebel in der Luft des Pflanzenhauses herrorzurufen, vielmehr die Glasscheiben und Fensterstäbe in ihrer nächsten Nähe mit Than beschlagen machen, welcher auf die Pfanzen herabtroptt und zu der, ,Verbremen" genannten Erkrankung derselben Veranlassung geben kann.

Wir werden uns eines anderen, ein wenig umständlicheren aber doch sehr einfitchen Mittels bedienen müssen.

Dieses Mittel wird darin bestehen, dass wir den Pflanzen, ebenso wie wir ilmen Wasser zutragen oder zupumpen, ihnen auch Luft zuführen, und zwar durch Pumpen, deren Röhren mit doppelien Wandungen an der Nordseite des Hauses unter genügenden Schutze zweckmässig angebracht sind, und in dem obern Rum des Pflanzenhauses, oberhalb der Pflanzei, ihre bransenförmigen Mündungen haben.

Diese Luft muss möglichst lialt und mit Wassergas gesättigt sein; man wird sie aus dem Eiskeller des Hauses mittelst eines Rohres heraufziehen, an welchem noch durch zweckentsprechende Krümmungen und Erweiterungen Vorrichtungen angebracht sind, die Luft mit Wasser zu waschen, um ihr den wrisstmöglichen Wassergehalt zu verschaften.

Die in neuester Zeit construirten sogenamnten Eis- 
maschinen, durch welche mit Hülfe rerdunstenden Ammoniak- oder Aethergases Kälte erzeugt wird, können diese Vorrichtung sehr vereinfachen und die Operation ablï̈zen, nur ist damn noch mehr darauf zu achten, dass die kalte Luft nicht unmittelbar die Pflanzen trifft.

Bevor man die kalte Luft aus den Brausen hervortreten lässt, aus denen sie mit Schnelligkeit herrorströmen muss, sind bis zu ihmen die Röhren mittelst der hineingepressten Luft völlig abzukühlen.

Es werden diese Luftpumpen in Bewegung gesetzt, nachdem durch Heizen und durch Spritzen von Wasser an Wänden und Fussboden und durch die Hülfe der Sonne die Luft des Hauses dem Sättigungspunkte mit Wassergas sich möglichst genähert.

Hierdurch werden sich je nach der Temperaturdifferenz beider Luftmassen mehr oder minder dichte Nebel bilden, welche sich auf die Blätter der benachbarten Pflanzen niedersenken.

Wird z. B. ein Cubikmeter Luft von $+20 \circ$, in welchem 17,6 Gran Wassergas gelöst sind, mit einem Cubikmeter Luft von $0^{\circ}$, welches fast 5 Gran Wassergas enthält, gemischt, so werden fast $2 \mathrm{Cub}$. met. Luft ron $10 \mathrm{Grad}$ entstehen, welche gegen 19 Gran Wassergas enthalten; es werden also $3 \frac{1}{2}$ Gran Wassergas als ziemlich dichter Nebel tropf barflüssig niedergeschlagen.

Das gleichzeitige Ausbreiten der oben beschriebenen Schattendecken wird die Wirkung dieser Operation unterstützen, welche nicht allein diesen heilkräftigen Cinchonen ein nothwendiges Lebensbedürfniss befriedigt, sondern auch allen übrigen mit ihmen gemeinschaftlich die Nebelregion der Hochgebirge bewohnenden Gewächsen, ron denen hier nur die Baumfarne und Hymenophyllaceen, die Andromedeen und Vaccineen, die Laplaceen, Escallonien, Canellaceen, die Pleurothallideen und riele der schönsten Melastomaceen, (Meriania, Schwerinia, Chastencea) genannt sein mögen. 
Die den oben besprocheneu medicinisch werthvollsten physiologisch wie morphologisch ganz nahe stehenden, gross- und weichblättrigen grübchenlosen Cinchonen, die C. cordifolic, ovata, Tucujensis, und deren Verwandte, welche die wärmeren Gegenden der oben bezeichneten Region inne haben, und zum Theil schon mit den Arten der Cinchonen-Tribus: Cascarilla Endu., Buena Poнt und Muzonic WEDD. zusammenleben, - denen sich hinsichts der climatologischen Verhältnisse ihrer Standorte die Gattungen Cosmibuena Rurz et PAv., Mcnettia Mut., Hillia JAcQ., Lasionema Dor anschliessen, - verlangen schon für längere Zeit wärmere und durchsichtigere Luft.

Diese trocknere, wärmere Atmosphäre fordern aber noch dringender die zum Tribus Remijia D. C. gehörenden Cinchonen, welche mit vielen Arten der Gattungen Coutarea Aubl., Exostemma L. C. Rich., Alseis Sснотт, Bourardia Salisb., Pinkneya Rich., Calycophyllum D. C. ete. ihr Territorium theilen.

Diese Pflanzen bewohnen meistens die heissesten Gegenden der Tropen, in denen heftige Regengüsse während des Sommers den im Winter ausgedörrten Boden tränken und die Pfianzendecke zu nener Thätigkeit anregen; sie vegetiren im Sommer bei einer mittleren Temperatur im warmen Hanse olne Schwierigkeit; im Winter dagegen ist es oft nicht möglich, ihmen die erforderliche Menge Sommenlichtes zu verschaffen; und da sie in geschlossenem Hause, bei der ihnen nothwendig zu gebenden Wärme, die trockene Luft entbelıren, die ihnen fur die volkommene Entwickelung ihrer Blumen ein nothwerdig zu erfüllendes Bedürniss ist, unterliegen sie leicht der Einwirkmng der feuchten Niederschläge, die sie für ihren Organismus nicht zu verwerthen im Stande sind.

In Hinsicht des Letzteren nähern sie sich den die trockensten Küstengegenden bewolnenden Capparideen, Amyrideen, Burseraceen, den meisten Brometiaceen, Bi- 
gnoniaceen und Malpighiaceen, ien Cacteen, Leguminosen und vielen anderu, deren erfolgreiche Cultur noch immer ein pium Desiderium ist.

Bei dieser grossen Verschiedenheit der Lebensbedingungen der Planzen dieser climatologisch so verschieden zu behandehden Gruppen, kömien sie in demselben Hause nicht cultivirt werden, wesshalb in grösseren Gärten schon längst für jede dieser Pfianzengruppen besondere Abtheilungen eingerichtet sind.

Aber auch dort, wo diese Sonderung wirklich ausgeführt ist, stellen sich, bei den bestehenden Einrichtungen der Häuser, der vollkommenen Entwickehung zur Blüthe und Frucht Schwierigkeiten entgegen, die man bis jetzt noch nicht zu überwinden gelernt hat. Ganz besonders ist es freilich das zur Fruchtentwicklung unentbehrliche Sonnenlicht, welches den Pflanzen fehlt, aber auch die Feuchtigkeitsverhältnisse sind bei den bestehenden Einrichtungen nicht der Art, wie es diese Pflanzen verlangen. Bei der Wasserheizung kann die nöthige Trockenheit der Luft nicht erzielt werden. Einigemmassen annähernd schon bei der Dampf- und noch mehr bei der Kanalheizung; doch bleibt es auch bei diesem Verfahren noch immer schwer, den Pfianzen einen erquickenden, von epiphytischen und parasitischen Schimmeh nicht gestörten Winterschlaf zu verschaffen.

Ein einfaches, freilich aber etwas kostspieliges Mittel wäre es das Haus, welches für diese Bewolner der heissen, trocknen Tropengegenden bestimnt ist, durch eine verschiebbare Wand in zwei Abtheilungen zu tremen, von denen die Pflanzen auf leicht transportirbaren Gestellen zur Zeit nur die eine bewohnen, und sobald die Atmosphäre dieser mit Feuchtigkeit, mehr als ihnen zuträglich, geschwängert ist, in die zweite inzwischen gewärmte und getrocknete transportirt zu werden.

Um die Cultur der Cinchonen vollständig zu besprechen, ist noch die Behandlung der ersten Entwick- 
lungszustände, diejenige nänlich der Samen und deren Keimung, zu berühren.

Im Allgemeinen ist die Aufbewalnung der Samen, je nach ihrer Natur, selr verschiedenartig. Während die Samen der Wasserpflanzen nur unter Wasser ihre Keimfähigkeit bewalıen, ist allen Landptlanzen Luftwechsel ein unumgängliches Bedürfniss.

In zu feuchter, nasser Erde verpackte Samen dieser letzteren Pflanzen gehen, ebenso wie die in luftdicht verlötheten Blechgefässen trocken verpackten, während längerer Reisen unfehlbar zu Grunde.

Aber ebensowenig wie die Pflanzen verschiedener Zonen auf gleiche Weise zu cultiviren sind, ebensowenig können die Samen der Landpflanzen alle nach ein und derselben Methode verpackt ind verschickt werden.

Im Allgemeinen gilt hier als Regel, dass diejenigen Samen, deren Embryo von einem austrocknenden, hornigen Eiweisse umgeben, oder gar im Umkreise eines solchen eingebettet ist, wie dic Rubiaceen, Palmen, Piperaceen, ebenso die eiweisslosen von zarthäntiger oder auch von fester, aber lockerer Schale umgebenen, nnit fleischigöligen Samenlappen versehenen Embryonen, wie diejenigen von Theobroma, Quercus, die der Artocarpeen, Capparideen, Tropaeolen, Ternstroumiacen, Magnolien, leicht ihre Keimkraft verlieren, wenn sie nicht in feuchter und zugleich dem Wechsel zugünglicher Luft aufbewahrt werden. (Am besten in Gemeinschaft mit Orchideen oder andern Knollen- oder Zwiebel-Gewächsen verpackt.)

Die meisten, wie es scheint alle kleinen Samen indess, wie z. B. die «ler Cinchonen, verlangen eine völlig. trockne Aufbewahrung. Wahrscheinlich ist diese merkwürdige Erscheinung darin begründet, dass die Embryonen dieser, wie es auch in vielen Fällen ersichtlich ist, durch ein schleimig-fleischiges Eiweiss oder durch eine ähnliche Hülle, wie z. B. die Datteln, oder durch dichte Samen- oder Fruchtschalen, wie die Leguminosen, Dru- 
paceen etc. vor dem Vertrocknen geschützt sind. (Die mit dichter, fester Umhüllung versehenen Samen der Palmen haben bekanntlich eine kleine Oeffinung, mit einem lockern Deckelchen in der harten Schale.) Solche Samen schickte ich in vorzüglichster Erhaltung, indem ich sie, in Papierbenteln lucker in ein Netz beisammengepackt und durch lockere Umhïllhung von Wachsleinwand vor Regen etc. geschützt, in den Mastlorb hing, also in die luftigste Position brachte, die ich ihnen am Schiffe geben konnte.

Von den so transportirten und im Frühlinge in Europa angelangten Samen schlugen keine fehl; alle keimten.

Auch die in Biiefen geschickten Samen erreichten alle stets keimfähig ihren Bestimmungsort.

Als Beispiel nenne ich hiefür nur die Samen der Cinchona lancifolia MUT., die ich anf diese Weise aus dem oberen Magilelenenthale (bei Neiva) der Königlich Holländischen Reyierung, über Bogota, durch die gütige Unterstützng ihres General-Consuls in Caracas, Herrn van Lansberge, zu vermitteln die Ehre hatte, und die von hier wahrscheinlich auf' gleiche Weise nach Java geschickt, dort keimfähig ankamen.

Das Keimen der Cinchonen geht am leichtesten auf feuchten Holzsägespänen vor sich, die sich in geschlossener feuchter Luft befinden und von der Morgen- und Mittagssonme beschienen werden.

Bei ähnlicher Behandlung vermehren sich auch die Cinchonen dureh Stecklinge mit Leichtigkeit.

Im dichten Walde trifft man zuweilen die kleinen Bäumchen umgeworfen auf dem feuchten Boden liegend und mit Luftwurzeln an diesem befes ${ }^{\perp}$ igt.

Die Tausende von Samen, welche auf einem Cinchonenbaume reifen, keimen aber nicht, oder nur sehr sparsam und atisnahmsweise, in dem Schatten des im Walde stehenden Baumes, sondern nur an Orten, wo sie von den Somnenstrahlen mehr oder weniger direkt getroffen werden, z. B. nach dem Fällen desselben. 
Mögen diese wenigen, gewiss schon in der Praxis berührten, aber noch nicht zur allgemeinen Erörterung gebrachten Andentungen genügen, die Fortschritte der Physik und Techmik für die Pfianzencultur nutzbar zu machen, denn nur bei naturgemässer Behandlung werden sich die Pflanzen unserer Gärten vollkommen entwickeln können.

VI. M. IMuller (de Bruxelles) dépose une ,Notic sur les plantes de pleine terre" (1).

VII. M. Westmael (de Mons) dépose, ,quelques considérations sur lcs Hybrides des cultures" (2).

VIII. M. le Président appelle l'attention des membres sur quelques opuscules de M. GLOGER traitant des insectes nuisibles, dont des exemplaires ont été déposés au bureau.

L'ordre du jour étant épuisé, la séance est close à $4 \frac{1}{2}$ heures.

(1) Voir le Supplément.

(2) Voir le Supplément. 


\section{SECTION DE BOTANIQUE}

\section{APPLIQUEE ET D'HORTICULTURE.}

SÉANCE du 10 Avril 186õ, à dix heures du matin.

LE BUREAU SE COMPOSE DE:

MM. KOCH, Président.

NISSON, Vice-Président.

DE BEUCKER, Secrétaire.

FRANCKEN, Secrétaire-aljoint.

M. Ie Président commmique à la section que M. de Cannart d'Hamale, vice-président, a dû partir pour Bruxelles, par suite de la convocation du Sénat. Il lit des lettres de la part de MM. Barral et Mulder, secrétaires, annonçant qu'à leur regret leur présence est exigée ailleurs.

La question à l'ordre est la continuation de la discussion sur la taille raisommée des arbres fruitiers. La parole est à M. DE BEUCKer.

M. de Beucker (d'Avvers) s'exprime en ces termes:

\section{Mijne Heeren !}

Wij hebben reeds over de belıandeling en regrelmatigheid der viuchtboomen gesproken. Elkeen verlangt dat zijn vruchtboom fraai en naar alle zijden grelijkvormig zij, en dat hij cen gelijk kiachtig takkengestel bekome; hoe dikwijls echter en bij hoevele boomen wordt men bij eene regehmatige bewerking der takken niet bedrogen; hoevele boomen zijn er niet die gedmrende hun eerste leven eene ongelijke groeikracht toonen, en bijgevolg niet dan bezwanlijk een regelmatige boom zullen 
worden! Van waar komt dit? Dit komt door niets anders dan door het onregelmatig gestel der wortels. Aan de eene zijde is de boom soms voorzien van menigvuldige gezonde en wijduitgespreide wortelen, terwijl er aan de andere zijde geene of slechts eenige kleine vezelwortelen bestaan; door zulk een ongelijk onderaardsch gestel, wordt het bovenaardsch gestel ook gedwongen tot ongelijkheid op te groeijen. De wortels, die de voedingsstoffen uit den bodem moeten opzuigen, doen aan de eene zijde van den stam meer sappen naar de hoogte stroomen dan aan den anderen kant; de takken worden aan die saprijke zijde ook meer verlengd, maken een grooter getal bladeren, en door cit grooter getal bladeren kumnen deze ook wederom meer genot hebben van de werking der lichtstralen, even als zij ook meer stoffen uit den dampkring zullen opnemen, en verwerkt terugzenden langs dienzelfden gunstigen en meer uitgegroeiden weg, waardoor de stam dan ook onregelmatig zich moet verdikken en bijgroeijen; eindelijk worden de aan die zijde bestaande sterke wortelen nog meer versterkt en verlengd, waardoor het evenwigt van jaar tot jaar meer en meer verloren gaat. Nu kan men dit ongelijkmatig leven door groote zorg en gedurige waakzaamheid soms wel hersteld krijgen, met aan de sterke zijde herhaalde malen kort te snoeijen en aan de zwakke de takken zoo lang: mogelijk te laten, om deze laatste door een grooter tal bladeren in sterkte te doen aanwinnen en de wortelen aan die zijde te doen ontwikkelen. Men kan ook aan de zwakke zijde, eenige duimen onder de aarde, eene diepe insnede maken in den vorm eener openstaande V. Gelijk men met eene insnede tot door de buitenste houtlaag, in den vorm eener omgekeerde $\Lambda$, juist boven een bestaand oog, dit tot scheut, later tot twijg en tak, kan doen ontwikkelen, zoo verkrijgt men ook onder de aarde, door de insnede eener $V$, een of meer wortelen. Maar vermits die bewerking niet altijd gemakkelijk te verrigten is, en 
veel ggeduld en verlies van tijd vordert, zoo heb ik een ander middel bedacht, dat dan ook de schoonste en zigtbaarste uitkomsten upgeleverd heeft. Ik neem in den herfst of winter van een gezonden boom eenige wortelen, die men altijd genoeg in den grond vindt, waar een boom verplant is; deze zet ik, door de schor's in het roorjaar wat op te ligten, onder de aarde in den wortelkraag; deze bewerking doet men, wanneer de schors gemakkelijk pelt, met één woord, wanneer de vormsappen (cambium) in werking zijn; die wortelen groeijen van hunne, en de boom van zijne zijde spoedig en dikwijls binnen 8 dagen tijdsverloop goed in elkander, en het evenwigt van den boom wordt dan door de behandeling boven den grond spoedig en gemakkelijk hersteld. Ziet hier, Mijne Heeren, een stuk stam, met zulk een ingezetten wortel. Het is nu reeds vijf jaren, dat ik die bewerking toepas, maar tot hier toe heb ik nog geen boek gevonden, hetwell daarover handelt, of zelfs door niemand over die gelukkige behandeling hooren spreken, en indien het geschreven staat dan is dit buiten mijn weten.

$\mathrm{Nu}$ dient men in aanmerking te houden, dat men den wortel van denzelfden boom of plantensoort kiezen moet, zoo als peerwortel op peer, appelwortel op appel, pruim op pruim enz. Door die wortel-inenting heb ik dan ook een ander gelukkig werk kumnen verrigten, namelijk om verouderde of te weinig groeijende boomen in levenskracht te herstellen. Tk had een boom, welke slechts scheutjes maakte van vijf tot zes duim; dit scheen mij te weinig toe om nog lang te kunnen leven, en ik nam 8 sterke worteIen en zette deze onder den grond in den kraag van den boom, en de boom bragt dat jaar scheuten voort van 1 ? tot 2 ellen lengte. Door deze bewerking, Mijne Heeren, ben ik nog op andere gedachten gekomen, en wel, wanneer men onvruchtbare en toeh nog al welgroeijende perenbormen heeft, kan men ze tot eene spoedige ruchtbaarheid helpen, door aan de einden der afgesueden 
leiwortels, op minstens eene el afstands van den stam, worteltrosjes van de kwee in te enten. Na 4 of 5 jaren sterven die kweewortels soms af; de ingetoomde peerwortel maakt nieuwe vergaffelingen en intusschen is de boom zeer vruchtbaar geworden; evenwel op sommige gronden blijft de ingeënte kweewortel voortgroeijen; alle wortels, die eenigzins in familieverwantschap met de perenboomen zijn, kunnen in deze geënt worden, zoo als de wortels van peren zelf, van kwee, witten doorn, haveresch (lijsterbezieboom of Sorbus), mispel, enz., maar de wortel van peer op peer blijft het best voor eene schoone groeikracht.

Bij boompjes evenwel die men uit de pitten wil opkweeken, heeft men die wortel-enting niet noodig; men kan hieraan eene andere regelmatige wortelvorming geven, met de schors vier of zes duim diep onder de aarde ringvormig uit te snijden op de breedte van eenen halven duim; daar blijven de dalende vormsappen (cambium) stil staan, en maken er eenen ringvormigen aanwas of boord; dit in 't voorjaar gedaan zijnde, zal die in Julij of Augustus al tamelijk verdikt zijn. Dan steekt men met eene naald rondom in den boord en hetzelfde jaar komen er nog, of anders in de volgende lente, rondom gelijkwigtige wortelen te voorschijn; het jaar daarna haalt men dit boompje uit den grond, men snijdt den ouderen spilwortel weg en legt de menigvuldige nieuwe wortelen evenwijdig en straalvormig uit elkander. Ziet, Mijne Heeren, hier is zulk een boompje, dat ik heb mede gebragt om het $u$ te toonen (1). Welnu, zijn de vruchtboomen aan hun wortelgestel gelijkkrachtig ontwikkeld, dan kan en moet men ook de boomen in hun takkengestel gelijkkrachtig en gelijkvormig bewerken. Wanneer men de natuur in haren oorspronkelijken staat waarneemt, dan ziet men in de peren-

(1) M. Kосн a obtenu de M. DE Beucker la permission d'emporter \& Berlin ces remarquables documents, afin de les montrer à ses disciples et a ses amis. 
en appelboompjes een regelmatigen vijfhoek, uit takken zamengesteld die regelmatig en evenwijdig van elkander staan. De mensch slaat geen acht op die schoone bouworde der schepping; hij vestigt er zijne aandacht niet op, en onkundig bederft hij alles, waaraan hij zijne handen steekt. Nooit mag men die aan den Schepper gehoorzame natuurwet willen verbreken of belemmeren; men moet die wet bestuderen, en door verstandige tusschenkomst moet men ze op haren weg helpen en ondersteunen, dan verkrijgt men overvloed van vruchten en wordt men voor zijn werk ruimschoots en aangenaam beloond."

Plusieurs membres en ayant exprimé le désir, M. van Hulle résume en français ce qu'a dit M. DE Beucker. A cet exposé l'orateur ajoute les considérations suivantes:

Nous nous expliquons facilement la possibilité de la greffe des racines, dont vient de nous entretenir M. DE BEdCKer; nous n'en avions cependant pas encore fait ni vu faire l'expérience qu'il nous a été donné de constater aujourd'hui. Mais je crois devoir faire remarquer, que je ne vois pas dans la greffe des racines tous les avantages que M. DE Beucker semble vouloir lui attribuer. D'abord l'opération ne s'exécute pas aussi facilement qu'on peut l'expliquer, et quand même il en serait ainsi, pour donner par la greffe à un arbre les racines qui lui manquent, il faut être en possession de ces racines. Or, pour se les procurer, il faut commencer par les prendre à un arbre sain qui en a de trop.. pour les greffer ensuite sur un arbre qui en a trop peu. Eh bien, ceci encore, l'enlèvement des racines ne se fait pas très lestement, et supposant même que cela se pourrait, ne s'exposera-t-on pas bien souvent, au lieu de faire d'un manvais arbre un bon, d'en avoir deux mauvais pour un seul? Mais, passons encore làdessus; du moment qu'un arbre a besoin qu'on lui greffe des racines, c'est une preuve que déjà il ne se trouve pas dans les meilleures conditions, ce qui est bien souvent 
notre propre faute. Si, en élevant nos jeunes arbres, nous avions fait un bon choix l'amnée après le lever ou l'enracinement, jetant au feu tout ce qui était rabougri (la perte ne serait certes pas considérable) et ne conservant que ceux qui dès lors étaient de bome venue; si à cenx-ci on avait bien taillé les racines et bien étendu celles-ci lors du repiquage, puis continué à bien les soigner pendant les premières années de leur existence, rarement, ou jamais, ils n'auraient eu besoin de la greffe des racines.

Enfin, il est incontestable qu'un arbre qui a de plus fortes pousses d'un côté, a aussi de ce même côté de plus fortes racines qui y correspondent, et que par conséquent l'équilibre est menacé si non détruit. Mais pour réparer ce défaut, au lieu de recourir à la greffe des racines, n'estil pas beaucoup plus simple de tailler plus court les rameaux forts, ou de les incliner plus ou moins, et de tailler plus long les rameaux faibles et de les relever au contraire? En agissant ainsi, les parties inclinées et défavorisées pousseront peu, et attendu qu'elles correspondent aux racines relativement trop fortes, elles les fortifieront peu, tandis que les parties redressées et favorisées donneront beaucoup de bourgeons, lesquels, correspondant aux racines trop faibles ou trop rares, rétabliront celles-ci dans la même mesure, et par suite rétabliront aussi l'équilibre dans tout le système radiculaire.

Voilà Messieurs, ce que j'avais à dire concernant la communication de M. DE BEпcKer, qui du reste m'a paru être sinon nouvelle du moins très-intéressante, et j'ose croire qu'il en sera ainsi pour vous tous. Appliquée dans quelques cas et conjointement avec d'autres moyens, cette méthode pourrait être utile, mais que l'on en fasse jamais grand usage dans les pépinières, je ne le pense ni ne le souhaite. 
II. M. le Président communique à la section que M. Robert Warner (de Dublin) a déposé une notice intitulée: Extract from communications of the experiences of Thomas Bowley Esq. from Dublin with double glasshouses, compiled by RoBert W ARNer (1).

III. M. NedzeIsky (de Moscou) obtient la parole pour traiter la question qu'il a annoncée au Programme (No. XXI, pag. 27) sous ce titre:

Sur les progrès de l'arboriculture fruitière et de la pomologie théorique, ainsi que sur l'avantage que l'on peut retirer pour la pomologie et la classification des fruits par l'écude de la physiologie et de l'anatomie des plantes, en particulier de la famille des Rosacées.

L'orateur s'exprime en ces termes:

„Alles was wir vorgestern und heute über Erziehung, Beschneidung, Pfropfen durch Zweige und Wurzeln, Oculiren der Obstbäume gehört haben, beweist, welche bedeutenden Fortschritte die Obstbaumzucht in letzter Zeit gemacht hat. Die Regeln, welche sich auf Physiologie und Morphologie begründen, gaben den Aufschluss, wie in verschiedenen Climaten und Lagen gesunde Bäume und geeignete Formen zu erziehen, ihr Leben und ihre Fruchtbarkeit zu verlängern und zu vermehren, und durch zweckmässige Behandlung, anstatt nutzloser Holztriebe, nützliche Fruchtzweige, statt kleiner, saftloser, unvollständig entwickelter Früchte, grössere und wohlschmeckendere zu erhalten.

Denken wir an das mühsame und doch gelungene Streben, passende Obstsorten für Anpflanzungen der Felder und Strassen zu finden, sowie geeignete Sorten für längere Aufbewahrung und dergleichen gut zu transportiren, ihre Anwendung zur Zubereitung des Apfelweines, als Futter der Hausthiere u. s. w., so können wir mit Befrie-

(1) Voir le Supplément. 
digung sagen, dass die Obstbaumzucht auf bestem Wege fortgeschritten ist und den Wohlstand der Haus- und Landwirthschaft, ja selbst der Landesöconomie, bedeutend gehoben hat. Dieses ist bloss im Allgemeinen gesagt. Welche praktischen Fortschritte aber hat die Obstbaumzucht in den letzten zwei oder drei Decennien gemacht!

Um die Herren Mitglieder nicht zu ermüden, erlaube ich mir nur kurz an die praktischen Vortheile zu erinnern, bei welchen die Physiologie und Morphologie unsere besten Führer waren:

a. Die verbesserte Methode der Beschneidung, wozu uns die Morphologie den Aufschluss gab, wie viel Zweige zur Bildung der Krone gelassen, und welche Entfernung derselben für Hochstämme, Pyramiden und Spaliere vorhanden sein muss.

b. Welche Wildlinge für gewisse Sorten des Kernund Steinobstes zur Oculation, Pfropfen u. s. w. gewählt werden müssen, um gewisse Formen zu erhalten und den Wachsthum zu befördern oder zu verhindern, was für die Obstbaumzucht in verschiedenen Climaten, auch für die Fruchtbarkeit, Einfluss hat.

c. Die passende Zeit der Beschneidung, Oculation, des Pfropfens u. s. w. für g'ewisse Arten und Sorten in verschiedenen Climaten, um angemessene Formen, gesunde Bäume und frühere oder spätere Fruchtbarkeit zu erzwecken.

d. Die Wahl der Sorten für verschiedene Formen der Bäume u. s. w.

Alles dieses muss uns veranlassen, den Herren, die durch ihre Studien uns Winke und Aufschlüsse für die Obstbaumzucht gaben, und Allen, die sich immer darum noch bemühen, den herzlichsten Dank darzubringen.

Unter den Herren, welche sich dem Obstbau und der Obstbaumkunde widmen, finden wir Bürger, Gärtner, Pastoren, Doktoren, Barone, Grafen und Fürsten. Von Jahr zu Jahr wird die Zahl der Obstgärten vermehrt, werden pomologische Vereine gebildet oder in 
landwirthschaftlichen Vereinen Sectionen dazu bestimmt. In allen Ländern werden pomologische Institute von der Regierung und von Privat-Gesellschaften gegründet. Frankreich, Belgien, England, Holland, Deutschland, Schweden und Norwegen besitzen Musterbaumschulen und Fachmänner, die nicht nur einheimische, sondern auch ausländische Obstsorten, in den verschiedenartigen Climaten dieser Länder zu acclimatisiren suchen. In Ländern, wo noch Fachmänner fehlen, werden solche aus dem Auslande herbeigerufen; z. B. in Preussen wurde unlängst vom Ministerium ein Cireular an alle inländischen Behörden erlassen, der Obstbaumzucht alle mögliche Sorgfalt zu widmen; und Herr Alexis Lepère dirigirt in Preussen nicht nur von ihm gemachte Anlagen in Privatgärten, sondern hat auch in den königlichen Gärten in Sans-Souci bei Potsdam und auf dem Babelsberg Anpflanzungen hergestellt, wo feinere Obstsorten nach seiner Methode herangezogen werden.

Bei uns in Russland besteht, schon seit den 5 Decennien dieses Jahrhunderts, in Jekaterinoslaff in Süd-Russland ein pomologisches Comité mit einer Obstbaumschule und einem Obstgarten von 50 Morgen Landes. Dieses Comité, welches jetzt nach Uman übergesiedelt ist, bemüht sich, nicht nur einheimische, sondern auch fremde Obstsorten zu acclimatisiren.

Ausserdem giebt es Baumschulen und Obstgärten mit Instituten in Kischeneff, Odessa, Nikita, Astrachan, Pensa, Woronesch, Konstantinograd, Orel, und seit drei Jahren wurde auch ein Centralgarten-Institut in Uman angelegt, unter Aufsicht des Generals Anenkofr.

Ausserdem beschäftigen sich landwirthschaftliche und Gartenvereine in Nord-, Süd- und Ost-Russland mit diesem nützlichen Theile der Landwirthschaft. Nebenbei beschäftigen sich viele Privatpersonen mit Obstbau, nicht nur im Süden, sondern auch im Norden, z. B. in Moskau, ja selbst in Petersburg, in Nischni, in Riga u. s w. 
Im Auslande sind nur 5 bis 6 unserer heimathlichen Obstsorten (Aepfel) bekannt z. B. Borowitzky (Borowinka), Charlamowsky, Nalivia (Noliff). Hiernach dürfte die Meinung im Auslande die sein, dass wir nur diese wenigen Sorten besitzen, und nach dem Böhmischen Correspondenten im Lucas- und Oberdieck'schen Journal, (Pomologische Monatschrift für 1865) könnte man glauben, wir wissen gar nicht, wie der Calville cultivirt sein muss; aber zur Beruhigung dieses Herrn muss ich sagen, dass der Calville bei uns in Süd-Russland in grosser Masse cultivirt wird. Nicht bloss der Gärtner, sondern sogar der Russische Bürger würde lachen, wenn er hörte dass der Calville in Glashäusern gehalten oder wie Ananas getrieben werden müsste.

In Moskau, wo das Clima nicht erlaubt, selbst unsere heimischen Sorten aus den verschiedenen Theilen unseres Landes zu cultiviren, besitzen Handelsbaumschulen und Privatliebhaber eine grosse Anzahl verschiedener Obstgattungen und hauptsächlich Aepfel. Desshalb erlaube ich mir auf die Obstbaumschule des Herrn Krasnograsoff in Moskau aufmerksam zu machen. Diese Baumschule besteht schon seit dem. 12 ten Jahre dieses Jahrhunderts; auch in neuester Zeit legte Herr Grest in Moskau, der sich viel mit Nomenclatur und Prüfung unserer Obstsorten beschäftigt, und ein pomologisches Werk mit von mir gemachten Abbildungen herausgiebt, eine grosse Baumschule nicht allein von heimischen, sondern auch von fremden Obstsorten in grossem Massstabe an.

Der Kampf mit unserm kalten Clima war das beste Mittel, durch welches misere Gärtner auf mannigfaltige Weise gute, zweckmässige Methoden erfanden, um auch durch Treiben frühzeitige Früchte zu erhalten.

In diesem letzten Theile der Obstbaumzucht können auch wir mit dem Auslande concurriren.

Unsere Regierung bestrebt sich, auch ausländische, für unser verschiedenes Clima geeignete Obstsorten, 
durch Bäume und Reiser einzuführen. Zu diesem Zwecke werden gewisse Herren bestimmt, Studien darüber im Auslande über Auswahl der Sorten und ihre Behandlung zu machen. Herr Basiner aus Kieff, von der Regierung abgeschickt, trat vor einigen Jahren solch eine wissenschaftliche Reise an, wurde aber leider nach einem Jahre der Wissensehaft durch den Tod entrissen. Und mir, dem das Glück zu Theil ward, Mitglied des Congresses zu sein, wurde der Auftrag, die mit bestem Erfolge angefangenen Studien des Herrn Bastner fortzusetzen.

Doch ist es nicht allein unsere Regierung, die in diesem Fache nützliche und praktische Kenntnisse aus dem Auslande einzuführen trachtet, sondern es richten die Behörden aller Länder ihre Aufmerksamkeit auf die Obstbaumzucht, um sie auf alle mögliche Weise selbst zu heben. In vielen landwirthschaftlichen Academien Deutschlands, Frankreichs und anderer Länder giebt es specielle Professoren für Pomologie, oder Pomologie und Gärtnerei zugleich.

Pomologische Congresse bilden sich, um nicht allein wegen besserer Wahlen der Sorten und ihrer Bestimmung wechselseitige Mittheilungen zu machen, sondern auch um die Cultur zu befördern.

Nach allen den Fortschritten, welche der Obstbau in letzter Zeit gemacht, wollen wir doch die Frage stellen: Sind wir mit diesem, was wir Fortschritt nennen, so weit gekommen, um ausrufen zu können: Dies ist die richtige Methode des Obstbaues, wodurch wir der Landesöconomie erspriessliche Eimahme zuführen? Wirklich, wie wir bemerkten, hat die Spalierzucht und dergleichen die weiteste Verbreitung gefunden, worauf wir auch den Schluss ziehen kömnen, da diese Methode von den Herren Congress-Mitgliedern vielseitig besprochen wurde. Trotz der vielen Anwendungen von Spalierzucht aber sind die Obstpreise immer noch enorm, was darauf 
hindeuten muss, dass diese Methode zur Hebung der Landesöconomie nicht die zweckmässigste ist.

Weiter noch diesen Punkt ins Auge gefasst, giebt uns diese Methode hinlängliche Sicherheit, die Lebensdauer der Bäume zu erhalten, vor Ausartung und Krankheit zu schützen, welches bei Anwendung dieser Methode eine grosse Rolle spielt. Davon abgesehen muss ich sagen, dass die Cultur der Hochstänme noch mehr Fortschritte machen muss, und wir noch mit allem Fleisse und Eifer zu studiren haben, sie praktisch zu verbessern und $\mathrm{zu}$ heben.

Ausser dem praktischen Theile der Obstzucht giebt es noch die specielle Obstkunde oder Pomologie. Wir wollen nun sehex, welche Fortschritte dieser Theil machte. Eine Antwort hierauf zu geben, dürfte sehr schwierig sein. Es ist noch nicht erklärt, und nicht alle jene Herren, welche sich mit Pomologie befassen, können uns sagen, auf welchen Grundsätzen die Studien über Pomologie beruhen. Wollen wir aber die vielen Meinungen, die hierüber herrschen, mit was sich die Obstkunde befassen muss, prüfen, so dürften wir kaum im Stande sein, einen richtigen Schluss zu ziehen, da wir vielen Sätzen begegnen werden, welche einander widersprechen.

Und doch erscheinen jedes Jahr sogenannte rein pomologische Werke. Ich will mich bemühen, im Kurzen geschichtlich darzustellen, was man über specielle Pomologie in den verschiedenen Werken findet.

In ältester und neuester Zeit war, wie in allen Theilen der Naturwissensehaft, so anch in der Pomologie, das Bestreben vorherrschend, eine Systematils der Obstsorten aufzustellen. In den ältesten Werken der Obstkunde tinden wir Aufzählung der Obstsorten, Beschreibung der Form, des Geschmacks, auch anderer äusserer Merkmale der Früchte und öfters Symmymen rer Surten. Nach dieser Beschreibung ist es ummöglich, lie Früchte fest und sicher zu bestimmen. Und diese Aufstellung der Obstsorten 
findet man in botanischen und dendrologischen Werken. Tournefort (1) macht in seinem bot. Werke die Aufzählung der Sorten, welche damals cultivirt wurden, zugleich mit der Beschreibung der wild wachsenden, von welchen er gewisse Arten und Unterarten aufgestellt. Dasselbe findet man bei Caspar Bauhin, Johnston und in mehr rein pomologischen Werken, wie das des Dumaneu du Monceau, und bei Knopp, JoH. Herm, wo schon die Reifezeit angegeben wird, um die Früchte in gewisse Klassen zu gruppiren.

Die Herausgeber pomologischer Werke in der Mitte und gegen das Ende des 18. Jahrhunderts bestrebten sich, ein pomologisehes System aufzustellen, hauptsächlich des Kernubstes, um die früher schon beschriebenen und in Cultur gewesenen Sorten mit vielerlei Benennungen in Ordnung zu bringen.

Um ein System zu gründen, wurde hauptsächlich die äussere Form der Früchte in Betracht gezogen, und um die Sache wirklich musterhatt darzustellen, wurden anch noch Zirkel und Geometrie angewendet. Alle Formen der Kemobstsorten waren mit Namen geometrischer Figuren bezeichnet. Nach der Form rund, platt, hyperbolisch, parabolisch u. s. w., wurde die Eintheilung der Klassen gemacht.

Die Verschiedenheit des Banes des Kerngehäuses, Kelchs und Stiels, damn Kelch- und Stielwölbung, Beschaffenheit des Fleisches u. s w. wurde nicht berücksichtigt. Demmach war es aber Zeit, dass Pomologen sich bemühten, einigermassen Licht und Ordnung in diese Sache zu bringen. Dass diese auf geometrische Formen gegründete Systene nicht genügend und vollständig waren, versteht sich. Jeder Obstzüchter weiss jetzt, dass die Früchte variiren, dass Boden, Clima, Alter und Wachsthum und selbst Cultur verschiedene Einflïsse auf

(1) Institutiones rei herbariac. Parisiis 1719. 
die Ausbildung der Frïchte hervorbringen und diss nicht selten Früchte von sehr ähnlichen Formen grossen Unterschied in ihren Eigenschaften haben.

Bei allem dem sind wir diesen Pomologen schon zu Dank verpflichtet, dem durch die Ungewissheit der Namen der vielen Abarten der Aepfel war ein solches Dunkel und solche arge Verwirrung eingerissen, dass man sich nicht mehr herausfinden konnte. Das, was jene Pomologen in Darstellung der äusserm Formen der Früchte geleistet, das komnte noch Anhaltspunkt zur Schaffung eines künstlichen Systems geben.

In derselben Zeit sind noch manche pomologische Werke erschienen, in welchen die Früchte ohne Classification beschrieben waren. Doch andere mehr scharfsinnige Pomologen haben die schon aufgestellten und in Druck erschienenen Werke über Classitication nicht olne Aufmerksamkeit behandelt.

Dies nun war der Anfang der vielseitigen Beschreibungen der Früchte, und gab demnach den Pomologen Anlass, auf diese Studien eine bessere Classification aufzustellen.

Ende des 18. und Anfang des 19. Jahrhunderts hat die Beschäftigung mit Pomologie, Beschreibung des Kern- und Steinobstes, um eine systematische Ordnung: aufzustellen, die höchste Stufe erreicht. Beinahe in jedem Werke, gross und klein, war zur Vorrede die Erklärung der Merkmale angegeben, nach welchen der Verfasser seine beschriebenen Sorten classificirte, und durch Tabellen die Erklärung des aufgestellten Systemes dargestellt.

Manche Pomologen beschrieben die Sorten nach dem Linneischen System, noch mehre nach ihrer Rejfezeit. In solchen Werken wurden nicht selten auch Geschmack, Farbe der Frichte und les Fleisches, Länge des Fruchtstieles als Merkmale zur Beschreibung angenommen. Geometrische Form jedoch war immer der Hauptanhaltspunkt. 
Zur leichtern Erkennmng der Sorten wurden verkleinerte Zeichnungen der Früchte, und auch in natürlicher Grösse, schwarz und colorirt, den pomologischen Werken angehängt. Manche dieser Abbildungen dienen noch jetzt zur Vergleichung bei Bestimmung der Sorten. Nun, was aber diese Aufstellung anbelangt, können wir sie heutzutage bloss als kritisches System betrachten.

Doch im Anfange des 19. Jahrhunderts erschien in Deutschland eine Classification der Kernobstsorten, die weit verständlicher war als Alles vorhergehende, und dieses war die Mutter aller später erschienenen Systeme; welche zur Beschreibung der Früchte in verschiedenen pomolugischen Werken in Deutschland angewendet wurden. Was ist aber dieses System jetzt? Ist es jetzt noch anwendbar? Ist es auf Merkmale begründet, welche bei allen Einflüssen unverändert bleiben, und können diese Merkmale, physiologisch untersucht, auch als natürliche für ein System dienen? Die Antwort wird, Nein" sein. Die Meinung vieler um die Obstkunde verdienter Pornologen ist: dass das Dielische System weder logisch, noch naturgemäss sei.

Man bemerkt, dass, so ausführlich und weitläufig auch die Beschreibungen sind, die angegebenen Kennzeichen doch nicht genügen, und dass die Einreihung der Sorten dem aufgestellten System nicht entsprecht; dass ferner das angebliche Charakteristische der Sorten bäufig auf sehr unwesentlichen und zufälligen Eigenschaften beruht, und dadurch viele Sorten zwei-, drei- bis viermal in verschiedenen Zuständen beschrieben sind. So sagen auch andere Kritiker, das Dielische System sei nach dem jetzigen Standpunkte iler Pomologie nicht mehr anwendbar. Und sie haben auch Recht.

Es ist wirklich ummöglich, alle jetzt in Kultur befindlichen Sorten, sei es Kern-oder Steinobst, in Classen gewisser Systeme einzureihen und bloss nach Beschreibung der Frucht selbst zu erkennen. 
Die Fehler dieser Systeme sind angezeigt, jedcch das Gute davon wurde nicht unbeachtet gelassen.

In nenester Zeit bestreben sich die Pomologen eines gründlicheri Studiums nicht bloss änsserer und innerer Kenmzeichen der Früchte, sonder'n selbst der Kennzeichen der Fruchtbäume, ihrer Vegetation, Form der Zweige und Blätter u. s. w. Ausserdem 'wurde beachtet, was die Frucht selbst sei, und was man unter vollkommenen und unvollko:umenen Früchten verstehe; welche Merkmale der Früchte constant und welche von diesen Kennzeichen zur Gründung einer Classification grebräuchlich seien; welche Einflüsse auf die normale äusserliche und imerliche Entwicklung der Früchte Clima, Boden und Behandlung der Bäume selbst ausüben.

Solche Studien sind von manchen Pomologen sehr ausführlich dargestellt, and andere gehen in rein botanisch-pomologische Aufstellungen über, so dasi diese letztern Studien weit entsprechender als die ersteren sind, und für den wissenschaftliehen Theil der Pomologie ein reiches Material darbieten.

Einige Pomologen haben, bei der Beschreibung der Früchte, als charaliteristische Merkmale nicht bloss die Grösse der Früchte, ihre Form, Charakter der Färbung, Eigenschaft der Schale, Grösse des Kelches und Stielwölbung, Länge des Stieles gebraucht, sundern anch die Verschiedenheit des Kelches, ob offen, halb oder ganz geschlossen, auch Grösse des Kerngehäuses, Ausbildung, Zahl und Farbe der Kerne, Eigenschaften des Fleisches; noch weiter bemühte man sich, Bäume, Zweige, Blatt und Frucht, Knospen, Blätter und Nebenblätter, Triebe und Blüthen u. s. w. auf das gentueste zu beschreiben. Andere bemühten sich darzustellen, welche Formen der Bäume und Früchte sich zur Beschreibung am besten eignen.

Die Meinung Einiger ist auch die, dass nur die grössten Früchte die vollkommensten sind und für die Sorten 
charakteristisch gehalten werden inüssen. Ebenfalls wurde auf Gleich- oder Ungleichheit der beiden Hälften der Frucht im Durchschnitte, auf die Grösse der Kammern, des Kerngehäuses, selbst auf die Grösse der Rippen und des Bauches grosser Werth gelegt.

Den Pomologen, welche Botaniker sind, war es ein mühsames Studium, über Entwicklung der Kern- und Steinobstfrüchte, über immere und äussere Struktur der Pomaceen überhaupt, Normalform der Früchte, ihre wesentlichen immeren und äusseren Eigenschaften, über die verschiedene Form des Kerngehäuses, u. s. w. Aufschluss $\mathrm{zu}$ geben.

Die pomologische Terminologie ist durch solche Studien vollständiger und reicher geworden, so dass andere Pomologen ihre Systeme darnach aufstellten; doch auch solche Systeme können nicht ganz befriedigen, sie bleiben für die Pomologie dasselbe, wie das Linneische System für die Botanik. Sie können bloss als Leitfaden zur Bestimmung der Sorten und auch zu Anpflanzungen nach Classen und Ordnungen dienen. Diese Classification ist bloss eine künstliche zu nennen. Nun aber, wollen wir ein natürliches oder noch mehr ein botanisch-pomologisches System gründen, so wird es eine ganz andere Sache; dann müssen wir nicht bloss die oben genannten, für ein künstliches System aufgestellten Merkmale studiren, sondern auch die Entstehung der Varietäten der Früchte von gewissen Species ermitteln, eine Verwandtschaft durch andere Kennzeichen aufsuchen, um die Classification nach verwandten Aehnlichkeiten zu gründen. Der Hauptgrund. solcher Studien ist das Studiren der Obstarten selbst, ihrer Ausartung und der Kennzeichen, welche bei solcher Ausartung von längster Daner sind, der Einflüsse, die bei der Ausartung die grösste Rolle spielen, und noch mehr des Unterschieds zwischen Früchten verschiedener Arten. Die Botanik selbst kïnnte für solche pomologische Arbeit die beste Führerin sein. 
Was die Arten des Steinobstes anbelangt, diese sind in botanischen Werken gründlicher bearbeitet, und daher die Classification derselben in der Pomologie besser als jene des Kernobstes, da für letzteres bei der Beschreibung der Arten in botanischen Werken sehr wenige Merkmale angegeben sind, und überhaupt eine Beschreibung der Früchte nicht aufzufinden ist.

Für Botaniker schien es unnöthig, den Unterschied zwischen Früchten der Arten genau zu studiren; für Pomologen aber ist es zur Classification der Früchte nothwendig. Wie kam man z. B. ein gutes System für Kernobst gründen, wo man unzureichende Kenntnisse über Frucht-Apparate derselben besitzt?

Bei Durchlesung botanischer, sowie pomologischer Werke findet man verschiedene Theorien angegeben und wird auf vielerlei Meinungen darïber stossen. Einige sagen, dass der Kelch bei Ausbildung der Frucht des Kernobstes die Hauptrolle spiele; andere, der Kelch, die Achse der Frucht, und der Fruchtknoten. Professor KarL Косн gründet seine Theorie der Entwicklung der Früchte der Pomaceen hauptsächlich auf die Wölbung der Spitzen der Blüthenstiele. Es kommt mir nicht zu, über die Theorie des Prof. K. Косн ein Urtheil zu fällen, ich empfehle aber Allen, die sich dafür interessiren, die Notizen des Pomologen von Flotow, in der pomologischen Monatschrift von OBErdieck und Lukas, zu lesen.

Wie kömnen wir aber sagen, was die Frucht der Pomaceen ist, da sich noch in jetziger Zeit manche Botaniker bemühen zu erklären, ob die Frucht der Pomaceen ober- oder unterständig ist. Es giebt auch Theorien über Entwicklung der Frucht; aber leider waren die Früchte der Varietäten hauptsächlich das Material, anf welches die meisten Theorien begründet sind.

In der Botanik ist schon lange bekannt, dass alle 
Früchte eine Unwwandlung der Blätter sind und zwar der Fruchtblätter, welche die Samen einschliessen. Bei einigen Pflanzenfamilien sind die Früchte aus einem Fruchtblatte gebildet, bei Anderen ans melneren.

Die Zahl der Blätter lat Einfluss auf die Zahl der Lagen, und die Zahl der Lagen stimmt meistens überein mit der Zahl der Blätter, aus welchen der Blätterwirbel der Zweige besteht.

Bei Kernobst ist die Zahl der Blätter, aus welchen der Wirbel bestehet, nit geringer Ausnahme, fünf. Diese Zahl fünf wiederholt sich in allen Bestandtheilen der Blüthe und der Frucht selbst. Dasselbe wiederholt sich bei Früchten der Species und auch bei Varietäten. Eine Ausnahme giebt es noch dann, weun die Blüthe von der Natur monströs ist und ihren Charakter darnach ausbildet. So z. B. werden die Blïthen von Pyrus spectabitis als gefüllte gehalten; es hat aber die Frucht immer $6-7$ und 12 (Fig. 1, $a, b)$ Kammern, so dass man bei einer grossen Zahl von Früchten nur wenige Ausnahmen findet, wobei keine Normalzahl der Kammern vorhanden ist. Bei andern, wie Pyrus coronaria, ist die Zahl der Kammern nie vollständig, und man findet selten Früchte mit 5 , meistens mit 4-3, oder auch bloss mit einer Kammer (Fig. 2, $a, b$ ). Bei normal entwickelten Früchten kommen nach der abwechselnden Stellung aller Theile der Blume und Frucht selbst die Kelchblätter mit den Kammern überein.

Nach dieser Stellung der Blumen und Fruchttheile ist es sehr leicht, einen Durchschnitt der Frucht zu machen, um jedes der fünf Fächer in zwei gleiche Theile im Längsdurchschnitt zu bringen.

Zu diesem Längsdurchschnitte, welcher zur Bestimmung der Sorten in Betracht gezogen werden muss, können bloss die änsseren Merkmale dienen, z. B. Kelchblätter und Kanten; das heisst, um den Durchschnitt des Kernobstes richtig und schnell zu machen, richtig im pomologischen Simne, muss man bei Aepfelu und Birnen eins 
von den fünf Kelchblättern oder eine von den fünf grössten Kanten (Fig. 3, a) gerade durch die Mitte schneiden, und den Schnitt fortsetzen bis zum Fruchtstiel, welcher ebenfalls genau durch die Mitte geschnitten werden muss.

Durch diesen wird eins von den fünf Fächern des Kerngehäuses, welches (Fig. 3, b) dem geschnittenen Kelchblatte gegenüber steht, auch in zwei gleiche Theile getheilt. Bei manchen Aepfeln, wie bei Calvillen und Rambour-Reinetten, bei welchen in der Nähe des Kelches die Erhöhungen oder Kanten sehr bemerkbar sind, braucht man bloss die letztern als Richtschmur zu nehmen, um den Längsdurchschnitt zu machen. Es sind desshalb die grössten Kanten zum Durchsehnitt zu nehmen, da sie mit der Fächerzahl übereinstimmen.

Dieses von mir aufgestellte Gesetz, welches ich in Berlin, in einer Sitzung des Vereins zur Beförderung des Gartenbaues in Preussen zum ersten Male vorgetragen und der Kritik der versammelten Mitglieder anheimgestellt, und später in Dresden in der Vereins-Sitzung der Isis, unter Prïsidium des Herrn Professor Reichenbach, wiederholte, hat überall Sympathie erregt und, wie wir vorgestern hörten, ist es von den Deputirten des Boskoop'schen pomologischen Vereines in Holland in dankender Weise anerkannt und zugleich den anderen Pomologen zur erleichterten Bestimmung der Sorten empfohlen. Auf diese oben angegebene Weise hat man auch den Vortheil, den Naturabdruck solcher Präparate zu erhalten.

Nach dieser Methode wird der Schnitt sehr glatt sein, und trägt man auf die erhaltene Hälfte bloss eine AquarellFarbe (z. B. Zinnober) auf, so kann man den gefärbten Theil auf Papier abdrucken. Auf diese Weise erhält man den richtigsten Natur-Abdruck von allen innern und äussern Theilen der Frucht, wie z. B. Stellung der Kelchblätter, Form der Kelchwölbung, Länge der Kelchvertiefung, Form der Achse, Länge-Form des Fruchtstieles; und wenn man die Fächer, welche das Kerngehäuse 
umgeben, mit einer Bleifeder oder einem stumpfen Messer etwas vertieft, so wird man zugleich den Abdruck ihrer Form mit erhalten.

Ich muss aber bemerken, dass dieses von mir angeführte Gesetz seine Ausnahme hat. Diese Ausnahme erstreckt sich nicht bloss auf Species der Pomaceen, sondern es findet diese Methode auch bei andern Sorten, welche als Varietäten aufgestellt sind, z. B. bei Sternäpfeln die, wie bekannt, von keinem Botaniker als Art beschrieben, sondern von Pomologen als Varietät gehalten werden, keine Anwendung.

Wollen wir einen Durchschaitt eines Sternapfels machen, um die Fächer in gleichmässige Theile zu theilen, so schneidet man nicht die Mitte des Kelchblattes oder der Kanten, sondern macht den Durchschnitt zwischen zwei Kelchblättern oder zwischen zwei Kanten (Fig. 4).

Diese letztere für Sternäpfel angeführte Methode bleibt auch bei anderen Arten: Pyrus prunifolia WrLld. (Fig. 5), Pyrus microcarpa D. C., Pyrus Malus paradisiaca L. (Fig. 6) und anderen, aber merkwürdiger Weise nicht bei Arten der Birnen, sondern bloss bei Varietäten derselben.

Nach diesem Gesetz für Arten muss der Sternapfel als Art der Aepfel betrachtet werden. Dies erklärt zugleich, dass die Wirbelstellung der Blumenbestandtheile, hauptsächlich das Innere der Frucht, verschieden ist bei Species und Varietäten der Aepfel.

Diese beiden oben angeführten Gesetze, welche ron Botanikern und Pomologen bis jetzt unbeachtet geblieben, geben uns die besten Kemnzeichen, Arten von Varietäten der Aepfel zu unterscheiden, und zugleich einigermassen die Verwandtschaft zwischen Arten und Varietäten zu ermitteln, welches für eine natürliche Classification der Früchte sehr nothwendig ist, wozu wir aber noch viel Mühe und Fleiss anwenden müssen, und nicht einzeln, sondern in Gesammtheit. Ich sage in Gesammtheit; denn 
es ist kein Pomolog im Stande, die verschiedenen Arten und Varietäten aller climatischen Lagen zu studiren. Sehr häufig kommt es vor, dass Pomologen sich nur mit der Praxis beschäftigen und weniger Zeit haben, sich mit botanischen Studien zu befassen. Hieraus sehen wir, dass zur Hebung des botanischen Theiles der Pomologie die Botaniker ihren Antheil beitragen müssen, und hauptsächlich Direktoren der botanischen Gärten, in welchen die Bestimmung der Familien doch die sicherste ist. Doch findet sich da noch mancher Mangel vor, besonders bei Pomaceen und Amygdaleen in Benennung der Species. Dass diese beiden Familien nicht vollständig studirt sind kommt daher, dass für diese noch nicht so viele Monographien erschienen sind, wie für andere Familien der Pflanzen.

Es haben einige Botaniker Studien darüber gemacht in rein botanischem Sinne, wodurch die Entwicklung der Frucht der Pomaceen und Amygdaleen klarer geworden ist, sich die Nomenclatur aber noch beträchtlich vermehrte. Diese Studien jedoch haben das Verdienst, dass sie verschiedene Merkmale für pomologische Studien darbieten.

Die Botaniker bemühen sich die Entwicklung der Frucht zu beobachten, um die Erklärung zu geben, an welchen Theilen der Pflanze sich die Früchte entwickeln; aber durch diese Studien sind wir nicht aufgeklärt, wie dieses Entstehen bei Arten und Varietäten geschieht, dass in ihrer innern Struktur ein oben angegebener Unterschied entsteht, und warum man den Unterschied bei Arten der Birnen nicht findet, obgleich Birnen eben so gut Kernobst sind, wie Aepfel.

Das zeugt auch, dass bei der Ausbildung der Birnfrucht noch andere Theile als Blätter Einfluss haben müssen. Bei Monstrositäten der Birnen kommt vor, dass aus einer Blüthe nochmals ein Zweig herauswächst, der mit Blättern versehen oder anstatt Blätter Blumen bringt, und nach Abblühen sich wieder Früchte bilden, 
(Fig. 7, $a-b$ ), so dass die obere wie aus dem Kelch der untern ansgewachsen ist, der Fruchtstiel der obern durch die Mitte der untern Frucht geht und mit dem unteren Stiel verschmolzen ist. So auch Monstrositäten, wo eine Birne zwei- bis dreifrüchtig wird, oder sich drei Etagen bilden aber ohne Stiele (Fig. $8, a-b$ ) und jede Etage auf der Spitze 5 Blätter, oder 2- und 3-mal 5 stiellose Blätter hat. Noch mehr: bei manchen Monstrositäten sind auf den äussersten Theilen der Frucht wirkliche Blätter nach dem Wirhelgesetz gestellt (Fig. 9). Bei manchen Birnen findet man zwei Etagen des Kerngehäuses (Fig. 10.)

Als Beweis erlaube ich mir, in Zeichmungen die Birnenfrüchte darzustellen, welche ich in Spiritus aufbewahre. Diese Monstrositäten waren von manchen Botanikern und Pomologen bemerkt und bilden sich ebenfalls auch bei Rosen sehr häufig aus, welches im Allgemeinen Prolificatio genannt wird (Fig 11). Bei Apfelfrüchten kommen solche Monstrositäten nicht vor, doch kommt die Verwächsung von zwei Früchten vor mit einem Stiele(Fig. 12). Prolificatio der Birnen bringt uns auf die Meinung, dass die Früchte der Birnen sich hauptsächlich durch den Zweig ausbilden und das, was bei der Frucht Fleisch genannt wird, nichts ist als die verdickte saftig gewordene Cambium-Schicht der Zweige. Diese Entstehung und Ausbildung der Früchte verlangt natürlich noch viele anatomische und morphologische Studien, wozu nicht jeder Pomolog die nöthigen Vorkenntnisse besitzt.

Das von mir aufgestellte Gesetz zur Unterscheidung der Arten und Varietäten bei Aepfeln kamn ausser dem Nutzen, welchen er für den theoretischen Theil der Pomologie darbietet, auch nützlich sein für die Wahl der Unterlagen für Obstsorten, welche bei Acclimatisation und Cultur der Obstbäume grossen Einfluss hat.

Zum Schluss tühre ich noch an, dass die geographische Verbreitung der Arten und ihrer verwandten Varie- 
täten in der Pomologie, ja sellust in der Botanik, noch sehr wenig Anklang gefunden hat, und ich rersichere, dass, wäre dies geschehen, die Obstzucht nicht bloss in landwirthschaftlicher Beziehung grösseren Aufschwung hätte erzielt, sondern auch die Wissenschaft von der Pomologie manchen grossen Nutzen hätte ziehen können, um die Entstehung der Arten und Varietäten im Pflanzenreiche zu erklären. Darüber giebt uns selbst DARwin in seiner Theorie über Entstehung der Arten und Varietäten für die Familien der Pomaceen und Amygdaleen keine besonderen Aufklärungen. Doch ist es nicht seine Schuld; es war ihm nicht genug Material geboten von Pomologen und Botanikern, welche sich mit wissenschaftlicher Pomologie und mit landwirthschaftlicher Botanik beschäftigen, weil die Obstbaumzucht ein Haupttheil der Landwirthschaft ist.

M. Koch recommait que l'orateur précédent a rendu de grands services ì la pomologie; il s'est surtout appliqué avec zèle à la classification qu'il reut baser sur la physiologie et l'anatomie des plantes. Ce système n'est cependant pas nouveau. M. Kосн l'explique et le démontre par des dessins sur la planche. Il a été, dit-il, le premier, qui, il y a plusieurs années, a traité cette question et le célèbre pomologne von Flotow, se ralliant à ses idées, les a encore développées. Il démontre que la marche du développement des pommes et des roses est la même. Une classification systématique ne pent être obtenue, que si l'on fait attention ì la manière dont les fruits se développent. C'est ici que les pomoloğues sont ómbés en erreur; ils se sont trop attachés ì la forme extérieure. Il faut d'abord examiner la fleur et ce n'est qu'alor's que l'on obtiendra des résultats satisfaisants. M. Drcaisne, de Paris, a beaucoup examiné, recherché et expérimenté sans obtenir le résultat désiré: depuis il a cessé ses recherches. M. Kucr expérimente et recherche depuis 20 ans, et quoiqu'il ne soit pas encore parvenu à obtenir une bonne classification, il ne veut pas cependant cesser ses recherches. 
M. Nedzelsky répond à M. Kocr, que le système de ce dernier n'est pas celui de M. von Flotow, lequel se rapproche davantage de celui de M. Nedzelskx. Il explique encore en quelques mots ce qu'il a développé précédemment et réfute brièvement les observations de $\mathbf{M}$. Косн.

M. Regel (de St. Pétersbourg) fait les remarques suivantes:

Obgleich ich während des Vortrags des Herrn NEDzELSKY nicht die ganze Zeit anwesend war, so erlaube ich mir doch auf die von Herrn Prof. Косн gemachten Bemerkungen einige Entgegnungen.

Wenn wir ron systematischen Eintheilungen der Aepfel, der Birnen, etc. sprechen, so liegt es, wie es mir scheint, in der Natur der Sache, dass keine der zur Eintheilung in Classen und Ordnungen angewendeten Merkmale, mögen diese nun von imnern oder von äussern Charakteren stammen, eine so scharfe Abgränzung dieser Gruppen geben können, dass es nicht immer eine Zahl von Formen geben wird, die mit gleichem Rechte zur einen oder andern Klasse oder Ordnung gestellt werden könnten. In der Natur der Sache, sagte ich, liegt das, weil wir es ja hier nur mit den mannigfachsten Formen der gleichen Art zu thun haben, die wir classificiren wollten. Diese Formen sind aber nicht etwa durch lang fortgesetzte äussere Einflüsse entstandene Racen, sondern, genau genommen, ist jede einzelne mit Namen belegte Form nur ein durch Aussaat gewonnenes Individuum, dessen individuelle Eigenheiten durch ungeschlechtliche Fortpflanzung festgehalten worden sind.

Die natürlichen Folgen dieser Entstehung unserer Obstsorten sind einerseits, dass Merkmale, die bei manchen Formen sehr ausgesprochen rorhanden sind, durch andere Formen allmählig zu den gerade entgegengesetzten Merkmalen übergeführt werden müssen. Dieses muss der Fall sein, weil alle Merkmale, die wir zur Unterscheidung z. B. der Aepfelsorten benutzen kömnen, ja nur solche 
sind, in denen der Apfelbaum als Art eben durchaus unbeständig und darum den nannigfachsten Abänderungen bei den aus Samen gezogenen Individuen unterworfen ist. Aber nicht bloss diese Theorie, sondern auch die Erfahrung lehrt, dass es nicht ein einziges Merkmal giebt, wodurch die Classen oder Ordnungen der Aepfel von einander unterschieden werden, welches nicht von manchen gleichsam in der Mitte stehenden Sorten übergeführt wird. Andrerseits sind aber die dem Individuum, sowie dem durch ungeschlechtliche Fortpflanzung von dem ersten Mutter-Individuum erhaltenen Nachkommen eigenen speciellen Charaktere, auch, je nach den äusseren Einflüssen, unter denen solche leben, veränderlich. So z. B. bleibt der rothbackige oder selbst rothe Apfel in tiefem Schatten grün. Ferner ist es bekannt, dass wir vom verwilderten oder schlecht gehaltenen oder nicht gehörig ernährten Hochstanme Früchte erhalten, die mit gut ausgebildeten von Spalier oder Niederstamm, oder selbst vom gut gehaltenen Hochstamme, gar keine Aehnlichkeit besitzen. Wenn nun ferner gar Obstsorten aus dem nördlichen rauhen Clina in ein milderes, oder auch ungekehrt, aus milderem in rauheres übergeführt werden, so verschwinden viele Eigenschaften oft ganz. Keiner von Ihnen würde den Belui Naliv (Belui Nalivia) der Petersburger Gärten unter die feinen und guten Calville rechnen, zu denen die in Deutschland gezogenen Früchte von den deutschen Pomologen mit Recht gestellt werden, während unsere in Petersburg am Hochstamme gezogenen Früchte eher zu den Schlotteräpfeln zu rechnen sein würden, da der edle Geschmack solchen meist ganz fehlt. Unsere nordischen Glasäpfel verlieren die Eigenschaft, bei der vollkommenen Buumreife ein durchsichtiges, saftiges, zuckersüsses Fleisch zu bilden, in den milden Climaten ganz. Ueberhaupt zeigen alle vom Süden Russlands bis zum $60^{\prime}$ und $61^{\circ} \mathrm{N}$. Br. reichenden Aepfel, wie z. B. der Aport (Kaiser Alexander des Auslandes), der Borowinka, etc. 
so bedeutende Unterschiede im Süden und Norden, dass man solche kaum als identisch nachweisen kann.

Aus allem diesem schliesse ich, - wovon ich mich auch bei meinen Arbeiten über die Pomologie Russlands vielfach überzeng๋ habe - dass es überhaupt keine durchaus unwandelbaren Charaktere für pomologische Systeme der gleichen Pllanzenart giebt, oder dass überhaupt je solche aufgefunden werden kömnen. Ich halte desshalb diejenigen Merkmale zur Aufstellung der pomologischen Systeme für am besten gewählt, die am leichtesten in die Augen fallen, und doch eine grössere Menge von Formen zusammenfassen. Solche Merlkmale sind schon von ältern Pomologen und in nenerer Zeit auch von Lucas ganz vortrefflich benutzt worden, sodass ich die Nothwendigkeit der Aufstellung ron Systemen nach andern Grundsätzen um so weniger einsehe, als damit nichts weniger als eine wissenschaftliche Aufgabe vollendet, sondern einfach eigentlich nur ein Schema gegreben wird, nach dem es möglich ist, einzelne Obstsorten mit annähernder Gewissheit $\mathrm{zu}$ bestimmen.

M. Koch aroue qu'il y amra toujours des difficultés, mais il est d'avis, qu'il fiut tâcher de parvenir à composer un système aussi complet que possible.

M. Nedzelsky fait observer, que les dénominations que l'on trouve dans les catalogues sont incomplètes et en général peu justes.

M. de Beucker dit, que les praticiens doivent venir en aide aux théoriciens, puisqu'ils sont leurs meilleurs soutiens. La forme des arbres en fait recomnaître l'espèce, parce que la forme de l'arbre est celle du fruit, même les feuilles et les pédoncules en rendent la forme. Tout cela est dans l'économie de la nature. Il démontre sa théorie par des dessins sur la planche. 
IV. M. le Président annonce', que M. D'Avouilh DE SALIEs désire soumettre au congrès la question qui suit:

,Si l'on veut avoir de beaux fruits en général, et spécialement sur des poiviers soumis à la taille, il y a un avantage incontestable i ne conserver que trois ou quatre fleurs clans chaque bouton; et de supprimer, en pinçant ou autrement, toutes les autres au moment oic le bouton s'ípanouit.

V. M. Entz-Ferenez (de Bude en Hongrie) traite la question suivante: Quelle est la destination véritable des plantes dans l'économie de la nature?

Il s'exprime en allemand en ces termes:

Nicht nur der Aufschwung der Dichtersprache nennt die Natur die schaffende, sondern auch die besomene des Naturphysiologen darf derselben dieses Prädicat nicht vorenthalten; denn gleichwie einst das schaffende ,Werde" des Schöpfers das wachsende und bewegte Leben aus seinem starren Nichts hervorrief, so ruft alljährlich auch heute noch durch ihren Herold, den milden Frühlingshauch, die Natur ihre bunten Kinder der Vegetation aus dem üden Chaos des Winters zu neuem Schaffen, Wachsen und Leben hervor, und fristet und sichert in dem schaffenden Wirken dieses ihres Erstgreborenen, d. i. der Vegetation, den Bestand und die Wohlfahrt ihres letzten Schosskindes: die Existenz der Thierwelt. Dass mithin die Natur schaffe und dies auch heute noch thue ist uns wohl Allen klar, und sollte selbst die Gewohnheit der täglichen Anschaumg den Nimbus des Wunderbaren von dieser ewig neuen Schöpfung und von der bereits gewohnteu Anschaumg derselben für uns abgestrichen haben.

Wenn nun aber auch erst ein grosser Naturforscher in einer Anwandlung trübseliger Begeisterung den Ausspruch gethan: ,Ins Inn're der Natur dringt kein erschaff- 
ner Geist," so sei es uns dennoch erlaubt, in aller Bescheidenheit wenigstens den Versuch zu wagen, den Schleier der keuschen Jungfrau Natur, unter dessen Hülle sie ihre Schöpfungen vollbringt, wenn gleich auch nur um einen kleinen Theil. zu lüften.

Seitdem uns das praktische Genie der Fraunnhofer die Simne geschärft, und die Neugierde und Ausdauer der Forschung lohnender geworden ist, taucht manches Räthsel und Geheimniss der Natur in einem von HaLLer noch ungeahnten Lichte ror dem erstaunten Blicke auf; und wenn gleich auch heute noch die Erfolge der Forschung keinesweg's befiriedigend erscheinen, so rechtfertigen sie doch wenigstens die Ahnung kommender, grösserer Erfolge, und schärfen den Sporn für dieses Ringen nach Klarheit und Wissenschaft. Ist doch der Tanz des Jünglings auch nicht anders als ermüdendes, meist unbelohntes Ringen nach dem süssen Lohn der Braut, und dennoch tanzen und tanztev wir alle so gerne. Des reifen Mannes Braut ist die Natur; ihr gelte sein Ringen; und glaubt er der scheuen züchtigen Jungfrau auch nur ein flüchtiges Lächeln abgerungen zu haben, so fand er, wemn auch nicht im Erfolge, doch selbst schon im Ringen des Forschens seinen Lohn.

Wenn wir den Zweck der Pflanzenwelt vom Standpunkte der heute bereits sämmtlich in einander greifenden Naturwissenschaften betrachten, so kann es wohl kaum mehr ein Räthsel bleiben, dass der Zweck der Pflanzenwelt, im Allgemeinen, die Fixirung der Elementarstoffe sei, um selbe in Raum und Zeit deutlicher und materieller in die Erscheinung treten zu lassen, und für jene höheren Stoffumwandlungen vorzubereiten, welche veiterhin die Thiervelt zu besorgen hat.

Sauerstoff, Kohlen-, Wasser-und Stickstoffi, als primäre Elementar-Bestandtheile der Luft und des Wassers, treten in ihren mannigfach combinirten Vereinigungen in den ersten Bildungen der Pflanze zuerst in die deutlichen Grenzen der 
Form, und in die des wirkenden und bildenden organischen Daseins.

Da aber die bereits in die deutliche Erscheinung getretene Organisation der Pflanze, im Bereiche der obigen vier Bildungs-Elemente sich nicht genügend erhalten könnte, so muss die Pflanze mit einem Theile ihrer bereits begonnenen, wenn gleich noch dürftigen Organisation, den sonnigen Luftraum verlassen. Sie senkt sich in das Imnere des Erdkörpers ein, wm in den Bereich der übrigen Elemente ihres Planeten zu gelangen, welche in ihren einfachen binären Verbindungen der einmal erwachten organischen Thätigkeit bereits die Bedingnisse einer höheren Organisation und den Grundstoff zu deren weiteren Entwicklung bieten; - und siehe da: die reiche Vorrathskammer der fossilen Elemente aller Art steht der Wurzel der Pflanze in all ihren Saugorganen von nun an offen.

Die Pflanzenzelle, der Erstlings-Beginn organischer Form, und das erste Resultat günstig combinirter ternärer und quaternärer Verbindungen elementärer Urstoffe, tritt nun einmal entstanden alsbald ihre organische Thätigkeit an, und bedient sich nun — im Verhältniss ihres speciellen Organisations-Charakters - der ihr zur Verfügung stehenden binären Combinationen zur selbstständigen Erzeugung höherer Verbindungen, in Gestalt von ternärer, quaternärer, ja quinternärer Formen; d. h. sie wird im Verhältniss zu ihrem specifischen organischen Charakter zur Werkstitte höherer und vollkommener Stoffverbindungen, deren einige wir, ihrẹr ferneren Aufgabe wegen, näher betrachten müssen.

Wir selıen nämlich, lass, z. B. eine ganze Reihe pflanzlicher Organismen, die ternären oder auch noch complicirteren Verbindungen von Stärkemehl, Zucker, Gummi, Oel, Kleber und Eiweiss unter dem Einflusse von Luft und Wärme aus den einfachen Elementar-Verbindungen der Luft und des Wassers faktisch erzeugt. Diese Reihe der Pflanzen erzengt and liefert mithin das pflanzliche 
Ernährrmgsmaterial für die Thierwelt; oder ich möchte sagen: diese Pflanzen fabriziren die Nahrstoffe für die Thierwelt, bilden die schaffende Vorrathskammer sämmtlicher pflanzlicher Nahrungsstoffe, und begründen die Möglichkeit der Existenz einer höheren und vollkommneren organischen Welt. Ja, analoger Weise liefern oder fabriziren verschiedene unserer mamigfach construirten Fabriken aus einfachen Rohstoffen die verschiedenartigsten Fabrikate complicirterer Art, und lieferm mithin das Material für die höheren Zweche unseres Daseins, z. B. elzengen je aus Weizen oder Miris, Stäremehl, Zucker, Weingeist, Essig u. s. w.

Eine zueite gleichfills sehr ausgebreitete Reihe der Pflanzenwelt umfisst jene Pflanzen, welche ihre schaffende Unbildung der primären Elemente vorzüglich auf die Erzeugung der Pflanzenfiser ausdelmen; sie werden mithin in Verholzung der Pfinzenfaser nicht uur zur Ersatzquelle von Wärme und Luft, sondern fabriziren auch dem Menschen das Material zu einer sich noch höher aufzuschwingenden Existenz. Der Mensch kamn sich mit den Erzeugnissen dieser Pfanzenreihe bereits ein behagliches Leben sichern, indem sie seinen Gewerbfleiss wecken und nähren; er kamn sich mit den Produkten der feineren Faserpflanzen bekleiden, sich aus den festeren Hulzarten sichere Wohnungen und Schiffe banen, kamn sich durch ihre Hülfe sein Amrecht auf die Herrschaft und den Vollgenuss der sämmtlichen Naturschätze seines Planeten begründen und sichern.

Eine dritte Reihe der Pflanzenwelt umfasst jene Organismen, welche die einfachen oder binären ElementarCombinationen zur Erzeugung solcher Alkalö̈le verarbeiten, welche die heutige Wissenschaft wohl in ihren Erfolgen bereits kemnt, deren nähere Elementar-Combinationen aber ihr noch gegenwärtig als unaufgelöstes Problem vorliegen. Wir sehen in diesem Bereiche jene pflanzlichen Organisationen, welche Arznei- oder Farbenstoffe liefern; 
oder solche, welche bereits wohl bei verschiedenen technischen oder industriellen Aufgaben in Verwendung kommen, jedoch über das wie ihrer Wirkung noch als unenträthselt anzusehen sind. Dies dürfte um so natürlicher erscheinen, da selbst die Erkenntniss ihres Daseins erst der jüngsten Entwicklungs-Periode der Wissenschaft anheim fällt, und die Ergründung ihrer dem Anscheine nach complicirteren Natur eine die hentige bei Weitem überragende Reife der Gesammtsumme der Naturwissenschaften vorauszusetzen scheint. Der Erfahrung nach sehen wir jedenfalls in der Pflanzenwelt dieser Reihe jene freundlichen Gaben der Natur, welche in den Händen kundiger Schüler Aesculaps den Segen gresunden organischen Daseins sichern und erhalten, so wie auch Krankheiten zu heilen im Stande sind. Andrerseits erzeugen und vervielfältigen sie, als Färberpflanzen, die Wunder der Sinnenreize, und scheinen mitunter ihren Aufgaben auch jene zu erfüllen: das Nützliche mit dem Schönen zu praren, und den Sinn für dasselbe in den Wesen höherer Organisation zu wecken und weiter fort zu bilden.

In einer vierten Reihe der Pflanzenwelt stossen wir auf jene, in welchen die Stoffumwandlung sich nicht mit der Erzeugung von Nährfaser, Arzneistoff, oder, mit einem Worte, mit der Bildung von, dem Gehalte nach, werthvollerer Materie befasst, sondern wo die Grazie und der Adel der Form, gehüllt in das Kleid wundervollen Farbenschmelzes, die Aufgabe oder die Mission bezeichnen. In den Blumen gab uns die Natur, nachdem sie uns mit Nahrung, Kleid und Wohnung, kurz mit allen materielleren Bedürfnissen bereits versehen hatte, auch die Musterkarte des Schönen, um den an ihrem Busen bereits gross gewachsenen, rohen Thier-Menschen zum Wesen höheren Sinnes zu veredeln.

In der Blumenwelt entfaltet sie für uns einen Band ihres grossen Buches ästhetischer Urformen; entrollt auf jedem Blatte desselben vor unseren Blicken ein neues 
gelungenes Urbild dieser Formen; predigt in jedem einzelnen Worte dieses unendlichen Buches den Sinn für das Schöne und für die Kunst ein; und zieht den Rohen, mit Banden aus Blumen geschnürt, aus dem Schlamme seiner Rohheit in die olympischen Höhen des Schönen und der Kunst empor. In den Blumen schuj sie uns eine der fruchtbarsten Mütter, aber auch eine der fieundlichsten Tüchter der Civilisation.

Wenn wix in dem Ueberblick der Zwecke und Aufgaben der Pflanzenwelt weiter schreiten, so sehen wir wieder eine grosse Masse derselben vor uns, welche den Aufbrauch der primären Elemente zur Erzeugung solcher Stoffe verwenden, welche für den Menschen, als kurzsichtigen Nutzzieher der gesammten Schöpfung, bisher nur von mittelbarem Nutzen und Interesse sind, ja ihrem Nutzen nach selbst nuch räthselhaft oder zweifelhaft erscheinen. Wir zählen in diese Reihe all jene PflanzenOrganisationen, welche die unmittelbare kleine Welt unzähliger pygmäenartiger Thierorganismen bilden, deren ganze Lebensaufgabe darin zu bestehen scheint, dass sie Mittelstufen in der Stoffumbildung darstellen, welche die ihnen in den Pflanzen ihrer Welt gebotenen, etwa binären und spezielleren Stoffverbindungen zu weiteren dem allgemeinen Zwecke der Fortbildung entsprechenderen empor zu potenziren oder heran zu reifen, bestimmt sind.

Noch stehen wir ferner vor einer grossen Reihe von Pflanzen-Organisationen, welche uns in Hinsicht ihres Zweckes als noch undurchdringliches Räthsel erscheinen; doch darf uns dieses, bei der Jugend der Blüthe, deren sich die Naturwissenschaften erst seit Kurzem erfreuen, nicht befremden; jedenfalls lässt sich aber in Hinsicht dieser Reilıe im Wege der Analogie und Induction im Allgeneinen das sagen: dass sie gleichfalls als Werkstätte dienen, mittelst welcher die Natur die einfacheren Elemente zu greifbasem Stoff umgestaltet, und in bestimmte Formen reiht, wenn auch deren spezielle und unmittel- 
bare Verwendung im Haushalte der Natur noch einige Zeit ein ungelöstes Problem verbleiben sollte. Wenigstens berechtigen uns die genügenden Erfolge der jüngsten Forschungen, welche die Wissenschaft seit der kurzen Zeit ihrer Entstehung vor sich gebracht, auch hier zu den besten Hoffnungen; um so vielmehr da wir uns gerade gegenwärtig in einem Lande zu befinden die Ehre haben, wo die Pflanzenkunde in der Person des unsterblichen CARL voN Linné ihre ersten Triumphe gelungener Forschungen gefeiert, und zwar sowohl in der Herausgabe seines ,Hortus Cliffortianus," als überhaupt in der Begründung seines Sexualsystems. Wenn wir dann ferner bedenken, dass selbst dieser entscheidende Schritt vor kaum mehr als einem kurzen Jahrhunderte erst gethan wurde, so darf uns wohl kaum die Trostlosigkeit darüber entmuthigen, dass wir auf diesem Felde noch einiges Räthselhafte vor uns finden.

Und wenn es mir nun nach obigen Prämissen einigermassen gelungen wäre, meine Ansicht über die eigentliche Aufgabe der Pflanzenwelt im Haushalte der Natur zu verdeutlichen, und wenn es daher vielleicht klar geworden wäre, dass die Pflanzen, die in Luft, Wasser und Erde enthaltenen Grundelemente, welche zwar zugleich auch das Grundmaterial für die Möglichkeit des organischen Daseins in sich schliessen, - welche Grundmateriale jedoch in den einfachen Binär-Verbindungen für das höher organisirte Thierleben unzugänglich sind; — wenn, sage ich, die Pflanzen diese einfachen, bloss auf den Gesetzen der chemischen Wahlverwandtschaft beruhenden Verbindungen, kraft ihrer spezifischen Organisation zu ternären oder noch complicirteren Verbindungen, als Stärkemehl, Zucker, Eiweiss $u$. s. w. zu verarbeiten und zu potenziren berufen sind: so nehmen sie, wie bereits gesagt, um mich nochmals bildlich auszudrücken, im Haushalte der Natur eben iene Stelle ein, welche im menschlichen Haushalte diejenigen unserer Werkstätten einnehmen, deren Aufgabe es 
ist, unsere Rohstoffe, wie etwa Wolle, Häute oder Körnerfrüchte u. s. w. zu höherem Zwecken zu verarbeiten.

Ich habe übrigens in der Beleuchtung obiger Ansichten zwar nichts Neues gesagt, aber jedenfalls durch das Detail derselben den Weg zu jenem Standpunkte herangebahnt, von welchem aus der Mensch von nun an die Pflanzenwelt zu betrachten und zu würdigen hat, wenn er sich all jene Vortheile durch sie sichern will, welche sich ihm, bei Berücksichtigung ihrer physiologischen Verhältnisse, sowohl im Haushalte der Natur als auch in seinem eigenen, zu gewähren im Stande sind.

Dass der Mensch die bei weitem deutlicher ausgeprägten physiologischen Verhältnisse der ihm verwandteren Thierwelt eher als die der Pflanzenwelt aufgefasst und in seinen Forscherkreis gezogen hat, darf wohl kaum befremden, müsse aber auch seine natürliche consequenten Erfolge in der Praxis eher als in jener der Pflanzenwelt zu äussern und zu verwerthen im Stande sein, d. h. musste deren Ausbeute in der Praxis schon lange her sichern.

Da nun der Mensch die nähere Bekanntschaft der ihm näher liegenden Thierwelt in Bezug auf ihre Aufgabe früher machte und die Bedingnisse ihres höheren Gedeihens auch früher und leichter erkannte, so brachte er dieselben auch, vom eigenen augenfälligen Vortheile angetrieben, früher in selbstbewusste praktische Anwendung; und siehe da sein consequentes Streben: die mit Selbstbewusstsein verfolgte Cultur oder Veredlung der ihm nützlichsten Thiere, des Pferdes, des Schafes, der Rinder, gelang ihm auch bereits in einem nicht zu verachtenden Grade. Kuxz, er erzeugt bereits heute mit selbstbewusster Consequenz Ausgezeichnetes an Adel oder Masse, an all jenen Thieren, welche er in den Bereich seiner Cultur aufgenommen hat; und er veredelt sie effectiv, je nach der Richtung seines Bedarfes.

Jedoch wie stand und steht es in dieser Beziehung mit den Pflanzen? 
Mit diesen steht es, wie wir zu gestehen gezwungen sind, bei weitem nicht so erquicklich, trotzdem dass die Pflanze eigentlich die Basis des besseren Gedeihens der Thierwelt ausmacht.

Zwar hat der Mensch vom Beginne seiner Existenz den unendlichen Werth der Pflanzen anerkannt und auch anerkennen müssen. Er musste dies, seit der hungrige Magen des rohen Wilden in ihren mannigfaltigen Erzeugnissen und Früchten Nahrung, und seine natürliche Nacktheit Material zu Bekleidung und Wohnung in ihnen gefunden; seitdem er mit ihren Erzeugnissen die Grauen des Winters und der Finsterniss mit Erfolg belrämpft; seitdem er ihre Blüthen zum Braut- und Todtenkranze seiner Lieben verwendet; und seit er sich in frommer Anerkennung ihres Werthes in der Pflanzenwelt sofort die Lieblinge seiner Götter, oder die milden Gaben ihrer Huld erschaut.

Der Mensch, später grösser geworden an Geist und Bedürfnissen, nachdem sich der Nomade eine stätige Heimath geschaffen hatte, sammelte diese Gaben der Götter um sein Haus, und beschützte sie, gleich jener Erde, die sie ihm erzeugt; und diese Erde ward ihm sein theures Vaterland.

Später, wieder grösser geworden an Geist und Bedürfnissen und im Genusse der Gaben seiner Götter, nach deren Besitze auch noch lüsterner geworden, befriedigten ihn die Naturschätze seines Heimathslandes nicht mehr, und er zog mit dem Wanderstabe oder dem Säbel in der Faust in die Ferne, um neue und wieder neue aufzusuchen, erbaute sich Schiffe, um diese reichen Naturgaben in der fernsten Ferne aufzufinden; jedoch standen seinem nimmer satten Durste nach Genüssen lange nur die äusseren Säume der ihm damals bekannten und benachbarten Welttheile für seine Durchforschungen offen.

Endlich drückten die Entdeckungen des grossen Columbus und später die von Magelhaens, Cook, u. s. w. 
dem Menschen den Schlüssel zur Erkenntniss der sämmtlichen Pflanzenwelt in die Hand, und mit seinem Besitze war die Kindheit der Pflanzenkunde beendet; der beschränkte Kräuterkenner wurde von nun an zum Botaniker; der Weg zu einer sich höher aufzuschwingenden Naturkunde und jener einer höheren Civilisation war gebahnt. Jetzt entrollte sich erst die ganze Fülle der Pflanzenwelt vor seinen erstaunten Blicken, ohne das Bruchstückliche, und die daraus entstandenen bisherigen Lücken und Räthsel, an deren Klippen die Forschungen eines Theophrast und Dioscorides in den Tagen älterer Culturs-Epoche scheiterten, und überliess der vollen Erkenntniss ihres reichhaltigen Materials den Raum, und erschloss vor dem Blicke glücklicherer Forscher das weite Thor umfassender Erkenntniss des Pflanzenreiches in allen seinen Organisations-Modificationen. CARL voN LINné war nun, wie gesagt, der erste, welcher die glücklichen Errungenschaften seiner Zeit gehörig auszunützen wusste, und nun von Holland aus die befriedigende Ausbeute seiner Forschungen in der Aufstellung seines Sexualsystems niederlegte, um in demselben den Forschern kommender Geschlechter die Basis glücklicheren Strebens anzubahnen, im Bereiche der Botanik, welche nun durch ihn zum Range einer systematischen Wissenschaft erhoben ward. Die Botanik hatte mit LinNÉ, und durch ihn, ihr hoffinungvolles Jünglingsalter erreicht.

Die Bahn war gebrochen; geniale Heroen weihten sich von nun an ihrem Dienste und bewarben sich um die Gunst der jugendlichen Wissenschaft. Jussieu, Decandolite, Endicher und viele Andere, unterstützt durch die mächtigen Hülfsmittel der gleichzeitig jugendlichen Schwestern, der Chemie und Microscopie, liessen sie unter unseren Augen zu einer erfreulichen Reife gedeihen, die, wenngleich noch nicht vollendet, dennoch die Morgenröthe der Mannesreife der Pflanzenkunde vor unseren Blicken aufdämmern lässt und uns ihre Segnungen, wenn- 
gleich noch nicht im Vollgenusse schenkt, jedoch bereits mit Gewissheit ahnen lässt.

Wissen ist immer schön, doch hat nicht jedes Wissen seinen praktischen Werth. Der praktische Werth unseres Wissens setzt immer vollkommene Kenntniss des Substrates unserer Wissenschaft voraus. Die allseitige Erkenntniss des Substrates der Pflanzenwelt wird uns täglich klarer. Wir kennen die Organisation der Pflanzen; und nach obiger Detaillirung kennen wir ihre eigentliche Aufgabe im Haushalte der Natur vielleicht auch deutlicher. Es bleibt uns mithin nur noch eine Frage weiter zu erörtern übrig, und diese lantet:

Welchen praktischen Nutzen darf sich nun der Mensch von der zur Mannesreife herangewachsenen Pfanzenkunde versprechen?

Ich glaube, der praktische Nutzen, den die Pflanzenkunde dem Menschen zu gewähren im Stande ist, dürfte kaum unter jenen zu setzen sein, welche die Praxis in Bezug auf das Thierreich bereits in selbstbewusster wissenschaftlicher Consequenz sich errungen hat.

Was England in Pferden, Holland in Rindern, Schlesien und Ungarn in der Veredlung der Schafe durch selbstbewusste consequente Kreuzung, Ernährung, Wartung und Pflege geleistet, bedarf keiner weitern Erörterung.

Was der günstige Zufall im Wege der Kreuzung und der übrigen Beihülfe der Cultur bei unseren edlen Obstsorten bereits erzielt, - er machte nämlich aus dem Holzapfel und der Holzbirne die Wunder an Grösse und Güte unserer heutigen edlen Obstsorten, - was selbst das Schwanken der Empirie, und theils der Zufall, und endlich eine bis jetzt noch nicht im Allgemeinen genügend verbreitete rationelle Cultur aus der Melone, dem Spargel oder der Mohrrübe, dem Kohl und den Karfiolarten, und mit einem Worte, aus den übrigen sogenannten Küchengewächsen gemacht hat, ist gleichfalls bekannt und braucht keiner weiteren Registratur. 
Was aber endlich als wahrhaft wissenschaftliches und praktisches Resultat die Neuzeit in dieser Beziehung geleistet, d. h. im Wege der mit Selbstbewusstsein geleiteten Kreuzung verwandter Pflanzenarten, und durch die Beigabe rationell geleiteter und consequenter Cultur z. B. in Beerenfrüchten, wie etwa Erdbeeren, Stachelbeeren und anderen; ferner in Blumen, z. B. Georginen, Levkoien, Nelken, Rosen der verschiedensten Art, hervorbrachte, kurz bei beharrlich systematischem CulturVerfahren in all' jenen Vegetabilien bereits errungen habe, welche sie mit wissenschaftlich gärtnerischem Fleisse ihrer Beachtung und Cultur unterzogen hat; auf diese Erfolge, sage ich, erlaube ich mir die Aufmerksamkeit der geehrten Versammlung zu leiten, und glaube, dass sie gleich mir zufrieden sei mit den Erstlings-Versuchen und Resultaten, deren Gelingen man auf diesem Felde rein nur den Fortschritten der Wissenschaft zu danken hat.

Es sei mir endlich im Verfolge des obigen zum Schlusse vergönnt, nur noch eine Frage zu thun, welche ich in der Vorahnung befriedigender und günstiger Erfolge nicht zu unterdrücken vermag, nämlich:

Was wird und muss aus unseren vorzüglichsten Nutzpflanzen noch werden, wenn auch der Oeconom einst, gleich dem Gärtner, deren Cultur nach dem Standpuntte und Fingerzeige der Wissenschaft betreiben wird?

Es wäre allerdings voreilig, diese Frage schon jetzt beantworten $\mathrm{zu}$ wollen; denn man müsste sich hierbei in den Bereich der Fantasie versteigen, und vielleicht heute schon Weizenkörner säen, etwa so gross wie Kornelkirschen u. s. w. Ich sehe aber mein Thema für zu ernst und wichtig an, als dass ich darüber zu scherzen mich getraute.

Jedenfalls haben wir aber Anhaltspunkte für die Statthaftigkeit und Ausführbarkeit dieser Idee, und dürfen in derselben durchaus nichts chimärenhaftes erblicken, wenn wir für jene Erfolge keine Kurzsichtigkeit affectiren wollen, welche unsere meisten Nutzgewächse 
auch bisher schon, unter dem Einfluss einer äusserst beschränkten Cultur-Anwendung, aufzuweisen vermögen.

Wer will den schlagenden Unterschied zwischen wildem und wohl cultivirtem Reps verkennen? Wer den Unterschied läugnen, der bei der Vergleichung in die Augen springt, wenn wir wild wachsende, oder schlecht cultivirte Cerealien mit jenen vergleichen, welche besser, oder nach den verschiedenen Culturs-Ansichten und Postulaten der heutigen Oeconomie gut cultivirt sind, u. s. w.? Was bei einem beschränkten Grade von Cultur bereits Handgreifliches leistet, muss bei deren Potenzirung oder Verdoppelung auch doppelte Erfolge liefern.

So war meine bescheidene Ansicht, als ich mein Vaterland verliess; jetzt da ich die Bodenculturs-Verhältnisse Hollands nur einigermassen zu überblicken Gelegenheit hatte, ist aus derbescheidenen Ansicht bereits ein Glaubensbekenntniss geworden; und selbst ohne Sehergabe wird es mir klar und klarer, dass eben Holland im Kreise der civilisirten Länder zu einem der ersten berufen sei, welches meine ausgesprochene Idee ins Leben übertragen wird. Denn was müsste aus unserem Geschlechte werden, wenn einst die Bevölkerung im Allgemeinen zu jener Dichtigkeit heranwüchse, oder bestimmter gesagt, heranwachsen wird, deren sich Holland oder Belgien bereits heute zu erfreuen Ursache hat.

Meiner Ueberzeugung nach müsste sich ungezweifelt, ohne praktische Durchführung oft besagter Ideen, der einst mythologisch berühmt gewordene Appetit SATURns an unseren Kindern wiederholen; und da dies, meiner Ansicht nach, nur durch die grösstmöglichste Ausbreitung der Horticultur auch auf die Nutzgewächse aller Art zu verhindern sein dürfte, so sei es mir vergönnt, auf das Gedeihen derselben und auf das ihrer Jünger und Verehrer ein kräftiges Lebehoch! anszubringen. Denn nur die erhöhte Boden-Cultur ist undbleibt unsere unversiegbare Vollquelle des Wohlstands und Reichthums, aus welcher 
jeder zu schöpfen berechtigt ist, ohne irgend Jemanden an Gut oder sonstigem Interesse zu beeinträchtigen."

M. Kolb (de Munich) dit, que dans la Bavière Palatine on a déjà fait des expériences pour cultiver le tabac de manière à ce qu'il contienne moins de nicotine; dans ce pays on est done déjà entré dans la voie que le préopinant indique.

M. Regel demande la parole; il dit:

In Bezug. auf die vom Herrn Entz-Ferenez geäusserten Ansichten möchte ich einige Bedenken gegen die Möglichkeit derartiger Erfolge erheben.

Derselbe glaubt, dass der Gartenbau der Landwirthschaft und dem ganzen Menschengeschlecht grosse Dienste leisten könne, wenn derselbe sich der Cultur unserer Getreide-Pflanzen annehmen, und darnach streben würde von unsern gewöhnlichen Getreidearten, in ähnlicher Weise, wie dies z. B. bei den Erdbeeren gelungen sei, neue Formen zu erziehen mit bedeutend grössern Früchten, wodurch der Ertrag unserer Erndten verdoppelt und verdreifacht werden könne. Dagegen erlaube ich mir einzuwenden, dass dies in zwei Richtungen wohl kaum gelingen dürfte. In einer Richtung nämlich dürfte der Gartenbau solch einen Dienst der Landwirthsehaft schondesshalb nicht leisten kömnen, weil, wemn es ihm durch den Einfluss der Gartencultur, dureh fortgesetzte Auswahl der grössten Kömer zur Aussaat, auch gelingen würde, Racen mit nur etwas grössern Körnern von unseren Getreiden zu erzeugen, diese Racen sich höchst wahrscheinlich auch nur bei fortgesetzter, gleich sorgfältiger Gartencultur als beständig erweisen würden, während solche, aufs Feld gebracht, auch bald wieder zurückkehren dürften. Es ist schon von unsern unter besondern Verhältnissen gezogenen Gemüseracen bekannt genug, dass solche schon unter andern Verhältnissen bei sonst guter Cultur schnell wieder ausarten, wenn die Samen nicht immer von Neuem von den Orten bezogen werden, wo solche entstanden 
sind. Ueberhaupt ist das Resultat, das beim Getreideanbau im Garten gewonnen wird, für keinen tüchtigen Landwirth massgebend. Wenn für den Feldbau constante neue Racen gezogen werden sollen, so muss das auf dem Felde geschehen, und der Landwirth hat ja mit den Maschinen, welche die grössten Körner des Getreides von den kleinen sondern, das Mittel in der Hand, durch sorgfältige Auswahl des Samens eine etwelche Einwirkung zu üben.

In anderer Richtung können die Erdbeeren gar nicht als Beispiel zur Vergleichung mit dem Getreide dienen. Die grossfrüchtigen Erdbeeren sind nämlich nicht bloss durch den Einfluss der Cultur entstanden, sondern dadurch, dass von Natur grossfrüchtige Arten eingeführt und nun durch Bastardirung neue Sorten gezogen wurden. Diese Sorten sind aber, ganz wie die Obstsorten, nur Individuen, die ungeschlechtlich fortgepflanzt werden, nicht Racen, die durch Samen fortgepflanzt werden können. Endlich ist es bekannt, dass selbst die grossfrüchtigsten Erdbeersorten nur durch den fortgesetzten Einfluss der sorgfältigsten Cultur erhalten werden können. Schon im Garten verlieren solche bei nachlässiger Cultur ihre grossen Früchte, und auf das Feld verpflanzt würden solche gar nicht mehr sich selbst ähnlich sehen. Endlich ist es bei der Erdbeere nicht etwa ein Theil der eigentlichen Frucht, der jene verhältnissmässige bedeutende Zunahme an Grösse zeigt, sondern vielmehr ein Theil des Blüthientheils (der Fruchtboden), in welchem die Früchte eingebettet sind. Ein Fall, dass es durch Einfluss der Cultur gelungen sei, die innern Theile des Samens (Eiweisskörper oder Cotyledonen) irgend einer Pflanzenart bedeutend zu vergrössern, ist mir überhaupt nicht bekannt. Es scheint mir daher, dass ein derartiger Einfluss des Gartenbaues auf die Landwirthschaft, so sehr er zu wünschen wäre, dennoch ins Reich der Unmöglichkeit gehört.

M. Entz-Ferenez fait observer, que plusieurs plantes en culture se trouvent également à l'état sauvage, et que 
l'on obtient de meilleur's résultats et par conséquent de meilleures espèces par une culture plus raisonnée. Plus la culture est perfectionnée, plus les résultats seront satisfaisants.

\section{M. d'Anouilh de Salies traite la question sui-} vante:

Des moyens d'arriver avec certitude dans un délai le plus restreint possible, à la destruction complète du Puceron lanigère, ce fléau des pommiers dans beaucoup de contrées. (Voir le Programme XXXV, 1, pag. 30).

Il dit que d'après des naturalistes, entre autres d'après d'Orbignx, le Puceron lanigère (Aphis lanigera) est vivipare pendant une partie de l'année et ovipare pendant l'autre. De là la nécessité de faire la chasse aux oeufs, aussi bien qu'aux individus; il n'a jamais vu les oeufs, quant aux individus, ils sont faciles à voir, à cause du duret blanc et soyeux qui les recouvre.

Chacun peut voir, sans être entomologiste, qu'à l'approche des froids de l'hiver, la plupart des pucerons lanigères descendent des parties aériennes du pommier, et vont chercher un refuge au sein de la terre, sur les racines les plus voisines de la surface du sol et à côté de ces racines. Quelques-uns cependant n'abandonnent pas la partie aérienne, mais se réfugient alors, soit entre des crevasses des écorces, soit dans l'anfractuosité des plaies. Sous ces divers abris, les pucerons passent l'hiver. Ils en sortent successivement au printemps dans un laps de temps, que M. D'Anouilh de Salies croit impossible de déterminer. Ainsi, dit-il, il ne suffit pas de les pourchasser sur les branches, il faut encore le faire sous le sol, et c'est ce qu'on ne fait point; au moins ne l'a-t-il vu pratiquer nulle part.

Les publications horticoles foisonnent de recettes indiquées comme propres à détruire les pucerons lanigères. Le bulletin de la Société d'horticulture de Lyon, de Juin 1864, en fait connaître 15. Malgré cela, les pucerons n'en 
continuent pas moins à exercer leurs effets pernicieux sur le pommier. Pourquoi cela? C'est parce que l'on opère mal en appliquant les recettes employées, ou qu'on se contente d'en faire usage une ou deux fois seulement, ce qui est insuffisant.

M. D'AnoullH DE SALIES affirme avoir fait disparaitre entièrement, sur une collection de 40 pommiers, le puceron lanigère qui les avait envahis. Ces arbres ne poussaient plus de bois, et ils étaient devenus improductifs. Voici la série d'opérations qu'il a, dit-il, successivement effectuées dans ce but:

1. Pendant l'hiver: il a enlevé les vieilles écorces mortes ainsi que le bois chancreux et inerte des plaies, qu'il a ensuite recouvertes de cire à greffer; quelques jours après, il a fortement mouillé la surface des écorces, restées sur l'arbre, au moyen d'une éponge imbibée d'un mélange corrompu d'urine et d'eau de savon noir, liquides auxquels il avait ajouté un peu de terre argileuse pour leur donner du corps; il a ensuite frotté fortement les écorces mouillées avec un linge de laine quand elles étaient lisses et vivantes, avec une brosse rude, lorsqu'elles étaient rugueuses et mortes. Puis, avant les premiers mouvements de la végétation et dans un diamètre de 30 à 40 centimètres autour du collet de l'arbre, sur une profondeur de $0^{\mathrm{m}} 10$ à $0^{\mathrm{m}} 15$, il a fait enlever sous ses yeux toute la terre, qui a été remplacée par une terre neuve; enfin il a rapproché les productions fruitières ruinées ou trop allongées, et a enlevé sur la tige les branches décrépites.

2. Pendant l'été: il a saisi le moment où les jeunes pousses latérales passaient de l'état herbacé à l'état ligneux, pour les casser au-dessus de deux bons yeux, et a ainsi supprimé beancoup de pucerons qui avaient paru, malgré les soins des précédentes opérations; il a écrasé avec un morceau de drap les pucerons qui pouvaient encore paraître sur les bourgeons réduits ou sur les branches charpentières. Cette série d'opérations longues et laborieuses a détruit 
entièrement les pucerons sur environ les trois quarts des pornmiers atteints. L'autre quart, sur lequel des pucerons ont apparu dans le courant de l'été de l'année suivante, a été traité comme les autres l'avaient été l'année précédente et les pucerons ont totalement disparu.

Dès la $1^{\text {re }}$ année de l'opération les pommiers ont passablement donné de beaux fruits et un peu de bois; les $2^{\text {me }}$ et $3^{\mathrm{me}}$ il y a eu abondance de fruits et de pousse de bois.

VII. M. le Président communique à la section qu'il vient de recevoir de M. Bossin (de Paris), la notice suivante:

Sur la nécessité d'adapter des adjectifs latins aux variétés fixes des plantes potagères. (Voir le Programme XXX, pag. 29).

M. Thielens en fait la lecture:

„Depuis longtemps les amateur's d'horticulture de tous les pays sont privés de faire venir directement une partie de leurs plantes potagères, faute d'une nomenclature suffisamment explicative pour les désigner. Les types et les premières variétés furent cependant décrits et dénommés avec soin par les savants qui nous précédèrent dans la science horticole, et nous regrettons vivement que l'on n'aît pas continué cette grande œuvre, si laborieusement commencée dans le siècle dernier. Les noms génériques ne suffisent plus maintenant pour distinguer entre elles les nombreuses variétés fixes et les sous-variétés lég'umières, conquises depuis un demi-siècle environ par les semeurs de tous les points du globe. Ces espèces ou variétés jardinières, étant rarement accompagnées de leur adjectif qualificatif latin, il en résulte une confusion permanente et mutuelle entre les vendeurs et les acheteurs, à laquelle, croyons nous, il est temps de remédier. Ces qualifications distinctives, que nous prenons la respectueuse liberté de soumettre à la savante appréciation des membres du Con- 
grès, seraient très-utiles dans les transactions commerciale et horticole surtout. Car il ne suffit pas de parler la langue d'un pays; il faut encore, dans l'état actuel des choses, commaitire les noms vulgaires des plantes potagères.

Exemple: comment un Français demandera-t-il à un marchand ou à un jardinier de Lundres, de St. Pétersbourg, de Madrid, de Philadelphie, etc. de la graine de chou de Bruxelles, de chicorée fine cl'Italie, de poirean de Rouen, de céleri de Tours, de haricot beurre ou d'Alger, de haricot flageolet, de haricot de Soissons, de l'oignon d'Egypte, de l'oignon sous terre, etc. etc. sans crainte d'être trompé, et évidemment s'il l'était, il ne pourrait pas en rendre responsable le marchand un le jardinier auquel il se serait adressé; puisque ni l'un ni l'autre ne se seraieut compris.

Il serait done temps, pensons-nous, d'apporter un remède à ce mauvais état de choses, et́t de mettre un terme à ce dédale, dans lequel vendeur et consommateur se débattent depuis trop longtemps. Non seulement les pois, les haricots, les salades, mais beaucoup d'autres genres ne peuvent être demandés en France par les correspondants étrangers et vice versa, qu'avec des peines inouies, et bien souvent, pour lever les obstacles, les marchands sont forcés d'envoyer ou de demander des spécimens en nature pour échantillon, afirr de ne pas être trompés. Comme on Je voit, ce genre de transaction n'est ni prompt ni commode, et il faut avoir été, comme nous, 35 ans dans le commerce de graines, pour en bien comprendre et en bien apprécier tous les inconvénients que nous venons signaler au Congrès. Pour un amateur, les difficultés sont encore plus fortes, cur il arrive très-souvent, qu'il ne connait que de nom ou de réputation le légume, qu'il veut cultiver et déguster. Si la demande a lieu par correspondance, il est encure bien plus exposé à recevoir une plante pour une autre.

C'est au Congrès international d'Amsterdam qu'il appartient d'aplanir les nombrenx inconvénients que j'ai 
l'honneur de lui signaler. Son comité des plantes potagères est composé d'hommes compétens, venus de tous les pays pour y discuter toutes ces questions; ils possèdent toutes les connaissances nécessaires, pour les traiter à fond et pour les mener à bonne fin. Il faut done du bon vouloir et se mettre à l'œuvre, et d'avance nous sommes convaincus que personne ne nous fera défaut.

Voici un essai de nomenclature sur quelques plantes; j'ai l'honneur de les soumettre au Congrès, en le priant de ne voir rien d'absolu dans ce travail rudimentaire, susceptible d'être modifié et remplacé par des adjectifs latins mieux appropriés que les miens aux variétés fixes des plantes potagères.

Ce travail est basé sur les noms français pour la plupart, et nous croyons qu'il serait nécessaire de bien s'entendre sur l'origine des plantes potagères, avant d'entreprendre et de perfectionner les dénominations nouvelles. Il conviendrait, il nous semble, de bien se mettre d'accord sur les adjectifs de la chicorée de Meaux, du poireau de Rouen, du céleri de Tours, du pois Clamart, du haricot de Soissons, etc. etc. que nous ne revendiquons en aucune manière comme plantes essentiellement françaises. Ceci est loin de notre pensée.

Essai sur la nomenclature des variétés fixes des plantes potagères.

Arroche rouge ......................... Atriplex rubicunda, ou rubra.

Betterave rouge longue ................ Beta rubra longior".

Betterave blanche ...................... Beta albida.

Betterave jaune longue ............... Beta longior flavens ou lutea.

Capucine d'Alger à fleurs brunes ...... Tropahım africanum.

Cardon de Tours ....................... Cynara Cardunculus Turonensis.

Cardon d'Espagne ................... Cynara Cardunculus hispanicus.

Carotte rouge Iongue ................. Daucus ruber longus.

Carotte rouge demi-longue.............. Daucus miber semi-longus.

Carotte jaune longue .................. Daucus flavens longus.

Céleri plein blanc........................ Apium solidum album.

Céleri Turc ........................... Apium trircicum.

Céleri plein rouge do Tours ........... Apium solidum rubrum Turonense. 
Céleri rave

Cerfeuil frisé

Chicorée frisée de Meaux

Chicorée fine d'été ou d'Italie.

Chicorée Scarole

Chou pommé blanc de St. Denis

Chou cavalier, à vache

Chou rave

Chou navet

Chou quintal

Chou rouge pommé

Chou vert nou pommé

Chou de Milan frisé

Chou frisé de Savoie

Chou à jets de Bruxelles

Concombre blane long.

Concombre jaune long.

Concombre à cornichon

Courge gonrde

Fève rouge

Fève verte

Fève large de Windsor

Fève julienne (petite)

Fève naine

Fève pourpre

Haricot de Soissons à rames

Haricot de Soissons nain

Haricot flageolet

Haricot rouge de Chartres

Haricot noir de Belgique

Haricot riz

Haricot beurre on d'Alger

Haricot Suisse gris de Bagnolet

Laitue petite.

Laitue d'hiver

Laitue de Versailles.

Laitue Palatine.

Laitue Passion

Laitue Batavia blonde

Laitue Batavia brune

Laitue de Malte

Laitue Chicorée

Laitue sanguine

Laitue Romaine verte
Apium Rapum.

Scandic crispa.

Cichorium crispum Meldense.

Cichorium crispum italicum.

Cichorium Scariola.

Brassica capitata alba.

Brassica vaccina, B. procera.

Brassica gonglioides, B. Rapa.

Biassica Napus.

Brassica centumpondii.

Brassica capitata rubra.

Brassica viridis.

Brassica Sabellica crispa.

Brassica fimbriata.

Brassica multicapitata, ou polycephala.

Cucumis longus albus.

Ciucumis aureus longus.

Cucumis condimentarius.

Cucurbita Lagenaria.

Faba rubra.

Faba viridis.

Faba macrocarpa.

Faba parva.

Faba nana.

Faba purpurea.

Phaseolus Suessionensis scandens.

Phaseolus Suessionensis nanus.

Phaseolus fistulatus.

Phaseolus ruber Carnutensis.

Phaseolus niger belgicus.

Phaseolus Oryza.

Phaseolus silico-pallidus.

Phaseolus helveticus.

Lactuca parva.

Lactuca hyemalis.

Lactuca Versaliensis.

Lactuca Palatina.

Lactuca Passio.

Lactuca batava favida.

Lactuca batava, rufula, fusca.

Lactruca Melitensis.

Lactuca undulata.

Lactuca sanguinea.

Lactuca longa viridis. 
Laitue Romaine panachée

Laitue Romaine rouge d'hiver

Navet long

Navet de Clairfontaine

Navet de Meaux

Navet d'Hanneucourt

Navet petit de Berlin

Navet noir long d'Alsace.

Navet jaune long

Navet jaune rond

Oignon rouge pâle

Oignon blond ou jaune.

Oignon rouge foncé

Oignon poire.

Oignon blanc hâtif

Oignon blanc tardif

Oignon très-gros de Madère

Oignon d'Egypte ou bulbifère

Oignon sous terre

Panais rond

Persil frisé

Persil à grosses racines

Poireau long

Poireau de Rouen.

Pois Prince Albert

Pois Michaux de Hollande

Pois de Marly

Pois de Clamart

Pois de Knight à rames

Pois de Knight nain

Pois à bouquet

Pois vert Normand

Pois vert nain

Pois sans parchemin

Pois carré blanc

Radis rose rond

Radis rose demi-long.

Radis blane rond

Radis violet rond

Radis jaune

Radis noir

Rave rose longue

Rave violette longue

Rave blanche longue
Lactuca longa variegata.

Lactuca longa mbicunda hyemalis.

Brassica Napus longa alba.

Brassica Napus Clarofontensis.

Brassica Napus Meldensis.

Brassica Napus Hanneucourtii.

Brassica Napus Berolinensis parva.

Brassica Napus nigra Alsatica.

Brassica Napus flava longa.

Brassica Napus flava rotunda.

Allium Cepa rubrum pallidum.

Allium Cepa flavidum.

Allium Cepa rubicundum, ou violaceum.

Allium Cepa pyriforme.

Allium Cepa album praecox.

Allium Cepa album serotinum.

Allium Cepa Maderense.

Allium Cepa bulbiferum.

Allium Cepa subterraneum.

Pastinaca rotunda.

Apium Petroselinum undulatum ou cris. Apium Petroselinum Napus.

Allium Porrum commune.

Allium Rothomagense.

Pisum Princeps Albertus.

Pisum praecox.

Pisum Marlyacum.

Pisum Clamartianum.

Pisum Knightii elatum.

Pisum Knightii nanum.

Pisum umbellatum.

Pisum viride elatum.

Pisum vivide nanum.

Pisum excorticatum.

Pisum quadratum.

Raphanus roseus rotundus.

Raphanus roseus semi-longus.

Raphanus albus rotundus.

Raphanus violaceus rotundus.

Raphanus flavus.

Raphanus niger.

Raphanus roseus longus.

Raphanus violaceus longus.

Raphanus albus longus.

(pum.

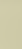


Depuis un an, notre proposition a fait son chemin, elle a rencontré des opposants, mais beaucoup plus d'adhérents. Parmi les botanistes, qui ont applaudi à notre projet, nous citerons M. DEs Moulins, président de la Société Linnéenne de Bordeaux et M. l'Abbé Brou, dont le nom est connu en botanique. M. Des Mourins nous écrivait de Bordeaux le 17 Février dernier: ..., ,Mais il n'en est pas moins vrai qu'en dehors de cette nomenclature générique, qui doit rester telle qu'elle est dans la science, vous demandez, Monsieur, une chose très-raisonnable et très-utile, lorsque vous désirez voir distinguées, à l'aide d'une nomenclature uniforme et qui soit comprise également de tout le monde, les variétés, races et formes, que les horticulteurs sont dans l'usage vicieux d'appeler espèces. Il est infiniment à désirer que vos vœux soient exaucés, dans l'intérêt de la science horticole et même botanique, et dans l'intérêt de l'industrie horticole et agriculturale. Mais aussi il est infiniment difficile d'atteindre ce but, parce qu'il n'y a pas de nomenclature officielle, légale, commandée par une autorité appuyée sur une loi pénale. Les particuliers n'y peuvent rien, les sociétés savantes de province n'y peuvent rien non plus, et pourtant tout le monde sent la gravité des inconvénients que vous avez signalés, les avantages qu'on recueillerait, si on réussissait à y porter remède enfin, la justesse du choix que vous avez fait en demandant une nomenclature latine et uniforme.

,Mes collègues m'ont donc autorisé, Monsieur, à vous faire part d'une réflexion que je leur ai soumise et de laquelle vous pourrez tirer peut-être quelque conséquence utile pour la direction à donner à vos efforts.

„C'est sous le patronage des plus grands noms de la science botanique et horticulturale à la fois que se poursuit au jardin des plantes de Paris une splendide et classique publication, nommée le jardin fruitier du Muséum. Cette publication est en dehors de la science, puisqu'il ne s'agit pour elle que de fixer les noms distinctifs et les caractères des 
diverses variétés ou races de pommes, de poires, de prunes, de pêches, de fraises etc., et n'est-il pas bien évident que tous les horticulteurs seront heureux, dans leur propre intérêt, de se conformer à cette nomenclature qui finira ainsi, non par sa force mais à l'aide de la persuasion et de l'intérêt individuel, à faire loi dans le monde horticole.

,Tel est, Monsieur, le sommaire des réflexions que votre bonne lettre m'a inspirées et que mes collèg'ues m'ont permis de vous exposer, en faisant mention de leur sympathique adhésion."

M. l'Abbé Brou nous écrit du 26 courant d'Oulins, Eure et Loir: „J'applaudis de tout cour, sans flatterie aucune, à votre projet persistant de classification adjective pour les plantes potagères. Sans être rigoureusement scientifique comme l'est celle des plantes spontanées, elle ne serait pas moins d'une grande utilité au commerce des différentes nations. Ce serait un lien de confiance entre acheteur et marchand. L'horticulture, si progressive de nos jours, se trouve grandement en retard dans sa partie la plus importante; il y a done là une immense lacune à remplir, et avec la persévérance et la ténacité qui ne vous manquent pas, vous atteindrez,j'espère, votre but. Déjà par des noms très-heureusement trouvés, comme Brassica multicapitata, Phaseolus silico-pallidus, vous avez très-bien caractérisé ces plantes, et il n'y a pas à s'y tromper. S'il vous est possible d'en former toujours d'aussi bons pour toutes les variétés, vous convaincrez vos contradicteurs, et il viendra un jour, j'aime à me le persuader, où le jardin potager aura sa nomenclature, comme l'a depuis longtemps le jardin fleuriste, ainsi qu'on peut levoir dans le bon jardinier et sur les catalogues de votre maison, où les noms latins jouent le premier rôle et sont aussi bien compris en Angleterre, en Allemagne, que dans notre France. Je sais, mon cher M. Bossin, que vous ne vous faites pas illusion. Vous ne prétendez pas, par votre méthode, arriver à la précision des caractères de la bota- 
nique proprement dite, ce qui serait impossible quand il s'agit de nommer tant de variétés si peu différenciées, mais ce que rous pourrez faire, ce sera d'établir cette méthode assez clairement pour mettre le commerce à l'abri de malentendus nombreux et journaliers. Vous aurez ainsi bien mérité de l'horticulture."

Nous avons reçu du président de la Société Royale d'horticulture de Londres les lignes suivantes en réponse à notre communication du 15 Mai 1864.

„J'ai reçu longtemps avant la séance votre proposition pour une uniformité de noms latins pour des légumes de cuisine. Nous voyons des difficultés considérables dans cette question, mais soyez persuadé que pourtant le résultat répondra à rotre attente." Londres 22 Juin 1864, South-Kensington.

Les adjectifs latins, que seuls nous avons adapté aux variétés fixes des plantes potagères, ne sont pas exempts de reproches; pour bien les appliquer à chacune des variétés, il faudrait connaitre l'origine de chaque plante, mais hélas, nous ne la connaissons pas chez toutes. Cette prémière liste si imparfaite qu'elle soit, sera suivie d'une autre, si MM. les botanistes et les latinistes veulent bien nous venir en aide pour celle-ci, et nous seconder dans nos premiers efforts.

Le congrès d'Amsterdam pourrait commencer à étudier cette question sur 40 ou 50 plantes potagères; l'année prochaine, un nouveau congrès s'occuperait de latiniser le reste. De la sorte, on arriverait facilement à adapter des adjectifs latins à toutes les rariétés de légumes, dans un laps de temps qui ne serait pas encore très-long. Seulement je supplie MM. les membres du Congrès d'Amsterdam, de voir dans ma proposition un travail difficile sans doute, mais qui n'est pas impossible. Si le grand Linné n'avait pas surmonté de plus grands obstacles, où en serait donc la botanique aujourd'hui? 
M. van Hulle fait les remarques suivantes:

Dans ma manière de voir, il faut conserver la langue du pays où elles ont été données, aux dénominations des variétés de plantes potagères, lesquelles, soit dit en passant, sont rarement fixes. Mais pour ce qui concerne les noms d'espèces et surtout les noms de genres, je crois qu'il serait utile et même nécessaire, que tout maraîcher sache le nom latin ou scientifique des plantes qu'il cultive. II apprendrait à comnaître par là, que souvent deux plantes qu'il avait considérées comme très-différentes, par ex. Andijvie (la Scarole, Endives, Cichorium Endivia L.) et Suikerij (chicorée sauvage, barbe de capucin, Cichorium Intybus L.) sont au contraire frère et soeur, comme on dit vulgairement, et peuvent par conséquent soit dégénérer comme portegraine, soit avoir à peu près les mêmes propriétés, soit exiger à peu près la même culture. Pour ces motifs aussi j'ai mis partout le nom latin à côté du nom vulgaire, dans la $2^{\mathrm{e}}$ édition en langue flamande de mon ouvrage sur la culture des plantes potagères; pour ces motifs aussi je dois remercier M. Bossin d'avoir attiré l'attention sur ce sujet.

VIII. M. Geitner (de Planitz en Saxe) s'était proposé de discuter la question de la consolidation de la profession du jardinier. L'heure étant trop avancée, il dépose un réglement d'un fonds de pension pour les jardiniers, basé sur le principe des associations mutuelles.

Le projet en question contient des détails sur le but de l'association et sur les conditions d'entrée, sur les devoirs et les droits des membres, l'administration des fonds, les assemblées générales, etc.

IX. M. Kolb (de Munich) dit, que, conformément à l'annonce du Programme (XXXI, pag. 29), il déposera 
une notice sur le système de vigne de Hoorbrink, principalement en rue des espèces d'engrais à employer (1).

X. M. le Président annonce que la parole serait à M. KraAijenbrink, du Loo, sur les questions qu'i] a proposées (voir le Programme pag. 26, XIII, 1, 2). M. KraAijenbrink n'assistant pas à la séance (2), M. le président donne la parole à M. D'ANodilh DE Salies, qui développe les questions qu'il a annoncées au Programme (pag. 30, XXXV, 2, 3 et 4), savoir:

a. Des avantages que peut offir dans bien des cas le greffage en fente, au coin du feu ou sur les genoux.

b. Des moyens de tirer partie, au point de vue de la production, des arbres fruitiers en général, et notamment de ceux à pépins, arbres plus ou moins âgés, arrivés à l'infertilité, même à une sorte de décrépitude, par l'effet de mauvais traitements ou par défaut de soins.

c. De l'habillage des racines dans la transplantation des arbres en général, et notamment dans ceux qui sont adultes et à feuilles caduques.

Sur la question du greffage au coin du feu, M. d'Anouilh de Salies s'exprime à peu près en ces termes:

Le greffage au coin du feu ou sur les genoux est une application commode de la greffe en fente ordinaire. Le très-petit nombre d'auteurs qui en parlent, ne font que l'indiquer. Je ne sache pas, dit-il, qu'il se pratique ailleurs qu'en France, notamment dans le Lyonnais. Je voudrais le vulgariser, afin d'inspirer le goût de l'horticulture à la jeunesse et réduire ainsi la masse de temps qu'elle emploie souvent dans l'oisiveté. Le greffage au coin du feu

(1) Nous regrettons vivement que, malgré notre demande réitérée, MI. KouB n’ait pu jusqu'à présent nous faire parvenir la notice annoncée.

(2) M, KraAijenbrink a déposé une notice, que nous publierons sous forme de supplément. 
ayant lieu dans une chambre, on pourrait s'y livrer en famille comme diversion agréable. M. D'AnouIlH DE SALIes a opéré dans sa chambre, pendant les premiers jours d'avril 1864, 38 poiriers sur lesquels il a obtenu 28 soudures; 4 coignassiers, sur lesquels ont repris les quatre greffons du genre poirier; 15 cerisiers, Ste Lucie, pruniers ou abricotiers, qui n'ont donné que deux soudures. Pourquoi ce mécompte sur ces derniers sujets? M. D'ANoumL DE SALIES n'en sait rien. Il croit que le greffage au coin du feu offre plus de chances de réussite quand il a lieu au printemps, lorsque la sève se remet en mouvement, que pendant l'hiver. Cependant, il n'a pas fait assez d'expériences pour oser se permettre à cet égard une affirmation absolue.

M. D'Anodilh de Salies en décrivant les moyens employés par lui pour coopérer au succès de la soudure de la greffe au sujet, a rapporté deux précautions, qu'il importe de relater ici.

10. Il avait entouré la portion fendue du sujet et la totalité de la greffe de deux tours au moins d'une feuille de papier, affectant après le placement, la forme cylindrique; il avait attaché ce papier au sujet par sa partie inférieure et l'avait fermé à sa partie supérieure par deux simples plis du papier, agissant comme préservatif de l'action désorganisatrice des hâles du printemps. On sait, a-t-il ajouté, que toute partie d'un végétal renferme en elle un principe vital propre, même après qu'elle a été détachée du pied-mère, et que dans cet état d'isolement elle ne peut recevoir aucun suc des racines. Mais ce principe s'éteint dans la partie détachée, lorsque la sève qui lui est propre et qui entretient sa vie, s'est évaporée. On peut dire, quand la soudure d'une greffe a eu lieu, que la sève, ou une partie de la sève qui lui est propre, s'y est maintenue jusqu'au moment où la sève du sujet $y$ a pénétré en plus ou moins grande quantité. Il faut done, jusqu’à la soudure, s'étudier à empêcher le plus 
possible l'évaporation de la sève propre à la greffe. Le morceau de papier enroulé est un moyen aussi simple que sûr de remplir ce but.

2. Les rameaux employés par lui pour greffer avaient été détachés en novembre 1863; il les a utilisés comme scions dans les premiers jours du mois d'avril suivant, c'est-à-dire, 5 mois après. Mais il les avait constamment tenus à l'ombre d'un mur. Malgré un hiver long et rigoureux, les rameaux n'avaient point été désorganisés, bien qu'ils eussent été gelés. Dans ce dernier état, ces rameaux auraient eu certainement perdu leur principe vital, si les rayons solaires étaient venus les frapper et produire par là en eux un brusque et très-sensible changement de température. Ayant toujours été tenus à l'ombre, ils se sont dégelés lentement, voilà pourquoi la désorganisation n'a pas eu lieu.

Sur la deuxième question M. D'ANourlm DE SAlies fait observer que, si les arbres, objet de la question, fleurissent, les fruits nouent rarement, et que s'ils nouent, ils n'arrivent pas à maturité, mais tombent avant terme.

Voici comment a agi M. D'Anouilh de Salies sur près de 700 arbres, la plupart pommiers ou poiriers: 10. Il a enlevé les vieilles écorces desséchées, pour permettre aux canaux séveux de se dilater et afin de détruire les insectes qui pouraient s'y trouver. 20. Il a fait travailler les pieds des arbres avec une fourche ou une bêche à trident, pour ne pas endommager les racines; en même temps, le terrain travaillé a reçu un paillis et a été arrosé avec un engrais liquide, mais pas trop puissant, pour que les radicelles ne soient pas exposées à être brûlées par lui. 3. Il a beaucoup rapproché la plupart des productions fruitières qui étaient épuisées, ridées et trop allongées. 4. II a rapproché sur leur empâtement, du $5^{\text {me }}$ au quart des branches charpentières, en choisissant les plus ruinées, et il a eu soin de mettre des emplâtres sur les plaies. 5. Enfin, il a badigeonné avec un lait de chaux délayée 
avec de l'urine et de l'eau de savon noir, sinon toutes les branches, tout au moins la tige et les parties les plus âgées des plus fortes branches.

Pendant la première année de l'opération, les arbres ainsi soignés ont déjà donné assez de fruit et ont poussé un peu de bois; l'amnée suivante, il y a eu plus de fruits et de bois; enfin, la troisième année, ces arbres sont rentrés dans l'état normal de fruit et de bois, et ils sont maintenant, dit M. D'ANourlm de SAlies, non pas beaux, mais productifs et en santé.

M. D'Anouilh de Salies assure que dans les trois ans qui ont suivi le traitement, il lui est mort une douzaine d'arbres sur les 700 qui ont été soignés. Il présume que la mortalité en aurait atteint plus d'une centaine, s'il avait laissé les arbres dans l'état pitoyable où ils étaient.

Passant à la troisième question, l'orateur fait les remarques suivantes:

Jusqu'à présent, quand il s'est agi de planter un arbre de tout âge, surtout adulte, on s'est efforcé de le mettre en terre avec toutes ses racines, ou avec le plus de racines possible. Maintenant, un habile praticien, M. VERRIER, jardinier en chef de l'Ecole Impériale de la Saulsay, en France, avance que ce principe est, sinon vicieux, tout au moins bien inférieur à celui qu'il préconise et qu'il a expérimenté, affirme-t-il, depuis 20 ans. Ce principe préconisé consiste à réduire, entre le tiers et le quart, la longueur totale des racines que l'arbre avait avant d'être déplanté. Il serait bon, dit M. D'Avourly DE SAlies, d'avertir le monde horticole de cette découverte, pour que des expériences comparatives puissent être faites sous divers climats et dans diverses natures de terrain, afin de savoir si le fait avancé par M. VERrIER et consigné dans le bulletin de la Société pratique d'horticulture du Rhône, se reproduit partout de même.

A la fin de l'analyse de la dernière question, M. D'Anouilh de Salies a dit en faveur du système suivi 
par M. Verrier: Théoriquement, il est généralement admis que la sève, absorbée par les spongioles des extrémités radiculaires, chemine par les canaux les plus jeunes de l'aubier pour parvenir jusqu'aux feuilles, où elle s'élabore et subit diverses modifications qui la rendent propre à produire de nouvelles couches d'aubier et de liber, ainsi que des spongioles; après cette élaboration dans les feuilles, la sève redescend jusqu'à l'extrêmité des racines, en formant chaque année une nouvelle couche d'aubier, une nouvelle couche de liber et de nouvelles spongioles. Ceci posé et admis, il adviendra, poursuit M. D'ANouizH DE SALies, que la sève, parvenue à l'empâtement de chaque racine, aura moins de chemin à parcourir pour arriver aux points extrêmes, où M. VERRIER dit de couper les racines, qu'aux extrémités sans sections des racines laissées intactes d'après le système suivi jusqu'ici. D'ailleurs, par la méthode VERRIER le cheminement est direct, par l'autre méthode il est toujours sinueux, par conséquent moins propre à la facile circulation de la sève. Donc, les nouvelles spongioles se formeront plutôt par le système VERrier que par l'autre méthode.

Contre le système Verrier, M. D'A fouilh de Salies dit que les arbres plantés avec la totalité des racines sont moins sujets à être renversés par le vent que ceux aux racines desquelles on a fait des suppressions. Ceci est évident et est, dans une certaine mesure, contre les idées de M. Verrier. Mais il faut dire aussi qu'il est facile, au moyen de tuteurs, d'obvier à cet inconvénient.

XI. L'ordre du jour étant épuisé et aucun orateur ne demandant la parole à cause de l'heure avancée, M. Ie Président passe en revue les questions pratiques qui ont été traitées dans cette section. Il déclare qu'il est heureux d'avoir assisté à ce congrès et d'avoir eu l'honneur 
de présider cette section. Il a beancoup profité des discussions qui ont eu lieu sur les questions d'arboriculture et surtout sur la nouvelle invention du greffage des racines, invention due à M. DE BEvCKen; il le prie de lui céder en souvenir les magnifiques spécimens de ce greffage, qu'il a apportés à la séance, afin de les montrer à ses élèves à Berlin pour tâcher d'introduire cette méthode en Allemagne. M. DE BEucker accède à ce désir.

M. le président déclare la séance close et les travaux de cette section du congrès terminés. 


\section{RESUME}

\section{DU COMPTE-RENDU DES SÉANCES DE LA SECTION DE BOTANIQQUE APPLIQQUÉE ET D’HORTICULTURE.}

SÉANCE du 7 Avril 1865, à 4 heures de relevée.

La séance est ouverte par M. RuUwexhofr (de Rotterdam) ............

Constitution du Bureau

Pag.

216.

M. Kooci étant absent, M. DE Caviart d'Havale occupe le fauteuil de la Présidence.

La section règle l'ordre de ses sémces. Il est arrêté que la question: „sur la taille des arbres fruitiers" sera abordée la première

La séance est close à 5 heures pour être reprise le lendemain à 9 heures du matin

216.

216.

217.

217.

SÉANCE du 8 Avril 1865, à 9 heures du matin.

Présidence de M. KOCH.

Ouverture de la séance

218.

Le procès-verbal de la séance précédente, lu par M. FrANCKEn, secrétaire-adjoint, est approuvé

$1 l$ est donné lecture d'une lettre concernant la nomination des viceprésidents du Congrès

M. De Cavnart d'Hamale fait communication d'une question proposée par MI. BrideL (de Gaimont).

II. vaN IIULLe (de Gend) traite, dles sistemes de taille d'arhres fruitiers."

MM. D'Avouili DE SALIES et Prvaert font quelques observations a ce sujet

M. DE Beucker (d'Ancer's) expose ses idées sur la taille.

M. Hooftuan prend la défense de lin taille hollandaise des pêchers et des abricotiers

M. VAN HulLe s'adresse spécialement aux pépiniéristes de Boskoop ...

218.

218.

218.

218.

228.

229.

232.

233 .

234. 
MNI. D'A vouili de Salies, De Cavinart d'Havale, Overexnder, BaLtet, vav Hall et DE Zavisis discutent sur la question. Lá discussion est close pour être continuée lundi

M. DE Cannart d'Havale communique à la section que M. Andersson (de Stockholm) met à la disposition des membres des greffes d'arbres fruitiers de Suède.

II. Triana (de Paris) traite des Mélastomacées et dépose son travail ..

II. Kocu développe lia question qu'il a posíe sur "la nomenclature des plantes."

M. Nedzelsky (de Moscou) veut que les idées énoncées par l'orateur soient appliquées aussi à la pomologie

M. vaN HALz fait une observation, à laquelle M. Косн répond; celui-ci s'étend encore sur le sujet en question et finit en faisant une proposition

MM. de Beucker, Battet, Overeynder et NeDzelsky discutent sur l'avantage que présente la proposition susdite. Le dernier s’adresse aussi aux délégués de la Société de Boskoop.

La séance est close pour être reprise à 2 heures de relevée .............. 244.

\section{SÉANCE du 8 Avril 1865, à 2 heures de relevée.}

\section{Présidence de M. KOCH.}

Ouverture de la séance. La lecture du compte-rendu de la séance précédente est renvoyée à celle de lundi

M. Baltet (de Troyes) traite la question posée par lui sur la dégénérescence des plantes bulbeuses

M. dE Cannart d'Hamale approuve ce qu'a avancé l'orateur concernant les poires

M. vaN HALL mentionne quelques cas de dégénérescence ................

II. de Cavinart d'Hamale dome lecture d'une lettre de la part de MII. HARDr, frères, contenant une question qu'ils proposent an Congrès ......

M. Pynaert (de Gand) développe sa question sur „la nécessité de recourir à la reproduction par voie de semis, pour créer des plantations rustiques."

M. D'ANouILII DE SALIEs considère le greffage comme le meilleur moyen de prévenir la dégénérescence

M. Kocir fait de même quelques remarques sur la nature de la dégénérescence

M. Nevzelsky discute sur la différence de l'espèce, de la sorte et de la variété. Il s'étend encore sur le discours de M. Prnaert

Autres observations faites par divers membres

M. Stelzaner (de Gand) dépose un travail sur „l'hybridation des Fougères. (Voir le Supplément.). 
M. Karsten (de Berlin) donne un aperçu de ses travaux sur ,la méthode de culture des Cinchona."

M. Mulier (de Bruxelles) dépose une „notice sur les plantes de pleine terre." (Voir le Supplément.).

305.

M. Wesmael (de Mons) dépose „quelques considérations sur les Hybrides des cultures." (Voir le Supplément.)

I. le Président appelle l'attention des membres sur quelques opuscules de M. Gloger

La séance est close à $4 \frac{1}{2}$ heures

305.

305.

305.

\section{SÉANCE du 10 Avril 1865, à 10 heures du matin.}

\section{Présidence de M. KOCH.}

Ouverture de la séance

306.

Continuation de la discussion sur la taille raisonnée des arbres fruitiers.

M. DE BEUCKER discute cette question

M. vaN Hulle résume en français ce qu'a dit l'orateur et y ajoute quelques observations

M. le.Président communique ì la section que M. WARver (de Dublin) a déposé une notice

M. Nedzelsky (de Moscou) traite la question XXI du Programme......

M. KocI fait ì ce sujet quelques observations .......................

M. Nepzelsky répond

M. REGEL combat l'utilité de la classification d'après les principes de I. KoCH

MM. КосH, NEDZELSKY et DE BEUCKEI font encore quelques remarques.

Question annoncée de la part de M. D'A vouILII DE SALIES....

306.

310.

312.

312.

329.

330 .

330 .

332.

333.

II. Evrz-Ferenez (de Bude) traite de ,la destination véritable des plantes dans l'économie de la nature.".

M. Коцв indique un fait en rapport avec les idées émises par l'orateur.

M. Regel combat la thìse développée par M. Entz-Ferenez, qui réplique brièvement

M. D'Anouili de Salies traite la question XXXV, 1, du Programme.

M. Thielens fait lecture d'une notice de M. Bossin de Paris...........

M. vaN HuLLE fait quelques remarques à ce sujet $\ldots . . . \ldots \ldots \ldots \ldots \ldots . . . . . .$.

M. Geitner (de Planitz) dépose un projet d'association mutuelle ......

M. Kous annonce qu'il déposera une notice

M. J'A vounli DE SALIEs traite les autres questions qu'il a annoncées.

L'ordre du jour étant épuisé, M. LE President passe en revue les questions traitées dans la section. La séance est close

346,347 .

348.

350.

358.

358.

358.

359.

363. 


\section{CONGRES INTERNATIONAL}

\section{DE BOTANIQUE ET D'HORTICULTURE.}

SECONdE RÉUnion générale. - SÉANCE DE cLôture.

Le 11 Avril 1865, à dix heures du matin, les membres du Congrès se réunissent dans la Salle des tableaux du Palais de l'industrie.

M. le prof. Oudemans, président, ouvre la séance.

M. Rauwenhoff, secrétaire-général, fait lecture du procès-verbal de la séance d'ouverture.

Aucun des membres n'ayant d'objection à faire, le procès-verbal est adopté.

M. le Président invite MM. les présidents des sections à faire leurs rapports; il s'adresse en premier lieu à M. le prof. FÉE, président de la première section.

M. Fée dit que la section de botanique pure a tenu trois séances, avec un auditoire très-assidu. On y a traité une foule de questions dont plusieurs même hors du programme. La section a parfaitement compris sa mission et s'est acquittée de sa tâche avec succès. Des mémoires d'un intérêt sérieux ont été présentés; la dernière séance surtout a été très-intéressante, et un public nombreux y assistait. Les discussions du reste se sont toujours distinguées par une bienveillance et une cordialité parfaites.

Ensuite M. le prof. Koch, président de la seconde section, a la parole. Il dit que dans la section de botanique appliquée et d'horticulture plusieurs questions très-intéressantes ont été traitées et discutées; il est persuadé que cette discussion portera de bons résultats. Le temps est trop 
court pour relater tout ce qui a été traité dans cette section. II ne relèvera donc que les questions les plus importantes, et en premier lieu celle de la taille raisonnée des arbres fruitiers introduite par M. van Hulue, de Gand; cette question a été vivement débattue par les horticulteurs français, belges et néerlandais; est venue ensuite la question, traitée par M. de Beucker, sur le greffage des racines, pour raviver un arbre dépérissant et en rétablir la force; puis celle de la dégénérescence des plantes bulbeuses, herbacées ou ligneuses, des arbres fruitiers ou d'ornement, présentée par M. Baltet; et la question de la nécessité de recourir à la reproduction par la voie de semis pour créer des plantations rustiques, traitée par M. Pynaert. M. Karsten a traité des Cinchona et son expérience en cette matière a rendu sa communication très-importante; M. NEDzELsky a présenté un travail intéressant sur les progxès de l'arboriculture et de la pomologie théorique; М. Косн lni-même s'est occupé de la nécessité de maintenir une nomenclature exacte pour les familles et les espèces des plantes. En général, les travanx de la section ont été très-importants et auront sans doute d'excellents résultats; les trois séances ont été bien remplies, et la discussion s'est toujours distinguée par un ton de la plus parfaite courtoisie.

M. Oudemans remercie an nom de l'assemblée MM. FÉE et Косн des rapports qu'ils ont faits, et prononce ensuite le discours suivant:

\section{Messieurs!}

Les questions qui ont été traitées dans les deux sections de notre Congrès, et dont il me semble superflu de vous rappeler la tendance, ont été bien numbreuses et bjen importantes; elles ont beaucoup contribué à étendre nos connaissances, ou à nous montrer le sentier qui seul peut nous conduire à la vérité. Je crois exprimer la conviction de tous ceux qui ont assisté à nos réunions, en déclarant que notre Congrès a été utile à la science; qu’il 
a resserré les liens d'amitié qui nous missent; qu'il a fait accroitre l'estime que mous nous portons mutuellement. Certes, Messieurs, ces résultats ne sont pas à dédaigner; qu'il nous soit permis d'espérer que le but, que nous nous proposions dans nos conférences scientifiques, a été complètement atteint.

J'espère que, dans le courant de l'année, notre secrétaire, M. Rauwenhoff, pourra vous faire parvenir le Bulletin, qui vous présentera un résumé tidèle des heures consacrées ici à l'étude, et que vous voudrez bien lui faciliter sa tâche en lui donnant des reuseignements, toutes les fois qu'il s'adressera à vous.

Avant de nous séparer, Messieurs, il nne reste à remplir un devoir bien agréable, celui de vons exprimer, au nom de la Commission Directrice de notre Exposition et de notre Congrès, la plus vive gratitude, tant du dévouement avec lequel vous avez voulu accepter le mandat de membres du jury, que de la part que rons avez bieli voulu prendre à nos délibérations scientifiques. Le secours puissant de tant d'hommes éminents, que ce palais a vu se réunir dans son enceinte, nous était nécessaire pour réaliser le projet d'une fête, digne des efforts infatigables de tous ceux qui, de près et de loin, sont venus nous offrir les résultats magnifiques de leur culture, témoins vivants de l'habileté avec laquelle ils savent forcer la nature à multiplier les moyens, dont elle peut disposer pour s'orner de sa plus belle parure. Vous avez répondu avec empressement à notre appel et nous avons applaudi à votre bienveillance et au désintéressement, qui vous fit quitter vos demeures pour venir à notre aide. Encore une fois, Messieurs, soyez convaincus de notre profonde reconnaissance, et veuillez croire que rien ne nous sera plus agréable que de vous témoigner de notre côté le grand prix que nous avons attaché à votre présence en ce lieu. Souvenez-vous longtemps de nos personnes, nous ne vous oublierons jamais! 
M. le prof. Meissner (de Bûle) demande la parole. Il dit:

En écoutant le discours d'adieu du président, je voyais repasser devant mon esprit toutes les belles images que l'exposition m'a présentées et auxquelles se rattachent de si belles, de si donces impressions. Jamais je n'oublierai l'accueil cordial, qu'on m'a fait, ainsi qu'à tous les membres étrangers du jury; jamais je n'oublierai ces jours heureux, ou j'ai pu faire à Amsterdam la commaissance personnelle de tant d'hommes distingués.

Aussi me semble-t-il un vrai devoir d'exprimer à la commission directrice la pleine satisfaction et l'admiration que l'organisation et l'exécution du projet conçu ont inspirées à tous les membres étrangers du jury, ainsi que notre reconnaissance profonde et unanime de toutes les jouissances qu'on nous a procurées. Je ne saurais passer sous silence l'intérêt et la part que la famille Royale, et spécialement S. M. la Reine et S. A. R. le Prince d'Orang'e ont daigné prendre à cette belle entreprise, et je désire vivement qu’au nom de tous les membres étrangers du jury grâce soit rendue ì leurs augustes personnes de l'appui et de la protection par lesquels Elles ont moralement et matériellement contribué à la réussite de l'exposition. Nous regrettons vivement qu'un deuil récent dans la famille Royale les ait empêchées de jouir Elles-mèmes du coup d'œil magnitique qu'offre l'exposition et nous ait privés de l'homneur de leur présence. Entin, comment ne pas firire mention du grand intérêt que l'exposition et le congrès nous ont offert sous le rapport scientifique? En terminant, j'ose assurer que nous grarderons tons le meilleur souvenir $\mathrm{du}$ bon accueil que nous avons recu et qui a si vivement touché nos cœurs. (Applaudissements.)

M. Oudemans remercie l'orateur des paroles bienveillantes et gracieuses qu'il vient de prononcer et déclare close la session du Congrès.

La séance est levée à 11 heures. 



\section{SUPPLÉMENT.}

\section{MÉMOIRES DÉPOSÉS AU BUREAU}

PT

\section{NOTICES PARVENUES AU SECRÉTARIAT}

APRÈS L'IMPRESSION

DU COMPTE RENDU DES SÉANCES. 



\title{
MÉMOIRES PRÉSENTÉS Ȧ LA SECTION DE BOTANIQUE PURE.
}

\author{
RECHERCHES SUR LE DÉVELOPPEMENT, LA STRUC'TURE \\ ET LES FONCTIONS DES TISSUS DE L'ANTHÈRE,
}

PAR

M. A. CHATIN.

(Voir le Compte Rendu de la Séance du 8 Avril 1865, pag. 59).

\section{I. État de la question.}

Les cellules des anthères, nommées par le docteur PuRkinje cellules fibreuses (cellulce fibros(e), sont l'objet essentiel de ce travail, dans lequel je comprends cependant aussi des observations se rapportant à quelques autres points de l'anatomie des étamines.

L'histoire du sujet sur lequel je ramène l'attention des botanistes, est presque toute moderne.

En effet, les connaissances sur les cellules fibreuses, qu'on désigne aussi par les mots cellules à filets (A. DE CANDOLLE), cellules spiralées, cellules élastiques, sont généralement reportées ì la date de 1830, époque qui rit paraître le grand travail: De cellulis antherarum fibrosis, nec non de granorm pollinarizm formis, du professeur PuRKIx.y, de Breslau. Dans ce travail, digne d'être cité pour le nombre et l'exactitude générale des observations, le savant naturaliste allemand expose, en l'accompagnant de dessins nombreux, le résultat de ses recherches sur environ 300 espèces végétales réparties dans un grand nombre de familles.

La structure propre de l'anthère, dans laquelle il distingue deux membranes, l'une épidermoïdale qu'il nomme exothèque (exothecium), l'autre intérieure, constituée par les cellules fibreuses, et qu'il désigne sous le nom d'endothèque (endothecium);

les formes qu'affectent les cellules fibreuses;

les fonctions de ces cellules (liées, assure-t-on, à la déhiscence des loges, qu'elles détermineraient par leur élasticité et leur hygroscopicité mises en jeu par les aiternatives d'humidité et de sécheresse);

enfin, les rapports qui existent entre la forme des cellules fibreu- 
ses des anthères et les groupes naturels, sont considérés par le sarant Purkinje dans des chapitres spéciaux.

Déjà cependant le sujet traité par PuRkinłe dans un travail resté classique, avait fixé l'attention de deux botanistes célèbres, Mirbel et Meyen.

PURKinje fit honneur à MEYen des premières observations (1). Mais les ohservations de MEYex ne dataient que de deux ans (1828), et c'est précisément à cette époque que remontait l'entreprise du travail de Purkinje, travail terminé et publié en 1830. L'honneur concédé par PuRkinje à son devancier ne diminuait ainsi que bien peu sa propre gloire.

M. Mirbel se plaignit d'avoir été oublié. Il rétablit ses titres en publiant, à l'oceasion de son premier travail sur le Marchantia, une note concise, mais concluante (2).

M. Miriel y rappelle que, dès 1806 et en 1815, dans ses Observations su un système d'anatomie comparée des végétaur fondé sur l'organisation de la fleur (travail imprimé dans les Mémoires de l'Institut pour 1808), il signala dans les anthères l'existence d'une lame de cellules découpées par des fentes horizontales ou verticales et se contractant par la sécheresse, se dilatant par l'humidité. Il lui paraît en particulier que la phrase suivante, empruntée à la première de ces publications, est le point de départ du travail de PuRkinse: „Il n'est pas facile d'apercevoir les ressorts délicats qui font mouvoir et ouvrir les anthères; mais ces organes sont d'une si grande importance et leurs formes si variées, qu'on ne saurait les examiner avec trop de soin. La nature du tissu qui compose les lames contractiles latérales et dorsales, mérite d'être connue: les premières font ouvrir' les valves, les secondes recourbent les anthères en arrière." (Mém. de l'Inst., 1808, p. 347).

Au tome I, p. 246, de ses Eléments de physiologie végétale, M. Mirbel s'exprime comme il suit: „J'ai remarqué que les valves des loges sont composées de deux lames cellulaires continues entre elles, mais distinctes par leur nature; que l'une, située a l'extérieur, a ses parois dilatées, entières et renflées en petits mamelons à sa superficie; que l'autre, située sous la première, a ses parois découpées verticalement, et qu'elle jouit, à un degré considérable, de la propriété de se dilater à l'humidité. de se contracter à la sécheresse. C'est

(1) J. E. I'urkinje, De cellulis antheramum fibrosis, præfatio, p. 1. MEYex, Anat. Physiol. Untersuchungen über den Inhalt der Pflanzen, Berlin, 1828.

(2) Mивв., Recherches anaton. et physiol. sur le Marchantia, dans les Nourelles Annales du Muséum d'histoire naturelle, p. 116. 
encore par le moyen de l'anatomie que j'ai reconnu que le nombre ordinaire des loges des anthères est de quatre au lieu de deux, selon l'opinion commune."

Je ne veux rien dire qui puisse amoindrir l'intéressant travail de Purkinje, mais il serait injuste, d'après les citations précédentes, de ne pas reconnaître que non seulement M. Mirber a reconnu l'existence et le siége des cellules fibreuses, et qu'il leur a attribué le rôle physiologique admis jusqu'à ce jour, mais aussi qu'il a distingué nettement les deux membranes auxquelles Purkinse a imposé les noms d'exothecium et d'endothecium.

Plus tird, les recherches de M. Mirbel sur le développement des anthères du Cucurbita Pepo, etc. (1), le portèrent à admettre que la transformation des cellules à parois simples en cellules découpées ou cellules fibrouses se produit brusquement vers le moment de la maturation de l'organe.

Il n'est pas superflu, afin de bien préciser l'état de nos connaissances sur les cellules dites fibreuses, d'emprunter quelques citations aux auteurs classiques. Ces citations ne sont d'ailleurs qu'un développement utile du travail de Purkinje.

M. Alph. de Candolue s'exprime ainsi (2): „La structure intime des anthères a été étudiée avec beaucoup de soin, dans ces derniers temps, par M. Purkinje. Quoiru'il ne le dise pas explicitement, on voit qu'il regarde chaque loge comme formée par l'un des côtés du limbe d'une feuille, en sorte que la fente ou le sillon longitudinal répondrait au bord de la feuille, et tout le contenu de la loge au mésophylle. Il distingue un épiderme, qu'il nomme exothecium, au-dessous un tissu cellulaire d'une nature très spéciale, qu'il nomme endothecium, et au centre le pollen."

L'endothecium se compose de cellules, que l'auteur nomme fibreu. ses (jibros(e), parce qu'elles sont entourées de filets (3) extrêmement

(1) Mrrbel, Complément des observations sur le Marchantia, suivi de recherches sur la métamorphose des utricules et sur l'origine, les développements et la structure de lanthère et du pollen dans les végétrux phanérogames. - Mémoires de l'Académie royale des sciences, t. XIII, 1835.

(2) Alpionse de Candolle, Introduction à létude de la Botanique, 1835, t. I, p. $143-144$.

(3) La mot filet (filum) doit être substitué, ce me semble, à celui de fibre, car ce dernier s'applique depuis longtemps aux faisceaux de vaisseaux et de cellules allongées, ce qui est tout à fait différent (AIPH. DE CANnoLLe, loc. cit., en note). - On peut ajouter que le mot fibre est souvent, comme le dit plus loin AD. DE Jussıev, employé pour désigner les cellules allongées elles-mêmes (surtout les cellules du bois). Nous nous servirons souvent du mot proposé par M. AlPir. De Candolle. 
petits, cylindriques et disposés en réseau. MI. Purkinje dit que l'on tronve quelquefois des cellules sans filets et des filets sans cellules, quoique d'ordinaire les deux choses soient réunies. L'endothecium contient un ou plusieurs rangs de ces cellules singulières dont la forme, naturellement arrondie, devient ellipsoïde, cylindrique ou polyédrique, suivant la pression qu'elles subissent.

ACHille Riciand dit en substance (1): „Il résulte de l'intéressant travail de Purkinje que chaque loge de l'anthère se compose d'un épiderme ou exothìque, et d'une membrane interne (endothèque) que composent des cellules à fibres internes élastiques. Ces cellules, de formes très-variées, sont semblables ou du moins fort analogues dans une même famille naturelle. Dans les Graminées, par exemple, ces cellules ont une forme rectangulaire, et ofirent des fibres courtes, droites, implantées sur leurs còtés à la manière de clous; tandis que dans les Cypéracées elles sont cylindriques et à fibres annulaires transversales. Les cellules de l'endothèque sont d'abord simples, ce n'est que plus tard que les fibres s'y manifestent"

AD. DE Jussiev (2) rappelle „que les anthères se composent d'une membrane épidermique souvent parsemée de stomates, plus d'une couche profonde d'un tissu particulier dont on conȩoit facilement la nature et la forme, en considérant qu'il a commencé par une réunion de cellules spiralées ou annulées, ou plus souvent encore réticulées (Ex. Cobcea). Mais ordinairement la membrane de ces cellules a complètement disparu aux approches de la maturité de l'authère. On a nommé cellules fibreuses ces cellules à claire-voie, ainsi réduites aux lames qui les doublaient, à leurs fibres, en attachant à ce mot, non l'idée d'une utricule allongée, mais celle d'un ruban plein. Cette couche fibreuse va en diminuant d'épaisseur à mesure qu'elle se rapproche de la ligne de déhiscence, point où elle s'interrompt complètement. Ces petites lames, très élastiques et hygrométriques, doivent se tendre, se détendre, s'allonger, se recourber, suivant que l'anthère est plus sèche ou plus humide, et ces variations doivent suivre,. d'une part, le développement de l'anthère, d'autre part, l'état de l'atmosphère, et c'est ainsi que la loge se rompt sur les points où la couche fibreuse est interrompue."

Quelques observations, éparses dans des travaux ayant un tout autre objet que l'étude particulière des anthères, sont ronnes confirmer les domnées de Purkinse et de Mirber, plutôt qu'ż ajouter. Cepen-

(1) Acir. Ricrard, Nonreaux éléments de botanique, 7e édition, p. 366, et Précis de botanique, 1852.

(2) AD. DE Jussiev, Botanique élémentaire, lre édition, p. 342. 
dant le fait de la localisation des cellules à filets le long de la ligne de déhiscence, constaté par M. Duchartre dans l'anthère du Lathroa clandestina, doit être spécialement mentionné (1).

Quant à nos connaissances sur l'anatomie du filet et du connectif, c'est encore à MirbeL qu'elles remontent: ,J'ai remarqué, dit cet illustre botaniste (2), que souvent les trachées du filet pénètrent dans le connectif." Ce qui est parfaitement vrai, contrairement à cette assertion deux fois inexacte, comme je l'établirai dans le cours de ce travail, D'ACHILle RichARD: ,Le filet est parcouru par un faisceau central fibro-vasculaire qui s'étend de la base au sommet, sans pénétrer dans le connectif (3)."

Les propositions suivantes peuvent être déduites des recherches de Mrrbei, de Meyen et de Purkinje:

1. La jeune anthère est d'abord à 4 logettes, puis à 2 loges.

2. Les anthères sont formées de deux membranes (signalées par Mrrbel, dénommées exothèque et endothèque par PurkixJe), jamais plus, jamais moins.

3. L'exothèque, épidermoïdale par son siége et de texture généralement délicate, ne manquerait jamais. Il n'est pas dit qu'ịl supplée ou aide, en aucun cas, l'endothèque pour la déhiscence des loges de l'anthère.

4. L'endothèque est formée généralement de cellules dites fibreuses par Purkinje, cellules découpées par M. Mirbel, cellules à filets par M. Alphonse de Candolle; les cellules à filets sont plus rares vers la ligne de déhiscence.

5. L'élasticité et l'hygroscopicité des cellules à filets formant la seconde membrane sont la cause de la déhiscence des loges.

J'allais omettre de citer le savant SchLEiden qui a figuré la troisième membrane, sur laquelle je croyais être le premier à appeler l'attention, jusqu'au jour où M. Ducirartre, compulsant les livres allemands à l'occasion d'un traité élémentaire de botanique, m'a fait perdre mes illusions. Toutefois SCHLEIDEN n'attacha pas d'importance à son observation.

6. La forme des cellules dites fibreuses est généralement la même dans les mêmes groupes naturels (4).

(1) P. Duchartre, Observations anatomiques et physiologiques sur la Clandestine d' Europe, pl. VI, fig. 86-89. Paris 1847.

(2) Brisseav-Mirbet, 1808 et 1815 , loc. cit.

(3) Acr. Richard, Précis de Botanique. Paris, 1852.

(4) Cette remarque de PURKINJE, généralement juste, peut être opposée à l'opinion des botanistes qui nient encore aujourd'hui l'existence de corrélations entre la structure anatomique des végétaux et leurs caractères morphologiques. 
7. La métamorphose des utricules simples de la seconde membrane en cellules dites fibreuses est si rapide, qu'elle ne peut être saisie par l'observation; elle n'a lieu que vers l'époque de la défiscence (Mirbel).

8. Les trachées ou vaisseaux spiraux du filet pénètrent souvent dans le connectif (MirbeL). Il n'est pas signalé queles trachées puissent ne pas exister sur toute la longueur du filet (ACH. Richand affirme même que les trachées s'étendrnt toujours de la base an sommet du filet.)

Cette analyse, que je me suis efforcé de rendre complète et fidèle, des travaux de Mirsen, de Meren et de Purkinje sur la structure de l'anthère, montre déjà avec les questions qu'on regarde comme fixées par ces savants botanistes, quelques-umes de celles qu'ils n'ont pas éclairées, quelques autres qu'ils ont mécommes.

Rien n'est dit touchant, non plus la forme, mais la disposition et les connexions des cellules à filets, soit au point d'attache des loges de l'anthère au connectif', soit dans l'épaisseur du connectiflui-même, soit par rapport aux groupes naturels des végétaux.

II. Premiers développements des tissus de l'anthère; des logettes.

Les recherches de Mirbei sur le développement de l'anthère peurent être ainsi résumées:

1. Le tissu de l'anthère est d'abord une masse utriculaire homogène.

2. Plus tard, les utricules situées vers le milieu de chacune des moitiés des deux lobes de l'anthère grandissent et changent de forme: ce sont les utricules polliniques (ou utricules mères du pollen) destinées à être résorbées après qu'elles auront donné naissance dans leur carité à des grains de pollen, généralement au nombre de quatre pour chaque utricule mère.

3. Vers la maturation de l'anthère, la portion de la masse utriculaire primitive qui avait persisté, séparant en deux logettes chacune des deux demi-anthères, disparaît, et chacune des demi-anthères n'offre alors qu'une seule loge.

4. Tout le tissu sous-épidermique se transforme, vers l'époque de la déhiscence, en cellules à filets.

5. La transformation des utricules simples en utricules ou cellules à filets est tellement brusque qu'elle ne peut être surprise au moment de son évolution.

Les propositions 1 et 2 , confirmées par divers observateurs, notamment par M. DucHalike (1), paraissent être hors de toute contestation; j'ai eu très-souvent l'occasion d'en vérifier l'exactitude.

(1) Observations anatomiques et physiologiques sur la Clandestine d'Europe, pl. VI, fig. 81 aे 85 . 
La proposition 3 est encore généralement vraie. Cependant j’ai constaté un assez grand nombre de cas dans lesquels la cloison de séparation persiste complète an milieu de chaque demi-anthère; celle-ei restant ainsi, jusqu'à sa débiscence, coupée en deux logettes. Alors le plus ordinairement (Lyeopersicon, Tradescantia, des Asclépiadées, Orchidées, etc.) la déhiscence a lieu, comme dans les cas où l'anthère est à deux loges ou deux demi-valves. La seule différence est que celles-ci reposent par' leur bord, jusqu'à l'instant de la déhiscence, sur la cloison des logettes. Ce sont les deux vantaux d'une porte qui, an lieu de répondre tous deux dans une chambre unique, donnent entrée à deux chambrettes contigues l'une à l'autre. Habituellement, l'extrémité de la cloison devenue libre à la déhiscence par le décollement des valves, qui jusque-là reposaient sur elle, se déjette, se contracte ou se détruit diversement, et à ce moment l'on pourrait croire que la destruction de la cloison a précédé la déhiscence elle-même an lieu de la suivre. Dans les Passiflora, Scabiosa, etc., ce sont les valves réfléchies et adossées de la loge qui, plus que la cloison proprement dite, forment les logettes.

Le nombre des plantes dans lesquelles la cloison de séparation des logettes ne persiste pas jusqu'au moment même de la déhiscence, mais ne se détruit qu'aux approches de ce moment, est considérable. I'un des meilleur's exemples est celui observé par M. Duchartre dans la Cltuntestine, qui présente une cloison encore épaisse après lak production des cellules fibreuses (1).

M. Mirbet avait d'abord professé que le nombre ordinaire des loges des unthères est de quatre et non de deux selon l'opinion commune (2). Plus tard, il crut pouvoir conclure de ses études organogéniques, limitées à un trop petit nombre d'espèces, que le nombre 4 des logettes, constant dans le jeune âge, fuisait toujours place à deux loges. C'est entre les deux opinions succersirement adoptées par le savant anatomiste qu'est la vérité.

On vient de voir que les proportions 1 et 2 , déduites du mémoire de M. Mirbel, sont absolument admises, mais que la proposition 3 n'est pas sans d'assez nombreuses exceptions. Mes observations établissent que les propositions 4 et 5 doivent ètre presque complìtement modifiées.

Et d'abord tout létissu sous-épidermique se change-t-il en cellules à filets (3)? On est conduit à l'affirmation en se reportant, non au texte

(1) Duchartre, loc cit., pl. VI, fig. 86.

(2) Brissfau-Mirbei, Eléments de Physiologie régétale, I, p. 247, et pl. XXX, fig. $6 D$ (c'est par erreur que l'auteur reuvoie à la pl. XXXI, fig. 9).

(3) Mirbel, loc. cit. 
muet à cet égard, mais aux figures de Mrrbel et de Meyen (1), représentant l'anthère dans son jeune âge, et plus tard lorsque ses cellules à filets se sont produites. Le contraire sera toutefois établi plus loin. On verra aussi que la transformation des cellules simples en cellules fibreuses, quoique rapide, peut être suivie.

III. De la première membrane ou de la membrane externe des anthères.

Développement. La membrane épidermique est dénommée eathecium par Purkinje. Elle est figurée par Meyen et Mirbel. Ce dernier ajoute qu'elle se présente sous la forme d'utricules relevées.sur la face externe en petits mamelons. Voilà tout ce qu'on sait de cette membrane. Mais son développement, sa disparition complète; les variations profondes de structure qu'elle peut offrir, le rôle qu'elle semble appelée à remplir dans certains cas de structure spéciale et surtout quand les cellules à filets viennent à manquer, n'ont aucunement préoccupé ces savants anatomistes.

Aux premiers âges de l'anthère, lorsque les utricules polliniques ne se dessinent point encore au sein des-masses cellulaires, et souvent longtemps encore après la formation de ces utricules, la première membrane n'est pas distincte du tissu qu'elle recouvre. Mais peu à peu, les utricules qui forment son assise (2), prennent une forme spéciale, grandissent en des directions variables, et le plus fréquemment, comme l'a dit MrrbeL, se relèvent en petites ampoules, ce que j’ai vu être aussi le caractère habituel des utricules épidermiques dans les pétales; chez quelques plantes, le relief des cellules épidermiques de l'anthère est même assez grand pour que celles-ci constituent de véritables poils (Lycopersicon, etc.)

C'est aux approches de la déhiscence que les cellules de la première membrane éprouvent les changements les plus remarquables, soit dans toute l'étendue de l'anthère, soit en particulier à certaines places nettement circonscrites vers la ligne de déhiscence et le point d'attache des valyes au connectif. On les voit alors tantôt prendre sur toute la surface-de l'organe (Pedicularis, Chironia, Cassia, Octomeris, etc.) une épaisseur notable, tantôt former, par un développement localisé excessif, une saillie dont la section verticale représente une sorte de crête de coq, soit des deux côtés de la ligne de déhiscence (Passiflorce spec., Aponogeton, Bignonia, Lycopersicon, etc.), soit à la base des valves (Aechmea), soit sur le connectif lui-même (Calen-

(1) H.J.F. MIEYen, loc. cit, et Pfanzen-Plyysiologie, 1837, t. III, fig. 1 et 7.

(2) Dans tous les cas où l'épiderme des anthères se distingue bien des autres tissusus, je ne l'ai vu formé que d'une seule couche d'utricules. 
dulu); quelquefois enfin (et ce cas doit d'autant plus fixer l'attention qu'alors l'anthère est réduite à une seule membrane) les utricules épidermiques disparaissent complètement. Au point de vue des balancements organiques, on ne manquera pas de remarquer que dans le Canna cette destruction de la membrane épidermique sur les valves de l'anthère correspond à un excès de développement de cette même membrane sur le connectif.

Mais c'est principalement chez les espèces dont l'anthère manque de cellules fibreuses, qne lit membrane épidernique prend des développements inusités, ainsi qu'on le voit dans le Lycopersicon, le Pyrola, le Melastoma et l'Octomeris. Du rapprochement de ces deux faits en coïncidence, manque de cellules à filets, développement considérable de la membrane épidermique, sort naturellement cette hypothèse: la première membrane ne supplée-t-elle pas, dans le phénomène de la déhiscence, la seconde membrane, quand celle-ci ne se transforme pas en cellules dites fibreuses? On se confirme en quelque sorte dans cette hypothèse, en considérant que le développement spécial de la membrane épidermique s'opère, comme celui des cellules à filets, vers le moment de la déhiscence et est parfois localisé comme lui sur les points, où le rỏle des tissus présumés actifs dans la déhiscence peut. s'exercer avec le plus d'efficacité. Je reviendrai plus tard sur les fonctions de la première membrane.

Généralité d'existence. La première membrane existe toujours (1); elle se distingue le plus ordinairement très-bien des tissus sous-jacents par la forme de-ses utricules; parfois cependant, surtout dans les premiers âges de l'anthère, elle ne peut être reconnue, quoique son existence ne puisse ètre révoquée en doute. Celle-ci est démontrée dans plusieurs des cas obscurs, soit par la transformation en cellules à filets de tout ou partie (Clandestina) du tissu sous-jacent, soit par la coloration de ce dernier, ou par celle des utricules épidermiques elles-mêmes.

Mais l'existence constante de la membrane externe n'est vraie que pour la jeune anthère, car il peut arriver que cette membrane disparaisse à peu près tout entière vers l'époque de la maturation (Calendula, Laurus nobilis, MIahonia, etc.); parfois sa destruction n'a lieu que sur la ligne de déhiscence (Schaneria, etc.).

Formes. La forme la plus habituelle des utricules est celle dans laquelle elles se relèvent en petits mamelons (ILeyenia, Aspidistra, etc.) ou en papilles, rappelant celles qui forment le velouté des pétales. Plus de longueur aux mamelons ou aux papilles constitue les poils

(1) Je ferai toutefois quelques réserves touchant l'existence constante d'une membrane épidermique superposée, dans le jeune age, d̀ la membrane fibreuse. 
généralement simples (Lycopersicon) qui se montrent surtout aux extrémités des anthères et sur le connectif. Le cas inverse du précédent, caractérisé par l'aplatissement des utricules, se présente dans le Balsamina, le Canne, surtout dans les Synanthérées (Cosmos, Dahlia, etc.).

Le plus souvent, quoique à peu près la même sur toute la surface de l'anthère, la forme des utricules de la première membrane peut différer beaucoup (autrement que par leur allongement en poils) par places. C'est ainsi que les utricules se relèvent considérablement, tout en restant soudées entre elles: dans le voisinage de la ligne de déhiscence (1) dans le Inyeopersicon, l'Aponogeton, l'Aechmea, le Peristrophe, l'Eranthemum, plusieurs Passiflora, etc.; sur le milieu même des valves dans le Solanum laciniatum et le long du connectif dans le CaTendula (2).

Coloration. La membrane externe se distingue souvent du tissu placé au-dessous d'elle par une coloration propre ou par le manque de toute couleur. Assez souvent de couleur verte ou incolore, elle est d'un jaune vert dans l'Octomeris, plusieurs Cassia, jaune dans les Tradescantio, les Solanum, Ranunculus, etc., plus ou moins brune dans les Evica, Rhododendron, quelques Cassia, etc., d'un rouge violet dans le Cyrtanthera magnifica, les Anemone, $P a$ paver, etc. Parfois, dans le Tradescantia par exemple, la coloration ie la membrane épidermique est semblable à celle de la troisième membrane.

Structure. Les utricules composant la membrane épidermique des anthères sont généralement d'une texture délicate. Cependant elles peuvent prendre une épaisseur considérable. Déjà résistantes dans le Pedicularis, l'Epimedium, beaucoup d'Acanthacées, etc., les atricules de la première membrane prennent ure notable épaisseur dans les Erica, Rholodendron, Pyrola, plus encore dans le Chironia, le Cussiu, surtout dans le Zamia et quelques Lobéliacées dont les utricules épidermiques rappellent assez bien les cellules scléreuses qui forment les granulations pierreuses des poires.

La cuticule peut elle-même former sur l'utricule une croûte épaisse; sa surface peut être comme chagrinée (Cassia, etc.).

La membrane épidernique est ordiuarement constituée par unc

(1) Jamais sur la ligne même de déhiscence, où la membrane s'amincit et peut même disparaitre.

(2) J'ai déjà fait remarquer que, par une sorte de balancement organique, le frand développement de lit membrane épidermique du connectif coïncide avec la destruction ou l'amincissement extrême de celle-ci sur les valves du Calendula. 
seule assise d'utricules. Je n'ai vu d'exception à cette règle que dans un Cassic (rapporté de Bahia par S.litzunanx et conservé dans l'herbier. Delessert), qui présente de deux à trois assises d'utricules trèsépaissies et colorées dans les côteśs ou crêtes qui bordent la suture. Ces utricules de la première membrane ne peuvent ici être confondues avec celles, aussi disposées en assises multiples, de la deuxième membrane, ces dernières étant incolores et ponctuées. Peut-être observerat-on quelques cas d'anthères à membrane épidermique formée sur toute son étendue de plusieurs couches d'ntricules, ainsi que cela est connu dans un certain nombre de feuilles.

\section{De lu seconde membrane des anthères.}

La seconde membrane des anthères, improprement dénommée endothecium par Porkinje qui pensait, avec Mirbel et Meyex, qu'elle représentait le tissu le plus interne, celui qui tapisse immédiatement et à tous les âges la cavité des valves, est la partie la moins incomplétement connue, on pourrait presque dire la seule un peu connue, des tissus qui composent ces organes.

C'est elle seule que Purkivje a eue en vue dans son grand travail, elle seule dont M. Mirber s'est attaché à suivre l'évolution. Comme la première membrane, la seconde membrane des anthères peut être considérée dans:

la généralité de son existence,

la coloration,

la forme,

la structure,

la direction,

et le nombre d'assises de ses utricules. Mais la formation des cellules fibreuses doit par-dessus tout être étudiée.

Transformation des utricules simples en cellules fibreuses. On a vu comment MirbeL, pour s'être trop attaché à l'idée que l'étude organogénique faite sur une seule espèce doit éclairer tous les faits du même ordre, ne vit pas que l'on peut suivre la transformation des utricules simples de la seconde membrane en cellules à filets sur un certain nombre d'anthères, tout aussi bien qu'on peut suivre une transformation analogue qui a lieu habituellement dans le tissu des feuilles des Orchidées épiphytes. C'est à tort aussi qu'il pensa que cette transformation des utricules se produisait toujours à un moment très-rapproché de la déhiscence.

En réalité, le passage des utricules simples en cellules à filets peut être suivi avec assez de facilité dans un très-grand nombre de cas, et l'on voit alors que lia transformation des tissus commence géné- 
ralement ou par l'attache des valves au comnectif, ou par un point rapproché de la ligne de déhiscence, ou par ces deux points à la fois.

Que, dans tous les cas, c'est le tissu bordant immédiatement la ligne de déhiscence qui se transforme le dernier, et que, même dans un très-grand nombre de plantes (Ptristrophe, Schaneria, Cyytanthera, Brillantesiu, Helleborne, etc.), un arrêt d'évolution aidant, les utricules marginales de cette ligne de déhiscence ne subissent pas la transformation.

Relativement à ce point: que la transformation des utricules simples en cellules à filets s'effectuerait toujours à un moment trèsrapproché de la déhiscence de l'anthère, je ferai observer qu'en général cette transformation commence et souvent se complète à une époque encore éloignée de la déhiscence (Helleborus, Belleperone, Bignonia, Lamium, Canna, Leculia, etc.).

Mais je dois, sur ce sujet qui a tant préoccupé le savant MirbeL, citer ses propres paroles et ajouter quelques détails de mes observations.

„Ce fut alors (aux approches de la déhiscence) qu'un changement extraordinaire se mauifesta dans une ou plusieurs couches d'utricules, placées immédiatement an-dessous de la membrane utriculaire superficielle. Les utricules s'agrandirent dans tous les sens et leurs parois se divisèrent en lanières ou en filets dont la position rappelait trèsbien la forme première de l'utricule. La métamorphose ne se faisait pas comme dans le Marehantia, par transitions appréciables; elle était si brusque, que je ne pus jamais surprendre la nature à l’œurre (1)."

Ces paroles de Mirbel devaient inspirer le désir de rechercher si, peut-être en suivant le développernent de l'anthère sur des espèces, autres que celles en très-petit nombre examinées par ce savant, on ne saisirait pas le moment de la transformation qui lui avait échappé.

L'Aechmea fulgens, le Chivonia fintescens, le Pedicularis syluatica, etc., se prêtèrent mal à mes recherches. Cependant il me parut qu'en plusieurs circonstances j'avais entrevu dans la seconde membrane de leurs anthères quelques états de transformation. Ces premières observations prirent plus de consistance dans le Canna nepalensis et l'Aponogeton distachyum, où je vis apparaitre les premiers linéaments des filets dans les utricules d'abord simples de la seconde membrane; mes expériences devinrent concluantes dans le Tradescantia virginiana, plante dans les anthères de laquelle la transformation commença par deux points de l'anthère: la ligne de déhiscence et le talon ou attache

(1) Mrrber, Méntoires de l'Acudémie des sciences, t. XIII, p. 394. 
des valves au connectif, pour de là envahir, rapidement saus doute, mais toutefois successivement, le reste des parois.

Fort de ces données, je m’adressai aux plantes à très-grosses anthères que Mrrbel avait soumises à son observation.

Le Cucurbita Pepo et le Passiflora brasiliensis, base du travail de M. Mirbel, se prêtent en eflet difficilement à l'étude du phénomène de transformation, mais celui-ci est plus aisément saisissable sur d'autres espèces de Cucurbita et de Passiflora. C'est même dans ces plantes que j'ai pu suivre le moins difficilement et la transformation des cellules et les points premiers de cette transformation, qui sont bien ordinairement le voisinage du connectif et de la ligne de déhiscence.

La transformation des utricules de l'anthère rayonnant alors de ces deux points en quelques plantes, rappelle ce qui se passe chez les animaux dans les os produits par divers centres d'ossification apparus sur des points opposés, puis marchant à l'encontre l'un de l'autre. Concluons donc en disant:

1. La transformation des utricules simples en cellules à filets, quoique rapide, peut être constatée dans son évolution;

2. La transformation commence sur des points divers, généralément vers la ligne de déhiscence et l'attache des loges, pour de là envahir successivement le reste des valves.

Généralité d'existence. L'existence de la denxième membrane peut être admise comme à peu près constante. Je dirais comme absolument constante, d'après mes observations, si je n'avais été conduit à douter de la présence de cette membrane dans le Thunbergia alata. Cette plante n'ayant, en effet, les valves de ses anthères formées que de deux assises d'utricules dont l'interne ne se transforme pas en cellules fibreuses, on est porté à penser que cette assise interne représente non la seconde, mais la troisième membrane, et l'on se confirme dans cette opinion par cette considération que, dans les autres genres d'Acanthacées, où les trois membranes existent, la seconde se change en cellules à filets.

Je ne tairai pas toutefois cette objection que, dans les Acanthacées, la troisième membrane se détruit après la production des cellules fibreuses, tandis qu'elle persisterait chez le Thunbergia. Mais je ferai aussi remarquer que l'objection perd beaucoup de sa valeur par ce fait, que la troisième menbrane persiste habituellement dans les anthères privées de cellules à filets comme celles du Thunbergia.

Coloration. Excepté dans les jeunes tissus, où elle est parfois teintée de vert, la deuxième membrane est généralement incolore. Mais à côté de la règle se placent des exceptions diverses pouvant être catégorisées sous deux chefs, comme suit: 
a. La seconde membrane offre la même couleur que l'épiderme; ainsi elle est jaunâtre dans le Ligusticum, jaune dans le Lycopersicon, verte puis jaune dans le Solanum Sisymbrium, rose dans le Butomus, bleue dans l'Erythronium Dens Canis et le Tulipa Gessineriana.

b. La seconde membrane partage la coloration de la troisième membrane; coloration qui est verdâtre dans le Rhamnus Alaternus (avant la complète maturation), jaunâtre dans le Salvia splendens (à première membrane d'un beau rouge), l'Aponogeton, le Gonolobus;

c. Les trois membranes sont uniformément colorées, exemple: plusieurs Anemone, dont les anthères ont toutes leurs cellules colorées en violet noir.

Je ne me souviens pas d'avoir observé des anthères ayant la seconde membrane colorée (autrement qu'en vert dans le jeune âge, comme dans le Soltum Sisymbritun), les deux autres étantincolores.

Forme des cellutes. Les cellules de la seconde membrane sont fréquemment arrondies-elliptiques; parfois, polyédriques (tabulaires, cuboïdes, etc.), dans un assez grand nombre de plantes (Solanées, Scrofularinées, Primulacées, Papilionaccées, Rosacées, Pulygonées, etc.), elles ont la forme générale d'une demi-sphère on d'une demiellipse à section appuyée contre la membrane épidermique, la convexité étant par conséquent tournée vers la troisième membrane ou l'intérieur de la loge.

Nous reviendrons sur la forme des cellules en recherchant les rapports de cette forme avec les groupes naturels.

Structure. Les cellules de la deuxième membrane offirent généralement, il n'est pas besoin de le dire, cette structure spéciale, dite fibreuse, avec filets développés dinıs les parois de faęon d'ailleurs fort diverses, comme dans le velamen des racines, dans les feuilles, etc., de beaucoup d'Orchidées épidendres. On a même admis jusqu'à présent que les cellules de la seconde membrane sont toujours fibreuses, opinion que les présentes recherches démontrent être mal fondée pour un assez grand nombre de végétaux, même pour d'im. portantes familles tout entières (Ericacées, Mélastomées, etc.).

Cette absence de cellules fibreuses chez des plantes varicies, absence que j'établirai plus loin avec détails, est bien de nature à surprendre les botanistes, labitués à admettre, sur l'autorité de Mirket. et de Purkisis, que la membrane dénommée par celni-ci rudothecinu est toujours fibreuse.

Quant aux cellules fibreuses elles-mêmes, elles diffèrent beancoup entr'elles par la disposition de leurs découpures, tantôt en arceaux, puis en spirales simples ou croisćes, ou en lanières isolées ou grou: pées, etc. Une disposition remarquable et assez commune, bien 
qu'elle paraisse ne pas avoir encore fixé l'attention des botanistes, est celle que j'appellerai disposition en griffe, et qu'on observe dans les cellules en forme de calotte dans les Polygala, Pyrus, Cerasus, Comzus, Scabiosa, Grevillea, Foeniculum, etc.

La structure des cellules fibreuses sera considérée plus loin avec tout le soin que le sujet comporte.

Nous renons d'indiquer que dans une catégorie de végétaux la seconde membrane est formée de cellules sans filets ou non fibreuses; que dans une autre catégorie, la plus nombreuse (et qu'on avait cru être la seule) les cellules de cette membrane sont au contraire fibreuses. Or, il existe une troisième sorte de membrane moyenne, caractérisée par ceci que les cellules n'y sont ni toutes fibreuses, ni toutes sans filets, mais participent des deux autres types. Dans cette troisième classe les cellules à filets ne forment qu'une portion des valves, l'autre portion restant constituće par des cellules à parois simples (Solanum, Rhinanthus, etc.); nous y reviendrons plus loin à l'article cellules fibreuses localisées.

Il est encore une catégorie spéciale de cellules, ni fibreuses ni à parois unies et semblables à elles-mêmes dans toute leur étendue, mais très-épaisses du côté de la membrane externe, extrêmement amincies ou manquant même de parois vers la membrane interne sur laquelle une coupe de dehors en dedans les montre s'appuyant comme le ferait un fer à cheral par l'extrémité de ses branches, le corps ou convexité étant adossé à la membrane épidermique. Généralement ponctuées dans les parties les plus épaisses ou les plus rapprochées de la membrane interne, ces cellules semblent être une forme de transition entre les rraies cellules fibreuses ou à filets et les cellules ordinaires. Les considérations suivantes viennent, du moins, à l'appui de cette hypothèse:

Ces cellules manquent, avons-nous dit, de parois du côté de la membrane interne vers laquelle elles vont en s'amincissant à partir de leur face adossée à la membrane externe (Cassio species); nous arons observé une structure et une disposition tout-à-fait semblables dans l'épiderme des racines de plusieurs Orchidées épidendres.

Dans les anthères de Cassia comme dans les racines d'Orchidées, ces cellules spéciales tiennent la place de cellules fibreuses ou spiralées existant chez des espèces voisines.

Dans les anthères, les cellules fibreuses dites en griffe, manquent de parois ou n'en ont que de très-minces, du côté où elles s'appuient à la membrane externe, comme les cellules ponctuées spéciales de quelques Cassia, là où elles reposent sur la membrane interne.

Les cellules fibreuses en griffe, comme les cellules spéciales du 
Cassid, vont en s'attenuant, de leur région dorsale qui est le point le plus épais, vers leurs extrémités appuyées à la membrane contiguë.

Il n'est pas douteux que dans les Orchidées les cellules épaissies du côté extérieur n'occupent la place des cellules spiralées des genres voisins; il paraît érident anssi que, dans quelques Cassia, ces cellules épaissies tiennent la place des cellules spiralées du Cassia Fistula.

Direction. Donnée par celle de leur plus grand diamètre, la direction des cellules de la seconde membrane est importante à considérer pour les cellules fibreuses, parce qu'elle est généralement constante dans un même groupe naturel et qu'elle paraît avoir un rôle dans la déhiscence des anthères.

Il importe de distinguer denx cas principaux dans la direction de cellules, savoir:

a. La direction ou le grand diamètre des cellules de la seconde membrane est parallèle aux valves (Erodium, Plantago, Silene, Centropogon, Brugmansia, Commelina, Encyclia, Limodorum.

b. La direction des cellules est perpendiculaire aux valves (Geranium, Mirbelia, Kennedya, Pyrus, Tiarella, Inychis, Malaxis).

Lorsque les diamètres d'une cellule sont à peu près égaux, il n'y a plus lieu de considérer la direction générale de la cellule, mais bien celle des filets que portent les cellules fibreuses, et ici encore on distingue ces deux cas:

a. La direction des filets est parallèle aux valves (Salvia);

b. La direction des filets est perpendiculaire aux valves, c'est le cas ordinaire (Tropceolum, Chelone, Ombrophytum).

Nombre d'assises. Il importe de considérer séparément la membrane moyenne, au point de vue du nombre d'assises des cellules qui la forment, en membrane fibreuse et en membrane non fibreuse.

Si la seconde membrane est formée de cellules sans filets, ces cellules se présentent le plus souvent sur une seule assise. Toutefois quelques plantes ont des assises de cellules multiples (2 à 3 assises dans le Pyrola, 4 dans le Solamum macrocarpum (portion des valves à cellules simples), 3 à 6 et même 7 dans certaines Cassia, jusqu'à 10 dans le Solanum Sisymbrinm (portion des valres lacuneuse et à cellules simples).

Si au contraire la deuxième membrane est constituée de cellules fibreuses, le cas le plus ordinaire est bien encore que cette membrane soit composée, an moins dans la plus grande partie de son étendue, d'une seule rangéc de cellules, mais ici les exceptions sont de beaucoup plus fréquentes que lorsque les valves de l'anthère ne renferment pas de cellules fibreuses. C'est ainsi qu'on compte deux assises de cellules dans plusieurs Passiftora, Hyoscyamu, Tropaoolum, 
Aspidistra, Atropa, Plantago, Crassula, Syringa, Chelone, Cinchona, Campanula, 3 à 4, et même darantage, chez les Cosmibuena, Siphocampylos, Bnugmansia, Encyctia, Uncidium, Limodorum, de 6 à 10 dans l'Agave, l'Iris, le Rhodea.

Dans les anthères à cellules fibreuses localisées ou n'occupant qu'une portion des valves, le nombre des assises peut être le même partout. C'est ce qu'on observe dans le Cassia Fistula, l'Halesia et le Chironia, dont les valves ne présentent qu'une seule assise de cellules, tant sur les points occupés par des cellules fibreuses que sur ceux formés de cellules sans filets. Mais il est d'autres cas où le nombre d'assises des cellules varie; nous donnerons comme exemple le Solarium Sisymbrium, qui, pour une rangée de cellules fibreuses vers le point de déhiscence, présente jusqu'à 8 assises de cellules simples dans les autres parties de l'anthère.

On peut prévoir que dans les anthères à cellules fibreuses localisées, les unes vers la ligne suturale, les autres près du connectif, c'est la forme de cellules la plus rapprochée du comnectif qui comptera le plus grand nombre d'assises. C'est ce qui résulte de la proposition suivante:

Dans une même valve, le nombre des assises cellulaires (que la deuxième membrane soit fibrense ou non), est toujours le plus grand vers le connectif et le plus petit dans la région suturale; ici leur nombre est à-peu-près constamment réduit à l'unité (1). C'est ainsi que parmi les valves à cellules simples le Solanum Sisymbrium n'a qu'une seule assise vers la suture, et en présente huit ou neuf auprès du connectif; que dans le Inycopersicon la deuxième membrane n'a qu'une assise ou même qu'elle manque sur la ligne de déhiscence, tandis qu'elle compte jusqu'à huit assises de cellules vers l'attache des ralves inférieures (les valves supérieures ont moins d'épaisseur).

Nous citerons, parmi les plantes à valves formées de cellules fibreuses : le Muscari et le Billbergia, qui ont une assise vers la suture, et trois assises près du connectif; l'Agave et la plupart des Iris, qui pour une ou deux assises à la suture en présentent de six à huit dans la région moyenne, et jusqu'à dix ou douze vers la base des ralves.

Lacunes. Je consigne ici, comme un fait auquel d'autres viendront se rattacher sans doute, l'observation que j'ai faite de lacunes dans l'épaisseur de la denxièmo membrane du Solanum Sisymbrium. Ces lacunes, disposées arec régularité comme dans le parenchyme des feuilles des plantes aquatiques, se forment, ainsi que dans celles-ci,

(1) La deuxième membrane peut même manquer vers la suture, que forment alors seules la première et la seconde membranes, quelquefois même une seule d'entre elles. 
dès le jeune âge de l'organe, et ne tiennent aucunement la place de tissus détruits; une assise des cellules de la seconde membrane les isole de la membrane épidermique et de la troisième membrane (1).

\section{Localisation des cellules fibreuses.}

Mirbel, Purkinge, Meren et les anatomistes renus après eux out admis, non seulement que les ralres des anthères sont toujours pourvues de cellules fibreuses, mais aussi que ces cellules constituent une membrane recourrant toute l'étendue des valres.

L'examen critique du premier de ces points (l'existence constante de collules fibreuses) m'occupera dans l'article suivant; je rais établir dans celui-ci que les cellules fibrenses, quand elles existent, n'occupent pas toujours l'étendue entière des valves.

Deux plantes voisines, peut-être congénères, la Squamaire et la Clandestine, ne présentent en effet de cellules fibrenses que sur la portion des valves voisine de la ligne de déhiscence (2).

C'est aussi vers la ligne de déhiscence des anthères que sont exclusirement placées les cellules fibreuses de l'Orobanche (3) et du Phelipcea, genres du même ordre que les Lathraa. Au même type d'organisation appartient le Rhinanthus.

Un mode de localisation des cellules, inverse de celui dont il rient d'être cité des exemples, c'est-à-dire avec localisation des cellules fibreuses, non plus vers l'extrêmité suturale des valves, mais du côté de l'attache au connectif, existe dans l'Halesia, les Chlora perfoliata et Chironia Centaurium. Telle est aussi la structure des valves du Cassia fistula.

Les Solanum ouvrent leur's anthères, non par une fente occupant toute la longueur de la loge, mais par une courte ouverture (sorte de pore) apicilaire. Or, si l'on fait de ces anthères des coupes transverses diversement étagées, on constate que c'est uniquement à la hauteur du point de déhiscence qu'existent des cellules à filets, toute la région inférieure de l'anthère en étant dépourvue. Si Purkinje a figuré les Solanum comme privés de cellules fibreuses, c'est sans doute parce que ses études araient été limitées aux portions inférieure et moyenne de l'organe.

Le Witheringia, genre peu homogène de Solanées, présente dans

(1) J'ai figuré ailleurs ces lacunes à divers âges ( $\mathrm{Pl} . \mathrm{V}$, fig. 3, 3a et $3 \mathrm{~b}$ ).

(2) On peut voir là un arrêt de développement, indiquant que la transformation des cellules procède (dans l'espèce) de la ligne de déhiscence vers le point opposé.

(3) J'ai vu les cellules fibreuses manquer complétement dans l'Orobanche Eryngii dont les valves se trouvaient réduites ì la membrane épidermique à l'époque de la naturation des anthères, sur quelques points même elle était représentée seulement par la paroi extérieure de ses utricules! 
l'anthère de ses diverses espèces, des différences anatomiques qui pourraient être l'indice d'états morphologiques correspondants. Le W. rubra est d'un intérêt spécial, au point de rue de cet article, par le type qu'il représente: il porte des cellules fibreuses sur toute l'étendue de la valre interne ou supérieure, tandis qu'il est tout-à-fait privé de ces cellules à la valve externe de la loge. Le W. crassifolia a des cellules fibreuses sur les deux ralres et un W. de Minas-Geraes, recueilli par Cladsex, en est partout dépour'ru.

Les Laurus ont cette organisation particulière de n'offrir des cellules fibrenses que sur les châssis qui, on le sait, se relèrent pour donner issue au pollen. Les Berberis, Mahonia, Epimedium diffèrent en ce que leurs anthères portent aussi des cellules à filets sur la portion des valves qui ne se soulève pas. Enfin, dans les Ophrys et Gymnadenia la production des filets est éparse et fort incomplète.

En résumé, on voit que les cellules fibreuses peuvent n'exister que sur une portion des valves des anthères et que leur localisation se rattache aux types suivants:

10. Les cellules fibreuses sont disposées vers la ligne de déhiscence sur toute la longueur de l'anthère (Phelipaea).

$2^{\circ}$. Elles occupent encore la longueur de l'anthère, mais senlement vers l'attache des valves au connectif (Chlora).

30. Elles n'occupent que le sommet des loges, au pourtour du point de déhiscence (Solanum).

4. Elles n'existent que sur l'une des deux valves (Witheringia nubra).

50. Elles sont localisées sur les châssis ou valvules de soulèvement (Laurinées).

6. Elles s'étendent au-delà des valrules de soulèvement '(Ber. béridées).

7\%. La production de filets sur les parois des cellules est incomplète, et les cellules fibreuses sont dispersées $\left(\mathrm{O}_{1} \mathrm{H}_{\mathrm{r}} \mathrm{rys}^{\mathrm{r}}\right.$, Orchis mascula, etc.).

\section{Absence de cellules fibreuses.}

Les livres dans lesquels j’ai appris la botanique m'avaient enseigné que dans la paroi des anthères entre toujours une membrane composée de cellules fibreuses; mais les observations auxquelles je dus me livrer pour le présent trarail, m'apprirent bientôt que dans bon nombre de plantes ces cellules fibreuses font au contraire complètement défaut.

L'absence de cellules fibreuses peut quelquefois tenir à des circonstances accidentelles. C'est ainsi qu'à Paris j'ai remarqué, depuis plusieurs amnées, que les anthères de l'Hypoxis erecta et du Pitto. 
sponn Tobir manquent de cellules à filets, en même temps qu'elles ne contiennent pas de pollen parfait; ces anthères stériles ont sans. doute été frappées d'un double arrêt de développement.

Mais dans les cas qui méritent plus de nous occuper parce qu'ils répondent à une constitution normale, les anthères, quoique contenant un pollen fertile, ont leur's ralves complètement dépourrues de cellules fibreuses.

Les Ericacées furent les premières plantes dans lesquelles je constatai l'absence de cellules fibrenses. Conjecturant alors que les autres groupes de végétaux à anthères s'ouvrant aussi par un pore pourraient offrir la même organisation, j'établis, à ce point de vue, une série de recherches dont les résultats répondirent à mes prérisions. Les Vacciniées, les Rhododendrées, les Pyrolacées, les Monotropées, les Epacridées, les Mélastomées n'offrirent pas de trace de ces cellules fibreuses qu'on arait cru ne faire jamais défaut.

Ces observations semblaient établir une relation constante entre le manque de cellules fibreuses et la déhiscence apicilaire. Les Cassia (non le $C$. Fistula), qui ouvrent leurs anthères par une courte fente apicilaire, et le Tetratheca confirmèrent ce rapport; mais une exception inattendue fut offerte par le Solanum, genre de plantes pourru de cellules fibreuses à la hauteur du pore terminal des anthères.

Ainsi la règle qui veut que les anthères à déhiscence terminale soient privées de cellules fibreuses souffre une très-curieuse exception dans le Solanum.

Par opposition à ce qui précède, on pouvait s'attendre à ce que les anthères à déhiscence longitudinale fussent toujour's munies de cellules fibreuses. C'est en effet la règle, mais une règle où les exceptions ne sont pas extrêmement rares.

C'est ainsi que le Lycopersicon, genre bien voisin du Solanum, a ses anthères privées de cellules fibreuses, quoique s'ouvrant longitudinalement, et l'on ne peut s'empêcher de faire la remarque que deux genres, assez voisins pour avoir été longtemps réunis en un seul, forment respectivement une curieuse exception aux rapports généraux qui lient la structure des ralres aux modes de déhiscence.

Les plantes qui, comme le Lycopersicon, manquent de cellules fibreuses, quoique offrant la déhiscence longitudinale, ne sont d'ailleurs pas tout-à-fait rares. Déjà mes observations permettent d'y comprendre les suivantes, appartenant à des familles fort diverses: Badula, Diospyros, Gonolobus, Thunbergia, Balanophora, Cycas, Zamia, Loroglossum, Orchis maseula, O. sambucina, O. fusea et plusieurs Orobanche.

Uné circonstance à noter, c'est que dans les anthères à'déhis- 
cence longitudinale, quoique privées de cellules fibreuses, les bords des valves, loin de s'écarter et de finir par se renverser au dehors, restent toujours rapprochés sur la ligne de déhiscence. A ce caractère on peut souvent reconnaitre la structure de l'anthère.

Il est établi par ce qui précède :

1: Que les cellules fibreuses manquent, en général, dans les anthères à déhiscence poricide.

2: Que les cellules fibreuses font défaut dans un certain nombre d'anthères à déhiscence longitudinale.

3. Que dans quelques plantes dont les étamines paraissent avoir subi un arrêt de développement, sinon morphologique du moins histologique, l'absence de cellules fibreuses coïncide aree la mauvaise conformation du pollen.

\section{Troisième membrane.}

A peu près inconnue jusqu'à ce jour, quoiqu'aperçue par Meyen et par Schleiden, la troisième membrane existe toujours dans le jeuno âge des anthères. Plus tard (vers l'époque de la maturation du pollen) elle disparaît généralement, ne laissant que peu ou point de traces. C'est à la troisième membrane, sorte d'épiderme (quant au siége, non pour les fonctions) interne des valves de l'anthère, que s'appliquerait avec juste raison le nom d'endothecium, que l'on donne depuis Purkinse à la membrane moyenne, et qui derrait aujourd'hui être dénommée mesothecium. On peut croire que si la troisième membrane n'a pas fixé l'attention des anatomistes, c'est parce qu'elle n'existe généralement plus vers l'époque de la déhiscence des anthères.

Cependant le tissu qui la forme, plus interne que l'endothecium de Purkinje ou membrane à filets de Mirbel, persiste dans quelques plantes.

J'ai observé au développement de la troisième membrane dans plusieur's centaines de plantes, et dans toutes j'ai vu les faits histologiques se suivre arec une grande uniformité. J'en donnerai une idée en disant ce qui se passe dans le Passiflora, genre à grosses anthères d'une étude facile et qui se trourent figurées aux diverses phases de leur évolution dans les deux premières planches de l'atlas (de 36 planches) consacré aux présentes recherches.

Formé dans le, Passiflora d'une assise d'utricules papilliformes généralement teintées de jaune et dirigées des parois des valves rers la cavité des loges de l'anthère, le tissu de la troisième membrane est déjà distinct des tissus roisins peu après l'apparition des utricules polliniques; il s'allonge à mesure que ces utricules, avanęant elles- 
mêmes dans leur érolution, prodnisent le pollen, puis disparaissent; son plus grand déreloppement répond en général au moment où le pollen, débarrassé de l'enveloppe que lui formaient les cellules-mères, est parrenu à ce degré avancé de son organisation qui précède immédiatement la production des filets dans les cellules fibreuses. Mais à mesure que ces dernières cellules complètent leur organisation et que le moment de la déhiscence des anthères approche, le tissu de la membrane interne se flétrit, se lacère, et à la fin disparait, complètement résorbé ou ne laissant pour témoin qu'une matière désorganisée et granuleuse appliquée sur les parois de la loge. Production transitoire dont les fonctions, sans doute nourricières, paraissent être liées à la fois à la nutrition du pollen et au développement si rapide des filets dans les cellules fibreuses ou sous-épidermiques, la membrane interne a ainsi, suirant nous, une grande importance biologique. Sa structure presque toujours délicate et souvent papilleuse (Passiflorae species, Colchicum, etc.) est en rapport érident arec cet ordre de fonctions.

Le caractère de la troisième membrane d'être résorbée lorsqu'approche l'époque de la déhiscence des anthères, n'est pas sans quelques exceptions. C'est ainsi que j'ai vu cette membrane persister à des degrés dirers dans les Pedicularis, Hyoscyamus, Evythronium, etc., et l'on peut dire la destruction en est rare danș les anthères privées de cellules fibreuses.

Coloration. Il faut compter la coloration parmi les caractères de la troisième membrane: elle est de beaucoup plus commune que celle de la membrane contigue; elle en est généralement indépendante; elle a an contraire des rapports intimes arec la coloration du pollen dont elle entoure la masse.

La deuxième membrane est généralement sans coloration, tandis que la troisième membrane est le plus sourent colorée. Si, en effet, celle-ci est d'un blanc satiné dans le Lycopersicon, presque incolore dans l'Echeveria, le Choetophora, l'Heterocentrum, elle est de couleur fauve dans l'Asclepias, verdâtre dans le Chimonanthus et l'Ulex, d'un vert jaune dans plusieurs Salvia, l'Epimedium, jaunâtre dans les Begonia, Thea, jaune chez les Daphne, Jasminum, Sparmannia, Aesculus, Vitis, Citrus, Fragaria, Canna, Aloe, orangée dans le Tropaolum et le Zygophyllum Fabago, parfois rouge, bleue ou d'un violet bleu (Phyterma), etc.

La coloration de la troisième membrane est généralement indépendante de celle de la membrane épidermique. C'est ainsi que dans le Plumbago scandens, l'Heterocentrum roseum, le Megazea alba et le Sylphium, tous à membrane interne jaunâtre ou presque incolore, on 
trouve que la membrane épidermique est bleue pour le premier, rose pour le second et le troisième, d'un blen noir dans le Sylphium.

- Quelquefois la seconde membrane partage la coloration de la troisième, comme on l'observe dans l'Aponogeton, le Gonolobus, le Salvia splendens.

Il ressort de ce qui précède que si de la troisième et de la seconde membrane une seule est colorée, c'est ordinairement la troisième; mais le cas contraire peut se présenter, comme si la nature diverse des deux membranes sous-épidermiques devait être établie par tous les genres de preuve. C'est ainsi que le Lycopersicon et le Cassia, chez lesquels la troisième membrane est incolore, ont la deuxième membrane, celui-là verte, celui-ci d'un jaune fauve.

Mais si la coloration de la troisième membrane n'offre que de rares analogies avec celle des deux autres membranes, cette coloration est au contraire directement liée, comme la cause à l'effet, à la coloration du pollen. Telle est même la généralité de ce rapport qu'on pent, soit remonter à peu près avec certitude de la couleur du pollen à celle de la membrane détruite, soit prévoir, par la coloration de la troisième membrane, quelle sera celle du pollen. Ces rapports constatés sont d'autant plus susceptibles d'applications que la formation et la coloration de la troisième membrane précèdent celles du pollen, et que le pollen reste après la destruction de la membrane elle-même.

Structure. Qu'elles s'avancent en papilles dans la cavité des loges ou que, plus ou moins aplaties, elles ne forment qu'une membrane étroite, sorte d'épiderme intérieur, les cellules de la troisième mem. brane sont généralement à parois minces, fort délicates, non ponctuées par conséquent. Quelques exceptions se présentent dans les Pyrola, Vaccinium, Rhododendroin et Cassia, plantes à anthères sans cellules à filets, mais qui, par une sorte de compensation, ont la membrane interne notablement épaissie.

Une seule assise d'utrieules constitue ordinairement la troisième membrane. Cependant celle-ci est composée de deux assises dans le Sparmannio, de deux à quatre assises dans le Viole, le Crassula orbiculcris, dans plusieur's Cassia et Canne, et jusqu’à sept dans le Cassia maiylandica. Comme pour la deuxième membrane, c'est vers l'attache des valves au connectif que les assises existent en plus grand nombre.

La membrane interne ne s'étend pas seulement sur les valves de l'anthère; elle tapisse toute la carité des loges, aussi bien la portion des parois des loges, formée par la cloison, le connectif et les placentoïdes, que la portion qui répond aux valves elles-mêmes. 
Contenu. Les substances que contiemnent principalement les utricules de la troisième membrane sont, avec des matières colorantes variées, des corps gras souvent réunis en gouttelettes et chargés de principes colorants, des substances azotées, du mucilage, du sucre et de l'alerone.

Fonctions. Les différentes phases du développement de la troisième membrane, son existence transitoire, son siége sur toute la paroi interne des loges polliniques, sa production précédant celle du pollen qu'elle isole de tous les autres tissus, sa destruction lor'sque le pollen arrive à son développement complet, et lorsque les filets se forment sur les cellules de la seconde membrane, les rapports de coloration qu'elle a de commun avec le pollen, les matières alimentaires qui y sont contenues; tout indique, qu'elle est la nourrice du pollen et sans doute aussi le réservoir où les cellules de la seconde membrane puisent les éléments de leur rapide transformation.

Les détails dans lesquels je viens d'entrer mettent, on l'accordera, hor's de doute l'existence, dans l'anthère, d'une troisième membrane, et assignent à celle-ci un rôle important. On ne saurait plus dès lors conserver à la deuxième membrane, par son siége vrai mesothecium, le nom d'endothecium que lui imposa Purkinje, dans la croyance qu'il représentait le tissu le plus intérieur de la paroi des loges. Ou ce dernier nom doit être abandonné, ou il faut l'appliquer à la troisième membrane.

Je ferai encore cette remarque que, si dans un certain nombre de plantes (Melastoma, Octomeris) la troisième membrane se distingue mal de la deuxième membrane, ce n'est pas une raison suffisante pour nier son existence dans ces plantes elles-mêmes. On comprend en effet que, comme la première membrane (l'épiderme) dont on ne contestera pas cependant l'existence, elle puisse se confondre on apparence avec la seconde membrane par une texture commune. Je citerai comme exemple le Chotophora et le Clandestina, plantes où sont confondues en un tissu homogène les utricules de l'épiderme avec celles du tissu placé au dessous de lui. Les faits de cet ordre ne sont pas rares dans le règne végétal.

\section{Cloison des logettes.}

Les deux loges qui forment habituellement l'anthère sont séparées par le connectif, dont la structure sera exposée plus loin. C'est uniquement de la cloison qui, à une certaine époque de leur développement, divise en deux logettes chacume des loges, que nous nous occuperons ici. 
Observée, mais dans sa première formation seulement, et complètement négligée quant à sa structure et à ses destinations par M. DE Mirber, la cloison des logettes prend forme au milieu des tissus de l'anthère à peu près en même temps que les utricules mères du pollen; puis elle achève ses développements, et enfin elle disparaît dans la plupart des plintes rers le moment de liu maturation du pollen.

Je rais considérer la cloison des logettes:

(t. Dans son organographie ou sa morphologie.

b. Dans son histologie.

c. Dans sa biologie ou ses fonctions.

A. Oiganographie.

Ordinairement il existe une cloison dans chaque loge, cette cloison est verticale, et par conséquent les deux logettes qu'elle sépare sont collatérales.

Parfois cependant la cloison, au lieu d'être verticale, s'est développée au travers de la loge, horizontalement, et dans ce cas les logettes sont superposées. (Laurus Cinnamomum L., Sassafiras).

Très-rarement (Nuphtai Zuteum, Launus nobilis, Berberidées), la cloison manque, et alors la loge est nécessairement indivise à toutes les phases de l'évolution de l'anthère.

Enfin une disposition, moins rare que la précédente, est celle où, par suite du développement inégal des valves (les postérieures étant généralement plus allongées que les antérieures), les cloisons (verticales) n'atteignent pas jusqu'aux extrémités de l'anthère, où les loges restent indivises. Dans ce cas, dont le Paratropic offre un bon exemple, des sections transverses menées par les diverses parties de l'anthère indiquent parfaitement les limites du développement des cloisons (1).

Quand on suit avec attention, jusqu'à 'ses dernières phases, le développement de la eloison des logettes, on est amené à lui reconnaître deux origines, lesquelles peuvent, ou présider respectivement senles à la formation de la cloison, ou contribuer à cette formation en des proportions diverses, ce dernier cas étant le plus ordinaire.

Dans ce qui ra suivre, nous prenons pour base cette définition incontestablement juste au point de rue du fait: la cloison des logettes est le tissu (quelconque) qui divise en derad chucune des loges de l'cuntlière.

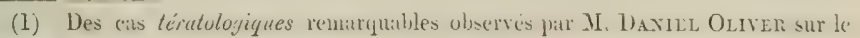
Loranthus europeens etc., et figurés dans le Vol. XXIII, tab. 4 , des Transact. Linn. Sos., montrent combien peut varier, dans ces cas anormaux, le développement relatif des valves. M. D. Otiver représente aussi des anthères privées des cloisons dans l'une des loges. 
La définition posée, nous sommes conduit à reconnaître les trois cas suivants:

a. La cloison des logettes est constituée uniquement par un tissu qui est la continuation du connectif;

b. La cloison est formée complètement par les valves réfléchies sur le connectif;

c. La cloison, de nature mixte, est composée en partie par la prolongation du connectif, en partie par la rentrée des valves.

Reprenons ces trois points.

a. Quand la cloison est formée tout entière par les tissus du connectif, ceux-ci se prolongent en une lame ayant toute la profondeur de la loge, et c'est sur l'extrémité de cette lame que viennent poser par leurs bords les valves à peine infléchies. On trouve des cloisons de cette origine dans l'anthère du Cucurbita Pepo, de l'didisia, de l'Hippuris, du Linum, du Jasminum nudiflorum, du Tradescantia, de l'Octomeris, du Melastoma, du Choetoplıo'a, de l'Heterocentrum, du Monochoetum. Peut-être la cloison exclusivement connectivale est elle plus commune parmi les plantes à anthères privées de cellules fibreuses que parmi celles d'une organisation différente.

b. Lorsque la cloison est formée par les valves seules, celles-ci s'infléchissent profondément pour renir, plus ou moins adossées l'une à l'autre, s'appuyer an connectif, constituant ainsi la séparation de chaque loge en deux logettes. Il n'est pas hors de propos d'ajouter que c'est au figuré que je m'exprime quand je dis: les valves s'infléchissent. Lue fait est que les valves se forment sur place, comme les cloisons vraies ou connectivales se taillent en quelque sorte dans la masse parenchymateuse de la jeune anthère, dont une portion devient membrane des ralres, d'autres portions utricules-mères du pollen, cloisons vraies, placentoïdes et commectif proprement dit.

Les cloisons d'origine exclusivement valvaire sont de toutes les plus rares, on peut les observer dans le Tellima, l'Habrothamnus, le Buxus, plusiemrs Passiflora et Sarifiaga.

c. Les cloisons dans la constitution desquelles les valves entrent pour une part et un appendice du connectif pour le reste, sont de beaucoup les plus communes. Elles offrent d'ailleurs toutes les combinaisons possibles quant à la longueur respective des parties fournics par les valres d'un côté et par le comnectif de l'autre (1). C'est ainsi que les valves entrent à peu près dans la formation des cloisons:

(1) Peut-être même, en procédant avec rigueur, trouverait-on que toutes les cloisons ont une origine mixte, ce qui ferait disparaître les catégories $a$ et $b$. C'est que à propos du tout on peut dire avec LrNNé: Natura hon facit saltus. Ėt cependant nous devons établir des eatégories. 
Pour 75/100 dans le Chironia fintescens, le Tulipa sylvestris et plusieurs Petssiftorct.

Pour 50/100 dans plusieurs Cinchone, Scebiose, Campanula, Phyterma, Minose, Tropreolum, Aechmea, Tritome.

Pour 25/100 dans les Chelone, Lathreea, Centropogon, Loperia, Cheiranthus, Corylus.

Pour 12/100 dans les Mioporum et Stylidium.

Pour $10 / 100$ ou même moins dans l'Atropa, le Beloperone, le Foeviculum.

Pour 5/100 dans l'Althaec et le ILalva, qui passent, le dernier surtout, aux plantes ayant les cloisons des anthères formées par le connectif seul.

Les divisions que nons venons d'indiquer sont purement organographiques, à savoir que nous attribuons aux valves les tissus qui se relèvent avec elles et en sont la continuation évidente, et au connectif la portion des cloisons qui lui reste adhérente, ou est résorbée vers l'époque de la déhiscence. Cette réserve est d'autant plus nécessaire que souvent la portion de cloison qui reste soudée au connectif se rattache par l'histologie (ou la nature intime des tissus) plus aux valves qu'au connectif. C'est ainsi que le bord des cloisons est recouvert de cellules fibreuses qui se continuent avec celles des valves (par l'attache de celles-ci) dans les Ployterma, Centropogon, Lopezia, Scabiosa et Knautia, Cheiranthus, Tropoeolum, dont la masse du connectif est privée de cette sorte de cellules. Et cependant il me parait qu'on ne saurait attribuer aux valves ces cloisons plus ou moins fibreuses.

\section{B. Histologie.}

La cloison des logettes étant le plus souvent, avons-nous dit, composée en partie par les bords infléchis des valves, l'histologie des cloisons de cette origine se confond avec celle des valves elles-mêmes; y revenir serait répéter ce qui a été dit des membranes constitutives des valves.

Mais nous devons entrer en quelques développements sur la nature histologique de la cloison que constitue une lame avancée du connectif, de celle qu'on pourrait nommer la vraie cloison.

Nous distinguerons dans cette vraie cloison: $a$. ses parois; b. Sa masse.

4. Parcis des cloisons. La paroi ou surface interne des cloisons est constituée par un repli de la membrane interne ou troisième membrane des valves, laquelle se prolonge sur les tissus en continuité avec le connectif. Lorsqu'approche le moment de la matu- 
ration du pollen ou de la déhiscence des anthères, la portion de la troisième membrane appliquée sur la cloison suit le sort de celle qui tapisse les valves, c'es̀t-à-dire qu'elle est le plus souvent résorbée, laissant à nu la cloison proprement dite, dont la nature histologique, très-rariable, peut toutefois être rattachée comme il suit, à quelques cas généranx:

1. Les tissus de la cloison ne sont jamais formés de cellules fibreuses. Ce cas très-commun peut être observé dans les Justicic, Mirbelia, Kennedya, Cydonia, Pyrus, Fragavia, Staphylea, Ribes, Sarifiaga, Rhem, Daphne, Gievillea, Cytisus, etc.

2. Les tissus de la cloison les plus rapprochés de la cavité des logettes sont formés seuls de cellules fibreuses; on observe cette structure, dans laquelle les cellnles fibreuses de la cloison semblent être une prolongation (toutefois avec une forme généralement différente) de celles de la deuxième membrane des valves, chez les Loperia, Cheiranthus, etc.

Deux cas se présentent ici quant au nombre d'assises des cellules fibreuses. Tantôt celles-ci sont disposées sur un seul rang, comme dans les Epilobium, Oenothera, Fuchsia, Kunutia, Sccubiosa, Pterocephalus, Hippuris; tantôt elles le sont sur plusieurs rangs, comme dans les Tropcueolum, Diclytra, etc.

Quelquefois il y a accord de nombre entre les assises fibreuses de la cloison et celles des valves. C'est ainsi que dans les Lopeziu et Epilobium il existe un seul rang de cellules tibreuses dans la cloison comme dans les valves, que dans l'Epidendrum ces assises sont au contraire au nombre de deux.

En d'autre plantes le désaccord est manifeste. Ainsi dans les Cinchona, Cheiranthus, Diclytra, les valves de l'anthère ont seulement une assise de cellules fibreuses, tandis que la cloison en offre deux dans le Cinchonc et Diclytra, et trois dans le Cheiranthus.

Un autre cas est celui dans lequel la masse entière ou à peu près entière de la cloison est fibreuse, les cellules à filets ne s'étendant pas sur le comnectif proprement dit: nous citerons comme exemples les Aponogeton, Colchicum, Lobelia, Forsythia.

Enfin, le tissu fibreux peut s'étendre des ralves et des cloisons an comnectif; alor's la masse entière de l'anthère est fibreuse, à l'excep)tion de l'axe que suivent les cellules conductrices ou nourricières entourant le plus souvent quelques trachées.

Une structure qu'on observe dans beaucoup d'Tris est celle où la masse des tissus étant de nature fibreuse comme il vient d'être dit, la cloison des logettes est seule formée de cellules simples.

Une autre disposition spéciale existe dans le Pontederia, chez 
lequel les cellules fibreuses des valves se prolongent, non sur les bords, mais sous la base même de la cloison, qu'elles coupent ainsi de ses communications arec le corps du connectif.

\section{Fonctions de la cloison.}

L'histoire physiologique ou biologique de la cloison peut être rattachée à l'architecture de la loge, à la nutrition du pollen, à la déhiscence des valves.

a. Architecture de la cloison. La cloison des logettes a une utilité architecturale qui ne saurait être niée. En effet, elle s'élève du connectif, sur lequel elle s'appuie solidement par une base évasée vers le point où viennent aboutir, et reposer sur elle, les extrémités ou bords suturaux des deux valves de la loge. Elle représente donc, dans la loge où elle porte les bords libres des valves, le mur de refend d'une maison sur lequel viennent porter les bouts de solives d'un plancher dont les bases sont engagées dans le mur d'enceinte.

Parfois, nous l'avons dit, la cloison vraie manque ou est rudimentaire, li séparation des logettes étant alors produite par les valves elles-mêmes, recourbées en are pour venir appuyer leurs bords sur le connectif:

On comprend très-bien que la cloison, soutenant les valves et les empêchant de s'affaisser dans la cavité des loges, conserve à celles-ci leur capacité et favorise par là-même et la production d'une quantité de pollen plus considérable que si li capacité de la loge était réduite, et un déreloppement plus régulier des grains de pollen, qui n'ont pas à souffrir de la compression qu'exerceraient sur eux les valves non soutenues.

b. Nutrition du pollen. Les cloisons des logettes concourent indirectement au déreloppement du pollen en empêchant la compression qui résulterait pour lui de la chute des valves. Elles favorisent directement sa nutrition en multipliant la surface de la membrane nourricière, ou troisième membrane. Nous avons dit, en effet, que celle-ci tapisse non seulement l'intérieur des valves, mais aussi la cloison. Que celle-ci soit supprimée, et du mème coup disparaitra cette membrane nourricière, qui par les deux faces de la cloison porte l'aliment au milieu de la masse pollinique.

Les cloisons de nature parenchymateuses (et c'est le plus grand nombre) peuvent aussi fournir des aliments au pollen (et à la transformation des cellules simples en cellules fibreuses), en proportion d'autant plus considérable qu'elles ont, comme dans quelques Iris, Anemone, Rumex, Tradescantia, une plus grande épaisseur. 
c. Rapports avec la déhiscence. La déhiscence des loges se rattache, non à une cause unique (les cellules fibreuses), comme on l'a admis longtemps, mais à des causes multiples que nous aurons plus loin à apprécier dans leur ensemble; mais nous devons noter ici celle de ces causes qui nous paraît tenir aux cloisons.

On a vu que la solidité de al'édifice des loges, ou chambres à pollen tient à la cloison même. Que celle-ci disparaisse, comme il arrive vers l'époque de la maturation du pollen dans la plupart deś plantes, et les valves se désuniront, par suite du porte-à-faux sur la ligne suturale, comme il arriverait aux deux moitiés d'un plancher dont les extrémités seraient suspendues dans le vide après l'enlèvement du mur de refend qui auparavant portait ces extrémités et les retenait ainsi en contiguité.

Le rôle ici attribué à la destruction des cloisons dans le phénomène complexe de la déhiscence s'appuie d'ailleurs sur cette observation, que dans les plantes à déhiscence poricide, (Cassia, Ericacées, ttc.) la cloison persiste généralement sur toute la longueur de la suture, le point de déhiscence excepté (1).

\section{Plucentoïdes (2).}

L'organe que nous allons faire connaitre n'avait pas encore été signalé. Le nom de placentoïdes, par lequel nous proposons de le désigner, rappelle les analogies de forme, et jusqu'à un certain point, de fonctions, qu'il a avec les placentas des ovaires. Nous la considérerons aux points de vue: (. morphologique ou organographique; $b$. histologique; $c$. biologique; $d$. taxonomique; $e$. philosophique.

a. Morplologie des placentoüdes. Les placentoïdes rappellent tout-à-ficit, par leur place dans les logettes et la forme générale qu'ils affectent, les placentas axiles des ovaires biloculaires. Que l'on fasse la coupe transversale de l'ovaire d'un Solanum et celle de l'une de ses anthères, ou trouvera dans chacuno des logettes de celles-ci, comme dans chaque cavité ovarienne, un corps charnu qui s'avance vers le milien des loges de l'ovaire, des logettes de l'anthère.

Par la place considérable qu'il occupe dans les logettes, le placentoïde r'éduit souvent beaucoup l'espace réservé au pollen, à peu près comme, dans beutucoup de Solanées et de Scrofularinées, on roit

(1) Il sera établi qu'aucune des cátuses de déhiscence n'est absolument générale. Aussi les anthères manquant de toute cloison (Nuphar) ou de cloison vraie (T'ellima) ne laisseront-elles pas que de s'ouvrir, les autres causes faisant alors compensation.

(2) Les Planches XXV, XXVI, XXVII, et la Pl.V (fig. 2-3) de notre Atlas sont consacrées aux placentoides. 
les graines resserrées entre de volumineux trophospermes et les ralves du péricarpe (1).

Quelquefois le placentoïde s'avance assez vers la valve qui lui est opposée pour venir la toucher par son extrémité, subdivisant alors chaque logette de l'anthère en deux sous-logettes. La coupe d'une jeune anthère ainsi conformée présentè celle-ci divisée en 8 logettes si l'anthère est complète (Hemitomus), en 4 logettes si, comme dans le Salvia, l'anthère doit être réduite à une seule loge.

J'ai observé quelques plantes dans lesquelles les placentoïdes, au lieu d'exister dans chacune des deux logettes, ne s'étaient développés que sur l'une des faces de la cloison, manquant par conséquent sur la face opposée et dans la logette correspondante: tel est le cas du Justicia flavicoma et du Brillantaisia.

La production du placentoïde a lieu généralement comme celle de la cloison, c'est-à-dire que les tissus se taillent en quelque sorte en plein drap, dans la masse parenchymateuse de la jeune anthère; parfois cependant on les roit s'accroître par une sorte de végétation utriculaire appliquée sur la portion tenant à la cloison.

La durée des placentoïdes est limitée, comme celle de la cloison dont ils peuvent être en quelque sorte considérés comme une dilatation; c'est-à-dire que, comme celle-ci, ils sont résorbés et disparaissent plus ou moins complètement vers l'époque de maturation du pollen, ne laissant tantôt aucune trace, se manifestant quelquefois encore par deux petites cornes ou appendices que la rétraction de la cloison a rapprochés du connectif.

En somme, l'existence des placentoïdes est, comme celle de la cloison des logettes et plus qu'elle encore, liée au développement du pollen; ils apparaissent avec celui-ci, disparaissent vers sa maturation, sans jamais persister; la cloison persiste au contraire dans un certain nombre de plantes. La résorption des placentoïdes commence d'ailleurs généralement un peu avant celle de la cloison.

b. Histologie des placentoïdes. J'ai toujours vu les placentoïdes formés par un tissu parenchymateux très-semblable à celui qui forme la cloison. Comme celle-ci, ils ne sont jamais traversés ni par des fibres, ni par des vaisseaux (et sous ce rapport leur parallèle avec les placentas des ovaires ne saurait être soutenu, de même qu'on ne peut comparer le pollen, libre dans la carité des logettes, aux graines attachées sur les placentas); mais de plus ils paraissent ne jamais

(1) On peut comparer le pollen, resserré dans la logette entre le placentoïde et les valves, aux spores des Mousses pressées entre la columelle et les parois de l'urne. L'analogie physiologique quant au mode de nutrition est d'ailleurs complète, comme leur formation 4 par 4 dans les cellules-mires, entre le pollen et les spores. 
compter parmi leurs éléments histologiques les cellules fibreuses ou à filets qui revêtent assez souvent la surface des cloisons, et parfois les forment tout entières. Ce dernier point me paraît important à noter au point de vue du rôle biologique des placentoïdes.

Je dois d'ailleurs faire cette remarque que je n'ai pas observé de placentoïdes dans les anthères à cloisons formées même partiellement de cellules fibreuses; toujours la présence des placentoïdes ayant coincidé avec l'existence de cloisons simplement parenchymateuses.

Les placentoïdes sont ordinairement, comme les cloisons, recouverts par un repli de la troisième membrane; toutefois j'ai ru quelquefois cette membrane manquer ou être peu distincte (Squamaria), la surface des placentoïdes étant alor's en rapport immédiat arec le pollen.

c. Biologie des placentö̈des. Les placentoïdes me paraissent avoir pour fonction de concourir à la nutrition du pollen. Ils naissent rers la même époque que lui, le suivent dans ses développements, disparaissent quand, sa maturation approchant, ils lui sont inutiles, leur persistance pourant même être un obstacle à sa facile et complète dissémination.

La structure essentiellement parenchymateuse des placentoïdes, la troisième membrane ou membrane nourricière, qui les revêt ordinairement et dont ils augmentent la surface et multiplient les poiuts de contact avec le pollen, dont la masse, déjà divisée par la cloison, est subdivisée par les placentoïdes qui la réduisent ainsi en tranches minces presque partout resserrées entre deux surfaces nourricières dont les produits ne peuvent lui arriver que par imbibition, voilà évidemment des conditions appropriées au rôle que nous attribuons aux placentoïdes.

On est d'ailleurs d'autant plus frappé de l'utilité d'une organisation ayant pour effet de mettre partont l'aliment à la portée du corps à nourrir, que celui-ci (le pollen) ne reçoit pas directement sa nourriture par continuité, comme les ovules la reçoivent des placentas, mais indirectement, par simple contiguité.

d. Des placentoüdes dans leurs rapports avec la taxonomie. Étant donnée, comme c'est le cas pour les placentoïdes, l'observation d'un organe nouveau dans les plantes, il est nécessaire à l'histoire de cet organe de rechercher quels rapports d'existence ou de développement il peut avoir avec les divisions naturelles, plus ou moins générales, du règne régétal. Ce premier point déterminé, il deriendra possible d'apprécier la signification de l'existence des placentoïdes dans ses rapports avec les degrés variés d'élévation organique des espèces végétales.

Il est digne de remarque que je n'ai constaté la présence des placentoïdes dans aucune espèce appartenant à l'em!)ranchement des plantes monocotylédones. Or, comme mes études ont porté sur 
presque toutes les familles de cet embranchement, on peut, je pense, tenir pour certain que les placentoïdes y font complètement défaut.

Les plantes dicotylédones ont été divisées par l'illustre dE CANDOLLE en quatre classes, dont denx, les Monochlamydées ou Apétales, les Thalamiflores ou Polypétales hypogynes, n'ont présenté, comme les monocotylédones, aucun restige de placentoïdes. J'en dirais autant des Calyciflores, si je n'arais obser'ré des placentoïdes dans 1. Cassia Marylandica.

La dirision des plantes calyciflores du célèbre botaniste de Genève, fondée uniquement sur l'insertion, réunit deux séries distiuctes, celle des polypétales et celle des gamopétales, et il est bien digne de remarque (en raison de ce qui va suirre) que ce soient précisément les Calyciflores gamopétales qui manquent absolument de placentoïdes. Je pense du reste qu'il ne faut accorder qu'une médiocre raleur au fait exceptionnellement offert par un Cassia, bien que ce genre soit l'un des plus parfaits d'une famille regardée comme des plus élerées de sa classe.

Reste la quatrième classe de dicotylédones, les Corolliflores ou Gamopétales hypogynes. Or, c'est aux familles qui en font partie qu'appartiennent les espèces dont les anthères sont pourrues de placentoïdes. J'ai obsercé ces organes:

chez les Gentianées, dans le Chlore prevfoliate, les Chironia fintescens et C. Centanium;

chez les Solanées, dans les Solanum, Lyeopersicon, Atropa, Datura, Habrothamnus, Hyoscyamus, Witheringia, Ulloa;

chez les Scrofularinées, dans les Verboscum, Hemitomus, Pedicularis, non dans les Veronica ni les Chelone;

chez les Labiées, dans les Salvia, Rosmarinus et Westringia, à anthères uniloculaires; dans les Lamium, Leonurus et Marubium; chez les Acanthacées, dans les Acantlurs, Beloperone, Brillantaisia, Cyntanthera, Peristrophe, Justicia;

chez les Orobanchées, dans les Clandestina et Squamaria, non dans les Orobanche ni les Phelipaca;

chez les Bignoniacées, dans les Tecoma.

Les familles qui suivent, rapprochées des précédentes à des degrés divers, ont au contraire paru être privées de placentoïdes:

les Gesnériacées (Gloxinia, Achimenes);

les Polémoniacées (Polemonium, Phlox, Cobaea);

les Apocynées (Asclepias, Allamanda, Nerim, Tinca) (1);

les Conrolvulacées (Convolvulus, Cuscuta);

(1) Je prends ici les familles dans leurs ancienues et grandes circonscriptions. 
les Primulacées (Primula, Cyclamen, Androsace).

Enfin, les Plumbaginées (Plumbago, Statice) et les Plantaginées (Plantagn, Littorella), familles qui forment le passage aux Monochlamydées, et devaient à ce titre, on pouvait le prévoir, être privées de placentoïdes.

On sera frappé de ce fait, que parmi les Corolliflores portant des placentoïdes, ce sont les ordres Labiatiflores qui dominent (Labiées, Acanthacées, Orobanchées, Scrofularinées), les Solanées étant ellesmêmes inséparables des Scrofularinées.

Quelques groupes fort complexes, comme les Scrofularinées, présentent à cet égard des différences en rapport avec les coupes qui semblent être les plus naturelles. De ces faits on peut conclure que la présence ou l'absence des placentoïdes est en rapport arec les caractères morphologiques et pourra, à ce titre, être invoquée comme caractère complémentaire dans la recherche des affinités naturelles.

e. Philosophie des placentoïdes. Sous ce titre on pourrait considérer les placentoïdes sous plusieurs côtés, revenir à leur rôle biologique, etc. Mais je circonscris la question à ce seul point, l'appréciation de l'existence des placentoïdes par rapport à la mesure de la gradation organique des espèces végétales.

On peut dire, en se reportant aux faits ayant déjà cours dans la science, que poser la question c'est la résoudre.

En effet, il est depuis longtemps admis, et les preures surabondent, que les monocotylédones sont moins élerées en organisation que les dicotylédones. Or, les monocotylédones n'ont pas de placentoïdes.

Pour les dicotylédones, la question, plus controversée, paraît être enfin arrêtée à cette solution (vers laquelle j'ai poussé, dans la mesure de mes forces, par mes écrits sur la mesure de la gradation mganique des espèces végrétales), que les plantes gamopétales sont plus élerées en organisation que les plantes dialypétales (polypétales) et apétales et que, parmi les gamopétales, les familles à oraire soudé au calice doivent prendre rang au-dessous de celles à oraire libre, c'est-à-dire, au-dessous des Corolliflores de. DE CANDOLLE.

Or, nous n'arons obserré les placentoïdes que dans les Corolliflores. Done ces organes sont un attribut des plantes les plus élevées en organisation; seule conclusion que nous nous soyons proposé d'établir ici.

Pressé par le temps, je termine par quelques mots sur les points suivants:

Structure du connectif et du filet;

Causes de la déhiscence; 
Rapports entre la constitution anatomique des anthères et les groupes naturels.

10. Connectif. Le connectif contient.il des trachées? Ordinairement oui, rers sa base du moins. Exceptionnellement non (Calendula, Cosmos, Heliantlurs et beaucoup d'autres Synanthérées).

En dehors de la ligne axile occupée par les trachées et les fibres minces ou cellules allongées, le connectif est ordinairement formé de cellules parenchymateuses. Mais il n'est pas rare que celles-ci soient remplacées, en partie ou même en totalité (Iris, Breynia, Limum, Doryanthes, Tulipa, Aspidistra, etc.) par des cellules à filets. Ces cellules peuvent ne former qu'une ceinture sur les faces du connectif, comme si elles se continuaient des valves d'une loge à celles de la loge opposée en passant sur les faces dorsale et antérieure du connectif (Tellina, Saxifraga). Le Viola ne diffère que par l'interruption des cellules fibreuses sur la face postérieure du connectif.

Les cellules fibreuses n'ont pas été observées sur le connectif quand elles manquaient aux ralves (Ericacées, Mélastomées); l'inverse est commun.

20. Filets. Ont-ils toujours des trachées jusqu'à leur sommet? A. Richard l'affirme, mais à tort, comme on le voit dans le Cosmos, l'Helianthus, etc. Inutile d'ajouter que si les trachées ne vont pas jusqu'au sommet du filet, elles manquent tout à fait au connectif. C'est un caractère de ces organes que les cellules fibreuses ne s'étendent pas du connectif, quand elles y existent, dans les filets (Dorianthes).

30. Causes de la déhiscence. Loin d'admettre que la déhiscence soit due seulement au jeu des cellules fibreuses, nous lui attribuons six causes dont aucune n'est absolument générale, savoir:

a. La destruction des cloisons des logettes, parce qu'elle détermine le porte-à-faux des valves;

b. le décollement des bords suturaux des valves;

c. la destruction des tissus occupant la suture des valves;

d. l'action des cellules épidermiques;

e. l'action des cellules à filets ou fibreuses;

$f$. la combinaison des effets de la première et de la seconde membranes; peut-être aussi l'action des connectifs formés de cellules ì filets.

40. Rapports avec les groupes naturels. Purkinse a dit que la même forme de cellules fibreuses des valves se retroure dans un même groupe naturel. C'est généralement vrai.

Mais ce que Purkinje disait des valres seulement, il faut l'étendre i toutes les parties de l'anthère, aux cloisons, aux placentoïdes, au connectif, quant à leur composition histologique générale. 


\section{DES MATIL̀RES COLORANTES DES FEUILLES,}

PAK

M. J. E. BOMMER.

(Voir le Compte Rendu de la Séance du 8 Avril 1865, pag. 157).

La matière verte des feuilles désignée sous le nom de chlorophylle, terme le plus généralement admis, a déjà été l’objet de nom. breux travaux scientifiques. M. Hugo Monl a traité la partie anatomique de cet élément important de l'organisme végétal. Pelletier et Caventou, Clamor Marquart, Berzelius, Mulder, Scilleiden, Morot, Martens, Fueur, etc. en ont fait l'objet d'études sérieuses qui cependant n'ont pas encore élucidé complètement la question. Le dernier mot n'a pas été dit sur ce sujet, car plusieurs botanistes et nous-même sommes convaincus que les matières colorantes nommées par M. Frexr phyllocyonine et phyllowanthine ne constituent pas à elles seules la coloration des feuilles.

Nous avons répété très-consciencieusement les expériences de M. Fremy, et jamais il ne nous a été possible d'obtenir ce qu'il annonce comme étant immanquable. En parlant de la sorte, nous ne voulons pas dire que le résultat obtenu par le savant chimiste français soit inexact; nous avons pleine foi en sa véracité, mais nous nous permettons de croire, que M. Freury s'est limité à une ou plusieurs espèces végétales dont il ne cite pas le nom.

Pour arriver exactement aux mêmes résultats que M. Fremy, il eût été important de savoir sur quelle espèce de feuilles ce savint avait opéré, car nous sommes convaincus que le choix d'un régétal n'est pas indifférent pour réussir de prime abord.

Nos expériences se sont portées principalement sur l'Mlex aquifolium qui, par son feuillage d'un vert foncé, semblait devoir contenir une assez forte proportion de phyllocyanine.

Ainsi que nous l'avons dit plus haut, outre la matière verte, Leaucoup de végétaux contiennent d'autres matières colorantes. Il en est une, non apparente dans beaucoup de cas, qui se rencontre 
souvent. Elle consiste en une matière colorante brune soluble dans l'eau; nous la désignerons sous le nom de matière extractive, ou mieux encore sous celui de phyllophaioüne (poís, brun), parce que c'est à cette substance que nous devons la coloration de la plupart des extraits employés en pharmacie.

Dans certains cas, lorsque l'on a fait une dissolution de matière verte, elle offre souvent: 1\% une vive fluorescence d'une couleur rouge de sang; 20. une teinte brunâtre à la lumière solaire transmise; 30. une teinte rouge à la lumière artificielle. L'intensité de cette teinte est en proportion de la quantité de matière extractive contenue dans la matière verte. On peut la séparer facilement en la précipitant par l'anmoniaque; c'est du moins le procédé qui nous a fort bien réussi pour la matière verte des feuilles de l'Ilex aquifolium: traitée par une partie d'ammoniaque et deux parties d'éther sulfurique, elle s'est séparée en deux matières colorantes, l'une verte dissoute par l'éther et l'autre d'un beau brun en dissolution dans l'ammoniaque. Cette matière verte ainsi obtenue n'offre plus de fluorescence. En la soumettant 'à l'action de 1 partie d'acide chlorhydrique étendue d'une petite quantité d'eau et de 2 parties d'éther sulfurique, on obtient deux colorations bien distinctes: la première d'un beau jaune dissonte par l'éther; la seconde d'un vert bleuâtre pâle qui, lorsqu'on laisse reposer le liquide, produit à sa partie supérieure une zône d'un bleu brillant. La phyllophaioüne traitée par l'éther et l'acide chlorhydrique ne donne lieu à aucune réaction.

Ce qui précède, indique suffisamment que, pour obtenir de la matière colorante rerte pure, il est nécessaire de s'assurer avant tout, si les feuilles que l'on veut soumettre aux expériences, ne contiennent aucune matière colorante soluble dans l'cau et qui puisse être dissoute sans attaquer la matière verte.

En poussant nos investigations sur les plantes à feuillage coloré, nous sommes arrivés à des résultats complètement différents. Le Coleus Verschaffeltii offre, comme on le sait, un feuillage maculé de rouge foncé plus ou moins bleuâtre par places, et les parties non colorées sont d'un vert pâle jaunâtre. Ayant fait macérer dans un mélange d'alcool et d'éther (parties égales) des feuilles de cette plante, les parties colorées en rouge se décolorèrent complètement en peu de temps, et la dissolution prit une teinte verte beaucoup plus fonccie que celle que possédaient les feuilles à l'état frais. Il $y$ avait ici évidemment une transformation de la matière colorante rouge qui, devenue blene, avait renforcé la teinte première de la matière verte. Ce qui semble nous le prouver, c'est que nous arons remarqué à plusieurs reprises que par l'évaporation spontanée la 
solution prenait en partie une teinte blene riolacée. Cette dissolution de Coleus Verschaffeltii, soumise à l'action du réactif indiqué par II. Fremy pour décomposer la chlorophylle (1), a subi les modifications suivantes: 10. l'éther a pris une teinte d'un jaune clair; 2o l'acide chlorhydrique, au lieu de prendre une coloration totalement bleue, indiquée par le chimiste français, s'est coloré en un beau rouge groseille.

Après plusieurs essais, nous arons reconnu qu'au moyen d'une dissolution de sulfate de zinc on pouvait séparer les matières colorantes de la matière verte, surtout si elles appartiennent à la série cyanique. Nous avons donc ajouté à la dissolution type du Coleus une solution concentrée de ce sel; elle s'est emparée d'une matière colorante bleue tirant sur l'indigo; la matière verte, par suite de cette réaction, a légèrement jauni et a pris une teinte analogue à celle de la couleur verte normale de la plante. En ajoutant à la solution précédente une très-faible quantité d'acide chlorhydrique ( 2 ou 3 gouttes) la matière colorante bleue est passée à un rouge brillant; tandis que la matière verte a pris une teinte $d^{\prime} u n$ jaune vif.

Il nous semble que M. Fremr á eu tort de généraliser l'action de l'acide chlorhydrique comme réactif de la phrllocyanine; nous venons d'avoir une preuve du contraire en l'employant avec la plus rigoureuse exactitude pour la chlorophylle du Coleus Verschaffeltii. En variant la dose de cet acide depuis les plus fortes quantités jusqu'aux plus mimines, nous avons toujours en le même résultat, si ce n'est une coloration plus pâle ou plus foncée.

Des expériences récentes ont été faites par M. G. G. Stokes sur le moyen de distinguer les substances organiques par leurs propriétés optiques (2). Voici ce que ce sarant dit au sujet des expériences de M. FREMY:

„Des solutions de chlorophylle dans des dissolvants neutres. tels que l'alcool, l'éther etc., présentent une rive fluorescence d'une couleur rouge de sang; et quand- la solution est examinée à la lumière du spectre, la fluorescence rouge, très-abondante dans les parties rouges du spectre, est comparativement faible dans la plus grande partie du vert, et se retrouve de nouveau très-vive dans le bleu et le violet. Or, une substance d'une simple couleur jaune, qui par conséquent exercerait une absorption sur les rayons plus réfrangibles,

(1) Penouze et Frnsr, Traité de Chimie générale, analytique, industrielle et agricole, Paris, 1860 , T. IV, p. 807.

(2) Journal de Pharmacie et de Chimie, Paris 1865, T. ler, p. 297. 
ne montrerait pas une simple fluorescence rouge. Ou bien elle ne serait pas fluorescente, ou bien la fluorescence de sa solution contiendrait, comme l'expérience le prouve, des rayons de réfrangibilité appartenant à la partie du spectre où commence la fluorescence, et par conséquent l'absorption; la lumière fluorescente ne serait donc pas simplement rouge comme celle de la chlorophylle, qui se montre même dans le bleu et le violet. La substance jaune séparée par M. Frewy, à l'aide de réactif's neutres, est en réalité non fluorescente. Il suit de là que la forte furorescence rouge dans le bleu et le violet ne peut être attribuée qu'à la substance qui exerce une puissante absorption dans le rouge et qui absorbe fortement aussi le bleu et le violet. Nous pouvons donc affirmer a priori que si cette substance était isolée, elle ne serait pas bleue, mais seulement d'un vert un. peu blenâtre. La solution bleue obtenue par M. Fremy doit en réalité sa couleur à un produit de décomposition qui n'est pas du tout bleu quand il est dissons dans des réactifs neutres, mais d'une teinte à-peu-près neutre, qui montre dans son spectre des raies d'absorption extrêmement prononcées."

La citation du passage ci-dessus nous a semblé utile, vu son opportunité pour la question qui nous occupe. Sans vouloir le commenter, nous devons cependant faire remarquer que M. STокеs a opéré sur de la chlorophylle qui contenait beaucoup de matière ex. tractive ou phyllophrioïne, laquelle n'aurait peut-être pas été sans influence sur le résultat obtenu. Il serait à désirer que ce savant opérât de nouveau sur une solution de matière colorante verte, pure de tout mélange.

La seule appréciation que nous nous permettrons sur la conclusion de M. SToKes, c'est. qu'elle tendrait à prouver la non-existence de la phyllocyanine de M. Frewr.

Nier l'existence de deux éléments constitutifs de la matière colorante verte nous semble impossible. Sans avoir pu le prouver, nous sommes convaincus qu'une matière colorante jaune et une autre bleue doivent former la matière verte des feuilles; mais pour arriver à décomposer celle-ci, nous croyons qu'il faudrait trouver un agent moins énergique que l'acide chlorhydrique, qui doit nécessairement modifier profondément la substance organique avec laquelle on le met en contact. Ensuite, nous ferons encore remarquer la grande altérabilité des solutions de chlorophylle exposées à la lumière solaire où elles deviennent jaunâtres; ce qui indique la disparition d'un principe colorant qui nécessairement doit appartenir à la série cyanique, puisque des deux couleurs qui forment le vert il ne reste que le jaune, c'est-à-dire la couleur la moins alté- 
rable dans tous les végétaux. Par conséquent la phyllocyanine existe.

Puisque nous admettons que la matière verte des feuilles est due au mélange de deux matières colorantes la phyllozanthine et la phyllocyanine, il faut nécessairement que cette matière complexe reçoive un nom qui la définisse mieux; nous croyons que le terme xanthocyane sera le plus convenable.

Certains gaz réducteurs tels que l'hydrogène ne font subir qu'une faible altération aux solutions de wanthocyane exposées aux rayons solaires; tandis que celles soumises à l'influence d'une atmosphère d'oxygène, et placées dans les mêmes conditions subisscnt rapidement une altération complète.

Pour arriver à obtenir de la phyllocyanine pure, il est, croyons nous, nécessaire d'opérer dans des conditions où la lumière ne puisse en aucune manière produire la décomposition de ce principe colorant qui est d'une altérabilité très-grande.

Au moyen des dissolvants neutres tels que le chloroforme, le sulfure de carbone, la benzine, le naphte, nous ne sommes parvenus qu'à extraire une matière colorante jaune; l'huile de pétrole ne dissont qu'une très-faible quantité de matière verte. De tous les dissolvants, l'éther sulfurique et l'alcool nous ont semblé les préférables. Dans la plupart des cas, nous avons employé ces deux liquides en parties égales, leur action simultanée étant plus énergique. Pour certaines espèces, l'alcool rend les dissolutions de xanthooyane très-foncées, mais il ne la dissout pas complètement; il semble porter principalement son action sur la phyllocyanine, tandis que les dissolutions dans l'éther sulfurique sont alors très-pâles, et ce dernier paraît agir surtout sur la phylloxantline.

Nous arons omis de mentionner la phyllowanthéine, matière colorante jaune soluble dans l'eau, dans l'alcool et dans l'éther, jaumissant fortement par l'action des alcalis et se décolorant par l'acide chlorhydrique. On peut séparer facilement la phyllaxanthéine de la xanthocyane en traitant cette dernière par un excès d'eau distillée.

Il nous reste encore à signaler une coloration due à une altération de la phyllophaioüne. Ce fait ne nous est pas personnel, nous le devons à un savant franęais, M. Chatis qui, lui aussi, a constaté dans les feuilles jaunies du Maronnier (Aesculus Hippocastanum), la présence d'une matière colorante brune mêlée à la xanthocyane et dont l'altération lui semble produire la coloration rouge qui pare les feuilles jaunies de certaines plantes en automne. Cela est parfaitement exact; nous arons pu le vérifier sur des feuilles autumno-hiver- 
nales du Ligustrum vulgare. Ces feuilles étaient fortement colorées en rouge vineux souvent sur les denx faces, mais tonjours d'une manière plus prononcée sur la partie supérieure du limbe. La lumière a dû intervenir dans la production de cette coloration, car bon nombre de feuilles recourertes jartiellement par des feuilles entièrement colorées, étaient rougies sur les places exposées au jour et vertes aux endroits soustraits à la lumière. Une certaine quantité de ces feuilles ont été soumises à une macération dans un mélange en parties égales d'alcool et d'ether, qui nous a donné une belle solution de anthocyane, offrant une fluorescence d'un rouge de sang qui, comme nous l'avons dit plus haut, indique la présence de la phyllophaioüne. Après une assez longue macération, les feuilles, quoique decolorées en grande partie, offraient encore une teinte rougeâtre très-prononcée. Voulant nous assurer de quelle nature était cette coloration, nous broyâmes une partie de ces feuilles avec de l'eau distillée qui se colora en un brun rougeâtre foncé. Cette matière colorante fut presque insensible à l'action de l'ammoniaque et de l'acide chlorhydrique, c'est ce que nous avons déjà constaté pour la phylloplacioüne provenant de l'Ilex aquifolium. La coloration rouge printanière qui se produit sur les jeunes pousses de plusieurs genres de plantes tels que Rosa, Rhus, Quercus, etc., nous semble avoir la même origine. Du moins, les solutions que nous avons faites de ces parties colorées nous ont donné un résultat analogue à celui obtenu avec le Ligustrum vulgare. C'est aussi à une forte proportion de pluyllophaioüne qu'est due en partie la coloration du Fugus jerileyilere.

Certaines fenilles totalement rouges paraissent au premier aspect dépourrues de ranthocyane. Parmi les plantes qui offrent une coloration semblable nous citerons quelques Amaranthus, l'Achyrontes Verschuffeltii, le Perilla nankinensis, le Dracaenu ferrea etc. Si l'on fait une solution alcoolique ou éthérée de l'une de ces espèces, de l'Achyranthes Verschaffeltii par exemple, l'on obtient une matière colorante verte très-foncée et une substance colorante rouge, soluble dans l'eau, très-sensible à l'action des alcalis et à celle des acides.

Cette matière colorante rouge est répandue dans beaucoup de végétaux chez lesquels elle accompagne la teinte verte des feuilles, comme cela se voit pour les Begonia. Dans les feuilles les plus intérieures du chou rouge (Brassica olerueca vubra) elle constitue à elle seule la coloration de ces parties.

On avait primitivement désigné la coloration rouge des feuilles comme étant produite par une seule matière colorante à laquelle on 
avaiu donné le nom d'érythropluylle. Ainsi que l'a déjà fait remarquer M. Martens (1) c'est à tort qu'on a confondu les deux espèces de rouge qui existent dans les feuilles ou dans les fleurs.

La matière colorante rouge des feuilles est due à deux substan. ces colorantes de nature bien différente: 1. à la phylloxanthéine et à la phyllophaioine qui se colorent en rouge par l'action de la lumière et de l'oxygène. A cette matière colorante rouge d'origine xanthique nous donnons le nom de anthérythrine. Elle se produit surtout dans les jeunes pousses des végétaux au printemps et dans les vieilles feuilles à l'automne. 2. La phyllocyanine, altérée par la présence d'un acide, donne lieu à une coloration rouge qui se rencontre dans les feuilles normales de beaucoup de végétaux. Nous désignons cette seconde matière colorante rouge sous le nom de cyanérythrine; son origine cyanique indiquant suffisamment le terme qui lui convient.

De l'ensemble des faits qui précèdent nous concluons:

1. Que le terme de chlorophylle, trop général, est devenu impropre pour désigner les matières colorantes contenues dans les feuilles.

2. Que toutes les colorations des feuilles ont pour origine deux matières colorantes types dont elles dérivent; ce sont la playlloxanthine et la plhyllocyanine qui, par leur mélange, forment la matière verte ou wanthocyane.

3. Qu'à la xantluocyane sont sourent mêlées d'autres matières colorantes, et que pour être pure, elle ne peut contenir que les deux principes colorants qui la constituent.

4. Que les matières colorantes mêlées à la xanthocyane sont de deux sortes: l'une jaune ou pluylloxanthéine, soluble dans l'eau, dans l'alcool et légèrement soluble dans l'éther', jaunissant fortement par les alcalis et se décolorant par l'acide chlorhydrique; l'autre brune ou phyllophaioüne, soluble dans l'eau, légèrement soluble dans l'alcool et insoluble dans l'éther', brunissant par l'action des alcalis et se décolorant légèrement sous l'influence de l'acide chlorhydrique.

5. Que la matière colorante rouge désignée autrefois sous le nom d'érythrophylle dérive: 1 . de la décomposition de la phylloxanthéine et aussi de celle de la phyllophaioüre, ce qui donne lieu à la formation du ronge xanthique ou duntherythine; 2. de l'altération de la phyllocyanine, ce qui produit le rouge cyanique ou cyanérythrine. Que si l'on voulait donner une désignation générale à la coloration

(1) Martens, Recherches sur les couleurs cles végétaux. Bulletin de l'Académie royale de Belgique, 'I. $\mathrm{XX}$, lre partic, 1853. 
rouge des fenilles, on devrait confondre sous celle de pluylloérythrine le rouge xanthique et le rouge cyanique.

6. Que si l'on admet que la fleur soit le résultat de modifications de la feuille, on doit admettre aussi que les matières colorantes des feuilles sont, à part quelques modifications, les mêmes que celles qui concourent à la formation des couleurs des fleurs.

\section{ERRATUM.}

Nous avions annoncé que la phyllophaioüne ne se modifiait pas sensiblement par l'action des alcalis et de l'acide chlorhydrique. De nouvelles expériences entreprises sur l'Tlew aquifolium, le Ligustrum vulgare, le Thea vividis, le Thé noir du commerce, le Camellia japonica, le Coffea arabica, le Rhododendron ponticum, etc., nous ont prouré, que la pluyllopluaioine extraite de ces plantes brunissait plus ou moins par l'action de l'ammoniaque et se décolorait sensiblement par l'acide chlorhydrique.

\section{LA LUNE ET LE RÈGNE VÉGÉTAL,}

PAR

\section{NARDY.}

(Voir le Compte Rendu de la Séance du 8 Avril 1865, pag. 158).

Les phases de notre satellite exercent-elles ou non une influence quelconque sur le règne végétal? Telle est, Messieurs, la question que je me suis bien souvent faite. Bien d'autres que moi ont dû se la faire, et, comme je l'ai fait souvent, la poser à diverses personnes supposées capables de résoudre cette question difficile, ou du moins de jeter quelque jour sur l'obscurité dont elle est environnée. Je doute toutefois que, plus heureux que moi, quelqu'un ait jusqu'à ce jour recueilli sur elle des données claires et sûres. 'Il serait pourtant très-utile pour la culture en général d'acquérir ces données, qui bien établies, donneraient le seul moyen d'apprécier à leur valeur deux opinions très-contradictoires. Suivant l'une, que soutient quelque peu la science, les phases de la lune n'exercent aucune influence sur le règne végétal, ou du moins n'en exercent que 
très-peu. L'autre est soutenue par la plupart des cultivateurs, mais ils ne peuvent pent-être l'asseoir que sur une routine longuement enracinée. Les phases de notre satellite, prétendent-ils, exercent une influence très-importante sur les végétaux, cette influence consisterait surtout à donner ou à retirer aux plantes, aux arbres, certaines propriétés, certaines facultés, selon les quartiers de la lune où s'opèrent le semis, la plantation, la coupe, etc. de ces végétaux. Qui a raison? J'aime et je respecte trop la science, sans laquelle la pratique ne serait qu'une aveugle routinière, pour lui adresser des reproches, quand elle repousserait même pour le moment du moins l'idée de toute influence lunaire sur le règne végétal. Mais sur quelles preuves cette opinion serait-elle fondée? Le savant Arago, parlant de la lune rousse, a défini et expliqué le roussissage qui souvent au printemps atteint les jeunes pousses des végétaux; ses définitions et explications à la portée de toutes les intelligences nous prouvent que le roussissage, dont on accuse la lune innocente, est dû au rayonnement nocturne de la chaleur du sol reers les régions élevées de l'atmosphère; rayonnement qui, comme on sait, n'a pas lieu si le ciel est couvert, mais est d'autant plus ì craindre que le ciel est plus serein. La lune n'est point cause du roussissage, dit Arago, puisque, fût-elle à son plein, le roussissage, les gelées blanches désistreuses d'avril-mai, n'ont point lieu si le temps est couvert, mais seulement si le ciel est serein, et que la lune paraisse ou non au firmament. L'explication d'Arago est admise par la science; je voudrais qu'elle le fût également par les praticiens. Mais si la science a fait dans ce cas reconnaitre l'innocence de la lune, s'est-elle jusqu'à ce jour sérieusement occupée d'étudier la valeur des assertions des cultivateurs sur l'influence des phases du satellite sur les végétuux? S'en fût-elle même bien occupée, la nature peut-être a pu lui refuser ses secrets, qu'elle ne dispense que peu à peu, et souvent, pour certains cas surtout, alors seulement que la science et la pratique agissent ensemble et de concert. Dans le camp des cultivateurs, l'opinion de l'influence est-elle assise sur des preuves que la pratique ait domnées? C'est possible. Mais où sont consignées ces preuves, et qui peut les citer? Et sans elles pourtant, comment établir sûrement, comment faire adopter l'opinion?

$\mathrm{Au}$ risque de passer pour un lunatique, je me permets bien d'entrevoir peut-être un appuii à la véracité de l'opinion dans la concordance avec laquelle on la rencontre professée partout dans la classe des cultivateurs. Tous aussi prétendent tenir cette opinion de leurs aieux. Ne pourrait-on pas penser que cette croyance générale ait dû aroir une raison de se produire? Si aussi nous nous reportions 
au règne animal, ne trouverions-nous pas que la science et la pratique paraissent reconnaitre quelques cas d'influence exercée par les phases de notre sateliite? La vue du cheval lunatique est plus fatiguée au retour d'une des phases de la lune. Et certaines maladies, certaines affections de notre humanité, les affections vermineuses de l'enfance par exemple, ne sont-elles point, selon le sentiment commun et de l'aveu même de quelques médecins, ainsi que j'ai pu m'en assurer, plus accentuées souvent au retour de certaines phases de la lune? Si l'influence lunaire sur le règne animal est reconnue ou soupçonnée dans certains cas, pourquoi n'existerait-elle point aussi sur le règne végétal, sur lequel même elle paraitrait devoir être plus puissante, les êtres de ce règne vivant toujours en plein air.

Mais ce ne sont là que des inductions sans valeur peut-être, en faveur de l'opinion de l'influence lunaire sur le règne végétal. Je le repète, il faut des preuves, il faut des faits. Ne pourrait-on, Messieurs, y avoir recours? Que ceux qui prétendent par exemple, que les haricots plantés en lune ascendante, ou du moins mis en terre en lune ascendante, assez tôt pour pouvoir entrer bien en germination avant le plein de la lune, que ceux qui prétendent, dis-je, que ces haricots donneront une production successive plus prolongée pour la récolte en vert que ceux au contraire plantés en lune déclinante, veuillent bien faire des essais comparatifs suivis pendant plusieurs années avec soin. Que ceux pour l'influence, et ceux contre l'influence fassent sérieusement, sans idée préconçue, et dans le but utile de découvrir la vérité, de pareils essais comparatifs, chacuns dans leurs cultures diverses, sur les semis, les plantations, etc. Ces essais peuvent seuls éclairer la question. Seulement, soyons patients, observons exactement, consignons de même; unissons aussi dans nos recherches, dans nos observations, la science ì la pratique, et, Dieu aidant, nous atteindrons le but que je voudrais จoir poursuivre.

Si le résultat des recherches était négatif pour l'influence, on pourrait alors faire en connaissance de cause une guerre fructueuse et fondée à une opinion reconnue n'avoir d'autre base que la routine. $\mathrm{Si}$, au contraire, le résultat était affirmatif, ne fût-ce que dans certains cas, profitant de ce point de départ, science et pratique poursuivraient de concert leurs recherches dont la culture en général pourrait avoir à profiter.

C'est là, Messieurs, le double but vers lequel je me suis proposé d'attirer votre attention en écrivant ces lignes. Je serai heureux, si elles réussissent à provoquer des essais comparatifs utiles et intéressants. 


\section{UEBER GICHT ODER DAS SOGENANNTE GICHTIG- ODER RADIGWERDEN (NIELLE) DES WEIZENS UND ANDERER GRASFRÜCHTE,}

vox

\section{Dr. JULIUS MÜNTER.}

(Voir le Compte Rendu de la Séance du 10 Avril 1865, pag. 208).

Nach einer in der Zeitschrift „Die Mühle” (1) enthaltenen, aus der Leipziger illustrirten Zeitung entnommenen Notiz, hat man im Sommer $1864 \mathrm{im}$ preuss. Osterlande Weizenkörner gefunden, welche nux $\frac{1}{2}$ mal so gross waren, als gesunde Körner, bei denen die Längsfurche verschwunden und deren Obertläche schwarzbraun gefärbt erschien. während das Innere derselben aus kaum erkennbaren Kileinen Wiumern bestand, (d. h. also Anguillulce enthielten) und folglich, wie man sagt, Ton der" „Gicht” befallen waren.

Einer im „Wochenblatte (2) der Annalen der Landwirthschaft in den königl. preuss. Staaten" befindlichen Mittheilung zufolge, sah Dr. Antox Schnemer in Berlin die .,Gichtkrankheit” in Salsitz (Kreis Zeitz), wo man zuror niemals dergleichen bemerkt hatte. Auf einem Magdeb. Morgen zeigten sich $\frac{3}{4}$ gesunde und $\frac{1}{t}$ von der Gicht befallene Aehren des Kolbentreizens; seltner kam die Krankheit beim Bartweizen ror. Der Beobachter, welcher die ron ihm nicht näher benannte "Nematode" als das veranlassende Moment zur Erzeugung der Gichtkrankheit ansieht, ist, nach dem von ihm am 18 October 1864 in der Gesellschaft naturforschender Freunde in Berlin gehaltenen Vortrage, der Ansicht, dass jene Würmer während der Blüthezeit in die jungen Aehren eingedrungen sein dürften, und er glaubt nicht, dass das Beizen der gichtkranken Körner dits Uebel zu beseitigen im Stande sei.

Aus einem kurzen Berichte, welcher sich im landwirthschaftlichen Centralblatte (3) findet, geht herror, dass der rühmlichst bekannte Entomolog, Dir. Dr. Löw in Meseritz, dieselbe Krankheit schon seit Jahren in der preuss. Provinz Posen beobachtet hat,

(1) Die Irühle, 1864. No. 42 , p. 176.

(2) Wochenblatt, 186t. No. 50, p. 447 .

(3) AD. Wilda und ANtox Krocker, Landzirthschaftl. Centralblatt für Deutsch. and. Berlin 1865, Januarheft, p. 54 und Marrzheft No. 3, p. 227. 
während Prof. Dr. Küıs (1) in Halle a./S. berichtet, dass unter dem Namen , Kinulbrand" die angegebenen Krankbeitserscheinungen in der Umgegend ron Mühlherg an der Elbe seit Menschengedenken bekannt sind, so dass bei der Stadt Strehla (Königr. Sachsen) bis nach Witteriberg (Pror. Sachsen) hin, namentlich zwischen Dommitzsch und Pretsch, der Kaulbrand so intensiv auftritt, dass nach Aussage dortiger Getreidehändler oft $\frac{1}{4}$ des geernteten Weizens , raden- oder kaulkrank" sein soll.

Insbesondere will man nach der ton Dr. TAschenberg (2) bekannt gemachten Mittheilung hemerkt haben, dass auf einem Land. gute (Köttlitz bei Mühlberg, Pror. Sachsen) sich so lange keine Spur der Krankheit zeigte, als man den gewöhnlichen braunen Weizen baute, dass aber sofort nach Einfübrung des sogenannten , weissen Weizens" schon bei dessen erster und zweiter Ernte die Krankheit erschien, während der braune Weizen bis dahin noch immer verschont blieb. Erst nach gänzlicher Abschaffung des braunen Weizens zeigte sich der nun allein angebante weisse Weizen überall krank. Man sah sich genöthigt, den weissen Weizen nunmehr gänzlich abzuschaffen und zum braunen Weizen zurückzukehren, weil dieser im Beginne der Krankheit verschont geblieben war; allein indem dieser nun auf Schläge kam, wo zuvor kranker Weizen gestanden hatte, wurde auch dieser afficirt.

Indem Dr. Taschexberg (1. c.) dieses bisher nicht bekannte Auftreten der "Gicht" oder Radenkrankheit veröffentlicht, erörtert er jag. 215 die durch Anguillula Dijsuci Kürx herbeigeführte Krankheit der IVeberkarde (Dipsurns Fullun!m L.), auf welche Kürs (3) zuerst aufmerksam machte; ferner auf pag. 217 die ron Davarne (4) und später von Kürrs (1. c.) ausführlicher beschriebene Anguillulı Tritici (Vibrio Tritici RofFredi) (5) und endlich die von Schacht (6) zuerst aufgefundene und ausführlicher beschriebene Rüben-Nematode, oder Rüben-Trichine genannt, welche sowohl in der Provinz Sachsen bei

(1) Zeitschrift des Central-Vereins der Proriñ Sachsen 1865, No. 1, und Schlesische landwirthschaftl. Zeitung, Jahrgang VI, 1865, No. 3 und $\$$ (19 und 26 Januar).

(2) Naturgeschichte der wirbellosen Thiere, die in Dentschland elc. den Feld-, Wiesen- und Weide-Culturpflanzen schädlich werden. Leipzig, 1865, So., Gekrünte Preisschrift.

(3) Kürn, Die Krankheiten der Culturgewächse. Berlin, 1858, p. 178, und v. Siebold und KöLliker, Zeitschrift für wissenschaftliche Zoologie. Bd. IX. Tab. VII, C.

(4) Comptes rendus de l'acad. des'sciences, 1855, p. 435-438 und 1856, 21 Juli.

(5) RozIER, Observations sur la physique, Tab. II, fig. 1, 2.

(6) Zeitschrift des Vereins für. Rübenzucker-Industrie, 1859, IX, p. 177-178 und 240. Taf. III, fig. 5-16. 
Stassfurth, als auch in der Prorinz Brandenburg im Oderbruche und in der Provinz Schlesien an den feinen Wurzelfasern der Zuckerrübe lebt. Auch einer Anguillula Phalaridis bei Phleum Böhmeri gedenkt Taschenberg 1'. c. pag. 282, und rindicirt deren Auffindung dem Prof. Kürs, während dieser selbst und mit vollem Rechte (1. c. pag. 183) deren Entdeckung Sterniuch (1) zuschreibt, welcher ausserdem an einem "Agrostis sylvatica" genannten Grase (Agrostis stolonifera diffusa Nitr.) eine Anguillula (Vibrio) Agrostidis aufland.

Eine Reihe ron Jahren vor diesen neueren Beobachtungen kam der berïhmte Wiener Helmintholog Dresing in seinem Systema Helminthum (2) nach sorgfältiger Prüfung der yos Gleichex (3), Dugès (4), Dujardin (5), Hexslow (6), Bauer (7), so wie von NeedнАл (8), beschriebenen und abgebildeten Anguillulue zu der Ueberzeugung, dass dieselben auf eine einzige Species zurückzuführen seien, welche er mit dem Namen: Anguillula graminearum Dies. belegte. Gleichzeitig freilich läugnet Diesing die von BAKER entdeckte und in dessen Beiträgen zum nützlichen und vergnügenden Gebrauche und zur Verbesserung des Mikroskopes (2 Voll. 8․, Augsburg 1754, Vol. II, Cap. 4, p. 327) beschriebene Reviviscenz mehrere Jahre lang trocken aufbewahrter, anscheinend todter Thiere, welche doch durch LEDERyïLLER (9) unzweifelhaft bestätigt worden war, indem er jene angeblichen actiren Bewegungen für blosse Molecularbewegungen ansah. Endlich bemerkt Diesing, dass der primitive Wohnort jener Anguillulae unbekannt sei; man wisse nur, dass sie sich fänden ,,in seminibus Tritici Uredine depravatis," wo sie NEEDHAM, BAUER und HeNsLow gefunden hätten, ausserdem fänden sie sich auch ,,in semine depravato Agrostidis sylvaticae” wo sie STEINBUCH zuerst erkannt habe.

Sind, wie weiter unten bewiesen werden soll, Diesing's Zwieifel in Betreff des Wiederbelebungsrermögens parasitisch-lebender A grillulae, schon nicht begründet und mithin unberechtigt, so bestätigt sich dessen Amnahme jedenfalls nicht, wonach die Amyuillulau, „,in seminibus Tritici Uredine depravatis" rorkommen sollen. Das

(1) Der Naturforscher. Berlin, Stück XXVIII, p. 241 und 257 , tah. V, fig. 1-7.

(2) Systema Helminthum. Vindeb. 1851, 80., Vol. II, p. 132.

(3) Micrographic, p. 61, Tab. XXVIII, 6.

(4) Annal. d. sc.nat., Sér. I, Tom. IX, p. 225.

(5) Histoire naturelle des Helminthes, 242.

(6) Microscopical Journal, 1841, p. 36.

(7) Phil. Transact. 1823, Tom. I, p. 1-12, Tib. I, II und Amales des scienc.nat. Súr. I, Tom. 1I, p. 154-167.

(8) Nouvelles déconvertes faites avec le Microscope, 17.77, Chap. VIII, p. 99.

(9) Amusement microscopique, Nürnberg, 1764, 40., pag. 41 et suiv. 
ron Neediaj, Ledermüller und in der neueren Zeit ron Davaine zur Bezoichnung der ron deutschen Gelehrten ,Gicht” oder ,Radenkrankheit” benannten Krankheit ,Niclle" hat allerdings im Deutschen die Bedeutung: „Flugbrand” und würde, wörtlich genommen, durch Ustilago segetum Fr. zu übersetzen sein; allein es beruht auf einer irrigen Voraussetzung, wenn Dresing behauptet, dass Weizenfrüchte oder überhaupt Grasfrüchte, welche von Anguillulis parasitisch bewohnt werden, zuror durch Ustilago, oder Tilletia caries TuL., mit welcher sie noch grössere Aehnlichkeit besitzen, zerstört oder krank gemacht worden seien. NeEDHaM gebraucht in der franz. Uebersetzung LEDERMüLLER's das Wort „Gangraine" synonym mit „Nielle" und äussert sich dahin, dass man darunter eine Krankheit des Weizens izu verstehen habe, bei welcher das Stärkemehl durch eine von Aussen eingedrungene Substainz verdrängt und ersetzt werde. Diese Substanz erscheine entweder: in Form eines schwarzen Pulvers, oder: in Form eines aus langen Fasern zusammengesetzten weissen Körpers (substance blanche), der, mit Wasser angefeuchtet, sich belebt zeige. Demnach ist "Nielle" nicht, wie es Diesing that, mit "Uredo" zu übersetzen, denn Needham selbst unterscheidet, wenn ich mich des Ausdrucks bedienen darf, veissen und schwarzen Brand; versteht unter dem weissen Brande den Zustand der Weizenfrucht, in welchem sie von parasitischen Anguillulis bewohnt wird und unter schwarzem Brande den Zustand, wo das Früchtchen Brandpilz-Sporen enthält. LEDERMüLLer theilt offenbar diese NeEnhar'sche wohl begründete Ansicht; denn auch ihm war das Vorkommen von Anguillulis aus Autopsie bekannt und weiss er auch, dass diese beim Mahlprocesse nicht zerstört werden, sondern in das Mehl übergingen und folgerecht sich dann im Sauerteige und im Kleister. (Cole, wie er schreibt, oder Colle, wie man jetzt schreibt) fänden: eine Ansicht, die von neuern Zoologen freilich nicht getheilt wird.

Aus dem Vorstehenden erhellt nun aber unwiderleglich, dass seit der Mitte des rorigen Jahrhunderts, sowohl in England, als auch in Frankreich und Deutschland mikroskopisch kleine Thiere aus der Klasse der Nematelminthes Gegenb. und zwar aus dem Genus: Anguillula Hempr, und Ehrenb. (Vibrio O. F. MüцL, Rhabditis DuJ.) in Weizenfrüchten rorkommen und sich nicht allzuselten sogar üher ganze Ackerflächen verbreitet finden; dass ebeufalls Anguillutce in den Früchten von Phleum Böhmeri WIB. und Agrostis stolonifera diffusa Neilr., ferner in den Blüthenständen von Dipsacus Fullonum L. und endlich eine verwandte Nematode an den feinern Wurzeln der cultivirten Zuckerrübe leben. 
An diese Thatsachen anknïpfend, glaubte ich es für zeitgemäss halten zu müssen, hier einige eignen ,Beobachtungen über das VorKommen von Anguillulis in Grasfriichtchen" mitzutheilen, um namentlich einige streitige Punkte zu erledigen, die bisher noch immer nicht ihre völlige Lösung gefunden haben.

Bei Gelegenheit einiger mykologischen Excursionen nach Mönchgut auf der Insel Rügen, so wie in den Demminer Kreis an der Mecklenburger Grenze in Neu- und Vorpommern im Anfange Juli fand ich Aehren von Phleum Böhmeri Wrв. und Koeleria glarec DC., welche durch eine beträchtliche schnabelförmige Verlängerung einzelner Aehrchen (spiculae) sich schon ron Weitem bemerkbar machten. In der Voluussetzung die gefundene Difformität als durch parasitische Fungi herbeigeführt, deuten zu können, eröffnete ich die um das dreifache verlängerten Paleno vorsichtig, fand aber weder Lodiculae, noch Stamina, noch Stigmata plumosa an dem wesentlich vergrösserten Ovarium, welches sich mit einem wenig rerlängerten Stylodium rersehen und dunkel-riolett gefärbt zeigte. Nach Entfernung der Paleae und rölliger Isolirung des Ovariums mittelst Nadeln unter einer Statis-Präparirloupe, wurde die Oberhaut des Ovariums der Länge nach aufgespalten und der Inhalt durch einen sanften Druck herausgepresst. Mit Wasser befeuchtet und unter dem Mikroskope bei 350-maliger Vergrösserung untersucht, zeigten sich zahlreiche Körper so eigenthümlich nach Gestalt und Inhalt, dass es unmöglich war, dieselben auf Sporenähnliche Bildungen zurückzuführen. Alle Körperchen waren völlig isolirt, ron länglich-riereckiger Gestalt, jedoch mit abgerundeten Ecken, scharf umschrieben, glashell durchsichtig und mit einem das Innere der Hülle unrollkommen ausfüllenden granulösen Inhalte erfüllt. Andere Körperchen zeigten ihren Inhalt wurstförmig gestaltet, theilweise mit etwas rerwaschenen Grenzlinien, theilweise aber auch schärfer contourirt. Bei noch andern Körperchen war die länglich-viereckige glashelle Hüllhaut ron dem sich heraus-windenden Inhalte weniger oder mehr entleert, und ein aalförmig-gestalteter sehr durchsichtiger Thierleib, welcher sich als eine "Anguillule" documentirte, bewegte sich in der bekannten peitschenförmigen Weise, bald mit Kopf und Schwanz-Ende nach links, bald nach rechts schlagend, oder schlängelnd sich auf und ab bewegend.

Bei den in der Entwickelung etwas vorgeschritteneren Individuen dieser lebhaft agitirenden Thierchen erkannte man unschmer die Anlage des Mundes, eine den Oesophagus andeutende helle Linie ron der Mundanlage zum schwach angedenteten Muskelmagen hin sich erstreckend, während in dem ïbrigen langgestreckten Leibe 
nur feine Granula, noch unverwendete Dotterzellen (?) zu erkennen waren. Vom Munde ab bis nahe zum Schwanz-Ende hin, war der Querdurchmesser der Thierchen wenig verschieden; allein nach dem entgegengesetzten Körperende hin verjüngte sich der a al-ołer schlangenartige Leib; allmählig zwar, doch so, dass der Schwanz sich in ungemein fein-zugespitzter Weise endete.

Berechtigten diese Befunde zu dem Schlusse, dass die vorliegenden Körper theils ans Eier'n mit uncntwickelten oder entwickelten Embryonen, theils aus jüngern, der Gattung , Anguillula" Hempr. und Eurexs. angehörenden Thieren beständen, so entstand natürlich auch der TVunsch, zu ermitteln, von wo diese zahllosen Gebilde ausgegangen und in die auf entschieden embryonaler Entwicklungsstufe stehen-gebliebenen Grasovarien eingedrungen sein köunten.

Wiederholtes Oeffnen und Durchsuchen anderer violettgefürbter Ovarien ergab die Ausgangsquelle der Eier und Thierchen zur Genüge; bald fand sich nur ein, bald ein doppeltes Elternpaar mit grössester Evidenz; niemals fehlten die gepaarten Erzeuger, niemals fanden sich aber auch drei Pärchen.

Das Münnchen, rielmal grösser und dicker als die grössesten jungen Thiere, gab sich durch das in der Nähe der Schwanzspitze, olngefähn $\frac{1}{s}$ der Körperlänge vor derselben befindliche stiftförmige Copulationsorgan deutlich genug zu erkennen. Das Weibchen aber übertraf das Männchen um das Doppelte, wenigstens in Betreff der Dicke, wäbrend es auch in Betreff der Länge das Männchen nicht unerheblich überragte. Das weibliche Zeugungsorgan öffnete sich so wie beim Männchen in der Nähe des zugespitzten Körper-Endes, olngefähr vor dem letzten Neuntel der Gesammtkörperlänge. Beim Männchen und Weibchen liess sich die Mundöffnung, der Oesophagus und der Darm neben den innern keimbereitenden Organen rollkommen klar erkennen. Die Männchen waren sehr lebhaft in ihren Bewegungen, die Weibchen viel träger, auch ihre Hant sehr weich und offenbar in Folge der sehr bedeutenden Ausdehnung, welche die zahllos entwickelten Eier herbeigeführt hatten, schlaffer als beim Männchen.

Konnten otwa Angesichts der geschlechtslosen Thiere noch Zweifel, über die Stellung derselben im System bestehen, so wiesen die geschlechtsreifen Thiere unzweifelhaft darauf hin, 1. dass es sich sowohl bei Phleum Böhmeri WIB., als auch bei Koclevia glauca DC. um das Genus Anguillnlu. Hexpr. und EnBG. handle, welehes offenbar ron Sternbuch (l. c.) bei Phleum Böhmeri DC. wenigstens, ebenfalls gekamnt und gefunden worden war; 2 ., dass Diestra, welchem die Stennucu'sche Alt bereits bekaunt war, die betreffende Species nicht 
mit Stemsuch Vibrio (oder Airyullula) Phalaridis benannte, sondern dieselbe zu seiner Anguillula graminearum Dies. zog.

Inwiefern jedoch diese A. graminearum mit der Anguillula tritici Roffred (BAUEn) oder der Anguillula glutinis EHRBG., respectire der Anyuillula des Sauerteigs, identisch sei, konnte aus mangelnden Vergleichungsmaterialieu nicht festgestellt werden. Nach BAKER (l.c.) sollen die im Sauerteige enthaltenen Anguillulae langsamer in ihren Bewegungen sein, nicht unaufhörlich sich mit Kopf und Schwanz gedreht, auch gern sich zusammengerollt haben, durchscheinender, an beiden Enden auch etwas länger gewesen sein und in der Körpermitte einen sehr hellen Theil besessen haben, welcher „dreieckig für das Auge" erschien.

Von Bedeutung bei der Beurtheilung der betreffenden Arten dürfte jedenfalls die Bemerkung des Prof. Grube in Breslau sein, dass alle nicht purtesitischen Anyuillulae die Oeffnung des weiblichen Zeugungsorgans in der Mitte des Körpers besitzen und lebendige Junge erzeugen, während alle parasitischen Anguillulae Eier legen und die Geschlechtsöffnung des Weibchens sich im hinterı Körpertheile, vor der Schwanzspitze findet. Hienach möchten die Anguillulae des Kleisters (glutinis EHBG.) mit der Anguillula graminearum Dies. nicht identisch sein, denn LederMüLler (1. c. pag. 42) giebt in seinem Resumé ausdrücklich sub No. 1 von seinen Kleisterälchen an ,qu'elles sont rivipares; c'est-à-dire, qu'elles portent leurs petits vivants duns le ventre, et qu'elles ne les mettent pas." Da nun KüHN auf Grund einer Beobachtung, zu welcher derselbe das Material rom Prof. Dr. CoHs in Breslau erhielt, (pag. 184 seiner Krankheiten der Culturgewächse) rücksichtlich der Anguillulv tritici Rorrr. sich dahin äussert, dass das Mutterthier zahlreiche Eier lege und dann stürbe, so möchte wenigstens die LEDERMüLLER'sche Vermuthung, dass die Weizenälchen, durch den Mahlprocess nicht getödtet, in den Kleister und Sauerteig übergingen, nicht ihre Bestätigung finden; allein ungeachtet Küns sich sehr ausführlich ïber Anguillulu tritici äussert, ist es mir noch nicht möglich, die Behauptung aufzustellen, dass 1. tritici Rofrr. und graminearum Dies. röllig identisch sind, obschon mancherlei für die Annahme der Identität spricht.

Hienach bleiben noch zwei Fragen zur Erledigung übrig; die eine betrifft die Wiederbelubugsfühigheit der anseheinend todten Thiere und die zweite: die etwaige Tervandtschaft der Gras-Anguillulae mit den Trichinen.

In Betreff des ersten Punktes, die Wiederbelebungsfähigkeit inscheinend todter Anguillulae anlangend, so ist dieselbe ron NEEDInM,- BAKER und Lederaülten im vorigen Jahrhundert zwar be- 
hauptet, aber von Diesing l. c. neuerdings auf Molekularbewegungen zurückgeführt worden. Zur Erledigung dieser Frage unterzog ich die am 6 Juli gesammelten und seitdem im Herbarium des bot. $\mathrm{Mu}$ seums der Universität Greifswald trocken conservirten Phleum- und Koelevio-Aehrchen, welche ihrer äussern Beschaffenheit wegen $A n$ guillulae vermuthen liessen, am 6 December desselben Jahres einer erneuten Untersuchung. Dieselben wurden in Wasser von $+15^{\circ} \mathbf{R}$. gelegt und zuerst nach 5 Stunđên, dann nach 12 Stunden und endlich nach 36 Stunden mikroskopisch untersucht. Schon nach 5-stündigem Aufenthalt im Wasser, nachdem die getrockneten Anguillulae Wasser aufgenommen hatten, liessen sich unverkennbare Zeichen wiedererwachenden Lebens bemerken, welches sich jedoch nach 12 Stunden so unzweifelhaft aussprach, dass zufällig anwesende Freunde der Botanik die lebhaften activen Bewegungen der geschlechtslosen Anguillulae unbedingt als solche mit mir anerkannten und kuum zu überreden waren, dass dies Thiere seien, welche unter gleichen Verhältnissen mit den ihnen vorgelegten trocknen Thieren so lange Zeit existirt hätten. Die Untersuchung der in das Leben zurïckgeführten Thierchen, nach 36 stündigem Aufenthalt in Wasser, bestätigte das 24 Stunden zuvor Gesehene vollkommen. Auf Grund dieser sicher gestellten Thatsache ist es aber nicht möglich, die Behauptung DiesING's zu unterstützen, wonach die von Baker, Neediam und Ledermüller constatirte Wiederlebungsfähigkeit auf Mfolecularbewegungen zurückzuführen sein soll. Es steht vielmehr, durch diese Beobachtung sicher gestellt, fest, dass ein rölliges Trockenliegen und Eintrocknen der geschlechtslosen $A$ Ifrillulne diese selbst nicht sofort tödtet, und erklärt sich durch dieses Vermögen, welches ja auch bei Mucrobiotus Hufelandii S. Scuvltze, Ecluniscus Creplini S. Schultze und andern Tardigraden nachgewiesen ist, das plötzliche und zahlreiche Erscheinen der parasitischen Thiere auf Feldflächen, wo man sie zuvor nicht gesehen hat.

In Betreff der zweiten Frage: die Verwandtschaft der Anguillulae graminearum DIEs. mit Trichina spiralis OWEN anlangend, so lässt sich heute auf das Bestimmteste behaupten, dass eine solche nicht besteht. Allerdings musste man sich die Frage vorlegen, was die Natur mil den parasitischen Anguillulis für Zwecke verbinden möge. In Grasfrüchtchen erzeugt, kamm man von ihnen unmöglich läugnen, dasss sie mit den Grasährchen in den Darmkanal der Grasfresser übergehen müssen. Auch ist es nicht unwahrscheinlich, dass diese Wanderung nothwendig ist, um die geschlechtslosen Thiere geschlechtsreif zu machen. Aus meinen Untersuchungen des Pferde- und Kuhdünger's, denen jedoch directe Fütterungs-Ver- 
suche nicht zur Seite stehen, geht herror, dass sich darin oft zahlreiche Anguillulae vorfinden. Dass aber eine Auswanderung derselben durch den Darm der Grasfresser in deren Leib nachgewiesen werden kömnte, habe ich experimentell festzustellen nicht rermocht, weil ich seiner Zeit genïgendes Versuchsmaterial nicht besass. Ebenso ist es meines Wissens aber auch noch nicht experimentell geprüft, ob die Rüben-Nematode, nachdem sie in den Darm der Schweine, Kühe, etc. gelangt ist, von dort in den Thierleib auswandere. Aber in Betreff der Rüben-Nematode ergeben die Beobachtungen VIRchot's (1), Kürrs's (2) und Sterrs's (3), dass dieselbe, weit entfernt, mit der übel berüchtigten Trichine identisch zu-sein, weder generisch, noch auch in Betreff der Familie mit derselben verwandt ist. Indessen blieb durch die letztgenannten Untersuchungen die Frage rücksichtlich der Anguillulae graminearum DiEs. noch offen, und daher benutzte ich die sich mir darbietende Gelegenheit sehr sorgfältig, ım diese Frage ihrer endgültigen Lösung zuzufübren.

Aus Gartz a/O. erhielt ich frisches Schweinefleisch mit eingekapselten Trichinen, rerfütterte ein halbes Loth desselben etwa an eine von einem biesigen Schlächter eingefangene Maus (IIus mus. rulus L.), tödtete dieselbe am 15 Tage nach geschehener Fütterung und fand num im Darm derselben : yeschlechtsreife Trichinen. Dieselben stimmten in allen wesentlichen Punkten mit den ron Pagevstecher (4) gegebenen Abbildungen rollkonmen überein und gestatteten eine durchgreifende Vergleichung mit den Anguillulae graminenrum Dres. Geschlechtsreife weibliche Trichinen führen nur einige Zeit kleine orale Eier; denn noch im Mutterleibe selbst verlassen die Embryonen die Eischale und werden mithin lebendig geboren. Die Eier der Ang. gram. werden rom lebenden Mutterthiere abgelegt, und das Junge entwrickelt sich ausserhalb des mütterlichen Körpers. Die neugeborne Trichina ist an beiden Enden ziemlich gleichgestaltet, die junge Anyzillula dagegen gleich ron Hause aus am hintern Körper-Ende zugespitzt. Die junge Trichine durchbohrt den Darm und gelaugt ins Blut (wo sie überall ron mir erkannt worden ist, auch im Herzblute), und von da in die Muskeln. Yon den parasitischen Anguillulis ist eine derartige Einwanderung in thicrische Muskeln noch nicht nachweisbar; wohl aber weiss man, dass

(1) Vrronow, Archiv fïr pathologische Anatomie, Bd. XXXII, p. 350.

(2) Mitheilungen des landwirthschaftl. Instituts der Univ. Ialle. Berlin 1865, p. 24.

(3) Vinciow's stenographischer Bericht der Verhandhngen über die Trichinenfrage. Berlin, 1865, p. 6.

(4) Die Trichinen, Leipzig, 1865, 80. Thf. II, fig. 1, 2. 
sie im geschlechtsumreifen Zustande sich zwischen den Blättern und Halmen der Giamineen fanden (cf. Tascuexberg l. c. Tab. VII, fig. 12) und paarweise in die noch offenen Ovarien einwanderten, um sich zu befruchten und die Eier abzulegen. Das männliche Thier von Tr $i$ chinc spiralis besitzt am hintern Körper-Ende die Austrittsstelle des Penis, umgeben von zwei hörnchen-förmigen Fortsätzen, welche der Anguillula ohnehin gänzlich fehlen. Die Geschlechtsöffnung der weiblichen Trichine liegt nahezu im ersten Sechstheile der Gesammtkörperlänge; die der Anguillutae in letzten Neuntel des hintern Körpertheiles.

Geht hieraus zur Evidenz hervor, dass die Trichinen zur Gruppe der Acrophalli Dies. gehören, so ist auch hiemit bewiesen, dass die. Anguillulae grom. nur zu den Hypophalli Dies. zu rechnen sind und mithin beide Genera ganz verschiedenen Familien, ja sogar verschiedenen Abtheilungen der Nematoden angehören.

Ergiebt sich aus Vorstehendem noch keineswegs ein Nachtheil für Thiere und den Menschen, wenn Auguillulcue mit Cerealien oder Grasfrüchten in deren Darm gelangen, so ist doch die Beeinträchtigung del Ernten bedentend genng, um Maassregeln zuzulassen, welche auf möglichste Beseitigung der Anguillulke selbst gerichtet sind. Es empfehlen sich daher Tasciexiberg's und Kürx's Torschläge, welche darauf hinauslaufen : 1. Gichtige Weizenkörner (blé niellé) mit verdünnter Schwefelsäure (1 Th. auf 150 Wasser) zn beizen, ehe sie auf den Acker gebracht werden und 2. dergleichen Früchte nicht auf den Dunghaufen zu werfen, sondern zu verbrennen, oder doch wenigstens zuvor einer Temperatur von $70^{\circ} \mathrm{R}$. auszusetzen, ehe sie dem Dunghaufen übergeben werden. Die in Grasähren vorkommenden Anguillutae dürften, wenn sie in Menge vorkommen, dem letzten Verfahren zu untorziehen sein. 


\title{
MÉMIOIRES PRÉSENTÉS A LA SECTION DE BOTANIQUE APPLIQUÉE ET D'HORTICULTURE.
}

\author{
L'HYBRIDATION DES FOUGÈES, \\ PAR
}

\section{A. STELZNER.}

(Voir le Compte Rendu de la Séance du 8 Arril 1865, pag. 292).

Parmi nos occupations de jardinage une des plus intéressantes, sinon la plus intéressante de toutes est sans contredit celle de féconder les plantes, dans le but de changer les espèces, d'obtenir de nouvelles formes ou des hybrides.

Pendant que nous observons avec un rif intérêt le progrès des plantes mutuellement fécondées, dont le procédé nous est parfaitement clair chez les phanérogames par leurs organes plus ou moins grands, mais toujours distinctement risibles, nous sommes encore dans l'obscurité concernant la fécondation et son procédé chez les cryptogames, resp. fougères.

Les études aussi profondes qu'intéressantes des sarants MüNter, Lechinsky, Wigand, Schacht et autres ont établi qu'il existe chez les fougères des organes reproducteur's des deux sexes (anthéridies et archégones) qui sont portés à la face inférieure du prothallion, c'est-à-dire, sur la lame foliacée qui résulte de la germination des spores. Conformément à ces études qui prêtent aux prothallions pour ainsi dire le caractère des fleurs, la plupart des physiologistes sont de l'opinion qu'une fécondation chez les fougères n'aurait lieu que par le rapprochement intime des prothallions de deux espèces différentes. L'opinion qu'une fécondation pourrait avoir lieu à la fronde même au moment où les spores se forment (ce qui serait analogue ì celle des phanérogames et me semblerait plus conforme aux lois de la nature), n'a jusqu'à ce moment guère trouvé d'adhé. rents, quoique, d'après ce que j'ai entendu, ScHachi aussi se soit déclaré pour cette théorie daus le dernier temps.

Cette opinion a été exprimée, je crois, dans la Bonplandia, il y a une dixaine d'années, par mon ami M. F. SraxGe, horticulteur à Hambourg; l'article en question ne m'est pas conuu dans ses détails. Les 
expériences que j'ai faites dans ma pratique sur l'hybridation des fougères, rapprochent peut-être ces deux opinions sans admettre ni l'une ni l'autre dans toute son étendue. Je suis tenté de croire qu'une sorte de fécondation a lieu au moment même que les spores commencent à germer, et quand la masse gélatineuse de différentes spores se touche et se transforme mutuellement longtemps avant que le prothallion soit formé.

Ce qui me fait douter principalement d'une fécondation des prothallions, c'est le fait qu'il s'est trouvé souvent un grand nombre d'hybrides ensemble, l'une à côté de l'autre. Un rapprochement des prothallions ne pouvait donc pas avoir eu lieu, et il me semble qu'on ne peut non plus admettre que toutes ces quantités auraient pu être fécondées par le saut mutuel des organes reproducteurs, qui s'ourrent comme on sait en sautant. Ce qui me fait douter d'une fécondation sur les frondes, c'est que j'ai gagné des hybrides d'espèces qui n'ont pas été en contact arec d'autres de leur genre, comme je vais rous le montrer tout à l'heure.

Il y a quelques amnées j’ai rapporté dans le Wochenschrift de Berlin (an 1859, pag. 183) que j'avais obtenu une nouvelle et intéressante hybride de Gymnogramme; elle provenait d'une fécondation de G. chrysophylla et G. lanatu. Nos savants amis ici présents, MLM. les proff. Kocir de Berlin et Reichenвaci fils, de Leipsig, ont bien voulu me le dédier en le nommant $G$. Stelzneriana (Wochenschrift 1859). 'Tous les sujets que j'en ai élévés, avaient la particularité de ne donner de spores nulle part, quoiqu'ils soient passés dans les mains des praticiens les plus expérimentés en Angleterre et en Allemagne; $\mathrm{j}$ 'en arais cultivé moi-même des exemplaires avec des frondes de trois pieds de longueur qui n'ont pas donné de spores non plus, et je crois que les plantes que j’ai élévées à cette époque sont disparues partout aujourd'hui. Il y a trois ans je réussis de nouveau à gagner cette hybride en semant les G. chrysophylla et G. lanata de la manière suivante. Je réunis sur un morceau de papier blanc les spores des deux Gymnogrammes, je les mêlai le plus possible, puis je les semai ensemble. J'obtins de cette manière une bonne partie de G. Stelaneriana qui avaient pourtant deux caractères différents; les uns entièrement semblables à ceux gagnés en 1859 , couverts à la face inférieure des frondes d'un enduit farineux doré dont les prenières développées étaient légèrement crêtées à leurs extrémités; les frondes de l'autre partie étaient plus étroites, plus velues et moins couvertes de cet enduit farineux et n'atteignaient pas la grandeur de celles de la première partie. Le phénomène le plus intéressant était, que toutes les plantes des deux catégories donnaient, en moins 
d'une année de culture, des spores parfaites qui reproduisaient en les semant les mêmes variétés; leurs frondes sont trois fois plus grandes que celles de leurs parents. Je fis encore d'autres essais avec les Gymnogramme gracilis, G. pulvenulenta, argyrophylla, G. l'Herminievi et G. Laucheana, semés pêle-mêle ensemble.

Les spores de G. gracilis provenaient d'une plante que j'avais élévée il y a une dixaine d'années, alors que cette fougère était toute nouvelle, éloignée non seulement de tout autre Gymnogramme, mais même de toute autre fougère, parce qu'elle était la seule qui se trouvât dans la serre; les autres espèces prorenaient par contre des exemplaires qui n'avaient été en aucun contact avec G. gracilis ni avec $G$. tartarea. Les résultats obtenus étaient les suivants:

G. pulverulento sulphurer; les frondes étaient couvertes sur les deux faces d'un enduit farineux d'un jaune pâle; je n'arais gagné que trois exemplaires que je dus malheureusement perdre en hiver.

G. gracilis hybrida, qui tient le milieu de G. grucilis et de G. Zanato, ressemblant à $G$. tartarea; il y en a dans le nombre qui sont converts d'un enduit farineux épais d'une couleur d'argent brillant, d'autres d'un enduit blanc-jaunâtre et d'autres enfin auxquels cet enduit manque totalement. Tous sont couverts à la base des frondes d'un fort duvet plus ou moins brun foncé.

G. gracilis elegantissima, aux frondes très finement découpées et couvertes sur les deux faces d'un épais enduit blane jaunâtre.

G. gracilis superba, la plus ormamentale et la plus précieuse de toutes; les frondes très-élégamment courbées sont bien moins finement decoupées, mais larges, d'un vert clair luisant, d'un lustre métallique à la face supérieure et couvertes d'un enduit luisant blane jaunâtre très-épais à la face inférieure; c'est un des plus rustiques de tous les Gymnogrammes.

G. Tybrida davalliaefolia, sortant d'une fécondation opérée sur le G. l'Heminieri; elle se distingue avantageusement par la multitude et la finesse de ses pimmules qui rappellent extrêmement certaines Duvulliu gracieuses; sa fructification aussi est des plus ornamentales.

Les Gymnogrammes se prêtent plus facilement à ces sortes de croisements. Les autres genres ne m'ont pas encore donné d'hybrides jusqu'à ce jour. J'ai bien obtenu quelques formes différentes, comme Davallia tenuifolia stricta, variété touffue de la Davallia tenuifolia; Pteris aspericaulis des graines de Pteris tricolor, ce qui prouverait peut-être que Pteris tricolor ne serait qu'une hybride ou variété - du Pteris aspericaulis, d'autant plus qu'il a absolument la même croissance que celui-ci. Ces formes rappellent celles des fougìres de pleine terre dont la plupart sont si intéressantes et 
si ornementales, et dont les spores reproduisent toujours les mêmes formes, comme je l'ai expérimenté, il y a quelques années, sur l'intéressant Athyrium Fil. focmine Frizellice, et maintenant de nouveau sur l'Osmunde regalis cristata.

Ces formes provenaient-elles aussi d'une fécondation? Je ne le crois pas, puisqu'il y a des exemplaires, quoique comparativement en très-petit nombre, qui retournent an type après avoir eu pendant toute une amnée l'aspect de cette forme particulière.

L'expérience m'a enseigné, comme elle l'a déjà fait à d'autres praticiens, que les prothallions peuvent se conserver aussi longtemps que les plantes mêmes, qu'ils peuvent être divisés et servir ainsi à la multiplication là où on n'a gagné que peu de sujets. On n'a qu'à détacher les plantes avec un couteau bien tranchant et à cónserver toujours les prothallions. Ce sont particulièrement les prothallions des fougères en arbres et d'autres qui deviennent très-larges, qui sont propres à cette expérience.

Mes expériences mont également enseigné qu'une chaleur excessive hâte et favorise la germination des spores ainsi que leur hybridation; je leur accorde toujours un degré de chaleur qui tuerait les graines de toute autre plante appartenant aux Phanérogames.

Comme horticulteur mes essais ont été naturellement restreints, ne s'étendant que sur les espèces très-décoratives recherchées par le commerce; il me manquait par la même raison le temps et les instruments nécessaires pour pouvoir chercher un résultat positif, s'il avait été possible.

C'est à nous praticiens, de faire nos travaux horticoles avec intelligence, d'en observer les faits et les phénomènes extérieurs qui puissent ensuite guider les hommes de la science dans leurs examens minutieux et profonds. 


\title{
NOTICE SUR LES PLAN'IES DE PLEINE 'TERRE,
}

\author{
PAK
}

M. F. MULLER.

(Voir le Compte Rendu de la Séance du 8 Avril 1865, pagg. 305).

En venant vous entretenir des plantes de pleine terre, je sens mon cœur plein de joie, et je me reporte, avec bonheur, vers le temps où elles étaient recherchées et comprises, et où l'on était fier de les montrer, comme on exhibe aujourd'hui un bean spécimen d'Orchiclée exotique. Combien de fois suis-je revenu portant avec précaution un exemplaire d'une de ces petites merveilles, aujourd'hui délaissées.

Cependant, à ces souvenirs des joies d'autrefois se mêlent aussi des regrets amer's, quand je pense à ceux qui, à cette époque, ont bien roulu guider mes premiers pas dans le domaine de Flore. La mort nous a enlevé, en peu de temps, le Dr. D'Avorne, de Malines, Syout, son compagnon et son ami, Themister de Liége, Donckelaer et D. SPAE, de Gand. Qu'il me soit permis, dans cette assemblée des illustrations de la science botanique et horticole, de jeter quelques Heurs sur la tombe de ces savants modestes, qui semblent avoir emporté avec eux le goût de la culture des plantes de pleine terre. En effet, il n'y a plus guère en Belgique d'amateurs qui cultivent en savants ce genre de plantes. Ceux dont je viens de vous rappeler le souvenir ne cherchaient ni la belle forme de la fleur, ni la richesse du coloris; leur jardin était un herbier vivant, et, comme le botaniste, ils sacrifiaient à la rareté plutôt qu’à l'éclat.

La perte de ces hommes distingués est certes bien grande pour cette branche de l'horticulture et pour la botauique elle-même, mais nous ne pouvons attribuer à leur mort seule l'abandon dans lequel ce gemre de culture est tombé depuis quelque temps; d'antres causes ont produit cet état que les amis de la botanique et de l'horticulture déploreront avec moi. Ne derons-nous pas, nous que le temps à épargnés, chercher à rendre à ces pauvres délaissées la place qui leur appartient, et permettre à celui qui doit borner ses dépenses à un chiffre minime, de briller aussi, par ses connaissances, dans la phalange éclatante des amis de l'horticulture. Il n'est pas donné à chacun de réunir en des serres opulentes les merveilles 
de toutes les parties du monde et de posséder un personnel nombreux de jardiniers. Encourageons donc le modeste amateur de plantes de pleine terre; celui-là, du moins, cultive le produit de ses efforts, et les espèces, moins splendides, il est vrai, qu'il entoure de ses soins, il ne les confiera jamais à des mains étrangères.

Et d'abord, d'où vient l'abandon que je vous signale; pourquoi tant de charmantes espèces n'ornent-elles plus nos parterres? Les motifs de ce fait sont nombreux; voici comment j'ai cru pouroir, en partie, résoudre cette question.

Ceux qui cultivent les plantes qui nous occupent, possèdent ordinairement leur jardin à proximité des maisons; en quelques années ils ont vu l'espace où ils les renferment entouré de constructions; les usines ont surtout porté un préjudice considérable à leurs cultures. Les rosées bienfaisantes, qui venaient désaltérer les plantes pendant la nuit, ne pénètrent plus jusqu'à elles, et tandis que le soleil du matin les a privées de ses rayons, il ne parvient plus à elles qu'à une heure assez avancée, alors que pour beaucoup il est devenu nuisible.

A côté nous trouvons une autre cause, l'épuisement du sol.

Je vais tâcher de développer ces différents points, vous parler du milieu que les plantes de pleine terre exigent, des premières bases de leur culture.

Comment espérer, en effet, que des végétanx qui ont été introduits dans nos tristes jardins, encaissés dans des murs élevés, entourés de maisons ou de fabriques, dont les cheminées et les ventilateurs laissent échapper toute la journée des gaz délétères, puissent vivre dans une atmosphère si viciée, eux qui nous arrivent des prairies ou des montagnes, où ils ont toujours respiré l'air le plus pur. Ce milieu convenable qui fait défaut est un des grands obstacles que rencontre le cultivateur; dans les jardins des villes; il nous explique peut-être la disparition de tant d'espèces remarquables.

J'ai connu, autrefois, dans les prairies des environs de Bruxelles, différentes plantes qui $n^{\prime} y$ existent plus aujourd'hui, entre autres l'Orchis latifolia et le Parnassia palustris, etc., et je n'attribue leur disparition qu'au voisinage de la ville, et non aux petits jardiniers qui en arrachent tous les ans un grand nombre, pour les vendre aux marchés, ni aux enfants qui se bornent à en couper les tiges.

Les rosées si nécessaires et si rafraîchissantes pour les plantes courbées sous les rayons ardents des canicules, remplacent la pluie, comme sous la zône torride: elle ne descend dans les villes qu'à la fin d'Août et de Septembre. 
Le soleil qui vient dès l'aurore x'échauffer, par gradation, la terre et les végétaux, ne pénètre dans les jardins des villes que brûlant et lorsqu'il a déjà parcouru une partie de sa carrière.

J'ai souvent observé que des serres qui avaient devant elles un horizon assez vaste pour permettre aux premiers rayons du soleil levant de pénétrer jusqu'aux plantes, pouvaient pour un grand nombre d'espèces se passer d'abri contre les plus fortes chaleurs.

Quant à la nature du sol, il est à remarquer que dans les villès ou les faubourgs il est presque toujours composé d'une couche épaisse de détritus de fumier, dont l'humus est déjà absorbé, ainsi que d'un mélange de bone, provenant des rues; c'est ce qui forme, dans la plupart des jardins maraichers, cette couche de terre noire qui convient à cette culture, mais qui comprend, généralement, des plantes annuelles ou bisannuelles.

Je crois pouvoir faire remarquer que ces dernières croissent la plupart spontanément dans les lieux cultivés par la main de l'homme, ou bien dans les bois et les prairies, là où la couche supérieure du sol est formée par un détritus d'autres végétaux, tandis que les espèces vivaces croissent pour le plus grand nombre dans les plaines incultes, sur les talus et les montagnes; la terre qu'elles trouvent dans ces conditions est plutôt amendée qu'engraissée. Telles sont les plantes des terrains calcaires, schisteux, calaminaires, etc.

Dès-lors il est facile de comprendre que les plantes alpines, par exemple, ne peuvent ni vivre, ni prospérer dans les jardins offrant une exposition et un terrain si défavorables. Voilà, en grande partie, les difficultés que rencontrent les amateurs, dans les jardins destinés à leur culture.

Les plantes vivaces de pleine terre, c'est-à-dire celles qui passent l'hiver à l'air libre dans le Nord de l'Europe, et que l'on nomme aussi plantes de plein air, sont indigènes du midi de la France, des Pyrénées, des Alpes, de l'Amérique, etc.; malgré leur rapprochement de l'équateur, il en est qui peuvent s'acclimater chez nous; cela dépend entièrement du degré d'altitude d'où elles sont originaires.

Cependant quelques-unes offrent certaines difficultés et réclament des soins dans la mauvaise saison; ainsi les plantes des hautes montagnes qui, pendant tout l'hiver, sont couvertes d'une couche de neige, jusqu'au moment où le soleil vient leur enlever cet abri, sont très-sensibles aux variations atmosphériques de nos pays. Ces variations brusques occasionment la perte de benucoup d'espèces. J'ai parfaitement bien réussi en abritant les plantes alpines par une couche de huit à dix centimètres de feuilles sèches, sans toutefois cacher la plante, puis en les recouvrant d'un pot à fleur's ébréché 
du côté où le rent et la pluie ont le moins d'accès, je laissais cet abri jusqu' au milieu du mois d'A rril. Il est aussi beaucoup de plantes qui craignent, pendant le moment du repos, l'humidité antant que le froid.

Un grand nombre de personnes cultivent les plantes alpines et des montagnes en terre de bruyère et les placent à l'ombre. Ce système est tout-à-fait contraire à la position qu'occupent ces végétaux dans leur pays. Par leur position, ils sont exposés au soleil et à un air très-rif; ensuite, le sol dans lequel ils croissent est une terre compacte. Je leur ai donc toujours donné une terre forte que je recouvrais de trois on quatre centimètres de terre de bruyère, pour empêcher le sol de se durcir par les arrosements ou par la pluie. Quand à l'exposition au soleil, elle me semble nécessaire; mais ici il faut user de quelques précautions, en considérant que les espèces délicates sont abritées par les sinuosités des rochers. Pour suppléer à cet abri naturel, j’ai enfoncé des pierres dans le sol, à vingt ou trente centimètres, en les tournant ver's le midi et le couchant, de manière à permettre à mes plantes de recevoir les rayons du soleil du matin.

Beaucoup de plantes exigent un abri pendant les fortes chaleurs du jour: celui qui a l'esprit observateur s'aperçoit que certaines plantes rampent sur le sol, non pour émettre des racines propres à leur croissance, mais pour se soustraire sous d'autres à l'action trop forte du soleil. Quelques espèces que j’arais rapportées des prairies avaient sans cesse les feuilles brûlées; en les plaçant avec d'autres, je suis parvenu à les faire prospérer au-delà de toute attente.

Les espèces croissant spontanément dans les bois réussissent dans les pares d'Azalées, de Rhododendrum; quelques Lilium, entre

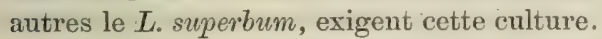

Il est fort difficile d'indiquer la station exacte de toutes les plantes dans un rapport aussi peu étendu; l'expérience doit guider le cultivateur; des notions de géographie botanique, ainsi que la connaissance de l'origine, de la patrie des plantes, science que l'on nomme climatologie, sont nécessaires à tout amateur consciencieux.

Les difficultés que j'ai énumérées plus haut, ne se présentent pas pour toutes les espèces; notons en passant qu'il en est de trèsrolontaires.

Pour la facilité du cultivateur on peut diriser les différentes plantes en quelques groupes, comme suit:

1. plantes croissant à toute exposition;

2. plantes des plaines et des prairies;

3. plantes alpines, des montagnes, des côteaux;

4. plantes des bois. 
L'amateur éclairé cherchera toujours l'origine des espèces et leur domnera une place convenable, semblable, antant que possible, à leur habitat.

Il n'est pas aisé de collectionner une même famille dans un parterre, à moins de donner à chaque individu la place qu'il occupe dans sa station d'origine. Citons pour exemple les fougères: Cystopteris germanica ne croît que dans les bois épais, et craint par conséquent les ardeurs du soleil, tandis que le Ceterach officinarum croît sur les rochers exposés au midi. Comme la première de ces plantes est assez grande, il est difficile de lui donner un abri convenable. Mais qu'importe la place qu'occupe la plante, pourru qu'elle soit dans un milieu qui lui est propre.

Il est un moyen de réunir les familles: c'est de les disposer autour de massifs d'arbres, ce qui permet de les ranger de manière que les unes soient ombragées et les autres exposées au soleil.

M. von Siebold m'a permis d'extraire de son Catalogue raisonné des plantes du Japon un moyen très-facile et très-savant de réunir les végétaux, et qui consiste en groupes physiognomiques.

Voici ce qu'écrit ce savant introducteur à ce sujet:

„AleXandre de HuмвoLdT, ce savant et ingénieux naturaliste, a avancé le premier, dans son ourrage connu an monde littéraire sous le titre de Cosmos, l'heureuse idée de faciliter et d'encourager l'étude de la nature végétale, en groupant soit dans des parterres en pleine terre, soit dans des serres chaudes ou froides, différentes plantes exotiques, propres à rendre un tableau caractéristique de la Flore des pays étrangers, et de récompenser ainsi de leur zèle et de leur dévouement pour l'horticulture les amateurs et les cultivateurs de plantes, attachés au foyer paternel, par l'aspect de groupes de plantes représentant la végétation des pays les plus éloignés de leur patrie."

On pourrait donc former différents groupes comme suit:

Plantes indigènes.

" du midi de l'Europe.

" alpines.

" américaines.

" japonaises, etc.

En tenant compte de la station d'origine, du mode de croissance, on parriendrait au moyen de cette classification générale à donner à chaque plante le mode de culture qu'elle réclame.

Malgré tous les soins et toutes les précautions, il arrive souvent, après un hiver rigoureux, que nous ayons à remplacer quelques sujets. Il est donc indispensable d'aroir des bâches dans lesquelles nous conserverons quelques doubles. 
En parcourant le catalogue de certaines collections on rencontre plusieurs plantes qui sont, à la vérité, fort remarquables par leur fleur ou leur feuillage, et qui figurent comme plantes de pleine terre, mais qui ne sont, en réalité, que des espèces de serre froide; l'amateur les excluera de ses collections.

Je dois me borner ici à ces quelques indications générales, l'espace me manque pour donner le mode de culture des nombreuses espèces qui sont dignes de figurer dans nos jardins.

On roit, en résumé, que celui qui forme l'intéressante collection dont j'ai voulu vous entretenir quelques instants, pourra $y$ trouver un vaste champ ì ses observations, et que ses soins ne seront bien payés que pour autant qu'ils auront été conduits d'une manière intelligente et raisonnée. 


\section{CONSIDÉRATIONS SUR LES HYBRIDES,}

PAR

\section{ALFRED WESMAEL.}

(Voir le Compte Rendu de la Séance du 8 Arril 1865, pag. 305).

Parmi les différentes questions soumises au Congrès international d'horticulture, il en est une sur laquelle je crois devoir attirer votre attention. L'hybridation, les croisements, les fécondations artificielles en général occupent une place de premier ordre dans les différent: problèmes sur lesquels l'horticulture demande des solutions positives.

La fédération des sociétés d'horticulture de Belgique avait déjà reconnu l'utilité de cette importante question, puisque au nombre de celles mises au concours en 1860 figurait la suivante: Exposer le phénomène de l'hybridation et en général celui des croisements naturels et artificiels entre les végétaux; les procédés à suivre dans ces opérations, les principaux résultats que l'hybridation a produits et l'influence qu'elle exerce en horticulture.

Un mémoire fut adressé; la commission chargée d'en apprécier la valeur conclut qu'il n'y avait pas de récompense à accorder.

La question fut maintenue au nombre de celles du concours de l'année suivante. En 1863 j'adressais un mémoire qui fut couronné.

L'académie des sciences de Paris proposa en 1860 une question analogue pour le grand prix des sciences physiques.

L'institut partageait la question principale en quatre questions secondaires:

1. Étudier les hybrides régétaux au point de rue de leur fécondité et de la perpétuité on nón perpétuité de leurs caractères.

2. Dans quels cas ces hybrides sont-ils féconds par eux-mêmes; cette fécondité des hybrides est-elle en rapport arec les ressemblances extérieures des espèces dont ils proviennent, ou signale-t-elle une affinité spéciale au point de rue de la génération, comme on l'a remarqué pour la facilité de la reproduction de ces hybrides euxmêmes:

3. Les hybrides stériles par eux-mêmes doivent-ils toujours leur stérilité à l'imperfection du pollen: 
le pistil et les ovules sont-ils toujours susceptibles d'être fécondés par un pollen étranger convenablement choisi?

observe-t-on quelquefois un état d'imperfection appréciable dans le pistil et les ovules?

4. Les hybrides se reproduisant par leur propre fécondation conservent-ils quelquefois des caractères invariables pendant plusieurs générations, et peurent ils devenir le type de races constantes, ou reviennent-ils toujours, au contraire, aux formes d'un de leurs ascendants, au bout de quelques générations, comme semblent l'indiquer des observations récentes.

Deux mémoires furent adressés: le premier avait pour auteur M. NaUdin, le second M. le docteur Godron; le premier de ces travaux fut couronné.

Le travail de M. NAUDIN ne nous est connu qu'en partie; l'auteur nous a communiqué un tiré-à-part de la deuxième partie qui a paru dans les annales des sciences naturelles. M. Godron nous a adressé son travail qui a été imprimé dans les mémoires de l'académie de Stanislas. Qu'il me soit permis, Messieurs, de faire une analyse très-sommaire de ces travaux importants.

Cette analyse est d'autant plus importante, que les auteurs sont en désaccord sur un des points principaux de la question des hybrides; je veux parler de leur fertilité ou de leur stérilité. M. NAUdIN reconnaît que chez les hybrides stériles cette stérilité provient de l'imperfection du pollen et de la mauvaise constitution des ovules.

La façon dont l'auteur pose ces faits doit nécessairement faire supposer qu'il y a des hybrides fertiles. Dois-je rous dire, Messieurs, ' $u$ 'en vous parlant ici d'hybrides fertiles, j'entends parler des hybrides fertiles par eux-mêmes?

M. NAUDIN reconnait que certains ovaires hybrides renferment à la fois de bons et de mauvais ovules.

L'action stérilisante de l'hybridité agit avec bien plus de force sur le pollen que sur les ovules. Ainsi done, si, comme le dit Monsieur NaUdis, l'organe femelle est plus souvent fertile que l'organe mâlẹ, l'horticulture est à même de se procurer bon nombre de plantes nouvelles en apportant à la surfice do l'organe femelle hybride du pollen spécifique, chaque fois que le pollen hybride est mal constitué.

Les différentes observations de M. NADDIN l'ont conduit à formuler cette règle: que les hybrides sont fertiles par euxmêmes toutes les fois que leurs anthères contiennent du pollen bien organisé.

Cette observation de l'auteur est singulièrement différente de celle de M. Godrox qui dit que, lorsque deux espèces incontestable- 
ment distinctes sont fécondées l'une par l'autre, elles donnent des produits constamment stériles, si ces produits sont séparés des parents au moment de la floraison.

Voilà, Messieurs, deux faits singulièrement contradictoires, sur lesquels je désire attirer toute votre attention. D'abord dois-je vous faire entrevoir que l'éternelle question de l'espèce est ici en jeu? Combien de travaux ont vu le jour sur la valeur de l'espèce, combien de théories différentes, combien de manières de voir autres ont été émises, et tous ces nombreux travaux ont-ils eu pour effet de mettre les naturalistes d'accord? Nullement.

Ne croyez pas que je veuille venir ici vous faire une profes. sion de foi sur la valeur du mot espèce. A quoi serviraient ces phrases? A vous convertir, à vous faire partager ma manière de roir? Bien au contraire; chaque botaniste, tout naturaliste raisonne à sa façon; convertir une opinion basée sur la philosophie est chose impossible. Si les produits de deux espèces bien distinctes sont toujours stériles par eux-mêmes, ils sont ordinairement fertiles quand ils reçoivent du pollen de l'un des ascendants.

Ceci est la théorie de M. Godron. Toutefois, certains produits hybrides resteront tonjours stériles puisque plusieurs hybrides sont frappés de stérilité par l'organe femelle.

Au point de vue de l'horticulture, il importe peu, au moins pour certains hybrides, qu'ils soient fertiles ou stériles; du moment qu'une forme intermédiaire est produite et qu'elle offre un intérêt horticole, ne possédons-nous pas assez de moyens de multiplication pour la conserver et la propager dans les cultures? Et puis, si la fertilité existe, quels sont les résultats que nous arons à attendre? Le retour probable à l'un des deux types spécifiques.

Cepeudant les différentes expériences consignées dans les travaux de MM. Godron, Grenier, Naudin et Lecoq nous montrent que, par suite du semis de graines d'hybrides de première génération. tous les descendants ne retournent pas d'emblée à l'un des types spécifiques; certaines plantes se montrent avec des caractères intermédiaires. Les individus de cette seconde génération sont fertiles et très sourent ì l'uniformité plus ou moins parfaite des premiers descendants ou hybrides de première génération succède une extrême bigarrure de formes.

Caractérisons les hybrides de deuxième génération.

Or, d'après l'opinion de M. NAUDIN, cette seconde génération aurait pour origine l'hybride lui-même, tandis que, d'après la théorie de M. Godron, cette descendance proviendrait de l'organe femelle hybride, fécondé par le pollen de l'une des deux espèces ascendanteg. 
Ne discutons pas les faits. Pour arriver à des conclusions positives, l'expérience est le seul moyen à employer.

Or, comme ces expériences n'ont pas été faites d'une manière tout-à-fait irréprochable de la part des différents auteurs, recherchons pour le moment dans un sens comme dans l'autre les ressources que l'horticulture peut attendre en se basant sur l'une ou l'autre théorie.

La création de variétés nouvelles, recherche constante de la part des horticulteurs, n'est pas chose difficile. Produisons des hybrides, fécondons ces hybrides par le pollen des parents, formons des quarterons, ces derniers sont fertiles et leur descendance retournera en partie à l'un des ascendants, tandis que d'autres se montreront avec des caractères autres; il y aura naissance de rariétés hybrides. Ce moyen de procéder est celui de M. Godron.

Quant à la méthode de M. NAUDIN, il s'agit de procéder un peu différemment. Mariez entre-elles deux bonnes espèces, semez les graines, elles rous produiront des hybrides de première génération dont le pollen sera peut-être bien constitué; dans ce cas, il en sera de même de l'organe femelle. Abandonnez ros hybrides à eux-mêmes et vous obtiendrez des graines.

Dans l'un cas comme dans l'autre, j'obtiens des graines d'hybrides, avec cette différence toutefois, que, dans la premiére expérience, l'organe femelle de l'hybride a reçu une fécondation spécifique, tandis que dans l'autre cas la fécondation a été hybridique. Comme je vous le disais tout-à-l'houre, Messieurs, ces hybrides de seconde génération varient beaucoup.

Or, ce sont ces variations que la culture recherche et qu'elle peut perpétuer à l'aide des nombreux moyens de multiplication dont elle dispose. En poursuivant l'analyse des deux mémoires, nous arrivons à la solution de la deuxième question posée: L’aptitude des espèces à se croiser et la fertilité des hybrides qui en résultent, sont-elles proportionnelles à l'affinité apparente de ces espèces? La réponse à cette question est nécessairement double, parcequ'il y a à considérer d'abord l'affinité entre les espèces qui s'hybrident, puis la fertilité des descendants.

Les deux auteurs répondent également oui à la première partie de la question posée, bien que cependant l'un et l'autre consignent des exceptions. Les différents hybrides que j’ai rencontrés dans la campagne provenaicnt tous d'espèces roisines, à une exception près le Cirsinm lanceolato-arvense.

Les différentes recherches que j’ai faites sur les hybrides des cultures, m'ont fourni la certitude que presque tous provenaient de 
parents ayant entre enx énormément de rapprochement, et toụjours entre espèces d'un même genre naturel.

L'horticulteur doit donc, dans la recherche des produits hybrides, faire usage d'ascendants chez lesquels les affinités sont les plus apparentes.

Quant à la solution de la seconde partie de la question, les deux auteurs marchent dans deux voies toutes différentes. J'ai déjà dit que pour M. Godrox les hybrides de première génération sont toujours stériles; manière de roir que je partage en tous points, du moins d'après les observations que j'ai faites sur les hybrides de Cirsium que j'ai décrits.

Quant à l'opinion émise par M. NaUdis, elle est autre. Sa réponse est oui dans le même sens que pour la première partie de la question. Voici en quels termes l'auteur résume ses conclusions :

,L'aptitude des espèces à se féconder réciproquement et le degré de fertilité des hybrides qui en naissent sont done véritablement le signe de leur affinité spéciale au point de vue de la génération, et, dans la grande majorité des cas, cette affinité est accùsée par l'organisation extérieure, en un mot par la physionomie des espèces.

Que pouvons nous conclure, Messieurs, de deux manières de voir aussi différentes, la fertilité ou la stérilité des hybrides de premier croisement? La seule chose à faire à mon aris, c'est, comme je vous le disais, de recommencer de nouvelles séries d'experiences dont les résultats permettront d'arriver à une solution décisive.

Cette question est plutôt du domaine de la physiologie végétale que de l'horticulture; néanmoins comme cette dernière est arrivée à l'état de vraie science, les horticulteur's pourront communiquer leurs observations aux botanistes.

La question de la fertilité ou de la stérilité des hybrides est résolue affirmatirement dans d'autres mémoires ou notices; je rais. Messieurs, vous en faire un coirt résumé.

M. REgEL rapporte le résultat d'expériences qu'il a faites sur plusieurs espèces de Begonia. Le B. rubrovenia fécondé par le $B$. vanthina à donné naissance à un hybride ayant beaucoup de rapport arec le $B$. vanthina marmorea et qui, comme celui-ci, était fertile par le pollen et par le pistil. Il résulte done de la première expérience qu'il y a des hybrides à pollen bien organisé.

M. Stange obtint des graines bien constituées de la fécondation du B. aunthina marmorea par son propre pollen. Une seconde preuve donnée par le même auteur est celle dims laquelle le $B$. splendida est fécondé par l'hybride $B$. úantlina argentea. 
M. Stange a reconnu que certains hybrides, d'inféconds qu'ils étaient dans les premiers mois de développement, pouvaient devenir féconds par la suite. M. LECoQ a observé la même chose chez les hybrides de Mirabilis.

M. Stange a démontré que certains hybrides sont féconds à la condition que certains types soient pris comme mère et d'autres comme père, et cessent de l'être quand l'opération est renversée.

Comme vous le voyez, Messieurs, cette question de stérilité ou de fécondité revient à chaque instant.

Ai-je eu raison à deux reprises différentes d'attirer votre attention sur la nécessité qu'il y avait de faire de nouvelles expériences? Encore une fois, je renouvellerai mes conclusions dans l'espoir de rencontrer parmi vous quelques zélés observateurs qui auront à coeur d'arriver à obtenir des données certaines sur cette question importante:

La physionomie des hybrides est une autre question sur laquelle je dois m'arrêter un moment.

Dans la majeure partie des cas, les hybrides d'un même croisement se ressemblent beaucoup entre eux; néanmoins on constate quelques infractions à cette règle générale. Malgré cette ressemblance assez apparente, M. Grenier reconnaît que deux espèces en s'hybridant réciproquement peuvent donner naissance à six formes, se partageant en deux sections, suivant que l'une des deux espèces a servi de porte-graines. Cette manière de voir est partagée par M. KLotscir. MI. NAUdin ne nie pas le fait d'une manière absolue, mais ce qu'il affirme c'est que tous les hybrides réciproques, qu'il a obtenus, tant ontre espèces voisines qu'entre espèces éloignées, ont été aussi semblables les uns aux autres que s'ils fussent provenus du même croisement.

Un point sur lequel ces hybridologistes sont d'accord, c'est que les hybrides sont des formes mixtes, intermédiaires entre celles des deux ascendants. Il arrive cependant que les hybrides ne se comportent pas toujours exactement comme je viens de le dire.

Ainsi on obscrve souvent que les descendants ont plus de caractòres de l'espèce paternelle que de l'espèce maternelle et vice versa.

M. LECOQ dit qu'en général le produit tient des deux, mais qu'il a remarqué dans un très-grand nombre de croisements que les hybrides tiennent plus des porte-graines ou de la mère que du père.

M. Grexier attribue ce singulier jeu de la nature à l'inégalité d'action du pollen qui, dans certains cas, opère une influence beaucoup plus grande que dans d'autres. Il résulte de ce fait que les descendants d'une espèce fécondée par une autre, et 
dont le pollen aura réagi avec force, se rapprocheront d'avantage au point de vue des caractères de l'espèce qui a fourni le pollen que de celle qui a servi de porte-graines.

Au contraire, si le pollen à réagi avec moins d'intensité, l'hybride se rapprochera davantagè de sa mère que de son père.

M. NAUDin croit que ces inégalités de ressemblance, quelquefois très-grandes entre l'hybride ct ses parents, tiemment avant tout à la prépondérance marquée qu'exercent beaucoup d'espèces dans leurs croisements, quel que soit le rôle de père ou de mère qu'elles y jouent. D'après les observations que j'ai faites sur les hybrides spontanés, j'ai remarqué que le port, l'aspect, la physionomie se rapprochaient plus de la plante yui avait servi de porte-graine que de celle qui avait fourni le pollen.

Cependant une très-singulière exception s'est présentée sur un hybride de deux espèces de Cirsium.

„Les Cirsium oleraceum et acaule ont donné naissance à un hybride que j'ai décrit dans les bulletins de l'académie de Belgique, 2e Série, T. XI, No. 1. J'ai considéré le Cirsinm acaule comme ayant été l'espèce qui avait servi de porte-graine. En effet l'hybride croissait au milieu d'autres Cirsium cacuule type. La tige de la plante adultérine n'était pas plus haute que celle des individus types. D'après tous les caractères l'hybride se rapprochait beaucoup plus de sa mère que de son père; aussi son nom, en adoptant la théorie de M. Grenier, devait-il être $C$. subolevaceo-acaule.

Tige de quinze centimètres environ, dressée, ferme, sillonnée, un peu aranéeuse, rameuse au sommet. Calathides au nombre de trois, grandes, solitaires au sommet des rameaux, entourées à la base de deux ou trois bractées inégales, linéaires, vertes, denticulées-épineuses, égalant les fleurs ou plus courtes. Péricline ovoïde-globuleux, non déprimé à la base, un peu concave à son point d'insertion sur le pédoncule; écailles étalées an sommet, subciliées, munies sur la moitié supérieure d'une faible nervure dorsale: les extérieures lancéolées, terminées par une courte épine; les intérieures linéaires, acuminées, terminées par une pointe molle au-dessous de laquelle elles sont un peu noiritres, scarieuses sur les bords. Corolle jaunâtre à limbe plus long que le tube. Feuilles moins épaisses que dans le C. acaule, se rapprochant pour la consistance de celles du C. olerareum, glauques à la fuce inférieure, à nervures couvertes de quelques poils, sinuées-pennatipartites ì segments étalés bi-trilobés, inégalement ciliées-spinuleuses; les caulinaires supérieures sessiles, arrondies à la base sans être embrassantes, les caulinaires inférieures et les radicales atténuées en pétiole cilié-spimuleux. La plante fut 
transportée dans mon jardin, et l'année suivante elle se montrait ayant un port singulièrement différent de celui qu'elle avait la première année. An lien d'avoir une tige haute de quinze centimètres, comme ì l'époque ou elle avait été rerueillie, elle s'est montrée haute d'un mètre cinquante centimètres, chargée de nombreuses ramifications et d'un très-grand nombre de calathides. Ces dernières étaient beancoup plus grosses que celles qui s'étaient montrées la première année. Malgré des différences d'une année à l'autre, l'hybride se montrait néanmoins avec plus de caractères du $C$. acaule que du C. oleraceum; ainsi forme et couleur des feuilles, vestiture de la tige, etc. Mais si j’avais observé cet hybride à sa seconde année de végétation, alors qu'il avait acquis cette grande élévation, j'eusse bien certainement cru à une action modificatrice du pollen très-forte, puisque l'hybride avait acquis la taille de son père; et dans cette hypothèse j'eusse baptisé ma plante du nom de C. superoleraceo-acaule, vu qu'elle rentrait dans le groupe voisin du père.

L'hybride à la seconde année présente les caractères suivants: tige de $1^{\text {m }} 50$ de hauteur, dressée, ferme, fortement sillonnée, couverte de quelques poils mous, crépus. C'alathides nombreuses, disposées en une espèce de corymbe simple, irrégulier, entourées à la base de quelques feuilles florales beaucoup plus développées que dans la plante âgée d'une année. Péricline ovoïde-globuleux, plus gros. Feuilles radicales aussi grandes que celles du $C$. oleraceum, de même consistance, mais d'un vert beaucoup plus foncé, pennati-partites, à segments pennatifides, bords fortement spinescents; les caulinaires pennatifides, à segments bi-trilobés.

La comparaison des deux plantes nous montre une singulière différence au point de vue des caractères qu'elle avait la première année et ceux qu'elle a actuellement.

Voilà donc une observation qui n'est nullement en faveur de la théorie de M. Girenier, puisque l'hybride, à l'époque de sa naissance, se rapprochait beaucoup plus de l'espèce mère que de l'espèce père, et, la seconde amnée, s'éloignait singulièrement du porte-graine pour conquérir plusieurs des caractères de son père.

En supposant qu'au lieu d'observer l'hybride l'année de sa naissance, je ne l'eusse récoltée que la seconde année, il va de soi, comme je le disais tout-à-l'heure, qu'il fallait supposer une action modificatrice très-forte du pollen, puisque lhybride se rapprochait davantage de son père que de sa mère; cependant les choses ne s'étaient nullement passées ainsi au moment de la naissance de l'hybride.

Dans mon mémoire sur l'hybridation, j'ai recherché sur les hy- 
brides des cultures quelle part de caractères arait été léguée par l'espèce prise comme porte-graine et par' celle prise comme père.

Mes differentes analyses m'ont conduit à formuler une règle à peu près générale: que les plantes hybrides et métis des jardins se rapprochaient du type paternel ynant aux organes de la reproduction, et du type maternel quant à ceux de la nutrition; la part léguée par la mère est donc plus grande. Puisque, dans la majeure partie des cas, l'hybride ressemble à son père quant aux fleurs, et à sa mère par rapport aux organes nutritifs, nous savons sur quelles données doit se baser le choix des ascendants ou parents. Je crois que la même règle est applicable pour le mariage entre deux variétés d'un même type spécifique.

En supposant que deux espèces s'hybrident, ou que l'on prenne deux variétés de même espèce, celle des deux qui présente la fleur la plus remarquable doit être prise pour père, l'autre pour mère.

Cette règle n'est applicable que dans le cas où l'on veut rechercher des corolles plus grandes et présentant des couleurs intermédiaires entre celles des parents. Si, au contraire, on recherche une modification dans le feuillage, c'est sur l'espèce ou la variété qui a servi de mère que l'on doit baser ses espérances; aussi, ne doit-on prendre pour porte-graine que les plantes à feuillage parfait, présentant toutes les conditions que l'esthétique exige. Ces différents préceptes sont rarement possibles pour la production des hybrides, mais entre variétés d'un même type ils sont communément réalisables. Si, dans l'éducation des animaux domestiques, on est si difficile sur le choix des reproducteurs, c'est que l'expérience a appris qu'avec des parents ne présentant pas tous les caractères d'animaux parfaits, on n'obtient; dans le plus grand nombre des cas, que des descendants ayant une conformation qui laisse à désirer.

Dans la pratique horticole, les choses se passent absolument de même, alors qu'on recherche des variétés nouvelles. Il s'agit ici de croisements et non pas d'hybridations. Si les porte-graines sont parfaits, si, en un mot, ces plantes portent le cachet du beau, il est à présumer que les produits seront également beaux.

Ainsi, lorsqu'on se propose d'obtenir de nouveaux métis, il faut rejeter, sans miséricorde, toutes les plantes qui ne sont point parfaites et ne prendre pour parents que celles qui sont aussi belles que possible, et surtout ne choisir que les variétés les dernières obtenues.

Si je recommande, Messieur's, de prendre pour ascendants les dernières varićtés obtenues, c'est que celles-ci sont plus sujettes à varier que celles déjà anciennes; ces deruières ont acquis une espèce 
de stabilité de caractères qu'il est plus difficilc d'ébranler que chez les variétés nouvelles.

Les plantes, comme tous les êtres vivants, sont soumises à l'habitude. Une variété que l'on vient d'obtenir dommera des graines qui produiront plus facilement des rariétés nouvelles que les graines des anciens types. Cette variété $n$ 'aura pas encore acquis cette stabilité que donne l'habitude. Ainsi nos jardins nous offrent des variétés que nous n'avons conservées qu'ì force de soins, en les maintenant toujours dans des circonstances farorables à leur stabilité, et nous leur arons enfin donné l'habitude. Tout ce qui est de création nourelle ne l'a pas encore acquise et varie avec la plus grande facilité.

Les caractères de beauté et d'élégance, qui font rechercher tant de Heurs de collection, doivent éprouver bien des améliorations par des croisements bien entendus, mais, dans tous les cas, il faut se garder d'employer des types inférieurs, c'est-ì-dire, des plantes qui ont déjà été dépassées de beaucoup par des gains nouveaux; on peut parvenir à en obtenir d'aussi beaux que ces gains nouveaux, mais on perd du temps pour y arriver; tandis que, si on les prend pour point de départ, on a la chance de les perfectionner encore.

La couleur chez les variétés est un point sur lequel je dois m'arrêter un moment. Toutes les parties pétaloïdes sont colorées par deux couleurs fondamentales, le bleu et le jaune. Ainsi le bleu passe au rouge par l'action des acides, et du mélange de ce bleu avec le rouge, en proportion variable, résulte toute une série de nuances ou de couleurs que les chimistes ont désignée sous le nom de série cyanique. Cette série de teintes peut se reproduire artificiellement ainsi, en ajoutant à la matière colorante bleue ordinaire des fleurs un acide faible, en quantité d'abord minime, puis en augmentant progressivement la proportion de l'acide jusqu'à ce que toute la matière blene soit passée au rouge. La série ayant le jaune pour base, ou série xanthique, peut également s'imiter en ajoutant progressivement an jaune une matière colorante rouge. Ainsi toutes les corolles rouges peuvent prorenir de deux couleurs types différentes, du bleu rougi par un acide ou bien du jaune par l'oxygénation du rouge de la série xanthique.

La chimie nous procure les moyens de déterminer auquel des deux rouges nous avons à faire dans telle ou telle corolle.

Le rouge de la série jaune ne passe pas au bleu lorsqu'il est mis en présence d'un alcali, la potasse par exemple, mais bien au jaune. D'après les observations de feu le professeur MAztexs, les neuf-dixièmes des corolles apparticnnent à la série xanthique. Chaque fois qu'une espèce a des fleurs rouges dérivant du jaune, il ne faut jamais espérer 
arriver à obtenir des variétés à fleurs bleues; le contraire aura lieu, si la corolle est rouge dérivant de la série cyanique.

Quelles sont les conséquences que nous pouvons tirer, Messieurs, de ces données dans la production des variétés et pent-être de quelques hybrides? Si deux espèces voisines capables de s'hybrider renferment du rouge appartenant ì la série xanthique, il ne faut pas espérer de voir se développer la couleur bleue; tandis que, si l'un des ascendants possède du rouge de la série cyanique, il sera très-possible que les hybrides auront des fleurs dans lesquelles le bleu dominera.

S'il s'agit du croisement de variétés d'une même espèce ayant des fleurs rouges dérivant des deux séries, il ne sera pas impossible que les descendants se montreront avec des fleurs tirant plus ou moins sur le bleu.

Les fleurs du Comellia japonicu renferment du rouge xanthique et du rouge cyanique. Aussi devons-nous espérer voir un jour apparaitre au monde horticole un camellia bleu; les mêmes rouges existent également dans les fleurs du rosier de Bengale. A vis aux rosomanes!

D'après les observations de M. Vimorin, il résulte que la panachure consiste uniquement dans un retour partiel d'une variété de nuance claire à la couleur fondimentale de la plante, de laquelle elle tire son origine, et que les lignes qui la constituent se montrent toujours faibles à la première génération, plus prononcées, plus larges et de ton plus foncé à la génération suivante.

Cette manière de voir de M. VILMoriv n'est pas à l'abri de toute critique, car', comme je l'ai démontré ailleurs, les choses ne se passent pas toujours ainsi. En effet, le Glowinic cuntictissimu fécondé par le G. caulescens coerulec donne des plantes toutes à corolles bleues, bien que la mère soit à fleurs du blanc le plus pur. La même variété de Gloxinia fécondée par le G. grandiftora rubre donne naissance à des variétés toutes à fleurs blenes.

Dans les expériences de croisement entre les variétés de Mirabilis faites par M. LeCoQ, nous voyons que sur six cents semis provenant de six pieds, dont trois unicolores et trois panachées, trois on quatre seulement sont à fleurs blanches. Les faits acquis à la suite d'expériences tendent à prouver que la plupart des variétés à fleurs panachées, dès qu'elles se montrent entièrement constantes pendant un temps assez long, étant nultipliées par graines, domnent ì leur tour naissance à d'autres variétés panachées, mais il y en a beaucoup qui résistent à tons les efforts ‘u'on fait pour les fixer et les rendre susceptibles de se reproduire.

Ainsi, au total, Messieurs, nous voyons qu'il règne encore 
beaucoup d'obscurité relativement à la couleur des fleurs et nous ne saurions trop recommander aux horticulteurs de chercher à soulever le voile dont la nature a couvert le phénomène de ces remarquables variations de couleur. Il est inutile, je crois, Messieurs, de vous dire tout ce que l'horticulture a encore à attendre des hybridations et de croisements faits judicieusement. Ce n'est pas uniquement la floriculture seule qui doit mettre en pratique ces moyens modificateurs; la culture maraîchère, la pomologie et la sylviculture ont beaucoup à faire en fait de croisements. La culture fruitière surtout est loin d'avoir dit son dernier mot, quant à la prodnction des variétés nouvelles. Si les fécondations artificielles n'ont pas été pratiquées jusqu'à ce jour sur une grande échelle, par la main de l'homme, elles l'ont été par l'intermédiaire des insectes, qui, à l'époque où les fleurs de nos poiriers, pommiers et autres arbres fruitiers sont épanouies, roltigent de fleur en fleur pour y récolter les matériaux nécessaires à la confection du miel et de la cire. Tous ces hyménoptères au corps velu sont les messagers du pollen; ce dernier est transporté de variété à variété, et j’ose avancer, que cette énorme variation dans nos fruits résulte de fécondations croisées.

Faisons comme les insectes, mais avee discernement; choisissons les porte-graines et les variétés pour récolter le pollen, et de semblables mariages nous produiront de nouvelles variétés sur lesquelles nous serons en droit de formuler les plus belles espérances.

La production des variétés de garde dans les poires et les pommes doit être le point de mire après lequel nous devons viser. Choisissons nos ascendants parmi les variétés les plus tardives, et il est à supposer que les descendants à attendre auront au moins une époque de maturité analogue à celle des parents, peut-être sera-t-elle retardée. En admettant que les variétés obtenues mûrissent à une époque analogue à celle des parents, on aura toujours gagné quelque chose de bon, nous posséderons une variété tardive de plus. Or, comme ces dernières sont en nombre très-limité, les résultats acquis auront bien leur mérite.

J'ai captivé assez longuement votre attention, Messieurs, sur ce sujet, pour que les amateurs et les horticulteurs se persuadent bien qu'il y a encore énormément à faire en fait de croisements, tant entre espèces qu'entre variétés de même espèce.

Ces opérations constituent une source féconde de plantes intéressantes à la fois au point de vue de la science et de l'horticulture. 
EXTRACTS FROM COMMUNICATIONS OF THE EXPERIENCE OF Thomas Bowley ESQ. OF DUBLIN WITH DOUBLE GLASS HOUSES.

COMPILED $\mathrm{BY}$

Mr. ROBERT WARNER.

(Voir le Compte Rendu de la Séance du 10 Avril 1865, pag. 312).

'The advantages of double glazing are at least fourfold.

Before we proceed to describe them we will state in what the principle of double glazing consists.

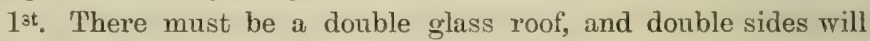
carry out the principle more completely.

$2^{\text {nd. }}$ It is absolutely necessary that the space between the two glass roofs should be perfectly air tight, as this encloses a body of air which becomes a non-conductor of heat or cold.

The roof is constructed with ordinary wooden rafters 4 feet apart, with 3 light iron sashes between. The inner glass is commonly 21 inches per foot; it is put in about one inch on the underside of the rafter, so that the rafter being about 6 inches depth, 5 inches of rafter. is outside the inner' glass. On these projecting rafter's the outer roof rests. The outer roof consists of simple framed sashes 4 feet wide, and for the convenience of moving are made in two lenghts. They are glazed with the same glass as the sides, viz. fine ribbed glass called Hartley's rough plate. These simple sashes are fixed on the rafters by screw bolts, so as to be easy to move. Thus we have the inner and outer glass 5 to 6 inches apart. The distance is not important either 4 to 6 inches will do, but it is of the utmost importance that they fit close so that there should be no circulation of air between. The glass is carried up to within 1 foot of the top of the ridge, which foot of space is fitted with wooden shutters for ventilation.

The Fernhouse is 48 feet long and 60 feet wide and 21 feet high, 2 rows of 4 inches pipe is sufficient to heat all this large body of air.

\section{Advantages.}

16t. Prevents any excess of heat by day.

$2^{\text {nd }}$. Affords a great protection from cold by night. 
$3^{\text {rd }}$. Prevents aridity consequent upon radiation and condensation in bright cold weather either day or night.

$4^{\text {th }}$. Almost total cessation of anmoyance by insects.

\section{Examples of advantages.}

1st. In hot summers the heat has never gone up higher than $70^{\circ}$ with a single roof, only one hot morning would have run it up to $85^{\circ}$.

2 nd. The cubic contents of the house is 46,000 feet. The heating power is only 430 feet of 4 inches pipe or about, 1 foot of pipe to every 100 cubic feet of air. This quantity of pipe in a single roofed house would only just keep out frost, but with the double roof it rarely goes down below $48^{\circ}$. The estimate is a gain of $14^{\circ}$ of heat. The loss of heat is very slow in cold weather. It would take 3 nights of severe frost to bring the heat down from $52^{\circ}$ to $48^{\circ}$ and probably 36 hours to cause the same reduction if the fires were to go out entirely. With a single roof this reduction would take place in 6 hours. It is a great comfort to know that if the fire was neglected or an accident happened to the boiler in winter for a whole night that the plants would receive no damage.

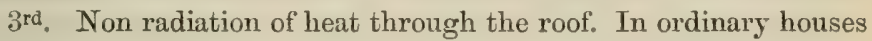
where moisture forms a requisite of good plant growing, no matter how freely we use the syringe in the evening, we find the house dry in the morning, arising from the great condensation of moisture on the roof and running off when thus condensed. With double roof there is very little condensation on the roof, the blanket of nonconducting air between the two roofs of glass preventing it; and consequently as the air cools down a little in the night, the condensation takes place on the plants themselres, exactly according to nature. The plants in the orchid house which has a double roof and sides are in the morning beautifully covered with dew like a plot of cabbages in a May morning. Is not this the perfection of plant growing? There is another fact connected with non radiation of great importance in plant growing. It is the curious fact, that there is a steady uniform increase of temperature in the honse according as we ascend from the floor to the roof, day and night. Thus in the Fernhouse hardy ferns and temperate plants grow below, while tropical ferns, plants and palms grow near the roof. In ordinary houses we remore tender plants from near the glass in severe weather; but in this double roofed Fernhouse the nearer they are placed to the glass the warmer they are.

The saving of fuel is 20 percent per annum, so that the extra cost of the doulble roof is soon paid for in fuel alone, but this a trifle compared to the enhanced value of the plants grown in a double roofed 
house. The effect is astonishing and would surprise plant growers in general.

Caution is needful not to give so much water as is done in single roofed houses.

$4^{\text {th }}$. Almost total cessation of insects. Formerly these pests abounded with me; now they rarely appear, and when they do, they are few in number and are easily got rid of. From this fact it may be inferred that when plants are grown in a condition of atmosphere favourable to perfect health, they are but little liable to attacks by insects which often spoil their beauty.

A remarkable instance of the value of double roofs to stove and orchid houses occurred in January 1864. We had 4 days and nights of hard frosts, the thermometer not rising to $32^{\circ}$ by day and falling to $18^{\circ}$ at night. Just as the frost began an accident happened to the heating apparatus and all the water ran out of the pipes and the accident could not be repaired until after the 3rd night of frost. The temperature was $56^{\circ}$ in the morning when the fire was discontinued; 24 hours after this, the reduction of heat was only $3^{\circ}$. The following morning or after 48 hours, only $3^{\circ}$ more, and in the 3 rd night $2^{\circ}$ more were lost: thus making a reduction of only $8^{\circ}$, viz. from $56^{\circ}$ to 48 , in 72 hours. Nothing was injured, which is a striking proof of the value of double glazing in winter, for without it, every plant would have been frozen and the loss would have been immense. With double glazing, gardeners and employers may in severe weather sleep in peace.

Another year's experience has fully confirmed all the adrantages before narrated.

A market-gardener at Colney Hatch in England has for some 3 years tried the system, for growing early cueumbers for Covent Garden-market, with great saving of fuel.

In Russia the system has long been used for planthouses.

I will conclude by enquiring whether in other continental countries the system has had a fair trial and with what results? 


\title{
OVER DE ORANJEBOOMEN EN ERICA'S,
}

\author{
DOOR
}

\section{J. M. KRAAIJENBRINK.}

(Voir le Compte Rendu de la Séance du 10 Avril 1865, pag. 359).

\section{De Oranjeboomen.}

De oorzaken op te sporen van de ongunstige resultaten, die wij bij de kweeking der Oranjeboomen waarnemen, zal wel niet tot het onmogelijke behooren, aangezien onze vroegere tuinlieden on bloemisten humne zorgen ten opzigte der kweeking van genoemde boomen altijd met den besten nitslag bekroond zagen. Daar dit thans het geval niet meer zoo is, rees deze vraag bij mij op, en ik achtte ze gewigtig genoeg; om ze op dit Congres behandeld te zien.

Ziet hier, Mijne Heeren, de oorzaken die, naar het mij voorkomt, de teelt der Oranjeboomen geheel en al tegenwerken. In de laatste 30 jaren zijn er een aantal planten in ons land ingevoerd, waaran om haar vreemden, nieuwen en zeker ook schoonen vorm, dubbele zorgen zijn gewijd, terwijl aan de Oranjeboomen niet die zorgen konden besteed worden, waarop zij zoo billijk aanspraak hadden; en wanneer het najaar aankwam, zagen zij de plaats, die vroeger alleen foor hen besteund was, grootelijks ingenomen door die planten, welke, om hare nieuwheid, meer de aandacht trokken en meer zorg wekten, terwijl tevens de atmospheer, waarin zij behoorden te leven, geheel en al gewijzigd werd naar de planten die hen verdrongen.

Als middel om dien toestand te rerbeteren, komt het mij dienstig voor, den Oranjeboom ran stek te kweeken, dewijl de ondervinding leert, dat zulke boomen gezonder blijven dan die waarbij men tot onderstam een citroen gebruikt.

Als voorbeeld zou ik u kunnen wijzen, Mijne Heeren, op de Oranjeboomen van Z. M. den Koning op het Loo, waarvan een groot aantal van stek gekweekt zijn. Geloofwaardige getuigen, die sinds ruim veertig jaren deze boomen behandeld hebben, kunnen bevestigen, dat de gezondheidstoestand dezer boomen niets te wenschen overlaat.

Uit dien hoofde ook heb ik er vijfentwintig te Amsterdam ten toon gesteld, om het geëerde publiek te overtuigen wat er van een stek kan groeijen. 
2. De Erica's.

Voornamelijk moeten wij weder, naar ons inzien, de aandacht vestigen op de min gunstige resultaten die de kweeking van deze plant oplevert, en eveuwel is het bij ondervinding gebleken, dat bij eene goede behandeling de resultaten gunstig te noemen zijn.

Niemand zal willen tegenspreken dat de Erica's, bij eene goede behandeling, de mecste gewassen overtreffen in het genot dat zij aanbieden, daar zij bij eene ruime sortering ons het geheele jaar door bloemen toonen; doch het is te betreuren, dat voor dit gewas zoo weinig liefhebbers worden gevonden. Dat de Erica's niet geschikt zijn, om vele dagen met hare bloemenpracht in de salons te prijken, zal ook wel een van de oorzaken zijn.

Eene zaak die, naar het ons voorkomt, de kweeking der Erica's grootelijks in den weg staat, is deze, dat wanneer het geval zich opdoet dat sommige soorten wegsterven, zoo als menigmaal gebeurt, men ze niet of zeldzaam kan terug bekomen.

Alle soorten van planten vindt men bij handeldrijvenden of kweekers, maar te vergeefs zoekt men die soorten van Erica's, welke een lief hebber zoo gaarne zou willen aankoopen, en wilde bij aan zijn lust voldoen om deze soort te bezitten, hij zoude zich gedrongen zien ze uit Engeland te ontbieden.

De weinige prijzen op tentoonstellingen uitgeloofd roor dit gewas schijnen ons ook daartoe bij te dragen. 


\title{
DISPOSITIO MELASTOMACEARUM,
}

\author{
AUCTORE
}

\section{J. TRIANA.}

(Voir le Compte Rendu de la Séance du 8 Avril 1865, pag. 237).

TRLBUUM CONSPECTUS.

ptis in Nelastomate et Otanthera, in quibus

est baccatus.

utica, v. rariusapicelaciniatav. setosa, exangulata. recta v. subrecta; - ovoidea, ovoideoangulata $\mathrm{v}$. subpyramidata.

Connectivum inconspicuum aut saepius infra loculos productum, et ultra insertionem filamenti antice in appendicem simplicem v. lobatum porrectum .........................

curvata; - cochleata v plus minus mani-

Neogea. 1. Microlicieat. feste reniformi-cochleata (in Rhexia glabella subpyramidata).

Connectivum ut plurimum infra loculos plus minus productum, cum filamento articulatum, ad articulationem saepissime antice varie appendiculatum

$\{$ Neogea. 2. Pleromeat. Connectivum postice in caudam porrectum

recta v. subrecta: - oblongo-pyramidata, pyramidata, scobiformia, linearia v. marginato-alata.

Connectivum postice basi in calcar saepius acutum $\mathrm{v}$, in caudam filiformem deorsum terminatum, interdumque appendicem dorsalem sursum expectantem gerens apice lobis latis, obtusis, membranaceis coro-

S Neogea. 5. Merianieaf.

Neogea. 4. RHEXIEAE. nata, saepius triquetra, angulata, v, subangulata.

Semina recta v. sulurecta, non cochleata... $\{$ Paleogea. 7. Sonrrileat. baccatus. Semina nunquam cochleata, recta. Connectivum $\{$ Neogea. 8. Bertolonicear. parum v. non productum, rarius appendiculatum. Antherae apice $1-2$, rarius 4 -porosae, rarissime $1-2$ rimosae.

Flores saepissime panieulati, non involucrati, antherae liberae

S Paleogea. 9. Dissochaeteat.

Flores axillares, pedicellati, bracteis 4 saepius peta- $\{$ Neogea. 10. Mrconiteat.

loideis, decussatis involucrati. Antherae connatae. Neogea. 11. Prxidanteae.

Fructus 2-4 locularis, drupaceo-baccatus, oligospermus, cotyledonibus magnis plano-convexis.

basilaris v. sublateAntherae rimulis duplicibus lateralibus v. poris apicalibus dehiscentes ralis.

Fructus plurilocularis, subbaccatus, irregulariter ruptilis, polyspermus. Semina parva, recta.

Antherae longitudinaliter 2-rimosae centralis. Fructus baccatus, 1-spermus. Embryonis cotyledones foliaceae contortuplicato-convolutue.

Antherae longitudinaliter 2-rimosae

Neogea. 12. Mouririeat.

Paleogea. 13. Astronieaf.

Paleogea 14. MEmecrleae. 


\section{MELASTOMACEAE.}

Genera et Synonyma.

1. MICROLICIEAE.

Flores-meri :

4. Castratella.

5 Eriocnema.

5 Pyramia.

5 Cambessedia.

5 Chaetostoma.

5 Svitramia.

4 Bucquetia.

6 Stenodon.

4 Meisneria. Syphanthera.

5 Microlicia.

5 Lavoisiera.

5 Rhynchanthera.

5 Trembleya.

4. Centradenia. Plagiophyllum.

5 Poteranthera. Onoctonia.

4 Tulasnea.

4. ?Lithobium?

2. PLEROMEIAE.

4-5 Acisanthera.

Dicrananthera.

Uranthera. Notherophila.

4. Ernestia. Dichaetandra.

4 Nepsera.

5 Desmocellis.

5 Ancistrodesmus.

4. Appendicularia.

4. Heteronoma.

Arthrostemma R. et Pav.

4. Heterocentrum.

4 Heria.

Schizocentrum.

4 Pterogastra.

4. Mecairia.

4 Arthrostemma DC. non $R$, et $P$.
Flores-meri :

4 Comolia.

Hostmannia.

Leiostegia.

Pachyloma.

Tetrameris.

5 Purpurella.

5 Pleroma. ref.

Lasiandra.

Chaetog. sp. Nnd.

Hephestionia.

Oreocosmus.

Micranthella.

4-5 Chaetogastra. refor.

4 Chaetolepis.

Happlodesmium.

Trimeranthus.

4 Marcetia.

4 Fritzchia.

4 Aciotis.

Spennera.

3. OSBECKIEAE.

4. Noterophylla.

4 Antherotoma. H.f. - g.n.

4-5 Osbeckia.

5 Otanthera.

Lacnopodium.

5 Tristemma.

5 Melastomastrum.

5 Dinophora H. f. - g. n.

5 Guyonia.

5 Melastoma.

5 Dissotis.

Osbeckiastrum.

Heterotis.

Lepidanthemum.

Argyrella.

4. Dichaetandra. 
4. RHEXIEAE.

Flores-meri :

4 Rhexia.

4 Monochaetum. Grischowia. Loevigia.

4 Urodesminim.

5. MERIANIEAE.

gen. n. 4 Acanthella Hook. f. g. n.

6 Behuria.

4 Huberia.

5 Centronia.

Brachycentrum.

Calyptraria.

Stephanogastra.

5 Axinaea.

Chastenaea.

5 Meriania.

Schwerinia.

Pachymeria.

Davya.

Adelbertia.

Notocentrum.

5 Adelobotrys.

4-5 Graffenrieda.

Cycnopodium.

5 Calyptrella.

4. Opistocentra H. f. $-\mathrm{g} . \mathrm{n}$.

6. OXYSPOREAE.

4 Oxyspora.

Homocentria.

Hylocharis.

Allozygia.

4. Barthea H. f. - g. n.

4 Allomorphia.

4. Roussauxia.

4. Blastus.

5 Orthocharis.

5 Veprecella.

4. Dionychia.
Flores-meri :

5 Anerincleistus.

4. Bredia.

4 Driessenia.

4 g. $n$.

7. SONERILEAE.

4 Phyllagatis.

4 Sarcopyramis.

5 Gravesia.

5 Amphiblema.

5 Calvoa Hook. f. - g. n.

3 Sonerila.

8. BERTOLONIEAE.

$5 / 3$ Bertolonia.

5/3 Salpinga.

5/3 Triolema.

5/3 Diolema.

5/3 Macrocentrum H. f. - g. n.

5/3 Diplarpea Tr. - g. n.

9. DISSOCHAETEAE.

4 Dalenia.

4. Dissochaeta.

Aplectrum:

4. Marumia.

Macrolenes.

Ptelandra.

5 Sakersia H. f, $-\mathrm{g}, \mathrm{n}$.

5 Dikellandra $\mathrm{H}$. f. - g. $\mathbf{n}$.

4 Creochiton.

4. Omphalopus.

5 Dactyliota.

$4-5$ Medinilla.

Erpetina.

Diplogenea.

Hypenanthe.

Triplectrum.

Pogonanthera.

4. Pachycentria.

6 Carionia. 
10. MICONIEAE.

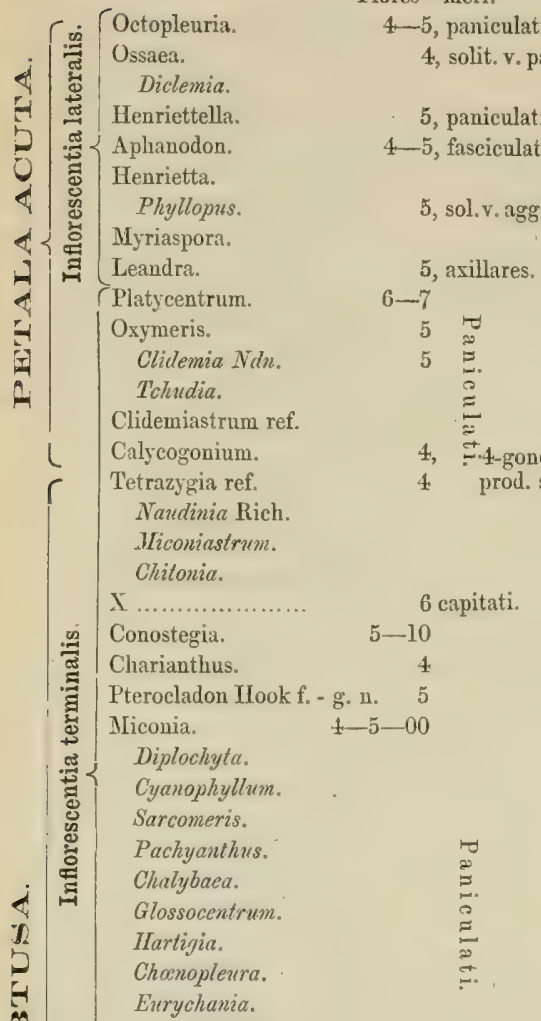

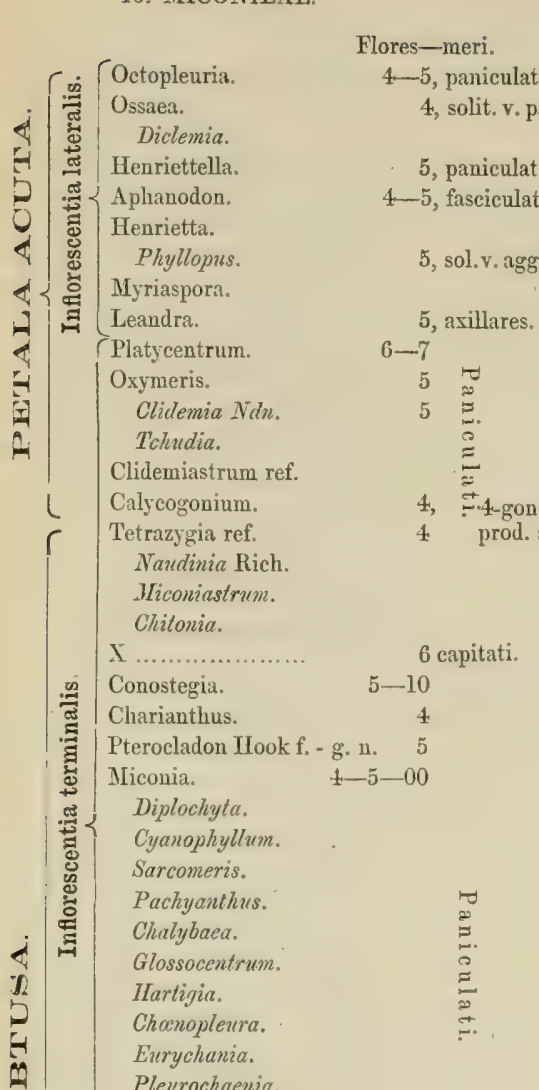

Flores-meri.

Calyx.

Antherae.

\section{Catachaenia.}

Schizanthera.

Tocoea.

Sphaerogine.

Pleiochiton.

Heterotrichum.

Octomeris

Capitellaria.

Æ $\begin{aligned} & \text { Clidemia Don. } \\ & \text { Staphidium } N d n \text {. }\end{aligned}$

Sagraea.

Staphidiastrum.

Calophysa.

Microphysa.

Maieta.

Mirmidone.

Loreya.

Bellucia.

Axinanthera.
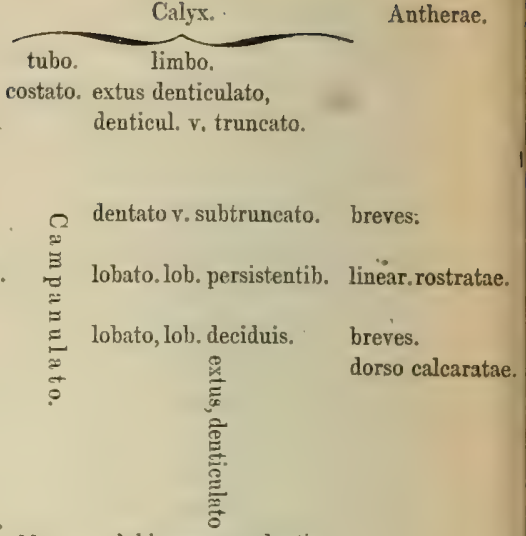

productis
subulatis.

lobato. lob. deciduis.

calyptriform. circumscisso.

alato.

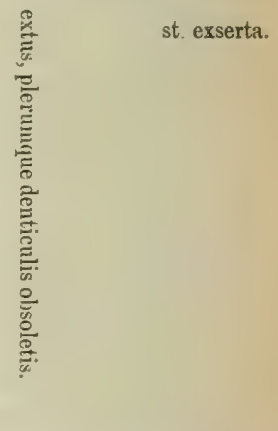

lob. ext. plerumque subulatis.

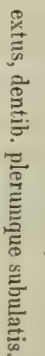

teapitati, involucrati.

5

4 节

$\Omega$
$\vdots$
$\vdots$
$\vdots$
$\Xi$
$\Xi$
$\vdots$
0
0

5 sol. v. 3 . involucrati.

6 solit. v, 3.

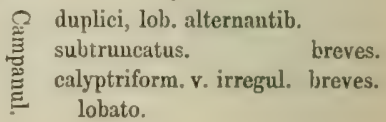

5 cymoso-paniculat.

5 cymoso - panicul lobato. 
11. PYXIDANTEAE.

Blakea.

Pyxidanthus.

Topobea.

12. MOURIRIEAE.

Mouriria.

Petalomu.

Guildingia.

Olisber.

13. ASTRONIEAE.

5 Astronia.

4 Pternandra.

Ewyckia.

Macroplacis.

Rectomitria.

4 Astronidium.

¿ Kibessia.

4 Naudinia Decas. mss.

14. MEMECYLEAE.

5 Nemecylon.

Spathandra.

Lijndenia. inflorescentia terminalis.

" axillaris.

" terminalis.

" axillaris. calyce extus muricato.

" terminalis. calyce calyptriform. antherae breves,

$n$ subulatae.

Genera dubiz, non satis nota.

Leucymmaena, Cymenolema, Aupetalum, Diplodonta. 


\title{
MÉMOIRES PRÉSENTÉS AU SECRÉTARIAT.
}

\author{
DU JARDIN BOTANIQUE DE BRESLAU ET DE SON \\ ORGANISATION INTÉRIEURE,
}

PAR

M. le Prof. GOEPPERT,

DIRECTEUR DU JARDIN BOTANIQUE, À BRESLAU.

1. Coup d'veil général.

En jetant un coup d'ceil sur les jardins botaniques de l'Europe, on n'en trouve qu'un petit nombre qui remplissent véritablement leur but. Celui-ci est de contribuer, par la facilité d'observation sur une vaste échelle, à l'avancement de toute la botanique, d'offrir aux étudiants en médecine et en pharmacie des matériaux suffisants pour leurs études dans cette belle science et de répandre en même temps l'instruction sur ce sujet parmi le public instruit et éclairé.

Les jardins botaniques de notre époque laissent encore beaucoup à désirer sous ces rapports. C'est ainsi qu'on n'y trouve sourent pas d'arrangements pour mettre en vue les plantes médicinales et importantes dans l'industrie. Que l'on n'objecte point que quelques. unes d'entre elles soient difficiles à acquérir. Aujourd'hui les rapports et les communications entre les diverses parties du globe sont trop bien établies, pour offrir sous ce rapport des difficultés sérieuses. Il n'existe, en outre, point d'ouvrage à l'usage des étudiants pour les instruire sur les plantes que renferme un jardin botanique, incovénient sérieux qui, à la vérité, trouve sa cause dans les progrès rapides de notre science.

Il est donc essentiel d’améliorer les jardins botaniques, de façon que les études puissent être non seulement facilitées, mais encore adaptées aux besoins et à la hauteur scientifique de notre époque. C'est ce que nous arons essayé à réaliser dans le jardin botanique de Breslau, comme le démontreront les détails qui vont suivre.

Le jardin botanique de Breslau renferme, sur une étendue de 26 arpents de Prusse (6 hectares 65 ares), environ 12,000 espèces de plantes classées d'après les familles naturelles, avec égard au facies, 
à l'habitus des plantes. Nous ne pouvons point, en effet, approuver la méthode de ranger les plantes d'un jardin botanique d'une manière trop absolue d'après le système seulement, et de placer par exemple, ensemble et à côté les unes des autres, les Légumineuses et les Rosacées herbacées avec celles qui forment des arbres et des buissons. Nous nous sommes contentés de n'y planter que des représentants des divers types et de les grouper d'après leur port.

Pour obtenir cependant un coup d'oeil général sur la végétation de la surface de la terre', nous avons essayé, depuis neuf ans, de grouper ensemble en pleine terre des plantes qui présentent tout un ensemble de végétation, en réalisant ainsi une des idées de notre immortel vox HuxBoLDT, qui attachait une si grande importance à la physionomie de la végétation. Nous arrangeons actuellement chaque été 84 groupes de ce genre, et un tableau synoptique, placé à l'entrée du jardin, en renferme les détails essentiels. Auprès de chaque groupe se trouve, en outre, un tableau plus petit qui renferme ses genres principaux; 56 de ces groupes se rapportent aux principales formes des plantes de toute la terre, et 26 à l'ensemble des plantes d'un pays ou d'une zône. Parmi les premiers se trouvent des groupes de Mousses, de Lichens, de Fougères dès zônes froide, tempérée et chaude, d'Aroïdées, d'Orchidées, de plantes grimpantes de toute espèce de famille, de Liliacées arborescentes, de Graminées des diverses zônes, y compris celles en forme d'arbre, de Bananes, de Zingibéracées, de Cannacées, diverses espèces et formes d'Ananas, d'Agave, de Begonia, de Casuarinées, de Palmiers, d'Ericées des diverses zones, de Conifères de l'hémisphère boréal et de l'hémisphère austral (les Abietinées, Cupressinées, Podocarpées, Taxinées), de Cycadées, de Protéacées, de Myrtacées, de Cactées, de plantes grasses (Crassulacées, Aïzoïdées, Mesembryanthemées, Aloïnées, Agavées, Stapéliées, etc.), d'arbres verts des diverses espèces et contrées, comme les arbres verts à feuilles qui tombent en automne, de la zône tempéréc de l'Europe, de l'Asie et de l'Amérique, de la zône temperée plus chiude de l'Europe et de l'Amérique, à feuilles simples ou composées; ensuite, d'arbres verts qui gardent leurs fenilles toute l'année, de la zône tempérée d'Europe, d'Amérique, d'Asie, surtout de la Chine et du Japon, des Alpes, de l'Asie subtropicale, de l'Himalaya, de la zône torride. Puis viennent des groupes d'Acacia, de Nimosa et de plantes de leur tribu, d'Ombelliféres, de Malvacées, de Solanées, etc.

Pour donner un aperęu général des formes des plantes des divers pays et des diverses zônes dans leur ensemble, il y a des groupes de plantes de la zône arctique, subaretique, de plantes des 
Alpes, dont nous cultirons environ $4(n)$ espèces; puis des groupes de plantes de l'Europe méridionale, de l'Amérique du Nord, du Mexique. des tropiques, du Chili, des iles Canaries, du cap de Bonne-Espérance. de la Chine, du Japon, de l'Australie, ete. Tous ces groupes et ces aspects de la végétation d"un pays pournont être beancoup plus com. plets ailleurs, selon la richesse des matériaux et selon les localités.

Dans notre jardin, l'étiquette de chaque plante porte non-seulement son nom systématique, mais aussi celui de la farnille, du pays, de son emploi médicinal ou technique, ainsi: Rhosades, Crucifercie: Cochlearia officinalis $L$. Offic.: Hb. Cochleariae. Europ. septentrinn. litore. En outre, pour les plantes cultivées en plein air, chaque famille possède un tableau spécial et synoptique, ce qui aide considérablement l'euseignement et l'instruction, et mérite, saus contredit, d'être appliqué dans d'autres jardins botaniques.

Bien que, comme nous l'avons dit, le système naturel ait servi de base et de norme à tous les arrangements, il a cependant paru désirable de trouver un moyen de donner' un coup d'oeil d'ensemble, rendu plus difficile par la grande étendue de quelques familles.

Pour atteindre ce but, 400 plantes dans des pots ont été placées sur les plates-bandes du jardin, représentant toutes les familles importantes; cet arrangement s'est montré très-instructif. Une disposition toute semblable a été appliquée aux serres du jardin; dans chaque division un tableau synoptique en rend compte. Des arbres officinaux toxiques, des plantes importantes pour l'industrie, ainsi que des plantes de tout climat et de toute famille sont groupées, autant que possible, de façon que pendant l'hiver aussi on puisse étudier, grâce à leur concentration et à leur xéunion, ce qui en été se trouve disséminé dans tout le jardin.

Les caractères anatomiques et physiologiques des plantes ne méritent pas moins une attention toute particulière. Dans ce but nous avons réuni toute une section physiologique, entourée de buissons protecteurs, destinée à montrer les détails de l'accroissement normal et de l'accroissement pathologique des arbres. Sur les lieux mêmes, des tableaux accompagnés de dessins rendent compte de chaque arbre, au nombre total de 60 . Un coup d'oeil général en donnera l'idée. L'accroissement normal est représenté en partie par des tables, en partie par des coupes verticales de chênes qui ont de 164 à 500 ans (Quercus pedunculata) et de sapins ronges (Rothtanne) Pinus Abies et P. Picea L. Parmi ces derniers, il y a une coupe d'un tronc provenant du Böhmerwald à 3500 pieds au-dessus du niveau de la mer, d'un sapin qui avait eu 175 pieds de hauteur avec 507 anneaux aunuels, pris à $5 \frac{2}{2}$ pieds au-dessus du sol et de 14 pieds de circon- 
férence. Un autre tronc, provenant de la même localité, offre 448 anneaux annuels de bois; ce tronc avait eu 186 pieds de longueur; un troisième, de la Heuscheuer en Silésie, offie 395 anneaux.

L'accroissement anormal est représenté par des soudures linéaires, tordues en forme d'anse, de rameaux de hêtres rouges et blanes, de troncs de chêne, de sapins, de tilleuls, par des soudures de racines de trones de 100 ans. Vient ensuite l'accroissement à la manière des pandanes et des palmiers, des sapins rouges et blanes, dont les troncs, supportés par des piliers de 4 à 5 pieds de haut, sortent de terre et ont poussé partout des racines aériennes; on y voit la formation des reines et des noeuds du bois sur une vaste échelle, à droite et à gauche des trones tordus, des lésions produites par les insectes, des formations remarquables de champignons, etc. Au milieu de cette partie se trouve debout, commeil a été trouvé, un trone fossile de Pinites protolarix Goepr. de 36 pieds de circouférence, provenant de la couche de lignite (Braunkohle) de Saurau en Silésie; l'intérieur est creux, mais d'après ce qui existe encore, il doit avoir eu de 4 à 5000 anneaux. Autour de ce tronc, unique dans son genre ainsi que dans son intérieur creux, se troure une plantation des fougères de nos jardins et de celles qui vivent en plein air dans les contrées septentrionales des deux hémisphères, et qui ornent tout ce groupe d'une manière fort agréable ì la vae. Dans le voisinage de ce trone s'en trouvent deux semblables de 8 pieds de hauteur sur 2 d'épaisseur, trouvés dans la même localité; ils sont encore pourvus de nombreux rameaux et conformes an trone plus volumineux sous le rapport anatomique; on peut les regarder comme les rameaux de cet arbre gigantesque. Tout près se trouvent encore deux autres troncs aplatis de 6 pieds de haut, contenus par des anneaux de fer appartenant au genre Cupressinoxylon ponderosum GoEPP.

A côté se trouvent sur des piédestaux un tronc pétrifié par du pyrite de fer et un autre par de la calcédoine, ainsi que des masses terreuses de houille brune. Tout ce groupe donne une idée des diverses manières d'être et des divers degés de conservation des végétaux de la formation de la lignite (Braunkohlenformation). Comme le trone principal ne renferme point de sulfure de fer, sa conservation, malgré l'injure du temps, parait d'autant plus assurée que neuf hivers en partie très-rigoureux et autant d'étés pluvieux n'ont pu l'endommager en aucune façon.

Nous avons représenté avec tout autant de soin dans le jardin botanique l'époque houillère ancienne, si importante en Silésie à cause de sa grande richesse en honille du commerce. Dans ce but nous avons fait dresser un profil de toute cotte formation dans nne 
roche de porphyre soulevée par du granit. La roche porphyrique est traversée par deux conches de houille de 1 à $1 \frac{1}{2}$ pied d'épaisseur. renfermant daus leurs rapports naturels les plantes qui ont composé la houille, comme les Conifères, les Sigillaria, les Lépidodendrées, et cela dans des exemplaires comme aucu musée paléontologique r'en possède. La longueur de ce profil, représenté par un mur solide, disposé en forme de terrasses, fait arec 22,100 pierres de construction, est de 9 à 12 pieds'sur 60 pieds de hanteur", la hauteur du cône de porphyre, à partir de la base de toute cette partie, est de 25 pieds. La surface de toute cette représentation du terrain houillier ancien, plantée d'Abiétinées, de Cupressinćes, de Fougères et d’Équisétums, parsemée de grands trones de ces plantes fossiles ayant jusqu'à 10 pieds de haut, est de 3/4 d'arpent de Prusse (de 19 à 20 ares). Le poids de toutes ces masses de pierre est de 4000 quintaux.

\section{Arrangements spéciaux.}

Nous venons de passer en revue tout ce qui se rapporte en fait d'arrangements à l'enseignement théorique dans son ensemble. Il nous reste à parler de ceux qui se rapportent plus particulièrement à la médecine et à la pharmacie.

II nous importait de réunir, non seulement les plantes actuellement contenues dans nos pharmacopées d'Europe, mais aussi d'autres plantes intéressantes sous le rapport physiologique, chimique, industriel, historique ou sous yuelque autre rapport impoitant.

Nos efforts ont été couronnés de succès en ce sens que de 900 plantes officinales, encore actuellement prescrites par les médecins, 780 se trouvent dans notre jardin, et les 120 qui manquent, aucun jardin botanique de l'Europe ne les possède.

Nous cultivons en outre 1800 espèces de la seconde catégorie, de façon que le nombre des plantes intéressantes sous l'un des divers rapports meutionnés, est de 2500 dans le jardin botanique de Breslau. Comme nous l'avons déjà dit plus hant, toutes sont étiquettées d'une manière complète avec noms de famille, geure, espèce, localité, patrie, avec indication même de leur emploi et des termes officinaux et techniques sous lesquels on les connait plus généralement, par

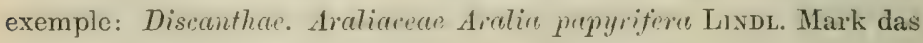
chines. Sammtpapier, China.

Bien qu'aucun jardin n'offre une pareille collection, elle me parait cepeudant encore insuffisante pour son but pratique, si l'on ne joint pas à l'aspect de la plante celui de son produit. C'est pour' cela que nous arons fait placer à còté des plantes en plein air leurs produits 
dans des exemplaires bien caractérisés, renfermés dans des verres fermés et étiquettés, soit sur des étagères de fil de fer, soit sur d'autres piédestaux, Nous avons étendu cette même méthode d'arrangement aux produits techniques; tels que matières pour la teinture, les tissus, etc., aimsi (qu'aux fleurs et aux fruits de plantes tropicales rares (Myristica, Caryophyllus, Theobroma, Cinchona, etc.), et enfin aussi aux représentants des familles et des geures, tous renfermés dans des bocaux. Nous avons ainsi établi un musée botanique de près de mille exemplaires, comme on n'en a placé nulle part, dans le proche voisinage des plantes correspondantes. Parmi ce nombre il y a des objects d'une grande rareté qui seraient l'ornement de tont musée. Nous avons eu le bouheur de ne pas en avoir perdu jusqu'à présent un seul exemplaire, tellement leur conservation a été faite avec soin. La population de Breslau a su apprécier l'abondante matière d'instruction qui est ainsi offerte à chacun, et pendant la bonue saison le jardin est constamment fréquenté par un public très-nombreux.

Nous savons bien que des jardins botaniques munis de dotations financières plus considérables pourraient fournir quelque chose de plus parfait, mais nous nous estimerions bien heureux déjà, si notre exemple était suivi et si nous pouvions avoir donné lieu à généraliser ce genre d'arrangement si favorable à une instruction toute pratique.

Nous ajouterons la liste de quelques plantes officinales rares:

Homantlus towicains dont, d'après Livingston, les Boschjes. manns se servent pour empoisonner leur's fièches en mélangeant le suc de l'oignon de cette plante avec le sue de l'Euphorbia arborescens qui ressemble à un Cactus; Vanilla planifolia ANDr. et $V$. guyanensis Spurg., qui fournit aussi bien la vanille du commerce que la Vanilla womatica.; Copenicia cerifere Ht. Amstel., qui fournit la cire du commerce, connue sous le nom de Carnauba.; Borassus flabelliformis, le principal palmier usuel dans les Indes orientales; Calamus verns Louk, une des plantes principales qui foumissent le sang-dragon. Chamacrops excelsc Tuunb. du Japon qui, en Angleterre, peut rester en plein air; les Palmier's-choux du Brésil, le Cocos oleracea Mart. et l'Euterpe olerace Mart.; le vrai Sabal Palmetto Lodo., plante rarement véritable; le Pandanus reflezus, encore peu répandu (Doruic reftexa DE VRIESE); le Pandanus fureutus, qui a actuellement dans le jardin des fenilles de 25 pieds de longueur. Arauearia Bidwillii, Dummara Brownii, alba, orientrulis ot australis, plintes-mères des diverses espèces de résine de Duminark.; Liquidambar imberbe AIT. de l'Asie Mineure, plante-mère du Styrais liquida; Artemisia Mroxa DC., plante médecinale japonaise; Cinehona condaminea LAN., ainsi 
que six autres espèces: C. ovata, succimbro, pubescens, Calisaya WEDD., etc. Puis, la belle Araliacée, Oldfieldia africana BentH. et Ноок., qui donne le bois de teak africain; les arbres brésiliens contre la fièvre intermittente, Cratreva 'Tapia L. et C. tapioüdes DC.; le cotonnier des tropiques américains, Bombax Ceiba; Hebradendron cambogioüdes Grum.u, la plante-mère de la gomme-gutte de Ceylan; Culopltuyllum Madrunno, la plante-mère Takamahakas du Brésil; le canellier blanc, Canella alba Murr., Yanthochymus pictorius Roxв., l'arbre de gomme-gutte de Mysore. Les arbres fruitiers des Indes déjà connus, mais peu répandus, de la famille des Clusiacées, comme le Garcinic mangostana, dont le fruit est le plus savoureux de toute l'Asie. Rheedia lateriflor L. et Mammea anericana des Indes occidentales, arbres des fruits du Mamméi. Carapa guyanensis AvBL., la plante-mère de l'huile anthelminthique de Carapa. L'arbre contre les fièvres des Caracas, Cedrela montana Karist. Croton Eleuteria Sw., le buisson qui fournit l'écorce de cascarille. Bursercu gummiferc J JCQ., l'arbre de la résine de Caranna, Guajacun arboreum D. C., l'arbre du bois d'acajou. Terminalia bellericu Roxв., l'arbre de Mysobalanes; Eucalyptus globulus, le blue gum-tree du van Diemensland, l'arbre à violettes qui atteint la hauteur énorme de 250 à 450 pieds, et qui est le rival de la Sequoia Wellingtonia. Melalenca Leucadendron, l'arbre qui fournit l'huile de cajeput, à feuilles de l'odeur la plus fine de cette huile. Lecythis ollariu, arbre fruitier en pot du Brésil; Bertholletia excelsa, l'arbre des noix de Para; Hymenaea courbaril et $H$. stilbocarpe Haxne, arbres mexicains et brésiliens, qui fournissent le copal; Copaifera officinalis, l'arbre du baume de Copahu; Cassia Fistule et brasiliensis, les casses officinales en tube et celles du Brésil; Iyyrtus Pimenta, Hacmatoxylon campechianum etc. (1).

(1) Conf. Goeppert, der botanische Garten der Universitiit Breslau, nebst einem Plan und einer Lithographie, Görlitz bei Remer; Govprint, die oftizinellen und technisch-wichtigen PHancen unserer Gärten inshesondere des botanischen Gartens der Universität Breslau, eine gedrängte Uebersicht derselben, unter Angabe ilırer systematischen Stellung, ihres Gebrauches und Vaterlandes, Gürlitz bei Remer, p. 106. 


\section{HANDELSPFLANZEN, WELCHE FÜR OSTINDISCHE COLONLSTEN WICHTIG UND VON GROSSEM WERTHE SEIN KÖNNEN,}

ION

\section{Herrn J. VON WARSZEWICZ,} IXSPECTOR DES KAIS. KÖN, BOTAXISCIIEN GARTENS IN KKAKAU.

\section{Smilax officinalis.}

Auf meiner langen Reise von zwölf Jahren habe ich mich überzeugt, dass das hoch geschätzte Smilex officinalis nur eigentlich sein Vaterland in Neu-Granada hat, besonders in den Landesstrichen, welche grenzen an den Magdalenen-Fluss, und die auch von Herm Alexander vox Humboldt beschrieben sind. Es existiren noch gegen zehn Species in der Republik Neu-Granada, aber von geringerem Werth. Am Magdalenen-Fluss habe ich viel gesucht, und man findet Stellen, wo es beinahe ausgerottet ist, und nach wenigen Jahren verschwindet vielleicht die richtige Species ganz und gar. Daher ist es wohl an uns, darübor nach zu denken, ob es nicht möglich sei, Smilax off'. nach Ostindien überzusiedeln und dort in Cultur zu bringen.

Die Königlich-Niederländische Compagnie bat schou viele wichtige und nützliche Pflanzei dorthin befördert und in Cultur gebracht. So viel ist gewiss, dass es mit Smilax off. nicht so kostspielig sein wird, als mit Cinchona; auch muss man bei Cinchona lange auf den Ertrag warten, welches bei Smilar off. nicht der Fall ist, wo man im dritten Jahre $4-5$ pro Cent Ertrag hat. Das echte Smilax off. ist nie unter 20-30 Dollar per Centner zu bekommen am Cartagen. Port.

Smilax off. nach Ostindien überzusiedeln ist weit leichter als Cinchona. Man muss in der trocknen Zeit die Strungwurzeln sam. meln und in lehmiger Lauberde in eine Kiste rerpacken. Hauptsache ist, dass den Strungwurzeln ein gewisser Grad ron Feuchtigkeit erhalten wird, $d x$ selbige eine harte und trockne Substanz besitzen, und, wenn sie einmal ganz ausgetrocknet sind, schon nicht mehr treiben. Sollte übers Jahr Smilas off'. in Ostindien in Cultur gebracht werden, so muss es an warme Stellen ausgepflanzt, regelmässig feucht gehalton und durch Schatten gegen Somnenbrand geschützt werden. Wenn dic Pflanzen anfangen zu treiben (bekanntlich bringt Smilar offi. viele 'Triebe), so nimmt man die 
Köpfe, zertheilt sie in so viel Stücke als Treibungen sind, und pflanzt diese in den zur Cultur bestimmten Platz, das heisst: in einen gut locker zubereiteten Boden, damit sich die Wurzeln gehörig bilden könuen. Es versteht sich von selbst, dass diese Operation nur in der Regenzeit vorgenommen werden kann. So kann man im dritten Jahre ron jeder Pflanze im Durchschnitt 2-3 Pfund trockne Laufwurzeln haben. Zum Abnehmen der Wurzeln bediene man sich eines scharfen Messers, und schneide selbige nahe an der Mutterpflanze weg, welche dadurch durchaus nicht gestört wird, und ziehe alsdann die abgeschnittene Wurzel, so weit sie reicht, langsam und rorsichtig hervor. Ich habe mich selbst oft überzeugt, dass diese Laufwurzeln 20-40 Fuss lang sind, und nur '3-4 'Zoll tief unter der Erdoberfläche laufen.

Das zweite, Smilax, Hanbury, wächst in der Provinz Chiriqui und Boca de Torro, und wird ron dort nach Jamaica rersandt, woselbst das berühmte Extract Sassaparilla daraus präparirt wird, welches dann nach Europa und Nord-America an grössere Laboratorien befördert wird. Der Centner hiervon kostet an Ort und Stelle in Boca de Toro 30-50 spanische Dollar. Es wird alle Jahre viel davon gesammelt, und es ist daher auch an manchen Stellen schon ganz ausgerottet. Es kommt viel ron den Central-Americanischen Provinzen unter dem Namen Boca de Toro, hat aber geringeren Werth; das Sassaparille von Chiriqui und Boca de Torro hat seinen besondern Character. Es enthält in seinen Laufwurzeln keinen Mehlthau, sondern, wenn man frische Wurzeln untersucht, so findet sich zwischen Haut und Holz der Wurzeln ein röthlicher Saft; die Wurzel selbst nimmt eine röthliche Farbe an, wenn sie trocken geworden ist. Ich habe selbst von einem Provisor aus dem Laboratorium des Herm Taylor ganz genau erfahren, dass dieses Sassapavilla, welches aus Boca de 'T'oro kommt, das meiste und beste Extract liefert. Es wird extra alle Jahre ein Schiff von Jamaica nach Boca de Toro geschickt, um dieses Sassaparilla zu kaufen. Es wäre auch sehr wünschenswerth, dass diese Species in Cultur genommen würde, da selbige auch schon in zwei bis drei Jahren Ertrag liefert.

Ich lege hier beide Original-Exemplare bei, rom Fluss Magdalena und von der Prorinz Chiriqui, von wo ich früher 1 Centner Sassaparilla zur Untersuchung an Herru Dr. DAxini Haxвтr nach London gesandt habe, welcher auch seinen rolkommenen Werth anerkannt hat.

Auf meiner ganzen Reise in Central und Süd-America habe ich nur dieses Sassaparilla in der. Provinz Chiriqui angetroffen. 


\section{Cinchona contaminea Нuмв. Ет Boxpt.}

Ich habe in verschiedenen Blättern gelesen, dass die KöniglichNiederländische Regierung Cinchona Culysayfo WEDD. (Cinchona floribunda Paron) nach Ostindien geln'acht hat, und dort grosse Anpflanzungen davon gemacht sind. Da ich nicht bestimmt weiss, ob die Species Cinchona condamine auch dorthin gebracht ist, so bemerke ich hier, dass letztgenamnte den Vorzug rerdient, da sie mehr Ertrag des Sulphates liefert als vorige. Bei der Cultur dieser $C$. condaminea ist es ganz zweckmässig, wenn man junge 2-3jährige Pflanzen bis auf 4-5 Zoll über der Erde zurückschneidet, so treiben sie von Neuen recht stark aus und bringen in der Regel $6-8$ junge Triebe. Diese lässt man 2 Jahre wachsen; im dritten Jahre kann man die starken Triebe schneiden und die Rinde abschälen; die gekröpften Stämme treiben wieder von Neuem, so im Vergleich wie unsere Weiden. C. cond. wächst im kühlen Klima, an Bergabhängen, in feuchten torfigen schwarzen Böden, gleich wie unser gewöhnlicher Eilerbaum, welcher bekanntlich auch am liebsten in solchem Boden wächst; erstere wächst das ganze Jahr hindurch, sie findet so zu sagen keine Ruhe und Rast. Ich fand sie nur in der Gegend von Loxa (Loha), Nord Peru und Chuarabomba. Die Indios nennen von jungen Trieben gesammelte Rinde Cusenvilla fina, und ron starken Stämmen gesammelte Rinde Cascarilla regia.

Wenn in Belgien der Wiuter wenigstens 2-4.0 Wärme Reaumur hat, so müssen sich jedenfalls diese Species Cinchona condaminea dort auch im Freien cultiviren lassen; deun wo ich selbige angetroffen habe, hat es mich manchmal gefroren im Sommerrock, besonders im Monat Juli, wenn der Wind aus Süden kommt, der oft Schneeschauer mit sich bringt. Der Schnee verschwindet jedoch gleich, so wie er auf die Erde fällt.

Die Cinchona Calysaya verlangt einen wärmern Temperaturgrad und lehmigen Bodeu. Auf meiner Reise durch Bolivia habe ich diese Species am wenigsten in der Prorinz Jungos rorgefunden.

Es scheint mir dass Dr. Weddell diese Pflanze nicht genau untersucht hat, welche Pavon beschrieben hat als Cinchona floribunda. Ich habe sellst das Manuscript in Lapas bei dem alten Dr. ZawaLL gelesen und auch ganz richtig so gefunden, dass diese Pflanze das ganze Jahr hindurch Blüthen und Früchte hat. Der Stamm ist selten dicker als 3 Zoll im Durchmesser, wenn er auch 15-20 Jahren alt ist. Die Pflanzen haben das Besondere, dass sie, wenn sie 2 Fuss hoch sind, schon Blumen bringen von schöner Rosenfarbe, doch nur an den Seitenzweigen; an den 
Spitzen kommen niemals Blumen. Sobald die Blume verblïlt und der Same reif geworden ist, sterben sie Seitenzweige ab, oder wachsen spärlich noch ein Jahr, wo sie denn doch bis an den Stamm absterben. Die Spitze dagegen wächst ungestört in grader Richtung aufwärts, an ihr bilden sich wieder neue Spitzenzweige welche wieder Blumen und Früchte bringen.

Das in früherer Zeit unter dem Namen Cascarilla Calysaya (Cinchona glabra Bexтн.) von Bolivia kommende wächst beim Tipurni Fluss, ist aber wenig und selten zu finden. Ich habe 8 Tage in den Wäldern herumgesucht, und fand endlich zu meinem Erstaunen einen Baum ron 50-60 Fuss hoch, gegen 2 Fuss Stamm-Durchmesser; die Rinde war ganz glatt und nicht dicker als 2-3 Linien. Wenn die Rinde getrocknet und in Stïcke gebrochen wird, sieht sie ans wie gebrochene Glasstücke; das Holz des Baumes ist sehr hart und fest.

Ich erfuhr nachher in Lapas beim alten 80jährigen Jose Karsero, dass er selbst früher alles rach England geschickt und einen hohen Preis dafür gezahlt erhalten habe. Die Rinden sind von rerschiedenen Species gesammelt worden. Ich habe selbst ron der Chilischen Grenze bis nach Quito 75 Species ron Cinchuna und Erostrmma gesammelt. Tch hatte es mir zur Aufgabe gemacht, diese Familie zusammen zu bringen, nach der Eintheilung des Herrn Dr. KLotscri. Ich sammelte Zweige mit Blüthen und Frïchten, dazu ein Stïckchen Holz, 1 Fuss lang, 3 Zoll dick, von jeder Species. Hauptsächlich sammelte ich solche Zreige, welche gut mit Flechten bewachsen waren. Die ganze Sammlung bestand aus 2 grossen Kisten, worin sich gegen 1000 Exemplare befinden, welche ich an ein englisches Haus Gibs \& Coms abgegeben habe, in Tacna Port, von wo sie auch richtig mit Segelschiff durch Cap Horn in Lirerpool, in 1852, angekommen ist. Von Liverpool nach London ist sie abhanden gekommen, vielleicht durch Nachlässigkeit der Expediteure, oder ob sie absichtlich entwendet ist, weiss ich nicht.

Für mich war es ein empfindlicher Verlust; denn wenn ich die ganze Sammlung glïcklich nach Deutschland bekonmen, so hätte man von jeden einzelnen Species ron Holz und Rinde chemische Untersuchungen rornehmen können, und daraus erschen, welche von allen diesen für die Cultur von Werth sein könnten. 


\section{Myrospermum punctatum KNTH ET KL.}

Obgleich dieser Baum sehr geschätzt wird, so ist es bis jetzt doch noch nicht in Amregung gekommen, Plantagen daron anzulegen, und wirklich weissen Balsam zu erziehen.

Myrospermum punctatum habe ich auf meiner Reise im südlichen Peru vorgefunden bei dem Dorfe Puyaga, 2-3 Leguas (1 Meile) ron den Niederungen des Flusses Maranhon, wo man schöne prächtige Bäume vorfindet, mit dunkelgrün glänzenden Blättern, von fünfzig bis hundert Fuss Höhe, und gegen zwei Fuss Stamm-Durchmesser. Die Rinde ist hübsch glatt und ron weisser Farbe, und wenn man in selbige Einschnitte macht, so quillt daraus ein weisses glänzendes Harz herror.

Myrospermum Pereira KLotscr, einheimisch in Central-America, Provinz Sonsonate, hat eine grau grünliche Rinde, und der darin enthaltene Saft ist von grüner ins braune schimmernder Farbe. Mit dem Sanmeln dieses Harzes beschäftigen sich Indios, und bringen dieses nach Sonsonate zum Verkauf. Die Kaufleute, welche hiermit Handel treiben, müssen dieses Harz noch erst abkochen lassen, damit es von Schmutz gereinigt werde. Dass dieser Balsam mitunter etwas bräunliche Farbe hat, ist wohl die Ursache des zu. langen Kochens. Auf meiner ganzen Reise sah ich nirgends dieses flüissige Harz sammeln, als nur in Sonsonate. In früherer Zeit liess Spanien Balsam sammeln, welches jedoch nach und nach verblieben ist; sie hatten viel Mühe und Arbeit damit und fanden vielleicht keinen genügenden Preis dafür gezahlt. Der rein weisse Balsam kann nur aus Myrospermum punctatum gewonnen werden, und ich bin überzeugt, dass er in den Parfumerie-Fabriken reichlich verbraucht würde, da er einen weit feineren Geruch hat, als der ron der andern Species.

Ich glaube auch gewiss, das wenn Myrospermum punctatum in den Ostindischen Ländern angebaut würde, es dort auch sehr gut gedeihen würde, denn es wächst in einer 'Temperatur von $20-30^{\circ} \mathbf{R}$. in einem feuchten Boden. Die Vermehrung geschieht am schnellsten durch Samen, welchen man nach der Reife noch 2-3 Monate ruhen lässt in ganz trockner Lauberde, wo man denselben nachher aussät in ein Beet, mit gut locker zubereiteter Lauberde ausgefüllt.

Die Samen gehen in einem feuchten warmen Boden schnell auf, ähnlich wie unser gewöhnlicher Ahornbaum. Nach meiner Ansicht kann Myrospermum punctatum in Ostindien eben so wichtig und nützlich werden, wie Vanilln arnmatica aus Mexico. Dabei werden 
diese Pflanzungen noch nicht so riel Pflege und Arbeit beanspruchen wie Vanilla aromatica. Nur in den ersten und zweiten Jahren nach der Verpflanzung; denn nachher, wenn die Bäume erst Wurzeln gefisst haben, thut die Natur das Nöthige. Den Herren Besitzern bleibt nur Sorge zu tragen, das Harz einzusammeln.

Von diesem Baume Myrospermum punctatum hatte ich dem Herm Danier Hanbury in London Originale von getrockneten Zweigen und Rinden zugesandt; auch hatte ich ein kleines Fläschchen roll von diesem flüssigen Harz-Balsam gesammelt; aber selbiges ist auf meinen Reiseplackereien zu meinem grössten Verdruss zerschlagen worden, da ich sonst hätte den Beweis liefern können von der Vortrefflichkeit des echten weissen Balsams, welcher den von den andereu Species an Klarheit und feinem Geruch bei weitem übertrifft. 


\section{OBSERVATIONS DE PHYSIOLOGIE VÉGETALE SUR \\ LES JACINTHES (HYACINTHUS ORIENTALIS) \\ CULTIVÉES EN CARAFES (1),}

PAR

\section{EUG. VAVIN.}

La culture des Jacinthes occupe en Hollande un rang distingué depuis près de trois siècles; aussi la passion pour cette belle fleur s'est-elle répandue arec raison dans le reste de l'Europe.

Aussitôt que la saison le permet, chacun s'empresse à Paris de placer ces beaux oignons dans des vases remplis d'eau, afin de jouir pendant l'hiver du coloris et de l'odeur de cette magnifique plante.

Un fait de physiologie végétale que je crois nouveau, a appelé mon attention sur elles; car, malgré toutes mes recherches, je n'ai rien trouvé dans les ourrages que j'ai consultés concernant le sujet de cette note.

Il $y$ a trois ans, j'eus l'idée pour un de mes oignons de Jacinthe placé dans une carafe remplie d'eau, de couper environ $0,03 \mathrm{c}$. sur les racines qui en avaient à peu près $0,08 \mathrm{c}$. Je remis cet oignon dans l'eau et je fus agréablement surpris de le voir, quelques jours plus tard, prendre une nouvelle végétation et la hampe se montrer un mois plus tôt que celles des autres oignons. Depuis, j'ai renouvelé cette expérience toujours avec le même succès.

Au mois de février de cette année, j’ai déposé sur le bureau de la Société impériale d'horticulture de Paris des Jacinthes cultivées par la même méthode; cette fois, afin de répondre à une objection qui m'avait été faite l'année dernière, j’ai voulu expérimenter sur des variétés de même espèce; le résultat a toujours été aussi satisfaisant.

Quelquefois même il m'est arrivé que, les racines ne se déreloppant pas assez vite et l'oignon paraissant fatigué, j'en coupais l'extrémité, sans attendre leur entier déreloppement.

Les oignous, dont le bout des racines est retranché, ont généralement des fleurons plus forts, la hampe s'élève au dessus des feuilles qui restent plus stationnaires que dans leur état normal, et la floraison surtout a lieu près d'un mois plus tôt.

A la simple vue, il est facile de reconnaître, par la beauté des fleurons, la force de la hampe et de la végétation, les plantes sur lesquelles l'opération du retranchement d'une partie des racines a été effectuée.

(1) Cette communication a été présentée au bureau par MI. LefÈvre-Pontalis, de Paris. 


\section{BETTRAG ZUR FERNEREN BEGRÜNDUNG DER LEHRE VOM GENERATIONSTECHSEL FÜR DIF GRLPPE DER PILZE,}

VON

\section{Prof. Dr. JULIUS MÜNTER,}

DIRECTOR DES BOTANISCIEN GARTENS DER KÖN, UNIVERSITÄT GREIFSWALD.

Indem man, die aus dem Studium der Phanerogamen resultirende Anschauungsweise der Pflanzenart (Species) rerallgemeinernd, den Grundsatz aufstellte: , die Species findet ihre zeitliche Grenze und ihren Gesammtausdruck in den mit der Embryobildung anhebenden und mit der Ablösung entwickelungsfähiger Embryone von der Mutterpflanze endenden Lebenserscheinungen," wurde für lange Zeit eine naturgemässe Auslegung und ein rolles Verständniss der Cryptogamen geradezu unmöglich gemacht. Nicht minder aber trug zu einer mangelhaften Erkenntniss derselben die auf unbewiesene Homologieen sich stützende Deutung der Organe bei und führte in nothwendiger Consequenz zu Widersprüchen der erheblichsten Art. Kein Wunder daher, wenn die auf zu eng gefasstem Princip errichteten sogenannten Arten der Cryptogamen durch ihre wahrhaft endlose und anscheinend unerschöpfliche Massenansammlung zu einem Material für Sisyphus-Arbeiten späterer Systematiker heranwuchsen. Es war daher in der eilften Stunde, als die von A. rox Chamisso zuerst erkannte, durch Sars und Steenstrup aber erst in die wissenschaftliche Zoologie factisch eingeführte Lehre rom Generationswechsel (digénèse?, génération alternante vas Bexedex?) auch in der Botanik Platz griff, leider aber gar bald nach ihrer Einführung in dieselbe, eine Ablenkung ron ihrem allein richtigen Wege erfuhr, die nicht zu den Resultaten führte, welche aus einer, der ursprünglichen adäquateru Anwendung dieser Forschungsmethode hervorgehen konnten.

Allerdings zwar nicht ganz vergebens sprach ich es am 21 December 1847 (1) zuerst aus, dass Pteris servulate L., der zufällig gewählte Repräseutant der Farn, in zwei verschiedenen Lebensformen aufträte, indem wenigstens der später von mir als Serualform aufgestellte und zusammen begriffene Organencomplex durch vos Märcklis und danach von vielen Andern eine glänzende Bestäti-

(1) Botanische Zeitung von II. v. Monl \& D. T. L. v. Schlecittendal, 1848. Jałrg. VI, No. 3, pag. 45 und KürıEn, Grundriss der Cryptogamentunde. Breslau 1848, pag. 163. 
gung erhielt, während jener, der Sporenbildung dienende, Organencomplex ja schon längst zur Genïge bekannt war und wenigstens durch mich keiner nähern morphologischen Erläuterung bedurfte; auch das darf' himzugefügt werden, dass meine spätem, leider nur unvollständig zum Abdruck gelangten Beobachtungen ïber Hepaticue, IIrsci, Seluginellen, Thirsocarpeen und Equiseten (1) in vollster Uebereinstimmung mit den gleichzeitig, aber völlig unabhängig von einander publicirten Entdeckungen Horraeister's, Milde's u. A. standen und das Gesetz begründen halfen, dass die Individuen der Arten jener Reihen in zwei fortpflanzungsfähigen Gestaltungen, ciner sexuellen und einer sporigenen Lebensform (wie ich sie namnte) mit Nothwendigkeit auftreten müssen. Allein ausser einer Erwähnung dieser sichergestellten Thatsache bei Grisebaci (2), hat die Physiographie jener (ehemals sogenamnten) Cryptogamen bis jetzt, ja selbst bis zu diesem Tage, noch nicht davon Act genommen; dieselbe fährt vielmehr nach wie vor fort, nur der sporigenen Formen zu gedenken und diese zu beschreiben, wie exact sonst auch immer diese einseitige Beschreibung sein mag. Anders verfaluren dagegen die neueren Zoologen, welche es vielmehr für eine wesentliche Aufgabe ihrer systematischen Forschungen halten, mit Consequenz und wissenschaftlicher Schärfe alle nachweisbaren Lebenszustände, gleichviel ob dieselben in den verschiedenen Gestaltungen zongungs- oder fortpflanzungsfähig sind oder nicht, festzustellen und bei der Beschreibung gesondert zu beachten. Indessen, um nicht ungerecht zu sein, auch in der", scientia amabilis" hat bereits dieses verbesserte Verfahren Platz gegriffen und wird dureh das Prachtwerk der Gebrüder Tulasse (3) jedenfalls auf das Glänzendste bekundet. Sind es doch deren Arbeiten, von welchen seit 1851 eine erleuchtetere Anschauung und Auffissung der wunderbaren Pilze überhaupt datirt! Allein wie rergebens deren Bemühen zu sein scheint, beweisen die Angriffe, welche in den letzten beiden Jahren von Bonordex (4), Steph. Schulzer (5), C. H. Schultz-Schulzenstein (6) und BARRLAX (7) ausgingen. Waren nun freilich die letztgenannten

(1) Comptes rendus hebdom. de l'Académie des sciences. Tom. XXXIII, 1851, p. 701.

(2) Bericht über die Leistungen in der geograpischen und systematischen Botanik während des Jahres 185̃ 1, Berlin 1854, 80 p. 108 n. ff.

(3) Selectn fungorum Carpoiogia, Tom. I, II. Paris, 1862.

(4) Abhandlungen der naturforschenden Gesellschaft $\approx u$ Malle. Halle 1564, to., Tom. VIII, pag. $16 \mathrm{u}$. ff.

(5) Oesterreichische botanische \%citschrift, 1865. Jahrg, XV, Wien, 8o. No. 8 und 9.

(6) Verhandlungen des Gartenban-Vcreins in Kocn's Wochenschrift 1865.

(7) Archix. der Pharmacie, Bd. XC, der ganzen Folge 156 Bd. Hannov. 1861, p. 31. 
Autoren nicht im Stande, die mit so grossen Opfern an Zeit und mühevoller Arbeit errungene Erkenntniss des Polyinorphismus, oder vielleicht richtiger gesagt, des Generationswechsels umzustossen und die ältere Forschungsmethode, wie sie uns in vollendetster Gestalt in den nichtsdestoweniger hochachtbaren Werker des unermüdlichen und geistrollen ELIAs FrIEs entgegentritt, wiederherzustellen, so beweisen doch eben deren neuesten Kundgebungen, wie nothwendig es selbst heute noch immer ist, den Widersachern einer geläuterteren und naturgemässern Umgrenzung der Pilz-species mit Thatsichen entgegenzutreten, welche einer anf' dem gleichen Wege gewonnenen bessem Erkenntniss, nicht aber einem unbegründeten Raisonnement weichen.

Als Paradigma zur Unterstiitzung der Lehre rom Generationswechsel mögen einige Sclerotiaceen mit den ihnen voraingluenden Hyphomyceten- und den ihnen nuchfolgenden Pypenomyceteri-, Discomyceten - und Hymenomyceten-Zustïnden dienen: Ptanzenformen, bei welchen zwar noch keine geschlechtlichen Zeugungen sich nachweisen liessen, bei denen abei eine Succession sporigener Generationen in unverkeunbarer Weise ausgesprochen ist.

Bekammtich grïndete der verdienstrolle Tode (1) sein Genus Sclerotium auf' eine läuglich-kugelige, derbe, nicht aufspringende, berindete Pilzmasse, deren ,finctificatio interna" ihm annoch unbekannt war, später'n Beobachtern es überlassend, dieselbe aufatunden. Die von ilm zu dieser Gattung gebrachten Formen: furpureum, inmersun, subteranewm, Semen, Mucor, rudicatrm, complanatum, und villosum beschrieb er mit möglichster Schärfe und bildete sie ab, so gut als es eben in jener Zeit (1790) greschehen konnte. . Von besonderem Interesse ist es uns aber hente, dass er in der" "Observ:" zu seinem Scl. subteraneum die Notiz nicht unterdrïckt, dass dieses Sclerotium einem Aguricus ans der Familie der Moucerons (Agaricus esculentus MURr.) zum Ausgangspunkte diene mud von demselben so gierig verzehrt werde, dass nur die Cutis des Sclerotium zuletzt noch übrig blieb. Für Tove also, den Begründer der nunmehr seit 76 Jahren im System erhalten gebliebenen Gattung Sclerotium, war der Mouceron eine Art von Parasit: eine Ansicht, welcher sich auch Persoon (2) anschloss, indem er sich auf Bulliard (Champ. tab. 256) stïtzend sagt: , ladicem saepe Agwrico tuberoso suppeditans."

Bereits in der Persoov'schen Synojsis erhebt sich übrigens die

(1) Fungi Mecklenburyenses selecti. Lumeburgi 1700, to., pag. 2.

(2) Synopsis metholica fungornu, I. I, Gottingae 1801, pag. 120. 
Zahl der von ToDe ursprünglich beschriebenen 8 Arten auf 16 , indem hinzukommen: Sel. crocorum BulL., lacunosum PERs., durum Pers., Brassicae Pers., varium Pers., pubescens Pers., Erysiphe Pers., quercinum Pers., populneum Pers., sphacroides Pers., während die 'ToDE'sche Species subteraneum in: Sclerot. fungorum und Scl. muscortun gespalten wird und die 'ToDE'sche Species immersum, Yrucor und villosum stillschweigend ignorirt werden.

Schumacher (1), welchem die gleichzeitig publicirte Persoos'sche Synopsis zwar noch nicht bekannt sein kounte, der aber doch mit den vor Erscheinen der Synopsis publicirten Persoox'schen Species sehr wohl vertraut war, fügte 1. c. Scl. ovatum Schux., Hypnorum Scrum., Fagi Scrum., album Scrum., perizceforme Scrum. (mit?), granulatum Scrux., flavum Schux., hirsutum Schum., fasciculatun Scrum. und Circene Schum. hinzu, jedoch ohne eine der ilm unzweifelhaft sehr wohl bekannten ToDE'schen Arten zu nennen, von denen doch sicherlich mehrere in Seeland rorkommen mussten, zumal er die Persoon'sche Species turum und Erysiphe als in Seeland heimisch nambaft macht. Atbertivi und Scinweiniz (2) bereicherten einige Jahre später das Genus Sclerotium durch die Species: vaporarium ALb. und Scrw., und seutellatum ALb. und Scrw. und beschrieben dieselben neben 6 bereits von PErsoos aufgefundenen Arten. Pyr. de Candolle (3) auf vorgenannte und andere Beobachter sich stützend, machte schon i. J. 1816 neun- und dreissig Arten namhaft, und nachdem ein Mougeot und Nestlek, ein Nees, Link, Schmid, Hofminn, Sowerby und Ehrenberg mit anerkennenswerthem Eifer Sclerotion aufgesucht und beschrieben hatten, wurde es Elias Fries i. J. 1823 möglich, in seinem berïhmten Fundamentalwerke (4) bereits 54 Sclerotium-Arten aufzuführen und zu beschreiben, obschon er dic Persoos'sche Species croconum ausschied und mit DF Caxpolle dem Genus Rhizoctonic DC. subsumirte; obgleich er die 'ToDE'schen Arten purpureum und Wucor nicht aufnahm, vielmehr einer weitern Untersuchung empfihl und die Scuvmacuen'schen Arten granulatum und persicolor der Gattung Aegerita zuwies, (1. c. pag. 264) obschon sie dort, wic aus Tom. III. p, 220 hervorgeht,

(1) Enumeratio plantarum in partibus Saclandiae septentrionalis et orientalis. Hafn. 1801. P. I, pag. 185.

(2) Conspectus fungorum in Lusatiac superioris ayro Nistiensi crescentium. Lipsiae 1805 .

(3) Mémoires du .Inséum dhistoire naturelle. Paris 181j, T'om. II, p. 401, pl. XV, fig. 8 .

(4) S'ystema mycologicum etc. Vol, II. Gryphiswaldiae 1823, 8o., pag. 246-264. 
keine Aufnahme fanden, sondern (1. c. 259) beim Illospurium coccineum gelegentlich erwähnt wurden. Die bedentende Anzahl neuer Formen gals E. Fries genügende Veranlassung zur Aufstellung ron vier Sectionen, und zwar: Scl. Tibera, crumpentia, adnata, Xyloma; nnter welche die ältern und die von ihm selbst zuerst aufgestellten Formen entsprechend rertheilt wurden. Vom Scl. fiungorum Pers. behauptet Fries ebenfalls, dass derselbe den Aguricus tuberosus ernähre, nicht aber den Agaricus racemosus, folglich aber zu demselben sich verhielte, wie der Apfelbaum zur. Mistel. Fortgesetztes Suchen nach Sclerotien durch Desmazières, Kuotzscir, Fräulein Libert und Andere mehrte indessen die Zahl der Arten so sehr, dass LÉYeILLÉ in sciner trefflichen MIonographie (1), ohme sich einer Uebertreibung schuldig zu machen, die Zahl der Arten auf hundert schätzen konnte und auch in der That die grösseste Artenzahl namentlich aufzuführen im Stande war.

Neben diesem mächtig auf'blühenden Geschlechte der Sclerotien, fristete das gleichaltrige ursprünglich mit 4 Arten ausgestattete Genus Acrospermum bis heute nur ein kümmerliches Dasein. Tode errichtete dasselbe 1790 (2) und zwar auf Grund der an der Aussenfläche der Spitze eines aulrechten cinfiachen Pilzkörpers auttretenden Fruchtbildung (apice extus fructificante), welche, wie aus der Beschreibung der dazu gezählten Arten compressum und pyramidale hervorgeht, in Form eines „pollen candidum” auftreten soll, während (1. c.) für die beiden andern Arten unguinosum und lichenoides die Fructificationsform nicht ausdrücklich angegeben wird. ScruMacher (I. c. pag. 441, T'om. I) erwähnt nur einer in der Flora danica Tab. 1076, fig. 4, beschriebenen Species Acrospermum cylindricum, die derselbe jedoch zu seiner Tremella zieht. Persoon gedenkt in der Synopsis meth. der Gattung Acrospermum überhaupt gar nicht; eben so wenig findet dieselbe in der Mycologia europaea Erwähnung. Indessen citirt E. Fries im Syst. mycol. (Tom. II, p. 244-245) eine Persoon'sche Schrift (3), in welcher Acrospermum compressum als Clavaria fabarum vorkommen soll. In der Mycol. europ. (Sect. I, p. 160 und fgd.) aber findet sich eine solche Clavaria nicht mehr beschrieben. El. Fries (1. c. p. 244) erhält das Tode'sche Genus Acrospermum mit den Species compressum und pyramidale aufrecht,

(1) Mémoire sur le genre Sclerotium. Annales des sciences natur. 1843. Tom. XX, Bot. p. 218 et suiv.

(2) Fungi mecklenburgenises selecti. Luneb. 1790, 40., Fasc. I, pag. 9. Tab. IT, fig. 13, 14.

(3) Conmentationes de fungis claraeformibus, pag. 68, Taf. III, fig. 4. 
bringt jedoch die Tone'sche Species unyuinosum zu seiner Coryne als sarcoüdes, das 'Tode'sche licluenoüdes nach Ausweis des Syst. mycol. (II, 246) für eine monströse Lichenenform ansehend. Ausserdem vermehrt FrIEs die Gattung Acrospermum um: sclerotioides, welche nicht mit Pistillaria sclerotioides DC. verwechselt werden soll, ferner A. conicum 1'ERs. (Myc. europ. tab. XI, fig. 5, 6), und endlich mit A. cornutum Bull., welche Bulutard für die Wurzel von Agaricus tuberosus hielt; eine Ansicht, die, wie aus Obigem hervorgeht, Persoon jedoch nicht theilte, indem er vielmehr sein Scluotium fungorum als das Substrat des Agar. tub. ansah, und wogegen Fries (1. c. II, p. 252) am Ende auch nichts einzuwenden hatte, indem er vom Scl. fungorum sagt: ,Intel lamellas Agar putrescentes, $\boldsymbol{A} g$. tuber. alens", während er doch gleichzeitig bei dcrospermum cornutum dieselbe Bulliard'sche Tafel citirt, welche Persoon bei Slc. fungorum (1. c. p. 120) allegirt und hinzufügt ,, saepius Ag. tuberosum enitens." Und doch hatte er bereits anderswo (1) sich dahin geäussert dass ,, in hac specie (nämlich Sclerotium comutum, welches er später als Acrosp. comutum beschreibt) et in nommullis aliis Sclerotiis parasitat $A g$. tuberosius Pers., ab omnibus tamen auctoribus pro basi stipitis hujus fungi habetur, at perperam."

Im Elenchus fungorum (2) erwähnt endlich Fries, dass Sclerotiumstercorarium DC. nach Angaben in den Acta Societatis Linneanae Paris., der Jugendzustand des Pilobolus cingstallinus und Sphaeria complanata Tode uach Desuazières die Ausgangsform des Agaricus Hudsonii PERs. liefern sollte, was. jedoch nicht wahrscheinlich erscheine.

Der Vollständigkeit wegen muss schliesslich zu dem Vorstehenden hinzugefügt werden, dass Elis Friss (der eigentliche Begründer einer wisseuschaftlicheren Auffassungsweise der Pilze überhaupt), zu der von ihm in J. 1823 aufgestellten Familie der Sclerotiaccae, ausser Acrospermum und Sclerotium, 1. das Genus Pachyma.Pers. mit den Species $P$. Cocos Schw. und $P$. Tuber regium zählt, welche letztere dem Agaricus Tuberis regii als matrix dient; 2. das Genus Pyrenium ToDe mit der Spec. terrestre; 3. Rlizoctonia DC. mit den Species: Crocorum DuH., Medicaginis DC. und Muscorum Fr.; 4. Periola Fries mit den Species: hispida Scrum,, pubescens FR. (= Scler. album Schum.) und tomentosa FrIES; 5. Acinula FrIes mit caudicans Fr. (= Scler. vestitum NeEs) und Anhangsweise; 6. Spermoedia Fr. (Sphacelia LÉv.) mit Sp. Paspali Scrw. und Clavus (Lonic.) DC.

(1) Fries, Observationes mycologicae. Havniae 1815, Tom. I, pag. 205.

(2) FrIEs, Elenchus fungorum, Gryphiswaldiae 1828, pag. V. 
Dieser mithin aus 8 Gattungen bestehende Ordo (Fam.) der Sclerotiaceen soll nach Friss (Syst. mycol. II, p. 269) den Coniomyceten oder epiphytischen Pilzen am nächsten verwandt sein, in Betreff der Vegetation der Tubercularien sich nähern; allein mit NeEs gegen DE CANDolle vereinigte er sie doch mit den Hymenomyceten. Im Character der Familie sagt Frres (1. c. p. 239), das Receptaculum sei verschieden gestaltet, fleischig, riugsum oder an der Spitze fructificirend; die Sporidien seien mit einander verbunden und bildeten fast das ganze Gewebe der Rindenschicht; bei einigen seien sie auch frei, an die Oberfläche tretend. Pag. 242 fügt er hinzu : „Uebrigens dienen die Sclerotien oft vollkommeneren Pilzen zur Anheftung (gleichsam wie eine Wurzel) so der Pexiž tuberosa den Typhulis, Agaricinen" und er bekräftigt diese Aussage abcrmals (1. c.p.273) indem er sagt: ,, Sed aliae species, quae tum fructificationem explicare nequeunt, ob nisum hymenium formandi, massam sporaceam in luce protrudunt, ubi, liberata, in Hymenomycetes rite evolutos (Pezizas, Clavarias, Agaricus) abit. Hinc multis speciebus ex citatis generibus Sclerotia matricem suppeditant; sed hae tantum secundum perfectissimam suam tructificationem in systemate citandae. Haec est causa, quare omittimus tubercula Typhularum, Pezizae tuberosae etc.; alias vero fructificatione etiam propria gaudentes (Avisyermum comutum, etc.) inter Sclerotiaceus citamus, fungos inmascentes parasiticos judicantes. Sed in his plurima observanda restant." Bei einem so unklaren Stande des Wissens über die Natur der Sclerotiaceen war es überaus erfreulich, eine Kraft, wie die eines LéveıLlé, der Lösung dieser Frage zugewandt zu sehen.

In dem oben citirten Mémoire theilt Lévenlé (1. c. p. 219) den Gesammtbestand des vorgefundenen Materials:

1. In solche Gebilde, die ausschliesslich pathologischer Natur sind, dahin zählend z. B.: Sclerotium femuineum. SchuLt\%; desgl. Spermaedice Clavus, Paspali et Muydis, welche nur Monstrositäten des Gramineen-Orariums seien: eine Ansicht, welcher auch E. F'ries(1) im Jahre 184 ? beitritt, nachdem dieciclbe in PHöвus (2) eine so kräftige Unterstützung gefunden hatte.

2. In solche, die nur als Anfänge oder als Hemmungsbildungen anderer Pilze anzusehen sind, z. B. Suter. fuy ScuU u.. welches nichts als das Mycelium eines Gastromyceten sei; ferner dcrospermum cor-

(1) Summa vegetabilium Scandinaviae. Holm \& Lips. 1849, pag. 519. „Omni fructificatione apud nos caret, at in eadem saepe parasitantur Mucelines, Fusaria, Sphacelia LEv, etc."

(2) Cryptogamische Giflgewähse, Tom. II, 1838, 40., pag. 97-110. 
nutum Fr. ein unentwickelter Agaricus (Nyctulis) parasiticus; sowie Acrospermum pyramidale TODE, welches den noch hutlosen Stipes des Agar. (Nyctalis) parasiticus darstelle.

3. In solche, welche anderen Gattungen einzureihen seien, wie z. B. Acrospermum sclerotioides FR., das eben nur Phacorrhiza sclerotioides Pers. darstelle, oder s'cl. laetum Enmens. = Pistillaria micans Fries, und viele andere.

4. In solche, welche nur Varietäten anderer Sclerotien sind. So sei z. B. Scler, ovatum Schum, nichts als eine Form des Scl. durum Pers., Sclerot. lacunosum Pers. sei nichts als Scl. fungorum var $\beta$. Fr.; desgl. Sclerot. minutum Diтtм. gleich Scl. varim Pers. Ueberhaupt aber ist Léveillé mit Conda (1) der Meinung, dass die Sclerotien ganz und gar nicht ächte Pilze, sondern nur unvollständige Gebilde fungöser Natur seien, die, so wie die Genera: Hypha, Himantia, Rhiromorpha, sterile verdicktete Mycelienformen ohne allen Samen darstellten oder eben nur Hemmungsbildungen wären. LÉveILLÉ sah das Sclerotium Semon, vulgatum, complanatum, compactum, Pustula, stercorarium, muscorum, pyrinum aus einem filamentösen Mycelium hervorgehen, welches aus haarfeinen ramificirten Langzellen bestand, in deren Mitte das zum rerschieden-gefärbten Sclerotium werdende anfängliche Knötchen auftrat. Mit einem solchen fadenförmigen Mycelium versehen, wird Rhizoctonia muscorum nambaft gemacht, welches ohne ein solches Mycelium im fertigen Zustande das Sclerotium muscorum darstelle, wie dies bereits Fries (l. c. p. 252 und 266) anzudeuten scheint. Diesen Anschauungen entsprechend, ist somit LÉventú schon 1812 der Meinung, dass die Sclerotien dienen , pour la conservation des espèces, mais qui, développée dans les circonstances peu fitrorables, en attend de meilleures pour arriver à son état parfait." Die Nöthigung zu einer solchen Auffassung derselben fand er in folgenden Beobachtungen:

1. Aus dem ruhenden Acrospermum cornutum sah er beim Eintritt fenchter Witterung den Alyuricus purasiticus BuLL. hervorgeben, auch schien ihm das Acrosprimum pyrumilale TODE nichts anders, als der jugendliche Zustand des Acrospermum cornutum zu sein.

2. Sclerotium mycetospora TIF. F. L. NeEs giebt dem Agaricus volvaceus seinen Ursprung, wie das:

3. Sclerotium fungorum PERs. dem Agavicus tuberosus BULI., der sich auch wieder ohne Sclerotium erzeugen kann, um als Agaricus ocellatus $\mathrm{F}$. zu figuriren.

4. Aus Sclerotium lacunosum Pers. erwächst nach Persoon,

(1) Icones fungorum. 
Sowerbi und Lévellué (1) Agarieus rocemosus, den Fries für eine Varietät des Agar. tuberosus hielt. Mit der Zunahme des Agaricus schwindet das gelbliche, bis erbsengrosse, mit Depressionen rersehene Sclerotium.

5. Das Sclerotium stercorariun DC. (dem Scl. Semen sebr ähnlich) erzeugt aus kleinen Auschwellungeu bei feuchtem Wetter 5-6 einzelne Agaricus stercorarius BuLL, erweicht und zersetzt sich endlich. Indessen besitzen einzelne Agarici gar kein Sclerotium, sondern entstehen sofort aus dem Mycelium.

6. Den Agaricus fusipes Bulu. findet man auch nicht immer mit einem Sclerotium; zuweilen aber kamn man ein solches erkennen, und dann bringt dasselbe mehrere Jahre hindurch den Agaricus fusipes.

7. Dem Agaricus grossus hängen weisse byssoide Fäden an, die aus einem haselnussgrossen schwarzen trüffelähnlichen Sclerotium kommen.

8. Clavaria juncea Pers. entsteht wahrscheinlich aus Sclerotium complanatum ToDe.

9. Clavaria minor Léveillé (sp. n.) aus kleinen gelben Gebilden, die an Scl. fungorum od. muscorum erimnern.

10. Pistillaria micans FR., (Sclerotium laetum EHнемв.) zeigte sich ebenfalls als aus einem hypophloeodischen Sclerotialgebilde hervorgegangen, welches auch fehlen kann.

11. Pistillaric sclerotioides Fr. od. Clavaria sclerotioides DC. verdankt einem Sclerotium seinen Ursprung, welches dem Sclerotium Semen am nächsten steht (2).

In Typhula gyrans Fr. hätte LÉvenLé ebenfalls in der Fries'schen Beobachtung (Elench. I, p. 236) eine Stütze dafür finden können, dass das Sclerotium-artige tuberculum bald fehlen, bald da sein kann, ohne dadurch das Wesen der Art zu alteriren. Nach

(1) Nach BAIL in Nota Acta Acad. Caes. Leopold. Carol. germ. naturae curiosorum. Tom. XXVIII', Jenae 1861, Abhdlng VI, „Die wichtigsten Sätze der neuern Mycologie etc." pag. 10, soll dieser Agaricus racemosus PERs. einen Dimorphismus der Fortpflanzungsorgane besitzen. Die traubig vereinten Knöpfehen, welche sich lateral frnden, sollen divergirende Astyphen und auf diesen länglich ovale weisse Keimzellen erzeugen, die sich später trennen.

(2) Nach Fries Elenchus fungorum, I, p. 238 und BAIL in dem von ihm bearbeite. ten NeEs'schen System der Pilze, Tom. II, p. 76, so wie in ter Heduigia, 1856 No. 14, ist es Phacorrhiza sclerotioides PERs. od. Pistillaria Phacorrhiza FR. welche ein dem Scl. Semen ähnliches tuberculum radicale (Sclerot. Puslula DC.) besitzt und nach BaIL einen keuligen Pilz producirt, welcher seiner Structur uach für eine Typhula anzusprechen ist. Dagegen geht nach Fries (1. c. p. 237) Pistillaria sclerotioides PERs. zwar aus einem ähnlichen Tuberculum hervor; aber die Keule ist nicht weiss, sondern braun. 
BaIl (1. c. pag. 76) ist es Sclerntium complanatum Tode, welches der Typhula gyrans Fr. zum Ausgangspunkte dient.

12. Peziza Candolleana LEv. (fuscescens subfasciculata, tenuis, cupula integerrima, planiuscula nuda; stipite gracili filiformi nudo passim basi villoso, tuberculo innato, 1. c. pag. 233) der. Periza subulipes BuLL. am nächsten stehend, entstand aus Sclerotium Pustula DC. der Hainbuche, Kastanie, etc. BuIn (Syst. d. Pilze II, p. 76) leitet von demselben Scl. Pustula DC. die Typhula sclerotioides ab. Nach RabenHonst (Deutschl. Crpt. F'lora I, 354) findet sich Peziza subularis Buct. od. subulipes Pers. in den Fruchtböden von Helianthus annuus, wo ich selbst zwar sehr häufig Sclcrotium Pustula DC. jedoch ohne Peziza sah.

13. Pilobolus crystallinus ToDe soll ebenfalls aus einem dem Scler. fungoim ähnlichen Sclerotialgebilde hervorgehen, während Durieu De Maisonneuve (1. c. p. 234) im Gegentheil den Deckel des Pilobolus crystallinus zum Sclerotium stercorarium.werden lässt.

14. In einer pulpa Tamarindorum entstand Penicillium glaucum, das am Grunde des Gefässes unregelmässig gelappte, rothe Körperchen erzeugte, welche ungeachtet alles Abwaschens und Zerschneidens Prnicillium glaucum an die Aussenfläche heraufbrachten.

15. Hatte Persoon (Mycol. europ. I, 33) Botrytis cinerea Pers. ausser auf verschiedenen Pflanzenstengeln auch ,passim parasitica supra Sclerotium durum" gefunden, so sah LÉveILLÉ denselben Hyphomyceten theils ohne Sclerotium, z. B. auf Umbelliferenstengeln, theils auf Solerotium durum in mehr oder weniger innigem Zusammenhange. Mit der Entwickelung des Botrytis schwindet das Sclerotium, und es ist dies einer der Wege, deren die Natur sich bedient, um Botrytis cinerea zu erhalten und unter dem Einflusse bestimmter Umstände wieder zu erzeugen.

16. Botrytis erythropus LEr. (1. c. 235 fasciculatus, floccis" erectis, ramosis tuberculo rubro innatis; ramulis subdichotomis acutis; sporis solitariis globosis, albis pellucidis). In todten Blättern von Caryota wrens erzengte sich ein Sclerotium (sous forme d'un tubercule aplati, de consistance presque cornée et d'une belle couleur rouge). Auf dem der Fenchtigkeit ausgesetzten Schotim entstand Botrytis erythrop̧us. (Hätte LÉrzilté das Sachverhältniss umgekehrt beschrieben, so wären die unten folgende Mittheilungen nunmehr überflüssig geworden).

17. Ausser den bereits oben angeführten Fällen citirt LÉVEILLÉ unter andern noch die Entwickelung des Agaricus arvalis Fr, auf Sclerotium vaporavium DC., welches vou Demoiselle Libert zu Malmédy in deren Cryptogames des Ardemmes sub No. 220 gegeben ward. 
Allein dieser Agaricus entsteht nicht immer und ausschliesslich aus einem Sclerotium, sondern auch aus dem einfachen fädigen MIycelium !

18. Nach Corda (1) entsteht Stachylidium characeum LK. auf Sclerotium Hippocastani.

19. Peziza tuberosa Hedw. und Bull, welcher nach Hedmig (2) von einer Anemonenwurzel, nach LéverLLÉ jedoch von einem Sclerotium scinen Ausgang nimmt, erweicht nach BdiLrarn mit fortschreitender Entwickelung der Pcziza. (Aus mehr als 30 directen Beobachtungen kann ich diese allmälige Erweichung und Aussaugung des schwarzen Knollens nur bestätigen; auch ich halte mit LéveILLÉ dieses basale tuberculum für ein Sclerotium, welches vielleicht in Heracleum Sphondylium oder ähnlichen Umbelliferenstengeln entstanden, in die Tiefe von 3-4" gelangt. Dies Sclerotialgebilde ohne Peziza tuberosa zu finden, ist mir noch nicht gelungen.

20. Peziza Tuba Batsch (Fungoides) fand schon Mrcheli (Nova plant. genera. Florent. 1729, pag. 205, Tab. 86, fig. 10) auf einer radix perennis, quae tribus plerumque tuberculis constat, quorum quidem, quod medio loco consistit, fungoidea (i. e. Peziza tuba Batsch) profert atque alit.

Aus diesen Thatsachen geht somit hervor (1. c. pag. 241), dass: , les Sclérotés sont des accidens de végétation, puisque les mêmes espèces en présentent quelquefois, et que dans d'autres circonstances elles n'en offrent point." Demnach spielen die Sclerotien: „le rôle de caïeux ou de rhizomes;" sie sind: "formes du tissu fongique, qui ont été décrites comme genres et comme espèces." Dieses Mycelium tritt in folgenden Formen auf: 1. als mycélium filcmenteux ou nématoïde; 2. als mycélium mémbraneux ou hyménoïde; 3. als mycélium. scléroïde und 4. als mycélium pulpeux ou malacoïde.

$\mathrm{Zu}$ dem mycélium nématö̈de gehören demnach die Pseudogenera: Fibrillaria, Ozonium, Himantia, Racodium, Xylostroma, Phlebomorpha, Hypha, Mesenterica, etc. Nur dem mycélium tuberculeux ou sclérö̈de (dem Sclerotium und Acrospermum nämlich), welches niemals primitiv ist, geht ein nématoïdes Mycélium voraus, (welches aber selbst aus Sporen entstand. MTR.)

Wäre LÉveillé nicht von der irrthümlichen Voraussetzung ausgegangen, dass Spermoedia Clavus DC. eine Monstrosität des Gramineonfrüchtchens sei, so hätte or auch für dieses unzweifelhafte Sclerotialgebilde die richtigere Auslegung finden müssen. Es ist

(1) Icones fungorum, I, p. 21, tab. VI, fig. 278 (nicht wie irrthümlich bei LéveILLÉ steht, p. 378).

(2) Musci frondosi, II, p. 31, Taf. 10, fig. 2. 
dies um so auffallender, als er sellsst in seinem frühern „Mémoire sur l'ergot" (1) die ron ihm Sphreelia seyetum genannte Schmarotzerpilzform entdeckt und beschriehen hatte, und nun später dieselbe Arbeit so weit ignorirte, dass er es unterliess, die Spermoedia Clavus Fr. damit in organischen Zusammenhang zu bringen. Auch hätte LÉVEILlé 1842 wohl wissen können, dass bereits in 1841 MEYeN) in seiner Pflanzenpathologie pag. 191-204) sich dahin ausgesprochen hatte, dass die gehirnartig erscheinende Sphacelia segetum im Innern der Gramineenfrucht sich ansiedelnd, dieselbe zerstört und dass das Mutterkorn die rollständigste Analogie mit der Bildung der festen Körpers der Sclerotiumpilze habe, welche ebenfalls aus einer Verfilzung der MIceliumfäden hervorgingen. Indessen Meyen ward nicht bloss von Iúveiclé, sondern auch von seinen eignen Landsleuten ignorirt und theilte insofern ein gleiches Geschick mit Quecкетт (2), welcher bereits im Jahre 1839 auf dem Ovarium der Gräser vor Eintritt der Spermoedia-Bildung und vor der Entfaltung der Grasblüthe eine Schimmelart gesehen und - zugleich nachgewiesen hatte, dass , unzählige kleine Fäden und winzige Körperchen das Grasfrüchtchen mit einem Ueberznge vollständig umkleideten, der Ergotactia ahortans nämlich; doch scheint er selbst kaum geneigt gewesen zu sein, einen Causalnexus zwischen seiner Ergotaetia und dem Mutterkorne anzunehmen.

Als Beweis, dass das Mutterkorn nicht übertragbar sei, galt der seit 1831 bekannte Versuch ron. Wiggens (3), welcher Mutterkorn unter Roggenpflanzen gesäet hatte, um in denselben Mutterkorn zu erzeugen; ein Versuch, der, wie wir jetzt wissen, begreiflicherweise niemals ein derartiges Resultat herbeifïhren konnte. Indessen die Fontana'sche Ansicbt der Fortpflanzungsfähigkeit des Mutterkorns veranlasste doch Meyen (4) , die Sporen der Sphacelia zwischen die Blüthen der Weizen-Aehren zu strenen, und schon nach einigen Tagen sah derselbe ein zartes Pilzgewebe daraus hervorgehen." Hätte sich MEYEN nicht von dem Vorurtheile leiten lassen, dass das

(1) Mémoires de la Société Linnéenne de Paris, V, pag. 365, 1827.

(2) Annals of natural history, 1839, March p. 54.

(3) Preisschrift über Secale cornutum, 1831, 40.

(4.) Pflanzenpathologie. Berlin, 1841. Nach d. Tode d. Verf. zum Druck besorgt ron Car. Gottrr. Nees v. Esexbeck, pag. 203. (Obschon Nees auf dem Titel genanut ist und er auch einige Anmerkungen dazu geschrieben hat, so kann ich doch nicht die Bemerkung unterdrücken, dass ich das Mauuscript zur Pflanzenpathologie bei Anordnung des Meren'schen Nachlass gefunden und die Herausgabe besorgt habe; indessen, weil ich seiner Zeit von Botanikern noch wenig gekannt war, bat NEES, seinen Namen der Schrift vorsetzen zu dürfen). 
Eindringen von Pilzfäden in geschlossene Pflanzenzellen unmöglich sei, so müsste er die Entistehung des Mutterkorns aus jenen flockigen Fäden der gekeimten Sphacelia-Sporen schon 1840 gefunden haben: eine Entdeckung, die erst Tulasse zu machen so glücklich war.

Im Anschluss an Rhizomorpla, Depazea, u. s. w. bezeichnet CoRda (1) i. J. 1842 die Familie der Selerotiaceen desshalb als dubiös, weil an deren namentlich aufgeführten Gattungen „noch keine Fruchtorgane aufgefunden werden konnten und sie demnach aus der Reilhe kritisch besser bestimmter und untersuchter Gattungen auszuschliessen seien." CoRDA erwartet, dass, bei genauerer Untersuchung und nach glücklichem Auffinden der Früchte, viele der Sclerotiaceenfamilie eingereihten Formen, ganz andern Fanilien anbeim fallen dürften. Folgerecht fordert er anch für Spermoedia (1. c. p. 186) „einen grossen Sarg." Da nun CoRDA vor dem 1 August 1842, an welchem Tage er die Yorrede zu jener „, Anleitung” schrieb, füglich nicht wissen konnte, was Lévellué am 21 März 1842 der französischen Akademie rorgetragen und erst 1843 in den Annales des sciences (Tom. XX) publicirt liatte, so geht wenigstens daraus hervor, dass der deutsche Mycolog auf dem Wege eigner Forschung ganz zu demselben Resultate gelangt war, wie der unabhängig von ihm forschende französische Mycolog.

Nichtsdestoweniger aber nalm RABENHonst in seiner Flora der Cryptogamen Deutschlands (Lpz. 1844. Bd. I, p. 238 fgd.) die Scleroticueen als zweite Section der Familie der Lycoperdacei in das System wieder auf, ertheilt denselben „, Sporen, die bisweilen unkenntlich, verwachsen, endlich aber frei werden und hervortreten," und spaltet sie in die Gattungen: Sclerotium ToDE und Acrospermum Tope, denen sich als Rhizoctonei RIchт. die Gattungen Anixia Fries, Mylitta Fries und Rhizoctonia DC. anschliessen, während WALLRoti in seiner Flora cryptogamica (Tom. IV des Compend. flor. germ. r. BuufF und Fingeruutri 1833, p. 187 und fgd.) die Gattungen Sclerotium TODE und Acrospermum ToDE seiner ersten Ordnung Aspromyceten einreiht und ,ad genesin paradoxa" erklärt hatte.

Den ersten von glücklichem Erfolge begleiteten Versuch, dies „Paradoxon," d. h. die Natur der Sclerotien auf dem Wege des Experiments zu lösen, machte TuL.sse (2). Aber derselbe pflanzte nicht Selerotinm Clavus DC. unter Roggen, wie es Wiggens gethan, sondern so in einen Topf ein, dass die. Producte beobachtungsfähig wurden.

(1) CORdA, Anleitung zur Mylologie, Prag 1842, pag. 137.

(2) Annales des sciences nalurelles, 3me. serie, Tom. XX, pag. 1-56. Zuvor in Comptes rendus hebd. des sénnces de l'acad. des sciences, 'Tom. XXXIII, pag. 645. 
Das auf dem Mutterkorn unter den gegebenen Bedingungen entstandene Gebilde, von ihm Claviceps purpurea genannt, war nun zwar an sich nicht neu, denn schon Schumacher hatte es (1. c. p. 174) unter dem Namen Sphaeria entomorhiza beschrieben, und Fries (Syst. myc. II, p. 325) dasselbe als Sphaeria purpurea (und zwar verschieden von der Dickson'schen Sph. entomortiza) in das System aufgenommen, auch WaLLRoTH bereits dasselbe Gebilde als Kentrosporium purpu. reum ausführlicher behandelt; allein die Zusammengehörigkeit, den nothwendigen Zusammenhang des Scler. Clavus mit der Sphaeria (Kentrosporium) purpurea hatte zuvor Niemand erkannt und nachgewiesen. Ferner ermittelte Tulasne, dass die Lúvemlü'sche „Sphacelia," welche dieser (1. c. p. 18) folgender Art beschrieben hatte: "ce champignon n'est d'abord qu'un corps mou, liquide, visqueux, fétide, sans forme determinée," von dem parasitischen Schimmel QUECKETT's, der Ergotaetia abortifaciens, zu sondern sei und ebenfalls im innigen Zusammenhange mit dem Sclerotium und der Sphaeria purpurea Fr. stehe, so dass man ,trois termes," unterscheiden müsse, ", dont chacun de nos Claviceps se compose," nämlich die „sphacélie, l'ergot und la sphérie," welche aber dennoch nur ein einheitliches Ganzes darstellten. Die LÉveILLÉ'sche sphacélie ist ihm eine Spermogonie, d. i. ein Organ, , dont le rôle doit correspondre à celui que les Cytispora et leurs analogues jouent chez d'autres Hypoxylées." Das ergot selbst ist ihm ein Selerotium und homolog dem Scl. complanatum Tope, scutellatum ALB, und Scrw., Pustula DC. u. s. w. (, qui naissent implantés sur les filaments à peine visibles, ou même entièrement cachés dans l'épaisseur des tissus végétaux, dont ils se nourrissent.") Endlich sagt er im Betreff der „splérie," „où l'appareil reproducteur par excellence en procédant de l'ergot confirme sa nature et complète l'être végétal dont il sert à unir les deux termes essentiels."

Vou eben so grosser Bedeutung als der durch vorstehende kurze Darstellung erzielte Fortschritt in der Erkentniss der Natur der Sclerotien war es, dass Tulasne nicht nur Claviceps purpurea aus Sperm. Clavus DC. erzielte, sondern dass es ihm auch glückte, die schon zuror von mehreren Mycologen rermuthete Differenz der Gramineen-Sclerotien thatsächlich nachzuweisen. Das Mutterkorn von Molinia, Corlamayrostis und Phraymites lieferte bei der eingeleiteten Cultur, Claviceps microcephala; das von Andropogon Ischaemum lieferte Claviceps pusilla und Mutterkorn von Scirpus-Arten lieferte Claviceps nigricans, von denen die beiden letzten jedenfalls ganz neue Formen der Keulen-Sphärie darsteltten, während ansser purpurea (Kentrosporium mitratum WALL.) auch $C l$. mierocephala schon 
von WALLnotm als Kentrosporium microcephalum WALL, und ron FrIES als Cordyceps purpurea beschrieben waren.

Das grosse Aufsehen, welches TulasNE's schöne Entdeckung herrorricf. veranlasste gar bald A. Braun (1), KüHn (2) und BaIL (3) die Aussaat von Sclerotium Clavus, etc. zu wiederholen.

Alle erzielten gleiche Resultate. Barc (l. c.) stellte aüch fest, dass Phncowhizo scterotioüdes Pers. (von welcher schon oben die Rede war) aus einem wahren Stengel-Sclerotium der Adenostyles hervorgehe; ermittelte, dass Sclerotium Semen. ThDe, selbst nach 2 jähriger Ruhe, einen keulenartigen Pilz, Typluula variobilis Riess erzengte, den Riess (Hedwigia No. 5) zuerst beschrieben hatte; auch fand BAIL (1. c. p. 200) $1855 \mathrm{im}$ Riesengebirge , kastanienbraune, innen weisse scleroticn-artige $4^{\prime \prime \prime}$ lange Körper in alten kohlschwarzen Hymenomycetenresten, die etwa die Gestalt länglicher Brutzwiebelchen hatten und aus der Spitze hie und da einen weissen, ihm noch unbekannten Ayaricus von $1^{\prime \prime}$ Länge, mit fädlichem Stiel und kleinem Hute hervorgetrieben hatten. Im Resumé (wo BAIL eine längere von Abbildungen begleitete Abhandlung in Aussicht stellt, welche aber nicht publicirt zu sein scheint) spricht sich der Verf. dahin aus: 1, dass alle Sclerotien nur unentwickelte Stadien anderer Pilze seien; 2, dass ein grosser Theil der bisher noch räthselhaft gebliebenen Sclerotien der Gattung Typh hula angehöre; 3, dass endlich auch einzelne Species anderer Gattungen eine Sclerotien-Form besitzen und deren Vor. kommen nur von der Individualität der Art abhänge und nicht auf den Character der Gattung influire. In der von BaIL besorgten Fortsetzung des Systems der Pilze von Th. Fr. Lodw. Nees rox Esenieck und Hexry, Bonn 1858, nennt derselbe die Sclerotien Druermycelien und unterschied damals drei Gruppen derselben:

a. die Gruppe Sclerotium Clavus ohne gesonderte Haut, deren zarte Fäden gegen die Spitze hin Spermatien abschnüren. Hiezu gehören Formen von Claviceps.

b. die Gruppe Acrospermum (Scl. cornutum Fr.) mit structurloser Hüllhaut und ohne Spermatien. Hiezu gehöre Agarieus tuberosus ButL.

c. die Gruppe der Blatt- und Stengel-Sclerotien, im Innern aus unregelmässigen, durch einander gewundenen Fäden bestehend; die Dauermycelien von Typhula-Arten. So gehöre Seler. cirustuliforme Roв. zu Typhula erythropus Fr., Sclerotium Pustula DC. zu Typhula

(1) Bolanische Zeitung von H. v. MoHL und v. Scirecritendat, 1854, p. 565.

(2) Küнs, in Dr. Haмm's Agron. Zeitung, Jahrg. X, No. 39, p. 612.

(3) Hedwigia, 1856, No. 14, und 34er Jahresbericht d. Schles. Ges.f. vat, Cultur, 1856,40, p. 48. 
sclerotiöides; ein früher nicht benannt gewesenes Sclerotium zu Phacomtize sclerotioüdss Pers. (welche ebenfalls eine Typhula sei); Sclerot. Semen nebst $\beta$., Brassicae FR. zu I'yphula variabilis Rress, und endlich Sclerot. compactum TODE wahrscheinlich zu Typhula gyrans.

$d$, , vielleicht wird diese Eintheilung noch durch neu hinzutretende Gruppen bezeichuet. Als Repräsentant einer solchen wird wohl das Sclerotium stercorarium DC. (aut lacunosum PERs. ?) anzusehen sein, welches zu Periza tuberosa BuLL. gehört."

BaIL schliesst mit der Bemerkung: ,Soviel steht fest: Die Sclerotien sind keine vollkommnen Pilze, sondern nur Dauermycetien ron solchen und können daher von jetzt ab im System nicht mehr für sich behandelt, sondern nur bei den einzelıen Pilzspecies erwähnt werden, denen sie als Grundlage dienen."

War nun somit durch BAIL in Rücksicht anf andere Sclerotialgebilde ein unverkennbarer Fortschritt gegeben, so geht doch unzweifelhaft aus dessen Arbeiten hervor, das es sich bei ihm nur um Metamorphose handelt, keineswegs um Generationswechsel, wie derselbe durch Tulasse angebabnt und durch KüHn (1) und Bonordex (2) für Sclerotium Clavus DC. dem Abschluss näher gebracht ward, freilich ohne das erzielte Resultat mit diesem Namen zu belegen und unter diese Categorie zu bringen.

KüнN fand das Mutterkorn sehr häufig im Waizen, auch in Triticum durum, turgidum und Spelta bei der Gerste, dem Roggen, Festuca arundinacea, Molinia coerulea und Phragmites communis. Die in dem süssen Schleime suspendirten Sporen sind für ihn nicht Spermatien, sondern Stylosporen (1. c. p. 130) weil sie , einen und selbst mehrere Keimschläuche zu entwickeln " vermögen, was bisher von Spermatien nicht behauptet werden konnte. Aus dem durch Keimung der Sphäriensporen des Clavierps herrorgehendem Mycelium entsteht zunächst ein Gewebe, welches die Oberfläche junger Gramineenfrücbte bedeckt, (cf. Tab. V, fig. 4) und von dessen freien Endästen die Stylosporen sich nebst einer schleimigen Substanz ablösen.

Diese auch durch gute Abbildungen erläuterte Thatsache drückt offenbar einen vollständigen Generationswechsel aus; denn nicht nur in der Lebensform des Claviceps, sondern auch in der Lebensform der Ergotaetir (C.) d. h. des Stylosporen erzeugenden Mycelium

(1) Kürv, Krantcheiten der Gevächse, 1858, 80, p. 113-132. Taf. V, Fig. 1-30.

(2) Botanische Zeitung von H. voN MoHL und von Schlecirtendax, 1858, 9 A pril, No. 15, Tab. IV, Fig. B. 
auf der Oberfläche der Grasfrüchtchen, ist das Gewächs zur Vermehrung durch keimfähige Sporen befähigt. Zwischen beiden Generationsformen liegt der ruhende Zustand der Sclerotialform mitten inne. (Ein unlängst in den ,Annales des sciences naturelles" von Wagsel und zuvor von PAgenstecher beschriebener Entwickelungsvorgang bei einer Cecidomyia (Diptere) giebt eine ganz analoge Reihe von Lebenszustïnden. Während Dipteren sonst nur die Formen rollkommener Verwandlung (Metamorphose) durchlaufen, ist für diese Dipterenform nachgewiesen, dass auch ihre Maden (Larven) einer ungeschlechtlichen Vermehrung fähig sind, was bisher von keiner Insectenlarve behauptet werden konnte. Diesem rermehrungsfähigen Larvenleben folgt der Zustand der ruhenden Puppe und dieser der Zustand der zeugungsfähigen Imago. Entständen die Claviceps-Sporen nachweislich auf dem Wege geschlechtlicher Zeugung, so würde jene merkwürdige Diptere den ganzen Lebensgang des Claviceps in der Gestalt eines Thieres wiederholen!)

Dem Sclerotium Clavus DC. gleichwerthig erachtet Küнs (1. c. p. 128) Sclerotium Semen var. Brassicae in Rapsblättern, sowie Sclerotim Brassicae in Rapsstengeln, faulenden Kartoffeln und Mohrrüben. Aus beiden sollen sich ,keulenförmige kleine Pilze entwickeln, die als Arten der Gattung Typhula angehören, eine Ansicht, die heute wohl nur zur Hälfte sich als zutreffend erweisen lässt, indem wenigstens das Sclerotium Brassicce der Rapsstengel Peziza Sclerotiom erzeugt, wie ich es ror Jahren gefunden und anderswo (s. unten) angegeben habe.

Den rou den Herrn Tulasse, Küin und Bonordex für Sclerotium Clavus DC. mit so glänzendem Erfolge betretenen Pfad weiter zu verfolgen, d. h. zu den übrigen Sclerotien mit ihren durch Cultur herbeiführbaren Hymenomyceten-Zuständen (Aymicus tuberosus Bull., Typhula variabilis Riess, ete.) die betreffenden Stylosprenenformen nachzuweisen, versuchten jedoch weder Tulasse, Küis und Bonorden selbst, noch auch Prof. Cons (1), obschon dieselben sämmtlich auf dem besten Wege dazu sich befanden. Kürs und CoHs hatten an Rapsstengeln, da wo Sclerotium Brassicae im Inner'n sich bildete, an der Aussenfläche nach unten zu "vergelbte" Stellen gesehen, ohne deren Entstehen zu rerfolgen und deren feranlassendes Moment zu ergründen. Auch Benkeler (2), welcher die Sclerotien für ,, compact masses of cellular tissue, which have arisen

(1) Sitsnngsberichte der Schlesischen Gesellschaft für voterlündische Cullur, 1856, 40., p. 50 .

(2) Introduction to cryptogamic botany. London 1857, pag: 267 . 
from the metamorphosis of an originally floccose mycelium" ansah, welches zuweilen wie bei Birnen und Aepfeln die Form eines Sporo. trichum annimmt, oder auch an den freien Spitzen fructificirend, Coremium, statt cines Sclerotium bildet, war weit enfernt, den Nachweis ron Hyphomyceten gefühnt zu haben, welche in Sclerotien sich umwandelnd, zu Hymenomyceten-oder Discomyceten-formen wurden. Erst Eugène Coemaxs (1) näherte sich der Lösung dieser Frage in seinem der Académie royale de Bruxelles überreichten Mémoire, betitelt: "Recherches sur la genèse et les métamorphoses de la Peziza Sclerotionum Lib." Herr Consuns unterscheidet (1. c. pag. 66) drei Phasen der Entwickelung: „L'état primitif ou némutoide représente, en effet, assez bien la larve des insectes; l'état sclésotien, état de la vie latente, rappelle leur' second âge, leur vie à l'état de nymphe; et l'état périzoüde, celui d'insecte parfait, ayant, comme celui-ci; toute la supériorité d'organisation, de forme et de couleur qui caractérise en zoologie les formes adultes." Durch Aussaat der Sporen von Peziza Sclerotiorum Liв. erzielte angeblich der Verfasser das Prothallium oder das „mycélium byssoide," aus welchem das mycélium sclérotique hervorging (ein Versuch, der mir nicht gelang, MTr.)

Unläugbar vermittelt diese Auffassung der Sclerotien einen weitern Fortschritt in deren Erkenntniss; allein schon der Titel der Abhandlung, so wie deren Inhalt weisen mit grosser Bestimmtheit darauf hin, dass es sich bei Coemaxs um Erledigung einer Frage der Metamorphose, nicht des Generationswechsels handeln soll. Und doch finden sich Andeutungen, dass der geistvolle Herr Verfasser auch auf die Erscheinungen des wahren Generationswechsels aufmerksam geworden ist, wie schon aus den Mittheilungen des Berichterstatters КІскх hervorgeht. Derselbe sagt nämlich in seinem Rapport im Betreff der Stylosporen des Sclerotium Cluvus DC. „Mr. Coevaxs les a aussi retrouvées chez le Sclerotium varium. Il y a observé, en outre, d'autres cor'ps reproducteurs qu'il hésite, peut-être à tort, à regarder comme des spermogonies, puisqu'ils paraissent en avoir tous les caractères." Auch Herr Krckx ist der Meinung, dass mit der Coemass'schen trefflichen Arbeit die Frage noch keineswegs erledigt ist. Wie, z. B., fragt derselbe, verhält sich eine Art, die zwar regelmässig ein Sclei'otial-Mycelium bildet, wenn sie ausnahmsweise diesen Zwischenzustand nicht entwickelt? Wird die Abwesenheit des Mycels durch eine ungewöhnliche Entwickelung:

(1) Bulletin de l'académie royale de Bruxclles, 29 année, 2 de série, 1860, I'om. IX, pag. $62-97$. 
von Pycniden oder Spermogonien compensirt? Herr Coemans beobachtete im März 1859 in den Kellern des Civilhospitals in Gent auf dortselbst conserrirten Mohrrüben, Rüben, Runkeln und Cichorienwurzeln eine grosse Menge ron Sclerotium vavium, die ihn bestimmten, die Metamorphosen dieses polymorphen Pilzes näher zu untersuchen und ermittelte, wie bereits oben erwähnt ward, drei Perioden oder Lebensformen. Die Periode nématoide oder sphacélienne sprach sich in drei fructificirenden d. h. Stylosporen oder Sporen-erzeugenden Formen aus: 1. in Form einer feuchten Warze (mamelon), von tremelloïdischer Consistenz und durchscheinend weiss: diese geht in Sclerotium varium über; 2. in Form unregelmässig-verlängerter Lappen: diese giebt das Sclerotium compactum DC.; 3. in Form eines feuchten weissen Häutchens, welches zur Entstehung des Sclerotium bullatum DC. Veranlassung bietet. Ausserdem kam aber auch ein trocknes nematoïdisches Mycelium vor, das nicht fructificirte, demungeachtet aber in Sclerotium überging. Die erste Form bestand aus farblosen, langgestreckten, dünnwandigen Zellen, welche die Hypha, die Basidien und sphorophoren Fäden der Pflanze darstellen; 9. aus grünen (?), runden, undurchsichtigen Zellen mit dickerer Wandung; an den Radicellen fanden sich die Hofruanx'schen Schnallenzellen. Das aufsteigende System der Hyphen bestand aus Stylosporen, die denen der Sphacelie des Cluvus ähnlich waren und Fäden, welche dem Fusisporium ähnliche „spores fusiformes ou cylindriques ordinairement bi-triseptées" trugen; 3. aus unregelmässigen Fäden, die dem Hormiscium ähnlich waren und, se départiculent facilement en spores rondes ou arrondies, mais de diamètre fort variable." 4. aus stets sterilen Fäden mit knotigen Zellen, die eine natürliche Tendenz zur Sclerotiumbildung an sich trugen. Der Beobachter spricht nun zwar selbst die Besorgniss aus, duss ihm vielleicht ver'schiedenartige Gewächre vorliegen möchten, indessen weil ja auch CAspary und Schacht verschiedene Sporen an ein und derselben Pflanze gefunden haben, so könnten auch wohl in diesem Falle die beschriebenen Sporenformen als einer und derselben Grundlage entsprossen angesehen werden. Aechte Spermatien fand er nirgends und tritt diher der Selbstverbesserung Tulasne's bei, der in seinen Spermatien (1. c. 8, note 2) später Stylosporen sah. Allein Tulasne, sowohl als Coemans, sehen in ihrer „,sphacélie" keineswegs eine den Hyphomyceten hornologe Lebensform.

Das erweist sich mit ziemlicher Bestimmtheit aus dem Capitel, welches überschrieben ist: „Parasites” die sich neben der Sphacélie fanden, nämlich Mucor mufus Pers. Ascophora Mucedo Tode, Fusarium roseum LIN K, u. S. W. Aerostalaymus cinnabarinus CoRDA, Oidium 
virescens Fr. und endlich ein kräftiger Polyactis vulgaris LINK, der jedoch nicht fructificirte, (,,jamais cependant je ne les ai vus fructifier 1. c. p. 80.") Bei dem von ihm gesehenen reinern Räschen von Polyactis auf Cichorienwurzeln und Rüben, wo sich im Innern Sclerotien gebildet hatten, komnte er nicht entscheiden, ob die sclerotiferen Filamente zu Polyactis oder zu der von ihm beschriebenen sphacélit gehörten. Somit ist Polyartis für Coemans auch ein blosser „Parasit," der neben der Sphacélie auftritt, aus welcher Sclerotien hervorgehen. Während dic Mucedincen in ihrer Fructification ihr natürliches Lebensende finden, geht bei seiner Sphacélie (l. c. p. 81) nur der, der Fructification dienende, Theil zu Grunde; der andere Theil aber wandelt sich in ein Sclerotium um. Der radicelläre Theil des Myceliums bildet anfangs die Sphacelie, die nach beendeter Fructification abstirbt, „c'est le rhizome central de la sphacélie qui donne naissance aux Selerotium." Ohne Tulasse würde Commans sicher das Rechte erkannt haben; aber indem er seinem Vorgänger nur allzustrict folgte, kim er von der richtigen Erkenntniss des Sachverhaltes ab. Hätte er den Polyactis nicht für einen zufälligen Schmarotzer gehalten, sondern dessen radicelläre Fäden verfolgt, so würde er deren Uebergang in Sclerotium bereits nachgewiesen haben; aber die périude lacrymante, die der période laineuse folgte, und die zu der süssschleimigen Exsudation des Clavus eine verführerische Parallele lieferte, bestärkte ihn um so mehr in seiner vorgefassten Meinnng. Glücklicher war Cokusss in der Nachweisung der Entstehung der schwarzen Rindenschicht der Sclerotien (1. c. p. 85), auch der Vergleich mit Korkzellen ist vollkommen naturgemäss, es sind dies dieselben Zellen, welche Fries einst für vereinigte Sporen hielt. Im Betreff' der "Période sclérotienne" welche Coemans ausführlich schildert, bleibt hervorzuheben, dass der Verfasser aus der Sphacelie der Peziza Sclerotion'un LiB. Sclerotienformen der mannigfiachsten Form hervorgehen sah: kugelige, gelappte, abgeplattete, convexe, cylindrische, so dass dieselben sich zurückführen liessen auf Sclerotium varium PErs. und dessen varietas: elongatum CHev. (bei Mohrrüben); ferner auf Sct. compactum DC. und tectum Fries (aü̈ Rüben und Cichorienwurzeln); sodamn auf tirl. bullate, DC. (auf Rüben und Runkeln); endlich sogar auf' Sclerot. spluacriaeforme LrB., kleinen Warzen ähnlich von 2 millim. bis $3-4$ centim. Länge. Die Sclerotien sind nach Coemaxs der' Wurzel einer biennen PHanze bomolog (1) und metamorphosiren sich gleich den Chrysaliden der

(1) Eine gleiche Ansicht sprach BAIL aus in seiner Arbeit über Sphlaeria lyphina PLRs. Acla acad. Ciaes. 1862, p. 13. 
Insecten nach einer Ruheperiode in eine neue Art von vegetabilischen Nymphen. Wurden Sclerotien in Wasser gesäet, so entwickelten sie sich selbst nach 7 Monaten-nicht; allein die am 16. März in Blumentöpfe gesäeten zeigten schon im Mai, also nach 2 Monaten, braune Knötchen, welche jungen 'Typhulis glichen. Im Juni abei wurden dentliche caneel-farbige Pesizen erkannt, die er für Pezizu Selerotion m LIв. halten musste. Das Sclerotialgewebe erweichte sich allmählig, ward kleiner und diente offenbar der Peziza zur Nahrung. Den Uebergang der Sclerotialgewebsfäden in das Gewebe des Pezizenstipes kounte er deutlich erkennen. ,Ici done la métamorphose est certaine, et on ne peut soupçonner une espèce de parasitisme, comme chez l'Agaricus tuberosus et l'Aguricus stercorarius, où les Sclerotium producteurs se courrent d'un mycélium blane, qui se transforme ensuite en stipe." In dieser Behauptung folgt CoEvans offenbar LÉveILté, ohne die Thatsache durch eigne Beobachtung constatirt zu haben. In Rücksicht der "période pezizéenne" bemerkt nun Herr Coemans, dass die von ihm durch Cultur erzielte Pezize zur Pezizu Sclerotionum Lin. ward, welche bereits durch Fräulein Libent in deren Plantae crypt. Arduennae sub No. 326 publicirt und vertheilt worden ist, ohne dass es sich dort freilich um ein Culturproduct handelte. Dieselbe stehe der Pexiza Cundolleana LÉveILlé (Annales des Sc. nat. XX, p. 233, 1843), wahe, welche sich auf Sclerotium Pustula DC. findet. Allein die Perizo Sclerot. variirt sehr und stellt sich dar als: var. clavarifonis, infundiuuliformis, subturbinata und hypocrateriformis. Der Durchmesser der Cupula varïrt von 1 Millim. bis 1 Centimeter; sie entwickelt sich in 8-10 Tagen, während sie 20-30 Tage ausdauert. Spermatien fanden sich nicht; auch ist die Entwickelung der Pezizensporen zu Sphacelien nicht beobachtet worden, obschon im Resumé und auch an einer andern Stelle die Behauptung aufgestellt wurde, diass aus den Pezizasporen das nematoïde Gewebe der Sphacelie hervorgehe, was doch wohl noch erst durch den Versuch zu erweisen bleibt.

Da ich selbst bereits im October 1857 (1) im Innern absterbender Stengel von Martynia fiagrans zahlieiche Individuen von Sclerotium varium PERs. gefunden hatte und deren weitere Entwickelung kennen zu lernen begierig war, so pflanzte ich im Monat December des. selben Jahres eine kleine Quintität in einen Blumentopt, etwa $1 \frac{1}{2}$ Zoll tief, erzielte aber el'st im Juli 1859 die ersten Pezizen, die ich, weil ich die Liber's'sche Art nicht kannte, für neu hiclt und dieselbe zum Andenken an meinen vieljährigen mycologischen Freund, Bataillons-

(1) Bulletin de l'académie royale de Belgique, 2e. série, Tom. XI, No. 2, p. 6. 
arzt Dr. Antz: Periza Antzii nannte und (l. c. pag. 8) abbildete. Offenbar ist dieselbe mit der von Cosuaxs erzogenen Periza Sclerofiorum LIB. identisch und kann daher nur als eine der Formen angesehen werden, wie sie Herr Commans ebenfalls erzielte und beschrieb. In jener Mittbeilung wies ich bereits auf den Generationswechsel der Sclerotiaceen überhaupt 'hin und musste damals nur mein Bedauern darïber aussprechen (1. c. pag. 6), dass es mir bis dahin nicht möglich gewesen war, die betreffende Hyphomycetenform angeben zu können, welche den Pezizen-Sclerotien der Martynia fragrans zu Grunde lng. Auch bei einer spätern Veranlassung hatte ich noch einmal Gelegenheit (1) auf die Sclerotiaceen zuriickzukommen und in Bezug auf Periza Sclerotion Lum. und andere zu bemerken , dass eine grosse Menge ron Schmarotzerpilzen die Eigenthümlichkeit besitzt, oine gewisse mehr oder weniger länger dauernde Periode ihres Lebens in einem ruhenden, durch schwarze Korkzellen nach aussen abgeschlossenen Zustande zu rerharren, um dann bei geeigneter Zeit und geeigneten Umständen neues Leben um sich zu verbreiten, indem sie in irgend welcher, aber stets gesetzlich bestimmten Gestalt keimfähige Sporen, d. h. Aequivalente der Samen erzeugen, um ibres Gleichen unmittelbar oder durch andere Zwischenformen, die selbst wieder zeugungsfähig sein können, hervorzurufen."

Alle diese vorstehend nnd möglichst mit den Worten der betreffenden Mycologen selbst anfgeführten Beobachtungen werden nun, wie Eingangs bemerkt ward, in einer nenesten Arbeit eines namhaften Forschers, des Herm Bonordex (2), in den wesentlichsten Punkten nicht nur angegriffen, sondern als auf Irrthum beruhend widerlegt. Die Widerlegung selbst aber fand bereits eine noch nenere Unterstützung in der ,, Einige Worte über die Abhandlungen aus dem Gebiete der Mykologie des H. F. Boxondex" betitelten Abhandlung von Steprax Schulzer von Müggenburg (3), so dass es wohl gegenwärtig gerechtfertigt erscheinen dürfte, dergleichen subrersiren Bestrebungen, die den historisch nachgewiesenen Entwickelungsgang unseres Wissens von den Sclerotien nicht nur zum Stillstande bringen, sondein ihn auf den Standpunkt ron 1822 zurückzuschrauben trachten, mit Thatsachen entgegen zu treten,

(1) Landvirthschaftliche Wochenschrift des baltischen Centralvereins, herausgegeben von Prof. Trommer und Oekonomie-Rath Dr. Ronde, 1863, 80. Greifswald, No. 8 und 9, pag. 112-119.

(2) Abhandlungen der naturforschenden Gesellschaft zu Halle. Halle 1864, "40., Tom. VIII, p. 1-168.

(3) Oesterreichische botanische Zeitschrift. Wien. Jalırg. XV, 1865, No 8, p. 241 und fgd. 
welche geeignet sein möchten, die gänzliche Unhaltbarkeit der jüngsten beiden Arbeiten über Sclerotien nachzuweisen.

In seinem 1851 publicirten Handbuche der Mykologie erklärt Herr Bonordex Sclerotien (1. c. p. 281.) für Pilzdegenerationen, "rüthselhafte Bildungen", die man verschieden gestaltet zwischen moderndem Stroh und andern Kräutern fände. Dieselben beständen aus einer festen hornigen Masse, welche in feinen Schnitten unter dem Mikroskop nicht zellig erschienen, sondern mit kleinen, länglichen, gekrümmten Höhlungen versehen seien. Zerriebe man aber die Substanz, so träte doch eine zellige Bildung herror; man fände alsdann auf der Glasplatte längliche gekrümmte Zellen, welche aber so innig verbunden wären, dass sie in Durchschnitten ein Continuum auszumachen schienen. Sclerotium Semen und fulvum hätten den Geruch des Scleroderma, weshalb er glaube, dass es degenerirte junge Sclerodermen sein möchten."

Musste eine solche Zeugenaussage von einem in der Mykologie seit Jahren heimisch gewordenen Forscher mindestens auffällig erscheinen, nachdem ein MEyen und LÉveILLÉ sich schon längst über Structur und Bedentung der Sclerotien so lichtroll und rerständlich ausgesprochen hatten, so erscheinen nicht minder auffällig die in den Hallischen Abhandhungen (p. 8-14) niedergelegten jüngsten Aeusserungen desselben Autors über Natur und Bedeutung der Sclerotien, nach allen jenen zahlreichen und tief eindringenden Arbeiten eines Tulasne, Bame, Coemans u. A. Herr Bonorden ist zunächst nicht mit Herrn BaIL in der Bezeichnung ,Dauermycelien" d. h. Knollen der Pilze für die Sclerotialgebilde einverstanden, indessen hat derselbe doch seit 1851 endlich im Jahre 1861 Gelegenheit gefunden, die ihm sehr merkwürdige Bildung der Sclerotien d. h. Sclerotium compactum an den faulenden Spitzen des Cheirant7us annu us und des Sclerot. Brassicce an einem monströsen, faulenden Kohlrabikopfe zu beobachten. Aus Büscheln von ästigen Hyphen erwuchsen lockere, aussen mit freien Fäden bedeckte Polster von weicher zarter Beschaffenheit, die sich allmählig rerdichteten und in deren Innerem bräunliche conrexe Linien erschienen, welche in der Form der künftigen Sclerotien, nach der Matrix hin, abgeplattet und undeutlicher wurden. Die Hyphenstämme und deren Aeste enthielten eine grosse Menge feiner runder Moleküle, die im Wasser moleculare Bewegungen zeigten. „, Die Ausschwitzung dieser Substanz zwischen die Hyphen bewirkt ohne Zweifel die künftige Verschmelzung derselben zu Sclerotien." Nach Bildungder dünnen gefärbten Rindenschicht fallen die ausserhalb der Rinde befindlichen Hyphen zusammen, verwittern und werden durch den Regen abgespïlt. Die Rindenschicht 
besteht aus ziemlich grossen cckigen bramen Zellen, welche parenchymatös verbunden sind; die Substanz des Sclerotium: aus einer soliden Masse mit innern kleinen Loculamenten. ,Sie sind also gleichsam Ansammlungen, Reservoire von Pilzplasma, somit auch ein gecigneter Boden für viele Pilze.” „Die aus dem Mutterboden hervorkommenden Polster kann man nicht Mycelium nennen, es sind vielmehr stromatische (thalloïdische) Zellen; denn dass Mycelium derselben ist in der Matrix verbreitet und bestehtaus wurzelförmig gekrümmten und kurz verästelten nicht septirten Fäden.” „Das Selerotium ist also kein Product des Myceliums, sondern des Myphasma, eine stromatische Pilzbildung, welche in Stellvertretung der gewöhnlichen Entwickelung und Fructification erfolgt, gleichsam ein Conidium compositum." Die Rindenschicht veranlasst Herrn Bononden zu dem jetzt, an Stelle der frühern Ansicht, getretenen Ausspruch, dass ", die Sclerotien normale Bildungen" seien.

Unter dergleichen vielfach wahren, aber nicht eben neuen Angaben, welche verbesserungsfähigen Anschauungen das Wort reden, ist es nun doch mindestens sehr befremdend, noch heute die Behauptung ausgesprochen zu finden, dass , die Pilze, welche man bisher auf Sclerotien fand, nur Gäste sein sollen," während wieder ganz richtig vermuthet wird, ,dass die Hyphenpolster, woraus die Sclerotien hervorgehen, augenscheintich den Hyphomyceten angehören." Dass Herm Bonorden die Coemans'sche Arbeit, sowie meine Mittheilungen, unbekannt geblieben sein müssen, geht genugsam aus der Aufzählung der ,Gäste" hervor, welche anf Sclerotien bisher gefunden worden sind. Wohl aber kennt er Kühn's und T'uLasne's Arbeiten über Sclerotium Clavus DC. und hat sich gleichzeitig mit KüHs, nicht vor Kürs, bereits 1858 (1), über Kentrosporium purpureum WALLR, ausgesprochen. Ungeachtet nun aber , kaum ein Myko. $\log$ existirt, der nicht bestätigende Versuche aufzuweisen," und Bonorden selbst das Kentrosporium erzogen hat, hegt derselbe den1. ch einen bescheidenen Zweifel dagegen, weil - (!) - , Kentrosporium capitatum und ophioglossö̈des ebenso constant aus der Hirschtrüffel (Elaphomyces) hervorgehen, die man doch nicht für eine Spermoedia erklären kann!" In der 'That, vom Bororden'schen Standpunkte aus lässt sich die Frage kaum anders msehen; jedoch wer nicht ron rlesien rorgefisster Meinung ausgeht, dass jede vor-

(1) Botanische Zcitung, $9^{`}$ April 1858, No. 15, Tab. VI, fig. B. (Im März 1858 schrieb Kürn indessen seine Vorrede zu den Krankheiten der Culturgewächse, nachdem derselbe bereits 1855 in Ham's agronomischer Zeitung Mitthcilungen über seine Beobachtungen niedergelegt hatte, die Herm Boxorden wohl entgangeu sind.) 
kommende Pilzform durchaus den Werth einer Species irgend einer Gattung besitzen muss, kann auch eine andere Ansicht über die Homologien einer Spermoedia, eines Sclerotium oder eines Elaphomyces haben. Aber wie auch nun Herr Bonorden die weitere Begründung seiner Meinung anstrebt, so wird es ihm doch nicht glücken, uns ron seinem längst überwundenen Standpunkte zu überzeugen. Weil die Sphacelia-Sporen, wie es Herr Tulasse berichtet, keimen und es Herrn Boxondex, so wie Merex, geglückt ist, aus den gekeimten Stylosporen Mutterkorn herrorzurufen, was mit Kentrosporium-Sporen nicht der Fall sein soll (bekanntlich ist dies Kürs doch geglïckt!), so kann das Kuntrosporium keinen Zusammenhang haben mit den Sclerotium Clavus, - denn - , nach bisheriger Erfahrung erzeugen die Sporen eines Pilzes immer nur den Pilz, durch welchen sie entstanden sind." Das aber ist gerade der Kerupunkt der ganzen Frage, zu deren Höhe sich Herr Bonordex noch nicht zu erheben geneigt ist. Von dieser seiner einseitigen Anschauung aus erkennt Herr Bonorden (1. c. p. 15), in der systematischen Entwickelung der Mykologie eher Rück- als Fortschritte", sodass die "Mykologie beginnt sich in ein wahres Chaos zu rerwandeln." Kann man, - wie Herr Bonorden in der III Abhandlung, betitelt: ,Ueber die Fortbildung des mykologischen Systems" genugsam schon durch die angeführten Worte, und wie es ausserdem fast jede Seite des langen Mémoirs darthut, seiner Zeit nicht mehr folgen und den Fortschritten der Wissenschaft gerecht werden, so dürfte es doch wohl angemessener sein, nicht über dergleichen Dinge also abzusprechen.

Wollte heute der sel. RudolpH, der Begründer der wissenschaftlichen Systematik der Entozoologie, mit Boxondex'scher Hart. näckigkeit seine Ordnung der Cystica rertheidigen, die nur deshalb aus dem System verschwand, weil die dazu gerechneten Cysticercen, Coenureu etc. als zeugungsfähige Formen gewisser Bandwürmer erkannt wurden, welcher letzterer Zeugungsfähigkeit Niemand bestreitet, so würde man allenfalls diesem rerdienten Forscher einen solchen Anachronismus rerzeihen, weil die Lehre rom Generationswechsel erst lange nach seinem Tode aufgestellt werden konnte; allein wenn ein wissenschaftlich-forscheuder Mycolog die Begründung derselben Lehre rom Anfange bis zur Gegenwart durchgelebt und mit den Phasen der Fortbildung derselben vertraut zu werden Gelegenheit gehabt hat, so muss man, wenn man wie Herr Bonordex streiten will, dergleichen Lehren nicht verstehen wollen. Herr Borornex möge nicht glauben, dass, weil er in Her'n Stepri. Schulzer (1)

(1) Oesterreichische botanische Zeitschrift, No. 8, 1865. 
und C. H. Schultz-Śchultzensters Unterstützung fand, er nun die Wissenschaft zur Umkehr nöthigen kann.

Worauf aber stützt sich denn Herr ST. Scmulzen? ,Wäre das Sclerotium, so sagt derselbe (1. c. p. 242), ein Mycelium, d. i. ein integrirender Theil des Pilzes, so könnte dieser nie ohne dasselbe crscheinen; dem widerspricht aber die Erfihrung. Wer sah je ein Knollengewächs bald mit, bald ohne Knollen regetiren?" Auf diese höchst naire Frage erwidere ich dem Herrn Fragesteller ganz einfach mit der in den Schriften des Gartenban-Vereins in Preussen von mir vor vielen Jahren niedergelegten Thatsache, dass ich und der Obergärtner ReInecke bei Hermu rox Deckel in Berlin an der sogenannten türkischen Bohne (Phastolus multiflons WiLld.) und zwar an deren unterirdischem Stocke grosse Knollen beobuchtet und durch Cultur erzielt haben, die wir dem Gartenbau-Vereine im Originale vorlegen konnten, während vielerfahrene Cultivateure Berlins desgleichen noch niemals gesehen hatten, und sicher auch Herr Schulzer nicht. Ferner class ich 1865/1866 in bot. Universitätsgarten eine Form der Begonia rex cultivire, welche gegen alle sonstige Erfahrung auf der Verbindungsstelle des Blattstiels mit der Lamina an jedem Blatte dicke Knollen erzeugi, die sich als ächte den Gesnerien-Blattknollen gleichwerthe und zur Fortpflanzung taugliche Fortpflanzungsorgane ausgewiesen haben. Die Einrede des Herru Stepr. Scrulzer ans Nüggenburg hat demnach gar keinen soliden Grund und Boden. Es giebt in der 'That Pflanzen, die bald mit, bald ohne Knollen regetiren; dess möge Herr Sciulzeк nux immer versichert sein!

Führt Herr ScHolzen ferner gegen die herrschende Lehre an, dass er selbst beim Agaricus (bei welchen?), BoNORDEN aber beim Kentrosporium die Beobachtung gemacht hätten, ,dass der Pilz (d. h. der Parasit) das Sclerotiun bis zur völligen Verkïmmerung parasitisch aussauge," so ist dies einestheils nur eben die Wiederholung einer bereits längst bekamnten Thatsache, anderentheils nur cin bestätigendes MLoment mehr für die Behauptung eines bestehenden organischen Zusammenhanges zwischen Siterotium und dem vermeintlichen Parasiten, so wie zwischen Kintoffelknollen und deren oberirdischer Axe. Wem Derselbe fermer im Augustmonate 1865 noch sagt, dass es ,Niemandem gelungen sei, aus KentrosporiumSporen Sclerotion hervorzubringen, so müssen ihm die ron glücklichem Erfolge begleiteten Impfrersuche Küus's ganz unbekannt geblieben sein, denen zufolge nach Aussatut von Stïckrhen der K̈̈̈pfchen eines Kíntrosporium auf Roggen Selcrotium Cluens DC. entstanden sind. Dass ein Gleiches für Aguicus nicht erfulgen kanu, 
werde ich unten selbst zu erweisen Gelegenheit finden; wenigstens werde ich versuchen, den Beweis zu führen, dass Agaricus tuberosus Bulu. nicht direct Acrospermum erzeugt, sondern zunächst einen Hyphomyceten, die Herr Bonorden (1. c. pag. 11), wie eben bereits angeführt wurde, als Veranlasser der Hyphenpolster ansieht.

Sind demnach alle in der neuesten Zeit vorgebrachten Gegengründe ohne jeden wissenschaftlichen Halt, so steht nichts entgegen, dass wir mutatis mutandis mit Herrn Scrulzer aus Müggenburg diesen Vorbericht mit dessen mur abgeänderten Worten schliessen: „Kurz, der auf einem Sclerotium erscheinende Pilz ist kein unwillkommener Gast desselben, vielmehr Ersteres sein naturgemässer Standort; denn er gelangt daruuf zur vollständigen Ausbildung durch Hervorbringung keimfähigen Samens."

Wie ich selbst zur Frage stehe, geht nicht nur aus den von mir früher publicirten Mittheilungen im Bulletiu der Könighlich Belgischen. Academie der Wissenschaften und in der landwirthschaftlichen Wochenschrift des baltischen Vereins, sondern auch aus verschiedenen obigen Bemerkungen bereits zur Genüge herror. Ich bin mit Kürs und DE Bary einverstanden, dass eine Precinie oder ein Uromyces nicht die einzig und allein vorhandene Lebensform ge. wisser Pilze ausmacht, sondern dass dieselben Sporidien auf sogenamnten Promycelien erzeugen und aus den Sporidiev Aecidien und deren Spermogonien hervorgehen könmen, um endlich wieder in die Form der 'Telenthosporen der Puccinien zurïckzukehren; d. h. dass verschieden gefurmte fortpflan:ungsfülvige Lebensüustionde cinander folgen mïsscn, ohne dass dadurch etwas Anderes, als unser auffalschen Voraussetzuugen basirtes und errichtetes mykologisches System leidet. Ich bin mit BarL (1) darin einrerstanden, dass der Erzeugung ciner Sphaevia tyllhina Pers. (Polystigme, typhinum DC.) ein Conidienbildendes, fädiges Lager vorausgehen muss, dass die Conidien keimen müssen, um ein Stroma zu bilden, welches, zum Sphaerien. gehäuse umgewandelt, in demselben sporenschläuche und Sporen producirt, d.h. also, dass eine mehrfiche Sporenzengung in verschiedenen Lebensformen desselben Pilzes nothwendig wird, um dessen cyclisches Dasein rollständig abzuwandeln. Ich hin auch mit'Tuldsxe und BAIL einverstanden, welche Tseria furinosa Fr. als Conidienform zu Cordyceps milituris Fr. (Krntrosponim militare) ziehen und ich begrïsse mit Freuden jede neue derartige Errungenschaft, welche hin-

(1) Acte ucud. Cacs. Lenpold.Curol. germ, nut. curios. Tom. XXIX, 1862, 40. $\Delta$ b. julg ivo. IY. 
reichend sicher gestellt, nur zum wahren Frommen der forschenden Wissenschaft führen kann. Auch Herrn Bonordes's Vermuthung, welche er 1. c. pag. 11 aussprach, , dass die Hyphenpolster, woraus die Sclerotien hervorgehen, angenscheinlich den Hyphomyceten angehören," rechne ich dahin und acceptire dieselbe bestens; denn sie trifft nach meinen 8-jährigen Erfahırungen das Rechte und soll daher auch zum Ausgangspunkte der nachfolgenden Mittheilungen über eigne Beobachtungen dienen.

Im Anfange des Herbstes, im Septenıber und Anfang October, sicht man, wie es bereits Conv (Schles. Ges. f. vat. Cultur 1856, p. 50) angegeben, auf verschiedenen Pflanzen, Tabacksstengeln, Georginen, besonders Somnenrosen (Heliantlus cinmuns L.) und dergleichen, namentlich nach fenchten, nebelreichen Tagen, zuweilen an deren unteren, zuweilen auch an höheren Steugeltheilen, ja auch auf der Aussenfläche des fleisehigen Receptaculums der Sonnenrose selbst, mehr oder weniger ausgedehnte vergelbte Flecken, Das Chlorophyll der Oberflächenzellen ist nicht mehr freudig grün, sondern vielmehr entfärbt und deutet auf eine Erkrankung des betreffenden Stengeltheils. Sind die erkrankten Stellen bereits weit ausgebreitet, so findet man im Centrum des Heerdes der vergelbten oder wohl gar schon vertrockneten Stelle nichts mehr, was einen sichern Schluss auf einen vorhanden gewesenen Parasiten zuliesse: eine Erscheining, die bei der sogenannten Kartoffelkrankheit seit melrr als 20 Jahren an jedem crkrankten Kartoffelblatte längst ebenfalls beobachtet und sattsam beschrieben worden ist. Will man die Peronospora trifureata Unger (Botrytis devastativiu Lib.) sicher finden, so muss man bekannitlich an der Grenze dor frendig-grïnen und der bereits gebräunt erscheinenden Blattfläche seine Untersuchungen anstellen, widrigenfalls eben nichts mehr von diesem Parasiten gefunden werden kann. Ganz ebenso muss man aber verfahren, wem man für derglcichen grössere vergelbte Stellen das veranlassende Moment finden will. An der äussersten und verwaschensten Gränze, ja oft darüber hinaus, gewöhnlich sogar auf den noch grün erscheinenden Stellen wuchert freudig irgend ein zierlicher Hyphomycet und stäubt seine massenhaft erzengten Sporen in dicken Wolken dem allzu lebhaft agitirenden Beobachter entgegen. Erfreut über den gemachten Fund, eilt man gerröhnlich schleunigst zum Mikroskope, um die Form des Gesammt-Gebildes und die seiner Sporen zu ermitteln und mit Hülfe der Conds'schen Icones, oder der Boxonves'schen Albildungen und dem sonstigen literarischen $A$ pparate Gattung and Art festzustellen, 
um dann den nenen schätzbaren Beitrag für das ,mycologische Herbar" vorzubereiten. Nach mehr oder weniger langer Zeit kommt man vielleicht wiederum an die ergiebig gevesene Fundstâtte, findet aber Alles wesentlich verändert. Die Herbstregen und Stïrme, auch wohl einige Nachtfröste hiben ihren zerstörenden Einfluss auf die Vegetation geltend genacht, und kaum findet man von dem Frühern irgend noch eine Spur. Hie und da aber tritt uns ein schwarzes Sclerotium durum Pensoos oder compactum DC. und dergleichen entgegen, das, soferm es der Sammlung noch etwa fehlt, in gleicher Weise behandelt und alsbald etiquettirt wird. Damit ist die nothwendige Arbeit gethan, um als Studium der Mycologie zu gelten. Die Gattungen und Arten häufen sich dabei ohne Ende und geben freilich gar Vielen, die daran Freude finden, Gelegenheit ihr ,, mihi" an den Mann zu bringen. Allein in der Mycologie ist es seit TuLASNE's Dazwischentreten höchst undankbar geworden, neue ,mihi's" aufzustellen; denn drei oder vier, oder wohl gar noch mehr dergleichen schmelzen zusammen, sobald das Studium der Art genetisch und auf dem Wege absichtlicher Cultur, d. h. auf dem Wege der Experiments und namentlich in Rücksicht auf den bedeutungsvollen Generationswechsel betrieben wird.

Verfolgt man, um auf die vergelbten Stengelflächen zurïckzukommen, den weitern Gang der Entwickelung, so sieht man gar bald auf den innern Oberflächen der röhrigen Internodien ein Gebilde, das dem unbewaffneten Auge, ja selbst noch unter der Loupe, baumwollenartig erscheint. Unter dem Mikroskop aber erkennt man ein melhr oder weniger dichtes Geflecht aus zarten, septirten und vielverzweigten Fasern gebildet und in deren Innerem ein sehr feinkörniges Plasma, zahlreiche Vacuolen bildend. Hie md da in diesen dichtgewobenen Filzen erhebt sich ein kugel- oder kissenförmiges Polster, dessen Fasern im Inuern noch dichter verfilzt erscheinen, indem sie, fort und fort neue dickwandigere Ramificationen bildend, sich in einander schieben und dicht und immer dichter, an einander legen; selten in parallelen Strängen, meist ein vielfach sich durchkreuzendes, fast lückenfreies, vielfuch gewundenes Geäst darstellend. Der dichter gewordene, noch immer weisse Kern vergrössert sich, oder hat wenigstens das Streben, nach allen Seiten hin zuzunehmen; allein nicht alle Körper erreichen das gleiche Zicl; denn mach mehr oder woniger längerın Bestehen dieser dichtern Mirk-oder Kernmasse, die noch in weisses lockeres Filgewebe eingehüllt ist und Tropfen einer Flüssigkcit absondert, schnïreu sich kurze scharf umschriebene polyedrische Kellen an der Grenze des Kern. und Fusergewebes ab, nchm’n allmählig bräunliche, cudlich dunkelere bis schwarze Farbeu 
an und bilden eine dem Kork wohl analogenere Rindenschicht fül das Kerngewebe, welches weder auf Läugen- noch auf Qucrschnitten späterhin regelmässige Zellformen erkemmen lässt. Der Anfangs noch in weisses Fasergewebe cingebettete dunkele Körper wird allmählig mehr und mehr frei, indem seine weisse, fascrige Hülle zusammenschrumpft, vertrocknet, oder durch Wasser und Luft zerstört wird. Das also entstandene Sclerotium bleibt aber bis zum endlichen Zerfallen des Mutterbodens, auf welchem es sich entwickelte, mehr oder weniger lange Zeit fest angeheftet, bis es mit dem verrotteten Mutterboden selbst zu Boden gelangt.

Zahlreiche derartige ,Dauermycelien”, wie sie Ball naunte, oder stromatische(thalloïdische) Pilzbildungeu, wie sie Boxon dex bezeichnet zu sehen wünschte, gehen, ohne eine weitere Entwickelung erfahren zu haben, unter, oder gerathen beim Umgraben des Gartens oder Umpflügen des Ackers in so grosse 'liefeu, dass ihre weitere Fortbildung vorläufig unmöglich wird. Nimmt man dieselben aber in ihrer vollen Entwickelung von ihrer Erzeugungsstätte ab, bringt sie in einen mit Erde gefüllteu Blumentopf und verliert auch 18 Monate lang nicht die Geduld, so hat man doch endlich die Freude, selbst nach so langer Zeit von seiner Aussaat weitere Erfolge zu erzielen.

Säet man das braune runde Sclerotium Semen Tode, (welches ich aus Tabacksstengeln gewann, die den Herbst hindurch bis in den Winter hincin auf dem Felde (bei El(leni) stehen geblieben waren),

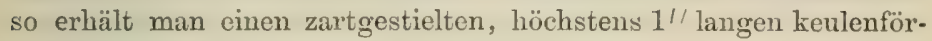
migen Hymenomyceten, die Typhula variubilis RIEss, dessen zarte Stielbasis auf dem Sclerotium nicht wie ein Schmarotzer aufsitzt, sondern aus einer durch Aufbruch der Sclerotialhüllzellen entstandenen offenen Ausgangsstelle des Markgewebes selbst hervorgeht; so wie man den Stiel von Claviceps pupurea Tul. ans dem Sclerotium Clavus DC. aussprossen sieht, als eine Fortsetzung der Sclerotialgewebe, deren Zellen nuch allen Dinensionen, insbesondere auch der Längo nach, zugenonimen haben.

Säet man aber jene vielgestaltigen und an Grösse so ausserordentlich variirenden Sclerotien, welehe unter dem Namen Scler. varium Pens. bekmunt sind, (die ich Anfangs nur in den Stengeln vou Martynia fragrans, später in Georginenstengeln, u. s. w. fand) so entwickelt sich nicht cin 'Hymenomycet während der Cultur, sondern ein Discomycet, nämlich Pezira Sclerotionum Liß. (P. Antsii Mrr.) (1). Auffallend verschieden aber ist die Zahl der Pezizen-Indivi-

(1) Abbildung im Bull. de l'Acad. royale de Belgique, 2me. Série, Tom. XI, No. 2, pag. 8. 
duen, die sich auf einem cinzigen Sctcrotirn bilden können. Ich zählte bis 16 Fruchtträger der kurzgestielten Form, während die langgestielte Form meist in geringerer Anzahl auf dem selcrutium sich fand. Die Basis dieser Sclerotien, gleichviel ob der Stiel lang oder kurz, war immer fast so dunkel gefürbt, wie die Sclerotial-Oberfläche selbst. Dem Becher näher, war der Stiel nehr lichtbräunlich, schmutzig gelblich. Während die jüngeren Fruchtträger sich im Betreff der Gestalt einer kleinen Keule näherten, zeigten weiter entwickelte Individuen sich in der Form eines Bechers mit einer nach längere Zeit noch erkennbaren centralen tiefern Depression, welche sich in einen Canal verlängerte, jedoch sich bei ganz ausgebreiteter wellig-geränderter Scheibe vollständig ausgeglichen und verwischt fand. Der Rand jüngerer, becherförmiger Pezizen zeigte sich meist gleichmässig gerundet, und der freie Becherrand war wie mit einem recht scharfen Mresser unschnitten. Eine lebhafte Handbewegung über die frei über die Erde hervorragenden Pezizenbecherchen betvirkte lebhaftes Ansstäuben der Sporen aus den ascis, in welchen sie entstanden waren. Der Zusammenhang der Stielzellen mit den Markgewebe des Selerotiums war' leicht und sicher nachweisbar, and nicht der geringste Grund vorhanden, in dem gestielten Fruchtträger der Periza einen Schmarotzer des Sclerotium anzunehmen. Der von mir erzogenen Pezizen zähle ich Hunderte, und alle, welche ich untersuchte, wiesen den gleichen organischen Zusammenlang mit dem Kerngewebe des Sclerotiums nach, während die dunkele zellige Rindenschicht keinen Zusammenhang mit der peripherischen Schicht des Stiels nachwies.

Säete ich endlich bereits in ihrer Ausbildung vollendete Acrospermum comutum Fr. mit lichtbraun gefärbter Oberhaut und von länglich-rundlich beiderseits zugespitzter Gestalt, (wie sie sich so vielfach auf moosreichen Flächen benachbarter Kieferwälder fanden, da wo Agaricus deliciosus und Russula-Arten faulten), so erhält man weder Typhula- noch Cordyceps- noch Peziza-Arten, sondern einen Agaricus, den ich Anfangs für Agaricus Amanitae Batcir. (Tab. XVIII, fig. 93 a-d), allein, nachdem ich die Bulliarn'schen Tafeln einzusehen Gelegenheit hatte, für Agaricus tuberosus BuLl. halten musste. Fries (l. c. I, p. 133) identificirt zwar beide, allein ich fand doch stets die länglich zugespitzten. Knöllchen an der Basis der Bulunadischen Form und suchte sie rergebens bei einer dem Agar. anzanitae BATscir ähnlicheren Form. Sind AcrospermumKnöllchen vorhanden, so findet sich auf denselben, wie gross sic auch sein mögen, nur immer ein mehr oder weniger langgestielter Hut; der Stiel zeigt sich, aus der geöflueten Rindenschicht hervortretend, stets an der Spitze des Lerospermum da, wo 'I'oDe den 
vermeintlichen Simen zu finden meinte. Eine weisse flockige Hülle begleitet die Stielbasis zuweilen bis zur halben Länge des hohlen Stipes, in andern Fällen fehlt diese Hyphen-itrtige Hülle. Der Hut. zuletzt horizontal ausgebreitet, hat $1-3^{\prime \prime \prime}$ im Durchmesser, ist genabelt, mit zahlreichen der weisslichen Hutoberfläche gleichfarbigen Lamellen. Fehlten die Arospermum-Kuöllchen, so fand sich ein dichterer Haufen der weissen Agavici, deren Hüte sich nicht so horizontal-schirmartig :usbreiteten, sondern mehr halbkugelförmig sich geformt zeigten, mit einem untern zusammengezogenen Hutrande, auch war der Durchmesser des Hutes erheblich kleiner, nämlich nur $\frac{1}{2}-1^{\prime \prime \prime}$; der Stipes, nicht von gleichmässiger Dicke, verjüngte sich nach dem kleineren Hütchen hin, so dass das ganze Gebilde verkümmerter erschien, als die mit kräftigen Acrospermumknollen versehenen Individuen.

Nachdem es mir gelungen war, die Entstehung des Sclerotium varium Pers. in einem grossen Glashafen, dessen Glasdeckel ich luftdicht aufgekittet hatte, im Arbeitszimmer täglich zu verfolgen und ab und zu genauer mikroskopisch zu untersuchen, so war es mir angenehm, auch die Bildung des Acrospormun comutum Fr, zu verfolgen. Es gelang mir in demselben Kieferuwalde, der im September so ausgiebige Mengen von entwickelten Agaricis tuberosis geliefert hatte', einige theilweise schon ziemlich verbröckelte, aber doch noch mit Stipes und mit mehr als dem lalben Hute versehene, Individuen von Agaricus (Gatorhous) deliciosus L. zu finden, auf welchen sich ein sehr blentend weisser kleiner Hyphomyret befand, ohne jedoch irgend eine Spur von Acrospermum-Knollen zu besitzen. Mit Vorsicht brachte ich die mit derartigen Hyphomyceten rersehenen Agaricus-Stücke, nebst Kiefernadeln, worauf sie lagen, nach dem botanischen Garten und cultivirte den kleinen Schimmelpilz mit grosser Sorgfalt. Unter einer Glasglocke auf feuchtem Saudboden verbreitete sich der Hyphomycet sehr rasch über die gesammte Kiefernadelmasse, ohne jedoch anf diesem Boden irgend welche weiteren Entwickelungen herlseizufülıren. Dagegen die ihm zur weitern Nahruug rorgelegten A!/aricus-reste zeigten nach einiger Zeit die Entwickelnug ron Arrospermum-Knollen, welche, von gelblich-weisslicher Farbe und lïnglich-walzlich-zugespitzter Form, sich auf ciner Länge von 3 '" entwickelten; grösser, als ich sie am OriginalStandorte gesehen hatte. Da nun diese Acrospormum-Knollen zwischen den Lamellen des Ayavirus driviosus $\mathrm{I}$. herrorbrachen, so wurden dieselben nit Vorsicht ausemander gelegt, mu die Anheftungsstelle des Acrospmm-Knollchens zu ermitteln. Allein dic* selben sasson nur locker auf and hingeu mit den Hyphen des 
Hyphomyceten nicht mit den Lamellen des Ayar. deticiosus zusammen. Nach vielfacher Vergleichung mit den CondA'schen Icones und den BoNorden'schen Abbildungen wies sich derselbe als eine dem Diploculium wrujor Bon. angehörige Hyphomycetenform aus. Das aus dem Mycelium desselben hervorgegangene Acrosjermum besass ein sehr grosszelliges lockeres Markgewebe, dessen vorwiegend in die Länge gestreckteren Parenchynzellen sich ohme sonderliche Mühe rücksichtlich ihrer Form erkenmen liessen. Zwischen den Zellen befanden sich Luftlïcken und hie und da waren lufterfüllte Höhlungen bemerkbar. Nach der Rindenschicht zu wurden die Durchmesser der Zellen immer kleiner, zeigten aber doch im Sinne der Längenaxe des Acrospmum sich immer noch vorwiegend gestreckt; auch waren diese Rindenzellen nicht so farblos, wie das Markgewebe, sondern licht bräunlich gefärbt und der Uebergang aus der farblosen grosszelligen MIark- in die gelblich-bräunliche kleinzelligere Rindenschicht war ein so allmähliger, dass eine scharfe Grenze sich nirgends ausgesprochen zeigte. Obschon der Bau des Acrospermum-Knöllchens ron dem Bau des Sclerotium Semen od. varim so bestimmt verschieden ist, so sind diese Gebilde doch einauder morphologisch so gleichwerthig, dass die Präsumtion Berechtigung findet, derartige Gebilde möehten auch wohl auf gleichem Wege entstanden sein.

Stellt man sich nun die Frage, ob auch für Sclerotium vavium, Scme'n und andere Sclerotialformen Hyphomyceten existiren, deren Mycelien sich zu derartigen „Danermycelien” umzubilden vermögen, so wird die Antwort nicht eben schwer.

Bereits im Jahre 1861 sprach ich mich bei Gelegenheit der am 20 September in Speier tagenden Section für Botanik dahin aus, dass mehrere Sclerotien gewissen Botrytis-Formen ihren Ursprung verdankten, die ich jedoch nicht vollständig mit der von LÉvenLLé in den Aunales d. Sc. (XX, pl. 7, fig. 5b) abgebildeten: Botrytis crythropus identificiren lionnte, die vielmehr der Form Botrytis cinerea näher kamen, welche LÉveillé auf Sclerotiun dưum Pers. wachsend gefunden habe, indem el von ihr sagt, ,il n'y a pas le moindre doute que le Sclerotium durum ne soit une partie constituante du Botrytis cinerea." Allein, da es sich zunächst um Sclero. tiun varium handelte, so komnte natürlich Botrytis cinerea, welche cinen constituirenden Bestandtheil des Selerotiun durum ausmachen sollte, nicht wohl füglich zu varium gezogen worden. Mit dem Lixk'schen Geuns Polyactis, wie es Boxordex (Hdb. d. Myc. p. 115) beschreibt, waren die Erzeuger des Seler, varium nicht zusammenzubringen; denn die Hyphen waren nur spärlich septirt und endigten 
sich am obern Ende auch nicht doldonförmig. Die Endäste waren auch nicht zweitheilig, sondern rielmehr rundlich angeschwollen und trugen ringsum eiförmige, fast farblose Sporen, währeud die am obern Hyphenende näher zusammengedrängten Aeste nach Art eines Racemus an der Hauptaxe sassen und keine eigentliche Cyma bildeten, wie BoNoRden (1. c. p. 101) anzunehmen geneigt ist, auch keinen Corymbus nachahmten, wie es FrIEs (1) zu wollen scheint, welcher die unserer Pflanze im Habitus am nächsten kommenden BotrytisForm, (eleyans Lk. nämlich), zu den corymbosen bringt. Die ron Freserius (2) fig. 16 abgebildete Botryfis acinonum Pers., welche derselbe neben den bereits bekannten Arten, welche Fries (1. c. p. 393-406) beschrieben hatte, unfstellt, würde für sich allein die Erzeugerin des Sclerotium varium Pers. am besten darstellen, allein Fries (1. c. p.400) führt dieselbe unter den "Umbellatae," und Fresenius und Fries lassen dieselbe nur auf faulenden Weinbeeren vorkommen. Mit Ausnahme dieses beschränkten Vorkommens und der Angabe bei Fresexius, dass die rerdickten Endzellen der Aeste die Sporev auf kurzen Spitzchen tragen sollen, die nach dem Abfallen stehen blieben, (was ich seiner Zeit nicht mit abgebildet habe), stimmt alles Uebrige so vollkommen mit der Botrytis-Form überein, aus deren basalen Hyphen ich 1860 Sclerotium varium PERs. erzog, dass ich dieselbe lieber mit dem Namen Botrytis acinorum PERs. belegt sehen, als ihr einen neuen Namen geben möchte, wie wenig ein solcher auch an und für sich noch heute Bedentung hat.

Die auf Tabacksstengeln von mir beobachtete Botrytis-Form, deren Mycelium Sclerotium Semen ToDe erzeugte, das bei fortgesetzter Cultur Ty/nhulev variabilis RIEss lieferte, gehört wegen der am Ende der septirten Hyphenhanptaxe zusammengedrängten Aeste erster Ordnung mehr den doldenförmigen Botrytideen an. Die Hyphen waren gran weisslich, die untern Hyphenäste erster Ordnumg horizontal oder bogig aufsteigend rerzweigt, die Endzellen derselben mehr oder weniger gestielt, kugelig angeschwollen und ringsum mit kugelig-eiförmigen weiss-graulichen Sporen dicht besetzt, so dass dieser Botrytis der Species umbellata Fries am nächsten kommen dürfte, dem nach Fries ehenfialls ein dichtes reichliches Mycelium zugeschrieben wird. Wie auch nun immer die sogenannte Botrytis-Species genannt werden mag, jedenfalls vermochte ich zu constatiren, dass die Mycelien von Botryfis-Formen durch die Stengel phanerogamer Pflanzen von aussen hindurchwachsen, auf der

(1) Syst. mycol., Vol, III, pag. 405.

(2) Beiträge zur MIykologie, Heft I, II. Frkft. a/M. 1852, 4o., tab. II, fig. 16. 
innern Stengeloberfläche verschiedener phanerogamen Pflanzen, dichte, weisse, filzige Mycelien erzeugen, aus denen Sclerotien hervorgehen und dass diese Sclerotien, welche verschiedenen Botvytis. Formen ihren Ursprung rerdankten, auch rerschiedene Endformen produciren, entweder Pezizen (z. B. Sclerotionum LrB. von Sclerotium varium Pers.) oder Typhlulae (z. B. variabilis RIEss von Sclerotium Semen); während Agaricus tuberosus PERs. dem Acrospermum cor-: nutum Fr. ihren Ursprung verdankt, welches durch das Mycelium eines Diplocladium major Bonond, erzeugt worden war, während zweifellos andere „Dauermycelien," wiederum durch andere Hyphomyceten entstehen und andere Gattungen und Arten im Sinne der ältern Systematik hervorrufen.

Obschon ich leider nicht die ganze Entwickelungsreihe, wie vorhin anzugeben im Stande bin, so kann ich doch nicht unerwähnt lassen, dass ich zum Beweise dessen z. B. eine eigenthümliche Form von Plygmatotrichum Bovord., welche dem gemellum Bovord, nahe steht, auf Symplyytum asperrimum fand, deren Mycelium auf der Stengeloberfläche des Symphytum Sclerotien erzeugte, die dem Sclerotium compactum nahe verwandt sein dürften. Die weitere Entwickelung dieses länglich-polsterförmigen Sclerotiums konnte ich leider nicht verfolgen. Unzweifelhaft würde die zum endlichen $\mathrm{Ab}$ schlusse gelangte Cultur wieder eine andere Endform hervorgerufen haben, so wie auch eine eigne Botrytis-Form auf Rhoum australe Dos ein dem Sclerotium durun verwandtes Sclerotialgebilde hervorbrachte, dessen endliche Form durch Cultur zu gewinnen, bis jetzt mir noch nicht glückte.

Aus diesen mehrere Jahre lang fortgesetzten Beobachtungen scheint sich zu ergeben, das die bisher in den Systemen der Mykologie geführte Orduung der Tryphomyeten, grösserentheils wenigstens, die conidientragenden Formen anderer Ordnungen sind und somit das Geschick der RudoLPH'schen Cystica theilen dürften, welche aus einem wissenschaftlich-exacterem „, Systema helminthum” verschwinden mussten. Theilweive sind die Hyphomyceten sicherlich nur eine besondere Lebensform anderer typischer Pilze, zwar in hohem Grade begalbt mit dem Vermögen Sporen zu erzengen und durch deren Keimung und Entwickelung ihres Gileichen hervorzurufen, jedoch um endlich in Formen überzngehen, durch welche sie in den Stand gesetzt werden, den rauhen Winter mit allen seinen Unbilden zu überdauern und in milderer Jahreszeit schliesslich in eine ueue fortpflan- 
zungsfähige Form sich umzugestalten. In derselben Weise erzengen die Daphniae unter den Cladoceren Crustaceen, nach neueren Forschungen, Winter- und Sommereier (die letzteren sogar unbefruchtet), um ihre Arten in allem Betracht zu rermeliren und zu erhalten.

Das Verhalten dieser besprochenen Hyphomyceten beweist, dass sie sich zeitweilig erhalten und ans sich selbst regeneriren können, so wie die aus nubefruchteten Sommer-Eiern hervorgegangenen Daphnien; dass sie jedoch, um sich dauernd erhalten zu können, in zahlreichen Fällen eine neue Lebensform annehmen, wenigstens zunächst eine wahre Metamorphose eingehen müssen, um aus dieser veränderten Gestalt (S.leiotium, etc.) nene multiplicationsfähige Lebensformen zu erzeugen, deren Producte (Sporen) dann die ursprüngliche Hyphomycetenform wieder hervorrufen. Ob nicht inzwischen noch abermalige neue Formen eingehend, bleibt weiterer Forschung vorläufig anheimgestellt.

Dass aber die Sclerotialform bei meinen Versuchen und Beob. achtungen nicht direct aus dem Mycelium keimender Ayarias. oder Peziza-Sporen hervorging, davon habe ich mich durch oftmalige vergebliche Aussaaten zur Genüge überzeugt.

Endlich sei es mir gestattet, darauf hinzuweisen, dass ich aus den Beobachtungen über die Entstehung des Acrospermum comutum Fries zu der Ansicht gekommen bin, dass zur Erzeugung eines Acrospermum das Mycelium einer Diplocladium-Spore nicht zureichendes Material liefert, sondern dass es dazu der Mycelien zahlreicher, gekeimter Sporen bedarf. Da nun aber aus jedem Acrospermum ein Agaricus hervorgeht, so kann man sich der Ansicht nicht verschliessen, das es zum Aufbau, selbst eines so kleinen Agaricus tuberosus BuLL., der Mycelien zahlreicher Hyphomycetensporen bedarf. Indem aber auch die Untersuchungen der Entwickelungsgenchichte der Perizu Sclerotionum Lin. aus dem Sclerotium varium Pers. und der dasselbe erzeugenden Botrytis-Form cine gleiche Schlussfolge zulassen, so dürfte sich als ein wichtiges fernerweites Resnltat vorstehender. Mittheilungen der Satz ergeben, dass Hymenomyceten sowohl, als Discomyceten, Pilzformen darstellen, welche aus der Keimung und Entwickelung zaImREICHeR Sporen hervorgehen uind nicht das Product einer cinzigen Spore sind: ein Satz, der jedoch noch aus nicht Sclerotien erzeugenden Mycelien seine fernerweite Begründung erfahren muss. 


\section{MEMBRES DU CONGRÈS.}

LISTE SUPPLÉMENTAIRE (1).

\section{Pays-Bas.}

MMI.

ALBARDA (Mr. Tr.), à Groningue.

BAKKENES (L. VAN), à Amsterdam.

BEELAERTS VAN BLOKLAND (M. A. A.), à Utrecht.

BERG (VAN DEN), maison van den Berg frères, horticulteurs, à Amsterdam.

BERG Jr. (J. F. VAN DFN), horticulteur, à Jutphaas (près d'Utrecht).

BOEKWEG (J. II.), jardinier à Veelzigt, Zeyst (près d'Utrecht).

BOER (D.), propriétaire du Bazar royal, à la Haye.

BONT (M. J. DE), fabricant, à Amsterdam.

BOS (A. J.), horticulteur, à Harlem.

BOS, maison Bos frères, marchand-grainier, à Amsterdam.

BROEKMAN (J. H.), horticulteur, ì Amsterdam.

BUNGE (J. W.), à Rotterdam.

BUYS Gz. (F.). jardinier, à Harlem.

COPIJN (J.), pépiniériste à Groenekan (près d'Utrecht).

DAGEVOS (P. J.), ì Rynsburg.

DIJKALAN (H. T.), horticulteur à Bloemenhoven (près d'Harleın).

ENKLAAR (E. C.), redacteur du „Vriend van den landman," ì 'Lwolle.

EYK (Mr. J. A. VAN), secrétaire-directeur du Palais de l'Industrie, à Amsterdam.

EYKEN SLUYTERS (J.), à Amsterdam

FRANCKEN (G.), à Amsterlam.

(1) Cette liste a été communiquée au secrétaire-général ảu Congrès après l'impression de la première partie du bulletin. Elle contient pour la plupart les noms des personnes, qui ont remporté des prix à l'exposition universelle d'horticulture, et qui, d'après l'article 33 du règlement, sont considérées comme membres du Congrès. D'autre part il s'y trouve répétés les noms et les titres de quelques personnes, dont aupararant les fonctions et les charges ne nous étaient qu'imparfaitement connues. On y a jjouté les noms de trois personnes, qui par erreur ne se trouvaient pas mentionnées dans la première liste.

Quelques personnes nommées dans les listes n'ont pas assisté au Congrès. Nous avons cru né:umoins devoir laisser leurs noms parmi ceux des membres, par la raison qu'ils ont contribué an succès de l'entreprise, soit par l'envoi de leurs produits, soit par leur adhésion au Congrès. 
MM.

GRAAFF (DE), maison de Graaff frères, horticulteurs, à Leyde.

GRAMPEL (J. VAN DE), jardinier au château Sandwyck (près du Bildt).

GRAMPEL (J. VAN DE), jardinier de Mad. Roüll, à Bloemendaal (près d'Harlem).

GROBBE (1.), jardinier de S. A. R. la P'rincesse Marianue des Pays-Bas, à Voorburg.

GROENEWEGEN, horticulteur, de la maison A. C. van Eeden \& Co., à Harlem.

GROOT Wz. (J. W. DE), horticulteur, ì Utrecht.

HARENCARSPEL (A. VAN), à Maestricht.

HOEFF (D. M. VAN DER), à Oegstgeest.

HOFFMAN (M. A. F. H), membre des Etats-généraux, à Voorburg.

HOOGEVEEN (G. W. F.), à Harlem.

HOOGEVEEN (K.), ì Utrecht.

HULSTER (A.), à Amsterdam.

HUNCK (Mad. la veuve J. F.), à Amsterdam.

JANSEN (W.), jardinier chez Mi. le Baron van Heeckeren van Brandsenburg, à Nieuweroord.

JONGELING (J. G.), jardinier de S. M. le Roi, au Loo.

JURRISSEN $(J$.$) , pépiniériste, à Naarden.$

KERSTEN (J. H.), horticulteur, à Harlem.

KIPP (P. J.), à Delft.

KLEINSTARING (H. J.), horticulteur, à Utrecht.

KLEYKANIP (P. J.), à Rotterdam.

KOOY (J.), Berckenhoven au Bildt.

KOSTER ( $\mathrm{P}$.), maison $\mathrm{P}$. Koster et fils, pépiniéristes, à Boskoop.

KRAAYENBRINK (J. H.), jardinier au château de Beerschoten (près du Bildt).

KRAMER (N.), horticulteur, à Harlem.

MLAAS GEESTERANUS, née Baronne van Dedem (Madume S. L.), à Delft.

MAURITZ (H.), à Dordrecht.

MEULEN (Madame la Veuve J. H. VAN DER), à Zeyst.

MEYER (F. W.), horticulteur, à Voorburg.

MIULDER (Dr. L.), redacteur du „Landbouw-courant”, à Deventer.

MUNSTER (D. H. VAN), à Amsterdam.

OROBIO DE CASTRO (S. H.), trésorier-directeur du Palais de l'Industrie, à Amsterdam.

OUDERMEULEN (Madame la Douairière VAN DER), à la Haye.

PREYDE (J.), de la maison Joh. Rosenkrantz et fils, horticulteurs, près d'Harlem.

RAVESTEYN (W. L.), à Amsterdam.

REELINK (J. H.), horticulteur, à Amsterdam.

REELINK Jr. (J. H.), horticulteur, à Amsterdam.

ROOZEN (A.), horticulteur, à Overveen (prés d'Harlem).

RIJN VAN ALKEMADE (J. VAN), professeur à l'ćcole militaire de médecine du Gouvernement, à Utrecht.

SARPHATI (Dr. S.), président-directeur du Palais de l'Industrie, à Amsterdam.

SASSEN (A.), à Maestricht.

SCHOLI'E (J. F.), à Amsterdam.

SIX (P. H.), à Hilverbeek.

SIXMLA VAN HEEMSTRA (Madame la Baronne A.), a V Venklooster (pri's de Leeuwarden).

SMIT (A. L.), jardinier chez Mademoiselle M. S. A. Borski, ̇̀ Overveen (près d’Harlem).

SPEET (J. D.), horticulteur, près de Leyde.

SWARTBOL (H. C.), ì Harlem. 


\section{4}

MM.

SYTHOFF (A. W.), libraire-éditeur, à Leyde.

TAK (Mr. S.), ì Soeterwoude (près de Leyde).

TEEUTVEN (Ti.), horticulteur, à Schoten (près d'Harlem).

TUBERGEN (C. G. VAN), élève-jardinier, à IIarlem.

VEERSSEN (P. VAN), ì Amsterdam.

VELSEN Jr. (F. VAN), horticultcur, ì Overveen (près d'Harlem).

VELSEN Fz. (P. VAN), horticulteur, ì Schoten (près d'Harłem).

VÖGE (H.), horticulteur, à Amsterdam.

VISSER (K. C.), jardinier, à Hoorn.

VOORHELM SCHNEEVOOGT (G. E.), professeur de médecine, à Amsterdam.

VREDENBURCH (Jhr. J. F. VAN), à Ryswyk.

WAVEREN (VAN), maison M. van Waveren et fils, horticulteurs, à Hillegom.

WAVEREN (VAN), maison L. van Waveren \& Co., horticulteurs, à Hillegom.

WERFF (G. VAN DER), jardinier de M. D. Borski, à Bloemendaal (près d'Harlem).

ZALME (W. A.), horticulteur, à la Haye.

ZLEGELER (J.), horticulteur, à Oegstgeest.

\section{France.}

BERNARD (A.), Heuriste, à Paris.

BRETON (L. L. LE), ì Paris.

BUCHETET (TH.), peintre mouleur de fruits, rue d'Enfer, No. 54, à Yaris.

MARGOTTIN (J. J.), horticulteur, à Bourg-la-Reine.

PAUWELS (TH.), à Paris.

UTZSCHNEIDER, ̀̀ Paris.

\section{Belgique.}

BEUKELAER (F. DE), horticulteur, à St. Josse-ten-Noode.

BISEAU D'HAUTEVILLE (A. DE), propriétaire, à Binché.

BODDAERT (L.), pépiniériste, à Deynze (près de Gand).

BOSSCHE (C. VAN UEN), à Gand.

DOUCHET ( $\left.\mathrm{L}_{*}\right)$, à Malines.

DOUCET (H.), délégué de la Société royale de Flore et de la Société royale d'horticulture belges, à Bruxelles.

EEKHAUTE (J. VAN), horticulteur, ì Ledeberg-lez-Gand.

HOUTIE (VAN), horticulteur, à Gand.

LEBRUN (A. F.), à Schaerbeek-lez-Bruxelles.

LEGRELLE D'HANIS (Mad.), à Berchem.

LEYS (Mademoiselle M.), tleuriste, à Gand.

MAENHOUT $\left(L_{\text {. }}\right)$, horticulteur, à Gand.

MOENS (Miademoiselle), à Anvers.

PALMANS (L.), à Lokeren.

ROELENS (D.), constructeur de chauffages de serre, à Gand.

SNET (L. DE), horticulteur, d̀ Gand.

VERVAENE (Jo), horticulteur, à Gand.

VERVAENE père (D.), horticulteur, ̀̀ Ledeberg-lez-Gand.

VERVAENE fils (J.), horticulteur, ì Gund: 


\section{MM.}

\section{Siuisse.}

FRÖBEL fils, horticulteur, à Zurich.

HEITZ (G.), jardinier-marchand, délégué de la société dlhorticulture, ì Bâle.

KRÜGER (L.), jardinier en chế du jardin botanique de Bâle et délégué de la société d'horticulture, à Bâle.

MEISSNER (1) r, C. F.), professeur de botanique, directeur du jardin botaniquue de l'université de Bâle, délégué du conseil tédéral de la Confédération suisse, à Bâle.

KAISER., à Berlin.

\section{Prusse.}

PÜTZ (W.), à Cologne.

SCHMIDT (J. C.); horticulteur, à Erfurt.

\section{Autriche.}

FENZL (Dr. E.), professeur de botanique, directeur du jardin botanique et du musće I. et R., vice-président et délégué de la société $\mathbf{I}$. et $\mathbf{R}$. d'horticulture de Vienne, délérgué de l'académie impériale des sciences de Vienne, à Vienne.

\section{Wïrtemberg.}

HOCHSTETTER (W.), jardinier en chef du jardin botanique, délégué du gouvernement, à T'übingen.

NESTEL $(H$.$) , jardinier, ì Stuttgart.$

SCHNEIDLI junior (FIRIEDRICII), jardinier, à Stuttgart.

SCHÜLE, jardinier, déléguć du gouvernement et de l'Institut de Hohenheim, à Hohenheim.

WEISS (VINCENZ), jardinier, ì Neuenburg.

WOLFF (ADOLPHE), jardinier, ’̀ Tübingen.

\section{Weimar.}

HAAGE (FELRINAND), jardinier de la Cour, à Weimar.

HARDWIG, jardivier de la Cour, ì Weirnar.

SKEELL, jardinier de la Cour, à Weimar.

WEBER, jardinier de la Cour, à Weimar.

\section{Russie.}

NORDMANN (Dr. A.), conșeiller d'état, nembre de l'Institut, professeur de botanique et directeur du musće d'histoire naturelle, ì Helsingyörs.

REGEL (Dr. E.), directeur du jardiu botınique imp., vice-président et délégué de la société russe d'horticulture, redacteur du "Garten-Flora," à St. Petersbourg.

\section{Grande-Fretagne et Irlande.}

BERKELEI (Rev. M. J), délégué du gouvernement, premier délégué de la société royale d'horticulture, à South-Kensington, ì Londres.

LEE (CH.), de lì maison John \& Charles Lee, horticulteurs, délégué du gouvernement et de lit société d'horticulture, à South-Kensington, royal Vineyard Nursery, llanmersmith, près Londres.

MOORE (TH.), directeur du jardin botanique de l'hôpital, à Chelsea, délígué du gouvernement et de la société d'horticulture, it South-Kensington.

PAUL (W.), horticulteur, Waltham Cronss, à Londres, délégué du gourernement et de la société d' horticulture, à South-Kensington. 


\section{TABLE DES MATIĖRES.}

Liste des membres de la Commission organisatrice an Congrès. Id. de la Commission directrice du Congrès, nommée par l'assemblée le 7 Avril $1865 \ldots . .$.

Bureau de la section de botanique pure. Id. le la section de botanique appliquée et d'horticulture

Pag.

1.

2.

Liste des membres du Congrìs......................................... 3-16.

Revue des questions soumises aux discnssions du Congrès ................ 17-30.

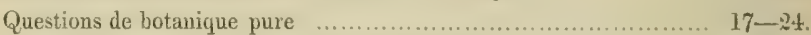

Questions de botanique appliquée et d'horticulture ............... 2t-30.

l'rojet de règlement du Congrès, adopté par les deux sections le 7 Avril 1865

31,32

\section{PREMIİRE RÉUNION GÉNÉRALE.}

SÉANCE D'OUVERTURE.

Discours de M. Oudenass, président

$33-39$.

Constitution de la Commission directrice. Proposition de M. $\nu^{3}$ ANovıu

DE Sarres. Le bureau actuel est maintenu et complété

$39-43$.

Discours de II. PIRÉ

$43-45$.

Clôture de la séance

45.

\section{SECTION DE BOTANIQUE PURE.}

\section{SÉANCE du 7 Avril 1865, à 4 heures de relevée.}

Ouverture de la séance

46.

Constitution du Bureau. Approbation du projet de règlement ...............

Clôture de la séance

46.

46.

\section{SÉANCE du 8 Aviril 1865, à 9 heures du matin.}

Lecture du procès-verbal, Proposition de li part du président relative à l'ordre des questions ì traiter. M. LeCOQ .

I. M. Incoq traite la le question proposéc par M. BELHomme

MiI. HofFuraviv

II. M. Kanstev discute lit IIe question proposée par le même $49-52$.

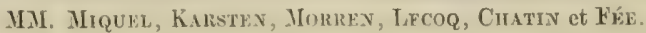


III. M. Levaine développe la IIIe question dn l'rogramme

Inta.

MII. HofEmaxi ef Miques.

$53-59$.

59.

IV. M. Cintis parle, sur la structure et les fonctions du tissu de l'anthère."

\section{Reichenbach}

Chatin

REICIENBACH

V. M. Passernis discute la IVe question du I'rogramme, le partie. MM. HOFHMAN...

('H.tix

LeCOQ

('Hitis

I'ASSERIXI

(30,61.

VI. M. Rercuenibacir entretient la section de la diffírence entre "Selenipedinin caudatum et Uropedium Lindenii."

VII. M. Casparx traite la XIe question du l'rogramme, section d'horticulture

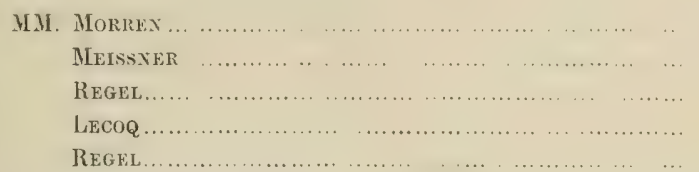

VIII. M. Oudemaxs lit une notice, sur l'origine des stomates de quelques espèces d'Aneimia."

$5 j-87$.

IX. MI. Passerini discute "sur la fonction des stomates.".

83,89 .

MII. MoRreis

X. M. Ifasskarl présente des communications, ,sur les Commelinarées.".

XI. M. le général JACoBI fait connaître le principe de sa classification des Agavées

MiI. ENTz-Ferenez, JACOBI

$108-115$.

115.

XII. MI. Corr présente ses observations „sur la culture des Algues marines."

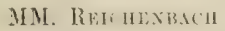

$116-129$.

Coms

129.

130.

Münt:e

130.

CoIrN

REGHI

CoItx 


\section{SÉANCE du 8 Arril 1865, à 34 heures de relevée.}

Lecture du procès-verbal

M. Wesmafic présente quelques opuscules

I. M. OU Demaxs dome lecture d'une notice, sur l'origine des spores daas certaines espices de Míncor"

II. M. Suringar présente le résumé de son travail "sur la Sarcina rentriculi"

$$
\text { M. MiñLel }
$$

III. M. vav Hulate déreloppe la question annoncée par lui ,sur la mission des jardins botaniques"

MM. FÉ

MonREx

LECOQ

Miquel

REGEL.

Iforfand Xy.

IV. M. Bommer traite de ,la coloration des plantes" $\ldots \ldots \ldots \ldots \ldots . . .154-157$.

MM. Cinatiz

\section{SEAANCE du 10 Avril 1865, ì 10 heures du matin.}

Lecture du procis-verbal.

I. M. REgri, prononce un discours, sur la valeur de l'espèce" $159-198$.

M. FÉE

II. M. DF Nordrarn discute sur les mérites du prof. Christiax STEVEN.

III. M. Coster adresse à la section une communication „sur la métamorphose des plantes"

MII. FéE

Morren

203.

IV. M. WrLlkomm prononce un discours "sur le chêne-liége de l'occident"

\section{Masters}

V. M. Müvrer communique d la section des observations, sur les maladies des plantes"

VI. M. Morren communique ses remarques, ,sur la panachure et li dıplicature"

Lecture de la proposition de la part de M. Kocr. Clôture 


\section{SECTIOR DE BOTANIQUTE APPLIQUÉE RT D'HORTICULTURE.}

SÉANCE du 7 Arril 1865, à 4 heures de relevée.

Ouverture de lis sítince

Pag.

216.

Constitution du Bureau. Rieglement de l'ordre des questions.

SÉANCE du 8 Avril 1865, à 9 heures du matin.

Lecture du procès-verbal. Questiou proposée par M. BrineL ........ 218.

I. M. vay ILuLle traite , des systèmes de taille d'arbres fruitiers" ... 218-228.

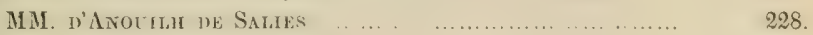

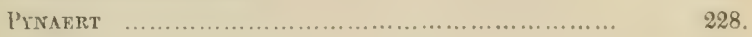

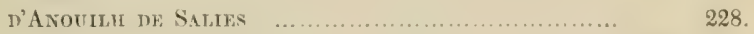

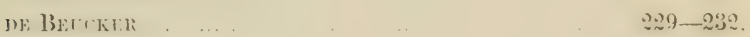

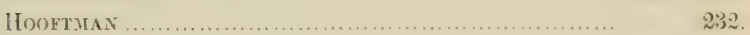

VAN HI L.LF

HooftMan ............................................... $234-236$.

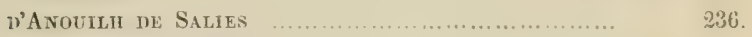

we Cannart d'Hamale ................................... $2: 36$.

OVEREYNDER ............................................. 236 .

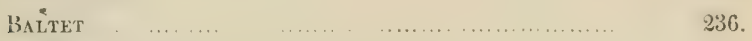

VA.T HALL . . . . . .

DE 'ZANTIS .....................................................

IE CANART WHAMALL:

II. Communication de la part de M. Axnerssox ..................... 237.

III. M. 'T'rtana traite „des Mélastomacées" …..................... 237.

IV. M. Kocri déreloppe ses idées sur "la nomenclature des plantes." 238, 239.

MM. NEDZWLSKY _.............................................

rAN HALI . .................................................. 240.

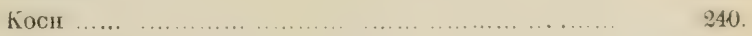

1) Beuckrir ............... . . . .

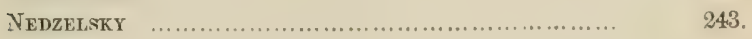

BAITET ...... .... ...................................

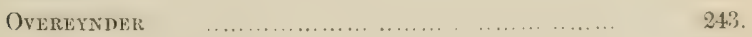

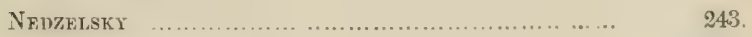

D'ANOUILH DE SALIFS .......................................

SÉANCE du 8 Avril 1865. à 2 heures de relevée.

I. M. Bartrơ traite "de la dégénírescence eher les plantes bulineness, ete."

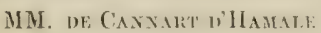


II. M. de Cannart d'Hamaiz donne lecture d'une lettre de la part de MM. HaRur frìres.

III. M. PrNafrt traite la IXe question du programme

MM. D'ANouILII DE SALIEs .............................

KocI

$249-285$.

285.

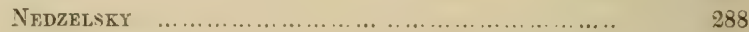

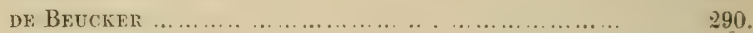

Pryatrt . . . . . . . .

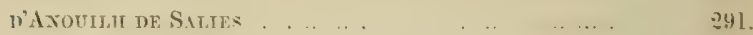

Prinamt

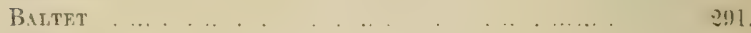

Kirн . . . . . . . . . . .

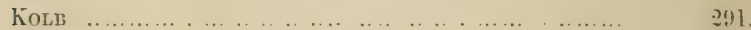

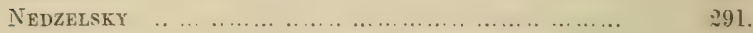

IV. MI. Stelzner dépose un travail sur l'hỵbridation des Fougères. 2?:?.

V. M. Karsten donne un aperçu de ses travanx „sur la méthode de rulture des Cinchona."

$292-305$

VI. M. Mulier dépose, une notice sur les plantes de pleine terre".

VII. M. Wesmafe dépose ses considúrations sur les hỵłrides des cultures...

VIII. M. le Président appelle l'attention des membres sur quelques 305. opuscules de M. GLOGER

\section{SÉANCE du 10 Arril 1865 , à 10 heures du matin.}

I. Continuation de la discussion sur la taille raisonnée, par M. DE BEUCKEI

MI. VAN HULIE $306-310$

II. Votice déposée de la part de M. Robert Warser $310,311$.

III. M. NeDzelsky traite Ia XXIe question du programme 312

MII. Kocil $312-329$

NEDZELSKY 329.

REGEL 330.

KOCH 330 .

NEDZELSKY

332.

DE BEUCKER

332.

332.

IV. Question soumise au congrès par M. p'A voumu dE SALIEs ... 333.

V. M. Entz-Ferenez traite "de la destination véritable des plantes dans l'ćconomie de la nature"

MM. KoLß3

346.

REgeL

ENTz-Frinlixez

VI. II. D'A roulur DE SALIEs traite la question XXXV, 1 du programme

VII. Notice de M. Bossta, lue par M. Turelens $350-357$. M. vav Hulit: 
VIII. M. Geitner dépose un règlement d'un fonds de pension pour les jardiniers.

IX. M. KoLB annonce une notice sur le système de vigne de Hoorbrink

X. M. D'Axourli be Surres développe les questions qu'il a annoncées

II. M. le Président passe en revue les questions traitées dans la section. La séance est close

\section{SECONDE RÉUNION GÉNÉRALE.}

\section{SÉANCE DE CLÔTURE.}

M. RauwenhorF, secrétaire-général, fait lecture du procès-verbal de la séance d'ouverture

Rapport de M. FÉE sur la sectiou de botanique pure

368.

Rapport de M. KocH sur la section de botanique appliquée et d'horticulture

Discours de MI. Ovdemays, président

Discours de M. Mrissine

Clûture de lì siance. $369,370$.

371.

371.

\section{SUPPLÉMENT.}

\section{MÉMOIRES PRÉSENTÉS À LA SECIION DE BO'IANIQUE PURE.}

M. A. Chatrix. Recherehes sur le développement, la structure et les fonctions des tissus de l'anthère

M. J. E. Bonuer. Des matières colorantes des feuilles $410-117$.

I. NArdy, La lune et le règne végétal $+17-419$.

M. J. MüNter. Ueber Gicht oder das sogenannte gichtig- oder radirwerden (nielle) des Weizens und anderer Grasfrüchte 


\section{MÉMOIRES PRÉSENTÉS À LA SECTION DE BOTANIQUE APPLIQUÉE ETT D'HORTICULTURE.}

M. A. Strlzner. L'hybridation des fougères Pag.

M. F. Muller. Notice sur les plantes de pleine terre.................43 13.49.

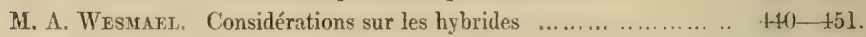

M. Rob. WARTER. On double glass houses ...................... $452-454$.

M. J. M. KraAijenbrink. Over de Oranjeboomen en Erica's ........ t55-456.

M. J. Triana. Dispositio Melastomacearum .................... $457-461$.

\section{MÉMOIRES PRÉSENTÉS AU SECRÉTARIAT.}

M. Gomppert. Du jardin botanique de Breslau et de son organisation intérieure

M. J. vow Warszewicz. Handelspflanzen, welche für Ostindische Colonisten wichtig und von grossem Werthe sein kömnen

$+62-468$.

$469-\$ 74$.

475 .

A. E. Vavin. Observations sur les Jacinthes cultivées en carafes ... Generations-Wechsel für die Gruppe der Jilze

$476-511$.

$512-515$.

$516-522$.

Table des matic̀res 


\section{NOMS DES MEMBRES}

\section{QUI ONT PRIS PAR'L AUX DISCUSSIONS.}

MIM.

Pag.

1'. LX SUILII DE SALIES.

$40,228,236,285,291,348,359$.

Bahtet (CiI.) $236,243,245,291$.

BarraL (J. A.)

de Beucker (J. J.)

$216,228,242,290,306,332$.

Bommer (F.)

154.

Bossiv

:3)

Bouchard-Husalu

+.

de Canvart d'Hamale (F.)

$42,216,218,236,237,248,249$.

Caspary (Prof. R.)

ij5.

Cinatin (Prof. A.)

$52,59,61,62,157$.

Conn (Prof, F.)

116,130 .

Coster (Dr. D. J.) 198.

DE ZaNTIS

ENTz-Ferenez (Dr. F.)

l'ÉE. (Prof. A.)

$47,49,52,130,152,158,198,202,210,368$.

Gritser (G.)

358.

vav HALL (Prot. H. C.)..

$216, \approx 37,210,244$

HASskaRl (Dr. J. K.)

Hooftman Pz. (J.)

\section{Hofrmann (Prof. H.)}

va. Hulle (H.).

$232,234$.

J ACOBI $48,6], 152$.

KARsten (Dr, H.) $146,218,232,310,358$. $108,115$.

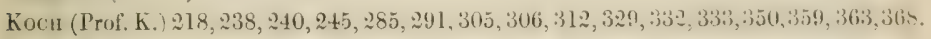
Kior.s (M.) LECnQ (Trof. H.) $291,346,358$.

LeMrirne (Prof. Ch.) $47,52,61,82,152$.

Misters 
MM.

Pag.

Nedzelsky (Dr. A.)

$217,239,213,288,291,312,330,332$.

NoRdMann (Prof. A.) 198.

Oudemars (Prof. C. A. J. A.) $33,39,42,45,85,139,368,369,371$.

Overzindek (C. G.) $236,2+13$.

Passerini (Prof. J.) $60,62,88$.

Puné (Prof. L.) 43.

Pynaert (Prof. E.) $228,249,291$.

RAUWENHOFF (Dr. N. W. P.)

$158,216,368$.

Regel (Dr. E.) $81,83,130,152,159,330,346$.

Reichenbach Fils (Prof. H. J.) $59,62,129,130$.

Scunizlein (Prof.) 139.

Stelzner (A.) 292.

Suringar (Prof. W. F. R.) $45,13.40$.

Tulelens (A.) 350 .

Triava (J.) 237.

WARNER (R.) 312.

WFamaEL (A.) $89,138,305$.

Willkомм (Prof. M.) 203. 


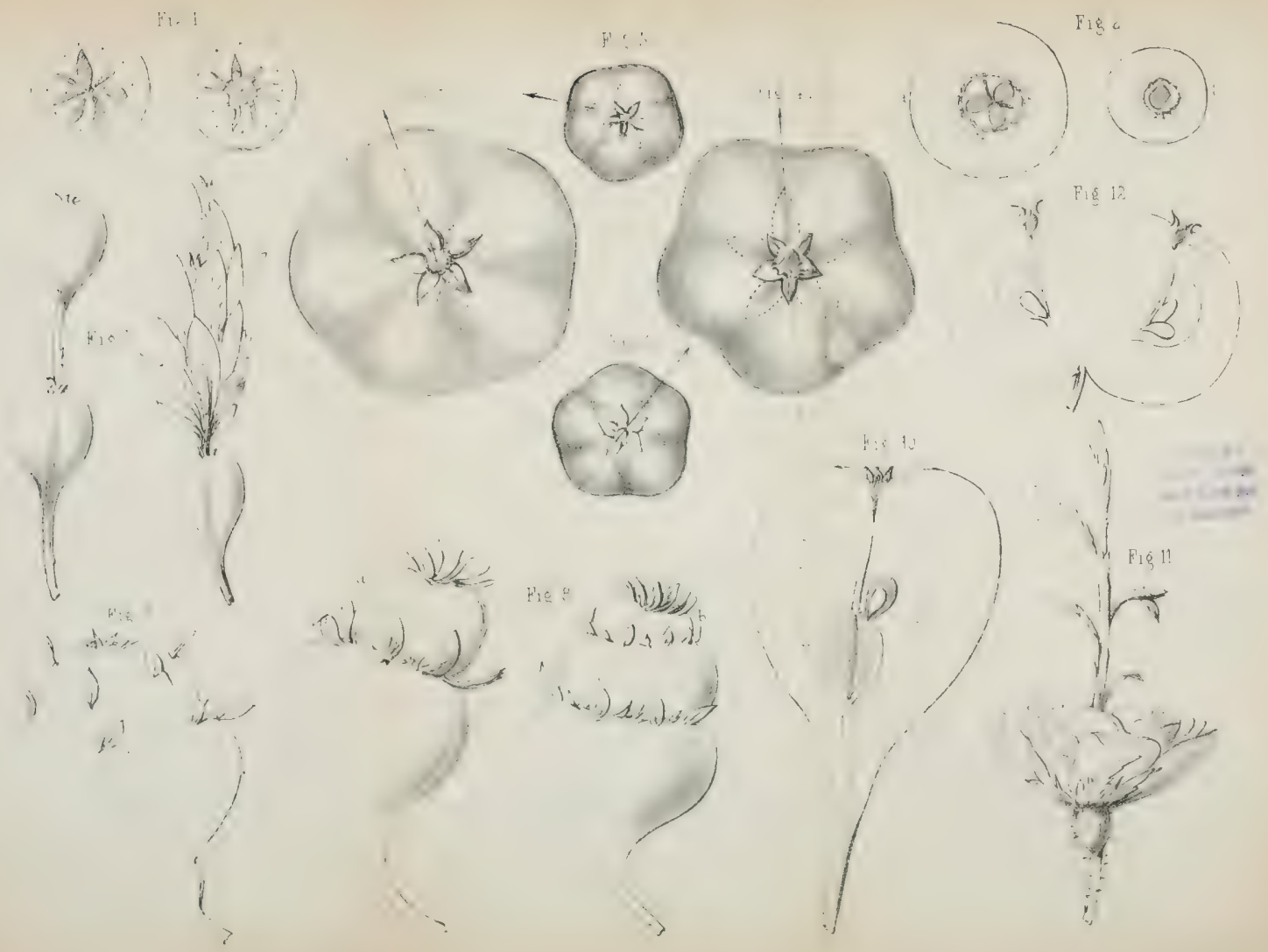





QK1.15 1865
International Botan/Bulletin du Congres

|| ||| || ||| || || || || || || |||||||||||||||||||||||||

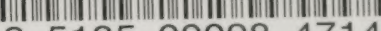

35185000984714 
